


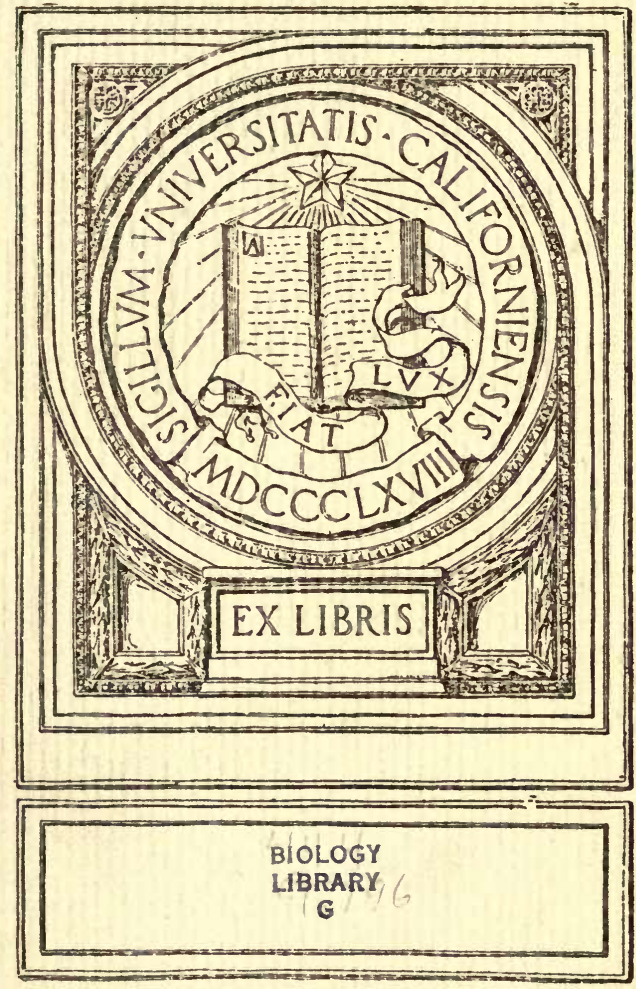


Wit $=$

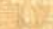

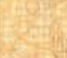

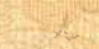

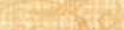

7.

Sintive

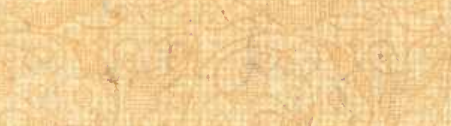

-

$\rightarrow$

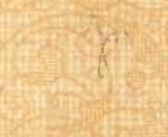

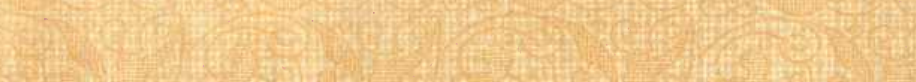

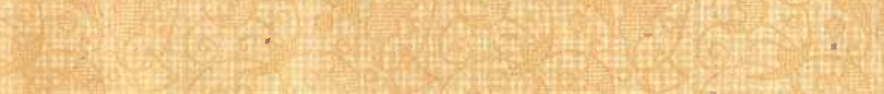

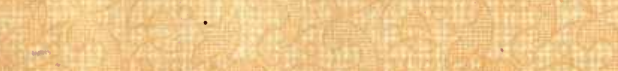

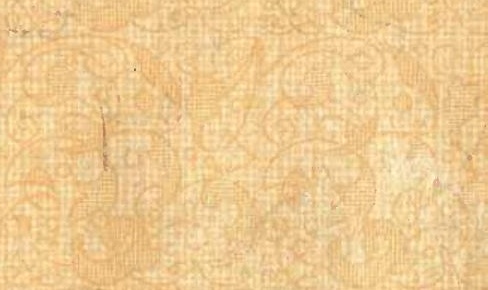
(1)

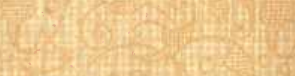
(2) 



\section{AN INTRODUCTION}

TO

\section{VEGETABLE}

\section{PHYSIOLOGY,}

BY

J. REYNOLDS GREEN, Sc.D., F.R.S., F.L.S.

TRINITY COLLEGE, CAMBRIDGE

PROFESSOR OF BOTANY TO THE PHARMACEUTICAL SOCIETY OF GREAT BRITAIN FORMERLY SENIOR DEMONSTRATOR IN PHYSIOLOGY IN THE UNIVERSITY OF CAMBRIDGE

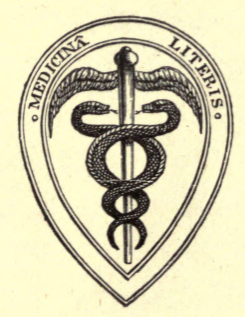

LONDON

J. \& A. C H U R C H I L L

7 GREAT MARLBOROUGH STREET

1900 
QK7II

G 7

BIOLOGY LIBRARY

\section{GENERAL}




\section{PREFACE}

Although during recent years considerable additions have been made to our elementary botanical textbooks, not one has appeared which deals solely, or at any length, with the subject of vegetable physiology. This has been either presented to the reader as a particular section in a comprehensive work, or treated of incidentally in connection with anatomical detail. This is the more strange, as an adequate and intelligent appreciation of the forms and structure of vegetable organisms can only be gained by a consideration of the work they have to carry out. It must be evident to the student of Nature that the peculiarities of external and internal form, of which any particular plant has become possessed, have arisen necessarily in connection with the need of mechanisms to do certain work, to overcome particular disadvantages, and generally to bring the organism into a satisfactory relationship with the surroundings among which it finds itself.

I have been led by these considerations to endeavour to fill this gap by writing an introduction to the subject, which, while putting physiology into its proper prominence among the branches of botanical study, shall serve to pave the way of the student and of the general reader to the more complete discussion of the subject which may be met with in the advanced textbooks of Sachs, Vines, and Pfeffer. 
With this view I have endeavoured to present the plant as a living organism, endowed with particular properties and powers, realising cerțain needs, and meeting definite dangers. I have attempted to show it to be properly equipped to encounter such adverse conditions, and to avail itself of all the advantages presented to it by its environment.

I have also set before myself another purpose, which, however, is naturally subordinate to the one just mentioned. When we consider the origin of the different organisms which we find around us, we are led irresistibly to the conclusion that the classification of living beings into animals and plants has been too strongly insisted upon in the past, and that while much has been made of their differences, their points of resemblance have been minimised. The fact that organisms exist, which it is difficult or impossible to refer with certainty to either kingdom, points to a fundamental unity of living substance. Protoplasm in short is the same material, whether we call it animal or vegetable. This being the case, its conditions of life and its immediate necessities must be practically the same, whatever its degree of differentiation in either direction. I have tried to bring out this identity of living substance throughout the book, and to indicate that apparent differences of behaviour and structural arrangement are to be traced rather to differences of environment and habit of life, than to those of constitution. The correspondence of the processes of respiration in animals and plants has long been recognised; many points of similarity in those of nutrition have been observed. The idea is, however, still prevalent that plants live upon inorganic materials absorbed from the air and from the soil. This seems to indicate a fundamental difference between the modes of nutrition of animal and vegetable protoplasm. I have 
endeavoured to show that this view is erroneous and that both are nourished similarly.

I have also tried to show that the sensitiveness of the plant and the animal is alike in properties, though differences are apparent in the direction of its differentiation.

I have avoided as far as possible the discussion of controverted points, feeling that this would be out of place in a work intended to serve as an introduction to the subject. Such matters are more properly treated of in the more comprehensive works to which I have already alluded.

J. Reynolds Green.

Cambridge: June 1900. 



\title{
CONTENTS
}

\author{
CHAPTER I \\ THE GENERAL STRUCTURE OF PLANTS
}

Unicellular plants ; zoogonidia, yeasts, bacteria ; multicellular plants; the protoplast, its structure and arrangements; characters of protoplasm; nuclei and nucleoli; association of protoplasts in colonies ; slime-fungi; cœnocytes; arrangements in multicellular plants-Needs of protoplasm; its relation to water; formation of vacuoles; relation of water to the plant in general; the aeration of protoplasm-Connection of protoplasts with one another in the body of the plant

\section{CHAPTER II}

\section{THE DIFFERENTIATION OF THE PLANT-BODY}

Division of labour the clue to differentiation of structure-Formation of protective tissues; epidermis, cuticle, periderm, bark-System of conducting tissues; vascular bundles and their distributionStrengthening tissues; collenchyma and sclerenchyma; the different arrangements of them which are met with-The stereome of the plant-The metabolic tissues-The arrangements for the aeration of the interior; stomata, lenticels . . . . . 17-35

\section{CHAPTER III}

\section{THE SKELETON OF THE PLANT}

Necessity of a skeleton to support the protoplasts; varieties of the skeleton-Development of the skeleton as the plant grows-Characters of the cell-wall ; cellulose, its properties and reactions ; pectose and related substances-Arrangement of the solid matter and the water of the cell-wall; hypotheses of Naegeli, Strasburger, and Wiesner-Differentiation of the substance of thickened cell-walls; 
stratification, middle lamella-Lignin and its reactions-Cutin-

Impregnation of cell-walls with various matters-MucilageDifferences between temporary and permanent portions of the skeleton

\section{CHAPTER IV}

\section{THE RELATION OF WATER TO THE PROTOPLASM OF THE CELL}

Dependence of the protoplasts on water ; function of the vacuoleRenewal of the water of the vacuole-Osmosis-Formation of the vacuole as the protoplast develops-Regulation of osmosis by the cell-protoplasm; the plasmatic membranes-Movements of water from cell to cell-Evaporation into the intercellular spacesTurgescence and its dependence on the protoplasm-Storage of water

\section{CHAPTER V}

\section{THE TRANSPORT OF WATER IN THE PLANT}

Varied needs of different plants in this respect-Transport in a terrestrial plant_The ascending sap-Condition of water in the soil ; absorption of water a function of the root-hairs; mechanics of the root-hair-Path of the ascending stream; forces causing the movement-Evaporation of water from the interior-Influence of the stream of water upon the development of the plant . . .

\section{CHAPTER VI}

\section{THE TRANSPIRATION CURRENT. ROOT PRESSURE.}

\section{TRANSPIRATION}

The ascending sap sometimes called the transpiration current-Its path; methods of demonstration-Rate of the transpiration current-Causes of the upward flow ; root pressure; transpiration; capillarity; pumping action of living cells; osmotic action of the parenchyma of the leaves-Root pressure; its nature and mode of action; bleeding of cutstems ; of entire plants ; measurement of root pressure ; conditions of the activity of roots ; diurnal variations of root pressure-Transpiration ; methods of demonstration; amount of water given off; negative pressure in the wood vessels ; character of the evaporation of transpiration ; regulation by stomata, their mode of action; variations in numbers of stomata; conditions affecting transpiration; light, temperature, 
moisture of air, rest-The Potometer-Suction of transpiration-

Osmotic action of the parenchyma of the leaves and its effect-

Regulation of all these forces by the protoplasm . . . 78-102

\section{CHAPTER VII}

\section{THE AERATION OF PLANTS}

Necessity of admitting oxygen to the protoplasts - The intercellular space system; its origin and development; condition in terrestrial plants ; relative extent in roots, stems, leaves-Air reservoirs in aquatic plants; in Equisetum, grasses, rushes, \&c.; mode of formation of the reservoirs-External orifices of the intercellular space system; stomata and lenticels-Relative dimensions of cellular tissue and intercellular spaces-Movements of air in intercellular space system-Composition of the air . . . 103-117.

\section{CHAPTER VIII}

\section{RESPIRATION}

Dependence of the plant upon oxygen; absorption of oxygen and exhalation of carbon dioxide; apparatus to demonstrate these processes-Loss of weight during respiration-Variations in the respiratory activity--Relation between the absorption of oxygen and the exhalation of carbon dioxide; the respiratory quotientExhalation of water during respiration-Respiration a function of protoplasm-Conditions affecting respiration; temperature, light, differences of gaseous pressure and of nutritive materials . 118-131

\section{CHAPTER IX}

\section{THE FOOD OF PLANTS. INTRODUCTORY}

True nature of the food of plants-Materials absorbed by plants, and their relationship to actual food-Differences between food and food materials-Construction of food from the latter-Assimilation of food-Intricacy of the metabolic processes of plants . . 132-139

\section{CHAPTER $\mathrm{X}$}

ABSORPTION OF FOOD MATERIALS BY A GREEN PLANT

Examination of substances absorbed from the soil; water-culture; destructive analysis-Classification of materials absorbed-The 
ash of plants-Conditions of absorption of substances in the soilPAGE Absorption of nitrogen by leguminous plants-Insectivorous plants and their behaviour-Absorption of metallic compounds ; siliconAbsorption of carbon dioxide from the air ; its mechanism . 140-154

\section{CHAPTER XI}

\section{THE CHLOROPHYLL APPARATUS}

Formation of organic substances from the inorganic materials absorbed-Chlorophyll-Structure of a chloroplast--Properties of chlorophyll ; its absorption spectrum-Xanthophyll-Erythrophyll -Composition of chlorophyll-Distribution of the chloroplasts-Relationship between the plastid and the colouring matter-Leucoplasts - Conditions of formation of chlorophyll ; light, temperature, iron -Formation of carbohydrates by chloroplasts ; conditions of their activity-Theories of photosynthesis-Relation of starch to the process-Rays of light made use of in photosynthesis; researches of Engelmann, of Tmiriazeff-Inhibition of the chlorophyll appara-

tus-Formation of organic substance in its absence

$155-172$

\section{CHAPTER XII}

\section{THE CONSTRUCTION OF PROTEIDS}

Complexity of the composition of proteid; its percentage composition -Classification of proteids ; albumins, globulins, albuminates, proteoses, peptones, coagulated proteids, proteids soluble in alcoholSynthesis of proteids in plants; various hypotheses-Locality of proteid construction in the plant

$173-183$

\section{CHAPTER XIII}

\section{THE CONSTITUENTS OF THE ASH OF PLANTS}

Nature and composition of the ash-Water-culture and the limitations of its usefulness in the study of the ash-Classification of the constituents of the ash-The selective power of plants-Sulphur and phosphorus-Potassium, magnesium, calcium, iron-Sodium, silicon, chlorine, bromine, iodine, manganese-Accidental constituents of the ash-Relation of nitrogen and potassium to herbage plants 


\section{CHAPTER XIV}

\section{OTHER METHODS OF OBTAINING FOOD}

Partial or entire absence of the constructive power-Nutrition of saprophytes - Insectivorous plants - Utricularia - The pitcherplants - Lathræa-Drosophyllum - Pinguicula-Dionæa-Drosera -Digestion of substances by Fungi-Commensalism-SymbiosisMycorhiza-Root parasites-Parasitism among green plants and Fungi

\section{CHAPTER $\dot{X} V$}

TRANSLOCATION OF NUTRITIVE MATERIALS

Conditions of the constructive processes; surplus production and storage-Necessity of circulation of food material in consequence of localisation of construction, and intermittence of consumptionMode of transport of substances in the plant ; osmosis and diffusion; temporary storage-Translocatory and storage forms of food-The so-called descending sap-The pathway of translocation 221-234

\section{CHAPTER XVI}

\section{THE STORAGE OF RESERVE MATERIALS}

Connection between transport and storage; forms in which food is stored-Reservoirs of storage ; stems, roots, floral organs-Storage of carbohydrates; starch grains and their formation by chloroplasts and leucoplasts, by the cytoplasm; glycogen; inulin; sugars; cellulose and similar compounds-Storage of proteids; aleurone grains, their composition and mode of formation; proteid crystals ; antecedents of gluten-Storage of asparagin ; glucosides ; fats and oils-Mode of formation of the last-named group . 235-255

\section{CHAPTER XVII}

\section{DIGESTION OF RESERVE MATERIALS}

Nature of digestion-Its localisation in plants-Agents of digestionSecretion of enzymes-Conditions of their action-ZymogensDifferentiation of glandular structures-Classification of enzymes - Diastase and its action on starch-Mulase-Invertase-Glucase - Cytase and cell-walls-Pectase-Proteolytic enzymes-RennetEnzymes which decompose glucosides-Lipase and its action on fats -Zymase and the production of alcohol_-Oxidases-Fermentative activity of protoplasm-Assimilation . . . . . 256-271 


\section{CHAPTER XVIII}

\section{METABOLISM}

Constructive and destructive processes; anabolism and katabolismConstructive processes depending on katabolism-Secretion-Byeproducts-Secretion of enzymes-Formation of cell-walls, of starch grains, of aleurone grains, of fat, of chlorophyll, of anthocyanFormation of resin, of alkaloids, of acids-Decomposition-products of cellulose ; colouring matters; nectar ; etherial oils . 272-287

\section{CHAPTER XIX}

\section{.THE ENERGY OF THE PLANT}

Preliminary considerations-The expenditure of energy in evaporation, in constructive processes, in movements, in radiation, in lightSource of the energy of plants the radiant energy of the sun; its absorption by chlorophyll; absorption of heat rays-Fixation of energy-Kinetic and potential energy-Distribution and liberation of energy-Relation of respiratory processes to local utilisation of potential energy-Oxidative actions other than respiration-Intramolecular or anaërobic respiration-Fermentation-Anaërobic plants

\section{CHAPTER XX}

\section{GROWTH}

Relation of growth to constructive metabolism-Definition of growth -Distribution of growth-Conditions necessary for growth ; plastic materials, turgescence, temperature, oxygen-The grand period of growth-Growth of a cell and of a multicellular organ-The region of growth in the latter-Daily period of growth in length-The Auxanometer-Variations in growth; hyponasty and epinasty; nutation and circumnutation-Tensions accompanying growthRectipetality

\section{CHAPTER XXI}

\section{TEMPERATURE AND ITS CONDITIONS}

Range of temperature through which the vital processes proceed; photosynthesis, germination_Causes and effects of fluctuations of 
temperature-Influence of the light rays on temperature; importance of anthocyan-Absorption of heat by conduction-Dissipation of heat in evaporation of water-Radiation-Nyctitropic movements-Loss and gain of heat by conduction-Regulation of heat-Power of resistance to extremes of temperature . . 320-329

\section{CHAPTER XXII}

\section{INFLUENCE OF THE ENVIRONMENT ON PLANTS}

Characters of aquatic plants; influence of a watery environment on structure-Xerophytes and their peculiarities-Alpine plantsEpiphytes-Parasites-Insectivorous plants . . . . 330-344

\section{CHAPTER XXIII}

\section{THE PROPERTIES OF VEGETABLE PROTOPLASM}

Adaptability of plants to their surroundings-Contractility-Ciliary and amœboid movement-Locomotion-Movements of rotation and circulation-Turgor and its maintenance-Mobile condition of protoplasm-Rhythm and its manifestations-Irritability and its conditions-Tone-Phototonus-Thermotonus-Tonic influence of light-Etiolation-Influence of too brilliant illumination; paraheliotropism, apostrophe and epistrophe-Photoepinasty-Regulating action of light on growth-Conditions of health-Acclimatisation

\section{CHAPTER XXIV}

\section{STIMULATION AND ITS RESULTS}

Response of an organism to changes in its surroundings-Nature of stimulation-Purposeful character of the response-Stinulation of light-Nyctitropic movements, their conditions and purposeIIechanism of the movements-Effect of incidence of lateral light - Heliotropism-Stimulus of gravitation; geotropism - The Klinostat-Knight's wheel-Stimulus of contact-Behaviour of various organs in relation to this form of stimulation-The root-Twining stems and tendrils-Hydrotropism-Chemical stimuli-Chemotaxis

-Induced rhythm . . . . . . . . . . . 


\section{CHAPTER XXV}

\section{THE NERVOUS MECHANISM OF PLANTS}

The purposeful responses of plants to stimulation ; relation of stimulus to effect-Nature of nervous mechanisms-Sense organs and their differentiation-Motor mechanisms of plants-Contraction-Regulation of supply of water to the cell-Glandular organs-Conduction of impulses; continuity of protoplasm-Co-ordination of impulses-Latent period of stimulation-After-effects-FatigueAnæsthetics-Comparison of nervous mechanisms of plants and animals

\section{CHAPTER XXVI}

\section{REPRODUCTION}

Distinction between the individual protoplast and the colony or plant - Process of multiplication of protoplasts ; gemmation, karyokinesis, formation of cell-walls; free-cell formation-Vegetative propagation-Formation of asexual reproductive cells, spores or gonidia; zoocœenocytes-Development of sexual cells or gametes : planogametes and conjugation; male and female cells; antherozoids and oospheres-Gametangia and their varieties-Fertilisation -Alternation of generations-Gametophytes and sporophytesHeterospory and its consequences-The seed and its formation 411-436

\section{CHAPTER XXVII \\ REPRODUCTION (continued)}

Pollination and its mechanisms-Advantages of cross-pollinationDichogamy, protandry, protogyny-Diclinism-Heterostylism or dimorphism-Prepotency-Self-sterility-Self-pollination ; cleistogamy-Mechanism of fertilisation; the growth of the pollen-tube - Hybridisation-Results of fertilisation; formation and ripening of fruits and seeds-Germination of the seed-Apospory; apogamy; parthenogenesis 


\section{LIS'T OF ILLUSTRATIONS}

FIG.

1. Zoospore of Ulothrix . . . . . . . . . . . 1

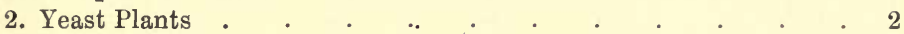

3. Bacteria . . . . . . . . . . . . 3

4. Plasmodium of a Myxomycete . . . . . . . . . 4

5. Vegetable Cells (Young) . . . . . . . . . 5

6. Vegetable Cells (Adult) . . . . . . . . . 5

7. Cells exhibiting Rotation, from Elodea $\quad$. $\quad$. $\quad$. $\quad$. $\quad$. . 7

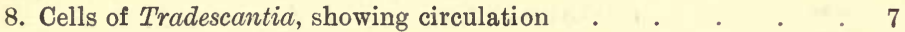

9. Structure of the Nucleus . . . . . . . . . . . 7

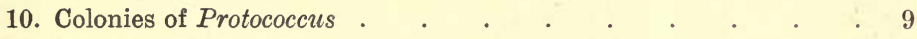

11. Volvox Globator . . . . . . . . . . . . 10

12. Cœnocytic Suspensor of Orobus . . . . . . . . . 11

13. Filaments of Nostoc . . . . . . . . . . . . 12

14. Pediastrum . . . . . . . . . . . . 12

15. Vegetable Cells (Young) . . . . . . . . . . 12

16. Vegetable Cells (Adult) . . . . . . . . . . $\quad$. 13

17. Continuity of Protoplasm in Seed $\quad . \quad$. $\quad . \quad$. $\quad . \quad$. 16

18. Continuity of Protoplasm in Seaweed . . . . . . . 16

19. Thallus of Pelvetia . . . . . . . . . . . . . 18

20. Stem of Sphagnum . . . . . . . . . . . . . . $\quad$. 19

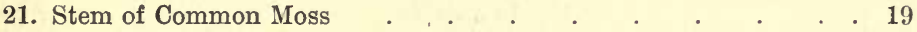

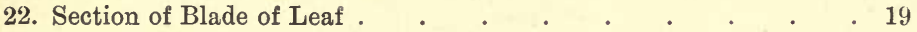

23. Cork Cells . . . . . . . . . . . 20

24. Bark of Oak . . $\quad$ : . . . . . . . . 20

25. Collenchyma . . . . . . . . . . . 21

26. Exodermis of Root . . . . . . . . . . 21

27. Diagram of Course of Vascular Bundles in a Dicotyledonous plant. 23

28. Venation of Leaf . . . . . . . . . . . 23

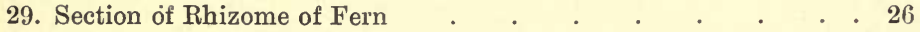

30. Section of Leaf of Pinus . . . . . . . . . . $\quad$. 27

31. Vascular Bundle of Monocotyledon . $\quad$. $\quad$. $\quad$. $\quad 28$

32. Different Arrangements of Stereome in Herbaceous Plants . $\quad$. 29

33. Chloroplasts in Cell . . . . . . . . . 30

34. Section of Stem of Potamogeton . . . . . . . 31

35. Cortex of Root . . . . . . . . . . 32

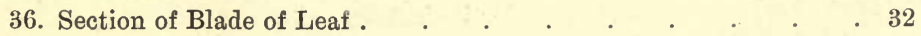

37. Stomata on Lower Surface of Leaf . . . . . . . . 33

38. Section of Epidermis of Leaf . . . . . . . . . 33 
FIG.

39. Section of a Lenticel .

40. Section of Dicotyledonous Stems of two ages . _ . . . . . 38

41. Embryo of Orobus . . . . . . . . . . . . . 39

42. Stratification in Cell-walls . . . . . . . . 44

43. Longitudinal Section of Vascular Bundle of Sunflower Stem . . 45

44. Wood-cells, showing Middle Lamella . . . . . . . 45

45. Section of Epidermis of Leaf . . . . . . . . . 48

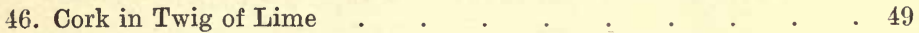

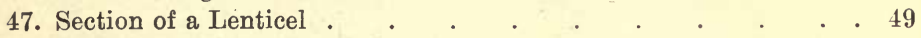

48. Crystals in Wall of Cell of Bast . . . . . . . . . 50

49. Cystolith of Ficus . . . . . . . . . . . 50

50. Apparatus to show the process of Osmosis . . . . . . 55

51. Young Vegetable Cells . . . . . . . . . . . . 56

52. Adult Vegetable Cells . . . . . . . . . . 57

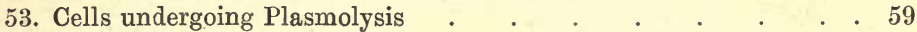

54. Rootlets with Root-hairs . . . . . . . . 68

55. Root-hair in contact with Soil . $\quad . \quad$. $\quad . \quad$. $\quad . \quad$. 69

56. Section of Young Root . . . . . . . . . 70

57. Diagram of Course of Vascular Bundles in a Dicotyledonous Plant 71

58. Veins of a Leaf . . . . . . . . . . . . . 72

59. Ending of a Vascular Bundle in a Leaf _ . . . . . . 73

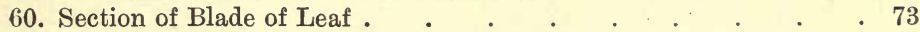

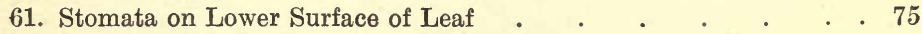

62. Apparatus for the Estimation of Root-pressure $\quad . \quad$. . . 84

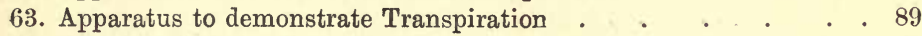

64. Section of Blade of Leaf . . . . . . . . . . $\quad .90$

65. Apparatus to show Dependence of Withering on Loss of Water . 91

66. Stomata on Lower Surface of Leaf . . . . . . . . . 93

67. Section of a Stoma $\quad$. . . . . . . . . . . . . . . 93

68. Darwin's Potometer . . . . . . . . . 98

69. Apparatus to show the Suction of Transpiration $\quad$. $\quad$. $\quad$. $\quad .99$

70. Ending of a Vascular Bundle in a Leaf . . . . . . . 101

71. Formation of Intercellular Spaces . . . . . . . . 105

72. Intercellular Spaces in Root . . . . . . . . . . 105

73. Intercellular Spaces in Leaf . . . . . . . . . . 106

74. Section of Leaf of Isoëtes . . . . . . . . . . 106

75. Section of Rhizome of Marsilea . . . . . . . . 107

76. Section of Stem of Potamogeton . . . . . . . . . 108

77. Section of Stem of Equisetum . . . . . . . . . . . 109

78. Section of Stem of Juncus . . . . . . . . . 110

79. Section of a Lenticel . . . . . . . . . . . . 111

80. Apparatus to show Continuity of Intercellular Spaces in a Leaf . 111

81. Section of Leaf of Heath . . . . . . . . . . . . 112

82. Apparatus to show the Absorption of Oxygen by a Green Plant $\quad 119$

83. Apparatus to show Exhalation of Carbon Dioxide by Germinating Seeds

84. Root of a Leguminous Plant with its Tubercles . . . . . 148

85. Section of Blade of Leaf . . . . . . . . . 153

86. Absorption Spectra of Chlorophyll and Xanthophyll . . . 157 
FIG.

87. Chloroplasts in Cell

88. Section of Leaf of Beta.

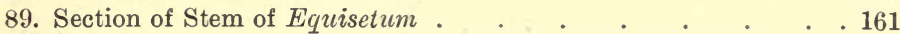

90. Apparatus to show the Evolution of Oxygen by a Green Plant . 165

91. Plants of Buckwheat cultivated in various nutritive solutions . . 186

92. Aleurone Grains in Cell of Ricinus . . . . . . . 190

93. Bladderworts (Utricularia) . . . . . . . . . 200

94. Traps of Utricularia . . . . . . . . . . . 201

95. Pitcher of Sarracenia . . . . . . . . . . . 202

96. Pitcher of Nepenthes . . . . . . . . . . . . . 203

97. Shoot of Lathraa . . . . . . . . . . . 204

98. Prison-chamber in Leaf of Lathrcea . . . . . . . 205

99. Leaf of Drosera $\quad . \quad$. . . . . . . . . . . 207

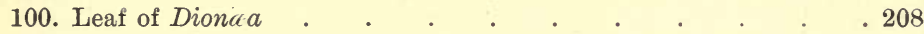

101. Section of Lichen . . . . . . . . . . . . 211

102. Mycorhiza on Beech Root . . . . . . . . 213

103. Plant of Thesium . . . . . . . . . . . . 215

104. Sucker of Thesium . . . . . . . . . . . . . . . 216

105. Plant infested with Dodder . . . . . . . . . . 217

106. Haustoria of Dodder . . . . . . . . . . 218

107. Haustoria of Phytophthora . . . . . . . . . 220

108. Starch Grains in Chloroplast. . . . . . . . . . 228

109. Section of Stem of Ricinus _ . . . . . . . . . . . . . . 239

110. Section of Stem of Tilia three years old . . . . . . 240

111. Starch Grains in Chloroplast . . . . . . . . . . . . . 242

112. Starch Grains in Cell of Potato . . . . . . . . . 243

113. Starch Grain of Potato . . . . . . . . . 243

114. Compound and Semi-compound Starch Grains . . . . . 244

115. Laticiferous Cell of Euphorbia . . . . . . . . . . . . . 245

116. Leucoplasts of Phajus . . . . . . . . . . . . . . . . . . . . . . .

117. Inulin Sphæro-crystals . . . . . . . . . 247

118. Aleurone Layer of Barley . . . . . . . . . . . . . 248

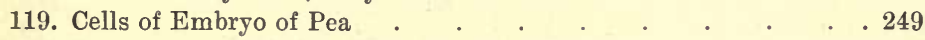

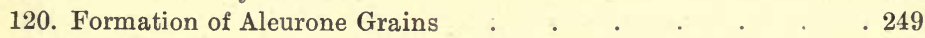

121. Aleurone Grains of Lupin . . . . . . . . . . . . 250

122. Aleurone Grains of Ricinus . . . . . . . . . . . . 250

123. Section of Oat Grain . . . . . . . . . . . . . . . 260

124. Epithelium of Scutellum . . . . . . . . . . . 260

125. Aleurone Layer of Barley . . . . . . . . . . . . 261

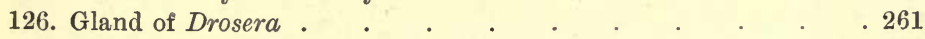

127. Corrosion of Starch Grains by Diastase . . . . . . . . 263

128. Glandular Hairs of Primula . . . . . . . . . . 283

129. Glandular Hairs of Hop . . . . . . . . . . . . . . 283

130. Oil Reservoirs of Hypericum . . . . . . . . . . . . . 286

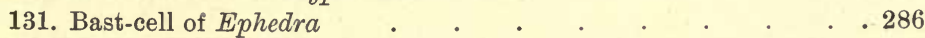

132. Crystals of Oxalate of Calcium in Cells . . . . . . . 287

133. Cystolith of Ficus . . . . . . . . . . . . . 287

134. Absorption Spectra of Chlorophyll and Xanthophyll . . . 293

135. Longitudinal Section of Growing Point of Root . . . . . 306 
FIG.

136. Section of Blade of Leaf

137. Section of Stem of Rush

. 307

138. Adult Vegetable Cells

308

139. Region of Growth in Root of Bean

310

31

140. Pfeffer's Auxanometer

141. Air Passages in Potamogeton

331

142. Leaf of Isoëtes

332

143. Petiole of Water-lily

333

335

144. Rhizome of Marsilea

337

146. Leaf of Heath

339

342

147. Suckers of Thesium

343

149. Zoospore of Ulothrix

346

150. Plasmodium of a Myxomycete

347

151. Cells from Leaf of Elodea

152. Cells from Hair of Tradescantia

350

153. Leaf of Telegraph Plant

355

154. Pulvinus of Mimosa .

356

. 373

155. Desmodium gyrans, day and night position

157. Pulvinus of Mimosa

158. Đarwin's Klinostat

159. Section of Sucker of Thesium . . . . . . . . . . . . . . . . . . . . . .

160. Haustoria of Cuscuta

161. Leaf of Dioncea

162. The same.

163. Continuity of Protoplasm through the Cell-wall

164. Yeast Plants

165. Stages in Karyokinetic Division of the Nucleus

166. Zoospore of Ulothrix

167. Gonidangia of Achlya

169. Part of Hymenial Layer of Peziza .

171. Filament of Ulothrix with Gametes escaping 


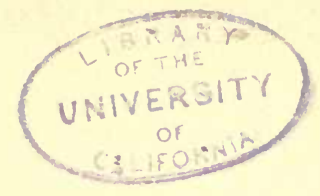

\title{
VEGETABLE PHYSIOLOGY
}

\author{
CHAPTER I \\ THE GENERAL STRUCTURE OF PLANTS
}

Examination of the body of every living organism shows us that it is composed of different materials, which exhibit a great deal of variety in the ways in which they are arranged. These different materials fall very naturally into two classes, which include respectively the living substance itself, and various constituents of the body which have been constructed by it. The relative proportions in which these two classes of materials exist vary very greatly in different organisms; in some of the simplest forms indeed we can discern nothing structural except the living substance itself. In others the materials constructed by the latter are much the greatest in amount.

When we study the life history of the simplest or the most complex plant with which we can become acquainted, we find that at some time or other in its existence it is found in the form of a minute portion of jelly-like material which is endowed with life. Sometimes this piece of living substance is motile, and can swim freely about in water by means of certain thread-like appendages which it possesses (fig. 1). Such structures occur almost exclu-

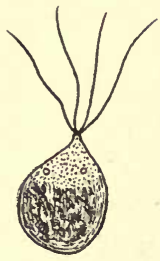

Fig. 1.-Zoospore of Ulothrix. $\times 500$. sively among the lowest forms of plants, particularly the seaweeds. They are known as zoospores, or zoogonidia, 
and are produced in large numbers. In other cases the little mass of living substance is not capable of locomotion, but may be found floating about in water, or enclosed in particular cavities in its parent plant.

The jelly-like substance of which these bodies are composed is living and capable of carrying out all the functions necessary for its life, growth, and multiplication. It is called protoplasm, and each portion of protoplasm which is thus capable of independent existence is known as a vegetable cell, or protoplast.

These free-swimming organisms are not protected by any coating, but every part of their surface is in complete contact with the water in which they live. This condition

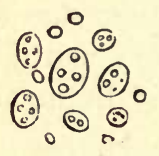

$a$

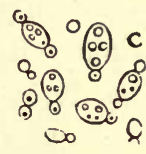

$b$

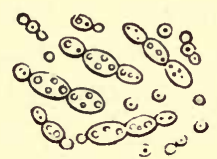

$c$

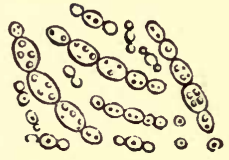

$d$

Fig. 2.-Saccharomyces Cerevisie. or Yeast-Plant, as developed DURing the process of Fermentation. $\times 300$.

$a, b, c, d$, successive stages of cell-multiplication.

is, however, exceptional. Usually the protoplast is encased in a colourless homogeneous membrane of extreme tenuity which is known as its cell-wall. Examples of unicellular organisms of this kind are found in great numbers among the fungi, the Yeasts (fig. 2) and the Bacteria (fig. 3) being exceptionally numerous. Such plants may be motile or nonmotile, a few of the bacteria being furnished with threadlike appendages, known as cilia or flagella, which are similar in most respects to those of the zoospores already mentioned. These plants show a little more differentiation than the others, the protoplasm being clothed by a kind of exoskeleton, the cell-wall, which is at once supporting and protective.

More complex organisms consist of two or more protoplasts united together in various ways. The number of 
these masses of protoplasm, or cells, may be as small as two, or may be enormous, as is the case in such plants as the gigantic seaweeds of the tropics or the tall terrestrial trees which abound all over the surface of the globe.

Whether the plant is simple or complex, we find the same fundamental arrangement of its parts: there is a certain number of protoplasts, in close relationship with each other, supported upon a framework or skeleton which shows a wonderful variety of arrangement, its details depending on the manner of life of the whole organism of which it forms so large a part. In such an organism each

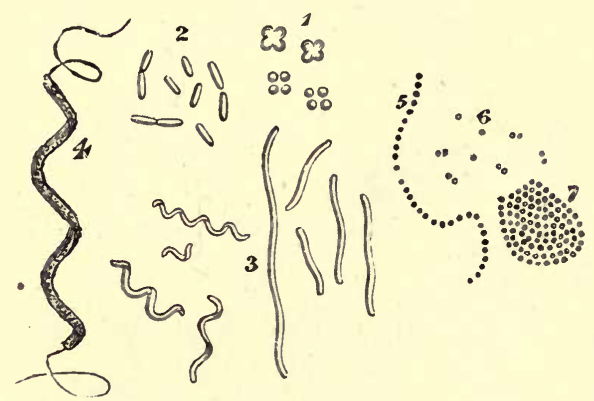

Fig. 3.-Figures of Different Bacteria. (After Cohn and Sachs. Very highly magnified.)

1, Sarcina ; 2, Bacillus; 3 , Spirillum ; 4, Spirillum with flagellæ; $5,6,7$, Micrococcus. (Single, in strings, and in groups.)

protoplast is usually found occupying a particular cavity which is formed by its cell-walls, and communicating with its neighbours on all sides by delicate prolongations of living substance which extend through the walls of contiguous chambers. Each chamber is often called a cell.

In dealing with the physiology of the plant, it is the living substance which should first engage our attention, though the arrangements of the supporting structures or skeleton exhibit the greatest variety. We have seen that in the simplest forms of plants the living substance may exist without any cell-membrane, and may be freely motile, swimming in water by means of cilia. The absence of the 
cell-membrane can also be observed in certain peculiar fungi, which are to be found creeping over moist surfaces without such appendages (fig. 4). These are known as the slime-fungi or Myxomycetes. In many respects they approach very near to one of the humblest animals, the Amcba. They have hardly any structure, appearing like a lump of transparent jelly, the whole mass being called a plasmodium. They have the power of extruding a certain portion of their substance in the form of a blunt protrusion

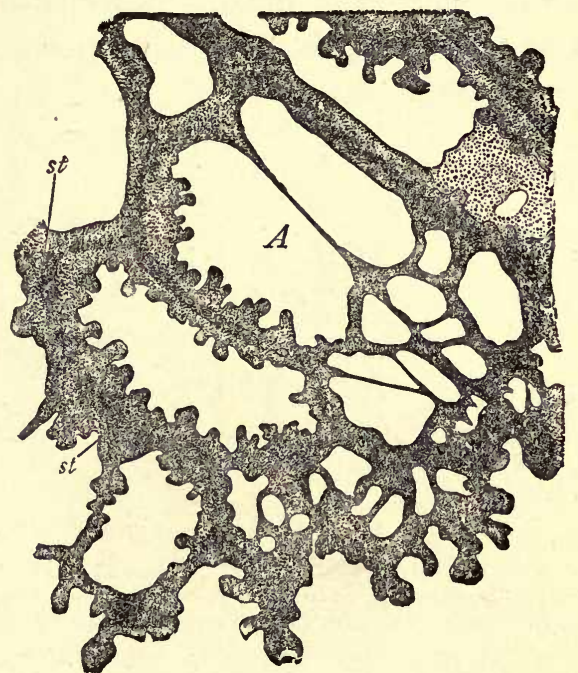

Fig. 4.-Portion of a Plasmodium of a Myxomycete. $\times 300$. (After De Bary.)

known as a pseudopodium, and by means of these pseudopodia they can creep slowly over the surface on which they are lying. The naked condition is, however, exceptional in plants. In most of those which are unicellular the living substance is covered by a delicate membrane or cell-wall, and it may either fill the space inside the latter, or may have in its interior a cavity or vacuole, which is filled with a watery fluid. In the multicellular plants each chamber during life contains its own protoplast or little mass of protoplasm, which jis connected, as already mentioned, 
with its neighbours on all sides. In such cells the protoplast when young usually occupies the whole of the interior (fig. 5, A), but when they are adult it generally lies as a

B

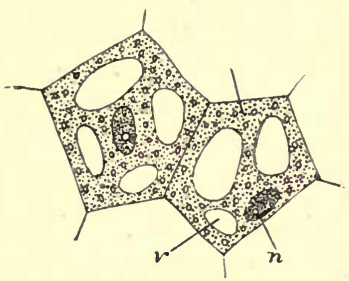

A

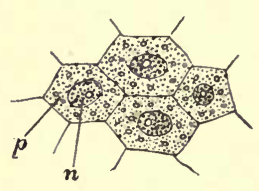

Fig. 5.-Vegetable Cells.

A, very young; B, a little older, showing commencing formation of vacuole. $p$, protoplasm; $n$, nucleus; $v$, a vacuole.

peripheral layer round the wall, to which it is closely pressed, while a central vacuole occupies the greater space of the cavity enclosed by the cell-walls (fig. 6). Sometimes the vacuole is crossed by a number of bridles or strands of protoplasm, which generally pass from a somewhat central spot to the periphery. The protoplasm is transparent, but somewhat granular in appearance, and is saturated with water. Somewhere in its substance, whether it fills the cell-cavity or not, there exists a specially differentiated portion called the nucleus. Sometimes, but only in particular cells, the protoplasm contains other differentiated portions, distinct from the rest of the substance, which are known as plastids. The bulk of the living substance, to distinguish it from these specialised portions, is

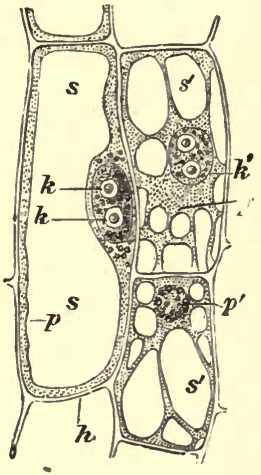

Fig. 6.-Adult Vegetable Cells. $\times 500 . \quad$ (After Sachs.)

$h$, cell-wall; $p$, protoplasm ; $k k$, nucleus, with nucleoli; $s s^{\prime}$, vacuoles. usually called the cytoplasm. It is not of the same consistency throughout, a generally firmer portion lying next to the cell-wall being known as the ectoplasm. A similar firm 
layer may frequently be detected round the vacuole. Sometimes these limiting layers are spoken of as plasmatic membranes on account of their specially dense character; they are not, however, to be confused with the cell-membrane or cell-wall, being particular layers of the cytoplasm.

The exact chemical composition of protoplasm cannot be ascertained, as analysis involves its death, and this is attended by changes in its substance. It contains carbon, hydrogen, oxygen, nitrogen, and probably sulphur and phosphorus, but we are quite unable to say in what different combinations they exist within it. Enclosed in it are always varying quantities of organic substances such as proteids, carbohydrates, and fats, and small quantities of various inorganic and organic salts. The substance of the protoplasm has been thought either to be arranged in the form of a network, these various bodies occupying the meshes, or to have a foamy structure much like that produced by vigorously stirring a mixture of oil and water. The various substances alluded to as occurring in close relationship to it are connected with the nutritive and other vital processes of the cell, or its metabolism, and hence differ greatly in nature and amount from time to time.

In the case of the free-swimming protoplasts, with which we began the study of protoplasm, we saw they were in active motion. As the protoplast becomes enclosed in cell-walls this motility is, of course, less and less obvious ; indeed in most cells it cannot be distinguished at all. There is reason to suppose, however, that protoplasm, wherever existing, is in active, though imperceptible, motion. In many of the constituent cells of even some of the higher plants this motility can be observed, particularly where the protoplasm has a granular appearance. In certain of the cells forming the leaves of many aquatic plants, e.g. Vallisneria, Nitella, Elodea (fig. 7), and others, a streaming movement of the granules the protoplasm contains can be detected under a high power of the microscope. In other plants of terrestrial habit, e.g. 
certain cells of Tradescantia and Chelidonium, a similar streaming of the protoplasm is observable (fig. 8); such

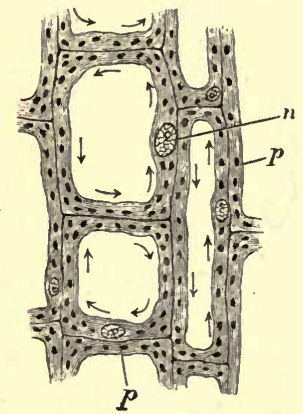

Fig. 7.-Cells from the Leaf of Elodea. $\times 300$.

$n$, nucleus; $p$, protoplasm, in which are embedded numerous chloroplasts. The arrows show the direction of the movement of the protoplasm.

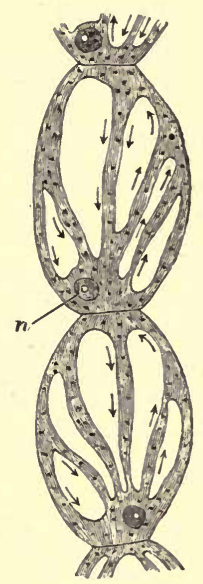

Fig. 8.-Two Cells from a Staminal Hair of Tradescantia. $\times 300$.

The arrows show the direction of the movement of the protoplasm.

movements are spoken of as rotation when the current flows uniformly round the cell, or as circulation when the path has a more complicated course.

It has been mentioned that, with very rare exceptions, all cells contain a specially differentiateả portion of protoplasm, known as the nucleus (figs. 6 and 9). This struc ture does not occupy a very definite position in the cell, but not infrequently is found almost in the Fig. 9.-Nucleus of a Cell, centre. If the whole of the space is not filled with protoplasm, the part in which the nucleus lies is con-

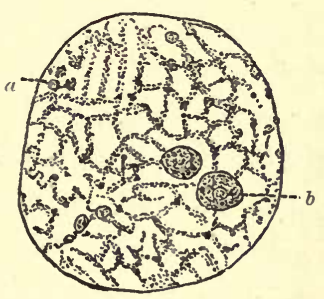
nected with the lining layer by means of strands or bridles. In other cases the nucleus is embedded in 
some part of the lining layer itself. This body has a more definite structure than the rest of the cytoplasm; it is bounded at the surface by a delicate membrane, which is thought, however, to be a denser layer of the protoplasm of the cell, rather than to belong to the nucleus itself. Within this nuclear membrane are found two substances which differ from each other in their power of staining with various reagents. The bulk of the nucleus is composed of a semi-fluid material known as nucleoplasm, in which is embedded a network of fibrils or a long much-coiled thread. The fibrils, or the thread, are composed of a hyaline substance in which lie, close to each other, a number of granules which stain deeply with many colouring matters. The threads contain these granules in such large proportion, that, except with very high magnification, the latter cannot be distinguished, and consequently the whole fibril appears stained. The fibrils are generally said to be composed of chromatin, the name having reference to nothing more than this reaction to stains.

One or more small deeply staining bodies, termed nucleoli, are found in each nucleus, sometimes being very prominent, and at other times hardly distinguishable from. the nodes of the fibrillar network or the crossings of the coiled-up thread (figs. 6, $k k$, and $9, b$ ). Chemically the nucleus resembles the rest of the protoplasm to a considerable extent. It contains, however, a material known as nuclein, of which phosphorus is a constituent. It is not known how the nuclein is related to the rest of the nuclear substance, but it appears to be present in the thread or fibrillar network and not in the general nucleoplasm.

It is of such protoplasts or aggregations of small portions of living substance that all plants are built up. There is, however, a wonderful variety in the relative arrangements of these units of construction, a variety which finds its expression in the multiplicity of existing forms, and the difference of dimensions which various organisms exhibit. 
The simplest plants, as we have seen, are unicellular, and many remain in this condition throughout the whole of their existence. When they have attained a certain size the cell divides into two. Sometimes these two become separated from each other, and we have two plants where but one existed before. Plants with this habit remain unicellular, and the division of the cell is equivalent to the reproduction of the plant. The unicellular condition in other cases is transitory, and the plant soon comes to consist of two, four, or more cells, in consequence of the products of each division remaining attached to-
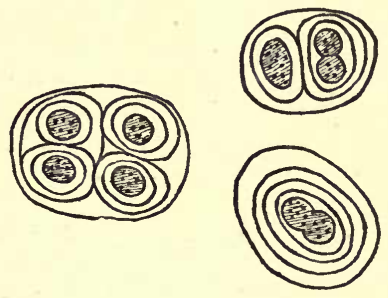

Fig. 16.-Colonies of Protococcus. $\times 750$. gether. We get in this way a small colony of cells, each like the others both in structure and in function. When the power of division is limited the resulting colony consists of a limited number of cells, and is often found surrounded by a common cell-wall or membrane. This condition is seen in such plants as Chroococcus, Protococcus, and other humble Algæ (fig. 10). A colony of somewhat higher type, though still of microscopic size, is found in the form of a hollow sphere (fig. 11), the wall of which is one cell thick (fig. 11, A). This organism, known as Volvox, shows a little higher differentiation than those last described, the cells being furnished with cilia by means of which the little sphere can propel itseif through the water.

In other cases the association of a number of protoplasts is not complicated by the formation of any cell-wall. Fig. $4, \mathrm{~A}$ shows an aggregation of a number of naked protoplasts which have combined to form a plasmodium. These organisms are found creeping about upon moist surfaces; they form the group known as the Myxomycetes or slimefungi. One species, Athalium, is found frequently among 
the refuse of tanyards and is known as 'flowers of tan.' These fungi pass the greater part of their life without possessing any cell-walls, only forming them indeed in connection with their processes of reproduction.

A
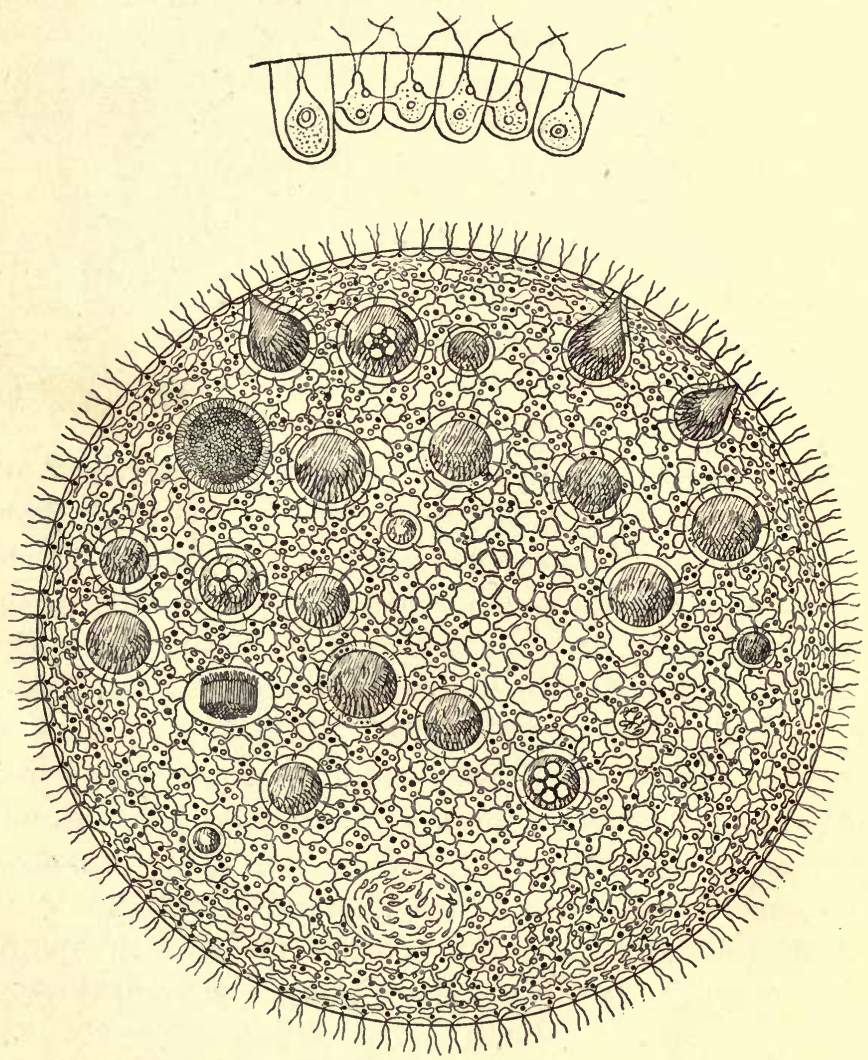

Fig. 11.-Volvox Globator. (After Kny.) $\times 120$.

$A$, section of a portion of the wall of the sphere. $\times 1000$.

A third mode of arrangement of a colony of protoplasts is found in the so-called Conocytes (fig. 12). In one of these plants, which are represented by several very important seaweeds and by a large number of fungi, we 
have a number of protoplasts arranged together over the inner surface of a common cell-wall. The separate protoplasts are often in such close contact with each other that their separate outlines cannot be detected. They have the appearance of a mass of protoplasm lining the wall of a hollow, generally tubular, cavity, and having a large number of nuclei embedded in the mass. The presence of a number of nuclei indicates that there are really as many protoplasts, as we have seen a nucleus is an essential part of one of the latter. Moreover, a single protoplast contains only a single nucleus.

The difference between a colony of this kind and one constructed like Chroococcus or Volvox is the absence of a cell-wall between the protoplasts. They are a stage higher than the Myxomycetes, as the whole colony is protected by an external membrane.

Other cœnocytes exist in which, besides the limiting wall, certain transverse walls exist, dividing up the chamber into compartments. This condition is intermediate between the cœnocyte already de- Fig. 12.-Embryo of Orobus scribed and the simple colony or the multicellular plant.

In most cases the division of

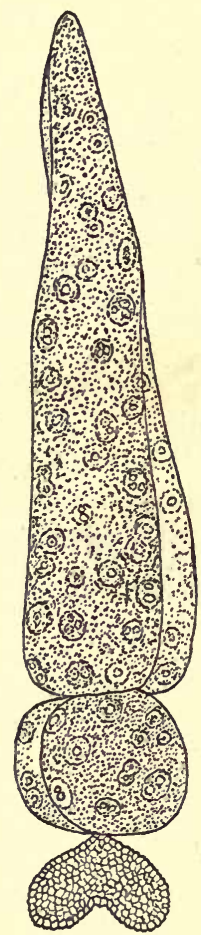

AT THE BASE OF A LONG Suspensor. THE LATTER shows a Ceenocytic StrucTURE. (After Guignard.)

the cells goes on for a considerable time and may continue almost indefinitely, the number of the constituent protoplasts becoming very great and the colony proportionately. large. According to the direction of the divisions we get filaments (fig. 13), plates (fig. 14), or masses of cells, 
the latter undergoing much subsequent differentiation according to their ultimate dimensions and the nature of their habitat or environment.

The protoplasm being the living substance of the plant is possessed of certain properties which are not shared by

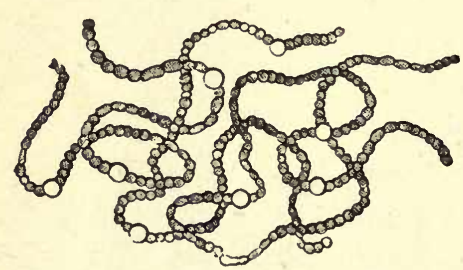

Fig. 13.-Filaments of Nostoc. (After Luerssen.)

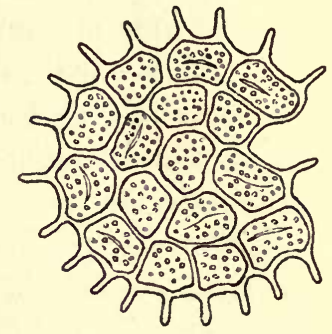

FIG. 14.-Pediastrum, consisting of a Plate of Cells.

the framework on which it rests. It is, indeed, the centre of all the activities which the plant manifests. It assimilates the food which the plant requires and earries out all the chemical processes necessary for life. It constructs

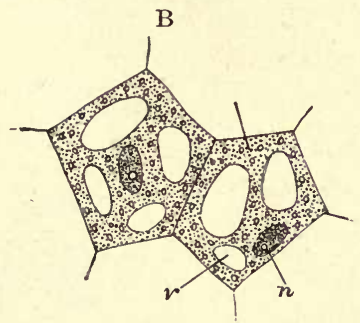

A

Fig. 15.-Vegetable Cells.

A, very young; B, a little older, showing commencing formation of vacuole. $p$, protoplasm; $n$, nucleus ; $v$, a vacuole.

the framework of the plant by which it is itself supported. It receives impressions from without and regulates the responses which the plant as a whole makes to those impressions, both by internal and external movements or changes of position. It is only by its powers of responding to such impressions that the whole organism is able to place 
itself in harmony with its environment. Finally, it carries out the processes of reproduction.

The primary needs of a plant are fairly simple. If we study the life and the behaviour of one of the free-swimming organisms of which we have already spoken, we see that its first requirement is water. In this it lives; from this it draws its supplies of nutriment and into this it pours forth its excreta. The arrangement of the protoplasm in the cell in one of the higher plants points to a similar need. If we regard the arrangement whether in the young or the adult cell, we notice particularly the very close relation of the protoplasm to water. The young cell enclosed in its cellmembrane speedily shows a tendency to accumulate water in its interior, and gradually drops appear in its substance which lead ultimately to the formation of a vacuole always full of liquid (figs. 15, 16). This store of water in the interior of a cell is of almost universal occurrence in the lowly as well as the highly organised plant. The constitution of protoplasm, so far as we know it, depends upon this relation, for the apparently structureless substance is always saturated with it. It is only while in such a condition that a cell can live; with very rare exceptions, if a cell is once completely dried, even at a low temperature, its life is gone, and restoration of water fails to enable it to recover.

The constancy of the occurrence of the vacuole in the cells of the vegetable organism is itself an evidence that such cells are completely

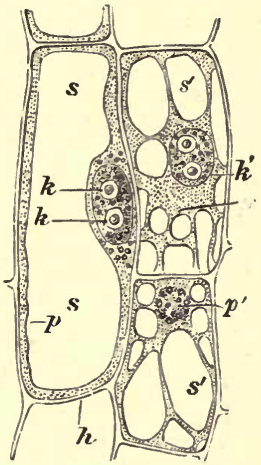

Fig. 16.-Adult Vegetable Cells. $\times 500 . \quad$ (After Sachs.)

$h$, cell-wall : $p$, protoplasm ; $k k$, nucleus, with nucleoli; $s s^{\prime}$, vacuoles. dependent upon water for the maintenance of life. The cell-wall, though usually permeable, yet presents a certain obstacle to the absorption of water, and so even those cells which are living in streams or ponds usually possess a 
vacuole. Cells without a membrane, such as the zoospores, already many times mentioned, can more readily absorb water from without, and hence they are not vacuolated to the same extent as the former ones; indeed, many of them have no vacuoles. Where the vacuole exists it always contains water, so that the protoplasm of the cell has ready access to it, as much so indeed as the cell which possesses no wall. The vacuole contains a store which is always available.

The advantages which water supplies to the plant are many. In the first place, we have seen there is a very close connection between it and the protoplasm, the life of the latter being dependent upon its presence. The information we have at present does not enable us to explain the nature of this dependence. There are other features of the relationship, however, into which we can enter more fully. The protoplasm derives its food from substances in solution in the water ; the various waste products which are incident to its life are excreted into it and so removed from the sphere of its activity. The raw materials from which certain cells construct the food which is ultimately assimilated are absorbed from the exterior in solution in water. Moreover, water is the ultimate medium through which gaseous constituents necessary for life reach the protoplasm.

Passing from the consideration of the protoplasm in particular, the plant as a whole shows a similar dependence on water. Many parts owe their rigidity to the distension of their cells by liquid; growth of the different members is dependent upon the same hydrostatic pressure. In many cases communication between different parts of a plant is brought about through the same instrumentality, and thus the response of the plant to various forms of stimulation is facilitated or indeed made possible.

Another primal necessity of the plant is air. Every living organism, with the exception of a few of the very lowly forms of microbes, is dependent on the access of oxygen for the maintenance of life. 
The oxygen is usually obtained by the plant through the intervention of water. The aquatic plant, whether free-swimming or stationary, unicellular or possessed of a highly differentiated body, absorbs the needed supply from the quantity which is dissolved in the water of the sea, stream, or pool in which it lives. The higher plant conveys it to the protoplasts in solution in the water with which its tissues or its walls are saturated. In such an organism there is need of a special mechanism by means of which the gases of the exterior may obtain access to the living cells in the interior of the mass.

A third requirement of the plant is food. Here ultimately, again, its dependence is placed upon the water it obtains. The food or the materials from which the food is constructed are absorbed by the plant in solution in water, whether the food material is solid, liquid, or gaseous in the condition in which it is presented to it.

Another condition is imperative in the case of a plant which is composed of a large number of protoplasts or cells. Not only must each have its own needs supplied, but it must be in a condition to influence others and be influenced by them. In such a plant we have, in fact, a community of individuals, situated differently with regard to the supply of individual and collective needs, and the well-being of the whole community must depend upon the co-operation of all in earrying out the different processes of life. The protoplasts of such a community must therefore be in organic connection with each other, so that such co-operation can be secured. The connection between contiguous protoplasts which are separated by cell-walls is not easy to determine. Special methods of preparation, and the application of particular staining reagents, will show, however, under very high magnification, that the living substance of one cell is continuous with that of its neighbour by fine delicate fibrils which perforate the wall (fig. 17). In a few cases, as in certain seaweeds, and in the sievetubes of the flowering plants, the connecting strands are 
sufficiently coarse to be visible under a comparatively low power of the microscope, and to need hardly any special preparation (fig. 18).

It will no doubt have been noticed that the term 'cell' is somewhat loosely used. A typical cell of a multicellular plant consists of three parts - the protoplast, the cell-wall, and the vacuole (fig. 6) ; of these the first is the most im-

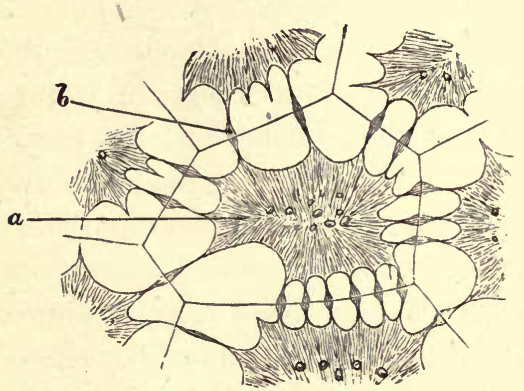

Fig. 17.-Continuity of the Protoplasi of contiguous Cells of the Endosperm of a Palm Seed (Bentinckia). Highly magnified. (After Gardiner.)

$a$, contracted protoplasm of a cell; $b$, a group of delicate protoplasmic filaments passing through a pit in the cell-wall.

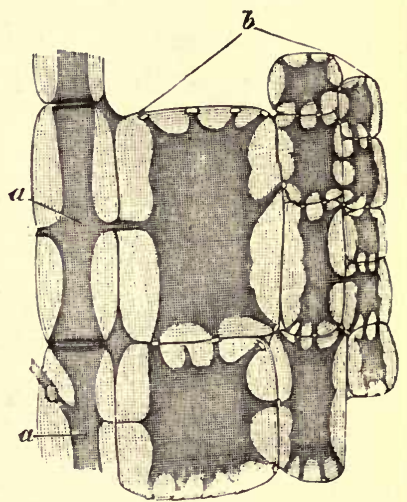

Fig. 18.-Semi-diagrammatic Longitudinal SECTION OF AN OLD AND STOUT PORTION OF Ceramium rubrum, SHOwING CoNtinuity Between the ProtopLasmic Contents of the Axial or Central Cells, $a$ a, at their ENDS, AND LATERALLY WITH THE Cortical Cells $b$, by means of Protoplasmic Threads. (After Hick.)

portant, being the living substance. A protoplast which has no cell-wall and contains no vacuole is still called a cell. The term is again often applied to a cavity which contains no protoplast, as in the case of old wood or cork. In such cases a protoplast once occupied the cavity, but it has been removed by death. These cells are consequently only the skeletons of dead protoplasts. 


\section{CHAPTER II}

\section{THE DIFFERENTIATION OF THE PLANT-BODY}

THE primary needs of a complex plant are the same as those of a single protoplast, the greater size of the former involving however a more elaborate method of supplying them. In multicellular plants we consequently meet with a considerable degree of differentiation of structure. Each protoplast, which is one of the units of the colony, has originally the same properties as the unicellular plant. With increase of number in the plant-body, and with the consequent increase of size, a certain division of labour soon makes its appearance, and particular groups of cells develop one property more than the others. A specialisation of powers is very quickly apparent, and we can recognise masses of cells devoted to the discharge of one function, others to that of another, and so on. Such limitations of the powers and properties of the individuals have for their object the well-being of the community of which those individuals are constituents.

Various groups of plants show this specialisation of function or differentiation of structure in very different degrees, any particular development having a special reference to the habitat or the mode of life which is characteristic of the community in question. A plant-body which takes the form of a long filament or a plate of cells shows little differentiation beyond the formation of a vacuole in each protoplast. The setting apart of special cells for purposes of reproduction is generally the first specialisation which takes place.

As soon as the cells of the plant begin to divide in 
three dimensions, so that a mass of protoplasts is formed, the progress of differentiation becomes marked.

In such a mass the necessity of supplying water to all the constituent units involves particular difficulties which vary according to the environment of the plant under observation. Those which live in water need rnuch less complex arrangements than those which are at home on land, as they can absorb water from the exterior by their general surface, and after absorption it can easily make its way from cell to cell. Those which derive their supply of water entirely from the soil, as is the case with nearly all terrestrial plants, need a specialised mechanism for transport of the water after it has been taken up.

On the other hand the supply of a suitable atmosphere to the interior of the plant for the service of its more deeply seated protoplasts is attended with more difficulty in the case of an aquatic than a terrestrial plant.

In cell-masses, therefore, such as are found in all plants possessing more than microscopic dimensions, we meet with considerable differentiation of the plant-body. 'The explanation of the details of such differentiation is to be found in the division of labour which the size and the mode of life of the particular plant demand.

The first indication of this differentiation in the vegetative body of the plant is a change in the character of the

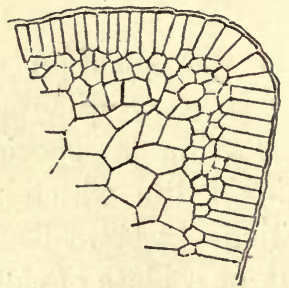

Fig. 19.-PIece of The

Thallus of Pelvetia, SHOWING CHARACTER OF The External and SUbJACENT Cells. $\times 300$. exterior, which has for its object the protection of the plant from external injurious influences. This can be seen even among the seaweeds, simple as is generally the structure of members of this group. Fucus and its allies, which form part of the class of the brown Algæ, have their external cells much smaller, more closely put together, and generally much denser than the rest of their tissue (fig. 19). In the group of the Mosses certain arrangements of this kind can 
be seen. The common bog moss (Sphagnum) shows its stem to have on the outside several layers of large empty cells whose walls are marked with spiral thickenings.

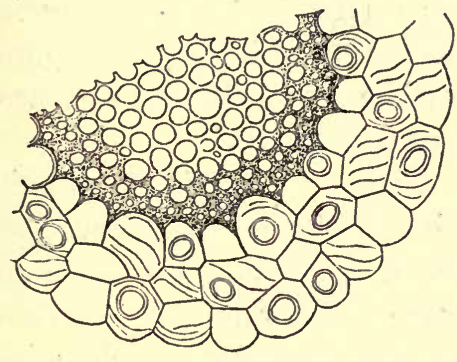

Fig. 20.-Transverse Section of Stem of Sphagnum.

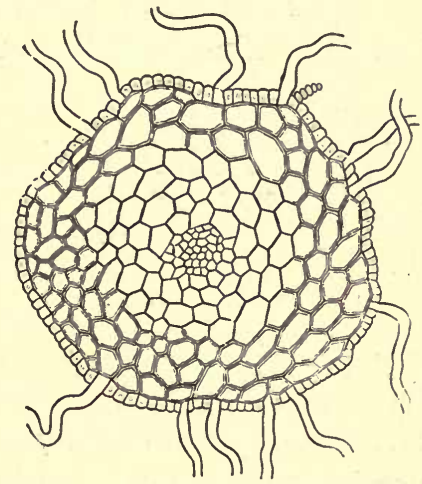

Fig. 21.-Section of Stem of Moss, showing Central Strand of THIN-WALled CELls SURROUNDED by Cortex and Epidermis. The Walls of the Outer Cells of the Cortex are constderably THICKENED. (After Sacbs.)

Inside these a further protective layer of small cells with uniformly thick walls is met with (fig. 20). In the smaller mosses the outer layers of the cortex are thickened (fig. 21).

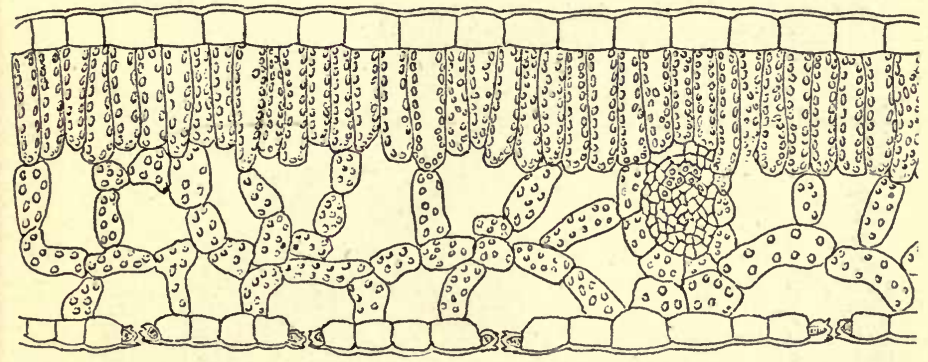

Fig. 22.-Transverse Section of the Blade of a Leaf, showing thy Outer Walls of the Epidermal Cells thickened and cuticuLARISED. $\times 100$.

In the higher terrestrial plants we have evidence of great specialisation for protective purposes, a special tegumentary system being developed, which varies in 
complexity in the different groups. In the smallest forms, which are only herbaceous in habit, we find the protective mechanism takes the shape of a thickening and cuticularisation of the outer walls of the cells of the epidermis (fig. 22). The protection secured is twofold; evaporation

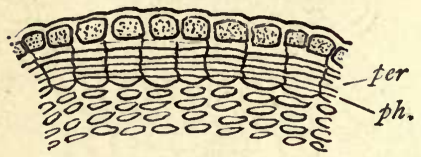

Fig. 23.-Outer Portion of Cortex of Young Twig of Lime. per, cork layer. of water is prevented, and so an economy of the supply is secured, while the dangers incident to cold or heat are minimised.

In plants of sturdier habit the protection afforded by the epidermis is replaced after a while by a more complicated tegumentary sheath. Certain cells become specialised and form layers of cork (fig. 23), which arise

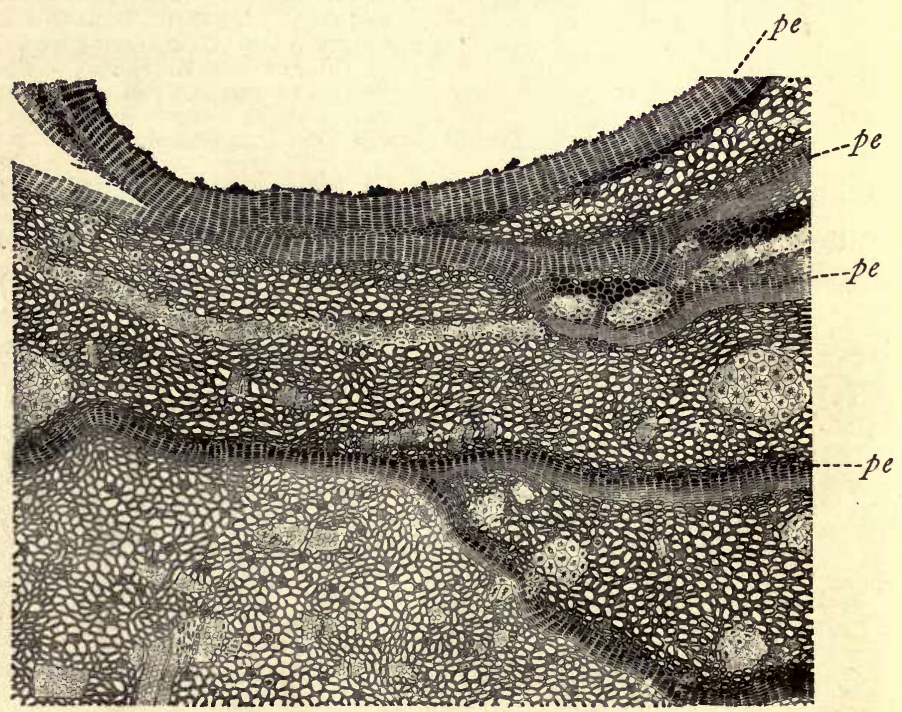

Fig. 24.-Section of BarK of Quercus sessiliflora. (After Kny.) $p c$, cork layers arising at different depths in the cortex.

successively at gradually increasing distances from the exterior, and in the case of trees finally lead to the construction of a bark (fig. 24). The corky formations are 
supplemented by masses or sheaths of hardened or sclerenchymatous parenchyma or even by sclerenchyma itself. In forms which are intermediate in requirements, such as the petioles of leaves, layers of collenchyma are developed below the epidermis (fig. 25).

Sometimes sheaths or layers of sclerenchyma are developed instead of cork; this condition occurs especially among the stouter Monocotyledons.

The protective mechanisms developed by roots also show a good deal of variety. There is

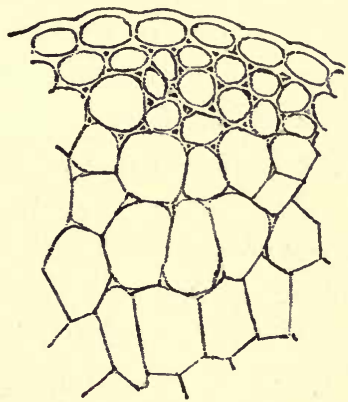

Fig. 25.-Collenchyma UNDER the Epidermis of Petiole. $\times 50$. not at first a provision of an impervious membrane composed of the outermost layer of cells; this would be inconvenient in view of the necessity for the existence of root-hairs. The second layer later undergoes modification, its cells becoming thickened in the peculiar manner characteristic of the exodermis (fig. 26). Other sheathing layers are also found more deeply seated, while eventually the pericycle becomes the place of formation of corky tissue.

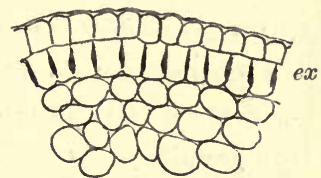

The second prominent differentia- Fra.26.-Section of OUter tion which presents itself is the formaREGION OF ROOT, SHOWING EXODERMIS, $e x$. tion of a system of cells and vessels for the transport of water through the plant and the circulation of nutritive and other materials. We may speak of this as the conducting system. A little reflection will show us the necessity for the development of some such system as this, which must be more extensive and complex as the size of the plant increases. We find that the source of water on which a terrestrial plant relies is the soil in which its roots are embedded. Even when it is young many of its protoplasts are placed at a considerable dis- 
tance from such a source of supply, and in the absence of a ready means of communication must die in consequence of their position. These moreover are among the most active of the protoplasts, discharging important duties in connection with nutrition, and needing for their purpose considerable quantities of the water from the soil with the salts dissolved in it.

The conducting system is formed by the collections of cells and vessels which are known as the vascutar bundles. These structures consist in most cases of two parts, the wood, which is the path for the ascent of water from the roots, and the bast, which is more concerned with the transport of the elaborated products of the metabolism of the cells.

The degree of development of this system varies very much in different plants. In an ordinary herbaceous Dicotyledon the bundles remain separate, and can be traced separately from the root, through the stem to the leaves (fig. 27) in which they form the branching network known as the veins (fig. 28). With greater size, however, more capacious channels are demanded, and we find more and more bundles developed, until we reach the condition of the oldest trees, nearly the whole of whose trunks are formed of tissue which either is or has been devoted to this service. In such trees the most actively living parts are found at the extremities, by far the greatest number of their protoplasts being situated in the twigs and leaves. Indeed, the greater part of the wood of the trunk of many trees is dead, and consequently functionless.

The same tissues serve for transport in the Monocotyledons, and in the Vascular Cryptogams, though the mode of arrangement of the elements is altogether different from that of the Dicotyledons.

In those vascular plants which live in water, and particularly in those which are totally submerged, there is no need for so elaborate a transport system, as water can be readily absorbed by the general surface. We find two 
modifications of structure in such plants; the epidermis is hardly at all cuticularised, so that water can pass into its cells; while the vascular bundles are comparatively feebly developed, the woody part of them being particularly small.

A third requirement of a plant of considerable mass, especially if it has a terrestrial habitat, is a power of resist.

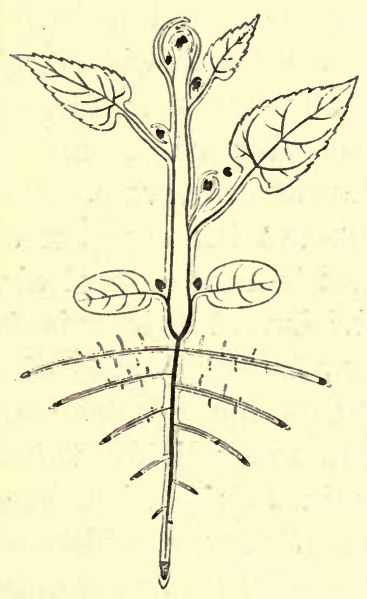

Fig. 27.-Diagram of the course of the Vascular Bundles in an Herbaceous Dicotyledonous Plant. ing such external forces as would lead to its uprooting, which must be combined with a considerable degree of flexibility, at any rate at the extremities of the body. This

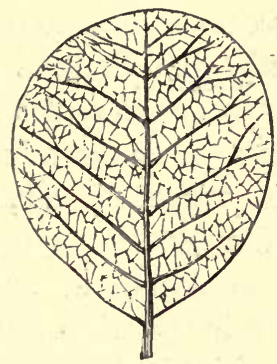

Fig. 28.-Distribution of thF Vascular Bundles or Veins in a Foliage Leaf.

combination of rigidity and flexibility has been secured in various ways, varieties of both the form and the structure of the plant being concerned in it. In the simplest plants but little differentiation of the body is needed; such forms as consist of single cells, or rows or plates of cells, living in water, need hardly any rigidity, and in their cases the unthickened cell-wall yields sufficient support to the protoplasm. Larger plants which grow in rapidly flowing water usually possess flexible stems and much-divided leaves, which consequently give way to the current, and escape damage. Small terrestrial plants or parts of plants, which have but 
a short life, resemble these aquatic forms in their general characteristics, though they show much greater variety in the forms of their leaves. The rigidity and flexibility of both depend upon the distension of their cells with water. We find this mechanism in succulent petioles, such as those of the rhubarb, and in certain herbaceous stems which contain little wood, such as those of the cabbage and lettuce. Plants of terrestrial habit which attain very large dimensions, such as the forest trees, need, however, much greater modification. Being exposed to winds and storms, they need a firm anchorage below the surface of the ground, and a more or less massive axis to secure stability when atmospheric disturbances are severe. For the needs of their protoplasts, to secure the exposure of the greatest possible number of them to the access of air, warmth, and light, a great subdivision of this axis is necessary, so that the form usually attained is that of a relatively very large head resulting from the repeated subdivisions of the trunk, and ending in finely divided twigs. The danger of too great rigidity in this portion soon becomes apparent, as it presents a very considerable surface to the wind. The rigidity needed for support must be combined with sufficient flexibility to enable the body, already helped by its fine subdivision, to give way before the force of atmospheric currents, and so to prevent the danger of uprooting the tree. In other forms a weak axis, quite incapable of supporting any great development of the plant-body, must be capable of obtaining support by clinging in various ways, and holding by various mechanisms to other structures, such as the trunks of trees, rocks, walls, \&c.

In many cases the strength and prominence of the tegumentary and conducting tissues supply the particular need. In most forest trees the anchorage is afforded by the strong much-branched root system, the centre of whose members is composed of great developments of secondary wood, forming part of the conducting system. The trunk 
and twigs are of similar composition, the former being strengthened very materially also by its bark. But there are many smaller trees and shrubby plants, as well as some herbaceous forms, whose requirements are similar, but which for various reasons have not a very great development of either primary tegumentary tissue or of vascular bundles. With no additional mechanism for support, they would be in great danger of either collapsing or being actually uprooted. In their cases we meet with a subsidiary development of supporting tissue, which shows a great variety in its arrangement and distribution.

We find that the tissue which most frequently subserves this purpose is either collenchyma, sclerenchymatous parenchyma, or true sclerenchyma. In a few delicate stems these tissues are much more prominent than the vascular bundles. We can notice three regions of the stem or axis where they may appear, and in these places they may take the form of isolated cells, or strands of tissue, or complete sheaths going round either the whole axis or separate parts of it. The first of these regions is the layer underlying the tegumentary tissue, which the new development supplements and strengthens. Most moss plants show the hypodermal cells of their axis thickened, while such a development is very common in many petioles and leafblades. The new development may occur in close relationship with the vascular bundles which, in such cases, are found among large-celled somewhat succulent parenchyma, and are not generally very strongly developed. The sclerenchyma by forming a separate sheath round each bundle gives it a rigidity which it could not derive from its own elements, and in addition prevents the whole stem from being crushed. This is seen in the stems of many semi-succulent monocotyledonous plants, such as those of the maize and the asparagus (fig. 31). The sclerenchyma may also occur freely in the ground tissue, at some distance from both tegumentary. and vascular structures. The bands of it which occur in the rhizome of the bracken fern are good illustrations of 
this mode of disposition. The two main ones form an interrupted cylinder (fig. 29), so arranged as to protect the delicate vascular tissue, which is in great part placed either within this cylinder or in some similar relation to other similar sclerenchymatous strands. In the case of a plant of humbler type, the common hair-moss (Polytrichum), a development of somewhat sclerotised cells forms a central core passing down the stem. In many of the flowering plants more complex distribution of sclerenchyma can be noticed, strands in the middle of the cortical tissue, or in the pith

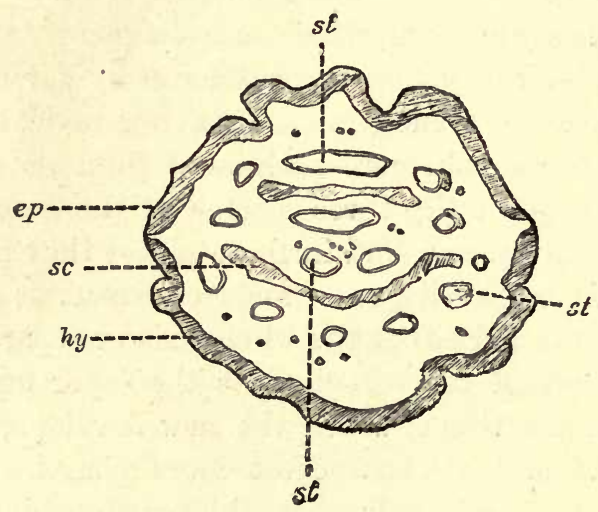

Fig. 29.-Transverse Section of Rhizome of the Bracken Fern. $\times 10$.

$s c$, bands of sclerenchyma; hy, hypodermal sheath of sclerenchyma; st, steles; $e p$, epidermis.

of the stem, being occasionally seen. Stems which are angular in section are usually found to have their angles strengthened in a similar way.

The arrangement of this sclerenchyma is generally such as to supplement the bundles, and to secure the greatest amount of solidity and sufficient flexibility, with the least expenditure of material.

Instances of various methods of arrangement of strengthening material may serve to illustrate this particular differentiation (fig. 32). In the simplest cases the sclerenchyma is developed in connection with only one of 
the three regions already alluded to. The stem of Equisetum and the leaves of Conifers are furnished with a layer of thick-walled cells immediately under the epidermis (fig. 30) ; the vascular bundles of many Monocotyledons are surrounded separately by a sheath of small cells of similar character (fig. 31); in Pennisetum a sheath is developed round the stem in the form of a hollow cylinder which lies between the bundles and the epidermis.

More frequent instances occur in which two of the

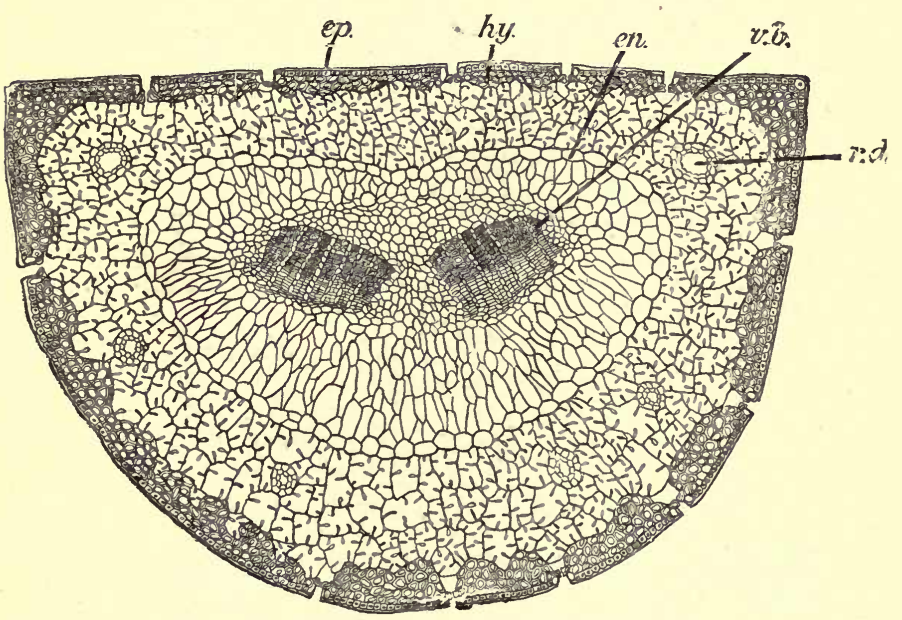

Fig. 30.-Leaf of Pinuis (one of the Conifers).

$e p$, epidermis; hy, layer of sclerenchyma ; en, endodermis; v.b., vascular bundle; r.d., resin duct.

regions in question are strengthened simultaneously. In the stems of Scirpus there is a development of sclerenchyma round the periphery, and strands occur also in connection with the bundles. Sometimes these are connected by bands of sclerenchyma lying between them. In Fimbristylis there is a ring of sclerenchyma in the cortex and patches around the periphery, which in other cases are joined like those of the former type. In the stems of Typha a band of sclerenchyma lies at the back of each bundle, and either a ring or some isolated strands may be 
found in the cortex. The stem of Juncus shows these two forms combined together.

Still more complicated cases show sclerenchyma arising in all three regions, sometimes the bands being all independent, sometimes united in various ways. In Cladium Mariscus those of all the regions are united into a continuous system which goes from the tegumentary

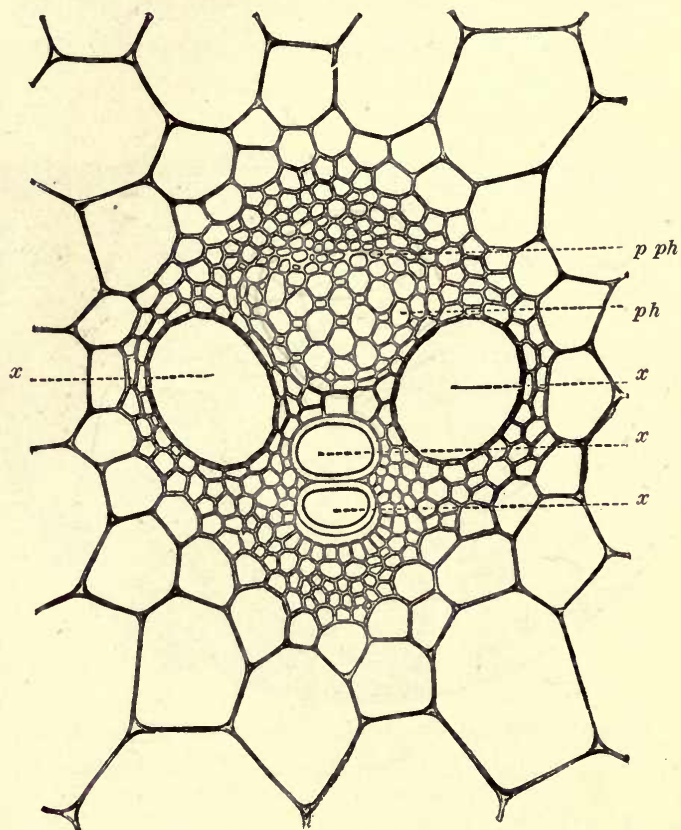

Fig. 31. - Vascular Bundle of Stem of Monocotyledonous Plant. (After Kny.)

$p h$, phloem ; $x$, xylem vessels ; $p p h$, protophloem. The bundle is surrounded by a small-celled sheath of sclerenchyma.

region towards the interior of the stem, embracing the vascular bundles and attaching them to each other.

Similar differentiation of the supporting system is found in many leaves, in which it subserves the same purposes. In many cases the veins afford sufficient protection against tearing or rupture in consequence of violent winds. The methods of their arrangement in many cases subserve 
this purpose very completely. In other leaves of tough leathery habit the delicate tissue of the mesophyll is frequently protected from crushing by isolated thick-walled cells of curious shape which extend from one epidermis to the other. Others show bands of sclerenchyma supplementing the veins and not infrequently enclosing them and reaching the epidermis on each side.

The supporting tissue is frequently known as the

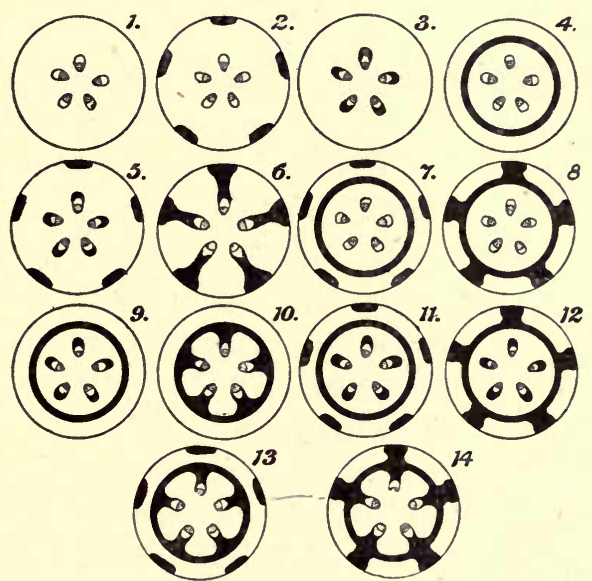

Fig. 32.-Diagram showing the Chief Dispositions of the Skeletal Apparatus in a Stem with Five Collateral Bundles (in Transverse Section).

(The sclerenchyma is black; the bast of the bundles is white; the wood is dotted.)

1, Type without accessory sclerenchyma; 2, Equisetum; 3, Bambusa; 4, Pennisetum; 5, Scirpus ; 6, Erianthus ; 7, Fimbristylis ; 9, Typha ; 10, Juncus; 14, Cladium. (After Van Tieghem.)

stereome of the plant. It forms, as we have seen, the most prominent part of the endo-skeleton.

The cells of which the masses of sclerenchyma are composed have been ascertained to possess almost as much power of withstanding longitudinal strain as the finest steel, and they are much more ductile than either this metal or wrought iron. Their arrangement in the different ways described has a very distinct relationship to the character of the strain they have to resist. In such 
structures as hollow stems where there is but little substance of tissue, but where they are required to resist lateral bending, the supporting tissue is situated near the periphery of the stem, and the latter is often still further strengthened by being furnished with ridges or flanges. An instance of an almost converse character is afforded by a young root. In its growth, while it must possess sufficient rigidity to enable it to penetrate the soil, it must be capable of frequent bending to enable it to avoid obstacles. This is most advantageously provided for by a central core of strong tissue, surrounded by more succulent material. The transporting tissue of the centre is comparatively little affected by the flexures of the structure, and its function is not interfered with.

Another kind of differentiation in such a cell-mass as we are dealing with, is the setting apart of particular groups of cells for various metabolic

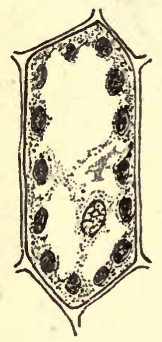

Fig. 33.-Chloroplasts Embedded in the ProTOPLASM OF A CELL OF the Palisade Tissue of A LEAF. purposes. We have the formation of glandular tissue, of the laticiferous systems, and so on. This differentiation may be marked also by the production of definite organs in the protoplasts, such as are seen for instance in the case of the chloroplasts of the leaves (fig. 33) and other green parts of plants.

The habit of life of a plant again may influence its structure and the degree of differentiation of its body to a very great extent. The great group of the Fungi afford us an illustration of the degradation of structure which accompanies a saprophytic or parasitic habit. Similar instances of degradation are met with among the flowering plants.

The needs of the cell-mass thus usually lead to the differentiation in its substance of at least four physiologically different regions - the tegumentary, the conducting, the supporting, and the metabolic. The latter includes all 
the parts in which the protoplasts are comparatively little changed, and consequently are most concerned in carrying out the vital processes.

The needs of the protoplasts forming the community of the plant embrace, however, as we have seen, something more than the arrangements so far described serve to secure for them. Each protoplast must be furnished with a certain

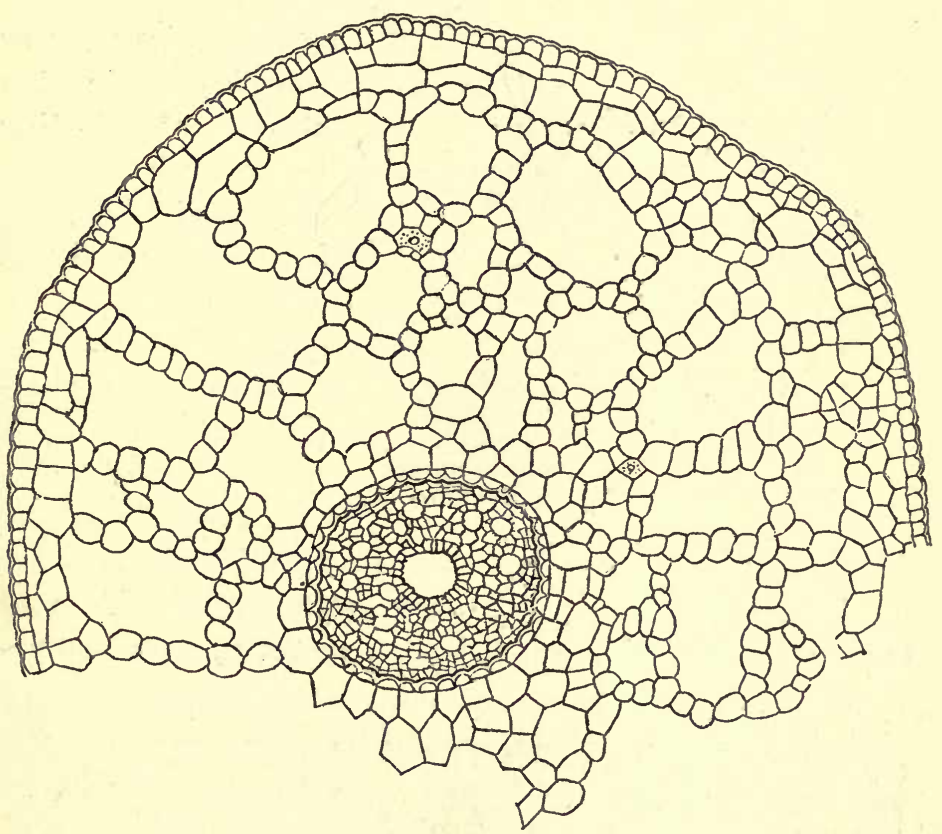

Fig. 34.-Section of Stem of Potamogeton, showing Air Passages in THE CoRTex.

amount of air, or rather oxygen. Almost all living substance must carry on during life the process known as respiration. The free-swimming zoospore to which we have so often referred obtains a supply of oxygen from the water in which it lives, the gas being dissolved therein. Aquatic plants also obtain their oxygen from this source, but many of them are composed of a large number of cells; mony of which are situated at some distance from the 
exterior. In such plants large cavities or reservoirs are constructed, in which a quantity of air is slowly accumulated and into which the respiratory products can be discharged. From such reservoirs the oxygen which the cells require is obtained. The composition of the atmosphere in these chambers or lacunæ is not accurately known, but it probably differs somewhat from that of ordinary air.

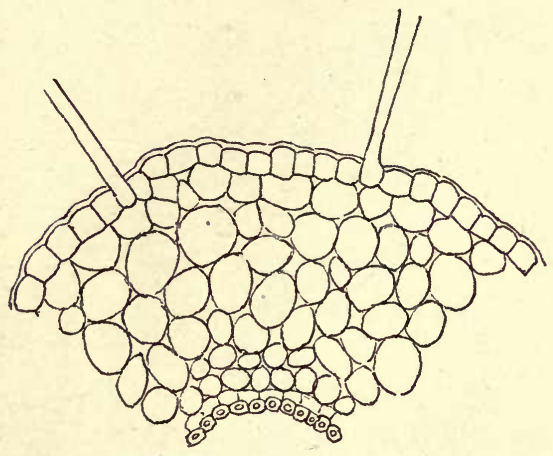

Fig. 35.-Cortex of Root, showing Intercellular Passages between the Cells.

These air passages or reservoirs are very conspicuous in the stalks of floating leaves such as those of the water-lily, and in the submerged stems of most aquatic plants (fig. 34).

A somewhat similar mechanism is provided in the case of terrestrial plants. At the time of their first formation, all the cells are in close approximation to each other at all points of their surface. This condition is, however, only tempo-

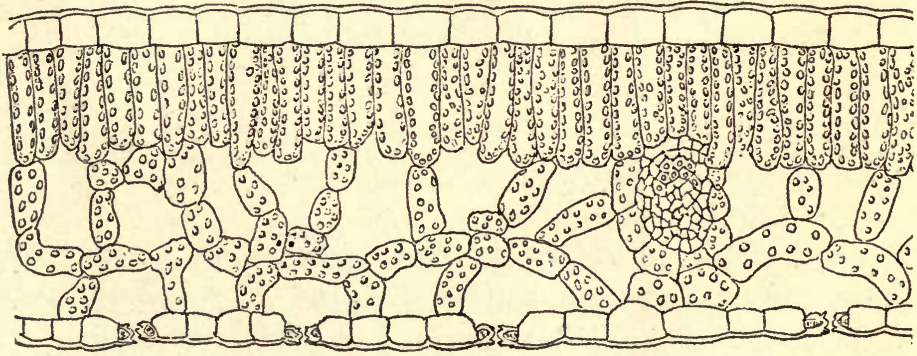

Fig. 36.-Section of Leaf showing the large Intercellular Spaces oF the Mesophyll.

rary; during the early stages of growth the cell-walls split apart at particular places, usually the angles of the cells. A system of intercellular spaces is thus formed 
which, as growth proceeds, become continuous with each other and form a system extending throughout the plant. They can be detected in the root, in the cortex of which

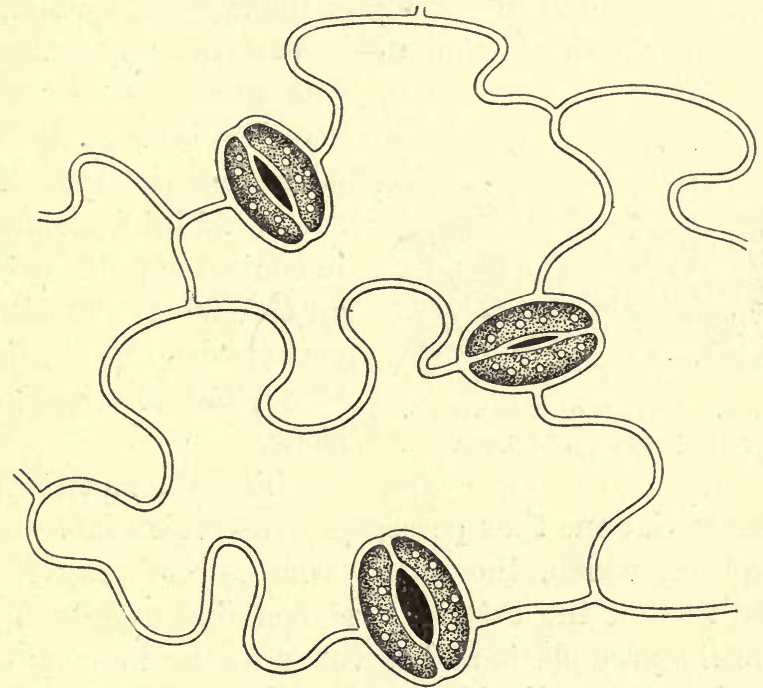

Fig. 37.-Part of Lower Surface of a Leaf, showing Three Stomata in Different Stages of Opening. $\times 300$.

they are conspicuous (fig. 35 ) ; they may be traced through all the ramifications of the stem, and are seen to form a very prominent feature of the mesophyll of the leaves (fig. 36). They communicate with the exterior in all the green parts of the plant, especially the leaves. In the epidermis of all such parts are small openings known as stomata (figs. 37, 38), which are provided with two guardcells by the behaviour of which the aper-

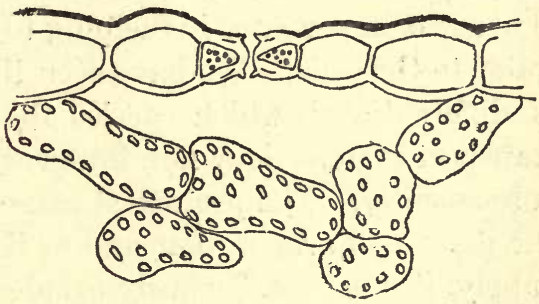

Fig. 38.-Section of Lower Epideris of a Leaf, showing a Stoma. $\times \$ 300$. tures can be opened or closed. In those regions of the axis where corky layers cut off the metabolic tissue of the 
cortex from the exterior, certain other special apertures, the lenticels, are present (fig. 39). The atmosphere consequently enters the plant by these orifices and circulates through the whole of the intercellular space system. As nearly every protoplast abuts in part upon a channel of

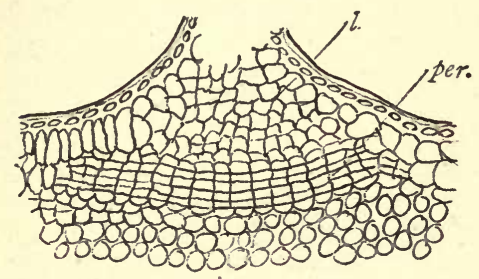

Fig. 39.-Section of a Lenticel.

$l$, lenticel; per, cork layer. this system, its necessary aeration is secured. Each protoplast is thus in a somewhat intricate manner in contact with the external air, though really situated perhaps deep in the tissues of a plant of large dimensions.

Like the aquatic plant, the terrestrial one thus possesses a reservoir containing an atmosphere, which, though its composition may not be exactly that of the exterior, yet contains oxygen for the need of the protoplasts and serves as the medium by which all surplus carbon dioxide is removed from them.

This intercellular space system not only subserves the purpose of the gaseous interchanges of respiration, but ministers in two ways to the metabolic phenomena carried out by the plant. It permits the access of the atmospheric carbon dioxide to structures in the leaves which make it available for the construction of food material. It further is of great importance in helping to regulate the supply of water to the cells. We have seen that a transport system is differentiated which carries the water to thém. This transport system does not, however, remove it from them subsequently. The protoplast can only get rid of water by the process of evaporation, and as it constantly needs a new supply, it must continuously exhale watery vapour to make room for the incoming stream. Such evaporation takes place into the intercellular spaces through the delicate cellwalls which abut upon them. The intercellular reservoir contains, therefore, an atmosphere which is charged almost, 
if not quite, to saturation by aqueous vapour, and under ordinary atmospheric conditions this is being continually exhaled as long as an excess of water is passing through the plant. The regulation of the process of exhalation depends mainly upon the condition of the guard-cells of the stomata which can permit it to go on freely or can check it by partially or entirely closing the apertures according to various internal and external conditions (fig. $37)$. 


\section{CHAPTER III \\ . THE SKELETON OF THE PLANT}

In the last chapter we discussed the differentiation of the body of the plant, and examined the constitution of various mechanisms which are associated with such differentiation. If we. study the arrangements which are peculiar to any plant, we shall find that almost all such differentiation as exists involves a modification of the non-living part, and particularly the walls of the supporting and conducting tissues, the living protoplasts having fundamentally the same structure or composition, whatever may be the nature of their immediate support. All the various dispositions of the non-living elements or structures are secondary in importance to the protoplasts.

We cannot, indeed, lay too great stress on the fact that the needs and conditions of the protoplasts are primarily the causes of the differentiation of the non-living structural parts, and such differentiation is the expression of the fact that division of labour has arisen among the protoplasts of the community.

We have seen that a protoplast in its simplest condition is capable of an independent existence without any form of mechanical support beyond that which it derives from the slight difference of density between its external layer and its interior. In most cases, however, this is not sufficient for protection during its whole life, and a membrane is subsequently formed around it. The membrane itself is a secretion from the protoplast, which in fact prepares its own defensive mechanism. In most cases the protoplast is always clothed by a cell-wall, the forma- 
tion of every new cell being completed at once by the membrane which is formed as soon as the protoplast has divided into two. This is particularly noticeable in cases where a cell-complex or community forms the plant-body. Each protoplast thus continually forms for itself a chamber to dwell in, the walls of which at first, at any rate, are probably all alike. We may thus recognise in the cell-wall an exoskeleton for the individual protoplast, which may or may not undergo subsequent modification.

In the case of a large plant consisting of innumerable protoplasts, the cell-walls of the separate units are found united together in different ways, and to a different extent in different individuals. The resulting network constitutes at first the skeleton of the whole plant. The modification of the cell-wall which was unnecessary so long as the protoplast was solitary, becomes imperative as soon as the needs of a large community are established, and secondary differentiations of such cell-walls result, the alterations being due, like the original formation, to the activity of the protoplasts. Not only are the walls changed in substance and in thickness after they are formed, but the protoplast itself frequently alters its shape, and consequently the form of the cavity containing it, by irregularities of subsequent growth. The slieleton of the plant is not therefore merely the hard tissues which will survive maceration and desiccation, not merely those coarser structures evidently set apart for protection and support, but it includes all the delicate cell-walls which form the cavities in which the protoplasts are living. We may indeed discriminate between the skeleton of the individual protoplast and that of the large community of which it forms a part.

The skeleton of a large plant such as a tree increases in complexity as its life continues. In such a plant growth continually proceeds so long as life lasts. Every year new branches or twigs with their associated leaves are constantly produced. With such continuous increase 
of size, new conducting tissue must be formed. The skeleton of a young plant is consequently much smaller

A

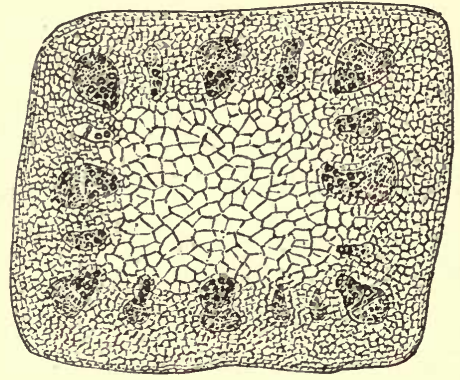

B

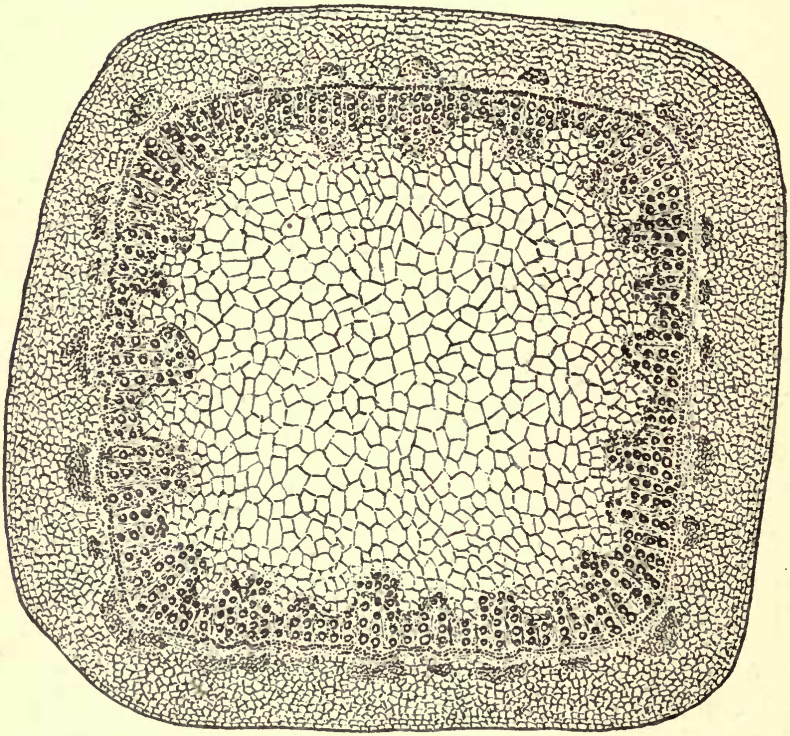

Fig. 40.-Section of Dicotyledonous Stem (Helianthus).

A, young condition, with the primary vascular bundles only developed; $\mathrm{B}$, older, after secondary bundles have been developed between the primary ones by the interfa scicular cambium, forming a ring of wood.

than that of an old one. The difference between the condition of a stem at two periods may be seen by comparing fig. $40, \mathrm{~A}$ and $\mathrm{B}$, the former of which shows the arrange- 
ment of the supporting and conducting tissue at an early stage of its life, and the latter indicates the condition after several months, during which a large formation of secondary vascular tissue has taken place.

The structure of a cœnocyte shows a similar mode of formation of the skeleton to that of a multicellular plant-community. In this case, however, the several protoplasts are not furnished with separating walls. The only skeleton is the external membrane which limits the whole structure, and which is formed by the conjoint activity of them all. In compound or septated cœnocytes we have in addition certain transverse walls crossing the interior and giving a greater degree of strength to the whole body. These separating walls have a similar origin.

The primary cell-wall which clothes the unicellular plant, and which serves as the original supporting membrane of the separate protoplasts of a community or colony, is, when first formed, a clear, transparent, extensible, and elastic membrane, which remains in contact with the protoplasm so long as the latter is living. Under certain conditions it is capable of absorbing considerable quantities of water, and in consequence swelling to a greater or less extent. Under ordinary conditions it is freely permeable by water. It is usually said to be composed of a substance termed cellulose, whose chemical composition is 
represented by the formula $n\left(\mathrm{C}_{6} \mathrm{H}_{10} \mathrm{O}_{5}\right)$, the value of $n$ not yet having been accurately determined. This substance is related to such bodies as starch, sugar, \&c., being a rnember of the group of carbohydrates. It is capable, under the action of hydrating reagents, of being converted into a form of sugar, and under certain circumstances it can yield nutritive material for the use of the plant. Cellulose possesses the peculiar property of becoming a deep blue in colour when treated with iodine in the presence of sulphuric acid, chloride of zinc, or other hydrating reagent. It dissolves with readiness in a solution of ammonio-cupric sulphate (Schweizer's reagent), but is not soluble in dilute acids or alkalis. Strong mineral acids, such as sulphuric or phosphoric, cause it to imbibe water and swell up, ultimately becoming gelatinous and dissolving. Certain soluble ferments affect it similarly.

When the cell-wall is examined by polarised light it is found to be doubly refractive.

When cellulose is oxidised with strong nitric acid it yields oxalic acid.

Cellulose is capable of existing in more than one condition. We find some kinds of it which will stain blue with iodine without previous hydration. Examples of this variety are found in the cell-walls of the bast of Lycopodium, the endosperm of the Pæony, the cotyledons of some of the Leguminosæ, \&c. The walls of the hyphæ of the fungi differ again, in that they will not give the blue colour with iodine even after treatment with hydrating reagents. Recent observations suggest that this variety of cell-wall approaches in composition the chitin of the animal kingdom.

The celluloses which have been so far examined have been divided into three categories, according to the ease with which they can be made to undergo hydrolysis, and to yield some variety of sugar by such treatment. The celluloses of cotton fibres are perhaps the most resistent of all, and maybe taken as representatives of the mostrefractory group. The cellulose found in the main mass of the fundamental 
tissue of the flowering plants is less resistent, giving very easily the reactions which have been just described. A 'third variety is hydrolysable with still greater readiness. It is to a certain extent soluble in alkalis and is easily decomposed by acids with formation of other carbohydrates of low molecular weight. Such cellulose is represented in the cell-walls of most seeds.

It is probable that cellulose is chemically combined with a certain amount of water, and that the degree of such hydration differs in the different varieties described.

Though, as already stated, the cell-wall is commonly said to be composed of cellulose, the latter material is always associated with other constituents. Among the latter we find various members of another group known as pectoses, which differ in many ways from cellulose. This group includes two series of bodies which vary among themselves as to the degree of their solubility in water. One of these series comprises bodies of a neutral reaction, while those of the other are feeble acids. In each series there are probably several members, which show among them every stage of physical condition between absolute insolubility and complete solubility in water, the intermediate bodies exhibiting gelatinous stages, characterised by the power of absorbing water in a greater or less degree.

Of the neutral series the two extremes are known as pectose and pectine. The former is insoluble in water, and is closely associated with cellulose in the substance of most cell-walls; the latter is soluble in water and forms a jelly with more or less facility. Pectose has not yet been obtained pure, in consequence of its close association with cellulose and the readiness with which it undergoes change in the process of extracting it. The reagents which separate it from cellulose convert it into pectine, or into pectic acid, the former being soluble in water, the latter in alkalis. The cell-wall can be shown to contain the two constituents by the action of Schweizer's reagent, which, when used with proper precautions, dissolves out the 
cellulose and leaves the framework of the cell apparently unaltered; it consists then, however, not of pure pectose, but of a compound of pectic acid with some of the copper of the reagent.

Pectine swells up and dissolves in water, forming a viscous liquid which soon becomes a jelly. It exists in considerable quantity in many ripe fruits and in some mucilages. It gives no precipitate with the neutral acetate of lead, but is thrown down by the basic acetate in the form of white flocculi. If it is boiled for some hours in water, it is converted into parapectine, which is precipitated by neutral lead acetate. Further boiling with dilute acids converts it into metapectine, which is precipitated by barium chloride.

The acid series shows peculiarities similar to those of the neutral one. Its most insoluble member is pectic acid, which will not dissolve in water, alcohol, or acids ; it forms soluble pectates with alkalis, and insoluble ones with the metals of the alkaline earths, of which calcic pectate is the most widely distributed. It dissolves in solutions of alkaline salts, such as the carbonates of sodium and potassium, alkaline phosphates and most organic ammoniacal salts, forming with them double salts which gelatinise more or less freely with water. Its solution in alkaline carbonates is mucilaginous, but when ammonic oxalate is the solvent it is perfectly limpid.

The member at the other end of the series is metapectic acid, a body with an acid reaction, freely soluble in water and forming soluble salts with all bases, especially those of calcium and barium, which precipitate pectic acid. Metapectates are coloured yellow when they are warmed with an excess of alkali. This body and its compounds are probably very prominent in the gums; when acted on by dilute sulphuric acid they split up, one of their products being a crystallisable dextro-rotatory sugar which is apparently arabinose. Metapectic acid does not form a jelly, its solutions always being limpid. 
The two series of pectic bodies are closely related to each other, for by the action of heat, acids, and alkalis the various members of both can be prepared from pectose. The final product of the action of the reagents is the freely soluble metapectic acid.

The cellulosic and pectic constituents of the cell-wall show considerable differences of behaviour. The former are soluble, the latter insoluble, in Schweizer's reagent; when oxidised with nitric acid the former yield oxalic, the latter mucic acid. The celluloses when partially hydrated stain blue with iodine ; the pectic bodies give no coloration with this reagent. They behave differently also to staining reagents and to dilute acids and alkalis.

Cellulose, as we have seen, is a member of the group of carbohydrates. Various writers are not agreed as to the relation of the pectic bodies to this group, some holding that their reactions separate them from it entirely, while others contend that they are closely connected with it, if they do not actually belong to it. It has been suggested that they are carbohydrates chemically combined with acids. Like cellulose they yield some form of sugar when hydrolysed with dilute mineral acids.

All unchanged cell-walls contain a varying quantity of water, and various views have been advanced as to the way in which the latter is held by the other constituents. It is probably not in a state of chemical union, as the quantity present can be easily increased or diminished.

Naegeli suggested that the wall contained particles of solid matter or micella, of crystalline form, the long axis of the crystals being arranged at right angles to the surface of the wall. He supposed each micella to be surrounded by a thin film of water. Every cell-wall is thus under some considerable internal strain, the micellæ attracting each other and tending to squeeze out the water. The latter, on the other hand, tends to separate the micellas

According to Strasburger, the particles of solid matter are arranged in a network, water occupying its meshes. 
On either hypothesis the quantity of water is capable of considerable increase or diminution, and the wall can be made to swell up by causing it to imbibe more fluid. This can be brought about by exposing it to the action of strong mineral acids, such as sulphuric acid. The water is held, however, by the solid particles with very great tenacity.

A different view of the composition of cell-wall has been advanced in recent years by Wiesner. He holds that the substance of cell-wall as it is first formed consists of rows of granular bodies which he terms dermatosomes; these are connected together by protoplasm which surrounds them.

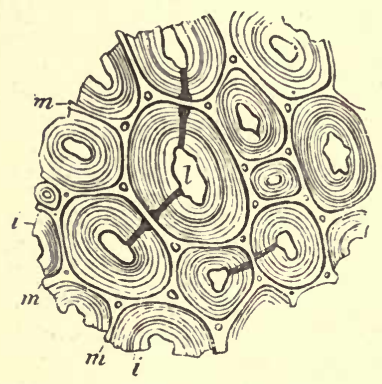

Fig. 42.-Thickened Cells of Wood, showing StratificaTION. (After Sachs.) On this hypothesis the cell-wall is living while young and growing. The protoplasm thus exists between particles of solid matter and holds water in its substance.

The thickening which always supervenes to a greater or less extent upon the first formation of the cell-wall is brought about by the protoplasm in a way similar to that of its original construction. Layers composed like the original one are continually secreted by the protoplast and are deposited upon its exterior in apposition with the wall already there. Hence walls which have a perceptible thickness show a certain stratification, which is most easily seen in transverse sections (fig. 42). When several such layers can be distinguished it has been found that pectic bodies are prominent in the layers furthest from the protoplasm and cellulose in those nearest the interior of the cell. The action of the protoplast is frequently irregular, so that the thickening layers are often seen as bands of various form, giving the surface of the membrane particular patterns, thin and thick places alternating in various ways (fig. 43). These are seen most conspicuously in the walls of the vessels of the wood. 
In cell-walls which have undergone considerable thickening the membrane shows a marked differentiation. The centre of the wall is found to possess a chemical composition very unlike that of the thickening layers. It marks

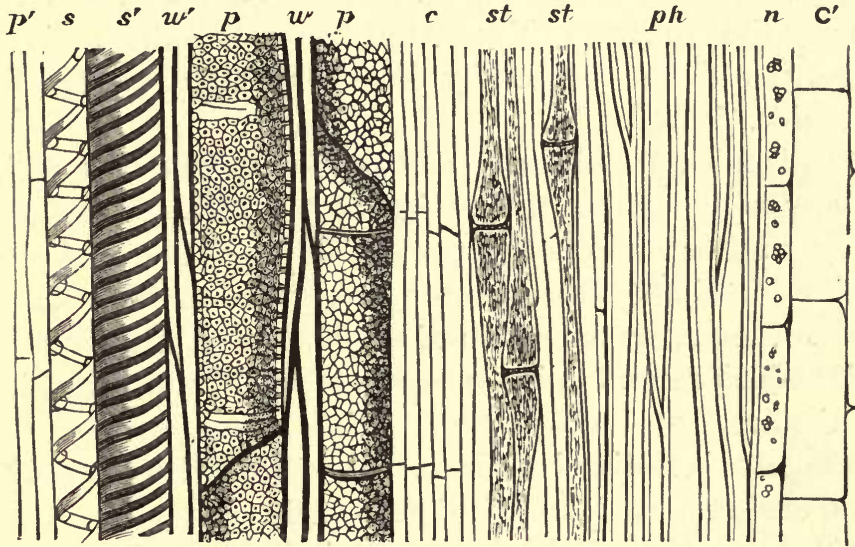

Fig. 43. - Longitudinal Section of Vascular Bunde of Sunflower Stem. (After Prantl.)

$p^{\prime}$, pith; $s, s^{\prime}$, spiral vessels ; $w^{\prime}, w$, wood-cells; $p, p$, pitted vessels ; $c$, cambium; st, st, sieve-tubes; $p h$, fibres; $n$, bundle sheath; c, cortex.

off the limits of the cells, occupying the position of the original thin membrane and looking as if it were the basis on which the thickening layers have been deposited. When a piece of tissue is warmed gently with a mixture of potassic chlorate and strong nitric acid, this layer dissolves and the cells become separated from each other. It has by certain writers been termed the intercellular substance and by others the middle lamella (fig. 44). Though it is most easily seen in thickened cells, it is probably not confined to

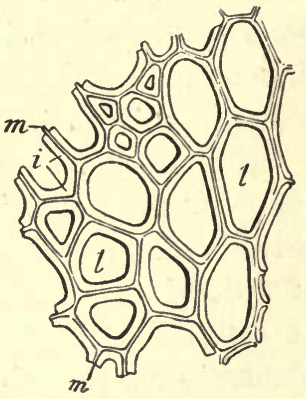

Fig. 44.-Thickened WoodCElls, SHowixg Middle Lamella. (After Sachs.) them, but exists in all cell-membranes, even when they are 
very young. Treatment with the reagent mentioned will disintegrate the tissue of even the growing points of stems and roots, and will cause their cells to become isolated. A thin layer of this nature must therefore exist even in the primary cell-wall. It is added to materially, however, during the growth in thickness of the walls, and in many cases it can be seen easily under a comparatively low magnification.

This middle lamella is composed of a material which is very unlike that of the rest of the cell-wall. It dissolves readily under the action of potassic chlorate and nitric acid which do not affect the inner layers of the membrane. It resists completely the action of sulphuric and other mineral acids which cause the inner layers to swell and ultimately to dissolve.

Recent investigations have led to the view that it is composed of a calcium salt of pectic acid.

Whether the primitive cell-wall is homogeneous or not is uncertain. If it is, it must be regarded as being formed of an intimate mixture or perhaps of a compound of cellulose and pectose. At a very early period in its development the middle lamella becomes differentiated, owing possibly to the conversion of the pectose into pectic acid and the interaction of the latter with some salt of calcium derived from the cell-sap which infiltrates the wall. The calcium pectate becomes deposited in this way halfway between the contiguous cells which are separated by the particular membrane in which the change is taking place.

If the cell-wall is not at first homogeneous, we must suppose that the original thin membrane is composed of three layers, a central one of calcium pectate, on each face of which is a layer composed of the mixture or compound of cellulose and pectose. We never find, even at the moment of cell division, that the membrane is formed of calcium pectate only.

It is possible to explain the growth in thickness of the middle lamella on either hypothesis. It is clear that the 
wall is the seat of a considerable chemical change which affects its whole substance, though the degree, and possibly the character, of the change may vary in the different layers of which the wall is built up.

Not infrequently it is noticeable that the intercellular spaces contain small concretions of various form, which consist of the same substance as the middle lamella. This is scarcely to be wondered at, as, when the intercellular spaces are formed by the splitting of the cell-wall, the region of the middle lamella, which is the central part of the membrane, must abut upon the space formed in the rupture. The calcium pectate which is formed or deposited in the central region, and which causes the thickening of the middle lamella, may well exude to a certain extent into the intercellular space that has been formed.

In such parts of the framework of a well-differentiated plant-body as need considerable rigidity, a conversion of cellulose into lignin takes place. This material is found conspicuously in the walls of wood-cells and sclerenchyma. It is formed in the substance of the cell-wall, and in partially lignified membranes the lignin can be dissolved out by appropriate reagents, leaving a cellulose basis. In its chemical characters lignin differs remarkably from cellulose. It does not stain blue with iodine and sulphuric acid, but can be recognised by its property of becoming red when treated with phloroglucin and a mineral acid, or yellow with anilin chloride under the same conditions. Its physical properties are also different, and bear a definite relation to the function of the tissue as a medium for the transport of water. It has no extensibility, nor can it absorb water and swell as can unaltered cell-wall; on the other hand, it allows water to pass through it with great rapidity and ease.

Lignin is probably not a definite chemical compound, but a mixture of substances successively formed from the cellulose.

Walls containing it subserve a double purpose. Its 
physical properties render it particularly adapted to serve as the material of which the tissues conducting the stream of water are composed. Its lack of flexibility or extensibility makes it suitable for the securing of rigidity in tissues or structures needing considerable power of resistance to winds or storms. It is thus a valuable material in the construction of sclerenchyma.

The protective tissues show a different modification of the original structure. In the simplest cases we have seen that the degree of protection secured is slight, and evidently only transitory. The epidermis is, in these cases, the seat of the changes which may be observed. The cells show their walls sometimes very materially thickened on the

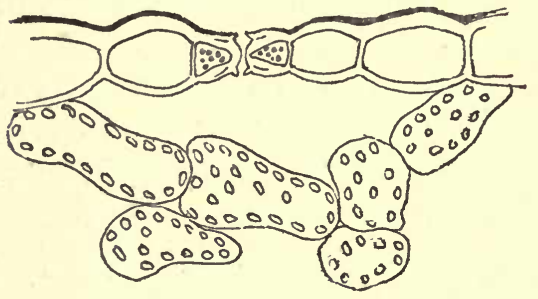

Fig. 45.-Section throvgh Epideruis of Leaf, SHOWING THE OLTER WALLS Materialy Thickened. exposed sides (fig. 45), though the thickness varies in different cases. Layer after layer of substance is deposited upon the original wall in these regions, the other parts of it remaining thin. The thicknèss itself secures a certain amount of protection against cold, but to prevent absorption or dissipation of water or of gases by these membranes, a chemical change ålso is brought about. The outer layers of the wall undergo a process known as cuticularisation, which generally extends about halfway through its thickness. This change in the outer walls of numbers of contiguous cells renders it possible to strip off from such a tissue a piece of apparently structureless membrane which is technically called the cuticle, and which consists of nothing more than these altered layers of the outermost walls of the contiguous cells. The alteration of the chemical character of this membrane in forming the cuticle of the epidermis is due to the transformation of its cellulose or pectose 
constituents into a substance known as cutin. Its properties are very different from those of the original cell-wall; it is but slightly permeable by water, and it is not easy for gases to pass into or through it. This difference of physical property is accompanied by characteristic reactions ; it stains yellow instead of blue when treated with iodine and sulphuric acid, and becomes brown under the action of strong alkalis, such as caustic potash.

More efficient and prolonged protection is afforded by the formation of sheaths of cork, certain layers of cells being differentiated as meristem tissue for the continued production of this material. The walls of true cork cells are thin, but the presence of cutin is a conspicuous feature

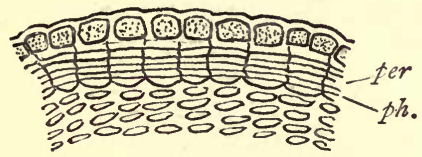

Fig. 46.-Outer Portion of Contex of Young Twig of Lime.

per, cork layer.

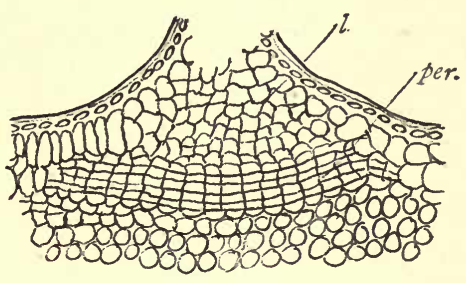

Fig. 47.-Section of a Lenticel.

$l$, lenticel; per, cork layer.

in them. They are very regular in form, and are closely arranged together without any intercellular spaces (fig. 46). Coming as they do between the exterior and the metabolic tissue of the cortex of stems, thus cutting off the intercellular space system of the latter from access to the air, they are usually penetrated by special structures known as lenticels, which are made up of corky cells very loosely arranged, and which consequently set up the communication needed (fig. 47).

In the corky cell-wall the cutin is frequently associated with a certain amount of lignin.

The thin corky walls possess almost exactly the same physical properties as the thickened cuticle of the epidermis, a fact which affords evidence that the primary meaning of both is the same. 
Like the substance of the middle lamella, both lignin and cutin are soluble in warm nitric acid containing potassic chlorate.

In some cases the cell-wall of the epidermal protoplasts is impregnated with various matters that do not proceed from its own disintegration. Among these are various fatty bodies, while wax is sometimes very conspicuous. The bloom of such fruits as the grape and the plum is composed of very fine waxy particles; the impregnation in their case having been so great that certain particles have passed beyond the walls and formed a layer on the

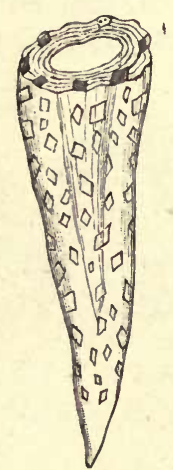

Fig. 48.-Crystals of Calcium Oxalate in Wall of Cell OF THE BAST OF Ephedra.

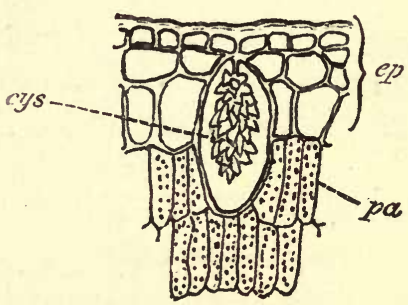

Fig. 49.-Section of Portion of Le.tF of Ficus, sHowing Cystolith (cys) in LARGE CELL OF THE THREeLAYERED EPIDERMIS $(e p$.

outer surface. The leaves of the wax-palm show an even denser deposit.

Mineral matters are also of frequent occurrence in the cell-wall. The chief of these are salts of calcium, usually the oxalate, but often the carbonate. Some cell-walls show a copious deposit of regular crystals of one of these-such are the cells of the bulb scales of the onion, the fibres of the bast of Ephedra and others (fig. 48). In many plants copious deposits of silica are formed in the cell-wall, especially in the epidermal cells of the Equisetacea, and in those of the cereal grasses. The value of this deposit to the plant is not very evident; it appears at first sight to be 
an adaptation enabling the plant to remain upright, but it is found that its absence does not render the grasses more liable to fall.

Some cells of the epidermis of certain plants, especially among the Nettle family, contain curious ingrowths of cellulose, in which there is a very large deposition of calcium carbonate. They are known as cystoliths (fig. 49).

The cell-walls of certain regions of particular plants are transformed into mucilage. This material is especially prominent in the large brown seaweeds, particularly the Fucacea, where it forms the bulk of the internal tissue. It occurs also in certain layers of the seed-coat of such seeds as linseed, and in certain regions in the sporocarps of Marsilea. It is of assistance in the dissemination of the spores of this plant, and possibly has a similar value in the cases of such seeds as contain it. It differs from cellulose by absorbing water greedily, and swelling up considerably. It gives a blue colour with iodine and sulphuric acid as cellulose does, differing from the latter chiefly in the ease with which the absorption of water is brought about. It is not clear at present whether mucilage is derived from cellulose only, or whether the pectoses take part in its composition, though the latter is probable. The gums are closely related to mucilage, and seem to represent a further disintegration of the cell-wall in that direction. Many of the gums yield derivatives much like those of pure pectic bodies, which suggests that their affinities are rather with the latter. In all probability, however, they are all mixtures of the two classes of constituents.

We see thus that in the construction of the skeleton of a complex plant, while its basis is the cell-membranes of the several protoplasts, which at first form an almost homogeneous tissue, not only does differentiation take place in the direction indicated in the last chapter, but this differentiation is accompanied by changes in chemical, physical, and mechanical properties, which fit the definite tissues formed for the functions which fall to them. 
Temporary structures possess a very different chemical composition from permanent ones. The transitory cuticle gives place to the more permanent cork, and this becomes strengthened by the introduction of sclerenchymatous elements as the cork formation becomes continuously more deeply seated. The strengthening tissue varies similarly ; the walls of collenchyma, though thickened in a particular way, are not chemically changed in the same manner as those of sclerenchyma or woody tissue, for their cellulose undergoes no conversion into, or impregnation with, lignin. The fibres of the bast differ from those of the wood in the same particulars. 


\section{CHAPTER IV}

THE RELATION OF WATER TO THE PROTOPLASM OF THE CELL

WHEN we regard the arrangement of protoplasm in the cells of the plant, or observe the environment of the freeswimming protoplast, we notice especially its very close relation to water. The naked zoospore is naturally saturated with the latter, being in the fullest contact with it. Unicellular plants which are not actually immersed in it are generally to be found in more or less moist situations, where they continually obtain supplies from dew or rain. Indeed in times of drought when moisture is not supplied to them they are seriously injured. The young cell which is clothed with a cell-membrane speedily shows a tendency to accumulate water in its interior ; gradually drops appear, which lead ultimately to the formation of a vacuole, which is always full of liquid. In a plant which consists of a complex of cells, such a vacuole is found in every adult cell so long as it is living. The healthy protoplasm is thus always in contact with water. Indeed the molecular constitution of protoplasm, as far as we know it, lends itself to this relation, for the apparently structureless substance is always saturated with it. It is only while in such a condition that active life can exist; with very rare exceptions, if a cell is once completely dried, even at a low temperature, its life is gone, and restoration of water fails to enable it to recover.

The constancy of the occurrence of the vacuole in the cells of the vegetable organism is itself very strong evidence that such cells are dependent upon water for the maintenance of life. The cell-wall, though usually permeable. 
yet presents a certain obstacle to the absorption of water, and so even those cells which are living in streams or ponds usually possess a vacuole. Cells without a membrane, such as the zoospores already many times mentioned, can more readily absorb water from without, and hence they are not vacuolated to the same extent as are those which possess a cell-wall; indeed many of them have no vacuole. This cavity when present being always filled with liquid, the protoplasm of the cell has ready access to water, as much so indeed as the protoplast which possesses no cell-wall. The vacuole contains a store which is always available.

The quantity of water which a vacuole can contain is very small, and as the needs of the protoplasm are somewhat extensive, a need arises for the continual renewing of its supply. This is evident when we consider that the protoplasm draws its nutriment eventually from the water, and that it must return to it such waste products as it gives off. Its oxygen must be drawn from the same source, for this gas can only pass into the interior of a cell by entering into solution in the liquid which it contains. In cells which are deep-seated this need can only be supplied by a slow passage from cell to cell of the gas which has been dissolved by those abutting upon a free surface. Similar considerations apply to the elimination of the carbon dioxide which accompanies the respiratory processes.

The life of a plant is consequently very intimately connected with the renewal of the water which the cells contain. Fresh liquid must be taken in, and that which is already there must be to a certain extent removed; the plant demands in fact a kind of circulation of water, and this becomes the more imperative as the mass of the plant increases, with the possible exception, however, of those massive plants whose habitat is marine.

In examining the way in which this circulation is set up and maintained, it is first necessary to inquire into the 
nature of the process by which water makes its entry into a cell. This is based upon a physical process which is known as osmosis.

When two fluids of different densities, such as water and syrup, are separated from each other by a homogeneous permeable membrane, they will tend to pass through the latter in both directions till there is a mixture of the two of equal density on each side of it. We shall thus have a stream of water passing through the membrane to the syrup, and a stream of syrup similarly passing to the water. The rate of flow of the two streams will not be the same however, and the first result will be a considerable increase of the volume of the liquid upon the side of the membrane in contact with the syrup, owing to the greater amount of water that will have passed through.

A convenient form of apparatus to exhibit this process of osmosis is represented in fig. 50. It consists of a bladder fastened to the end of a narrow tube which is immersed, as shown, in a vessel of water. The bladder and part of the tube are filled with syrup, and the height at which the latter stands in the tube is noted. After some time the contents of the tube will be increased in consequence of the entry of water being greater than the escape of syrup, and

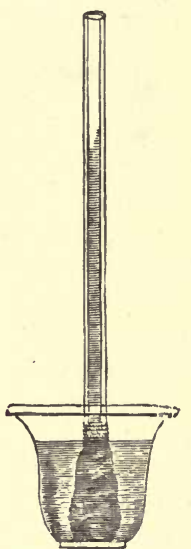

Fig. 50.-Apparatus TO SHOW THE PROCESS OF OsMOSIS. the liquid will stand at a higher level in the tube. If the positions of the water and the syrup had been reversed, the liquid would have fallen in the tube, showing that the greater osmotic stream was in the opposite direction.

The relative difference in the rate of the two streams will vary with the concentration of the syrup.

Other substances than sugar have a similar power of setting up osmotic currents, which indeed is especially pro- 
minent in those which are crystalline in character, though it is not confined to them. Solutions containing different substances in equal degrees of concentration do not, however, possess equal osmotic powers; each one has its own special ability which is often spoken of as its osmotic equivalent. With any particular osmotic substance, however, the osmotic efficiency varies with the concentration of the solution.

Though the process of osmosis as illustrated in the experiment just described is far simpler than that which we have reason to believe takes place in the vegetable cell, we can apply it to explain the original formation of the vacuole. Consider the case of a young non-cuticularised

B
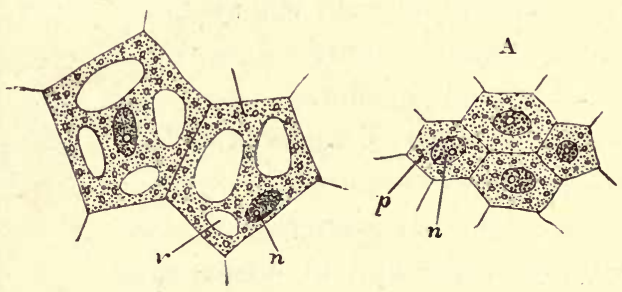

Fig. 51.-Vegetable Cells.

A, very young; B, a little older, showing commencing formation of vacuole. $p$, protoplasm; $n$, nucleus ; $v$, a vacuole.

cell of the external layer of a plant which is immersed in water. It is full of protoplasm, and limited or clothed by a cell-membrane which is permeable more or less readily by water. The protoplasm is saturated with water, but there is no separate accumulation of the latter in its interior. Part, at least, of the cell-wall is in contact with water on the outside. The protoplasm is actively living, and in the course of the chemical changes which are incident to vital action certain substances are produced by it, which, like the syrup in the experiment already described, have an affinity for water, or, to use a more technical phrase, have a fairly high osmotic equivalent. Water consequently passes into. the cell, at first only in such quantities as to distend it somewhat. As the process goes on, more liquid is taken up 
than can be stored in the molecular interstices of the protoplasm. Drops consequently appear, and these gradually run together until a distinct though small vacuole, and later a number of such vacuoles, are apparent in the protoplasm (fig. 51). These soon run together as the amount of water still increases, and after a time the protoplasm forms a layer round the cell-wall, enclosing a single large cavity in which the surplus liquid is held (fig. 52).

But, as has already been said, the process is not a simple physical one. Though the conditions of the first experiment are approximated to, they are not altogether realised. The syrup in the bladder finds its representative in the osmotic substances formed by the protoplasm and dissolved in the water in its substance; the water outside the cell is much the same as the water in the outer vessel. But there is a great difference in the membrane. The bladder of the experiment is replaced by a

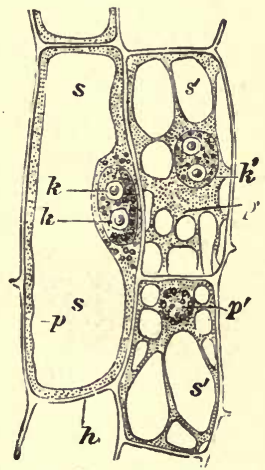

Fig. 52.-Adult Vegetable Cells. $\times 500 . \quad$ (After Sachs.)

$h$, cell-wall; $p$, protoplasm ; $k k$, nucleus, with nucleoli; $s s^{\prime}$, vacuoles. film of cell-wall substance, which we may speak of in general terms as cellulose, and this is lined by a delicate coating of protoplasm. This again is not homogeneous, but has on its surface, which is adpressed to the cellwall, a very thin dense layer which forms a kind of membrane known as the plasmatic membrane. As soon as the vacuole is recognisable its cavity becomes lined by another similar membrane, and between the two lies the nearly homogeneous protoplasm. These plasmatic membranes are immediately formed as soon as the surface of the protoplasm comes into contact with water. The membrane of the cell, therefore, through which osmosis must take place, is composed of four different layers. In the. experiment we have assumed that the outer liquid was pure 
water; this is not, however, the case with the fluid in which the plant is living, for all such water contains a large number of various inorganic salts dissolved in it, though of course the concentration of these salts is extremely small. While all the layers of the cell's membrane are permeable to water, they are not at all equally so to the salts which it contains. In such a weak solution these can pass freely through the cell-wall, but the plasmatic membranes of the protoplasm offer a variable resistance to their passage further. A further experiment will show a very important modification of the process depending on this property of the protoplasm, and demonstrating that the entry of both water and its dissolved saline contents into the cell is very largely under the regulation of the latter, when what is practically a dilute saline solution is presented to it.

Take a cell of the cortex of a plant and put it into contact with a liquid of higher osmotic power than that which is contained in its own vacuole; for instance, a solution of common salt of about 10 per cent. concentration. Watch its action on a slide under the microscope, and let the salt solution be coloured with some vegetable dye which will not injure the living substance. As the salt solution reaches the cell, the protoplasm of the latter gradually retreats from the walls (fig. 53), at first at the corners and then all round the sides, till it appears as a rounded or irregular mass in the centre. The salt solution has abstracted the water from the vacuole, and the protoplasm, relieved of the pressure outwards caused by the liquid in the latter, has shrunk away from the walls. The outward stream has been accompanied to a certain extent by an inward one, as in the first experiment. The coloured salt solution will be visible inside the cell-wall, betweeis it and the protoplasm. There has been an osmotic stream therefore through the cell-wall inwards. But it will be seen that the colour will not penetrate the protoplasm, which in fact retreats before the coloured salt solution. The latter has no power to pass the external plasmatic 
membrane, even in the condition of dilution which must result from its mixing with water which has been withdrawn from the vacuole. If now the salt solution is replaced by water, the latter is gradually attracted again, of course osmotically, into the cell. It passes through the whole thickness of the protoplasm, the vacuole is reestablished, and the protoplasm again comes to line the cell-wall, pressed against it by the water.

The protoplasm by means of its plasmatic membranes thus can oppose the passage through it of various osmotic

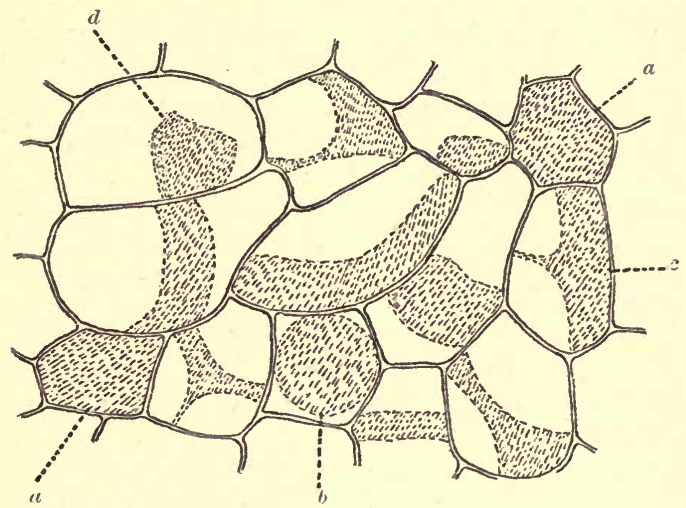

Fig. 53.-Cells of Parenchyma cndergoing Plasmolysis $a, b, c, d$ represent successive stages. The dotted area in each cell represents the protoplasm.

bodies with which it may be brought into contact, though it allows the water in which they are dissolved to permeate it freely. In the experiment just described the strong salt solution failed to pass through the external plasmatic membrane; the re-entry of the water into the vacuole showed that the internal one prevented the osmotic substances, originally present in the water which the cell contained, from escaping in the issuing osmotic stream. - These substances must have been left behind, or there would have been no osmotically active material to draw the water back, when it was allowed to replace the salt solution. outside the cell. 
That this behaviour is dependent on the vital activity of the protoplasm can be shown by repeating the experiment after killing the living substance by a short immersion of the cell in alcohol. Then the process of osmosis goes on exactly as in the first experiment described. The salt solution penetrates into the vacuole as if only a cellulose septum were present, the dead protoplasm exerting no regulating influence.

We must not conclude from this experiment that inorganic salts in all degrees of concentration are kept from entering the cell by the protoplasmic membranes. If extremely dilute solutions are employed, the protoplasm permits their passage together with a certain appropriate amount of water. Similarly, extremely dilute solutions of bodies found in the fluid of the vacuoles, the so-called cell-sap, can make their way out of the cells. The protoplasm exerts a definite regulating influence, however, upon both the entry and the escape of these different substances.

The modified osmosis which is thus the mode of entry of water into a cell containing no vacuole, and which causes the growth or completion of the vacuole after its first appearance, continues after its formation is finished. This can be studied most favourably in aggregations of cells, such as we find in the cortex of a stem or the loose mesophyll of a leaf, as in such cells there is a more evident renewal of the water of the vacuoles than in those of tissues which are surrounded by liquid. In such tissues as those just mentioned we can demonstrate with ease what is more difficult to detect in the others, that not only is water admitted to the cells, but it is also given off from them. This does not depend on osmosis in the stem or leaf, but is due to evaporation, which takes place from the surfaces of the cells abutting on the intercellular spaces, whence the watery vapour is exhaled through the stomata, or, in the case of a woody stem, through the lenticels. In a cell surrounded by water such removal must depend upon osmotic currents. 
This removal of water occasions a need for a continuous replenishment of the liquid in the vacuoles, which is brought about by the same modified osmosis which has been described. We can see that this process must be continually taking place in a complex of succulent cells. If we consider two which are contiguous and are separated from each other by a common cell-wall, it is evident that unless the proportion of water to osmotic substances in the vacuoles of both is the same, osmotic currents will flow from one to the other till this equilibrium is reached. Any disturbance taking place in one cell of a complex will hence spread from cell to cell until the composition of the fluid contents of them all is uniform. When we consider the differences, sometimes very slight, sometimes more extensive, which are continually taking place in the metabolic activities of the separate cells of a community, it is evident that, so long as life lasts, osmotic currents of this lind must be continually passing from cell to cell in various directions, and frequently at very different rates.

Evaporation from a cell into an intercellular space must lead to a certain increase of the concentration of the solution of osmotically active substance in its vacuole. This then attracts water from the contiguous cells, and consequently, independently of metabolic changes affecting the quantities of such osmotic bodies, evaporation itself must help in causing movements of water from cell to cell.

The quantity of these osmotic substances which are present in any particular cell will depend upon the behaviour of the protoplasm from time to time. Such substances are usually being continually produced in all growing cells, and in most others in which chemical changes are proceeding. Hence such cells are continually absorbing water, and are consequently so full that a certain stretching force is exerted on the cell-wall which bounds them. Cells in such a condition are called turgid, and the condition itself is known as turgor or turgescence. The equilibrium which is attained by such a cell is reached 
when the distension caused by the entering osmotic stream is balanced by the elastic recoil of the extensible cellulose wall. In some cases the tension set up in a tissue by the turgescence of the cells is sufficient to force the water, by a process of filtration, through the walls of the outermost ones, so that it escapes in drops or in a slow stream. This may often be seen on the edges or apices of blades of grass in the early morning. It is of great use also in forcing water into the axial woody cylinder of roots, as will appear later. Occasionally the turgescence becomes so great as to lead to rupture of the cell-walls, as is the case in some pollen grains, and sometimes in certain fleshy fruits.

That the condition of turgescence in cells is attended by a stretching of the cell-walls can be seen by taking a piece of a plant which is turgid, such as the stalk of a rhubarb leaf, and, after carefully measuring its dimensions, steeping it for some time in a ten per cent. solution of common salt. On removing it, it will be found to have become flaccid, and a remeasurement will show that both its length and thickness have diminished. Turgescence is not, however, due simply to physical causes; the protoplasm which lines the cells has a regulating influence over the passage of the water into and out of them. When a turgid pulvinus of such a plant as Robinia or Mimosa is stimulated by rough handling of the leaf, the latter falls backwards from its expanded position, and the fall is found to be due to the escape of water from the cells of the lower side of the pulvinus. The original state of equilibrium has been disturbed by the shock to the protoplasm administered by the stimulation, and the latter allows or compels the water to pass outwards.

The active influence of the protoplasm is seen also in another class of phenomena. Certain structures known as nectaries occur conspicuously in many flowers. They are aggregations of cells of a particular kind which exude a sugary fluid upon their surface. The liquid in the cells contains a little sugar, and this weak solution is capable of passing through the protoplasm, not by osmosis, but by 
a kind of filtration. Its concentration is usually increased by subsequent evaporation of the water in which it is dissolved, so that the secretion when collected has a distinctly sweet taste. When the petals of certain flowers bearing these nectaries are cut off, and their cut ends immersed in water, the glands continue for some time to exude the nectar. There can be no question here of a gross filtration of water under pressure through the tissue, as there is no such pressure acting on the base of the cut petal. The protoplasm causes a stream of water to flow into the cells of the gland by producing osmotic substances inside them, in this case chiefly sugar. The turgescence thus set up in the gland cells exerts a strong hydrostatic pressure on the limiting membranes of these secreting cells, which ultimately so stimulates the protoplasm as to cause the sugary solution to exude upon their free surfaces. We can discriminate between two forces at work in the excretion of the nectar. The absorption of water by the gland cells is due to osmosis; the excretion from them on to the exterior of the gland is more a question of filtration under pressure from the turgid cell. This is shown by the fact that if the surface of the gland is carefully dried, the exudation shortly recommences. Osmosis is not possible under these conditions. If the gland is killed by alcohol, the sugar already there is retained in the cells, and no exudation of nectar, or even of water, takes place.

The vital activity of the protoplasm is thus seen to be intimately connected with the presence of water in its substance. The importance of the ready access of the latter is seen further from other considerations. We have incidentally alluded more than once to the fact that the liquid concerned in these osmotic currents is not pure water only, but should rather be regarded as an extremely dilute solution of various salts. Though the protoplasm opposes the passage of anything like a strong solution of inorganic salts, it allows very dilute ones to enter the cell; much as it does pure water. In this way the slowly dif- 
fusing stream brings to the protoplasm of each cell the inorganic materials which are absorbed from the earth, and enables the matters elaborated or formed from them by the protoplasm to pass from cell to cell. The feeding or nutrition of the various cells, together with the construction of the substances which minister to that nutrition, is thus dependent on the transit of fluid about the plant in the way described. The access of various gases is similarly made possible, for these are dissolved in the liquid stream. The oxygen upon the presence of which life depends is thus transported to each cell, and the carbon dioxide of respiration is removed from the seats of its liberation.

The condition of turgescence is necessary also for growth, and for various movements of different parts, enabling them to adapt themselves to varying conditions of their environment. Some plants, particularly those which are aquatic in habit, and such parts of terrestrial plants as contain but little woody tissue, are dependent on the turgescence of their cells for the rigidity which enables them to maintain their position in the medium in which they live. The maintenance of the turgid condition of the cells is further of the highest importance in enabling: the interchange of water between contiguous cells to take place as freely as possible, and without intermission. Flaccid cells do not effect such interchange with sufficient readiness. Flaccidity of an organ is attended by a partial collapse of the tissue, which involves a diminution of the volume of its intercellular spaces, and hence often a serious interference with its processes of gaseous interchange, particularly respiration.

The importance of the water supply, and indeed its necessity to the plant, explains the existence of certain subsidiary mechanisms for its absorption and storage which are occasionally met with. These will be considered in detail in a subsequent chapter, but a few of such adaptations may be noticed here. We frequently find particular aggregations of cells set apart for storage of 
water. The epidermis of certain parts frequently subserves this purpose, and many plants possess a considerable development of aqueous tissue, variously disposed, which forms a similar storehouse. The cells of this tissue contain little else than water, and thus serve to supplement the vacuoles of the ordinary cells. In plants that inhabit dry arid soils such as sandy deserts there are often other adaptations relating to water storage. Such plants are often covered with large bladder-like hairs which hold a considerable quantity of liquid. Plants which are exposed to conditions threatening too copious evaporation are generally furnished with a very prominent cuticle tending to check undue escape. 


\section{CHAPTER V}

THE TRANSPORT OF WATER IN THE PLANT

We have seen that it is necessary for the life of a plant that all its living cells shall be freely supplied with water. According to the habit of life of plants the mode of supply must necessarily vary. Those which are so constituted that water finds free access to all the cells, such as the unicellular or filamentous Alga, which live in streams, pools, \&c., present no difficulty, as osmosis can go on freely in each cell, water entering its vacuole from the exterior. Sturdier plants of aquatic habit are almost equally easily supplied; the water enters by osmosis into the vacuoles of the epidermal cells, the walls of which in these plants are not cuticularised, and from them it can pass from cell to cell all over the plant-body. No force in addition to osmosis is necessary in these undifferentiated plants. Others, which have a terrestrial habitat, from the nature of their environment require a more elaborate mechanism which is found, as we have already pointed out, in the well-differentiated system of conducting tissue, composed largely of lignified vessels, fibres, and cells. Throughout all such plants a stream of water passes, entering at the roots, passing along the woody axis, and so rising up the stem into the leaves, where a very large part of it is evaporated. This stream of water is often known as the ascending sap. In addition to this comparatively rapid stream, slow currents of diffusion from cell to cell are also maintained, as in the plants of humbler type. These diffusion currents, depending mainly on osmosis between contiguous cells, have not the definite direction of the 
rapid current, and play quite a subordinate part in the supply of the whole plant with water. They are, however, supplementary to the ascending sap, and effect interchanges in regions which the latter does not immediately reach. The cortex of the axis of the plant is especially dependent upon them, as various mechanisms exist in the different regions of the stele to guard against too free an escape of water from its tissues into the cortex.

Except in some special cases the water which passes through the body of an ordinary terrestrial plant is obtained from the soil in which its roots are embedded. The soil itself is composed of minute particles of inorganic matter of very different degrees of solubility, derived originally from the breaking down of rocks, together with decaying animal or vegetable matter mixed with the inorganic constituents. This organic matter is known as humus and is of very varied composition. The soil thus consists of a loose matrix of granular character, the interspaces of which are normally filled with air. The air is in most cases mixed with a certain quantity of carbon dioxide which is being evolved from the humus constituents of the soil, and which is slowly exhaled from the surface. The interspaces are capable of containing varying quantities of water; indeed the soil may be so saturated with it that they are all full. We find soils of all conditions in this respect, from the dry sands of deserts to the mud of bogs. The water may be held with greater or less tenacity, clays and sandy soils affording instances of two extremes in that particular. When the interspaces of the soil are filled with water, the plants which it is supporting are very unfavourably placed for absorbing the liquid. By the excess of water their roots are deprived of the air which they need for purposes of respiration; their structure does not enable the absorption of water to take place all over their surfaces, as their external cells are more or less cuticularised ; they are consequently hindered and not helped by the superfluity of liquid. When a soil is properly drained, its 
interspaces are filled with air, and a delicate film o surrounds each of its particles and adheres closel This water, often spoken of as hygroscopic water: source of the plant's supply. The presence of ai interspaces supplies the wants of the root and frees the difficulties which have been pointed out.

The hygroscopic water adheres so closely particles of the soil that it escapes ordinary obs: when, however, soil that has been allowed to $d$ ordinary temperature till its interspaces are empty, is exposed to a heat approaching that water, a considerable quantity of vapour is gi to the volatilising of the hygroscopic films.

The difficulty of the eutry of the water in of the outermost layers of the young roots

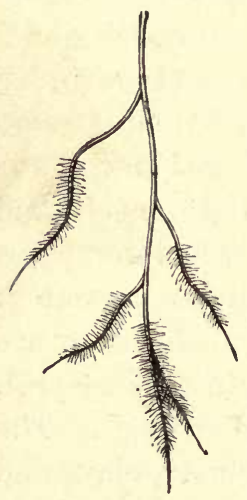

Fig. 54. - Ultimate Branches OF A Root, showing PoSITION OF ROOTHAIRS, development of a specia mechanism upon them. I form of a number of delica of the external cells, whi thin-walled hairs (fig. 54, not distributed all over ff" $\mathrm{su}_{\mathrm{s}}$ the young rootlets, but a a particular region not $f$ apex. As the delicate $b$ root grow, the root-hairs g] more being formed conti. the same distance from : is thus a continual renew. tion of hairs, which is ma as the root system exten

functional. The interspaces of the soil are penetrated by the young roots, the $1^{\text {r }}$. iner of whose growth involves a very close approximaticn of their substance to the surface of the particles of $\mathrm{w}$. wh the soil consists. The delicate hairs standing out at right angles to the surface of the roots are consequently brought into very close and intimate relations with these particles and with 
the $4 m$ of hygroscopic water which surrounds them. In so yeases the pressure between the two is so close that f 7 ticles become embedded in the membrane (fig. 55). If 1 rygroscopic film of water is thus separated from the inte of the root-hair by a most delicate pellicle of cellwall bstance, lined by an almost equally delicate layer of prot asm. The vacuole of the hair contains a somewhat acick ' l-sap, by virtue of which osmosis is set up; the osmot equivalent of the acids of the sap be considerable, the cell quickly become turgid and distended, such turges continuing so long as the conditi remain favourable. The roothairs ar $\exists r y$ numerous, and their united action c es a considerable accumulation of $\mathrm{w}, \mathrm{pr}$ in the cortex of the root, for it pas $"$ into the cells of this region by osmosi, frough the base of the hair. This, being one of the cells of the external layer impinges upon one or more of the cortis cells, which have a similar reaction to the root-hair itself. Osmotic cr snts are thus set up from every hair, sid a gradual accumulation of water tak place in the cortex of the

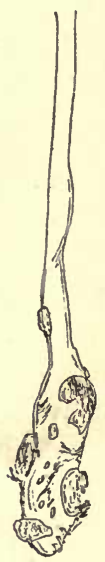

Fig. 55.-Root-HaIR IN CONTACT WITH PARTICLES OF SoIL. young root, rmaking all its cells turgescent and causing a considerabl a hydrostatic pressure in the tissue. This turgescence with its consequent pressure extends all along the axis of the joung root, though it is originally set up only by the region mhich is clothed by the absorbing hairs.

The central portion of the axis of the root is occupied by a cylindric mass which extends throughout its whole length, and widieh is known as the stele (fig. 56). It is generally marksd off sharply from the cortex, the cells of whose innermost layer, the endodermis, are often peculiarly thickened. This thickening is not, however, usually very marked in the region of absorption. At certain places 
round the periphery of the stele of the root, the woody strands (iig. 56, Sp) may be seen. These are in contact with the succulent and turgid parenchyma which has been filled with water in the way described, and consequently the hydrostatic pressure which has thus been set up is brought to bear upon the walls of the woody vessels which constitute the greater part of those strands. These form the lower portions of continuous open, or nearly open, tubes, which extend from the roots to the leaves; at the time when the absorption of the root-hairs and cortex is greatest these vessels are empty, or nearly so, and the effect of

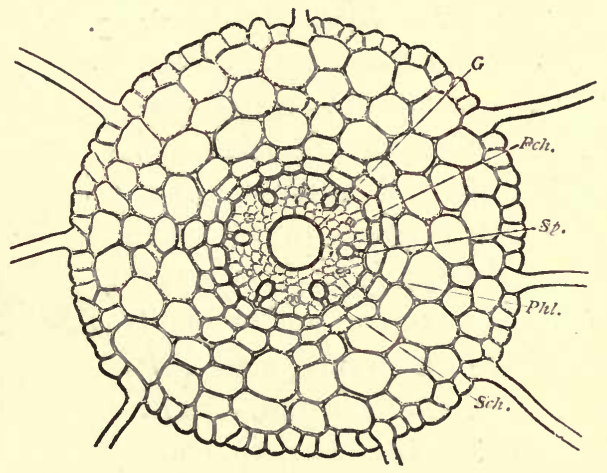

Fig. 56.-Section of Root, showing Root-hatrs abutting on the ParenChyma of the Cortex, and the Woody Strands, $S p$, of the Stele. (After Kny.)

the hydrostatic pressure on their walls is to force the water from the turgid cortex into the walls and cavities of the vessels. How the water is distributed is not fully known; we have seen that lignified cell-walls have a certain power of taking up water, and of passing it on with considerable rapidity, so that part of it may be expected to remain in the walls. Part, however, passes through into the cavities of the vessels, and in the early part of the year, before the leaves of the plant expand, they thus become filled with liquid. This filtration into the vessels tends to relieve the pressure in the cortex, and additional 
water can then be absorbed from the soil as before. The consequent increase of the turgescence is followed by further filtration into the vessels, and these two factors continually acting together, the water is made to rise gradually in the axial stele. The root-hairs and the turgid cortex, in fact, exert in this way a kind of continuous pumping action, forcing it along the axis. The force, which is the expression of the elastic recoil of the cell-walls of the overdistended cortical cells, and which is brought to bear upon their fluid contents, squeezing a quantity of liquid through the cell-walls into the vessels, is known as root-pressure, and is one of the main factors in the transport of water through the plant.

The turgescence not only leads to the rise of the sap in the axial stele, but it spreads throughout the whole of the cortical tissue of the plant, stem as well as root, reaching indeed every cell into which osmotic diffusion can take place. The action of the root-hairs is thus responsible not only for the rapid ascent of the sap, but also for the maintenance of turgidity outside the region supplied by the ascending stream.

The stele of the root is directly continuous with that of the stem, and though the disposition of the woody elements is somewhat different in the two regions, there is no doubt that

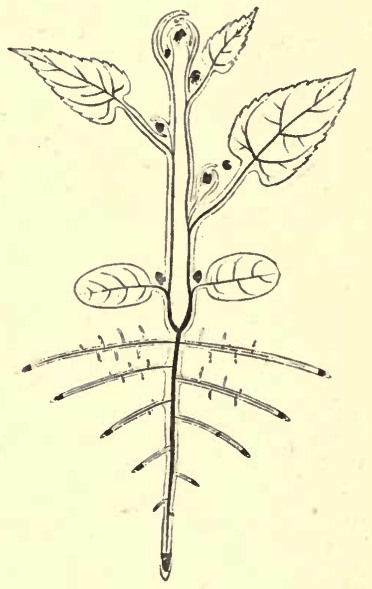

Fig. 57.-Diagram showing Course of the Vascular Bundles in a DicotyleDonous Plant. they also are continuous throughout (fig. 57). The stream of water consequently passes up the woody tissue of the stem so long as the cells are living. The stream in young plants passes along the whole substance of the wood; which in most cases forms a central mass of some size. 
In herbaceous plants the bundles do not usually form a continuous cylinder, but are more or less isolated in their course. In old trees the water-conducting area is limited to the outer regions of the central woody mass, which are known as the alburnum or sap-voood. The central portion of the wood is dead, and the cell-walls are often very much altered in chemical composition. This region is known as the duramen or heart-wood; it takes no part in the conduction, the tissue always remaining dry.

The vascular bundles are seen to be continuous from the axis to the leaves, where they are no longer found arranged in a cylindrical manner, but are disposed in

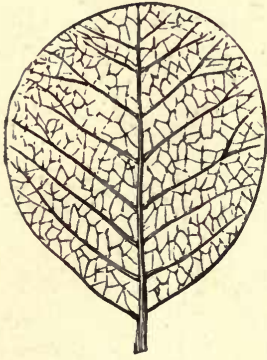

Fig. 58.-VAScular Bundles (Verns) of LeaF. various ways as a much-branched network (fig. 58). The separate ramifications are known technically as veins, and they are distributed in the various ways known, largely through the method of branching of the leaf axis. The latter, however, with very rare exceptions, is flattened or winged throughout the whole or part of its length, and the wings or flattened portions are supplied with veins continuous with those of the branched or unbranched axis. The vascular tissue, therefore, if traced from below upwards, is seen to exhibit a separation of its constituent bundles, which continually appear to subdivide until they form a series of delicate ramifications of considerable tenuity which permeate the whole of the flattened portions of the leaves or other parts. The tenuity of the ultimate endings of the vascular bundles is attended with certain changes in the character of the constituent cells, but they remain woody and irregularly thickened as they are lower down in the axis. In the leaves these endings of the bundles, which are sometimes free, and sometimes disposed in the form of an open network, are surrounded by delicate parenchymatous tissue, whose cells are in immediate contact with the woody ele- 
ments, as they are in the root (fig. 59). These delicate cells are also in contact with the special parenchyma of the leaf, which is in part very loosely arranged and provided with a great development of the intercellular space system (fig. 60 ), which we have seen to be characteristic of the whole

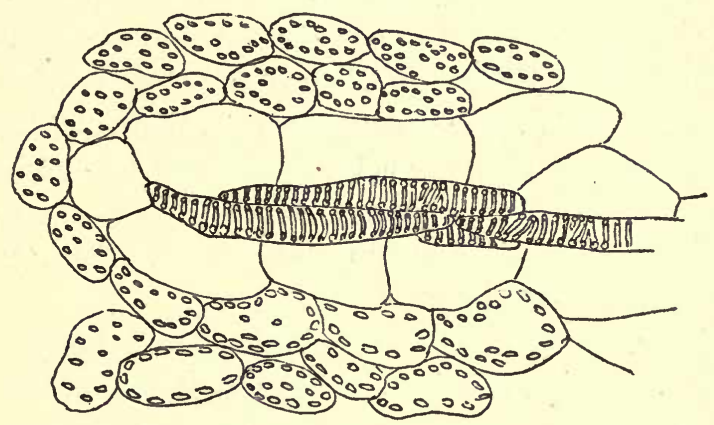

Fig. 59.-Ending of a Fibro-yascular Bundle in the PaRenchyma of a Leaf.

of the tissue of the plant. The cells abutting on the bundles are filled, like the root-hairs and the cells of the cortex, with a watery sap which contains substances possessing a relatively high osmotic equivalent. The woody

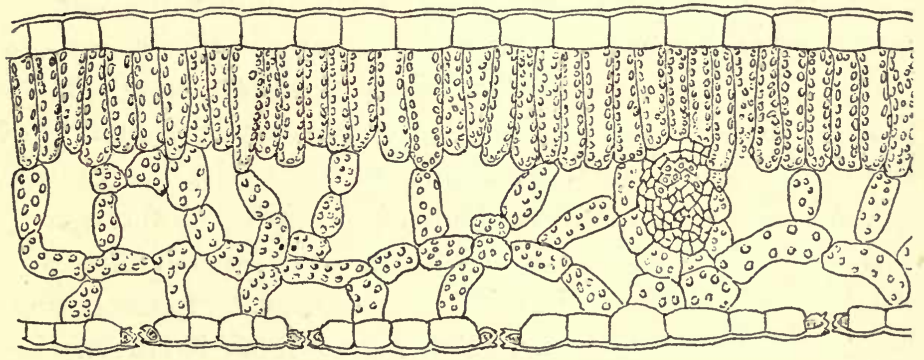

Fig. 60.-Transverse Section of the Blade of a Leaf, showing the INTERCellular Spaces of the INterior. $\times 100$.

elements of the veins are not completely empty; their walls, at any rate, are saturated with the water ascending from the roots. We have consequently here a resumption of the osmosis which we noticed to play so conspicuous a 
part in the original absorption of water. The water is drawn from the woody elements into the parenchyma of the leaf, and as it passes from cell to cell the leaf tissue is made turgescent. The turgescence is very largely due to the ascending stream, whose progress we have traced; at the same time we must remember that the turgid cortex of the root is continuous through that of the stem with the soft tissues of the leaves, and hence the slow movement of diffusion assists in its maintenance. In plants which have but little woody tissue, such as the greater number of herbaceous annuals, this slow movement plays relatively a more important part than in those trees which have a conspicuously woody trunk.

As we have seen, the turgid mesophyll tissue has a great part of the surface of its cells abutting on the intercellular spaces of the leaf. The cortical cells of the axis are also similarly placed, though the spaces are much smaller in that region. The intercellular spaces of the plant are in communication throughout, and the cells which abut upon them are in most places, and particularly in the leaves, furnished with very delicate cell-walls which readily allow a process of evaporation to take place, watery vapour passing into the passages. The whole intercellular space system thus becomes charged with vapour, the process of evaporation from the cells being, however, much more marked in the leaves, owing to the greater development of the spaces there. At particular spots in the leaves and other green portions of the plant, these intercellular spaces or canals communicate with the external air by means of small openings or crevices in the outer layer of cells, which are known as stomata (fig. 61). Each stoma is surrounded by two cells of peculiar shape, known as guard-cells, which, by being approximated to each other to a greater or less degree, enable the extent of the communication to be varied from time to time according to the conditions of the plant. The ultimate escape of the watery vapour from the interior of the plant is subject by means of these 
stomata to a very delicate regulation. So long as the apertures are open the watery vapour diffuses outwards into the external air. We may thus have a copious exhalation taking place from the surfaces of the leaves and other green parts, which plays an important part in causing the flow of water through the plant. This evaporation or exhalation from the surface is known as transpiration; it will be discussed more fully in a subsequent chapter.

Little or no evaporation takes place from the surface

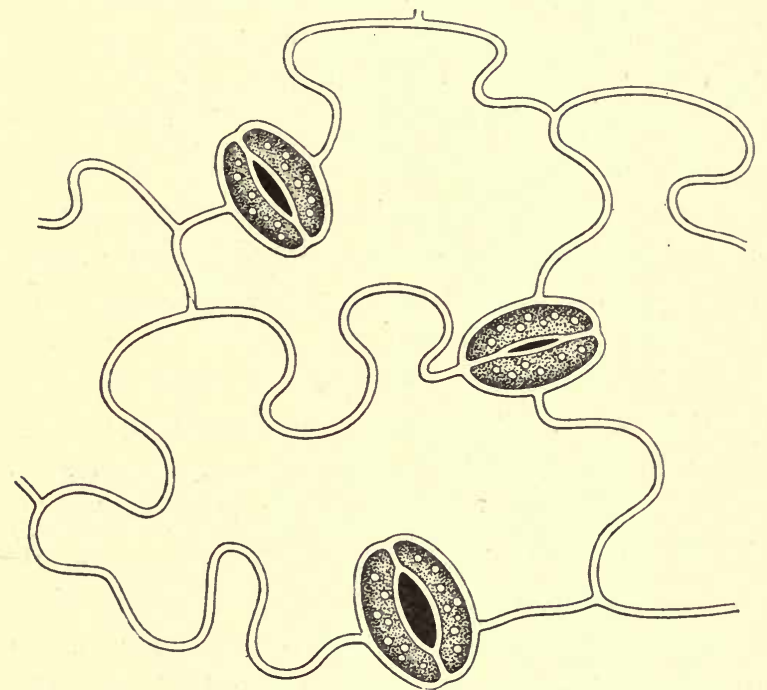

Fig. 61.-Three Stomata on the Lower Surface of a Leaf, showing different Degrees of Closure.

of the epidermal cells of the leaves, which have their outer walls generally cuticularised to a greater or less extent, the cuticle offering considerable resistance to the passage of water or watery vapour through them in either direction.

The escape of watery vapour by transpiration is supplemented in some cases by an actual excretion of water in the liquid form. This happens when the hydrostatic pressure is very high at times in herbaceous plants, water being forced out at the tips of the leaves. It is not infrequently 
seen in the case of grasses, the edges or apices of whose leaf-blades may show drops of liquid standing upon them in the early morning. Similar drops are often to be seen on the surfaces of the leaves of Alchemiilla when they have ceased to transpire during the night, while the absorption of water by the root has continued actively. The escape of liquid in this way is due to a filtration similar to that by which the water is forced into the woody elements of the stele of the root, as previously described.

A subsidiary mechanism allowing the escape of watery vapour from the cortex of stems and roots is provided by the lenticels. We have seen that these are loose aggregations of corky cells which are developed in connection with the sheaths of cork that form part of the secondary tegumentary protective tissue of a thickened axis. They are not, however, so intimately connected with evaporation as the stomata, probably being more concerned with the aeration of the tissue (fig. 39).

The stream of water thus passing through the plant has a very important influence upon its development. We have seen how important a factor in its growth is the maintenance of a condition of turgescence, which in turn depends on the constant absorption of water to take the place of that removed by evaporation. The quantity passing is correlated with the amount of leaf surface which the plant possesses; where there is a large leaf area there is copious transpiration; this necessitates a large path for the ascending stream, and a consequent development of the axial portions of the plant.

The greatest increase in the number of the protoplasts takes place at the so-called growing points which are situated at the terminations of the twigs, and which give rise continuously to additional leaves and branches. The development of new material of this kind and of the new protoplasts which they contain is largely dependent upon another feature of the water supply to which attention has already been called. A considerable part of the 
material from which the food of the plant is constructed is absorbed from the soil in solution in the water, and is transported by means of this stream to the regions of cell-formation. The fact that the quantity of the nutritive salts in the water is extremely small is a further reason for the transport of such large quantities of water as pass through the plant; for by the gradual concentration of the solution in the cells of the leaf enough new material can be obtained by the protoplasts for the construction of the food necessary for their nutrition, growth, and multiplication. Where there is a large flow of water, as in a tree, there is a continuous formation of new cells and of the various mechanisms their life demands; where the transpiration is but slight, as in a Cactus, or where the supply of water is limited, as is the case with such plants as grow in deserts or in rocky situations, there is but little formation of new substance. 


\section{CHAPTER VI}

THE TRANSPIRATION CURRENT. ROOT PRESSURE.

TRANSPIRATION

In terrestrial plants, so long as circumstances are favourable to the vital activity of the organism, we have, as we have seen, a stream of water passing from the roots through the axis to the green twigs and leaves, where the greater part of it is evaporated. This stream, which we have spoken of as the ascending sap, is often called the transpiration current. Its path through the axis of the plant has been determined to be the xylem vessels, which are in complete continuity from the young rootlets to the veins of the leaves.

In thick tree-trunks, in which the wood can be seen to consist of alburnum and duramen, the stream is confined to the former. Proof of this can be obtained in various ways. If an incision is made all round the trunk of a tree and a ring of tissue removed, everything being cut away down to the outermost ring of wood, the leaves of the parts above the wound continue to be turgid. If, on the other hand, the woody cylinder is cut through, while the continuity of the cortex and that of the pith are allowed to remain intact, the leaves very speedily droop and become flaccid.

If a plant in a pot is watered with a solution of a dye which has no noxious action on the protoplasts, the colouring matter is absorbed in the liquid which the roots take up, and its progress can be traced by a subsequent microscopic examination of the various tissues of the axis. The colouring matter will be found to have stained the wood for a considerable distance; in the case of a small plant, 
indeed, it will be coloured quite up to the veins of the leaves, while the pith and cortical tissues will remain unstained. An isolated branch can be taken as the subject of the experiment, its cut surface being placed in a solution of the dye.

The dye in these cases passes with the current of water, as may be seen by the difference in its rate of passage when transpiration is vigorous, and when from severance of the leaves of a branch it can penetrate only by diffusion.

A good deal of controversy has been excited with reference to the manner in which the transport of the water in the wood takes place. Sachs originally suggested that the path was altogether the walls of the cells, and that their cavities were empty. This view was based partly on the fact that the vessels undoubtedly contain a quantity of air during the period of active vegetation, and that this air is at a less pressure than that of the atmosphere. Another reason advanced for it was based on the nature of lignin and its relation to water. While refusing to absorb much water and swell as cellulose can be made to do, lignin can contain a certain quantity, which it will part with very easily. On this view the walls of the lignified vessels may be regarded as a column of water held together by the molecules or micellæ of lignin. A very little water removed from the top of such a column would be immediately replaced from below so long as a supply existed there.

Such a remarkable conductivity, however, is probably not possessed by the walls of the vessels. Many observations made in recent years tend to negative this view, and to support the hypothesis that the water passes in the cavities of the vessels. Sachs's opinion that these are always free from water during active transpiration has been shown not to be well founded, for various observers have proved that their cavities are occupied by a chain of water-columns and air-bubbles, the air having been originally absorbed from the intercellular space system. If the end of a transpiring branch is injected for a short. distance with a viscid fluid, which will penetrate the 
cavities of the vessels, and subsequently solidify, these passages can be occluded for a distance of a few centimetres. Gelatin or paraffin can be used for the experiment, being injected at a moderately low temperature such as will not injure the vitality of the tissue. If after it has solidified a fresh surface is made by a clean cut a very short distance from the end, and the branch immersed in water, the leaves very soon flag, even if some pressure is applied to the water in contact with the cut surface. If the path of the liquid were the cell-walls, no obstacle being offered to the transfer of water to them, the upper portions ought to remain turgid. The experiment shows that the normal channels are blocked by the paraffin or gelatin used, and flagging results.

A similar demonstration that the water passes by the cavities or lumina of the cells is afforded by the experiment of compressing the stem in a vice; if the pressure is carried so far as partially or entirely to obliterate their cavities, the rate of flow is materially interfered with.

The progress of a dye injected into the surface of a cut branch also points to the same conclusion. If such a stain as fuchsin or eosin, which colour wood very rapidly, is forced up into a stem and sections made almost immediately, the lignified walls will be found to be in process of staining, and the colour will be seen to be deepest on the side of the wall abutting on the lumen, often only penetrating partly through the thickness. If the wall itself were the path of the pigment solution, its thickness would be stained uniformly as far as the dye penetrated at all.

The rate at whlch the transpiration current naturally flows varies a good deal, plants showing differences among themselves as to facilities of transport. In a fairly vigorous tree it may be talien to be about 1-2 metres per hour, though in some plants it has been observed to be three times as rapid. In other cases as low a speed as 2 metre per hour has been found. It is a little difficult to measure in most cases; the plan generally adopted has been to 
immerse the cut ends of branches in a solution of such a dye as eosin, and notice how far the dye penetrates in some unit of time. The objection to this method is that very frequently the water of such a coloured solution will travel faster than the dye dissolved in it. Sachs used instead a solution of a salt of lithium, which he found was free from this objection. He detected the rate of progress of the lithium by means of spectroscopical examination, ascertaining how far the metal could be traced in the stem when pieces were cut out and burnt after a definite time, during which absorption had proceeded.

The causes of the transpiration current are not fully known, but there is no doubt that it is due to the cooperation of many factors, not one of which by itself is sufficient to account for it. Two of the main influences which are at work have been incidentally alluded to, which must now be discussed in greater detail. These are the constant pumping action of the cortex of the root, giving us the force known as root-pressure, and the modified evaporation into the intercellular spaces, and its exhalation from the surfaces of the green parts of the plant, which we have spoken of as transpiration. Recent investigations make it probable that we must add to these the force of osmosis in the parenchyma of the leaves, which apparently brings about the passage of the water from the veins into the cells of the leaf-substance.

Besides these, other factors have been held to cooperate, though much less certainly than they. . The walls of the vessels having an extremely narrow calibre, capillarity has been suggested as playing a part. This cannot, however, have much effect in a system of closed tracheïds, like those of the secondary wood of the Conifers, which, nevertheless, conduct the water. It has been thought that the living cells of the parenchyma, which abut upon the woody tissue of the stele, may play a part similar to the pumping action of the root. The medullary rays of the stele in tall tree trunks have been held to play a 
similar part. Against this theory we have the fact that, if the transpiration current is made to contain substances that are poisonous to the living cells, and the latter are consequently killed, the current still goes on. Considerable lengths of a stem have been killed by heating it to the temperature of boiling water, and the dead part has proved to be no obstacle to the transport. Nor do differences of gaseous pressure within and without the plant, or at different portions of the axis, explain the matter more satisfactorily.

Root-PRESSURE.-We have seen how the absorption of water osmotically from the soil by the root-hairs leads to a great turgescence of the tissue of the cortex of the root, not only in the regions of absorption but along the whole length of the younger portions, which turgescence exerts considerable pressure on the sides of the vessels and tracheids of the xylem of the stele. By this means water with various salts and other constituents in extremely small quantity is forced into the fibro-vascular tissue. The process is not a purely physical one of filtration under pressure, but is regulated to some extent by the protoplasm of the cells which abut upon the xylem. When these are distended to their greatest capacity, their protoplasm appears to be stimulated, perhaps by the very distension, and in consequence to allow water to transude through its substance. This mode of response to stimulation is not infrequent in vegetable tissues ; indeed it appears to correspond to the response of a muscle to stimulation by the process of contraction. We must not push this comparison. too far, for the protoplasm of the vegetable cell seems to respond not by contracting but by modıfying its permeability, so that the hydrostatic pressure existing in the cell is able to force the water through the living substance with greater facility than it could before the stimulus was appreciated. By thus modify-) ing the turgor of the cell, the protoplasm relieves itself of the over-distension, and we get an intermittent pumping action set up, which has a certain rhythm. By it large 
quantities of liquid are continually being forced into the axial stele. This rhythm, which is comparatively rapid, must not be confused with another rhythm which is much more gradual, and which constitutes what is called the periodicity of the root-pressure.

When transpiration is not taking place, the water may accumulate in the vessels, and its presence can then very readily be demonstrated, and the force of the root-pressure measured. If a vine stem is cut through in the early spring before its leaves have unfolded, a continuous escape of water takes place from the cut surface, and the vine is said to bleed. The phenomenon is not peculiar to the vine, but is exhibited by most other terrestrial plants.

In plants which have a large woody system the accumulation of water in the vessels can only be demonstrated while the absence of leaves renders transpiration impossible. Many herbaceous plants show a similar phenomenon daily, owing to the intermission of transpiration during the night. In these cases it is not necessary to cut the axis at all; the accumulation of water extends to the whole of the plant. In the early morning the plants show a certain exudation of water from the tips or apices of the leaves, drops accumulating on their surfaces. Alchemilla and Tropeolum especially display this phenomenon, which is due to the over-turgescence of their tissues, brought about by the pumping action of their roots.

This phenomenon of setting up a hydrostatic pressure causing an exudation of water is not confined to roots. Whenever the active living cells of the stem, or even of the leaves, force water into the vessels, the same exudation can be noticed. It can be shown by burying the cut ends of young stems of grasses in wet sand; after a time drops of water ooze out of their projecting upper ends. If the leafy branches of some trees are immersed in water so that only the cut ends project, the leaves can absorb water and force it through the stem, so that an exudation after a time can be noticed to take place from the cut surface which is 
not immersed. A similar exudation can be caused to take place from the hyphæ of fungi and from the tissues of mosses.

We must, however, be cautious not to attribute every escape of water from a plant to this cause. When a tree trunk is wounded or cut on a warm sunny day in winter, there is frequently an exudation of water from the wound. This is generally due to purely physical causes, being brought about by the expansion of

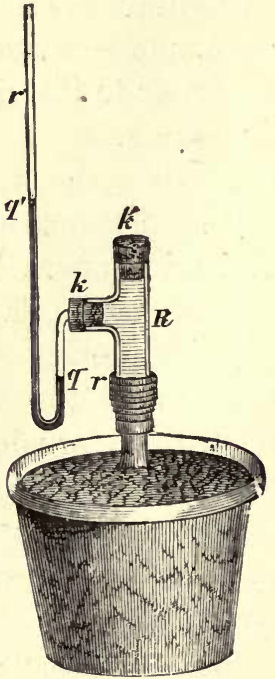

Fig. 62.-APParatus FOR THE Estimation of Root-PREsSLRE. the air which is contained in the vessels of the wood. It can be artificially produced at any time in winter by warming a freshly cut piece of wood; and its cause in this case can be seen to be physical by the fact that as the wood cools the water in contact with the cut surface is again absorbed, owing to the contraction of the air, which was expanded by the warming.

To measure the root-pressure in a plant the apparatus shown in fig. 62 may be used. It consists of a $\mathrm{T}$-piece of glass tubing $(R)$, which is fastened by indiarubber rings $(r)$ to the top of a cut stem, such as that of Helianthus. To the side arm of the tube a manometer $(q)$, with a capillary bore, is attached by a tightly fitting cork $(k)$, and the $\mathbf{T}$-piece is filled with water from the upper end $\left(k^{\prime}\right)$. Mercury is poured into the manometer till it stands at a level a little below the cork $k$, and the aperture $k^{\prime}$ is then tightly closed. As the root continues to take up water, it forces it into the tube $R$, whence it overflows into the proximal arm of the manometer, causing the mercury in the two limbs to be at unequal levels. By the displacement of the mercury therforce of the root-pressure can be 
estimated. A variation of the apparatus can be used, in which the manometer is replaced by a glass tube bent at right angles. The water will be forced through this, and can be collected in a suitable receiver, and its amount ascertained.

In performing the experiment it is best to allow the apparatus to stand for some time before closing the tube at $k^{\prime}$, as, if the plant is taken while transpiration is proceeding, the vessels of the stem will contain air at a certain negative pressure, and a certain amount of water will be sucked back until the vessels are full. As soon as this condition is reached, the pumping action of the roots will become evident, and the root-pressure will make itself obvious.

The root-pressure of various plants has been measured by different observers; an idea of its amount may be gathered from the fact that a medium-sized Fuchsia in a pot has been found able to send a column of water up a tube of the same diameter as the stem to a height of twenty-five feet.

The activity of the roots will depend upon various conditions, of which temperature, both of the air and of the soil, is one of the most important. The exudation of water has been observed at temperatures as low as freezing point, but most plants will not show it below about $5^{\circ} \mathrm{C}$., and as the air becomes warmer the quantity of water given off increases. Warming the soil of the pot in which is the plant under observation also increases the flow.

Of other influences which exert an effect upon the activity of the roots may be mentioned oxygen. Like all other vital actions, the absorptive power of the root-hairs depends upon their being in a healthy condition, and this cannot be maintained in any protoplast without the due performance of respiration. The character of the soil must also be considered. Without a due supply of moisture. the process, of course, cannot go on, and a disturbance of the normal constituents of the soil will lead to modifications 
of the process. If there is too great a preponderance of neutral salts such as sodium chloride or potassium nitrate, so that the liquid presented to the roots is practically a saline solution, the exudation will cease ; indeed, under such circumstances water may actually be withdrawn from the plant.

Root-pressure is continually at work while the transmission of water is going on ; but it is not easily seen later in the year when the development of the leaves has caused an active transpiration to proceed. If the stem of the vine is cut in July instead of in March, no bleeding follows the wound. This is not, however, due to the absence of activity in the roots, but to the fact that the copious evaporation of transpiration prevents the necessary accumulation of water in the cavities of the woody elements. In the experiment in the early spring the conditions were different; there were no expanded leaves, and the water absorbed and sent upwards by the root consequently remained in the vessels of the stem, escaping at once when the latter was cut. In July the vessels have been emptied by the transpiration, and there is no accumulation of water to overflow. The apparatus described will show, however, if the experiment with it is continued for some time, that root-pressure is still at work, even though transpiration is vigorous until the stem is severed.

The force of root-pressure must therefore be regarded always as a factor in maintaining the transpiration current. It is continually forcing water into the vessels of the axis, and the fact that transpiration prevents an accumulation there does not show that the influence of root-pressure is done away with as soon as it ceases to be easily demonstrated.

The root-pressure, though always considerable, is not the same at all times of the day and night. It can be measured by observing the output of water in the second form of the apparatus described above, measurements being taken every hour, or in the first form of the 
apparatus the manometer can be fitted with a float carrying a pen, which can be made to trace a continuous line on a slowly rotating recording surface. The line will be found to describe a curve, showing points of activity varying from maximum to minimum. The general features of the curve will be the same for all plants, but all do not give the maximum at the same time of the day. In the case of Cucurbita Melopepo the minimum point occurs in the early morning; the curve rises slowly during the forenoon, reaching its maximum soon after midday. From this point it falls; sometimes a second smaller rise takes place towards evening, and then it sinks continuously all night. The time of the occurrence of the maximum point varies in different plants, but in all it appears to be during the afternoon. In Prunus Laurocerasus it is much later than in Cucurbita. The points of maximum and minimum activity appear, however, to be about twelve hours apart, so that there is a complete diurnal cycle.

There may be noticed in some trees also a variation which suggests a yearly periodicity. The power of exuding water is lost for a time during the winter, the lóss being noticeable at different times in different trees. Vitis vinifera does not show any exudation usually in January; Acer platanoides is passive in November ; many plants will not bleed at all during the winter.

The causes of these variations in the activity of the absorbing mechanisms of the roots are still obscure. The annual periodicity, when it exists, appears to be connected with conditions which lead to the discontinuance of growth during winter. The trees pass in fact into a state that may be compared to hibernation. The daily periodicity does not appear to depend upon variations in the surroundings of the plant, but to be due to some cause or causes inherent in its constitution. It has been suggested that it has been induced in plants by long-continued variations of external conditions, particularly those of illumination, involved as these are in the alternation of day and night. This alter- 
nation, affecting successive generations of plants through an enormous length of time, may have impressed upon the protoplasm a peculiar rhythm of greater and less general activity, which has become ultimately automatic and independent of the immediate surroundings. Of this the varying action of the roots may be a particular expression.

It is remarkable, however, that very young plants do not exhibit this diurnal variation, but they gradually acquire the power of doing so as they develop, subject as they are under normal conditions to the alternation of light and darkness. In many cases, again, the diurnal periodicity is not manifested at all.

The effect of the periodic alternation of light and darkness cannot in any case have been originally appreciated by the roots, as they are implanted in the soil and so escape its influence. If it was originally due to such variations, these must have been impressed upon the general organisation of the plant.

Transpiration.-The modified evaporation by which the protoplasts get rid of water and enable the contents of their vacuoles to be continually renewed takes place ultimately from the surfaces of all the succulent parts of plants, and to a less extent from portions of the exterior which are covered by a layer of cork. Like the activity of the absorbing organs of the root, it is essentially a vital process and is regulated by the protoplasm of the cells which take part in it. As we have seen, it is usually spoken of as transpiration.

It is easy to demonstrate the fact of its continuous existence during daylight by enclosing a plant, or part of one, in a dry glass vessel which can be closed so as to admit no air. Very soon the surface of the glass becomes covered by a fine dew, which is the condensed vapour that has escaped from the plant. The same thing may be seen when a vigorous plant is covered over by a bell-jar, the water condensing copiously upon the sides of the latter.

A more elaborate method of demonstrating transpiration 
consists in placing the end of a cut branch in a small glass vessel, preferably a $U$-tube, filled with water, as shown in fig. 63. The branch passes through the cork of the vessel in such a way as to prevent any escape or evaporation of water at that point. Communicating with the other arm of the $U$-tube is a side tube bent at right angles, which dips into the water through a perforated cork. This tube is also filled with water. As transpiration proceeds the water is gradually drawn from the horizontal tube, and its progress can be noted by arranging a scale behind it. The

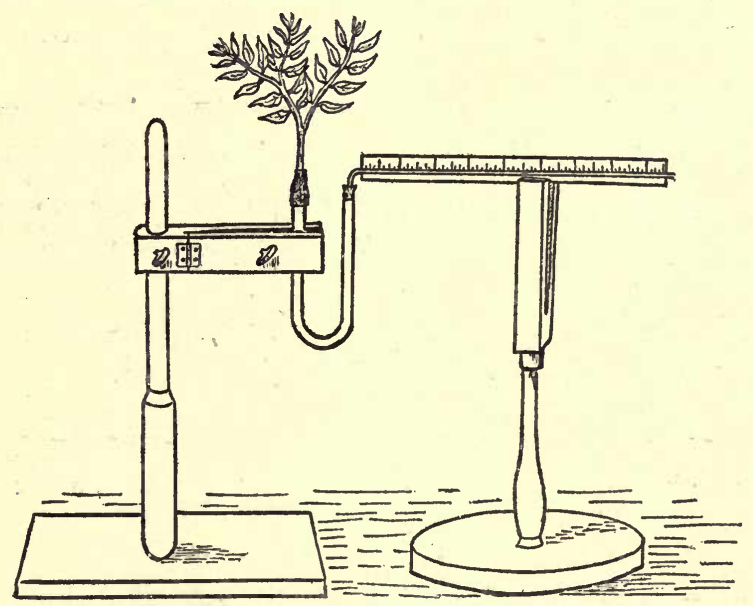

Fig. 63.-Apparatus to demonstrate Transpiration of a Branch.

stem or branch should be kept with its cut end immersed in water for several hours before being placed in the apparatus, as its vessels contain air at a negative pressure when it is cut, owing to the transpiration which has been taking place from it before its separation from the plant. The existence of this negative pressure will lead to an immediate absorption of water, which might be mistaken for an active transpiration.

The evaporation takes place to a certain extent through all the epidermal cells of the transpiring organ, but not to 
a very great one, the degree of the development of the cuticle having considerable influence upon its amount. It is carried out much more freely through the thin walls of the cells abutting upon the intercellular spaces, which, as we have seen, communicate with the external air by means of the stomata and the lenticels. Very little watery vapour is given off by the latter, so that by far the greater amount that is exhaled passes through the stomata. Transpiration is consequently most copious from the leaves, the structure of the lower side of which, in dorsiventral forms, is especially favourable to it (fig. 64). If a leaf is taken which has stomata upon its under surface only, and the rates of watery

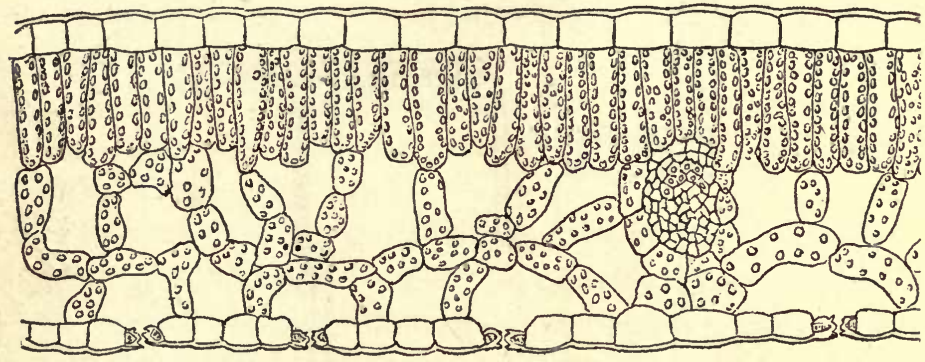

Fig. 64.-Transverse Section of the Blade of a Leaf, showing the Intercellular Spaces of the Interior.

exhalation from the two sides are compared, it will be found that the stomatal gives off considerably more vapour than the other surface.

A method first introduced by Stahl enables us to prove with considerable facility that the escape of vapour through the stomata is much greater than that through the cuticular surface. It consists in applying to each side of a leaf which has stomata only on the under surface, a piece of filterpaper which has been impregnated with a solution of cobalt chloride and dried. When dry this paper is blue in colour, but it rapidly becomes pink when exposed to moisture. A fresh dry leaf is taken and placed between two pieces of the cobalt-paper and the whole put between 
two dry sheets of glass of somewhat larger area. In a very short time, often in less than a minute, the paper in contact with the lower side of the leaf becomes pink, while the other piece remains blue for a considerable time.

The amount of water given off by transpiration varies in different plants. In the sunflower (Helianthus) the amount has been stated to be $\frac{1}{163}$ cubic inch of water per square inch of surface in twelve hours. V. Höhnel has computed that a birch-tree with about 200,000 leaves may transpire 60 to 80 gallons of water during a very hot day. Doubtless, however, individual plants show a considerable variety in the amount. This copious evaporation readily explains why the bleeding of plants from wounds can seldom be observed when the leaves are expanded and active.

When transpiration is excessive the leaves and branches lose their turgescence, become flaccid, and droop. A branch which has reached this condition may be revived by forcing water into it, which can be done by fastening it into one arm of a U-tube containing water (fig. 65), and pour-

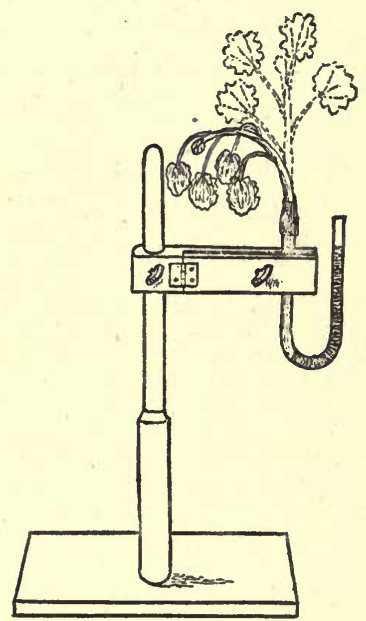

Fig. 65.-Apparatus to show Dependence OF Withering UPON LOSS OF WATER. ing mercury into the other. T'he restoration of the water restores the turgescence of the tissues, and the branch regains an erect position.

The exhalation of the water accumulated by rootpressure in the closed system of the vessels leads to a diminution of the pressure of the air which they contain in addition to the water. Indeed it is by such a suction that the air is originally enabled to enter the vessels, being drawn into them from the intercellular spaces. Consequently, while transpiration is active, there is a negative 
gaseous pressure existing in the wood vessels. This continues after transpiration ceases, and no doubt, like the evaporation itself, it is of assistance in maintaining the upward flow, acting as it does in the same direction as the turgid cortex, upon which it exerts a considerable suction. It continues until the entry of water from the root causes the pressure of the air in the vessels to be equal to the atmospheric pressure. This negative pressure is of considerable importance also in assisting the movements of gases in the plants.

The exhalation of watery vapour from the surface of the cells is not a process of simple evaporation. As in the /other phenomena which we have examined, the protoplasm exercises a regulating influence upon the escape of watery vapour from the cell. If the amount given off from a measured area of leaf-surface is compared with the quantity evaporated from an equal area of free water, the latter is found to be much the greater. This area is probably much less than the area of the cell-walls actually involved, which abut upon the intercellular spaces opening by the stomata included in the measured area. That this difference is due to the life of the leaf, and consequently to the protoplasm, is seen from the fact that a dead leaf gives off its water and dries up more rapidly than a surface of freely exposed water. The cuticle of the living leaf and its cell-walls are consequently not the causes of the differences observed.

The ultimate exhalation of watery vapour, we have seen, is chiefly carried out through the stomata of the green parts, at any rate in those plants which possess them. Each stoma is situated above a somewhat conspicuous intercellular space, to which it forms an outlet. The stoma originates by the vertical division into two of one of the cells of the epidermis which is usually somewhat elaborately differentiated from the rest. The partition which is formed between the two daughter cells thickens slightly and splits so as to form an opening between them, which does not, however, extend the whole length of the 
wall, so that the two cells remain attached to each other by their ends (fig. 66). The split constitutes the stoma, and

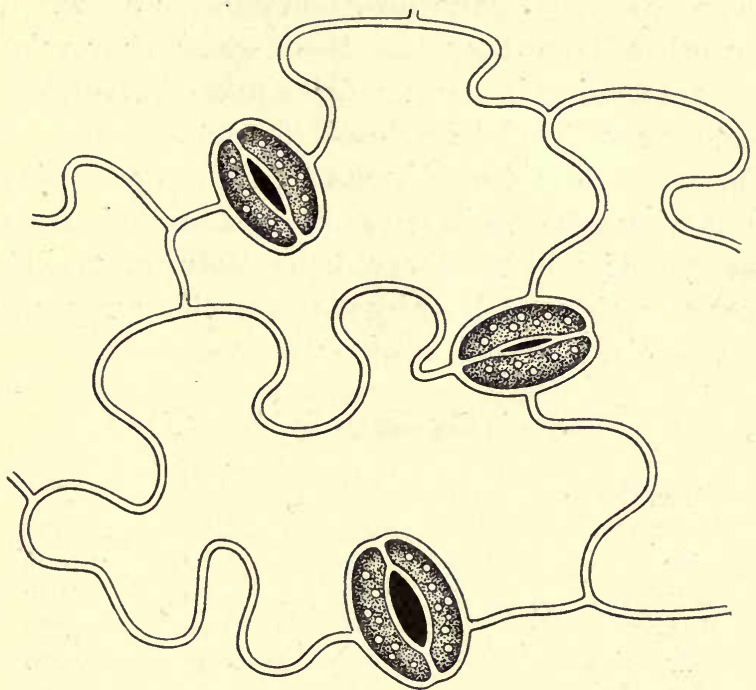

Fig. 66.-Surface View of part of the Under Surface of a Leaf, Showing three Stomata in DIfFerent Stages of Opening and Closing.

the two cells are known as the guard-cells. They are commonly of a more or less semilunar form and contain some chloroplastids, a point in which they differ from the other cells of the epidermis in the higher plants. Their walls become thickened and cuticularised, particularly those which abut upon. the slit and upon the intercellular space (fig. 67); the wall which is in contact with the other epidermal cells, however,

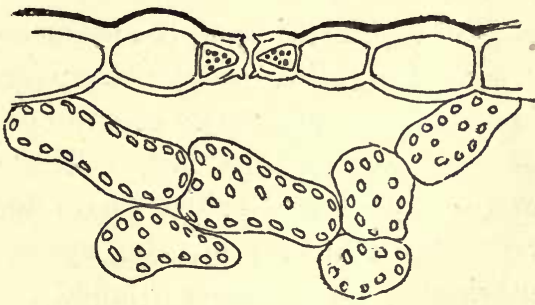

Fig. 67.-Section of Lower Epidermis of a Leaf, showing a Stoma. $\times 300$. remains thin. When the guard-cells are full of water, their form and mode of attachment cause them to become 
curved so that the orifice is widely open. This is helped by the thickening of the free edges, which makes it difficult for them to swell in the direction of each other. When, on the other hand, they lose their water, they relax, and their edges coming into contact, the aperture between them is more or less completely closed (fig. 66).

The number of the stomata varies very considerably. The following table will give some idea of their abundance in leaves, and it will be observed that the number of stomata is usually greatest in those leaves from whose upper surface they are entirely absent.

\section{Stomata in One Square Inch of Surface}

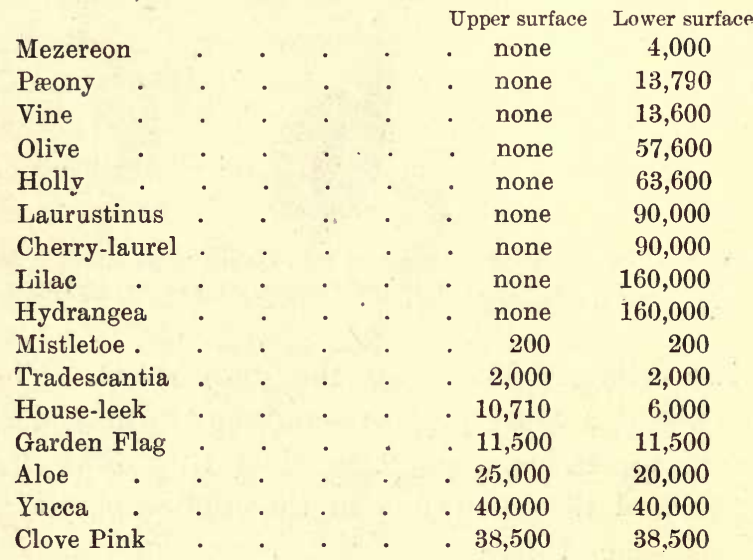

The modification of the turgescence of the guard-cells is caused by the osmotic transference of water between them and the other cells of the epidermis from which they are separated by thin walls. The vapour which is in the intercellular space below them does not penetrate them, the walls abutting on the space being thick and cuticularised. The osmosis alluded to may be associated with the presence of the chloroplasts in the guard-cells, which are instrumental in the production there of various substances, so that their contents have a higher osmotic equivalent than those of the epidermal cells which are con- 
tiguous to them. When, therefore, the epidermal cells are charged with water, this is osmotically drawn into the guard-cells, which become turgid, and consequently separate, opening the aperture. When the contiguous epidermal cells lose their water, the osmotic constituents of their contents become more concentrated, as these do not leave the cells with the water. The direction of the osmotic stream is consequently reversed, the guard-cells lose some of their turgidity, so that their edges fall together and partially or wholly close the slit. Thus the escape of watery vapour is accelerated or retarded by their action.

Transpiration is markedly increased by sunshine, rising to many times its original amount when a plant is transported into it from a dim light. No doubt this is due in a very large measure to the heat rays which then fall upon the plant, and which would raise its temperature very dangerously were they not applied to the evaporation of the water. But it is not due entirely to them, nor to the higher temperature of the air accompanying their passage. The light has, indeed, an influence apart from the heat. No doubt, so far as the visible rays of the spectrum are converted into heat vibrations after absorption, they must influence transpiration indirectly in this way. Besides acting thus indirectly, light has a direct effect upon the process, for it influences the size of the stomatal apertures. These have been observed to be open during the day and more or less completely closed during the night. The gaseous interchanges which light induces, in causing the decomposition of carbon dioxide and the evolution of oxygen, on the whole favour the exhalation of watery vapour. When green plants are exposed to light of various colours the most marked increase of transpiration is caused by the light of which the plants absorb most. This can be observed not only in the green parts of plants, but in those which are not green, as in the petals of the flowers.

The fact that the rays which are absorbed by chlorophyll are the most active in promoting the process has 
some significance when it is remembered that the guardcells of the stomata contain this pigment. The nature of the action of chlorophyll in this direction is not, however, fully understood.

Apart from direct radiation, the temperature of the air, and its hygrometric condition, are important factors in causing an increase or a diminution of the watery vapour exhaled. They act principally by exerting an influence directly upon the evaporation from the cells, but several indirect effects can also be noticed. The general movements of water in the plant, as well as its absorption, are influenced particularly by variations of temperature, and the latter has also an effect upon the width of the stomatal orifices. A rise of the external temperature causes the saturated air in the intercellular passages to expand, as the air acquires the new temperature more rapidly than do the tissues of the plant. The escape of vapour is consequently accelerated as the temperature rises, even though the rate of evaporation from the cells into the intercellular spaces is not at first affected.

The influence of the hygrometric condition of the air, apart from changes of temperature, can be seen when a plant which has been exposed to a dry atmosphere till its leaves have become flaccid is transferred to one saturated with moisture. After a short time the drooping leaves again become turgid. This is not due to an absorption of water in the form of vapour by the leaves, but to a diminished loss by the checking of transpiration. The return of turgidity is caused by the accumulation of the store drawn from the earth by the roots. This can be shown by comparing the behaviour of two plants treated in the way described, one of which is allowed to remain rooted in soil, while the other is taken up from the earth and exposed in that condition to the saturated air. There is in the latter case no recovery of turgescence.

The temperature of the soil in which the roots of a plant are embedded has also an influence upon the exhala- 
tion of watery vapour, which increases as the soil is warmed and diminishes as it becomes cooler.

If the protoplasts of the cells of the turgid leaves of a branch are stimulated by violently shaking it, the leaves become flaccid. The protoplasm under the stimulus allows more water to pass through it to the cell-walls, and hence evaporation is promoted. The effect may be compared with that which has already been mentioned as set up in the cells of the cortex of the root by their over-distension by the water which accumulates in them in consequence of the continuous osmotic activity of the root-hairs. The stimulus of this distension is responded to by the protoplasm by its becoming more permeable by the water of the vacuoles of the cells. The response made by the protoplasts of the leaves to the stimulus of shaking may help to explain the flaccid condition observable in the foliage of certain trees after the prevalence of a high wind. Besides this effect upon the protoplasm, the continuous removal of the air around the transpiring organs has, no doubt, a considerable influence upon the removal of the watery vapour from their intercellular passages.

The effect of alteration of the external conditions upon transpiration may be investigated by means of Darwin's potometer, which enables approximately accurate determinations of its amount to be made from time to time. This instrument is shown in fig. 68. It consists of a glass tube with a side arm which is bent upwards so as to be parallel with the tube itself. A capillary tube of about $\cdot 2 \mathrm{~mm}$. bore is fastened by an indiarubber cork into the lower opening of the tube so as just to project beyond the cork. A convenient length of the capillary tube is about $20 \mathrm{~cm}$. Its lower end dips into a small vessel of water, arranged so as to be easily withdrawn from the tube. The upper orifice of the potometer is closed by a tightly fitting cork, and the plant whose transpiration is to be observed is fitted into the side arm by means of an indiarubber band or tube which embraces the glass arm and the end of the cut 
branch so as to make a water-tight connection. The whole apparatus must be filled with water, and care must be taken that no escape of liquid can take place at any of the junctions. Any air that finds its way into the instrument during the arrangement of the branch in its position can be removed by causing it to collect at the upper portion

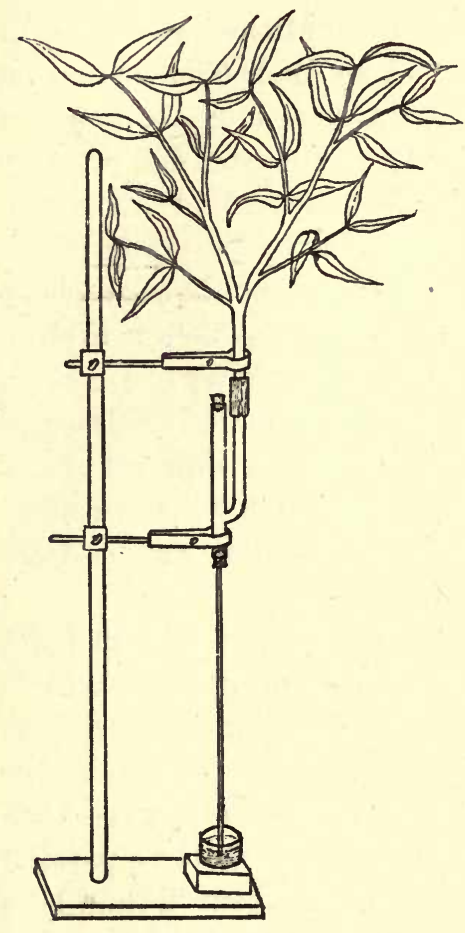

Fig. 68.-The Potometer. of the straight tube of the potometer. To take an observation of the rate of transpiration of the branch, a bubble of air must be admitted into the capillary tube by momentarily removing the vessel into which it dips, and replacing it as soon as the transpiration has caused the air to enter. The bubble of air must be of uniform size in successive readings, to ensure that the latter shall be strictly comparable with each other. The bubble will rise in the tube, and finally make its way to the upper part of the straight limb of the instrument, the rate at which it travels serving as an index of the rate of the transpiration. The capillary tube should be marked by a transverse line a few millimetres from its lower end, and by means of a stop-watch the time taken by the bubble to rise from this mark to the free end of the tube should be observed. The branch may be covered by a bell-jar, so that the variations of temperature, moisture, sc. of the air surrounding it can be controlled during a 
series of observations. Less accurate observations can be made by substituting for the capillary tube a tube of wider bore bent at right angles a little below the orifice of the potometer, and affixing to it a scale by means of which the rate of passage of the column of water in the tube can be observed (fig. 63).

According to the variations in the external conditions of the plant, including all the features already alluded to, the amount of watery vapour transpired is continually changing. The most favourable conditions being afforded in summer, it is not to be wondered at that transpiration attains an annual maximum during that season. It does not, however, entirely cease during the winter, though it is reduced to a minimum, especially in the case of such trees as shed their leaves in the autumn.

Apartfrom such changes in the external conditions, transpiration appears to show no independent periodicity, differing in this respect conspicuously from root-pressure. It is, how-

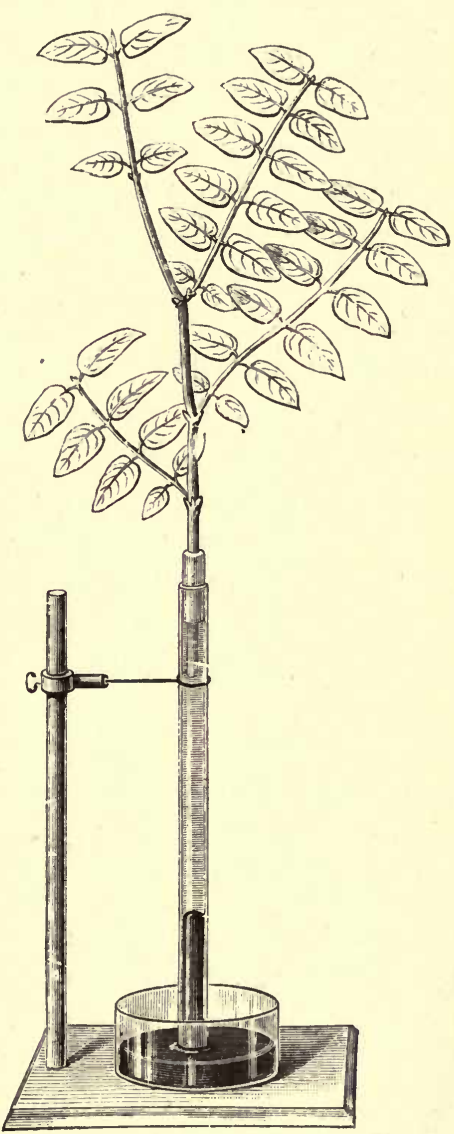

Fig. 69.-Apparatus to show thy Suction caused by TranspiraTron. (After Detmer.) ever, very sensitive to only slight changes in the environ-. ment.

It was mentioned in an earlier part of this chapter that 
the force of transpiration was of considerable assistance in maintaining the upward flow of water from the roots. The apparatus shown in fig. 69 enables this to be demonstrated. The cut end of a branch is connected by an air-tight joint with a glass tube filled with water, the lower end of which dips into a vessel of mercury. As the water is transpired, a certain quantity of mercury enters the tube, and is drawn up for some considerable distance by the suction.

The evaporation from the cells takes place, as we have seen, not immediately into the external air, but into the intercellular passages of the plant. The force causing this suction, so far as it is due to evaporation, is therefore localised in the surface film formed in the evaporating cell-walls. Such an evaporation has been shown by Strasburger to be capable of raising a current of water through pieces of dead wood which have been soaked and injected with water.

There is reason to believe, however, that a third factor in the ascent of the stream is interposed between the forces of root-pressure and the evaporation described. The water is passed from the wood-vessels or conduits to the evaporating cells through a varying thickness of parenchyma (fig. 70), which is kept turgid during active transpiration. The turgid condition of the cells is maintained by osmosis, just as is the similar condition in the roots. The vessels abutting on the parenchymatous cells are well supplied with water, which is in their cavities and which saturates their walls. The cells contain substances of an acid reaction which possess a high osmotic equivalent. We cannot doubt that osmosis takes place through the walls of the cells, and that the turgidity of the tissue of the leaf is due to it as much as is that of the cortex of the axis. Researches carried out by Dixon show that this osmotic force plays a very important part in supplying the water to the evaporating surfaces. If the end of a cut branch is immersed, in any of the forms of apparatus described, in a solution of a salt which will plasmolyse these cells by destroying their turgescence, such as the sodium chloride which we have 
already seen capable of doing so, the rate of transpiration continues without much, if any, diminution till the salt can be detected in the leaves, when it suddenly falls off. This takes place though there is no interruption of the continuity of the fluid in the channels of the transpiration current. From this point onward, instead of evaporation sucking up water from the root, it gradually leads to a drying of the leaf.: A similar result is brought about by raising the temperature of the transpiring branch to such a point as will kill the protoplasm of the cells. As these die the evaporation is unchecked at first, but gradually the water is taken from their interior and no more is supplied.

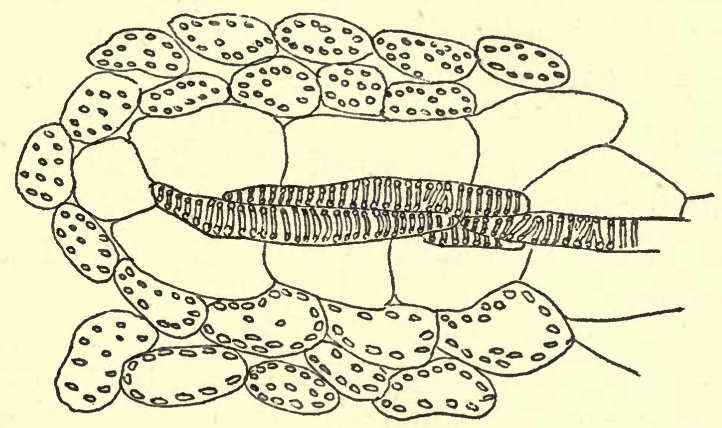

Fig. 70.-Ending of a Fibro-vasculat Bundle in the Parenchyma of a Leaf.

The cells rapidly become flaccid, the leaves droop, and the total quantity of vapour exhaled is materially lessened, the intercellular passages soon becoming partially obstructed by the collapse of the cells abutting upon them. The experiment does not interfere with the continuity of the water-stream, but as soon as the cells are made unable to retain their turgidity by the interference with osmosis which follows the death of the protoplasm, the evaporation empties the cells and no more water enters them to replace what has been lost. As we have seen in other cases, the death of the protoplasm is followed by the escape of the osmotic substances, which do not leave the cells during 
their life. The mechanical effects which follow the collapse of the tissue are the consequence of the assumption of a flaccid condition, and they intensify the check to the escape of watery vapour from the affected organ.

The course of events in a normal leaf during active transpiration appears to be, then, the setting up of a tension in the parenchymatous cells of the leaf by evaporation from their surfaces which tends to cause them to collapse and become flaccid. This tendency is opposed and overcome by a greater force excited by the turgescence of those cells whose osmotic properties exert a traction upon the water in the conduits or wood-vessels. Water is thus supplied through the inner walls of the evaporating cells as quickly as it is lost by eváporation from the surfaces which abut upon the intercellular passages.

Dixon ascertained that the osmotic pressure in the leaves of transpiring branches of the Laburnum amounted to between six and eight atmospheres, a force which is capable of raising a column of water to a height of more than 200 feet.

Careful consideration of the facts recorded in this chapter shows us that although we cannot fully explain the ascent of the transpiration current, we can see that it ultimately depends upon the behaviour of the protoplasm. All the factors which aid its progress, root-pressure, transpiration, osmosis in the cells of the leaves, are largely under the control of the living substance, and are particularly influenced by the power it possesses of allowing more or less water to pass through it, according to its condition. Moreover all the external influences which we have examined, which are brought to bear upon these factors, are mainly efficient in as far as they affect the protoplasm in the exercise of this power. 


\section{CHAPTER VII}

\section{THE AERATION OF PLANTS}

Is the study of the vital processes carried on by the protoplast we have seen so far how entirely it is dependent upon the free access of water. Another factor necessary for its existence is a supply of air. With but few exceptions, and those occurring among the lowliest plants, every living organism carries out a series of gaseous interchanges, a feature of which is the absorption of oxygen. In nearly all cases a corresponding amount of carbon dioxide is exhaled. In the case of many plants, of all, indeed, that are green, another gaseous interchange takes place, carbon dioxide being absorbed and oxygen simultaneously eliminated. Every protoplast must consequently be afforded facilities for carrying out gaseous interchanges, the nature and extent of which vary according to its constitution. The water with which it has such a close relationship serves as the medium through which such interchanges take place, for it is only in solution that gases are able to penetrate into the living substance.

In the case of those protoplasts which live in a watery environment, the latter supplies them with the gases they absorb and receives those which they exhale. If all air is withdrawn from the water in which they are living, death speedily ensues. The gases enter the naked protoplasts by diffusion through the film of water which is in contact with their free surfaces. In the case of those which have a cell-wall the same means are made use of. Gases in solution can diffuse through the cell-wall, which, as we have already seen, is saturated with water. If we turn to 
those unicellular or filamentous plants which live on the surfaces of rocks or tree-trunks, the process is only slightly modified, for the gases of the atmosphere readily dissolve in the water which the cell-walls contain and diffuse thence into the interior of the cell.

In the cases of those more bulky plants which we have especially been considering in the last chapter, a further mechanism is necessary, as the external air cannot gain access into the interior of a large mass of cells without special arrangements for its admission. This is especially the case with such plants as are possessed of protective mechanisms like the corky layers of the bark, or the strongly developed cuticle of the leaves. The arrangements of the structural elements in these plants we have seen to include a very complete system of intercellular spaces, passages, or canals, by means of which almost all the constituent cells are placed in nearly or quite complete relation with the external air. The intercellular space system has consequently a very important function to discharge in this particular, as well as to serve as the means of carrying off from the interior the aqueous vapour exhaled from the cells.

The intercellular space system begins to appear at a very early period in the development of the young plant. While all its cells are merismatic, as is the case when it begins to emerge from the seed, they are united together entirely, a condition which persists at all the growing points of the plant as its age increases. During this condition the aeration of the internal cells is provided for by the slow diffusion of the gases from cell to cell, absorption from the exterior by the external cells being possible so long as their walls are not cuticularised. Some of the cells situated deep in the interior of the adult parts are dependent upon a similar process, but the majority of the protoplasts are provided with access to the air by the early formation of spaces due to the splitting of certain of the cell-walls, and the subsequent partial separation of the 
cells. Air makes its way into these spaces by a process of diffusion outwards from the cells abutting upon them, and very soon external orifices in the shape of stomata make their appearance. The various constituents of the air make their way into and out of the cell by a process of diffusion, being dissolved in the water of the cell-wall or escaping from such a moist membrane according to the conditions existing, and the relation between the internal and external pressure of the particular gas in question.

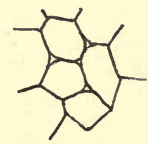

As soon as the differentiation of Fig. 71.-Cells splitting the tissue in the growing part of an AT THEIR ANGLEs To Form organ begins to take place, the formation of the intercellular spaces can be observed. In these regions they begin by a splitting of the wall between two contiguous cells or at the angles where three cells join (fig. 71). The crevice soon extends and may make its way for a considerable distance round any particular cell. The cavities so come into communication among the cells, each of the latter abutting upon a single one or upon several. While the tissue is young these are very narrow and slit-like, or are only visible at the angles when the cells are polyhedral. They rapidly become larger

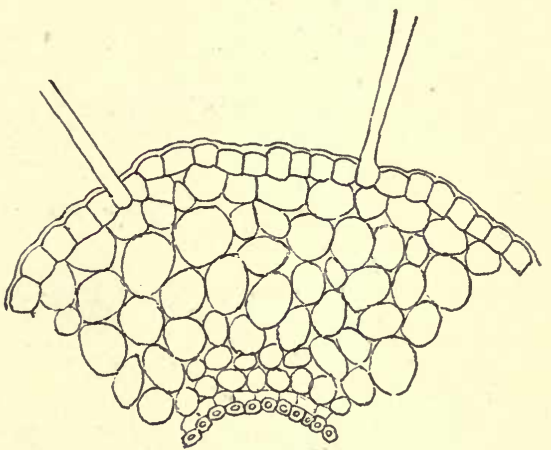

Fig. 72.-Cortex of Root, ShowiNg INTERcellular Passages between the Cells. (fig. 72), and in some parts, particularly in the interior of the lower strata of the mesophyll of dorsiventral leaves, they may occupy more space than the cells themselves (fig. 73). Light appears to influence their development somewhat, though no definite relation can be shown to exist between 
the degree of the illumination and the eapacity of the cavities formed. Light is, however, not the only factor,

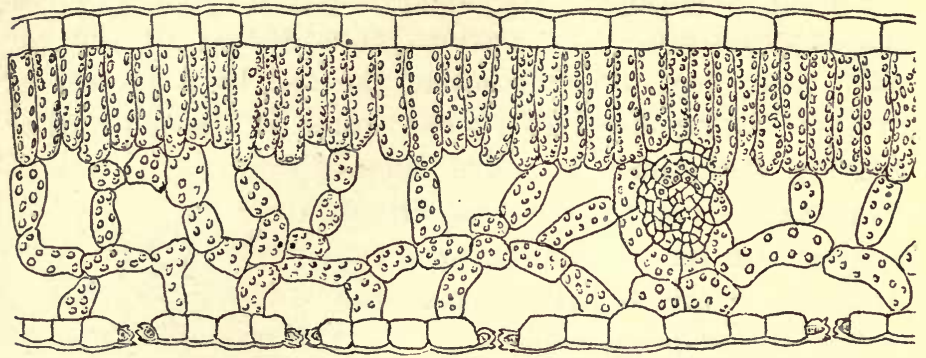

Fig. 73.-Section of Leaf Showing the LaRge INTercellular Spaces of the Mesophyll.

and probably not the most important one, in determining their extent, for they are usually prominent in the cortex of roots, which receive but little illumination. The

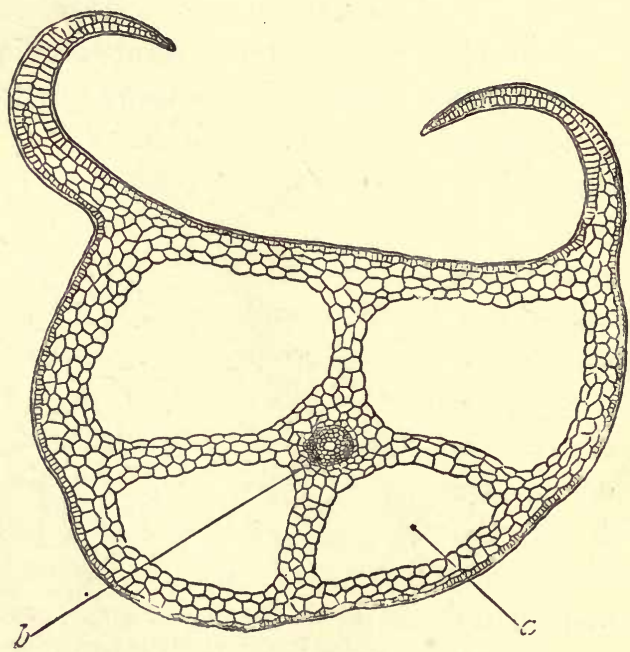

Fig. 74.-Section of Leaf of Isoëtes.

$a$, lacunar cavities; $b$, vascular bundle.

explanation of the relatively large development in this region may lie in the fact that the intercellular cavities there have very little communication with the outer air, as 
stomata do not exist upon roots. There is thus a necessity for a larger reservoir of air than in parts where gaseous interchange is more readily effected.

Besides these comparatively narrow channels we find cases where reservoirs of large size are specially developed. Such structures occur in the leaves, rhizomes, and roots of aquatic plants which are nearly or entirely submerged.

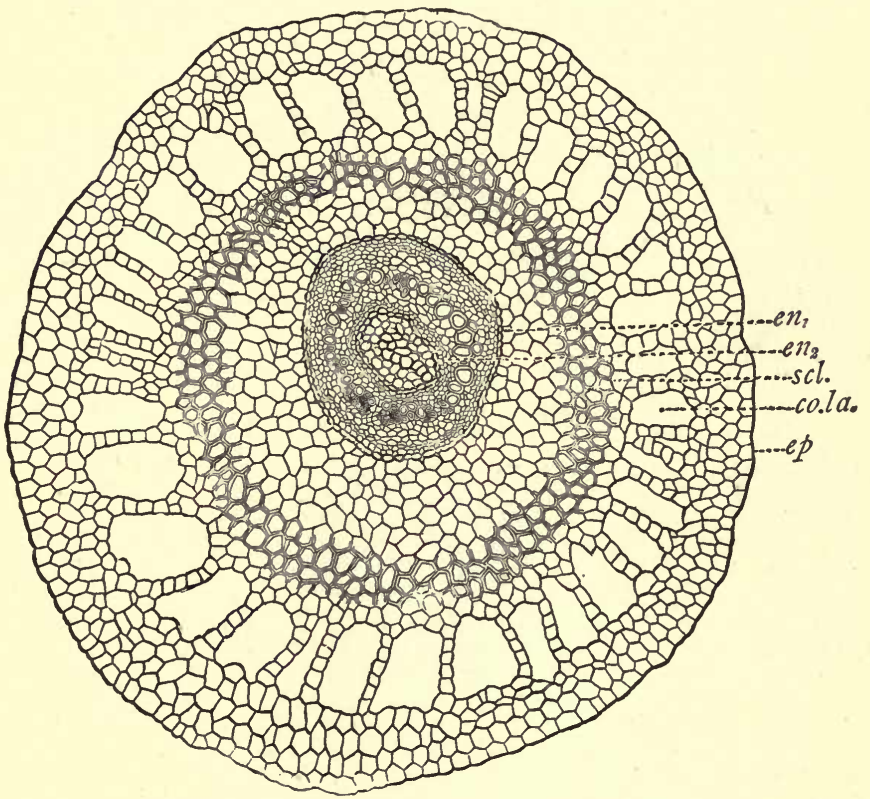

Fig. 75.-Section of Rhizome of Marsilea.

co.la, lacunæ in cortex.

Among them conspicuous examples are afforded by the leaves of Salvinia and Isoëtes (fig. 74), the rhizome of Marsilea (fig. 75), and the leaf stalks of many of the aquatic Phanerogams. These are developed in a similar manner to those already described, and they are so prominent in the structure that a section shows them separated from each other by rows of cells not more than one cell thick (fig. 76).

In some cases where large cavities of this kind occur 
the mode of formation is different. A mass of tissue lying in the position of the subsequent cavity does not keep pace in its development with the growth of the cells surrounding it, and consequently becomes ruptured, and the cells of which it is composed are gradually destroyed, leaving a cavity of some size. Instances of this mode of formation are afforded by the stems of Equisetum (fig. 77),

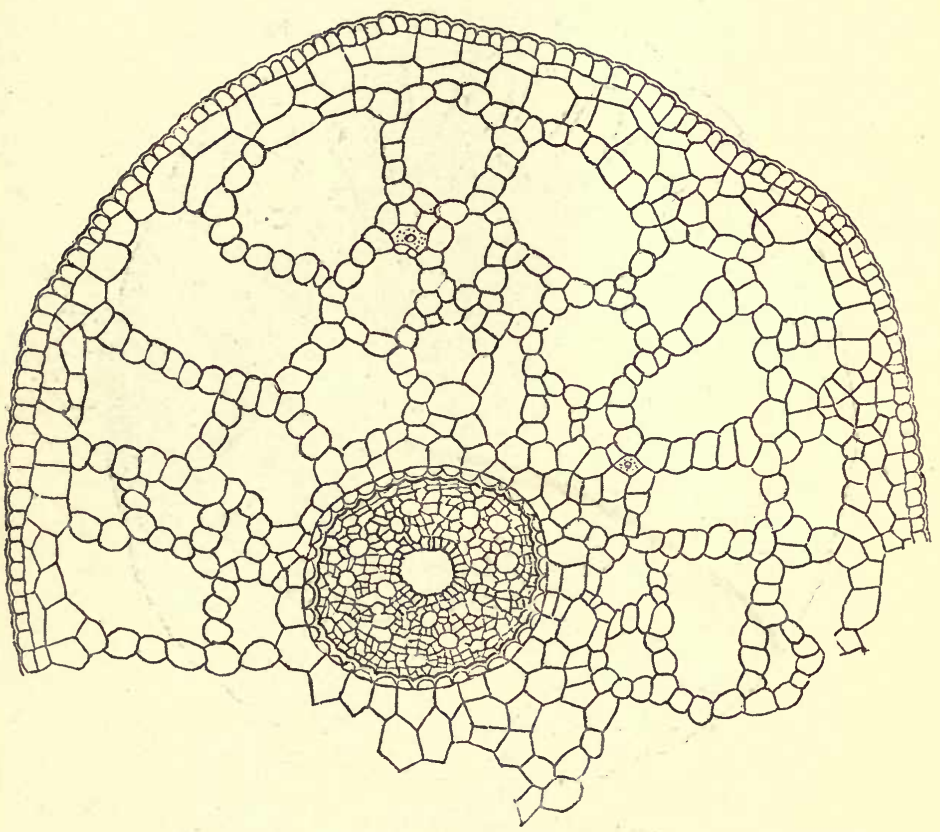

Fig. 76.-Section of Stem of Potamogeton, showing Air Passages iN THE CoRTEX.

the haulms of grasses, and the hollow stems of the Umbelliferæ and other plants.

The occurrence of these large air-containing cavities in partially submerged plants may be explained by a consideration of their habitat. The plant is in contact with the air by only a very small portion of its surface ; the leaf-stalk of Nymphæa, for example, is always submerged, and only the floating lamina can obtain a direct supply of air. The 
stomata are placed upon the upper surface, and afford its only means of entrance. The stems and roots are also cut off from air by being placed either in water or in mud. The protoplasts of such a plant are almost entirely dependent upon the reservoir of air which the body of the plant can contain, a small quantity only entering by diffusion from the water into its epidermal cells.

The air cavities which arise in the stems of terrestrial

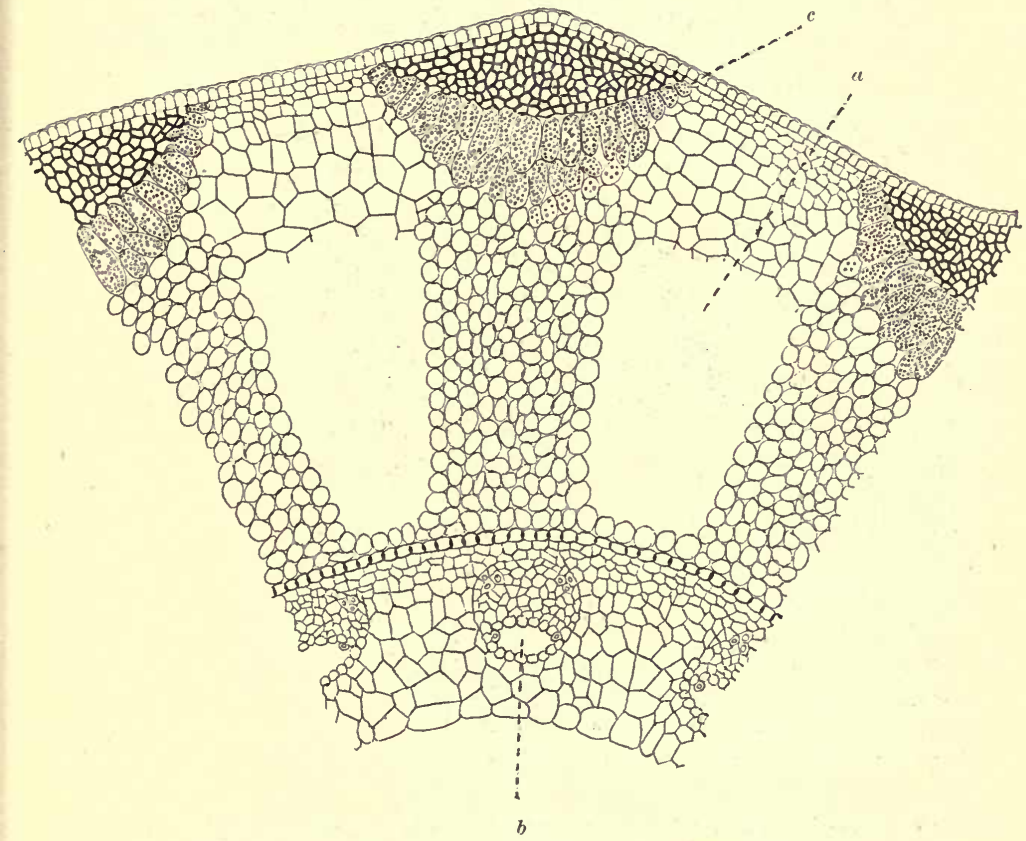

Fig. 77.-Portion of Aerial Stem of Equisetum.

$a$, cortical lacuna; $b$, lacuna in vascular bundle; $c$, chlorophyll-containing cells.

plants, such as the grasses, are probably not primarily developed with a view to the aeration of the plant, but are rather intended to economise the material used in construction. The hollow stems with a rigid periphery, strengthened at intervals by diaphragms, such as occur at the nodes of these organs, are especially adapted to maintain 
an upright position with comparatively little expenditure of material. A somewhat similar mechanism is met with in the stellate parenchyma of the stems of the Rushes (fig. 78). There is little doubt, however, that these spaces

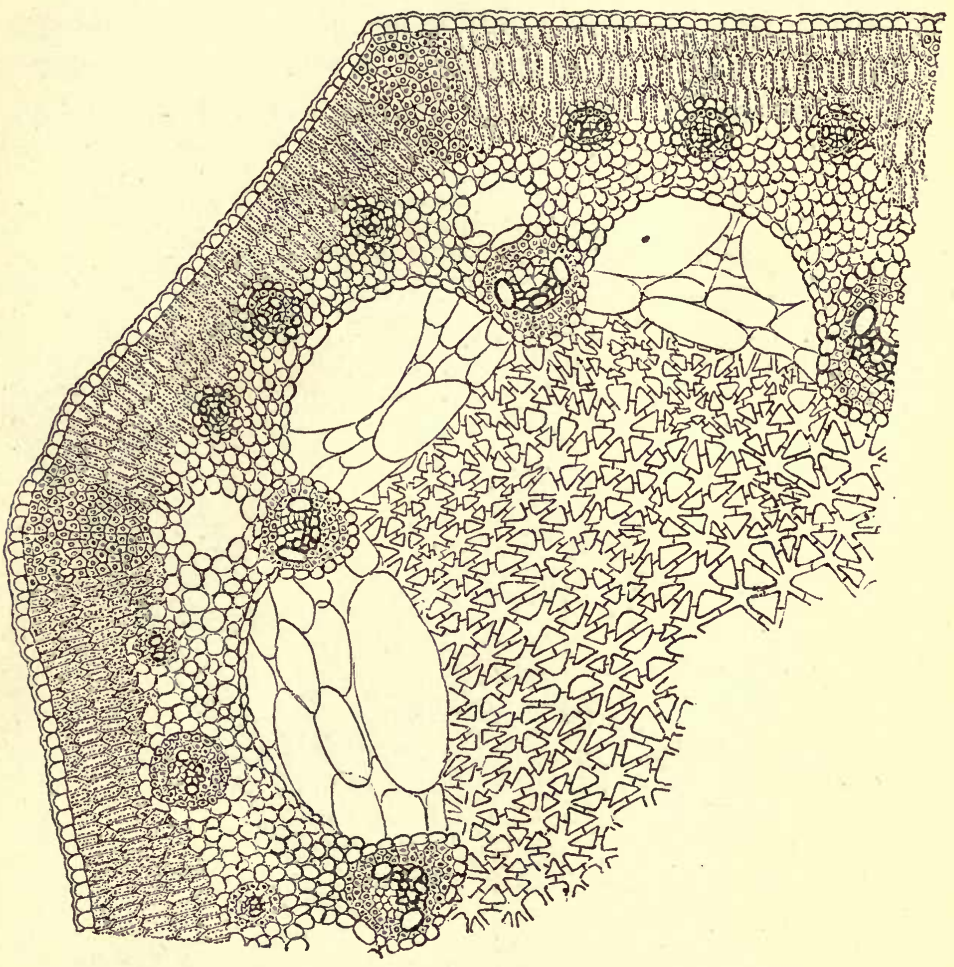

Fig. 78.-Portion of Section of Stem of Rush, showing Stellate Tissue of the Pith, with large Intercellular Spaces.

are of great assistance in promoting the aeration of the whole structure.

As has been already mentioned, the external orifices of the system of the intercellular spaces are the stomata of the leaves. In woody and corky parts these are supplemented by the lenticels. The evidence for this statement does not consist only of microscopic examination of the 
tissues. A direct proof can be afforded by a simple experiment. If the lamina of a leaf is immersed in water, air can be driven through it by subjecting the cut end of the petiole to gaseous pressure by means of an air-pump, or even by the effort of the lungs of the observer, and can be seen

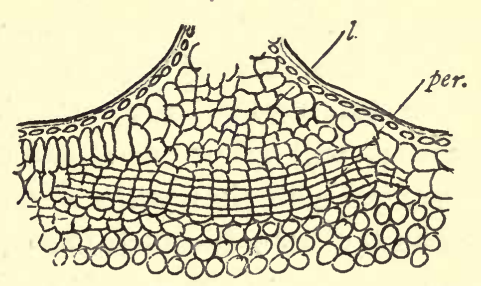

Fig. 79.-Section of a Lenticel. $l$, lenticel ; per, cork layer. to emerge from the surface of the leaf on which the stomatal apertures are situated. If a petiole is passed into a glass bottle through a tightly

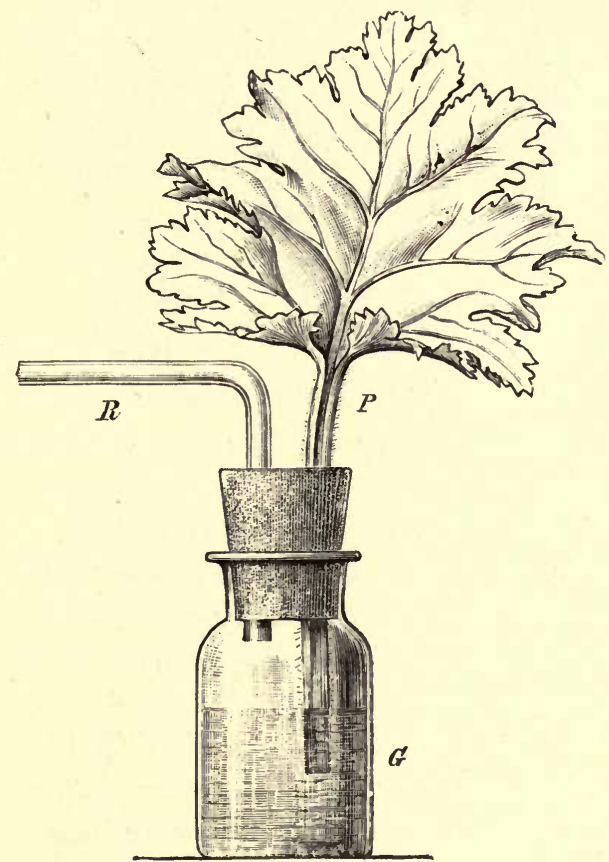

Fig. 80.-Apparatus to show Continuity of Intercellulak Spaces in the Leaf. (After Detmer.)

fitting cork, and covered with water, while the lamina remains in the air outside (fig. 80), bubbles of gas can be 
made to emerge from its cut surface in a continuous stream by reducing the pressure above the water by means of an air-pump.

The facility of the interchanges will largely depend upon the number, size, and position of these orifices. A lenticel will allow more gas to pass between its loosely arranged cells than will a stoma, but their relative numbers make the stomata much more important than the lenticels. In most cases there is a free passage through the stomatal pore, but in others considerable difficulty is afforded by the aperture being sunk in the epidermis or situated in a

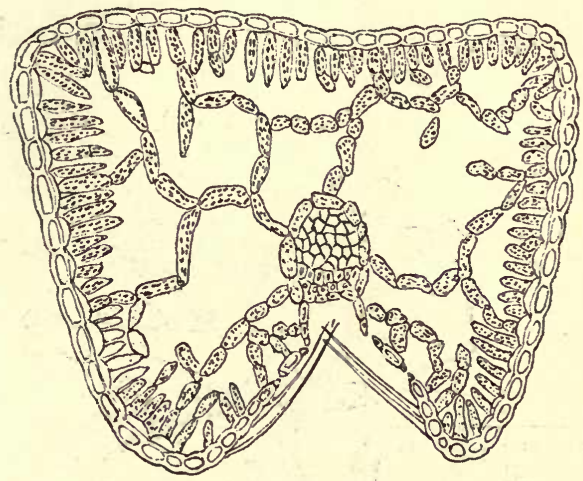

Fig. 81.-Transverse Section of Rolled Leaf of Heath.

depression of the leaf. In the rolled leaves of heaths and certain grasses this difficulty is frequently partially compensated by the lacunar character of the parenchyma, which is in the immediate neighbourhood of the stomata (fig. 81).

It must be noted in this connection that the stomata and the lenticels are passive with regard to the process of aeration, and do not exert an active influence upon it. The variations in the width of the stomatal apertures which are of so much importance in the regulation of transpiration must be regarded as bearing upon that function alone, being caused by fluctuations in the amount 
of water in the plant. They serve automatically to preserve the plant from excessive loss of water, but they have no direct regulating influence upon the interchange of gases. Indeed, when, from flaccidity of the leaves or from other causes, they close, the aeration of the plant is, to a certain extent, interfered with, if not suspended-a consideration which will help us to understand why a plant needs to contain so large a reservoir of air as is afforded by its intercellular spaces. The volume of this reservoir varies considerably in different plants, as has already been shown. Unger has put on record measurements of the relative volumes of air and cellular tissue in the leaves of forty-one species of plants. These were found to range from 77:1000 in Camphora officinalis, where it was least, to $713: 1000$ in Pistia texensis, in which it was greatest.

The movements of the air in the intercellular space systems of plants depend almost entirely upon the physical processes of diffusion. The entrance and exit of air from the exterior are generally possible, occasions when the orifices are completely occluded being very rare. It does not, however, at all follow that the atmosphere in the spaces has the same percentage composition as the external air. When we consider that it is the source of the supply of the gases used in the metabolism of the plant, and the recipient of those which are from various causes exhaled, it becomes evident that this is not the case. Nor is its composition uniform for even a short time, as the various processes which subtract from or add to it take place in different parts with very different rapidities. At the same time there is a tendency for it to become uniform according to the laws of the diffusion of gases.

The amount of nitrogen varies but little. This gas has a certain feeble solubility in water, and a small quantity goes into solution in the water which saturates the cell-walls; but as such nitrogen is not made use of in the cells, its absorption very speedily ceases, the cell-sap not being able to contain more than a trace of it. The 
percentage of nitrogen in a volume of gas obtained from a plant may not correspond with the percentage in an equal volume of air, but this will result from an interference with the amount of oxygen and carbon dioxide, and not be due to an absorption or exhalation of nitrogen, neither of which takes place to an appreciable extent.

The variations in composition which are noticeable are due to two processes which are characteristic of the vital processes of green plants. As we shall see in a subsequent chapter, all the green parts of plants are during daylight engaged in absorbing carbon dioxide from the air, and exhaling oxygen into it. In such parts this interchange takes place with considerable energy, and the composition of the air in their intercellular spaces varies accordingly, becoming relatively much richer in oxygen than it is in the deeper parts which are not illuminated, and which contain no green colouring matter. A change in the opposite direction goes on continually wherever there is living protoplasm, for this is always absorbing oxygen so long as it lives, while a good deal of carbon dioxide is simultaneously exhaled. This process, unlike the other one, is not confined to any particular part of the plant, nor is it ever in abeyance. Thus the plant shows a continuous and universal production of carbon dioxide, and a partial and local consumption of this gas. At the same time it exhibits a constant demand for oxygen everywhere, and a temporary production of it in places. The composition of the air in the intercellular spaces must therefore vary from time to time, and from place to place, according to the intensity and the localisation of these changes.

The process of diffusion, which is one of the phenomena characteristic of gases, leads to a constant occurrence of gaseous currents in plants. These currents may be influenced by various properties of the gases concerned, and by other factors, both internal and external. The rate at which carbon dioxide is absorbed by the cell-wall is very different from the rate of absorption of oxygen. If an atmosphere 
containing a good deal of the former gas is in contact with wet cell-walls, the result of the active absorption will be to set up a stronger current to that spot than would be the case if oxygen replaced it. Any cessation in the absorption of carbon dioxide by the green cells owing to diminution of light must be attended by a certain variation in the gaseous stream. The ways in which alterations in the absorption of oxygen will affect the currents will also be readily apparent. During bright sunlight, when both processes are proceeding in the same and in different parts of the plant, local positive pressures of either oxygen or carbon dioxide may occur, and it is evident that the direction of the gaseous currents will be very varied in consequence.

The structure of the plant has a certain influence on the composition of its internal atmosphere. The epidermis of most terrestrial plants is strongly cuticularised, while there is but little cuticle to aquatics. The entry of gases into the latter is accordingly easier than it is into the former, penetration into which must take place through the stomata. Moreover, the larger reservoirs in the interior of aquatics serve to equalise the composition of the internal atmosphere, and to cause it to resemble more closely that of ordinary air.

Such plants again as contain no green colouring matter-for example the bulkier Fungi, which require provision for the supply of air to their interior-have only the one metabolic process in which the interchange of oxygen and carbon dioxide is involved, the former being absorbed, and the latter exhaled. To a corresponding extent, therefore, the gaseous currents are simplified, though even in these plants the direction and the amount are never constant for long together, the metabolism continually varying.

In another important respect the internal air of plants differs from that of the atmosphere. It is always charged with aqueous vapour, frequently even to the saturation point, as we have seen in connection with the process of transpiration. 
The external conditions to which a plant is exposed have a considerable influence upon the gaseous currents. The effect of light upon a green plant has already been alluded to. The influence which it exerts is an indirect one, affecting the consumption of carbon dioxide and the liberation of oxygen. Nearly all the vital processes are subject to modification by the various external conditions. Transpiration we have seen to be very largely influenced thereby, and the varying amounts of watery vapour exhaled introduce variations in the amounts of the purely gaseous interchanges. The influence which the variation of the quantity of water in the plant exercises takes the form especially of modifying the width of the stomatal apertures, and hence of favouring or checking the entry and exit of gases into and from the leaves.

Mechanical disturbances due to wind are of some importance, generally increasing the gaseous interchanges. Diminution of the turgidity of the tissues, amounting sometimes to flaccidity, interferes at times to a serious extent, the intercellular spaces becoming narrowed by the falling together of the cell-walls, a phenomenon which is noticeable also in the partial or complete closure of the stomatal orifices, due to the flaccidity of their guard-cells.

Variations of barometric pressure and of temperature also influence to a considerable extent the process of diffusion within the plant, as well as the interchange between the interior and the external air.

The movements of the air in the plant are subject to disturbance also by the setting up of the negative pressure in the cavities of the vessels of the wood which we have seen to be caused by active transpiration. This negative pressure can be demonstrated with considerable ease in the cases of woody stems, but it can be seen also in plants in which the development of wood is only very slight, having been observed in some cases in the elements of the central cylinder of some of the stouter Mosses.

To demonstrate the existence of the negative pressure 
in the vessels of the stem, a young plant should be removed from the soil and allowed to become flaccid. The stem should then be partially immersed in mercury and cut across below the surface of the latter. The mercury will immediately rise to some distance in the vessels, being drawn up by the suction exerted by the negative pressure therein.

An actual positive pressure can under certain conditions be observed in the intercellular air-reservoirs of particular plants. This can be shown by cutting the stems of submerged plants such as Myriophyllum, when, if they are brightly illuminated, bubbles of gas may be seen to emerge from the cut end. This positive pressure appears to be due to a considerable production of oxygen by the green parts of the plant under the conditions of illumination, as it varies with the intensity of the latter, and ceases entirely in darkness.

It is well that we should lay some stress upon the relation which the stomata show to the processes of gaseous interchange. Though they are the chief means of the entry of gases into and their exhalation from the plant, it is misleading to speak of them as the organs of such gaseous interchange. The actual processes of interchange take place between the protoplasts and the air of the intercellular reservoirs, so that the latter are the special organs devoted to such functions. The stomata and the lenticels are merely the openings by which the air of these internal formations communicates with the outer atmosphere. The true gaseous interchanges which subserve the life of the protoplasts, and hence of the plant, take place not at the stomatal orifices, but completely throughout the interior of the substance of the plant. 


\section{CHAPTER VIII}

\section{RESPIRATION}

OF the gaseous interchanges which were mentioned in the last chapter as characteristic of living protoplasts, the most widespread is that which is marked by the absorption of oxygen. With the exception of a few of the lowlier organisms, all of which are members of the group of Fungi, every living protoplast must be constantly absorbing this gas in order not only that its vital activities may continue to be discharged, but that its life itself may be maintained. Withdrawal of oxygen from the environment of the protoplast is after a longer or shorter interval followed by its death. It is true that under certain conditions which we shall discuss in a subsequent chapter the interval may be prolonged, but death ultimately ensues.

This absorption of oxygen is in most cases associated with an exhalation of carbon dioxide, which is generally given off in a volume approximately equal to that of the oxygen taken in.

The universality of this process is not always easy to demonstrate. It can be ascertained without difficulty in the case of almost all animal organisms, and of such of the vegetable ones as possess no chlorophyll. In the case of those plants which are green, however, there is, as we have already mentioned, and as we shall discuss more fully later, a converse gaseous interchange occurring so long as the green parts are exposed to sunlight, carbon dioxide being absorbed and decomposed, and an equal amount of oxygen being exhaled. This interchange is usually more vigorous than the first one, and the latter is therefore diff- 
cult of detection under conditions which allow both to take place simultaneously.

The absorption of oxygen can be easily observed in the case of a large fungus, such as a mushroom. If one of these plants is placed in a closed receiver containing air, and is left there for several hours, at the conclusion of the experiment the mixture of gases in the receiver will be found to be almost devoid of oxygen, that which was there originally having disappeared. An almost equal amount of carbon dioxide will be found to have replaced it, so that the volume of gas in the receiver will be unaltered.

It is possible to devise an experiment which will show that a green plant has the same absorbing power. If the light is excluded from one placed in a similar vessel, no evolution of oxygen will take place from it, and that the oxygen present in the air at the commencement of the observation will diminish to the point of extinction can be made evident, just as in the case of the mushroom.

We have evidence, however, that this is not caused by the exclusion of the light, but that the gaseous interchange in question proceeds in the light as well as in darkness. An apparatus which was originally devised by Garreau, and which can be easily arranged to show the absorption of oxygen, even when a green plant is exposed to a bright sun-

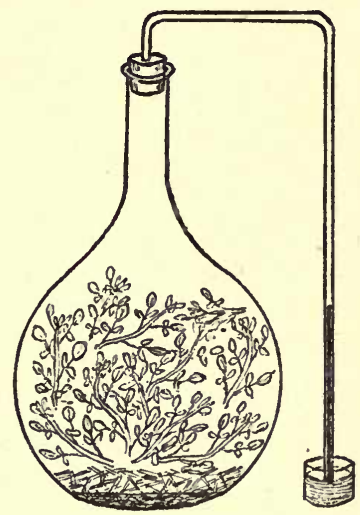

Fig. 82.-Apparatus to show the Absorption of Oxygen by a Green Plant. light, is shown in fig. 82. It consists of a glass vessel which can be closed by a cork through which a bent glass tube of small calibre is passed. The tube is carried over and made to dip into a small dish containing mercury. The bottom of the vessel is covered with finely broken glass, upon which is poured a strong solution of caustic potash. 
Above the latter, supported by the glass so as not to be in contact with the alkali, is placed the plant to be examined. Watercress or any other herbaceous plant will answer very well. The potash will absorb the carbon dioxide of the atmosphere originally admitted as well as whatever quantity of this gas is given off during the experiment. As the experiment progresses the temperature must be kept constant, when the mercury will be found slowly and gradually to rise in the small glass tube, indicating a diminution of the volume of the air in the flask. If the experiment is continued till the mercury ceases to rise in the tube, and the gas remaining in the vessel is measured at the ordinary atmospheric pressure, and at the temperature at which the experiment was started, it will be found that its volume has been diminished by about twenty per cent., and that what is left consists of nitrogen. The oxygen will have been completely removed by the green plant, even when the apparatus is left exposed to the sunlight during the daytime. If the caustic potash is examined, it will be found to have gained considerably in weight, and to contain a quantity of carbonate of potassium, derived necessarily from the plant during the experiment. The weight of this. will enable the volume of the evolved carbon dioxide to be ascertained. There will have been proceeding during the experiment an absorption of oxygen, attended as before by, an exhalation of carbon dioxide, the latter having combined with the potash.

The evolution of carbon dioxide by the plant can be more easily demonstrated by the use of the apparatus shown in fig. 83. The jar A in the centre contains the plant to be examined, which may preferably be represented by a number of germinating peas. It is closed by a cork, which is perforated in two places. Into one hole a tube is inserted which passes to the bottom of the jar, and serves for the admission of air. An outlet tube passes through the other hole from the upper part of the jar, and leads to 
another jar, c, which is partially filled with baryta water. The final outlet from $\mathrm{c}$ can be attached to an aspirator by which a stream of air can be drawn through the apparatus.

Before the incoming air reaches the jar $\mathrm{A}$ it is made to pass through another jar, $F$, containing a solution of caustic potash which frees it from all traces of carbon dioxide. To ascertain that this is secured, it passes next through a jar B which contains baryta water. A stream of air is then passed slowly and continuously through the whole apparatus, and as it bubbles through the baryta water in $\mathrm{c}$

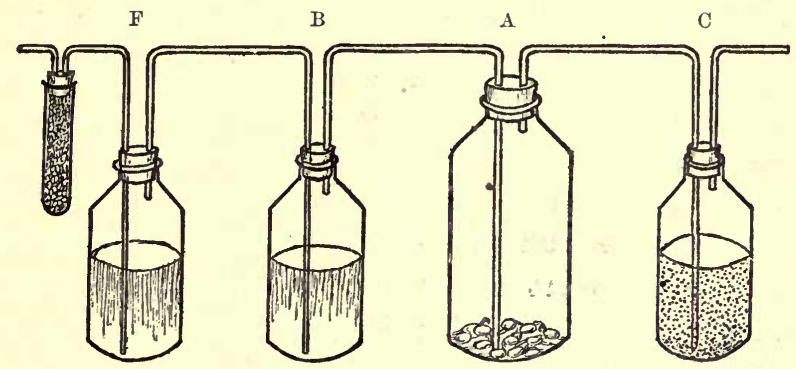

Fig. 83.-Apparatus to show the Exhalation of Carbon Dioxide by Germinating Seeds. The air enters through the tube on the left; ITS CARBON DIOXIDE IS ABSORBED BY THE POTASH IN F. IT PASSES THROUGH A, IN WHICH THE SEEDS ARE PLACED, AND THE CARBON DIOXIDE GENERATED THERE IS CARRIED OVER INTO C, WHERE IT IS PRECIPITATED BY THE BARYTA WATER.

it causes the formation of a white precipitate, which analysis shows to be barium carbonate. The formation of this body proves the evolution of carbon dioxide from the seeds, as the entering air contains none. By using a known strength of baryta water in c, the amount of the gas evolved in a definite time may easily be ascertained.

These two processes, the absorption of oxygen and the exhalation of carbon dioxide, are characteristic of what is known as respiration. As already stated, it is a normal process of the life of almost all protoplasm, and is continually going on so long as life lasts, although it is not easily observed while the converse process, the absorption 
and decomposition of carbon dioxide, is proceeding, accompanied by the evolution of oxygen. It is frequently said that during daylight the process of the respiration of a green plant is masked by that of carbon dioxide decomposition. To put this statement into somewhat different terms, the carbon dioxide which is liberated in the course of respiration by the green plant, and which is in comparatively small amount, is reabsorbed by the green parts of the cells, and undergoes the same decomposition as that which is brought to the plant by the surrounding air. It thus escapes observation unless special means, such as those detailed, are adopted to bring it into evidence.

The respiratory processes are easily observed in the case of all plants, and parts of plants, that are not green, as there are in such cases no gaseous interchanges that would interfere with their manifestation.

If a plant be carefully weighed at the commencement and at the end of such an experiment as has been described, it will be found to have lost weight during its stay in the receiver, so that respiration is associated with a loss of weight to the plant. This may readily be inferred from the fact that the oxygen absorbed and the carbon dioxide exhaled are approximately equal in volume, carbon dioxide being perceptibly heavier than oxygen. Besides the carbon dioxide, however, there is always also a certain exhalation of watery vapour which takes place quite independently of any supply from the root or the cut end of the stem. The nature of the metabolism, or the vital processes, is such that the living substance gives off both water and carbon dioxide, while it coincidently absorbs oxygen. This is quite independent of any constructive processes, for it can be observed when no nutritive material of any kind is supplied to the plant.

Though respiration is constantly proceeding wherever living substance is found, the activity of the process is by no means uniform. With care it can be detected in such quiescent parts of plants as resting seeds, or buds 
during their winter suspension of development, but in such cases the gaseous interchange is reduced to a minimum. In growing shoots or germinating seeds in which vital processes such as the growth of protoplasm are going on rapidly, and life is very active, it reaches a maximum. In ordinary adult leaves and branches the activity of respiration is intermediate between the other two conditions. It is more intense, again, in the floral organs during the time of their maturation. We may say in general terms, wherever protoplasm is abundant, and the chemical processes connected with the manifestation of its life are going on most vigorously, there respiration is most active. It is connected especially with the vital processes, and is not associated directly with the presence of food materials. A proof of this is afforded by an estimation of the activity of respiration in seedlings, which, in the case of wheat, has been found to increase steadily for about a fortnight, and then to decline. Further evidence is afforded by the fact that if seeds are thoroughly dried they do not respire. In this condition the protoplasm is completely quiescent, so far as we can ascertain. If, however, only a little water is supplied to them, which, as we have seen in an earlier chapter, is a condition necessary to set up changes in the protoplasm, respiration commences, and increases as the proportion of water present rises up to a certain limit.

When the respiratory processes are carefully measured and compared with the weight of the organism, it is found that under appropriate conditions they are more intense in plants even than in warm-blooded animals. The respiratory activity is as great in many seedlings as it is in the human body, provided that both are maintained at the same temperature. There is, however, a very great variability in this respect, and the maximum activity is never maintained very long in any particular plant. As maturity succeeds to development its amount falls materially, being marked at or near the original rate only in the regions of the active meristems. 
All seedlings, again, are not alike in the vigour with which they carry on their respiratory processes.

We may pass on to inquire what is the relation between the absorption of oxygen and the formation and elimination of carbon dioxide and water. It is conceivable that the oxygen may unite in the plant with carbon and with hydrogen to produce at once the exhaled compounds. A study of the living organism at work, however', soon shows us that the process is not of this simple nature. We have said, in the course of what has already been advanced, that the amount of the carbon dioxide exhaled and that of the oxygen absorbed are approximately equal. This, however, is only true within certain limits; if each is measured accurately, they are not found to show an exact correspondence. The ratio $\mathrm{Co}_{2}: \mathrm{O}$ is usually spoken of as the respiratory quotient. When the two processes are equal the value of the respiratory quotient is unity; when the carbon dioxide is in excess it is greater, and when the oxygen is in largest amount it is less, than unity. The respiratory quotient has been found to vary to a greater or less extent in different plants, and in the same plant under different conditions. If its value is determined in the case of germinating seeds, these differences are soon evident. With starchy seeds the quotient is unity; with oily seeds it is much lower. That is, in the former case, the seeds absorb a volume of oxygen equal to that of the carbon dioxide they exhale; in the latter case they take up more.

Various observers have shown that in certain cases succulent leaves, such as those of the Agave or of particular plants belonging to the Saxifragacea and the Crassulacea, or again the phylloclades of Opuntia, one of the Cactacere, are capable of absorbing oxygen without the simultaneous evolution of carbon dioxide. Nor is the oxygen absorbed in these cases any more than it is in others without entering into some form of chemical combination, 'for it cannot be extracted by the air-pump. The latter also fails to extract any carbon dioxide from the plants. The oxygen 
enters the plant, and is in some way fixed or combined; the other process which usually accompanies this absorption does not take place, the carbon dioxide not only not being exhaled, but apparently not even formed.

Conversely, earbon dioxide may be thrown off from a plant without any simultaneous or even antecedent absorp. tion of oxygen. When a seed is made to germinate in a vacuum over a column of mercury, carbon dioxide is found to be evolved. Ripe fruits have been found to give off this gas in an atmosphere quite devoid of oxygen. Too much stress must not, however, be laid upon these latter observations, as we have certain evidence which points to a different mode of formation of the carbon dioxide in the presence and in the absence of oxygen respectively.

Again, it is found that the respiratory quotient varies according to the temperature at which the observations are made. Evidently the two processes are not directly dependent upon each other.

In making the estimation of the respiratory interchanges we are apt to lose sight of a fact to which attention has already been called, viz. that carbon dioxide is not the only respiratory exhalation. The watery vapour which accompanies it must also be accounted for. On the hypothesis of the direct oxidation of carbon and hydrogen, if the volume of carbon dioxide is equivalent to that of the oxygen, there cannot have been the absorption of sufficient of the latter to unite with hydrogen to form the water. Even when the respiratory quotient is less than unity, the same consideration has a certain value. The idea of such direct oxidation cannot therefore be accepted.

It is evident from the foregoing considerations that the vital activity of the protoplasts is somehow associated with the two factors in the gaseous interchange. In the absence of oxygen this vital activity gradually ceases, the living substance being in fact slowly stifled or asphyxiated. During its life one of the manifestations of its vitality is 
the formation and exhalation of two fairly simple compounds, carbon dioxide and water. To ascertain what is the true relation of the two processes, it is necessary to look closely at the nature of the chemical changes going on in the protoplasm itself, or what is usually spoken of as its metabolism.

Respiration in the strict sense is therefore a process going on in the living substance itself. The gaseous interchange observed is the expression of the beginning and the end of a series of complex changes in which the molecules of the living substance are involved. The details of the absorption of the oxygen of the plant from its environment, and its presentation to the protoplasm, together with those of the ultimate evolution of the carbon dioxide and water from the plant-body, should be regarded rather as belonging to the mechanism of respiration than to respiration itself, which is a function of the living substance only. The former corresponds to the entry of the oxygen into the lungs of an air-breathing mammal, and its transport to the tissues, together with the return of the carbon dioxide and water therefrom ; the latter is strictly comparable to the changes taking place in those tissues after the entry of the oxygen into them.

The variation of the respiratory quotient which we noticed in starchy and oily seeds respectively points to a varied metabolism, according to the nature of the food supplied to the living substance.

We see, then, that the two processes are not immediately connected in the sense of the carbon dioxide and water coming at once from the direct oxidation of carbon and hydrogen, but that they are ultimately associated there can be no doubt, though they are separated in time by a series of chemical changes taking place in the living substance. This ultimate association is shown by the fact that, if the access of oxygen to a plant is prevented, after a longer or shorter period the evolution of carbon dioxide ceases.

To get a true view of the nature of the process of 
respiration we must therefore turn our attention to the metabolic changes which are taking place normally in the living substance. From the instability which we have noticed in the protoplasmic material, we can infer that its own molecules are in a constant state of decomposition and reconstruction, new material being incorporated and certain other substances cast off. Besides these, we are probably not wrong in concluding that other changes also take place in the various substances which are contained in it, into which its own molecules do not enter. Processes of slow oxidation and gradual reduction are taking place there continually, excited, however, in all probability by the changes in the protoplasm itself. Even these, however, are by no means simple, and the direct oxidation of either carbon or hydrogen has probably no place among them. An instance of them may be seen in the oxidation of alcohol in the cells of Mycodermi aceti, a fungus which converts alcohol into acetic acid. This process, into which the molecule of protoplasm apparently does not enter, can only go on in the living cell. Other similar instances could be quoted.

The probable course of events is that the oxygen in some way unites with the protoplasm, rendering it unstable, and initiating a series of decompositions which result in the successive formation of many bodies of less complex composition, each successive decomposition producing simpler ones, till finally carbon dioxide and water are formed. Simultaneously, reconstruction of the protoplasm goes on, many of these residues being in whole or in great part built up again into its substance, together with new material supplied to it in the shape of food. If the temperature is low, the breaking down of the protoplasm proceeds but slowly, and reconstruction is rapid, so that under these conditions the quantity of oxygen absorbed or fixed as intramolecular oxygen by the protoplasm is greater than the quantity of carbon dioxide formed by its decomposition. At a higher temperature decomposition is 
much more easily carried on, and its products are more numerous and simpler. The decomposition and recomposition go on side by side, simpler bodies being gradually produced, either by their splitting from the protoplasm directly, or by their being formed at the expense of the more complex decomposition-products during processes of slow oxidation in the substance of the protoplasm, till finally a certain production of carbon dioxide and water is arrived at. So long as the protoplasm remains alive the amount of these is relatively small, reconstruction continually taking place. When, however, the protoplasm dies, simple bodies, such as carbon dioxide, water, and possibly ammonia, in addition, are produced abundantly from the decomposition which attends its death.

It is possible that such processes as these are supplemented by others, in which the living substance is not so intimately concerned. The protoplasm may combine loosely with oxygen, and transfer it to other substances present in it, which are consequently more directly oxidised, the protoplasm in some way inducing the oxidation of compounds which oxygen alone cannot attack. In this way the protoplasm may act as an oxidising agent, without itself undergoing the decompositions suggested.

The carbon dioxide is thus the final term in a series of decompositions, of which the living substance is the seat and into which it may actually enter, the decompositions themselves being promoted by the access of oxygen. In some cases, such as those of the succulent leaves of the Crassulacere and the tissues of the Cactus already alluded to, this final term is not reached, no carbon dioxide being exhaled. We have no reason to think that in these cases a fundamentally different series of changes is set up. De Saussure found that a piece of stem of Opuntia absorbed a quantity of oxygen, which could not be extracted from it by the air-pump. The fate of this oxygen must have been similar to that which is absorbed by other plants; it must have entered into some form of combination, probably with 
the living substance. The resulting decompositions, though taking at first the same course as in other cases, did not go so far. Instead of the liberation of carbon dioxide, there was found a considerable increase in the amount of certain organic acids, chiefly malic and oxalic acids, which remained in the cells, and which probably represented the ultimate products of the decompositions.

We have mentioned in the earlier part of this chapter that there are a few lowly forms of plants which do not require oxygen for their vital processes, and which do not absorb it. Many of these so-called anaërobic plants are killed by exposure to this gas. We must not, however, necessarily conclude that their metabolism is of a totally different kind from that of others, but rather that they set up the decomposition and reconstruction of their protoplasm in a different way from those plants which need a supply of oxygen to determine them. We shall return to this point in a subsequent chapter, in which we shall consider also the meaning of the complex metabolic processes, which we have so far discussed.

Though respiration is always proceeding wherever there is living protoplasm, the activity of the process is modified by different physical conditions. Of these, temperature is one of the most important. There is a lower limit, beyond which it appears to be suspended, though life is not destroyed. This limit varies in different plants, but is generally one or two degrees below the freezing point of water. In a few eases, such as Conifers and Lichens, it may' even be $-10^{\circ} \mathrm{C}$., but this is rare. As the temperature rises from this minimum point, the activity of respiration increases up to a certain optimum, point, which is usually not well defined, and which varies considerably in different plants. If the temperature is raised only a little higher than this, the living substance is rapidly injured, and its respiration is checked. Variations 
in temperature do not affect equally the absorption of oxygen and the exhalation of carbon dioxide. At low temperatures the latter is smaller than the former; at high ones the reverse is the case.

The effect of light upon respiration is not very marked, and is probably indirect. Plants which grow in shady spots usually manifest less respiratory activity than similar ones growing in bright sunlight, but this may be the result of the difference in the amount of nutritive material they obtain, which is incident to the difference in their situation. As we shall see in a subsequent chapter, light has a very marked influence on the metabolic processes, and its indirect effects may be very farreaching.

Respiration is considerably affected by variations in the amount of oxygen which the environment of the plant contains. The protoplasts can absorb even the last traces of the gas which reach them, but a certain amount is necessary for them to maintain a healthy condition. Great variations are not usually met with, but on the summits of high mountains there is much less available for them than at the sea-level. If the amount of oxygen in the atmosphere from any cause falls below about 5 per cent., respiration is seriously impeded. Similarly plants cannot thrive in the presence of too great an amount. When the pressure of the gas attains the amount of twenty to thirty atmospheres, respiration becomes very difficult and after a short time ceases, and death ensues.

The process of respiration is also affected to a considerable extent by the nature of the substances which serve as nutritive material for the reconstruction of the protoplasm. It has already been pointed out that seeds containing oil absorb more oxygen during germination than those which contain principally starch. Fungi which are fed with carbon-compounds that contain relatively little oxygen, give off relatively less carbon dioxide than others which are 
supplied with food containing a large percentage of this constituent. Organs which contain much proteid matter respire more copiously than others which contain but little. The nature of the inorganic salts absorbed also influences the process to a certain extent, though probably these only act indirectly. 


\section{CHAPTER IX}

THE FOOD OF PLANTS. INTRODUCTORY

A GOoD deal of misconception exists as to the nature of the food of plants. The character of their environment, and the absence in most cases of any means provided in their structure for the taking in of any material having a composition in any way approaching that of living substance, have led to a not unnatural idea that they feed upon simple inorganic compounds of comparatively very great simplicity. This idea has found considerable support in the fact, which is easily ascertained, that such bodies are those which are absorbed in the first instance. By their roots when they live fastened in the soil, or by their general surface when they are inhabitants of water, comparatively simple inorganic salts are found to enter them with the water which they take up. By their green parts, and especially by their leaves, carbon dioxide is absorbed, either from air or water, according to their habitat. A study of the whole vegetable kingdom, however, throws considerable doubt upon the theory that these compounds are, in the strict sense, to be called their food. Fungal and phanerogamic parasites can make no use of such bodies as carbon dioxide, but draw elaborated products from the bodies of their hosts. Similarly those fungi which are saprophytic can only live when supplied with organic compounds of some complexity, which they derive from decaying animal or vegetable matter. We have no reason to suppose that the living substance of these non-chlorophyllaceous plants is so radically different from that of their green relations that it has a totally distinct mode of nutrition. 
In the flowering plants we find a stage of their life in which the nutritive processes approximate very closely to those of the group last mentioned. When the young sporophyte first begins its independent life - when, that is, it exists in the form of the embryo in the seed-its living substance has no power to utilise the simple inorganic compounds spoken of. Its nutritive pabulum is supplied to it in the shape of certain complex organic substances which have been stored in some part or other of the seed, sometimes even in its own tissues, by the parent plant from which it springs. When the tuber of a potato begins to germinate, the shoots which it puts out derive their food from the accumulated store of nutritive material which has been laid up in the cells of its interior. Considerable growth and development can take place without the access of any of the inorganic substances which the parent plant was continually absorbing. Fleshy roots, corms, bulbs, and all bodies which are capable of renewed life after a period of quiescence, show us the same thing; the young shoots emerging from any of them are not fed upon simple inorganic bodies, but upon substances of considerable complexity, which they derive from the tissues of the structures from which they spring.

In adult plants of the most considerable complexity we find instances of the same thing, though in these cases it is generally rather more difficult to determine it; the living substance is nourished by materials which have been constructed by it and stored at various places in its tissues till their consumption has been called for.

What, then, are these substances which, in the strict sense, constitute the food of plants? We can ascertain what are necessary by inquiring what are the materials which are deposited in the seed for the nutrition of the embryo during the process of germination. This process is the most favourable for the elucidation of this point, because, in its early stages at any rate, the nutrition of the young plant is not complicated by any absorption from 
the surrounding medium, such as sometimes rapidly supervenes on the emergence of a shoot from a tuber or a fleshy root. We find the seed contains in some part or other of its substance, sometimes even in the embryo itself, examples of great classes of food-stuffs which are the same as those on which animal protoplasm is nourished, and whose presence renders seeds such valuable material for animal consumption. As these disappear during the development of the young plant, which thus evidently grows at their expense, we cannot doubt that they form its food, and that vegetable protoplasm is essentially identical with animal, at any rate so far as its methods of nutrition are concerned. Proteids, carbohydrates, fats or oils, together often with certain other bodies which are less widely distributed, are the materials which, in various forms, are met with.

If we study the protoplasm of a living, active, vegetable cell, and treat it with appropriate solvents, we can extract representatives of these, or of some of them, from its substance, in the interior of which they are held sometimes in solid amorphous form, sometimes in fine suspension or in actual solution. The nutrition of the protoplasm can only take place when these substances are brought into the most intimate relations with it; from them, no doubt, in ways not yet discovered, it builds itself up, and by its own decompositions it reproduces many of them. The details, however, of the interchange of matter between the living substance and its food, the way in which the latter is transformed into the former, are points about which almost everything essential remains still to be discovered.

But while we recognise that the ultimate nutrition of protoplasm is dependent upon its receiving a supply of such materials, we are face to face with the fact that, with a few exceptions, the consideration of which may be deferred, they are not furnished at all from the environment to the ordinary green plant, and often only partially 
so to the saprophytic fungus, though they are freely obtained from their host-plants by parasites. On the contrary, we find the ordinary green plant taking in by ordinary physical processes carbon dioxide from the air, and water containing a variety of salts from the soil. The saprophytic fungus may, and frequently does, obtain from its surroundings certain compounds of ammonia, together with some carbohydrate bodies, such as sugar. We can ascertain that if these different compounds are supplied under suitable conditions to the groups of plants mentioned, the latter can flourish and develop. While we have the strongest grounds for holding that the protoplasm is essentially similar in all these cases, we see marked differences between them with regard to the materials which they absorb. The substances supplied to the green plant are utterly unlike what we have seen to be the actual food ; the saprophytic fungus can make use of the compounds of ammonia, but absorbs carbohydrates as such, while the parasite, whether fungus or phanerogam, obtains the materials which we see are directly capable of feeding it.

If we say that the food of these various groups of plants varies in the degree of its complexity, we must carefully consider in what sense we use the term food. In the nutrition of the green plant there are clearly two very different processes combined, which should be kept carefully distinct. We have the absorption of food materials rather than of food in the true sense, and we have, following such absorption, the expenditure of a considerable amount of energy upon these food materials, with the result that they are worked up into the complex compounds which we find protoplasm can assimilate, and which are those which are stored away in the substance of the plant for the nutrition of vegetable substance and the development of embryo, bud, or growing point.

In the case of the green plant this power of constructing food extends to all the classes of food-stuffs; in that of the saprophytic fungus it only applies to the proteids 
and the fats, the carbohydrates needing to be supplied to it as such, as we have seen.

This difference between food and the crude materials from which it is constructed can be made clearer by examining whether such simple inorganic bodies as the green plant absorbs are capable of nourishing protoplasm when freely supplied to it. If they are the true food, plants everywhere should be able to make use of them. But if we consider only one of them, the carbon dioxide of the air, we find this is not the case. The plants which are not green-that is, which contain no chloroplasts-can do nothing with this gas. So long as a seed is in the early stages of its germination, it is surrounded by carbon dioxide, which is given off by its own protoplasm. But it can make no use of it, and if the store of nourishment provided for it in the endosperm or cotyledons is cut off, it inevitably dies of starvation. A saprophytic fungus in like manner is dependent for its life upon the absorption of such a compound as sugar, and carbon dioxide cannot aid at all in its nutrition.

Another fact throws a certain light upon the relation of carbon dioxide to the feeding of a green plant. If such an individual, in good health and endowed with ample vigour, is removed from light to darkness, though this gas be supplied in appropriate quantity, it can make no use of it. The gas is evidently useless for immediate nutrition, and its ultimate utility is dependent upon its being submitted to the action of some mechanism in the plant which is called into play under certain conditions, of which adequate illumination is one.

Similar considerations apply to other constituents of the materials from which the true food of the living substance is elaborated. They are absorbed in quantity, but they do not become food until a considerable amount of work has been done upon them by the plant itself.

In the strict sense, it thus appears that the ordinary green plant does not absorb its food from without. It takes 
in various raw materials from which it manufactures its food in particular parts of its own tissues.

In connection with the nutrition of plants we have thus to deal with the absorption of the crude food materials, and to study the changes which they undergo after such absorption. But this is not all ; the food which is manufactured from them is not merely prepared in answer to the immediate requirements of the moment. A considerable excess is usually constructed, and the surplus quantity is stored in various parts of the plant's body for subsequent consumption.

The food which is thus laid up in seeds, tubers, bulbs, \&c. is not deposited there in exactly the condition in which the living substance requires it, so that there remains for us to consider the processes of storage and the changes which the stored materials subsequently undergo for the purpose of feeding the living protoplasm.

The construction of food from the materials absorbed is one of building up complex bodies from simple materials.

The utilisation of the stored surplus is comparable with the digestion which is so marked a feature of animal alimentation, and is one of breaking down of complex bodies into simpler ones.

The actual nutrition of the protoplasm shows again two distinct phases : the incorporation into its substance of the ultimate constituents of the food, or its assimilation, is a constructive process; it is in turn associated with a destructive one, by which, from the protoplasm itself, and by its own activity, simpler bodies are produced.

The whole round of changes which embraces all these operations is called metabolism, the constructive processes being grouped together under the name of anabolism, the destructive ones under that of katabolism.

The absence of well-differentiated organs set apart for the discharge of these separate functions makes it rather difficult at first to appreciate their independence. In mosit animal organisms such a differentiation is easily seen, but 
in plants the cellular structure is so prominent, and the life of the protoplasm is so closely related to its condition in the cell, that attention needs to be specially directed to the point. Each protoplast is dependent upon the contents of its own vacuole, and the early constructive processes in the metabolism, including the manufacture of food in such cells as carry out this process, may take place in it side by side with the digestive changes and at almost the same time. True, a certain division of labour can be noted, but it is not very clearly associated with particular organs. Thus the leaf is especially concerned in the manufacture of food, but it is mainly so by virtue of the chloroplasts which its cells contain, These processes can go on perfectly well in other parts than leaves; indeed wherever there are chloroplasts we know they do. Thus, though we associate the leaf with this manufacture, it would be wrong to speak of it as the organ to which this process must be referred. We can say with greater accuracy that the chloroplast is the organ which conducts these preliminary constructive processes, and that they take place wherever the chloroplasts are found. The wide distribution of the latter, however, shows us that there is no specially differentiated member of the plant set apart to be an organ for this function. In the same way the digestive process, or the utilisation of stored products, goes on wherever there are reservoirs of such bodies, and takes place in the cells of which such reservoirs consist. There, and there only for the most part, unorganised ferments or enzymes are found, instead of being located in particular glands, as in the animal body. These reservoirs, as we have already seen, and shall see again later, are found in the most varied regions of the plant's substance, regions moreover which differ considerably in situation in different plants. We cannot therefore speak of a differentiated organ of digestion.

Starting, then, with the intricacy of the metabolic processes placed before us, and with their relations to each 
other, we may begin the consjderation of them in detail with an inquiry into the preliminary absorption of the materials from which the food is ultimately made. Even here we meet with some complexity, as the ordinary green plant shows marked differences in behaviour from its parasitic relative and from the great class of Fungi, which possess no chlorophyll. We have already pointed out that the construction of food does not follow exactly the same course in green plants and saprophytic fungi, the chief point of difference being seen in connection with the carbohydrates. It will be best to consider first the ordinary terrestrial green plant, noticing in passing differences in behaviour shown by aquatic and epiphytic forms. 


\section{CHAPTER X}

THE ABSORPTION OF FOOD MATERIALS BY A GREEN PLANT

$\mathrm{WE}_{\mathrm{E}}$ have seen that the materials which protoplasm is eventually able to assimilate or incorporate into its own substance, and which, therefore, constitute its food, are of a similar nature to those deposited in seeds and other storehouses of nutriment. We know further that these are not the materials which an ordinary green plant takes into itself from the environment in which it lives. We know also that its structure prevents its taking in anything in a solid form, and that everything entering it musi either be in solution in the water which it is almost constantly absorbing through its roots, or must become dissolved in the liquid which permeates the walls of the cells which line the intercellular passages. The only substances that can be taken up under these conditions are certain gaseous constituents of the air, and various inorganic salts which are present in the soil. Between such raw materials, and the complex products which are needful for the nutrition of its substance, there is a great difference, and the manufacture of the latter from the crude materials absorbed constitutes a very important part of the metabolic processes.

There are several ways in which we may proceed to discover what a green plant absorbs from the soil, two of which especially have been made use of by various observers. The first is known as the method of water-culture. It consists in cultivating plants with their roots inserted in water containing various salts in solution, and observing what effect upon their growth and development is pro- 
duced by the addition of eertain compounds to the culture fluid, or how the absence of any particular salt affects their well-being.

In carrying out experiments in this way, it is usual to sow some large seeds, such as those of the broad bean, in damp sawdust, and allow them to germinate. When the radicle of the seedling has elongated to the extent of about an inch, the seed is placed upon a perforated cork inserted into the neck of a bottle containing the liquid which is the subject of the investigation. It is so arranged that the radicle dips down through the cork into the liquid. As growth proceeds the radicle develops a root-system in the way appropriate to the particular plant used, which absorbs from the liquid the salts which are required by it, so far as these are present. At the same the plumule grows upwards, and soon a shoot appears, which develops pari passu with the root.

By this method various plants can be cultivated with different degrees of success ; in some cases not only leaves, but flowers and even fruit can be produced. The progress of the plant, and the readiness with which it will develop, will depend upon the salts which are supplied to it in the water, if it is maintained in normal conditions of light, temperature, and aeration. In preparing the solution, particular mixtures can be employed, and the most favourable one ascertained, while subsequent analysis of the liquid will show to what extent the various constituents of the culture fluid have been abstracted from it.

This method is, however, only of use in determining particular points, such as the effect of the presence of certain metals in particular combinations, or the influence of different concentrations of particular substances. It does not give an account of what is happening to a plant with its roots embedded in the soil, for the composition of the latter cannot be compared with that of a solution definitely made up for purposes of experiment. The composition of the soil, as we have seen, is very far 
from uniform, and the constituents which are within the reach of the roots of two plants growing almost side by side naturally may be materially different in their proportions. This consideration makes it almost or quite impossible to ascertain, by observation of the soil and the plant growing in it, what are the substances which are entering its roots.

The other method, which is of much more general application, consists in making an analysis of the whole body of the plant after its removal from the soil, and so ascertaining what chemical elements it contains. A plant gives off no solid excreta, and consequently whatever it absorbs remains in its substance. The ultimate composition of the true nutritive matters, proteids, carbohydrates, fats, \&c., is known. Such an analysis having shown what elements enter into the composition of a plant, and of the food which it has stored in its tissues, it is easy to inquire into the manner in which each is supplied to the plant under examination, and into the work which is done upon them in its cells.

As already noticed, the structure of the plant demands that all the materials of a solid character shall be in such a solution that they can enter its substance by means of the physical process of osmosis taking place though the cell-wall. This is equally true of gases, of which there is considerable absorption by all plants, whatever may be the nature of their habitat.

The details of absorption vary to some extent, however, according to the environment of the plant. Aquatic plants can absorb water, and whatever is dissolved in it, whether of gaseous or solid character, by all parts of their surface. Those which grow with their roots embedded in soil, and their shoots exposed to the air, show a certain division of labour in this respect. The mineral constituents obtained from the soil are taken in by the root-hairs with the stream of water; those of a gaseous nature mainly find entry through the leaves and other green parts. 
If we examine the food-stuffs described as being essential, we find that proteids contain carbon, hydrogen, oxygen, nitrogen, sulphur, and perhaps phosphorus. Carbohydrates and fats contain only the first three of these elements. To make a destructive analysis of the plant, it must be dried at $110^{\circ}-120^{\circ} \mathrm{C}$. to drive off the water it contains, and it must then be carefully burnt, and the residue of the combustion collected. The volatile products given off can also be absorbed by appropriate methods, and their nature and amount ascertained.

The incombustible residue, which is known as the $a s h$, is composed of several metals and some other elements, which vary in nature and amount in different cases. An analysis of this ash will reveal the nature of its constituents, but it will not tell us in what condition or combination they existed in the living plant, on account of the various chemical changes which go on during the combustion.

The ash of plants when analysed is always found to contain the four metals potassium, magnesium, calcium, and iron. These are not present in the metallic condition, but are in combination with various acids, forming nitrates, sulphates, chlorides, carbonates, phosphates, \&c.

The presence of these nitrates, sulphates, \&c. must not lead us to infer that they have all been absorbed as such from the soil and retained unaltered in the plant. Part no doubt may be accounted for in this way, but much of the nitrogen, sulphur, and phosphorus which formed part of the substance of the plant entered into combination with the different metals and with oxygen during the combustion. Some of the carbon of the carbonates found may have had a similar origin.

Besides the four metals mentioned, various plants may individually contain larger or smaller quantities of many other elements variously combined. We find sodium very generally present; less frequently so, aluminium, copper, zinc, manganese, silicon, bromine, iodine and others. All of these are derived from compounds present in the soil, 
or the water with which they are in contact; indeed the composition of the soil in which a plant grows determines to a very great extent what minerals enter it. If a particular substance is soluble in the liquid which the roothairs absorb, and is capable of osmosis through their membrane, a certain quantity will, by ordinary physical processes, be taken up by them.

It does not, however, follow that, if the conditions alluded to are realised, absorption of a particular salt will go on indefinitely. The quantity of any substance which a plant will absorb will depend upon whether it is made use of in any way, or can be deposited in its tissues in an insoluble form. This can be seen most easily by studying the behaviour of a single cell. If any substance which enters the cell by osmosis is used in its metabolism, it will be quickly removed from the sap in its vacuole, and more will enter. If not, the cell-sap will soon have taken up as much of it as it can contain, and the absorption of that particular substance will cease. This is equally true of such a complex of cells as constitutes a plant, though the time of the absorption will be more prolonged. As soon as all the cells of the complex attain a condition of equilibrium with regard to the particular salt in question, no more will be taken up. This follows from the nature of the process of osmosis. If the substance under examination is withdrawn from the sap in any part of the plant, and made use of for any purpose, or deposited in the cells in an insoluble form, the condition of equilibrium will not be attained so long as such a withdrawal at any point takes place, and a stream of the substance will flow continuously to the point in question, so that the process of absorption will be continuous also.

Some of the materials found in the soil are readily soluble in the water which surrounds its particles. We have already seen that it is only this hygroscopic water which finds its way into the root-hairs. Such salts dissolve in this water and can enter the plant without diffi- 
culty if they are capable of passing through the plasmatic membranes which are the limiting layers of the protoplasm of the root-hair (page 57). The solution of the salts is always very dilute, and, on account of the ready diffusion that takes place, their concentration is approximately uniform in any particular soil. Other salts are insoluble in pure water, and their absorption presents more difficulty. Many are soluble in water which contains carbon dioxide, and as considerable quantities of this gas are continually being generated in the soil, the water there is charged with it, and bodies, otherwise intractable, are thereby brought into solution and absorbed.

The power of water containing carbon dioxide to effect the absorption of such substances is capable of easy demonstration. One of these salts is calcium sulphate or gypsum. If a plate of this substance is placed at the bottom of a flower-pot and the pot then filled with moist earth, a plant caused to grow in it till its root system is well developed will have some of its roots closely adpressed to the gypsum plate. After a time, examination will show the surface of the plate eaten away at all points except where the roots have become adpressed to it, and the regions covered by the latter will stand out in slight relief. The whole surface will have been subjected to the action of the water and the carbon dioxide it contains, except where it has been covered by the roots, and the solvent action will consequently be recorded.

A third factor which must be considered in the process of absorption is the acid sap which the root-hairs contain. Not only does the acid cause water to enter the hair osmotically, but a little of the sap exudes in the same way, and this has a certain solvent action upon the particles to which the root-hairs cling. Thus certain salts can be absorbed, though they may be soluble neither in pure water, nor in water containing carbon dioxide.

A similar experiment to the one just described will demonstrate this property of the acid sap. If, instead of 
gypsum, a polished plate of marble is inserted into the flower-pot, after a certain time of growth of the plant contained in it, the plate will exhibit a tracing of the course of the roots which have come into contact with it, but, instead of being in relief as in the former case, it will be etched to a certain depth. The solvent influence can thus be seen to come from the root itself, and not the water in the soil. It will, in fact, be the acid sap which makes its way out of the root-hairs.

Certain constituents of the soil can be absorbed which are made available in neither of the ways mentioned. Soils contain many constituents which cannot pass through the plasmatic membranes of the protoplasm, but which, in the presence of water, react with one another, producing new compounds which are capable of such osmotic entry and which are consequently absorbed.

The solutions taken in are excessively dilute. We cannot make a plant take up a greater quantity of any salt by bringing its roots into contact with a strong solution of it. There is a certain relation necessary between the substance and the water, which has been the subject of considerable investigation. Every salt is absorbed by a particular plant in a certain strength of solution, or in other words with each molecule of salt there is a certain invariable quantity of water taken in. The quantity is not the same, however, for each salt.

The salts which different plants absorb, in like manner vary in amount. If two species are growing in the same soil, side by side, under exactly the same conditions, the amounts of the several salts present in the soil which are absorbed by the plants of the different species will not be the same. In each case the quantity will vary according to the use the plant can make of it. This is well illustrated by the amounts of silica which can be taken up by grasses and by leguminous plants respectively. In an ordinary pasture there are always found several kinds of grasses, together with clover and other allied plants. An 
analysis of these will show that the ash of the grasses may contain many times the percentage of silica that is found in that of the leguminous plants. The grasses accumulate silica in their epidermal cells, while the leguminous plants do not. Hence the absorption of that substance soon ceases in the latter case.

Again, if a particular soil contains several different salts, a plant growing in it will not absorb them in equal proportions, nor in those in which they exist in the soil. An illustration of this fact is afforded also by marine Algæ, which accumulate in their tissues much greater amounts of potassic than of sodic salts, though sea-water contains much larger quantities of the latter than of the former. This fact admits of a similar explanation to that given in the case already mentioned. The absorption of a salt will cease as soon as the cell-sap attains exactly the same degree of concentration as the entering stream. In this case there will be no further osmotic action as far as the salt is concerned, though there may be a continuous entry of water into the absorbing cells.

We have seen that the continuous absorption of water by the root-hair will depend upon certain external conditions, such as the temperature of the soil, the activity of transpiration at the time, the degree of illumination the plınt receives, \&c. These conditions affect also the absorption of the substances in solution.

The substances which are absorbed by the roots in this way are naturally very varied. The most important of them in the metabolism of the plant are the compounds of nitrogen. In the soil these exist in the form of nitrates or nitrites of the metals mentioned, and as compounds of ammonia. Green plants take in little or none of the latter, which are however made available for their use by the action of certain bacteria which the soil contains. These humble organisms have the power of converting the ammonia compounds into nitrites, and the latter into nitrates, in which form they are taken up. This process 
of nitrification is the special property of two different bacteria, one of which forms nitrites from the ammonia compound, and the other transforms nitrites into nitrates. Certain fungi differ in their behaviour from green plants, absorbing ammonia compounds without such conversion.

It is in the way described that a normal green plant absorbs all the nitrogen which it uses for the construction

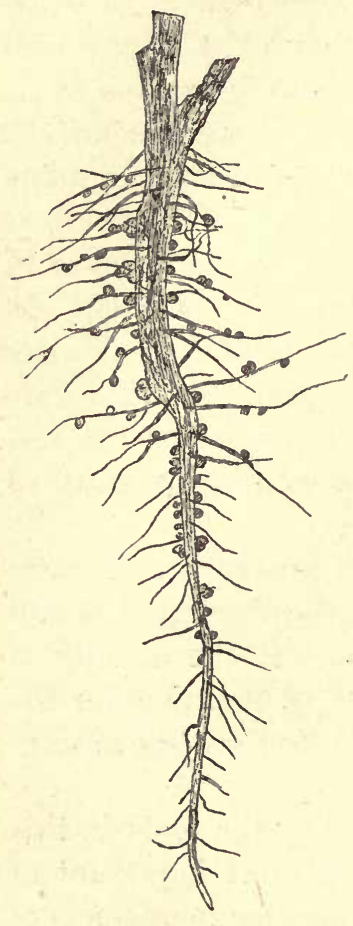

Fig. 84.-Root of a Leguminous Plant, Showing the TUBERCLES ATTACHED TO THE MaIN ROOT AND TO ITS Branches. of food substances. The nitrogen of the air is made use of only in very exceptional cases. Certain lowly Algæ are said to have the power of using it, but the process is not fully understood. Some of the bacteria in the soil appear to be able to cause the nitrogen of the air to enter into some form of combination, probably yielding either nitrates or compounds of ammonia. A few plants belonging to the Natural Order Leguminosa can also use atmospheric nitrogen, but their power depends upon the association with their roots of certain fungi or bacteria which infest the cortical tissues and develop peculiar tubercular structures upon the roots (fig. 84). The actual mode of absorption in this case also is obscure; the parts played by the root and the fungus or bacterium respectively are not at all determined. The atmospheric nitrogen apparently is made to enter into some form of combination, and is then absorbed by the root, probably through the tissue of the fungus. It is not absorbed by the leaves of the plant.

Organic compounds of nitrogen are seldom presented to 
the roots of plants, so that the amount of the element which is absorbed in such a way is very small. Indeed it may be said that such an absorption is almost entirely exceptional. It has been found that plants are able to utilise urea and other amides when these are present in the soil. In very rich soils, or those containing a large quantity of humus, such compounds are to be met with, and there is a probability that they are more easily worked up into actual nutritive substance than the inorganic compounds which have been spoken of.

A few plants obtain a more fully elaborated material in a very different way. These are the so-called insectivorous plants, which have the power of utilising proteid matters. Among them may be mentioned the pitcher plants, Nepenthes, Sarracenia, \&c., and the fly-catching plants, Drosera, Dioncea, and others. In the pitchers of Nepenthes, \&c., which are specially modified foliar structures, there is an accumulation of water, in which insects are from time to time drowned. Their bodies decay, or are digested by a peculiar secretion, which is prepared in the tissue of the pitchers, and excreted into the water they contain. The products of the decomposition or digestion are absorbed by the tissue of the pitchers in the same way as similar products are absorbed by the stomachs and intestines of animals. Drosera and Dionca bear certain glandular structures on their leaves which pour out a fluid, by which insects become surrounded after alighting on the laminæ. This secretion possesses digestive properties resembling those of the gastric and pancreatic fluids of animals, and by the action of this juice the bodies of the captured insects are digested, and the nitrogenous material is subsequently absorbed by the leaf surface. A fuller discussion of these mechanisms will be found in a subsequent chapter. These plants are generally found growing in such a situation that they are not brought into contact with inorganic compounds of nitrogen, and hence are cut off from the supplies which are afforded to the roots of 
ordinary terrestrial plants. The mechanisms described afford instances of special adaptations to particular environments, and will therefore be considered in more detail later.

Besides compounds of nitrogen, the materials absorbed by the roots of normal green plants include the constituents of the ash. Of these the more prominent are the compounds of potassium, sodium, magnesium, calcium, and iron. The sulphur and phosphorus which enter into the composition of the protoplasm are also taken in by the roots, in combination with the metals mentioned, and with others whose occurrence is not so general. The sulphur is absorbed in the form of suiphates, and the phosphorus in that of phosphates, of these metals.

Potassium is present in the soil in various combinations, principally as the sulphate, phosphate, chloride, and probably the silicate. After the nitrate the chloride appears to be the salt which is the most advantageous to plants. Calcium and magnesium exist in similar combinations, all of which, except the chloride, appear to be suitable for absorption. The chloride is, on the whole, deleterious. Iron can be absorbed in almost any inorganic combination. Sodium is absorbed in similar forms to those of potassium, the nitrate being the most valuable. Sodium chloride is frequently present in considerable quantity in the plants which are found on the sea-shore.

Silicon is present in many plants, being especially prominent in the grasses and the horsetails. It is taken up from the soil in the form of soluble silicates, and possibly to some extent in that of soluble silicic acid.

The other occasional constituents of the ash, which have not so general a distribution as those already mentioned, include a number of metals which play no part in the nutritive processes. They are usually present in very small amount, and appear to be of accidental occurrence, being absorbed by reason of the solubility of their salts and their power of entering the root-hairs by the 
ordinary process of osmosis. They are taken up in very various combinations. Their presence is not generally constant, and appears to depend entirely on the composition of the soil.

The water which the plants take up is the chief source of the hydrogen and oxygen which enter into the composition of the substance of the plant. A little of both these elements is taken in in the several combinations of the metals; phosphates contain both, nitrates and carbonates contain oxygen. The amount of them absorbed in these forms is, however, relatively small. As we shall see later, the water plays a very prominent part in the constructive changes which take place in the plant.

The gases present in the water of the soil also make their way into the root-hairs with the stream, but the quantity is very slight compared with what is absorbed by the sub-aerial parts. The gas carbon dioxide, which we have seen to be present in the earth in considerable quantity, is, however, not made use of in the constructive processes. All of this particular food material is taken in from the air. A little carbon is absorbed in the form of carbonates. Many complex organic compounds of carbon are taken in by those roots with which fungi, such as the mycorhiza of certain trees, are living symbiotically, but this is exceptional. The root-hairs are capable of absorbing such organic compounds as sugar, but these materials are rarely presented to them.

The absorption of gases from the air takes place in the leaves and other green parts. They enter freely through the stomata so long as these are open, and find their way into the interceliular space system, the importance of which we have already examined. These intercellular spaces contain, as we have seen, a mixture of gases which, though approximating to the composition of the atmosphere, yet differs from it in the relative quantities of the constituents. We have seen that the composition of this mixture of gases tends to become uniform by the currents 
which circulate in the intercellular' cavities, and by the slower processes of diffusion, which are set up in consequence of local production or abstraction of particular constituents. So long as the stomata and the lenticels are open, the composition of the atmosphere within the plant tends to become identical with that of the external air. The actual absorption of the gases takes place almost entirely from this internal reservoir, very little finding entrance into the cells of the epidermis. A certain amount is, however, taken in by the very young parts which have not become modified by the development of a cuticle.

The cells which abut upon the spaces in the leaves and other green parts are those which are principally concerned in the absorption of gases. Their walls are very thin and delicate, and are saturated with water. The different gases present dissolve in the outermost film of this water, according to their degree of solubility, and thence diffuse slowly through the membrane into the cell sap, which saturates the protoplasm, and fills the vacuoles. The quantity of each taken up depends, as in the case of the metallic salts already discussed, upon the ability of the protoplasts to make use of the gas, and thus to withdraw it from the sap. If it can be combined in any way with other bodies in the cell, or with the living substance itself, it is thus withdrawn from the water, and room is made for more to enter. If not, the limit of saturation of the sap is soon reached.

The only gas which is absorbed from the air for the purposes of food-construction is carbon dioxide. This exists in the atmosphere in very small amount, only about four parts in ten thousand being normally present. The very large green surface which an ordinary terrestrial plant possesses renders, however, a considerable amount of absorption possible. If the general conditions are favourable, the absorption is continuous, for carbon dioxide is at once decomposed or made to enter into some form of 
combination in the cells of the green tissues, and so a stream is always entering.

Both nitrogen and oxygen are soluble in water, though to a different extent. It has already been stated that the nitrogen so taken in is not used in the constructive processes, and accordingly a mere trace is absorbed in this way. A larger amount of oxygen enters, but experiments have proved that it is not used for the manufacture of nutritive substances, being applied to other purposes.

The absorption of carbon dioxide takes place usually at the ordinary atmospheric pressure. In some parts of

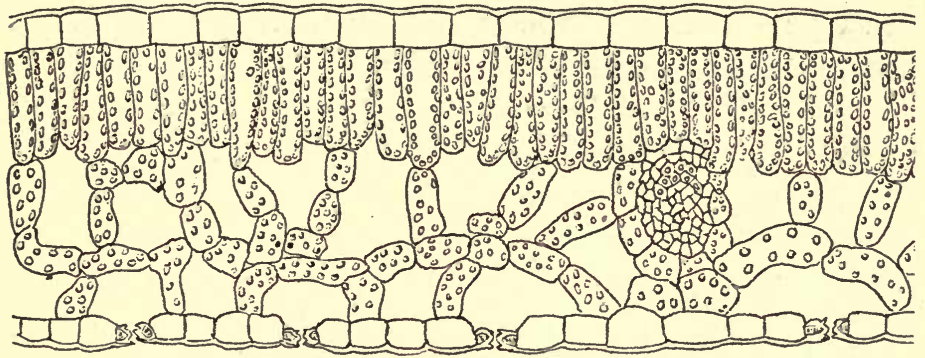

Fig. 85. - Transverse Section of the Blade of a Leaf, showing the DIFFerent aRrangement OF THE MEsophyll ON THE TWO SIDES. $\times 100$.

the internal reservoirs it exists at a slightly higher pressure, in consequence of a local production in the tissues. Plants can, however, absorb this gas when it is present in much larger quantities than it is in air. Too much, however, is possible, and then the cells are unable to take it in at all.

The continuous absorption of carbon dioxide is possible only under certain conditions; the cells which contain chloroplasts are the only ones which can take it in in any quantity, and they can only do so when they are exposed to light, preferably that of bright sunshine, and when the plant is maintained at an appropriate temperature. Its absorption. is accompanied by the exhalation of a volume of oxygen which is equal to the volume of the carbon dioxide absorbed, and it is attended by a continuous increase in the weight of the plant. 
We have seen that most of the water absorbed by the roots is conveyed regularly through the axis of the plant until it reaches the leaves, in which, after traversing the cells of the mesophyll, it is evaporated into the intercellular spaces. Into these cells of the interior of the leaf, all the materials used in the construction of food are thus at once transported, both those entering the tissues from the soil, and those absorbed from the air. These mesophyll cells have generally a different arrangement on the two sides of the leaf (fig. 85), but they all agree in containing chloroplasts. In these cells takes place the work of construction of organic nutritive substance, such as the plant can live upon-work which is carried out mainly through the instrumentality of the chloroplasts. 


\section{CHAPTER XI \\ THE CHLOROPHYLL APPARATUS}

THE food materials whose absorption we have now discussed are built up in the body of the plant into such substances as are capable of being assimilated by the protoplasm, and consequently of ministering to its nutrition. They undergo a striking series of changes before they are capable of subserving this purpose, and of becoming incorporated into the plant-body. The great object to be attained is the construction and growth of the living substance, which itself subsequently produces the more permanent material that we find stored in the shape of the masses of wood and bark and the other substances which an adult plant contains. The green plant contains a mechanism for the formation of organic substance from these simple inorganic materials, and it is to the activity of this mechanism that we owe almost the whole of the organic matter which is found in nature, whether exhibited by animal or by vegetable structures. This mechanism is known by the name of the chlorophyll apparatus, and our attention must now be turned to its nature and its mode of working.

Chlorophyll is a green colouring matter which is generally found associated with definite protoplasmic bodies known as plastids. These are usually considered to possess a reticulated structure, and the pigment, in some form of solution, occupies the meshes of the network. From their being coloured green by the pigment they are known as chloroplastids or chloroplasts. The solvent of the pigment which is in these bodies is of a fatty nature, and is probably some kind of oil. Alcohol, chloroform, ether, 
benzol, and a few other liquids can extract the chlorophyll from the plastids and leave them colourless. The pigment can be obtained from them also by treatment with dilute alkalis, such as potash and soda. By whatever solvent it is extracted, however, it appears to undergo decomposition, so that the solution does not yield it up in the form in which it exists in the vegetable cell.

A solution of chlorophyll in alcohol or chloroform shows the curious property of fluorescence ; if regarded by transmitted light it appears green, whatever may be the degree of concentration of the solution; if a strong solution is looked at by reflected light, it has a blood-red coloration.

When a beam of white light is allowed to pass through a prism, and is then made to impinge upon a screen of white paper, it gives the appearance of a band in which all the colours are represented in the following order :-red, orange, yellow, green, blue, indigo, and violet. This is due to the different degrees in which the rays which produce the sensations of those colours are bent or deflected by the prism. This coloured band is called the spectrum of white light. In order to get it exhibited to the greatest advantage, it is best to admit the beam of light to the prism through a narrow slit. The spectrum may then be regarded as a succession of images of the slit, each ray giving its own image of the aperture and producing that image in its appropriate colour. If a solution of chlorophyll is placed in the path of the beam before it reaches the slit, the resulting spectrum is found to be considerably modified. Instead of showing a continuous band in which all the colours are represented, it is interrupted by seven vertical dark spaces. The rays which would have occupied these spaces in the absence of the solution of chlorophyll have no power to pass through the latter, and consequently their images of the slit are represented by dark lines, which together constitute the black bands. In other words, chlorophyll absorbs these particular rays of light which are missing. 
In fig. 86 is a representation of the spectrum which such treatment produces and which is called, from the facts just narrated, the absorption spectrum of chlorophyll. The uppermost figure is that which is exhibited by an alcoholic solution or extract of leaves ; the middle one is given by chlorophyll dissolved in benzol. The first band on the left is the darkest, and is found to be in the red part of the spectrum. The three bands on the right are broader, but are not so well defined. They cover nearly all the blue end. The three thinner and lighter bands are in the yellow and green parts of the spectrum. Chlorophyll therefore has

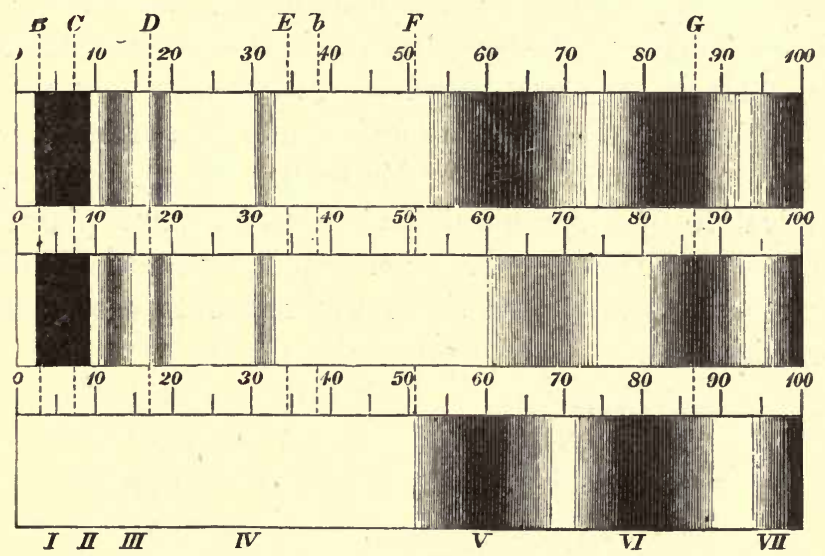

Fig. 86,-Absorption Spectra of Chlorophyll and XaNTHOPHYLl. (After Kraus.)

the power of absorbing a large number of red rays, a good many blue and violet ones, and a few of the green and yellow. The distinctness with which these absorption bands are seen depends upon the strength of the solution, those in the red and blue being, however, always prominent. Careful experiments have proved that chlorophyll is a single pigment and not a mixture of two, as has often been stated. It is, however, easily decomposed, and the products of its decomposition are generally found with it in the chloroplast. One of these, Xanthophyll, which is of a bright yellow colour, is always extracted with the 
chlorophyll by alcohol. It can be separated from the extract by appropriate means, and its solution yields the absorption spectrum represented below those of chlorophyll in fig. 86. Another pigment, Erythrophyll, is present in those leaves which are found upon the trees in autumn. Like xanthophyll, it appears to be a product of the decomposition of chlorophyll, and it has a spectrum which differs from both the others.

It is extremely difficult to say what is the chemical composition of chlorophyll, on account of the readiness with which it is decomposed. In all the processes which have been adopted for its extraction it undergoes decomposition, and conséquently no definite conclusions as to its chemical nature can at present be arrived at. It can be made to yield definite crystals by appropriate methods of treatment after extraction, but it is probable that these crystals are a derivative of chlorophyll and not the pure pigment. Analyses of the crystals have been made by Gautier and by Hoppe-Seyler, who give them the following percentage compositions :-

$\begin{array}{cc}\text { Gautier } & \text { Hoppe-Seyler } \\ \text { C } 73 \cdot 97 & 73 \cdot 34 \\ \text { H } 9 \cdot 8 & 9 \cdot 72 \\ \text { N } 4 \cdot 15 & 5 \cdot 68 \\ \text { O } 10 \cdot 33 & 9 \cdot 54 \\ \text { Ash } 1 \cdot 75 & 1 \cdot 72\end{array}$

According to Hoppe-Seyler the ash contains phosphorus and magnesium.

From his analysis Gautier came to the conclusion that chlorophyll is related to the colouring matter of the bile; Hoppe-Seyler considered, on the other hand, that it is a fatty body allied to lecithin.

Except in the lowest unicellular plants, the chlorophyll is always attached to some form of protoplasmic body known as a plastid. These are small masses, of varying size and shape, which are embedded in the general cyto- 
plasm of the cell (fig. 87). Even in the lowly forms it is apparently never uniformly distributed through the body of the protoplast. The form, dimensions, and structure of the chloroplast differ considerably in the different groups of plants. In some of the filamentous green seaweeds it may appear as variously shaped bands or plates. Spirogyra shows it as a spiral band passing round the cell ; in $Z y g$ nema it has the form of two star-shaped masses which are attached to the cytoplasm by bridles extending to the cell-wall. In the brown and red seaweeds the plastids are not green, but have the appropriate colours of the plants. These plastids contain other pigments in addition to the chlorophyll, but the latter can be made apparent by extracting the cells with cold distilled water, in which the other pigments are soluble. In all plants higher in the scale than the Algæ the chloroplasts

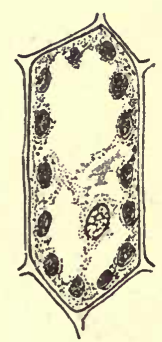

Fig. 87.-Chloroplasts kMbedded in the ProTOplasm of a Cell of the Palisade Tissue OF A LEAF. are found as round or oval bodies embedded in the cytoplasm. They never occur in the vacuoles of the cells. Though normally green, they can assume other colours, such as yellow, brown, or red, but this is due to an alteration of the pigment they contain. Examples of this change are afforded by the assumption of the autumnal tints by foliage leaves, and by the changes in colour which are characteristic of ripening fruits.

In the Mosses the chloroplasts are found throughout the cells of the leaves, in the outer parts of the sporogonium, and in certain cells of the axis. In the Ferns they occur chiefly in the leaves, occupying the cells of the epidermis as well as those of the mesophyll. In the higher plants they are found most prominently in the mesophyll of the leaves, the epidermis as a rule being free from them. When the leaves are dorsiventral in structure, the chloroplasts are more numerous in the palisade parenchyma which lies just below the upper epidermis than they 
are in the spongy tissue which occupies the lower half of the thickness of the leaf (fig. 88). The guard-cells of the stomata, however, always contain them. The green cortex of young stems and twigs also exhibits them. In such plants as the Casuarinas and the Equisetums (fig. 89), in

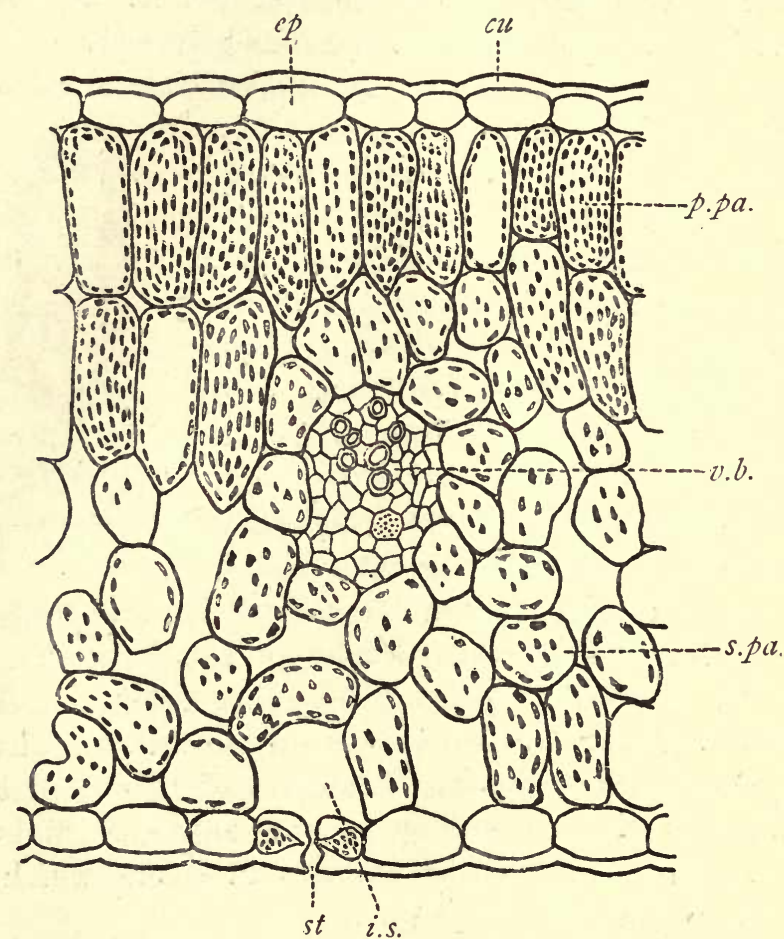

Fig. 88.-Transverse Section of portion of the Blade of the LEAF of Beta.

$c u$, cuticle ; $e p$, epidermis ; $p . p a$, palisade tissue ; s.pa, spongy tissue ; $v . b$, vascular bundle; st, stoma; i.s, intercelfular space.

which the leaves are rudimentary, definite longitudinal bands of cells in the young stems contain them.

The structure of such a chloroplast as is characteristic of one of the higher plants has not been very completely investigated. There is undoubtedly a protoplasmic basis with which the colouring matter is in some way associated. 
As already stated, many botanists consider the protoplasm to be arranged in a network, whose meshes are filled with a solution of the pigment. Others consider the protoplasm to be homogeneous, but honeycombed with vacuoles which are filled with the solution of the chlorophyll. By the action of dilute acids, or by treating the chloroplasts with steam, the colouring matter may be made to exude from the framework in viscid drops, leaving the latter colourless.

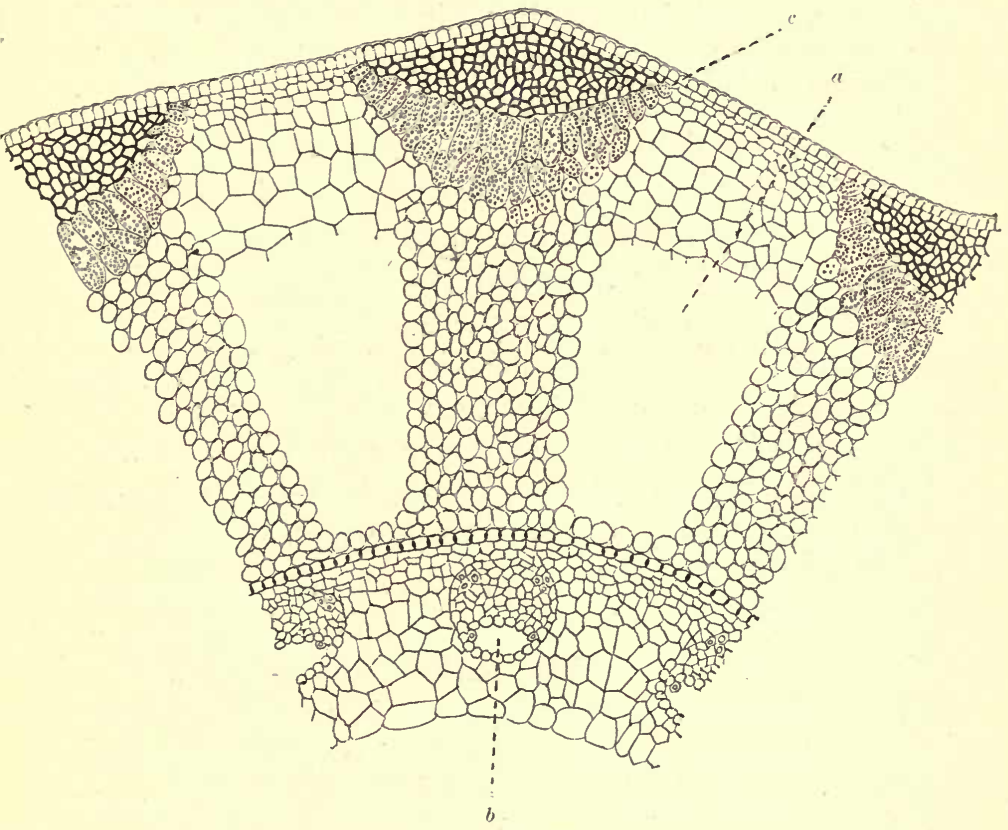

Fig. 89.-Portion of Aerial Stem of Equisetum.

$a$, cortical lacuna; $b$, lacuna in vascular bundle; $c$, chlorophyll-containing cells.

It then appears to have a reticular structure, but how far this condition is brought about by the action of the reagents is uncertain. The chlorophyll, however, is certainly not uniformly diffused through the body of the plastid.

In the process of the formation of the chloroplast it is not difficult to see that its two constituents are not 
inextricably connected. The plastids are not, as already mentioned, differentiated out of the ordinary protoplasm of the cell, but are formed by the division of other plastids. In many cases they are found without the colouring matter, as in underground organs such as the tubers of the potato. They are then known as leucoplasts. Plants which are grown in darkness have no green colouring matter in their leaves, but the cells of the mesophyll contain the plastids much as normal ones do. They are pale yellow in colour, containing another pigment known as etiolin, which appears to be an antecedent of chlorophyll, and to. be transformed into the latter when brought into the presence of sunlight. Exposure to light is almost universally a necessary condition for the formation of the green pigment. Exceptions are known among the Ferns and the Conifers, particularly the seedlings of Pinus; also in the seed of Euonymus europaus, the embryo of which is green, though it is buried in the interior of the endosperm and surrounded by a thick testa covered by an arillus. If a green stem is withdrawn from the light, the chlorophyll slowly disappears, as is shown in the process of the bleaching of celery. The disappearance is, however, very gradual. It is probable that in the living chloroplast the colouring matter is continually being decomposed and reconstructed, and that the reason of the bleaching is that the reconstruction cannot take place in darkness. Light of too great intensity causes the destruction of the green colour.

Chlorophyll can be developed only when the temperature rises above a certain point, which varies with different plants. It is a matter of common observation that the leaves of young Hyacinths which emerge from the soil in the early spring are often colourless or pale yellow. The chloroplasts are found to be present in such leaves, but they are yellow, owing to the presence of etiolin instead of chlorophyll. The leaves which are produced later, when the temperature of the air is higher, have the normal green appearance.

Chlorophyll is not developed in a plant unless the latter 
is supplied with a certain quantity of iron, but the relation of the latter to the pigment is not known. It does not enter into its composition. The influence of the metal can be ascertained by cultivating a seedling, by the method of water-culture, in a solution which is free from iron. The seedling assumes a sickly yellow appearance, not unlike that presented by a plant grown in darkness. It is said to be chlorotic. The addition of a very small quantity of an iron salt to the culture-medium causes the appearance of chlorophyll in the plastids. The presence of oxygen is also necessary for the formation of the pigment.

The chlorophyll apparatus of a plant is primarily concerned with the production of carbohydrate bodies, such as the various sugars which the plant contains, and it is to the formation of these that attention must first be given. It carries out this constructive process only under particular conditions, the most important of which is light. We have seen that a certain degree of illumination is necessary for the formation of the chlorophyll. The pigment once formed may continue to exist for a time in darkness, but it is quite incapable of exercising any constructive power unless light be admitted to it. Consequently the formation of carbohydrates is an intermittent process, being quite in abeyance during the night. The effect of light is thus twofold, its access causing the original formation and the subsequent activity of the chlorophyll apparatus. The illumination need not be very intense, though it is probable that the greatest activity is manifested in direct sunlight. Plants which grow in deep shade are however capable of decomposing carbon dioxide. It must be remembered, too, that the chloroplasts are situated some little distance within the leaf or stem, at any rate in phanerogamic plants, and there must be a certain loss of light as it penetrates through the epidermis.

The activity of the chlorophyll apparatus is also considerably influenced by variations of temperature. The lower limit beyond which no carbohydrates are constructed 
lies probably a little below the freezing point of water, at which point, however, activity is not long maintained, and then only by alpine forms. Jumelle has stated that in certain plants of hardy type it can proceed at as low a temperature as $-40^{\circ} \mathrm{C}$. Plants which normally live in hot climates cannot manifest any power of action below about $4^{\circ} \mathrm{C}$. The optimum temperature for the plants of temperate climates is from $15^{\circ} \mathrm{C}$. to $25^{\circ} \mathrm{C}$., above which activity diminishes, though not very rapidly, ceasing when about $45^{\circ} \mathrm{C}$. is reached. These high temperatures affect the living substance of the chloroplasts very injuriously. The activity of the chlorophyll apparatus is dependent also to some extent upon certain of the mineral salts present in the cells. According to Bokorny it cannot be called into play in the absence of compounds of potassium.

As the activity of the chlorophyll apparatus is so essentially dependent upon light, the process of construction of carbohydrate substances from carbon dioxide and water, which is its primary object, may appropriately be called photosynthesis. This term has certain advantages over the older expression, the assimilation of carbon dioxide, as the term 'assimilation' may preferably be reserved for the process of the incorporation of food materials into the substance of the protoplasm.

Photosynthesis consists, then, in the formation of some form of carbohydrate from the carbon dioxide which is absorbed from the air, and the water which is present in the cells. When these simple bodies are exposed to the action of the chloroplast in presence of light and moderate warmth, the carbon dioxide disappears, and a volume of oxygen equal to that of the carbon dioxide is evolved.

The apparatus shown in fig. 90 will enable this interchange of gases to be seen. Into a glass jar is poured some water containing carbon dioxide in solution. Some watercress is put into the water and a funnel inserted above it, the end of which rises into a burette filled with water and closed by a stopcock. The whole apparatus being 
placed in sunlight, bubbles of oxygen will be given off by the leaves and will rise into the burette. If no carbon dioxide is in the water, no oxygen will be given off.

There is nothing certainly known at present as to the details of the changes which connect these two phenomena. It has been suggested by Baeyer that the carbon dioxide is decomposed with the formation of carbon monoxide and oxygen, according to the equation $2 \mathrm{CO}_{2}=2 \mathrm{CO}+\mathrm{O}_{2}$. At the same time there is a decomposition of water, possibly in the way denoted by the equation $2 \mathrm{H}_{2} \mathrm{O}=2 \mathrm{H}_{2}+\mathrm{O}_{2}$. The oxygen is given off, the volume being found, when carefully measured, to be equal to the volume of carbon dioxide undergoing decomposition. The carbon monoxide and the hydrogen are then thought to unite, producing formaldehyde, a body represented by the formula $\mathrm{CH}_{2} \mathrm{O}$, or preferably $\mathrm{HCOH}$. This suggested series of reactions agrees fairly closely with the observed facts, but it must

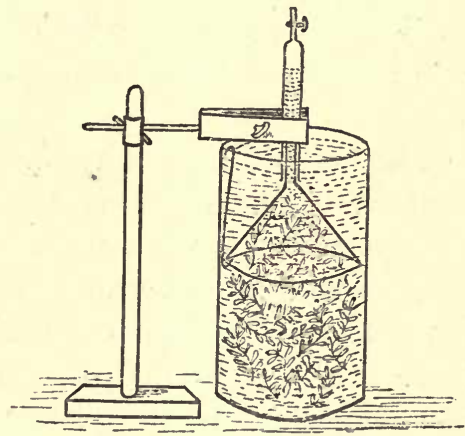

Fig. 90.-Apparatus to show the Evolution of Oxygen by a Green Plant in Suxlight. not be regarded as anything more than an hypothesis. Indeed there are considerable difficulties in accepting it as it stands. There is no evidence that carbon monoxide is formed. Experiments have shown that this gas is quite useless to a plant; if it is supplied in the place of the dioxide, the formation of carbohydrates does not take place. Nor has any formation or liberation of hydrogen ever been detected so long as the plant is maintained in normal conditions.

The formation of formaldehyde, again, is very difficult of proof. It very readily undergoes change, and therefore is difficult to detect in a plant. If administered directly to a 
green cell such as one of a filament of Spirogyra, the plant cannot make any use of it. It has been found, however, that if Spirogyra is fed with a compound of formaldehyde and sodium-hydrogen-sulphite, which slowly evolves the former in the presence of water, a formation of carbohydrates occurs. This cannot, however, be accepted as proof that formaldehyde normally subserves this purpose.

If we concede that formaldehyde is very probably the first stage in the photosynthetic process, a consideration of the probable decomposition seems to lead us to the view that the carbon dioxide and the water are made to interact without the liberation of carbon monoxide, and that the reaction may be represented by the equation $\mathrm{CO}_{2}+\mathrm{H}_{2} \mathrm{O}=$ $\mathrm{HCOH}+\mathrm{O}_{2}$, which agrees equally well with the observed facts.

The formaldehyde may give rise without much difficulty to a form of sugar. It is a property of the aldehydes to undergo readily what is known as polymerisation, or condensation of several molecules. Such a condensation of formaldehyde would lead to the formation of sugar thus: $-6 \mathrm{HCOH}=\mathrm{C}_{6} \mathrm{H}_{12} \mathrm{O}_{6}$. There are many sugars of this composition in the plant, especially glucose or grape sugar, and fructose or fruit sugar.

That some such process takes place is extremely probable, for sugar is present in the mesophyll cells very speedily after the absorption of the carbon dioxide and the beginning of the exhalation of oxygen. Sugar of some kind appears to be the first carbohydrate to be formed; it is not very readily detected, being freely soluble in the cell-sap. Almost as quickly as the formation of sugar we have the appearance of starch in the substance of the chloroplasts, and as this is easily visible, it was long thought that starch was the culminating product of the photosynthetic process. We shall find reasons shortly for suggesting a wholly different meaning to the appearance of the starch, that it is indeed only a temporary store of carbohydrate in an insoluble condition, due to the production of sugar being in excess 
of the quantity needed by the cell for immediate consumption.

If we accept the view of the polymerisation of formaldehyde to give rise to the sugar, we cannot withdraw this operation also from the activity of the chloroplast. Sugars are what are called optically active compounds; that is, they possess the power of deflecting a ray of polarised light to the right or to the left as the latter is made to pass through either crystals or a solution of them. Formaldehyde has no such power. There is no process known by which an optically active compound is formed from an optically inactive one without the intervention of living substance. Consequently we must suppose that the polymerisation is brought about by the chloroplast as certainly as is the original decomposition of the carbon dioxide.

We have so far assumed that a sugar having the formula $\mathrm{C}_{6} \mathrm{H}_{12} \mathrm{O}_{6}$, and known as a hexose, is the first carbohydrate formed. This, however, is not certain. Some experiments carried out in 1892 by Brown and Morris point rather to cane-sugar as the first carbohydrate synthesised. Cane-sugar is a more complex substance, and has the formula $\mathrm{C}_{12} \mathrm{H}_{22} \mathrm{O}_{11}$. This conclusion is based on repeated observations that when leaves of Tropaohum were plucked and then exposed to sunlight for twelve hours, there was a great accumulation of this sugar in the leaf, while the simpler hexoses did not increase in quantity. The severance of the leaves from their stems prevented the transport of the sugars to any other part of the plant, so that they accumulated at the seat of their formation.

Further investigations on this point are, however, necessary before a definite conclusion can be arrived at.

This theory of the processes of photosynthesis is by no means the only one which has been advanced, though on the whole it is that which has been received with most favour. A modification of Baeyer's view was advanced by Erlenmeyer, who suggested that the first interaction of carbon dioxide and water leads to the formation of formic 
acid and hydrogen peroxide, according to the equation $\mathrm{CO}_{2}+2 \mathrm{H}_{2} \mathrm{O}=\mathrm{HCOOH}+\mathrm{H}_{2} \mathrm{O}_{2}$, and that then they are decomposed, yielding formaldehyde and water, and giving off oxygen, $\mathrm{HCOOH}+\mathrm{H}_{2} \mathrm{O}_{2}=\mathrm{HCOH}+\mathrm{H}_{2} \mathrm{O}+\mathrm{O}_{2}$.

An hypothesis of a different nature was put forward by Crato many years later. He suggested that the carbon dioxide after absorption becomes orthocarbonic acid, $\mathrm{C}(\mathrm{OH})_{4}$, which exists in solution in the cell-sap. The orthocarbonic acid has the structure of a closed benzene ring in which six molecules are linked together. This becomes decomposed, liberating six molecules of water and six molecules of oxygen, and forming a hexavalent phenol : $-6 \mathrm{C}(\mathrm{OH})_{4}=\mathrm{C}_{6} \mathrm{H}_{6}(\mathrm{OH})_{6}+6 \mathrm{O}_{2}+6 \mathrm{H}_{2} \mathrm{O}$. This new body then undergoes a molecular rearrangement and becomes glucose, $\mathrm{C}_{6} \mathrm{H}_{6}(\mathrm{OH})_{6}=\mathrm{C}_{6} \mathrm{H}_{12} \mathrm{O}_{6}$.

This view is purely hypothetical, and cannot claim to be based on experiment.

The most recent suggestion on this subject is that made by Bach in 1893. He points out that when sulphurous acid, $\mathrm{H}_{2} \mathrm{SO}_{3}$, is exposed to light, it becomes transformed to sulphuric acid, sulphur and water being split off, $3 \mathrm{H}_{2} \mathrm{SO}_{3}=$ $2 \mathrm{H}_{2} \mathrm{SO}_{4}+\mathrm{H}_{2} \mathrm{O}+\mathrm{S}$, and he argues that a sirnilar process analogous with this reaction takes place in a leaf. The carbon dioxide unites with water and forms carbonic acid, which is then split up in the same way as the sulphurous acid, $3 \mathrm{H}_{2} \mathrm{CO}_{3}=2 \mathrm{H}_{2} \mathrm{CO}_{4}+\mathrm{H}_{2} \mathrm{O}+\mathrm{C}$. The carbon' and water are not set free separately, but in combination as formaldehyde. The new acid, $\mathrm{H}_{2} \mathrm{CO}_{4}$, splits up into carbon dioxide and hydrogen peroxide, and the latter is decomposed into water and free oxygen.

All these views must be regarded rather as ingenious speculations than as sound hypotheses resting upon observation and experiment.

A theory of a totally different nature was advanced some years ago by Vines. Starting with the observation that a carbohydrate substance (cellulose) is produced or secreted by protoplasm in the process of the thickening of 
cell-walls, and noticing the formation of starch grains in the chloroplast almost as soon as the photosynthesis has been established, he argues that the earbohydrate is not directly formed from the simple materials absorbed, but appears as a secretion product of the chloroplast. He suggests that a body possibly allied to formaldehyde is first formed according to Baeyer's theory, and that this is used in the construction of proteid, by combining with the nitrogen and sulphur absorbed in the form of salts from the soil, or with nitrogenous substances derived from previous decompositions of proteid. This proteid then is assimilated by the protoplasm of the chloroplast, and from the latter the carbohydrate (starch) is secreted.

This view, while no doubt, in the main, accurate as far as the mode of formation of starch is concerned, cannot be regarded as explaining the formation of earbohydrates from the simple compounds absorbed. The leucoplast of the tuber, as well as the chloroplast itself under certain conditions, ean form starch grains when supplied with sugar in the absence of carbon dioxide, and in all probability the appearance of the starch is the result of the presence of an excess of sugar in the leaf-cells. Regarded as an explanation of the photosynthesis of carbohydrates, it, like the others, must remain hypothetical. Moreover it is based upon the assumption that starch is the highest term reached in the plant in the series of carbohydrate bodies. This assumption, however, is not supported by the evidence at our command, the construction of sugar and not starch being the completion of the photosynthetic process of the chlorophyll apparatus. Though starch is a very general accompaniment to this process, it never appears till a certain amount of sugar has been formed, and in many plants, particularly the onion and certain other Monocotyledons, it is not produced at all, however active photosynthesis may be. To this point we shall return in a subsequent chapter.

Though the production of starch is apparently not the ultimate aim of the photosynthetic processes, its ready 
occurrence affords us an easy method of demonstrating the activity of the chlorophyll apparatus. If a leaf is partially covered by a piece of opaque material, such as tinfoil, and is then exposed to the light, starch rapidly appears in the illuminated portion. Its presence can be shown by bleaching the leaf with boiling alcohol, and then immersing it in iodine, which forms a blue colour with starch. The blue tint only appears where the light has reached the chlorophyll apparatus.

These processes are carried out by the chlorophyll apparatus under the conditions set forth. It is evident that such changes as have been described cannot be accomplished without the expenditure of a considerable amount of energy. In this need we have the explanation of the composite nature of the chloroplast. The chlorophyll absorbs certain rays of light which fall upon it, and the energy which is liberated by the extinction of their vibrations is taken up by the protoplasm of the plastid and applied by it to effect the decompositions that take place. A very ingenious method of demonstrating that the energy is derived from the rays of light absorbed by the pigment was devised by Engelmann. He observed that certain bacteria were excited to active movement only in the presence of free oxygen. He placed a filament of a green alga upon a glass slide in a fluid containing a number of the bacteria, covered it with a glass cover-slip, and sealed it with wax. He kept it in darkness till the microbes had come to rest, and then by the aid of a microspectroscope he threw a spectrum upon the filament and observed in what parts of it the bacteria accumulated as soon as they began to move. These places corresponded with the positions of the absorption bands which we have seen to be characteristic of the chlorophyll spectrum, the maximum effect being produced by the deep band in the red region. These were evidently the places at which the chlorophyll apparatus of the filament was at work, the movements of the bacteria showing that oxygen was liberated there. Tmiriazeff 
proved the same thing by throwing the spectrum of solar light upon a darkened leaf, when he found that starch was produced only in the positions of those same absorption bands, indicating that those were the only places of photosynthetic activity.

The process of photosynthesis has been found to proceed under certain circumstances in light which is too feeble in intensity to cause the development of chlorophyll. It is effected in these cases by the etiolin, which we have seen to be the antecedent of chlorophyll. The photosynthetic power of etiolin is, however, exceedingly small.

The percentage of carbon dioxide admitted to the chloroplasts has some influence upon the activity of the process. Normal air contains a mere trace of the gas, about 4 parts 'in 10,000. A more copious supply is, however, distinctly advantageous, and the activity increases as the percentage rises. The optimum quantity appears to be about 10 per cent. with light of the ordinary intensity. More than this gradually exerts a paralysing influence on the chloroplasts, and sets up consequently an inhibition of the apparatus. A similar inhibition can be caused by the accumulation of the products of its activity, a concentration of the sugar amounting to 8 per cent. being sufficient to bring it about.

The mechanism is an exceedingly delicate one and can be thrown out of gear by various external agencies. Ewart has shown that it can be inhibited by heat, cold, desiccation, partial asphyxiation, prolonged insolation, and by the action of dilute alkalis or mineral acids.

We mentioned at the commencement of this chapter that the chlorophyll apparatus is concerned in the manufacture of almost the whole of the organic material of the globe. In a few humble organisms the construction of such material can proceed without its help. These are certain bacteria which can transform ammonia compounds into salts of nitrous and nitric acids, growing and multiplying at the expense of the products they thus obtain 
together with carbon dioxide. There are two kinds of these bacteria, one of which oxidises ammonia to nitrous acid and the other converts this into nitric acid. They grow freely in the soil and multiply with considerable rapidity, the result being the formation of certain quantities of organic substance. They cause the carbon dioxide to enter into combination, this gas being normally the only source of their supply of carbon. They possess no chlorophyll and consequently cannot utilise directly the radiant energy of the sun. Their energy is apparently derived from the oxidation of the nitrogenous compounds which they attack. Nothing is known at present of the steps by which the synthesis of the organic matter takes place.

A process which at first appeared to involve a mechanism resembling that of the chlorophyll apparatus was discovered some years ago by Engelmann. Certain bacteria which contain a purple pigment were found to possess the power of photosynthesis. The pigment was thought to be allied to chlorophyll and to possess the same power of absorbing and utilising the radiant energy of light. Recent researches make it probable that, like the red seaweeds, these organisms contain a certain amount of chlorophyll, together with the purple pigment.

Saprophytic and parasitic fungi, which contain no chlorophyll, have no power of photosynthesis. They are compelled to absorb their carbohydrates from the medium in which they grow, and they take them in chiefly in the form of sugar. Parasitic phanerogams depend upon a similar source of supply. 


\section{CHAPTER XII}

THE CONSTRUCTION OF PROTEIDS

The simple compounds containing nitrogen which we have seen to be absorbed by the roots of green plants, are as unavailable for direct nutrition as the carbon dioxide taken in from the air. The nitrogenous organic material which is actually assimilated by the protoplasts always takes the form of proteid. With very few exceptions, and these occurring only among micro-organisms, gelatin and similar bodies cannot be made to support vegetable living substance, though they can be made use of by animals to supplement, but not to replace, their proteid supplies.

In studying the story of the construction of proteids from the nitrates and ammonia compounds taken into the plant, we meet with even greater difficulties than those which are presented by the photosynthesis of carbohydrates. These difficulties are connected with the stages which occur in the course of the construction, with the mechanism which is concerned in the transformation, and with the conditions under which the building up of proteid takes place.

At the outset of the study we find ourselves in complete ignorance as to the chemical nature of proteid. We know that it is the most complex material found in the plant, with the exception of the living substance itself, but we know nothing about its molecular structure or the arrangement or grouping of its constituent atoms. Destructive analysis has revealed its percentage composition within certain limits, although, as there are many kinds of proteid and all of them are extremely difficult to prepare in 
a pure condition, too much stress must not be laid upon the results obtained. These, moreover, are, as we should expect, not altogether concordant.

Analysis of a crystallised proteid prepared from the seed of the hemp showed it to have the following percentage composition, which may be taken, within somewhat wide limits, to be fairly typical of all.

\begin{tabular}{|c|c|}
\hline Carbon & \\
\hline Hydrogen & \\
\hline Nitrogen & \\
\hline Oxygen & \\
\hline Sulphur & \\
\hline
\end{tabular}

Besides containing these essential constituents, many proteids leave on ignition a certain amount of ash. This consists of small amounts of the chlorides, phosphates, sulphates; and carbonates of sodium and potassium, with traces of the corresponding salts of calcium, magnesium, and iron. It is not certain that these ash constituents are an integral part of the proteid molecule in any case; the balance of evidence points rather to their being impurities which are very difficult of removal.

Most of the proteids found in plants exist in an amorphous condition, and are very closely incorporated with the protoplasm. In a few cases they are met with as definite grains, and in certain reservoirs of food material they occur as crystals. Some of them can be made to crystallise after extraction from the organism, but many forms exist which do not possess this property, so far as we know at present. It is not certain, however, that the crystals are always composed of pure proteid only.

The proteids vary very much among themselves as to their solubility in water and other neutral fluids. Some are soluble, others insoluble, in water: some are soluble in solutions of neutral salts of various degrees of concentration. Nearly all are insoluble in alcohol and ether; they all dissolve in strong mineral acids and in caustic alkalis, 
but they are decomposed during the process. Their solutions have generally a power of deflecting a ray of polarised light to the left.

The best known groups into which proteids have been divided are the following :--

1. Albumins.-These are soluble in distilled water, and if the solution is heated, the proteid is converted into a peculiarly insoluble form, known as coagulated proteid, and deposited as a granular or flocculent precipitate. As the temperature rises the liquid becomes markedly opalescent before the separation of the proteid. The change takes place at a point which lies between $65^{\circ}$ and $80^{\circ}$ C., its exact place depending upon the nature of the albumin and the reaction and concentration of the proteid solution. This point is known as the coagulation temperature. Albumins can be precipitated unchanged by saturating their solutions with sodio-magnesic sulphate. They are not of frequent occurrence in plants, but can be extracted from certain roots.

2. Grobulins.-These differ from albumins in not being soluble in distilled water. They can be dissolved by adding a little neutral salt, such as sodium chloride. Their solutions are coagulated on heating, but they show a considerable variability as to the coagulation temperature, which in the case of some is as low as $55^{\circ} \mathrm{C}$. Most of them, however, remain unchanged below $75^{\circ}-80^{\circ} \mathrm{C}$. They can be precipitated by saturating their solutions with magnesium sulphate. If sodium chloride is used instead of the latter, an incomplete precipitation usually takes place. Different members of the group show different degrees of solubility in solutions of sodium chloride; some require only a trace of the salt ; others need 8-10 per cent.; and a few are soluble only in saturated solutions.

The proteids found in plants belong chiefly to this class. Globulins can be readily extracted from most seeds, and probably this form of proteid is the one which occurs in the green parts of plants. 
3. Albuminates, or Derived Albumins.--These are insoluble in distilled water or in solutions of neutral salts. They are readily soluble in very dilute acids and alkalis, and their solutions do not coagulate on boiling. They are precipitated by carefully neutralising their solutions, and when they are boiled in the resulting state of suspension they are converted into coagulated proteid, and will not re-dissolve on the addition of either dilute acid or alkali.

They are readily prepared from either albumins or globulins by warming them in the presence of a little acid or alkali, preferably at about $60^{\circ} \mathrm{C}$. Alkali-albumin may be prepared by'acting on albumin with fairly strong caustic potash in the cold.

The derived albumins are not of frequent occurrence in plants, but may be met with in certain seeds.

4. Proteoses or Alburioses.-These are generally soluble in distilled water, though some are less so than others. They can be precipitated from their solutions by saturating the latter with neutral ammonium sulphate. They differ from the members of the first two classes by not being converted into coagulated proteid on boiling. Their characteristic reaction is that they give with nitric acid, or with potassium ferrocyanide in the presence of acetic acid, a precipitate which dissolves on warming the liquid and reappears as it cools. Unlike any of those of the preceding groups, they have the property of dialysing through a parchment membrane, but only very slowly.

5. Peptones. - These are much like albumoses, but do not give a precipitate with nitric acid or with potassium ferrocyanide in the presence of acetic acid. They are not precipitated by saturation of their solutions with ammonium sulphate, nor are they coagulated on boiling. Their power of dialysis is much greater than is that of the albumoses.

Neither peptones nor albumoses occur very plentifully in plants, and they are probably formed in them only from the decomposition of the more stable forms of globulin and 
albumin. There is no evidence at present that they are stages in the constructive process.

Some of the albumoses occur in certain seeds in association with some of the globulins. Both the albumins and the globulins, and probably the albuminates as well, are transformed into albumoses and peptones by the action of hydrolysing agents, such as dilute mineral acids and certain secretions of the protoplasm known as enzymes, whose action will be treated of in a subsequent chapter.

6. Coagulated Proteids.-These are the products of the action of heat on the members of groups 1,2 , and 3 . They may also be formed by the action of alcohol on the same bodies. They are insoluble in water, but are dissolved with some difficulty by strong acids and alkalis, probably undergoing decomposition during the process.

Besides these classes of proteids, another occurs which presents the curious peculiarity of being soluble in alcohol. Proteids of this group have been extracted from the endosperm of some of the cereal grasses. Examples of them are found in the zein of maize; and the gliadin and glutenin of wheat flour. Zein differs from the crystallised proteid of the hemp in its comparatively low content of nitrogen, which amounts to only $16 \cdot 13$ per cent. It dissolves easily in alcohol of about 820 specific gravity, but is insoluble in absolute alcohol. It is insoluble also in water, but in mixtures of alcohol and water it dissolves to a greater or less extent, being most easily soluble in a mixture containing about 90 per cent. of the spirit. In one of a lower concentration than 50 per cent. it is very sparingly soluble. Zein can be dissolved by glycerine if heated to $150^{\circ} \mathrm{C}$.; also by glacial acetic acid and by dilute solutions of caustic potash. Like other proteids, it is converted into peptone by pepsin and hydrochloric acid.

The original construction of proteid matter, like that of carbohydrates, seems to be carried out only by vegetable protoplasm. It does not, however, appear to be dependent upon the same conditions as the process already 
described. It cannot be classed with the latter as a process of photosynthesis, and it is only indirectly dependent upon the action of the chlorophyll apparatus. Unlike the construction of carbohydrates, it is not confined to green plants-indeed the fungi can commence the synthesis at a lower stage than the latter, beginning the construction with compounds of ammonia, which have to be converted into nitrates before green plants can utilise them.

For the synthesis of proteids we have accordingly two certain starting points, to which may be added another which is confined to a small group of plants, if not indeed to a single organism. We have already alluded to the fact that certain of the Leguminosæ have the power of using the nitrogen of the atmosphere for the purpose of constructing organic food. This utilisation of it is, however, not carried out by the green plant independently, but only when its roots are associated symbiotically with a fungus which forms peculiar tubersular outgrowths upon the rootbranches. It is apparently the latter organism which effects the first fixation of the nitrogen. The leguminous plant alone is as powerless in this direction as any other green plant. How the fixation takes place, what part of it is due to the direct metabolism of the fungus, and how far the protoplasm of the green plant is concerned in the early stages, are at present quite uncertain. It seems, however, probable that the fixation is carried out by the fungus alone, without any influence or aid derived from the green plant. A few other similar organisms can under appropriate conditions carry on a similar fixation without being in symbiotic union with any green plant. If this view is correct, the leguminous plant is supplied by the fungus with a food material which has already been worked up from the simple form in which the elements of it are absorbed ; but how far the manufacture has proceeded - that is to say, in what condition the nitrogenous material is actually presented to, and absorbed by, the tissues of the root-is at present uncertain. 
The power of fixation of free nitrogen thus possessed by the organisms mentioned has been stated by several observers to be shared by certain lowly Algæ, but the evidence as to their activity in this direction is conflicting. It may be that they are capable of a similar symbiotic relationship with certain of the nitrogen-fixing bacteria of the soil already mentioned, but it is more probable that bacteria living simultaneously, but not symbiotically, in the soil with them, carry it out.

When we turn to the method of construction of proteid by a green plant we find ourselves in possession of very little accurate information as to the stages which are involved. We find that nitrates especially are absorbed by the root-hairs from the soil, and that a continuous stream of them passes into the plants. This naturally is associated with a transportation of the nitrates through the root and stem. They can be detected in varying quantities in these regions, but the amount seems to diminish as the termination of the stem is approached, and none can be found to be present in the leaves. It may be inferred that a gradual decomposition takes place as they pass along the axis, and that this is completed in the leaves.

A theory has been advanced to explain this disappearance, which may be mentioned here. It is that the nitrates are decomposed by the organic acids of the plant, and in particular by oxalic acid. Simultaneously the sulphates which are absorbed undergo a similar fate. The resulting bodies, the nitric and sulphuric acids, unite with some form of non-nitrogenous organic substance, possibly formaldehyde, or a fairly simple carbohydrate, to form proteid. From what has already been advanced, however, it is evident that this scheme of construction is purely hypothetical.

When we search for a form of nitrogen compound that is nearer proteid in its composition than these simple salts, it is natural to look at the products of the decomposition of proteid material to see if these furnish any clue to a possible 
constructive process. When proteids are digested in the animal organism under the influence of the strong ferment of the pancreatic secretion, we find that among the products of the decomposition certain nitrogenous compounds occur which are crystalline and capable of diffusing through animal and vegetable membranes. These substances, the chief of which are tyrosin and leucin, with a little asparagin, are known technically as amido-acids, owing to their containing the group $\mathrm{NH}_{2}$ (amidogen), replacing one atom of hydrogen in the hydrocarbon group of an organic acid. It is extremely probable that these compounds are made use of again in the subsequent reconstruction of proteids in the cells. Many of these substances have been found to occur in plants, and among them asparagin is extremely conspicuous. It can be detected in seeds and seedlings, and in older plants it is not infrequently present in the leaves. There is considerable probability that these substances occur as a stage in the original construction of proteids, though they may no doubt also be formed during its digestion in the vegetable as well as in the animal organism. This probability is supported by the observation that green plants are able to absorb from the soil and utilise many such amido-acids when artificially supplied to them.

Another hypothesis of proteid construction has been advanced which takes account of these substances as stages in the process. We have seen that salts of ammonia are converted into nitrates in the soil before being absorbed. The first step in the construction is thought to be the reconversion of the nitrates into ammonia, which interacts in some way with formaldehyde or one of its polymerides to form one or other of these amido-acids. This subsequently combines with some kind of non-nitrogenous organic substance together with some compound of sulphur, to form proteid.

This view of the construction, though of course only hypothetical, associates certain processes which apparently occur in nature. The formation of proteid seems to involve 
the simultaneous presence in the cells of some amido-acid, frequently asparagin, and some carbohydrate such as sugar. If shoots of plants which exhibit no accumulation of asparagin during normal growth are eut off and kept in darkness for some time, a gradual accumulation of the amido-acid can be observed. This in all probability is the expression of the decomposition of proteid taking placeduring the life of the shoot, and is presumably a normal occurrence. The reconstruction which would explain its nonaccumulation during illumination is prevented by the nonformation of the needed carbohydrate in the darkness.

The probability of a combination or interaction of these two classes of substance in the synthesis of proteids is supported by the fact that at the active growing points, where protoplasm is energetically formed, and where consequently abundant supplies of proteids are needed, neither sugar nor amido-acids can be detected, though they can be traced quite readily up to a short distance below the place where this active growth is proceeding. This fact is easily understood if we admit that proteid is constructed there at the expense of these two constituents; supplemented, of course, by the necessary compound or compounds of sulphur. If either of these supplies ceases to be available, the growth of the plant at that point stops.

Though we have seen reasons for thinking that nitrates and amido-acids form two stages in the normal process of proteid construction, we must not conclude that they invariably do so. In one plant, Pangium edule, which was examined by Treub in 1894, the nitrogen needed for proteid construction appears to be supplied in the form of hydrocyanic acid. In the shoots of this plant, cells occur in the cortex which contain this acid. In those nearest the apex the latter occurs alone, but as they grow older, a little proteid is found to be mixed with it. In still older ones the proteid preponderates, and at some distance behind the seat of growth it occurs alone, the acid having disappeared. Certain fungi can utilise nitrogen-containing derivatives of 
methane or benzol for the same purpose. It is probable, therefore, that more than one pathway to the proteid molecule may yet be found in different plants.

Probably the construction of proteid is not confined to any definite tissue or series of tissues in the plant. It is certainly only connected indirectly with the chlorophyll apparatus, and that in so far as carbohydrates are necessary for its formation. At the same time, there is a certain amount of evidence which points to its synthesis being in the first place effected in the leaves. The fact that nitrates can be traced towards these organs, and that they nevertheless do not appear to be present in the mesophyll cells, makes it probable that they are manufactured into something else there. The occurrence of amido-acids in the leaves is more in harmony with the view that they are built up there, than with the assumption that they arise from the decomposition of already existing proteids, though, no doubt, the latter is the case in the tissues of seeds, and possibly of seedlings, which are being nourished at the expense of materials stored in the seed. The proportion of proteid to dry weight of tissue has been stated to increase gradually and progressively from the roots to the leaves, in which it attains a maximum. Moreover, proteids are continually being removed from the leaves. If, however, the process does primarily go on in the leaves, it does not take place under the same conditions as the construction of carbohydrates. It goes on quite well in green cells in darkness, so that it is not, as already mentioned, a process of photosynthesis. It has recently been claimed that the construction of proteid in certain plants is favoured by light, and more particularly by the ultra-violet rays, though the luminous ones have a certain feeble effect. Whether or no the energy for the construction is derived therefrom is not, however, certain.

Sachs held that the sieve-tubes of the fibro-vascular bundles of the axis of the plant are also the seat of the construction of proteid. Though this is possible, it seems 
more likely that they are concerned in the transmission of organic nitrogenous material from the leaves to other organs. In whatever form proteid material travels about the plant, which for the present we cannot discuss, it is almost certain that it passes by the sieve-tubes, and it may well be that too great an accumulation of the travelling form may be attended by its conversion into an insoluble condition, and its deposition in the cells. There is no conclusive evidence pointing to the sieve-tubes as the places where it is originally synthesised.

The same considerations apply to the various growing points or zones. No doubt proteid is constructed there, but it is equally certain that it is so built up from bodies which have resulted from the digestion or decomposition of proteid that has already been synthesised elsewhere, and which has undergone such decomposition solely with a view to transport or translocation.

We judge it probable on all these grounds that the great seat of proteid construction in a green plant is its leaves, and this not on account of the possession of the chlorophyll apparatus, but because of a property inherent in the cell-protoplasm. Whence the energy is derived is not clear, but many writers hold it to be supplied by accompanying chemical decompositions.

Subordinate synthetic formation of proteids may take place in the roots, for nitrates appear to be used up there, though in much less quantity than in the leaves.

The construction of proteid by fungi is an additional proof that it is altogether independent of the chlorophyll apparatus, if not that it is unconnected with the access of light.

The third group of foods, the fats or oils, are probably not directly synthesised in plants, but are products of the decomposition of proteids, or perhaps of the living substance itself. 


\section{CHAPTER XIII}

THE CONSTITUENTS OF THE ASH OF PLANTS

WE have seen in a previous chapter that when a plant is carefully burned and the residue collected, the latter, which is known as the ash, is found to contain a number of elements which vary in different cases and which always include certain metals, as well as some non-metallic elements. The occurrence of this ash being universal, we can conclude without any difficulty that some of its constituents at least must be of importance to the organism, though it cannot be denied that our information is exceedingly incomplete. In the study of the nutritive processes of animals we meet with similar phenomena. How far any of the constituents of the ash can be regarded as actual food is uncertain, nor can we solve this question until we know something more about the composition of living substance. Whether any of these bodies actually enter into such composition is doubtful, but several of them appear to be necessary for the assimilation of the food which is either manufactured or supplied, as well in the case of the vegetables as in that of the animal organism.

Many of them, again, while not serving as food or even as materials for the formation of food, no doubt play important parts in the general metabolism of the organism. At present we are not in a position to say definitely how most of them are concerned in any or all of these processes.

From the nature of the plant-body and the absence of 
the localisation of different functions in particular organs which is so much more clearly characteristic of the animal organism, it becomes very difficult to ascertain the exact nature of the part played by any of these ash constituents. We can more easily determine what is the effect produced by variations in the amount supplied or by the total absence of any of them. This effect is usually, however, only the general effect upon the plant, and the experiments leave us still quite in the dark as to the way in which any general effect is produced, whether directly, or indirectly by affecting the health of the plant and thus leading to -secondary changes in its tissues.

The experiments in question are preferably carried out by means of water-culture, the general nature of which we have already explained. Plants will grow very well in water containing small quantities of various inorganic salts, and these can be varied at will for the purpose of definite inquiries. The composition of such a culture-solution is given by Pfeffer as under :-

$\begin{array}{lll}\text { Calcium nitrate } & \cdot & 4 \mathrm{grms} . \\ \text { Potassium nitrate } & \cdot & 1 \mathrm{grm} . \\ \text { Magnesium sulphate } & \cdot & 1 \mathrm{grm} . \\ \text { Potassium acid phosphate } & 1 \mathrm{grm} . \\ \text { Potassium chloride } & \cdot & 5 \mathrm{grm} . \\ \text { Ferric chloride solution } & \cdot & \text { a few drops } \\ \text { Water } & \cdot & 7 \text { litres }\end{array}$

Or a convenient fluid may be prepared by dissolving 20.5 . grms. magnesium sulphate in 350 ce. of water, and 40 grms. calcium nitrate, 10 grms. potassium nitrate, and 10 grms. acid phosphate of potassium in another 350 cc. ; 100 cc. of each of these solutions should then be added to $9 \cdot 8$ litres of water. This culture-medium will contain . 2 per cent. of salts, and will need only the further addition of a few drops of ferric chloride solution.

This percentage is generally satissfactory, though the concentration may be increased twofold without affecting 
the plants injuriously. Too great a quantity of salts, however, becomes deleterious.

The effect of omitting any particular constituent can be examined by making up the culture-fluid as required.

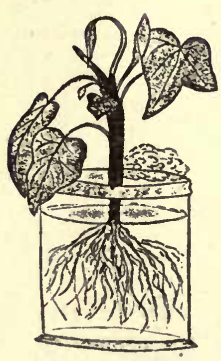

4

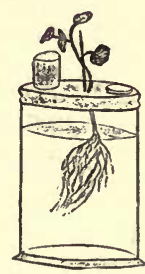

3

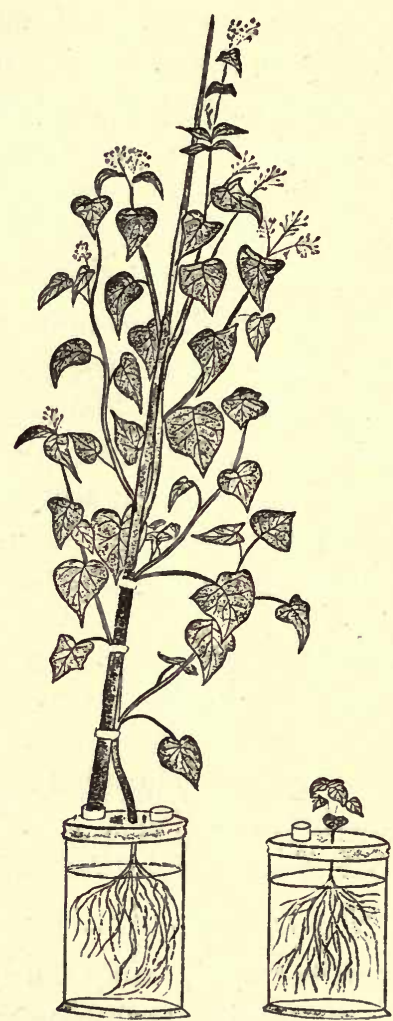

1

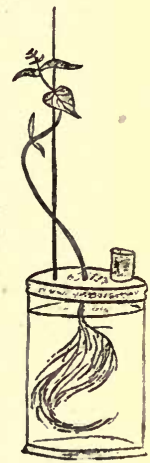

5

Fig. 91.-Plants of Buckwheat cultivated in various Nutritive Solutions.

1, normal solution containing all necessary salts; 2 , solution containing the same salts as 1 , except potassium compounds; 3 , solution of same composition as 1, except that sodium salts have been substituted for potassium compounds; 4, solution of same composition as 1 , except that no calcium salts are present; 5 , solution containing no compounds of nitrogen.

Fig. 91 shows in (1) a plant growing in such a fluid as is described above; (2) shows a similar plant cultivated in a 
medium from which potassium is absent, (3) one in which sodium is made to replace potassium, (4) one in which there is no calcium. The general character of such experiments can be seen by comparing the relative development of the plants under these conditions, and it is at once evident that the different metals and other elements employed have a certain functional importance. Deprivation of any of those mentioned affects all plants injuriously, though in different degrees.

We can, however, say very little as to the way in which the injurious effects are produced in different cases. We can, as a rule, only guess at the functions of the different ash constituents by studying the effects thus made evident. In a very few cases we can associate an element with some definite metabolic process. An instance is afforded by the behaviour of iron, in the absence of which, as we have seen, there is no development of chlorophyll in the chloroplasts. We cannot even here say very definitely how this inhibition is caused. It seems unlikely that it directly causes the failure of the etiolin to be converted into chlorophyll, for all analyses of the latter show that iron does not enter into its molecule. It is probably an indirect effect arrived at through faulty nutrition set up in the absence of the metal.

At first sight it seems as if the absence of inorganic salts may be effective by interfering with the maintenance of the turgid condition of the cells, as all the compounds mentioned have osmotic properties. It is evident, however, that this cannot be the only or even the main cause of the disturbance of nutrition, as the salts are not interchangeable, and a salt of sodium in concentration quite sufficient to maintain the condition of turgor is unable to replace the salts of potassium normally required. Moreover, turgescence can be maintained by organic acids in the total absence of the normal constituents of the ash.

We can divide the latter into four groups which subserve different purposes. Of these the members of the 
first are essential, because they enter into the constitution of the living substance. They are sulphur and phosphorus. All analyses of proteids show that sulphur is an essential constituent of them, and as proteids are immediately applied to the construction of protoplasm, there can hardly be any doubt that sulphur is contained in living substance. Phosphorus does not seem to be present in the ordinary cytoplasm, but it is undoubtedly associated with the nucleus. The nature of the connection is not very clear, but all nuclei contain a constituent which bears the name of nuclein. This can be extracted from it by appropriate treatment, and analysis shows that phosphorus enters into its molecule. Nuclein occurs also in the substance of many cells, either as nucleic acid, or associated with certain proteid bodies.

The second group comprises certain metals which are essential to the development of a plant, but which apparently do not ever form part of the living substance. There is some little doubt about this, as the fact cannot be ascertained by analysis. The members of this group are potassium, magnesium, calcium, and iron.

The third group includes several elements which are not absolutely essential, but which are useful in many cases, and which are very widely distributed, although not universally present. Among them are sodium, silicon, manganese, chlorine, bromine, and iodine.

The fourth group includes many other elements which are only occasionally present, and which probably play no part in the metabolic processes. They appear to be absorbed because they are present in the particular soil in which the plant happens to be growing, and have the power of osmosing through the walls of the root-hairs, and passing their plasmatic membranes. Many of them have only been found in a few plants. Among them may be mentioned aluminium, zinc, copper, cobalt, nickel, zirconium, fluorine, and lithium.

What is frequently spoken of as the selective power of plants is often misunderstood. If a substance is present 
in a soil, can be made soluble in the hygroscopic water permeating that soil, and can dialyse through the semipermeable membrane of the root-hair, absorption of a certain quantity of it will take place. How much is ultimately absorbed is a question of the power of the plant to decompose or utilise it after absorption. Many substances which are useless or even deleterious to the plant which takes them up are absorbed continuously until a very large percentage of them is present, because other constituents of the plant decompose them, or because their power of dialysis is such that they are easily removed from the absorbing cells. The possibility of the dialysis by which they are originally taken up is perhaps a question of relationship between the size of their molecules and that of the meshes of the plasmatic precipitation membranes which bound the cytoplasm on its two faces, abutting on the cell-wall and the vacuolar cavity respectively. This possibility of penetrating these membranes, and the power of subsequently removing the substances from the absorbing cells, are the special features of the so-called selective power of the plant, and it is evident that this power is particularly associated with the disposition of the materials after absorption, more than with the absorption itself.

We may now turn to the consideration of these varied constituents of the ash, and examine them in detail. The first group, we have seen, is composed of sulphur and phosphorus. Its importance lies in the fact that these elements enter into very close relationship with protoplasm, the former being in all probability a constituent of its molecule.

Sulphur is only taken up by the higher plants in the form of sulphates of some of the metals of the other groups or of ammonia. Fungi can also utilise salts of sulphurous and hydrosulphurous acids when they are presented in dilute solutions.

Phosphorus is associated with the nucleus rather than 
with the cell-protoplasm. It is contained in the substance called nuclein to the extent of about 6 per cent. The nuclein is apparently chiefly in the chromatin substance of the nucleus. Phosphorus is also a constituent of some proteids, and is probably present in the enzymes which are concerned in the true digestive processes of the plant. It occurs in chlorophyll also, according to Hoppe-Seyler, whose analysis of this pigment has already been quoted

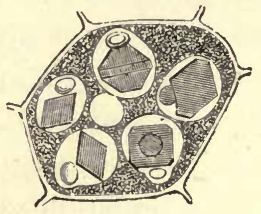

Fig. 92.-Cell of Ricinds

Seed, containing five

Aleurone Grains. (page 158). In a few plants phosphorus is temporarily stored in the seeds. Examples are presented by the Brazil nut (Bertholletia) and the Castor-oil plant (Ricinus), whose seeds contain stores of proteid material in the form of complex grains. In the substance of these grains there is a small, usually round, accumulation of mineral matter composed of a double phosphate of calcium and magnesium (fig. 92), which lies side by side with a crystal-like proteid body. Lecithin, a complex fatty body containing phosphorus, is present in actively growing cells of many plants.

Phosphorus is absorbed by the plant usually, if not entirely, in the form of soluble phosphates, most frequently a phosphate of calcium. Besides being important as an integral part of the living substance, certain observations tend to show that it materially assists in the construction of proteids.

The second group of ash constituents includes four metals which are essential to all plants, viz. potassium, magnesium, calcium, and iron. Probably these act only indirectly in the constructive processes, though there is some evidence that they may be integral constituents of living substance. They do not enter into the composition of proteids.

Potassium is absorbed in a variety of compounds, of which the nitrate and the chloride are the most advan- 
tageous. The part which it plays is not at all well understood. It may enter into the composition of protoplasm, for it is especially abundant in embryonic tissues. It has been thought to be connected with the construction of carbohydrates, but in what way it affects it is not known. It occurs in greatest quantity in the organs in which the formation and storage of these bodies are most actively carried out, viz. leaves, tubers, seeds, \&c.

Magnesium has a distribution much like that of potassium, and, as well as calcium, is thought by some botanists to enter into the composition of protoplasm. It may be absorbed in various combinations, but the chloride is the least advantageous. Calcium is essential to all green plants, but fungi do not always require it. Little of it relatively is found in young tissues, but greater amounts are present in adult ones. Its function is not understood, but it is useful in neutralising oxalic acid. It is prominent in cell-walls, part of which even in the very young state consist of calcic pectate. In older cells the middle lamella appears to consist entirely of this substance until lignification is complete. Calcium may be absorbed in the same combinations as magnesium.

As has been already mentioned, the most evident function of iron is to assist in the formation of chlorophyll. As it is not contained in the pigment, its influence here can only be indirect. It may be associated in some way with the protoplasmic basis of the plastid, so that the latter in its absence is thrown into a pathological condition and ceases to form the colouring matter. The influence both of the metal and of light in this particular may consequently be similar. That it is associated with the plastid does not appear improbable in view of some observations of Macallum's that iron is always found in direct relationship with the chromatin of the nucleus, of which it appears to be an integral part. There is here evidence of a close association between the metal and some forms of living substance. 
Iron can be absorbed with advantage apparently in any soluble combination.

The third group of elements contains several that are of importance to particular plants but are not universally necessary. Others usually included here are not known to be functionally important at all, except that they have a certain power of replacing to some extent the more important metals which have been already spoken of.

Of the metals of this group, sodium is the most widely distributed. It exists in all soils, and it is capable of absorption in considerable quantities. Experiments by means of water-culture show, however, that its beneficial influence is extremely slight. It can be omitted from the culture-fluid without entailing any harm to the plant, and its presence in any quantity will not compensate for the absence of potassium (fig. 89, 1 and 3 ). If compounds of sodium and potassium are present together in sufficient quantity, the latter is always absorbed in far the largest amount, indeed almost exclusively by many plants. Sodium seems able, however, to effect a certain economy in the use of potassium. If a cereal plant is supplied with too little potassium, and with a certain amount of sodium, development is normal, and an examination of the distribution of the two metals in its tissues shows that the potassium is accumulated in the flowers and seeds, while the sodium replaces it in the vegetative parts. It is absorbed in the same combinations as potassium, but the chloride is not, as in the latter case, a valuable salt. Indeed, sodium chloride is generally deleterious, except, perhaps, to the plants of the sea-shore, in which it brings about their peculiar succulence.

If we compare the influence of potassium, sodium, and calcium, on the development of a crop of herbage plants, we find that the presence of potassium leads to a development of stems, flowers, and fruit, or to what may be regarded as the maturing of the plants, while in the absence of sufficient potassium and the presence of calcium and sodium 
vegetative growth is more directly favoured, but the crop remains backward and immature.

There is a possibility that all these metals serve another purpose as well as some particular functional one. We have seen that the nitrogen which the plant obtains is derived from the soil, being most favourably supplied by the latter in the shape of nitrates. In the soil the nitric acid is combined most frequently with the metals under discussion, and a not inconsiderable quantity of the latter may be taken up solely for the sake of the nitrogen which they can thus carry into the plant. The varying amounts of sodium and calcium which plants contain have been found to bear a certain relationship to the amounts of their compounds which occur in the particular soils in which the plants have been growing. When calcium and sodium nitrates are taken up for the sake of the nitrogen, they are probably decomposed by the organic acids formed in the plant, and the nitrogen is made to enter into further combination, leading to the construction, possibly of amido-acids, and eventually of proteids.

Of the other elements which are included with sodium in this group, silicon is one of the most prominent. It is absorbed almost entirely in the form of silicates of potassium and sodium, the latter combination being the principal one. It is difficult to say what purpose it serves. It is usually found deposited in the epidermal cell-walls, and as the grasses and the horsetails contain it in greatest abundance, it has been suggested that its utility consists in its contributing to the rigidity of their weak stems, and consequently to the maintenance of their vertical position. This is, however, not the case ; their rigidity is dependent on the degree of development of their harder tissues, and the absence of silica makes but little difference to them. Silicates, when added in quantity to the soil in which green crops are growing, have no marked effect upon the amount. of silica which is subsequently present in the straw. It is uncertain whether the silica enters into the metabolism of 
the plant, or whether the silicates are decomposed at once and the silica deposited in the cell-walls in which it is prominent. As it is most readily taken up in combination with sodium, this is unlikely, the sodium being, as we have seen, of very little, if any, use. It has been said that oats mature less fully and completely in the absence of silica, so that in the case of that particular plant there is some evidence of its aiding in metabolism, though no suggestion has been made as to the way in which it exerts its influence. It is possible that it may be of value also by protecting the plant from the depredations of animals or from the attacks of fungi, as it is mainly accumulated in the epidermis.

The other elements of this group include chlorine, bromine, and iodine. A little of the former is of universal occurrence, but it may be due to its being taken up in conjunction with potassium. Water-culture experiments show, however, that in many cases it cannot be omitted altogether without injury to the plant. It has been associated by some writers with the translocation of earbohydrates, particularly in the buckwheat, a view which is based upon the observation that in its absence the chloroplasts become abnormally filled with granules of starch. Bromine and iodine are chiefly found in marine plants, but their function is unknown.

Manganese is a constituent of many plants. Till quite recently nothing was known about its influence on metabolism, but it now appears probable it plays a part in various oxidative processes, which are carried out by a somewhat widely spread enzyme known as Laccase, whose normal function is, however, at present obscure.

The elements of the last group are numerous; they vary with the composition of the soil in which the plants are growing, and appear to subserve no useful purpose. Many of them in even moderately dilute solutions are extremely poisonous, so that they must be absorbed in a high state of tenuity. Their presence shows that the 
selective power of plants is not necessarily connected with the development of normal metabolic functions, but is mainly physical and only to a slight extent physiological.

From what has already been advanced, it is evident that the time is not ripe for a detailed discussion of the parts played by the constituents of the ash of plants. Nor will it be till we have ascertained much more fully how the various metabolic processes are carried on. Certain broad statements of a somewhat general character are all that are at present justified, and these concern only some of the mineral matters which are absorbed. The metabolism not only depends on the presence of certain elements, but is largely influenced by the relative quantities of each which the active cells contain.

The vegetative activity of, at any rate, herbage plants is associated with a plentiful supply of nitrogen. In the absence of sufficient potassium vegetative luxuriance is obtained, but the degree of development of the plant is limited by such deficiency. In the event of sufficient supplies of potassium being afforded, the relative abundance of the nitrogen has an important influence on the forma. tion of carbohydrates, which are then produced in greater quantities. Coincidently the plants go on to maturity; the luxuriance of the leafy parts becomes curtailed, and the development proceeds normally, leading to the formation of the flowers and subsequently the seeds. Thus the composition of the supplies in the soil determines largely the character of the development of the plants growing in it. It has also considerable influence upon the variety of the species of these plants, owing to the various ways in which particular constituents may influence different individuals.

In the absence or the deficiency of particular salts, others may be absorbed in proportions very different from those which would have been found had the missing ele. ment or elements been present. 


\section{CHAPTER XIV}

\section{OTHER METHODS OF OBTAINING FOOD}

Is our introductory considerations of the true nature of the food of plants, and of the manner in which they obtain it, it was pointed out that there are stages in the lifehistory of all plants during which it is imperative that they shall be supplied with food in a form in which they can assimilate it at once, constructive mechanisms either being altogether absent from them or not having been developed at the particular time under consideration. There is thus in every plant a power of assimilating organic food so supplied, a power which in some cases is permanently relied upon, sometimes completely, sometimes only partially, and which in other cases is laid aside as soon as the chlorophyll apparatus becomes developed. The need for the supply of the organic food is always felt by every protoplast, and the latter cannot be nourished except by it. We may contrast in this respect the individual protoplast and the colony of which it is a member, the latter being able through the co-operation of its individuals to construct the organic food which must be provided for the use of every member, even of those to which the work of construction is allotted.

The constructive power may be partially or wholly lost or undeveloped; in such cases the loss must be compensated for by the supply from outside of the material the plant is not able to synthesise for itself.

Examples of plants possessing different powers of such absorption are supplied by every class of the vegetable kingdom. They are most conspicuous among the Fungi, 
because in them there is no chlorophyll apparatus, and hence constructive processes must be very rudimentary. Distinct differences can be seen, however, in this group. Certain lowly forms appear to be able to utilise very different compounds of carbon and to synthesise carbohydrates therefrom. Many can grow and multiply in solutions of simple acids such as formic or acetic. A much larger number need for their nutriment a supply of carbohydrates in the form of sugar, and if this is given them, together with certain relatively simple compounds of ammonia, especially ammonium tartrate, they can construct therefrom proteid and fatty bodies.

Others need the nitrogen to be supplied in the form of amido-acids, as they have no power to utilise the simpler ammonium salts; others again need their proteids as well as their carbohydrates to be supplied to them as such, for they possess scarcely any constructive ability.

A similar power of utilising carbohydrates and allied bodies is exhibited by many green plants. If their roots are watered with a solution of sugar, they can take it up and economise by its aid the sugars which the chlorophyll apparatus is constructing. Various bodies also from which sugar can be formed are absorbed when presented to the roots. and serve as forerunners of sugar in the plant. Among these may be mentioned Glycerine. The process of the synthesis of proteids also may be shortened by supplying the roots with material such as asparagin, leucin, or urea. Proteid as such can only be utilised by a few flowering plants which possess special mechanisms for its preliminary digestion.

Among what we must regard as these abnormal methods of food supply we must include certain processes in which two organisms are associated, for the well-being, in some cases, of both, in others for that of only one. The two organisms are brought into very intimate relationships with each other, in some cases a very complete union of their tissues being effected, so that transport of elaborated food materials 
can readily take place between them. In those cases in which this close association is of benefit to both the organisms it is spoken of as symbiosis; in those in which one flourishes at the expense of the other, the relationship is called parasitism. While there are many cases which can be definitely referred to both these categories, they seem to blend one into the other, cases being known in which it is very difficult or impossible to say whether the advantages are all on one side or not.

The plants which differ least from the normal habit which we have described are those which are known as Saprophytes, their characteristic feature being that they derive at least part of their food from decaying animal or vegetable matter, absorbing it in some cases as actual foodstuffs, and in others as organic compounds which require relatively little expenditure of energy to build them up again into proteids or carbohydrates.

Numerically the fungi are the most prominent in this group, but the green plants also afford many instances of the habit. Among the mosses Splachnum grows upon lumps of dung, and various species of Hypnum flourish in water which contains various compounds derived from the decomposition of once living matter. Among higher plants still, the soil of woods and pastures affords many examples of individuals which depend partly upon the humus of the soil and partly on their own chlorophyll. Among the ferns we have notably the moon-wort, Botrychium Lunaria, and among the club-mosses some species of Lycopodium, while numerous flowering plants show this peculiarity.

The chlorophyll apparatus is found in nearly all of them, though in some cases it is so reduced as to be almost functionless. Some of our native Orchids are remarkable in this respect, that they are almost, if not altogether, dependent upon their saprophytism. Neottia, the so-called bird's-nest orchis, has a flowering stem above ground, on which are only a few rudimentary leaves. At 
the base of the stem there is found a cluster of fairly stout roots which intertwine with each other to form a mass sometimes as large as a man's fist. These are developed only in masses of humus, from which they absorb the products of decay. These plants differ thus from normal phanerogams by their method of absorbing food. Their roots are not provided with the system of short-lived roothairs which are so characteristic of the ordinary roots. They are not in need of such close contact with continually fresh particles of soil as are the latter, lying as they do embedded in a mass of humus. In some cases all their external cells absorb material from this ; in others special absorptive cells are present, but these are not localised like the ordinary root-hairs, and they are not being continually renewed, but remain active for long periods. Frequently they are only found on the roots at the points where contact with the humus is effected. Many of these saprophytes have the cells of their cortex infested with the hyphæ of a fungus.

The food which is thus absorbed from the decaying organic matter is not necessarily in a fit condition for immediate assimilation by the protoplasts. It may, and frequently does, require alteration before being available for nutrition.

The plants of the next group which we must consider differ from the saprophytes in an important particular. Like them they are provided with a chlorophyll apparatus, and are consequently capable of carrying on carbohydrate construction. Indeed they are generally more active in this respect than the members of the last group. As in the case of the greater number of the latter, it is chiefly their nitrogenous material that they obtain nearly or quite ready for assimilation. They appear to need this nitrogenous food in the form of proteids, and they obtain it by capturing and killing various animal organisms whose putrefying bodies yield them what they want.

The Utricularias, which are members of this group, are 
plants which live floating in water (fig. 93); they have a much-branched stem which bears a number of leaves, the

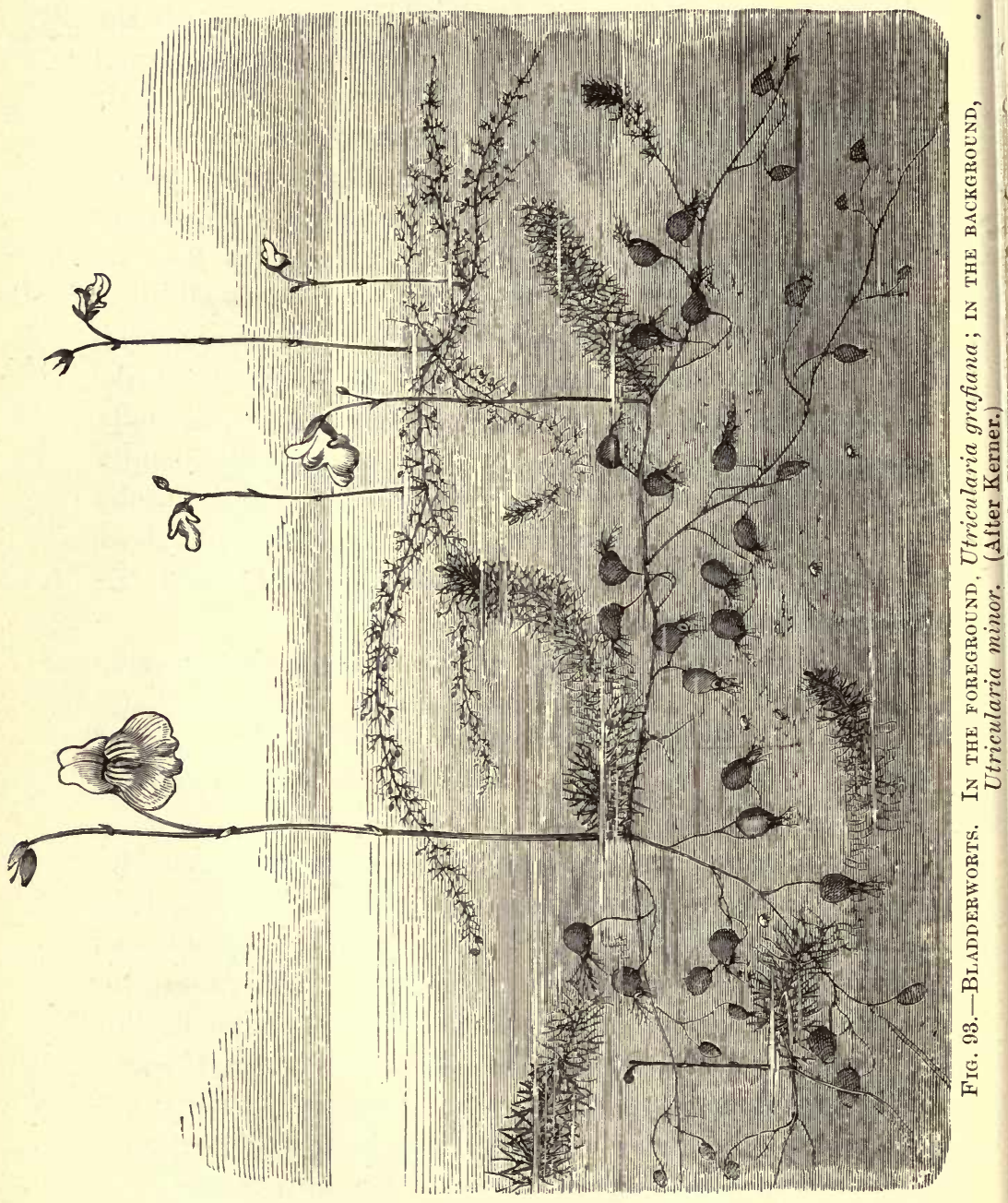

shapes of which differ in the case of different species; they possess no roots. Growing out of the stems are numerous small bladder-like bodies, each with a small opening at its 
apex. This orifice is guarded by a number of stiff tapering bristles, and is closed by a sort of trapdoor which opens inwards and shuts again with a kind of spring. A small animal such as an aquatic insect can easily open it by pressing against it, and thus can enter the bladder. The trapdoor immediately closes by virtue of its own elasticity, and cannot be opened by pressure from within. The insect accordingly finds egress, impossible, and after a short
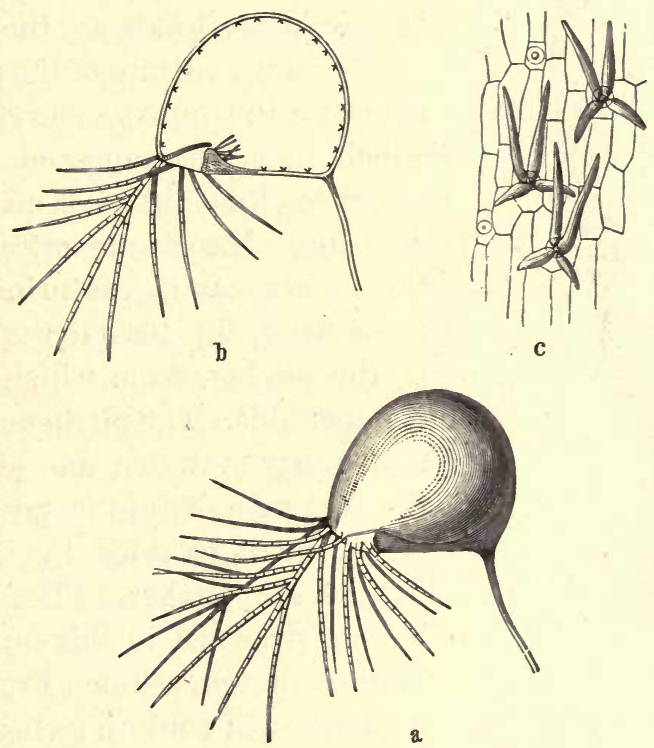

Fig. 94.-Traps of Utricularia neglecta. (After Kerner.)

$a$, a bladder magnified ( $\times 4) ; b$, section of a bladder ; $c$, absorption-cells on the internal surface of the bladder $(\times 250)$.

time, usually ranging from one to three days, it perishes and its body decays, yielding to the plant the products of its decomposition, which are absorbed by particular cells growing from the internal wall of the bladder (fig. 94).

Some of the ascidia-bearing or pitcher-plants show a somewhat similar mechanism and utilise corresponding organic substances. The Sarracenias afford good examples. 
These are marsh plants having their leaves arranged in rosettes, which spring apparently from the surface of the soil, and from the centre of which arises a single flower stalk. Each leaf is modified to form a curious pitcher-like

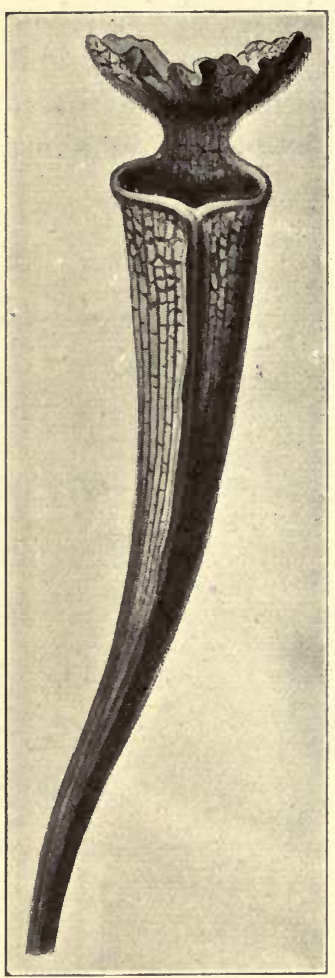

Fig. 95.-Leaf of Sarracenia, MODIFIED TO FORM A PITCHER. (After Kerner.) body (fig. 95), furnished with a kind of lid. The pitchers are generally conspicuously coloured, while the lid, which is the lamina of the leaf, often bears hairs which secrete honey to attract the prey.

The inner surface of the pitchers is lined with slippery recurved hairs which make it impossible for an insect to climb out of it after once entering. Insects are attracted by the honey, and, venturing upon these hairs, slip down to the bottom of the pitcher, from which escape is impossible. The pitcher contains a quantity of water, due either to the entrance of rain or possibly to some extent secreted by the surface of the pitcher. The insects become drowned in this liquid and undergo decomposition. Frequently a pitcher will contain so many that the products of their putrefaction become offensive. They are absorbed by the cells of the interior.

Certain other pitcher-plants show a still further advance in their method of obtaining proteid supplies. They possess similar means of attracting insects and alluring them to their death, but they do not depend on the slow process of putrefaction for the decomposition of their prey. Instead of this, they secrete and pour out a definite digestive fluid possessing properties like those of the secretions of the stomach and pancreas of the higher 
animals, by the instrumentality of which the insoluble proteids of their prey are converted into peptones, and possibly partially into amido-acids, prior to actual absorption. Among these Nepenthes and Cephalotus may be mentioned.

The pitchers of Nepenthes (fig. 96) are in the main similar to those of Sarracenia. They possess means of attracting insects to them, of seducing them into the interior of the pitcher, and of preventing their subsequent escape, all of which are comparable to those already described. The pitchers contain a watery liquid, which is secreted by their interior surfaces, and which has a faintly acid reaction. When an animal is captured and falls into the liquid, it sets up a further secretion, which is more strongly acid, and which contains a peculiar body known as an enzyme or ferment, the properties of which will be discussed in a

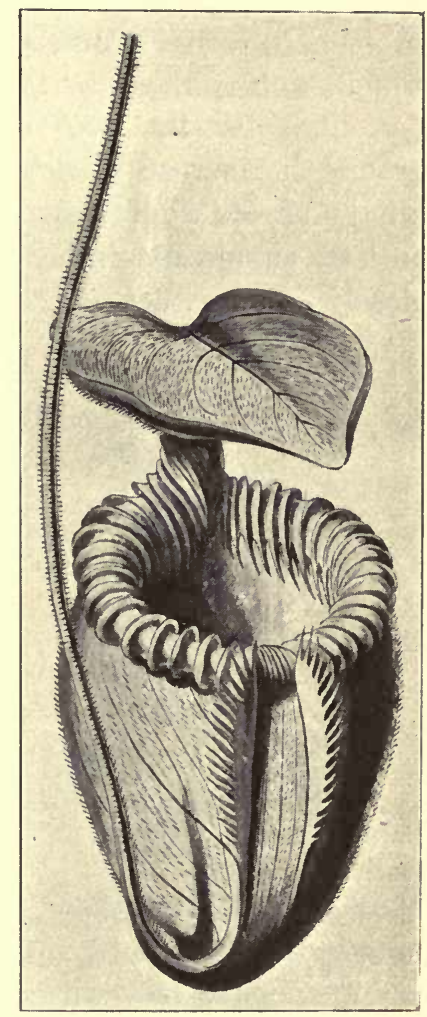

Fig. 96.-Modified Leaf (Pitcher) of Nepenthes. (After Kerner.) subsequent chapter. This ferment somewhat closely resembles the active principles of the gastric and pancreatic juices of the human body, and in the acid medium is capable of converting the proteids of the prey into peptone, leucin, and tyrosin, products which are all soluble and diffusible. This secretion is prepared by special glands, which are plentifully distributed over the lower portion of the internal face of the pitcher. 
The mechanism of Cephalotus is essentially similar to that of Nepenthes.

The members of another group of plants, represented by Bartsia and by Lathraa, effect the absorption of proteids in rather a different manner. Lathræa is a plant which, unlike those so far described, has no chlorophyll, but lives for the most of its time parasitically underground on the roots of certain trees, only sending up into the air the shoots which bear its flowers. Its stems, which are entirely subterranean, bear a number of thick fleshy leaves closely set together (fig. 97), which are quite colourless.
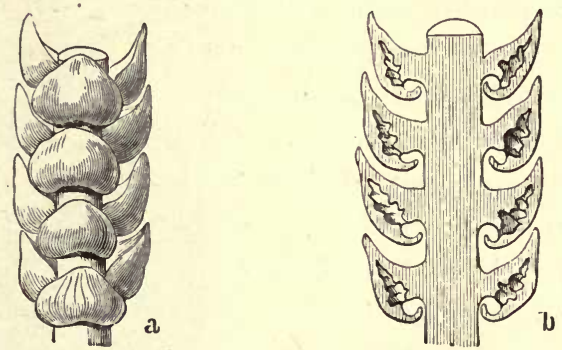

Fig. 97.- $a$, Piece of an underground Leaf-Shoot of Lathraa squamaria. $b$, Lovgitudinal Section THROUgh SaMe, SHOWiNg the Cavities in the LaMina. (After Kerner.)

They are curiously bent, so that the under surface is of very small dimensions, and where the two surfaces join are the openings of several cavities which are hollowed out in the thickness of the lamina. These chambers are irregular in shape (fig. 98), and on their surfaces are curious stalked hairs which project slightly above the other cells which bound the lacunar space. When an insect or other small animal makes its way into one of these chambers and comes into contact with the heads of these hairs, the latter are stimulated, and they then send out, through openings in their walls, a number of delicate protoplasmic filaments, which seize upon the intruder, killing it and ultimately digesting and absorbing its nitrogenous constituents. There 
are other stalked gland-like structures growing from the walls, which probably serve to hinder the escape of the prey. Bartsia possesses a somewhat similar mechanism, which is associated with its subterranean buds.

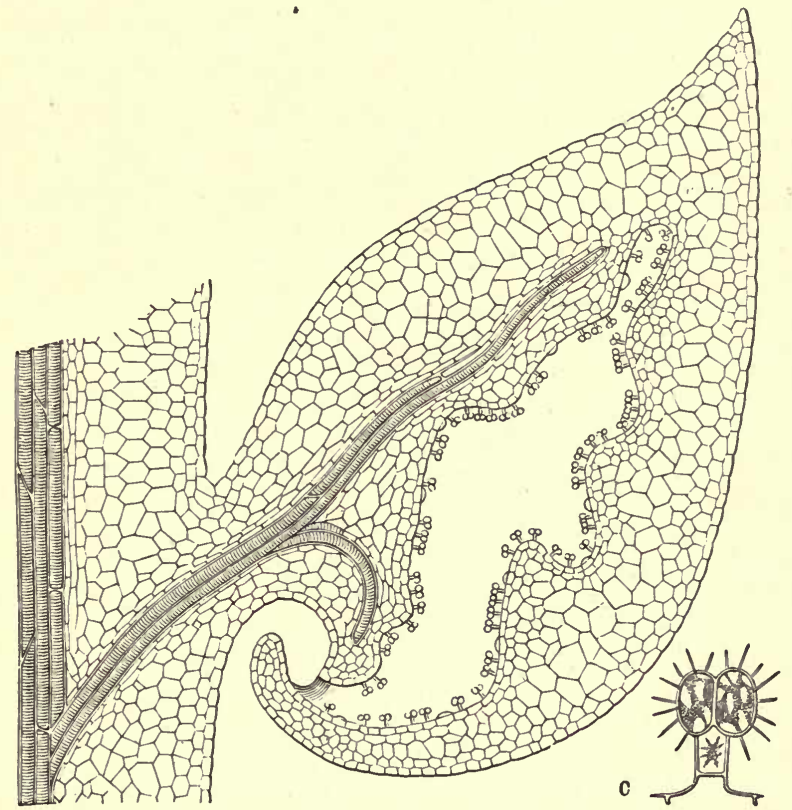

Fig. 98.-Leaf of Lathrca enIarged, showing the character of the Cavity OR PRISON-CHAMBer. $\times 60$. $e$, Plasid Threads Radiating FROM THE CELLS OF THE LITTLE HEAdS. $\times 540$. (After Kerner.)

There are other plants which effect the capture and digestion of insects in other ways. Drosophyllum, which is found in part of the Mediterranean region, is furnished with a number of long filiform leaves, which are closely set with stalked glands. These pour out a peculiar mucilaginous secretion which forms a drop of very glistening appearance round their swollen heads. There are other sessile glands among them which exude an acid digestive secretion resembling the gastric juice of the stomach, when they come into contact with proteid animal matter. An 
insect, attracted to the leaves by their glistening appearance, is at once entangled in the viscid mucilage and is presently suffocated. It is speedily digested by the secretion of the sessile glands.

Pinguicula, the butter-wort, has a mechanism of a somewhat similar nature. It bears, resting on the ground, large fleshy green leaves, the edges of which are slightly curled over towards the upper surface, forming a kind of open trough. All over the upper surface are distributed glands which pour out a viscid mucilage. On contact with any small mass of proteid, or with an insect or other small animal, these glands also pour out an increased amount of mucilage, mixed. now with a digestive fluid similar to that of Drosophyllum. If an insect alights upon the margin of the leaf, not only is the secretion poured out, but the edge slowly curls over more strongly, either covering the intruder, or pressing it towards the centre of the trough. Here it is suffocated and digested as in other cases. Pinguicula is peculiar in that its secretion has the power of curdling milk in the same way as the gastric juice of animals.

In some cases a yet more elaborate mechanism is found to effect the same purpose. We find associated with the power of digesting and absorbing animal food, a mechanism for the capture of the prey which involves a movement of either the leaf-blade itself or of the glands which it produces. The former is exhibited by Dionaa, the Venus's fly-trap; the latter by the different species of Drosera (the Sundews).

Drosera is a small plant which is found growing upon a substratum of bog-moss (Sphagnum). Its dimensions are small, the plant not being more than a few inches in height. It bears a rosette of leaves, from the middle of which rises a single scape of flowers. The leaves are covered with stalked glands (fig. 99), which stand out from the surface. Each gland has a somewhat substantial stalk, containing a rudimentary vascular bundle. At the top of the stalk is a rounded head which is always covered by a viscid secretion 
that it pours out. From the shining appearance of the glands with their drops of mucilage, the name of the plant, sundew, is derived. When an insect alights upon the leaf it is entangled in the secrestion, and, struggling to be free, is brought into contact with more and more of the drops, becoming hopelessly captured. The stimulus of contact provoles a

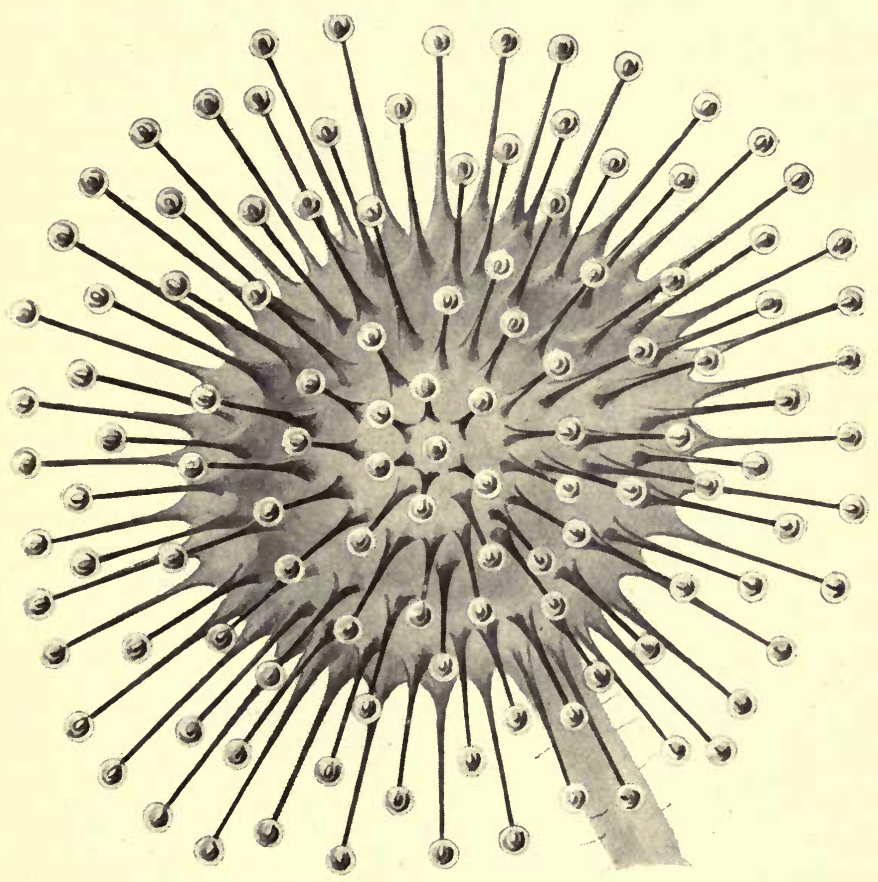

Fig. 99.-Leaf of Drosera, showing the glandulak tentacles.

movement of the stalked glands, all of which slowly bend over and bring their viscid heads to bear upon the struggling insect. The same disturbance causes an outflow of acid enzyme-containing secretion, which surrounds the prey, and digestion and absorption follow as before. After a time the glands unfold again and resume their normal attitude, and the leaf is ready to receive another visitor. 
Dionæa affords an instance in which the movement of capture is effected with greater rapidity. Like most of the insectivorous plants it possesses a rosette of leaves which rest upon the ground, and from the centre of the rosette it gives off a single inflorescence. The leaves are very different from those of Drosera. They have a flat expanded petiole, at the end of which the lamina is attached by a sort of joint. The lamina is roundish and is divided

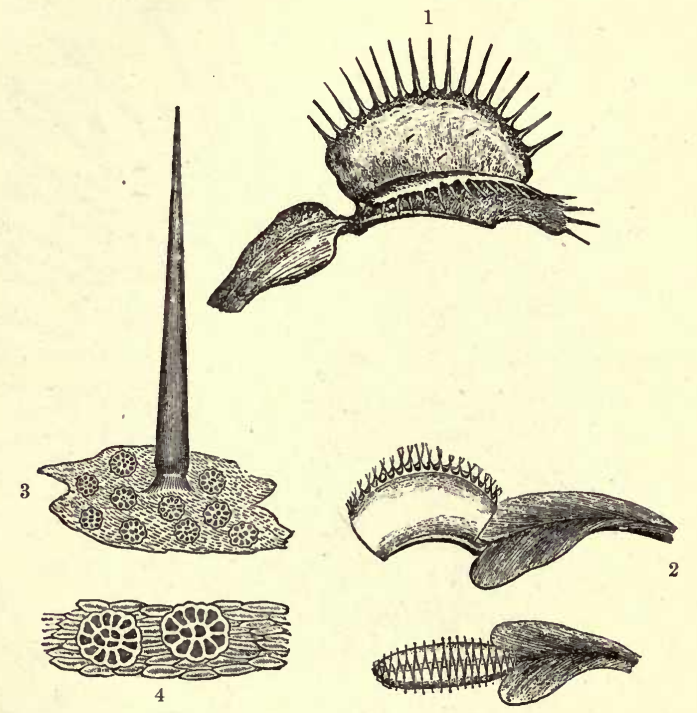

Fig. 100.-Leaf of Dioncea muscipula.

1 , open; 2 , closed ; 3 , one of the sensitive spines $(\times 50) ; 4$, glands on the surface of the leaf $(x 100)$.

into two almost exactly similar halves, which are separated by the midrib (fig. 100). The edge of each half is furnished with a number of rigid teeth, and when the two halves are folded together on a hinge which the midrib forms, the teeth interlock with each other and a closed cavity is prepared. On the upper surface of each half of the leaf, about in the centre, are three short spines which project outwards and upwards. When either of these is touched twice in rapid succession, the two lobes of the lamina become 
slightly concave and fold over quickly, the teeth interlock, and the cavity is closed. If the contact has been made by an insect, it is captured and imprisoned between the lobes. The closing is fairly rapid, taking perhaps ten seconds. All over the upper surface of the lamina secreting glands are found, whose secretion is similar to that of Drosera. If the leaf encloses nitrogenous digestible matter, such as the body of an insect, the prison remains closed for some considerable time, and the glands surround the prey with the digestive fluid, the products of its decomposition being absorbed by the gland-cells.

These mechanisms for the digestion and absorption of proteid substances are seen to be extremely complex. Evidence of such digestion and absorption is shown also by far humbler plants without any differentiated structure. Many Fungi and Bacteria when cultivated in solutions containing native proteids, such as albumin or globulin, are able to effect their digestion by the secretion of a similar enzyme to those of the plants already described. They subsequently absorb the peptone or the amido-acids which result from such action. Nor is proteid material alone affected in this way by these humbler plants. They derive their carbohydrate supplies from their environment in the same way as their proteid ones. Many of the filamentous fungi possess the property of forming digestive enzymes, which attack sometimes starch, sometimes inulin, sometimes various sugars which are not immediately available for nutrition, sometimes other more complex substances, all of which undergo this external process of digestion, the resulting bodies being subsequently absorbed.

In the earlier pages of this chapter we drew attention to the fact that it was not at all uncommon to find two plants closely associated together, with different degrees of completeness, with a view to their co-operation in carrying out some of these abnormal processes of nutrition. We may now study these relationships a little more fully.

The simplest cases of the dependence of one plant 
upon another are afforded by the so-called epiphytes, representatives of which are supplied by many members of the Orchidacere and the Bromeliacece which inhabit tropical forests. The dependence in these cases is merely one of situation. The epiphyte grows upon the external surface of some supporting tree, to which it clings by various arrangements, without penetrating into its tissues. Frequently the long roots of the epiphyte are attached closely to the crannies of the bark of the tree, and the dust and débris which accumulate there are utilised for the purpose of supplying it with nutriment. In other cases the supporting plant does not give it even so much assistance.

An almost. equally simple relationship is seen in the cases of Anthoceros and Azolla. Cavities in the tissues of these plants are inhabited by numerous cells of an Alga (Nostoc). Beyond affording them shelter and a certain degree of protection, the higher plant does nothing for its guests. The relationship is sometimes called commensalism.

A more complete association, attended by distinct advantage to one or both of the plants taking part in it, is known under the name of symbiosis. By some writers this term is confined to such an association as is of benefit to both organisms, and does not profit one at the expense of the other. Where the latter is the case the relationship is said to be one of more or less complete parasitism. Others speak of reciprocal and antagonistic symbiosis, to indicate these two different kinds of association.

One of the best known cases of symbiosis in the strict sense is that of the Lichens. These are lowly organisms which are epiphytic upon tree trunks, old walls, rocks, and other supporting structures. They are composed always of two distinct plants, an Alga and a Fungus, which are closely united together to form a kind of thallus (fig. 101). The relative modes of arrangement differ in different species, and many algæ and many fungi are found to be capable of entering into such an association. The advantages which result to the two constituents of the lichen are consider- 
able. The alga, which possesses chlorophyll, is able to construct earbohydrate materials by its instrumentality, and these are shared after their formation by the fungus, which has no such constructive powers. The fungus is able to condense aqueous vapour, which is very necessary in the dry situations lichens occupy. It can thus dissolve much of the dust and other débris of its resting place, and so carry raw material to the constructive algal cells. It also attaches the thallus to the substratum. Both partners can no doubt take, part in the construction of proteids. The relationship affords a further advantage, for the compound organism is much better

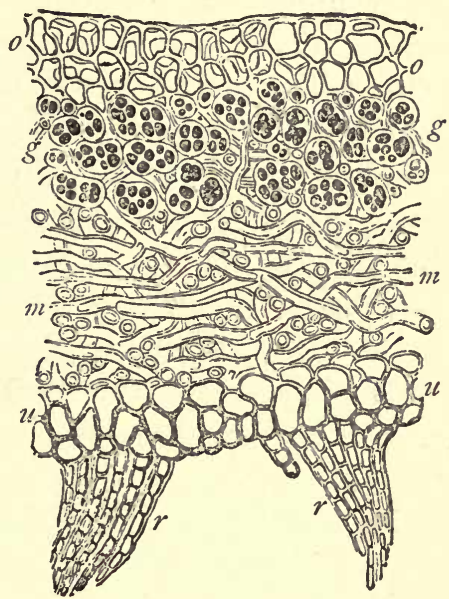

Fig. 101.-Section of a Lichen showING ALGAL CELLS $(g)$ IN THE MIDST OF A NETWORK OF FUNGAL HYPHE $(m)$. After Sachs.) able than either of its separate constituents to resist adverse conditions of temperature, drought, \&c.

A similar symbiosis is met with in the so-called kephir organism and others of the same kind. In these cases the two constituents are a yeast and a bacterium, the former of which is closely surrounded by chains of the latter, making a fleshy mass of irregular shape, and sometimes of comparatively conspicuous dimensions. The parts played by the two organisms are not very well understood, but there seems to be no doubt that the association is mutually beneficial.

In a former chapter mention was made of a property which is possessed under certain conditions by various plants belonging to the Leguminosa--that of being able to utilise the free nitrogen of the air in the construction of proteid food-substances. The power was shown to be connected with the formation of certain tubercular structures 
upon the roots of the leguminous plant. These tubercles are swellings of the cortex of the root, the cells of which are inhabited by a particular fungus, which breaks up in their interior into curious bacterioid bodies. The exact nature of the fungus has not been accurately determined. The soil contains many of these bacterium-like bodies, which make their way into the interior of the leguminous plants by penetrating their root-hairs, and growing down them into the cortex of the root. In the cells of the latter the penetrating filaments bud off the bacterioid bodies in great numbers. The stimulus resulting from the invasion causes a considerable hypertrophy of the roots at the points attacked, and the tubercles are the result. The fungus appears to have the power of fixing atmospheric nitrogen, bringing it into some combination, the exact nature of which is unknown, but which serves as the starting point of proteid synthesis, either by the green plant or by the intruder. The relationship is clearly of great advantage to both organisms, the fungus obtaining its carbohydrate supplies from the green plant, much as is the case in the lichens already described.

Many of our forest trees, among which the members of the Cupuliferce are conspicuous, exhibit another symbiosis which is of the greatest interest and importance. The roots of these plants grow down into soil which is infested with the mycelia of different fungi, with which they become entangled. The hyphæ of the fungi continue to grow together with the root, and form an investment over it, which is in some cases met with in the form of an open network, and in others in that of a dense feltwork (fig. 102). The fungi in some cases perforate the external cells of the roots and form a network in the interior. From the outside of the investing mantle hyphæ grow out into the soil in a similar way to the root-hairs of ordinary plants. These take the place of the root-hairs, which cease to be developed, and serve the purposes of the roots as absorbing organs for the water and the salts of the soil. The fungus 
is benefited by drawing its own nutriment from the cells of the root into which it has penetrated. The fungoid mantle is known as a mycorhiza; it is present not only on the roots of the Cupuliferæ, but on those of Poplars, and many Heaths and Rhododendrons.

A curious case of this kind of relationship is shown by Monotropa, a member of the Heath family which possesses no chlorophyll. Monotropa possesses a

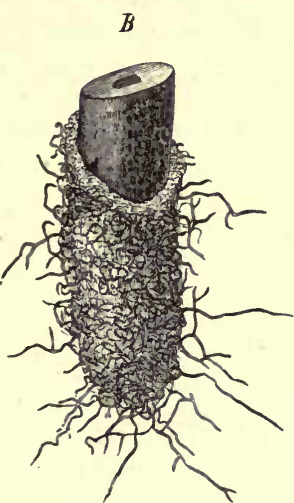

$\boldsymbol{A}$

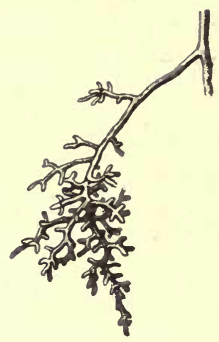

Fig. 102.--A, Epiphytic Mycorhiza of Fagus sylvatica $(\times 2)$; B, Tip of Root Partially DENUDED OF THE INVESTING MANTLE ( $\times$ 30). (After Pfeffer.) rhizome, from which rise sub-aerial stems from ten to twenty centimetres high, bearing succulent membranous leaves. From the rhizome are given off crowded masses of roots which are covered with a mycorhizal mycelium, and are embedded in humus. There being no chlorophyll apparatus, Monotropa is dependent entirely on the mycorhiza for its nourishment. The latter is entirely saprophytic. We have here a curious case of the complete dependence of a higher plant upon a more lowly one.

A complete symbiosis between two green plants is occasionally met with. A good instance is afforded by the Mistletoe and the plants upon which it grows, usually either the Poplar, the Silver Fir, or the Apple-tree: The seed of the Mistletoe is left by a bird upon a branch of one of these trees, and under appropriate conditions it germinates. The root of the seedling penetrates into the bark of the tree and grows inwards till it reaches the wood. It makes its way no further, but maintains its position there, and as the branch gradually thickens by the activity 
of its cambium, the intruding root is by degrees impacted in the secondary wood, its own growth preventing its being cut off and buried by the latter. The root branches in the substance of the tree, and the secondary roots make their way along in the bast, growing parallel with the exterior. These branches also put out small vertical outgrowths, which make their way to the wood just as the primary root did. A very complete fusion of the tissues of the two plants is thus ultimately arrived at. The advantage of the alliance is on the side of the Mistletoe, which derives a great part of its nourishment from the host. It possesses evergreen leaves, however, which serve for the construction of carbohydrates, and as it manufactures these during the winter, when the host plant has no leaves, the latter is able to benefit in its turn during that season.

Passing on to notice the association of two organisms which is known by the name of antagonistic symbiosis or parasitism, we find various degrees of completeness in the dependence of one form, the parasite, upon the other, the host. As in the case of the insectivorous plants, there are members of this class which are provided with a chlorophyll apparatus. and which are therefore indebted to their hosts for proteid substances only, or perhaps also for certain of their ash constituents. As these almost without exception fasten themselves upon the roots of the host plant, they are frequently spoken of as root-parasites. From their general structure and their relationship to the host plant, they evidently have much in common with the Mistletoe, and it is not very easy to distinguish between their semiparasitism and the symbiosis of the latter with the trees on which it lives. They are, however, usually herbaceous forms, and can-therefore be of no use to the host plant in the winter. Moreover, most of them ultimately destroy. the root on which they have fastened.

These root-parasites are mainly members of the Scrophulariacea or the Santalacea. As a rule, they are herbaceous annuals, though there are some perennial 
species. They grow from seed with fair rapidity, the root of the seedling attaining a length of an inch in two or three days. Shortly after penetrating the soil, the main root puts out secondary branches, which make their way parallel to the surface. As they grow chiefly in woods or among herbage, they speedily encounter the roots of other plants, and on contact being made between one of these

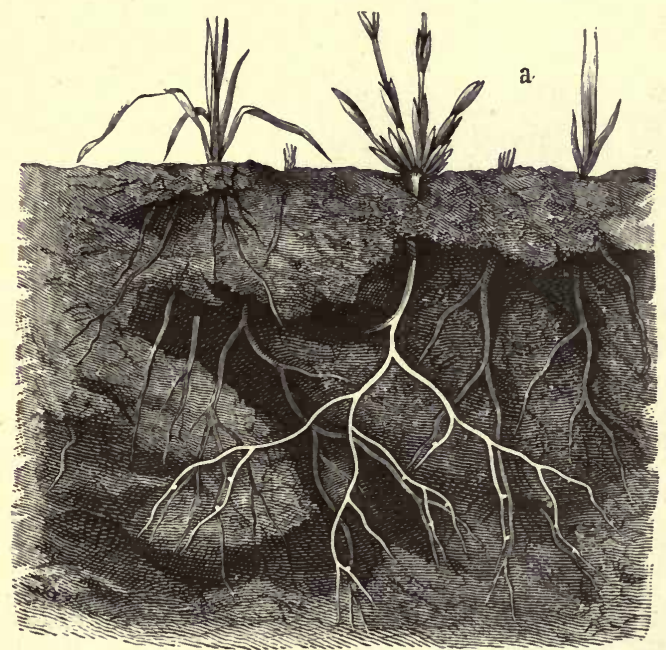

Fig. 103.-Thesium alpinum, SHOwING THE suckers oN THE ROOTS.

(After Kerner.)

root-branches and a root of a suitable host, a curious suckerlike body is developed at the point of contact (fig. 103). This is a kind of parenchymatous cushion, which partly surrounds the host, and from the inner side of its concavity certain absorption-cells grow out and penetrate into the former, pushing their way until they reach the centre of the invaded root (fig. 104). These absorbing organs are often erroneously spoken of as roots. They cannot properly be so called, as they are developed from the cortex of the rootlet, and not, as root-branches are, from the tissue of the pericycle. They are best spoken of as haustoria, a term which is purely physiological, and carries with it no anatomical significance. 
While the root is setting up this relationship with a host plant, the shoot of the seedling is growing normally. Its leaves and other sub-aerial parts are well developed and discharge their appropriate functions. The plants would not be recognised at all as in any way parasitic without an examination of the subterranean parts. They absorb certain nutritive materials from the roots on which they fix themselves, and generally destroy them. The damage is, however, local, and does not involve the death

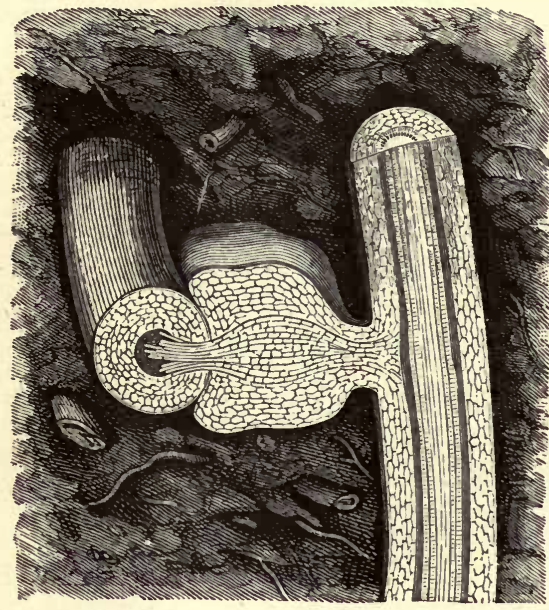

Fig. 104-Thesium alpinum. Piece of a ROOT WITH SUCKER IN SECTION. $\times 35$. (After Kerner.) of the host plant. In$b$ deed, many of these root-parasites do so little harm to the latter that an affected host is often not noticeably different in appearance from a neighbouring plant of the same species which is not attacked.

The perennial forms produce fewer suckers or haustoria which only function for one year. The rootlets usually bear only one sucker each, and when it has ceased to act as an absorbing organ it dies. The rootlet grows on, and in the next year develops a new sucker, and makes a fresh attachment.

Some of these root-parasites are also saprophytic in their habit, bearing, besides the suckers, absorbing hairs on their underground stems, which come into relationship with the humus of the soil.

There are many other plants which are parasitic upon roots, but they must be distinguished from those we have just discussed, on account of the greater degree of their 
parasitism. They include such forms as Lathrea and Orobanche, which are members of the British Flora. Lathræa has already been mentioned as a plant which draws proteid material from small animals which it captures and kills. It supplements this method of obtaining food by becoming parasitic on the roots of trees, to which its roots attach themselves by suckers, much in the same way as the semi-parasites already described. The host plant in this case is drawn upon for carbohydrates as well as proteids, as Lathræa possesses no chlorophyll.

Orobanche resembles Lathræa in exhibiting the same degree of parasitism. It shows certain differences of structure, and it does not attach itself exactly in the same way. It derives

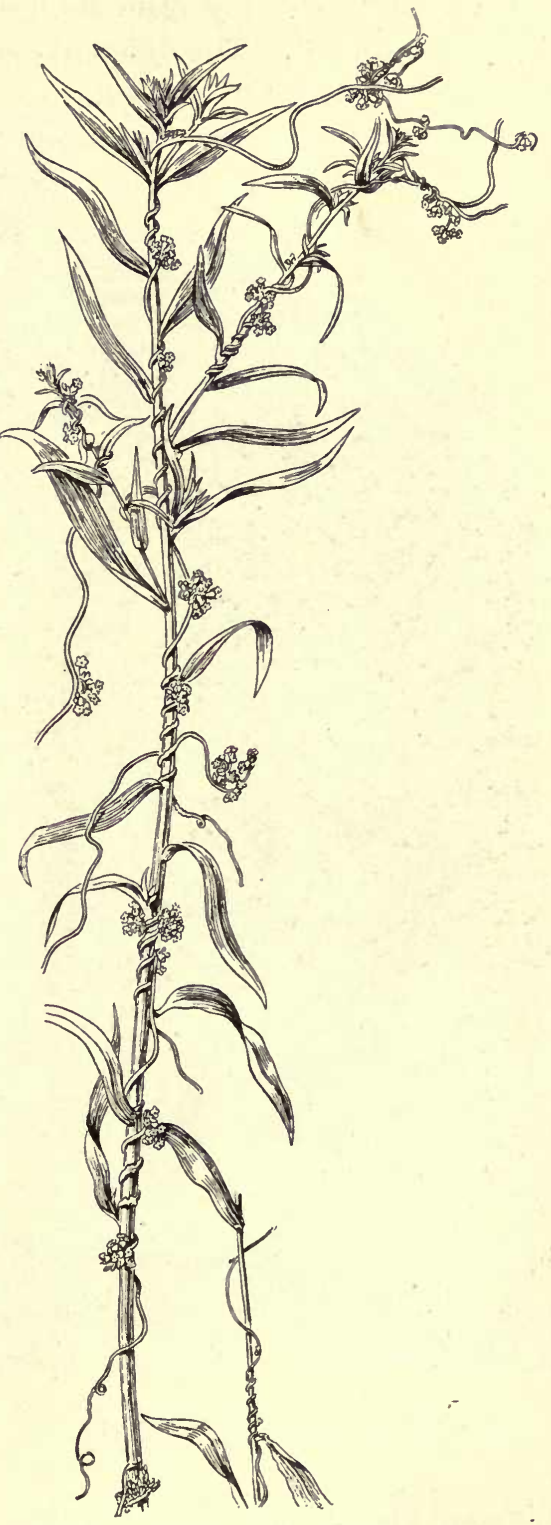

Fig. 105.-Plant of Melampyrum INFESTED wiтн Cuscuta. 
its nutriment entirely from its host, which is frequently a herbaceous plant. The different species of the genus infest

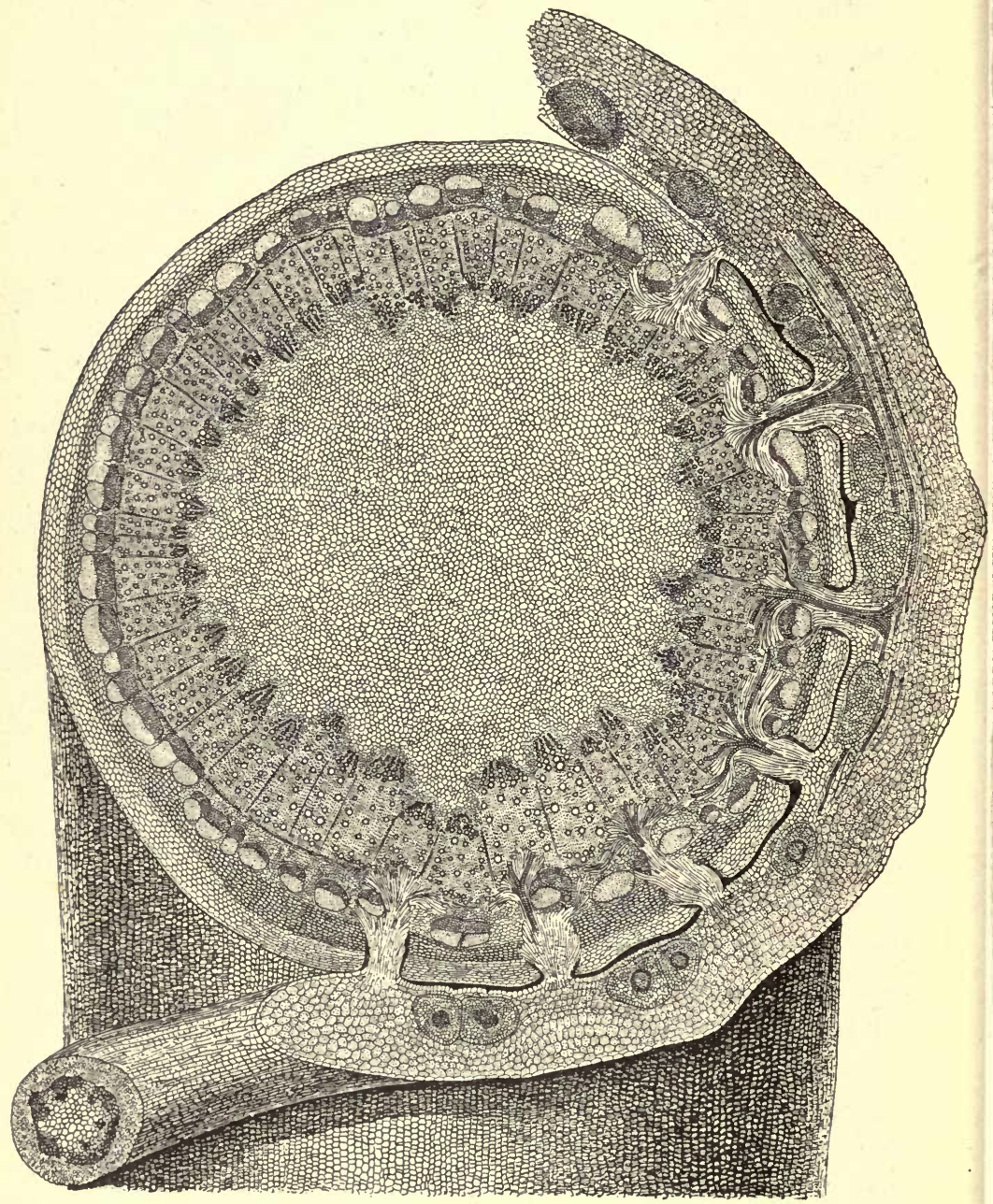

Fig. 106.-Section of Stem of Dicotyledonous Plant attacked by Haustoria of Cuscuta.

different plants, each having only one suitable host. None of the species are like Lathræa in being partially carnivorous. 
Some curious parasites which are met with in the tropies show a very peculiar method of attaching themselves to their host plant. They constitute the natural order Rafflesiacea. The embryo, after emerging from the seed, penetrates the cortex of its host, usually a root, though not always, and gradually forms a hollow cylinder surrounding its woody centre. This sheathing structure is composed of rows of cells, and in appearance resembles the mycelium of a fungus. Buds arise upon this investment, which eventually burst the cortex above them, and protrude through the host plant. These, in Rafflesia itself, develop a single flower which, in some eases, is of enormous size. The plant produces no outgrowths of any kind except the buds described. Other genera show some modification of this structure, but exhibit exactly similar physiological peculiarities.

Certain other parasites which resemble these in many respects differ in attacking only sub-aerial portions of their hosts. The most easily observed of these is the Dodder (Cuscuta), which often attacks the clover (fig. 105). The seed when germinating puts out an embryo which bears no cotyledons. Germination takes place on the ground, and the embryo grows to a length of about an inch. Its apex attaches itself to the ground, and the free portion moves round, describing a sort of spiral in the air. If it comes in contact with a suitable host, it twines round it after the fashion of a tendril, and numerous suckers are developed in rows at the points of contact. Haustoria spring from these suckers and penetrate the host, extending inwards till they reach the wood (fig. 106). The part below the attachment dies shortly after this relationship, has been established, and the parasite is left attached to the host. In its further growth it continues to twine around the latter, putting out numerous branches, which also form similar coils, so that the host is completely immeshed in the twining stems of the parasite. The latter bears no leaves and possesses no chlorophyll in any part, so that it derives 
all its food in fully elaborated form from the tissues of the host. Cuscuta produces numbers of flowers on its branches, and from them are developed fruits and seeds. The parasitism is complete, and the relationship frequently leads to

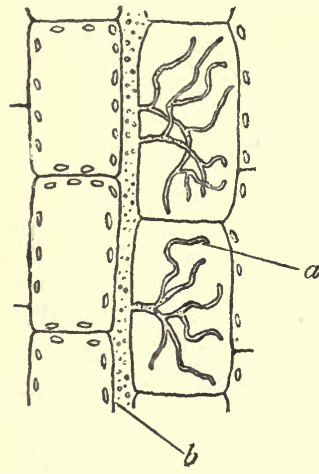

Fig. 107.-Cells of Potato Plant infested With Phytophthora.

$b$, hypha running between the cells and sending haustoria (a) into their interior. the death of the host which has been attacked.

Parasitic plants are very frequently met with among the Fungi and the Bacteria. The former penetrate the living cells of the plant they infest, or in a few cases ramify between them, sending haustoria into the interior of the cells between which the mycelium grows (fig. 107). They make use of the contents of the cells, destroying and absorbing the living substance as well as any formed materials which may be present. In many cases also they destroy the cell-walls, and utilise the carbohydrate materials of which the latter consist. Their ravages only cease with the death of the organism.

The power of living plants to assimilate the food manufactured by others is taken advantage of in the processes of grafting and budding. In these operations a slip of a particular plant is inserted into a wound made in another nearly related one, and the two are closely bound together. The graft or scion comes into such close connection with the stock that the food which is contained in the cells of the latter passes into the tissues of the graft, which thus receive their nourishment. After a longer or shorter time the two become so completely united that they live subsequently as a single organism, and the processes of carbohydrate and proteid construction proceed as in a normal plant. 


\section{CHAPTER XV}

TRANSLOCATION OF NUTRITIVE MATERIALS

We have so far traced the ways in which plants receive their food, and have examined the processes by which it is appropriated. In some cases, indeed in the vast majority of instances, it is constructed in the interior of the plant by certain of the protoplasts from simple inorganic materials which are absorbed from the environment. In green plants this construction extends to all the substances which can be termed food. In plants without a chlorophyll apparatus this construction is partial only, never going so far as the formation of carbohydrates, though, when these are supplied together with inorganic compounds of nitrogen, proteids and fats can be manufactured. In other cases the constructive processes are supplemented by the absorption of food in a suitable condition for nutritive purposes, while in others, again, the last method is the only one observable, all constructive power being absent.

There are other considerations, which must be briefly stated, which have a bearing upon this subject. The conditions of life of an ordinary green plant involve a great extension of the original constructive process. It has no definite and regular times at which it can take in a certain quantity of food, which are regulated partly by the needs of the organism and partly by the mysterious factor which we call appetite. Its absorptive processes are much more under the influence of natural phenomena, such as the degree of illumination, the amount of warmth, moisture, \&c., which it is receiving. Periods of intermission of irregular duration are caused by differences in these respects, 
even during an ordinary day, and still more by the alternation of day and night; in the case of perennial plants yet greater disturbances are caused by the succession of the seasons of the year, and the alterations these produce in the amount of foliage which the plant preserves; weather and its vicissitudes form a series of disturbing influences. We have thus the certainty of failure to survive in the struggle for existence unless the initial absorptive and constructive processes are supplemented by others, which in some way shall make the organism indifferent to these changes and intermissions of supply, and capable of carrying out true nutritive work, though the initial stages of such work are checked or suspended. In other words, suitable conditions for the construction of food being intermittent, the plant must accumulate a reserve store on which it can subsist during the periods, short or prolonged, when no such manufacture is possible.

We may view the matter from a slightly different standpoint, and yet come to the same conclusion. The processes of absorption in a plant depend, as we have seen, almost entirely upon physical conditions. Given a certain amount of carbon dioxide in the air, and a certain amount of water in the plant to which that air has access, the carbon dioxide will be dissolved according to the power of the water to dissolve it, or-putting it more technicallyaccording to its coefficient of solubility. In the presence of the chlorophyll apparatus, with the access of sunlight, the other subsequent changes which we have discussed lead to the continuation of the absorption of the gas. This is the case again with the root and its relations to the soil. The process of absorption of water with its dissolved substances will proceed as long as certain physical conditions obtain. Thus the plant is, on the whole, rather passive than active in the initial stages of its own feeding, exercising no inhibitory power, such as that which in an animal is attendant upon a failure or cessation of appetite.

These considerations lead us to the conclusion that when 
the absorption of food or food materials by a plant is proceeding, the probabilities are decidedly in favour of such an absorption being much greater than the immediate need for direct consumption. The constructive process, followed by the accumulation of its products, is certainly the leading one in the history of the different members of the vegetable kingdom. The increase of the framework which attends upon the multiplication of the protoplasts, which we commonly speak of as growth, proceeds for such long periods, moreover, that there is stored up in such a structure as a forest tree an enormous amount of material and of potential energy.

But this latter form of storage, devoted especially to the production and maintenance of a very large plant-body, differs materially from the accumulation of a quantity of food which is temporarily a surplus, but which is destined for subsequent consumption by the protoplasts. This is a feature of the life of all plants in varying degrees, whether they form a large plant-body or not. We must turn to examine this surplus production in more detail.

In an earlier chapter we alluded to the very marked division of labour which we can observe in such a community of protoplasts as form a large plant. We have since studied certain of the different processes which are carried on by particular tissues or collections of protoplasts, rendering them unable to perform other necessary duties. It is evident that to enable them to discharge their special functions they must be fed and nourished. It is equally clear that they are not living under conditions which enable them to construct food for themselves. We see that it is consequently necessary for food to be transported to them from the seat of its construction.

There is in every green plant a localised, though fairly widespread, region in which construction is taking place, and there are other equally well-defined regions which must be supplied with food transported from the seats of its manufacture. The cell or protoplast, which contains a 
portion of the chlorophyll apparatus, has thus not only to provide for its own nutrition, but to prepare a part of the nutritive material required by other protoplasts which are set apart for the discharge of other work.

But this is not all. We find, from a study of plants, that in almost all cases, so long as life lasts, growth is proceeding. This may result in a continuous increase in the dimensions of the plant-body, or may only lead to the replacement of parts which have a brief existence, and need to be renewed. This is the case, for instance, in forest trees that have attained their full dimensions. Growth in the vegetable organism is very definitely localised. Growth in length takes place at or near the apices of stems and roots; it has a definite though variable localisation in leaves of different kinds. Growth in thickness is confined to sheaths or bands of cells in different regions of the axis, such as the cambium, and the different phellogens met with in the cortex.

Growth and nutrition differ in another respect: the former is intermittent, the latter needs to be constant, though the intensity of the requirements may vary.

These considerations show us that there must exist in the plant a very complete mechanism by which the different food-stuffs can be circulated about its body. Each protoplast must be in receipt of a continuous, though perhaps small, supply of nutritive material; the demands of growth must be satisfied by the transport of considerable quantities of formative material to the growing regions. The intermittence of growth makes a further demand. Consider one among many places at which a large consumption of such formative material is proceeding : a stream is travelling there to supply the need. Suppose that some temporary check to the growth at that spot takes place. The stream will be diverted elsewhere by the demands of the other growing parts, and when the hindrance is removed and growth should again proceed, there will be no stream of constructive material, and much time will be lost 
before it can be restored. To prevent this there should be a storage of food close to the seat of its consumption, so that, with the awakening need, the required supply may be at hand.

This temporary storage of food must play an important part in the metabolism of an organ whose vital processes are subject to such numerous and often rapid checks as befall young stems, leaves, and roots. Still more necessary is it to the floral and fruiting organs during the time of their maturing.

We have seen again that plants set apart particular structures for periods of longer quiescence, especially in connection with their reproductive processes. Seeds may remain for several years without germinating, and they generally do so at least for months. The embryo in the seed is, however, ready to resume its growth as soon as all conditions are favourable. It is evident that it is in a practically helpless condition with regard to the manufacture of food, and it must depend upon a previously stored supply for the resumption of vital activity. The parent plant must, therefore, store quantities of its manufactured products in or about the embryo in the seed, stores with which it will itself have no further concern, but which will be altogether the property of the new organism. The same thing is seen to be the ease with tubers, bulbs, and other organs of vegetative propagation.

A condition intermediate between the two we have so far described is presented by the large fleshy roots and rhizomes of biennial and perennial plants. For an illustration we may consider an ordinary carrot or beetroot. Though these plants propagate themselves by the preparation of flowers, fruits, and seeds, they do not enter on this task during the first year of their lives. During this time they are in full foliage, and their constructive processes are at their best. They store in their roots a large amount of the food so prepared, and these towards the close of the first year's vegetation become enormously swollen by the 
development of succulent parenchyma. During the second year they have a much smaller foliar development, but each sends up its flowering stem. The constructive activity is much less than during the previous year; the root gradually dwindles as the fruit and seeds develop, the store deposited in the succulent parenchyma being applied to their formation and maturity.

Based upon considerations such as these, we may make a further classification of the nutritive substances which exist in the body of the plant. We can speak of those which are used in the cells where they are formed, and of those which are removed therefrom for the feeding of the other protoplasts. These, again, may be devoted to immediate use, or may be stored as reserve materials for deferred consumption. We can recognise in every plant some kinds which are suitable for transport from cell to cell, and others which are not able to pass through cell-walls, but must remain in the position in which they are formed. These two classes of circulating and stored food-stuffs have an intimate relationship to each other, and must be mutually interdependent, each being reinforced by the other according to the needs of the particular moment.

If now we turn from these general considerations to the course of the events that are normally taking place in the cells which contain the chloroplasts, we can form some definite idea of the course of the processes of construction of the carbohydrates and removal of the products. In such a cell we have seen that there is, during favourable conditions, a manufacture of sugar which is continuous and rapid. The cell itself needs a certain amount of such sugar for its own nutrition, but only a very small part of what is being made. The sap of its vacuole soon becomes saturated with the sugar, and if nothing further transpired the process of manufacture would stop. But the cell is in contact with others, in many of which, perhaps in all, a similar manufacture is taking place. The ordinary processes of diffusion tend to equalise the amounts in any contiguous 
cells, so that very soon the whole of the parenchyma of the constructive region is saturated with the sugar. This parenchyma abuts however on other cells which contain no chloroplasts, especially the sheaths and the bast of the fibro-vascular bundles. Diffusion of sugar into these takes place, and proceeds from cell to cell, especially among the delicate bast tissue, so that a stream of sugar is soon diffusing all along the bast. As a rule it does not penetrate very far beyond this tissue, owing largely to the anatomical arrangements of the parts and the great facility which the structure of the bast affords for this diffusion. So long as the manufacture goes on, therefore, there is an outflow of the manufactured carbohydrates from the region of its formation, the ultimate and even the temporary direction of the stream being determined by other factors which we shall consider later.

This removal of sugar from the leaf can be proved by several observations. We find but little of it in the mesophyll of the leaf, though we know it is being continually produced there. We find it fairly easily in the bast of the veins, and if a leaf is cut off from the stem while construction is going on, so that it cannot be transported away, it can very soon be detected in the mesophyll cells as well.

This, however, is not all. The process of diffusion is a slow one and does not serve to remove the sugar as fast as it is formed. The excessive formation of sugar would soon lead to such saturation of the sap as would at any rate temporarily inhibit its construction, were it not for another agency at work. The chloroplasts are endowed with another property than that so far described, which is now called into play. This is a peculiarity of the body of the plastid, and is quite independent of the colouring matter, being shared by other quite colourless plastids which occur in other parts of the plant. These structures have the power of converting sugar into starch, a power which we must examine more fully in a subsequent chapter. The transformation is apparently a process of secretion. 
Part of the sugar consequently gives rise to numerous minute grains of starch, which the plastid forms within itself and deposits in its own substance. This formation of a temporary store not only relieves the over-saturation of the sap in the cell, but supplies the need of the protoplasm when the formation of sugar from carbon dioxide and water is interrupted by the failure of the daylight. These minute granules are of very small dimensions, three or four of them being formed within each plastid. They have no apparent structure, but can be detected by treating the cell with a solution of iodine, which stains them blue. If a chloroplast so treated is examined with a high power of the microscope, it presents the

(9.9)

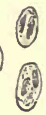

FIg. 108.-STARCH Grains IN THE BODIES OF CHLOROPLASTS. $\times \mathbf{2 5 0}$. appearance of fig. 108, the little grains of starch lying as blue specks in the green substance. They can be seen more distinctly if the leaf under examination is bleached by warming it in alcohol, which dissolves out the chlorophyll. A leaf so treated turns blue wherever the light has had access to it, not only showing the formation of the starch but allowing its exact locality to be determined with absolute precision. In fact this test may be applied to ascertain whether the chlorophyll apparatus of a part is at any time active, the deposition of the starch taking place within a few minutes of the commencement of carbohydrate construction. This rapidity of appearance led indeed to the old view that the construction of starch rather than sugar was the immediate object of the chlorophyll apparatus. The reasons we have given lead us preferably to the view that the starch is the expression of the superabundant supply, requiring that a certain portion shall be deposited in an insoluble form as a temporary reserve material, to allow the process of carbohydrate construction to proceed without intermission so long as the conditions are favourable. At the same time we cannot but notice that the appearance of starch in the chloroplasts is so rapid when the conditions of carbo. 
hydrate formation are realised, that it may be relied on as a test for the absorption of carbon dioxide by the tissue in which it appears.

In connection with the manufacture and fate of carbohydrates, we can now see that they may be met with in two different conditions: the one suitable for retention in the cell and hence capable of functioning as reserve, but not immediately nutritive, material; the other capable of diffusion, and hence serving as a translocatory form, or one in which it can pass from cell to cell, remaining all the time in a suitable condition to minister to the nutrition of any protoplast which it reaches.

The same considerations affect the manufacture, transport, and storage of proteids. We have already seen reason to believe that these, like the carbohydrates, are in the first instance constructed in the leaves, if not by the chloroplasts. Our information about them is, however, very incomplete; we do not know even what form of proteid is first formed, nor which kind is needed for assimilation by the protoplasm. Probably it is a soluble and diffusible form, such as a peptone or a proteose, but our only reason for thinking so is that such properties characterise the travelling forms of carbohydrates. We can, however, readily believe in the construction being greatly in excess of the immediate need of the cell, and hence in the chain of events being similar to that in which the carbohydrates are concerned.

The different properties of the two classes of bodies involve, however, some differences in their behaviour, and we can therefore expect similarity only and not identity. The diffusibility of peptone even is very greatly below that of sugar; and we can hardly suppose therefore that peptone is the translocatory form of proteid in the plant. It seems on the whole more probable that nitrogenous plastic material is transported in the form of some amido-acid such as asparagin, and that the latter is subsequently worked up into proteid at the place where it is 
assimilated by the living substance. This view is supported by observations made upon the utilisation of the reserve stores of proteids found in seeds, which have been found to give rise to similar amido-acids before being transported from the site of storage. To this point we shall return in a subsequent chapter.

We cannot say either in what form proteids are temporarily stored in the cells of their first formation. Probably, like starch, they are made indiffusible and so retained in the cell. But whether they are thrown into a solid form we do not know. If so, they are amorphous and are hidden away in the substance of the protoplasm. They may be kept in solution in the sap which saturates it. Different forms of globulin and albumin have been found in the cells in different regions. It is possible again that the manufacture of proteid may be only so great as to provide for the needs of the cells in which such formation takes place, together with the amount that can diffuse during such manufacture, so that there may be no occasion for a temporary storage there.

The translocation of food has no very determinate direction. On leaving the cells which are the seats of its formation, its path is dependent on physical processes taking place in different parts of the plant. We can study it most simply by taking a special case, which as before may conveniently be that of sugar. We have seen that it passes from cell to cell under the influence of osmotic diffusion--that is, that when two contiguous cells contain sap of different degrees of concentration, an interchange of the liquids takes place till the sap of both cells becomes uniform. The fibro-vascular bundles, the bast of which we have seen forms its principal path, extend in complete continuity throughout the plant, so that any travelling compound can be transported from the leaves to the growing points of the stem and root. So long as it is being used by the protoplasm in these regions, the sap of the cells of the tissue there, which are using it in the construction of 
living substance, becomes continually weaker in that constituent, and hence more and more diffuses into them as the construction goes on. The utilisation or consumption of the sugar so acts as an attracting force, directing the stream to the points where it is required. The same principle applies to the consideration of the deposition of the large reserves of carbohydrates in seeds, tubers, or other organs. The withdrawal of it from the travelling stream which is the result of the formation of the quantities of starch or cellulose which those reservoirs contain, leads to fresh quantities being transported slowly but continuously to those cells, owing to the physical process of diffusion already described. The stream passes in fact in both cases exactly in proportion as the consumption takes place, whether the consumption takes the form of construction of new protoplasm, or the transformation of the travelling carbohydrates into the insoluble resting forms.

This passage of the sugar about the plant need not demand a coincident transport of water, so that the old idea that there was an actual stream of fluid along the bast, or in the old nomenclature a stream of descending sap, need not have any foundation in fact. The principle of diffusion alone will suffice to explain the passage of the sugar. Disturbances of the fluid contents of the cells do no doubt occur, as osmosis is continually taking place in both directions between the contiguous cells. A definite flow of water need not, however, coincide in either magnitude or direction with the passage of the stream of sugar.

The translocation of the sugar, we see, thus varies in direction and in magnitude according to the varying processes which are from time to time proceeding. As the variations in these processes, particularly those of growth and nutrition, are often sudden and considerable, we find the translocation is generally accompanied by changes of the carbohydrate from the labile to the storage forms, and vice versa. It is very usual to find temporary accumulations 
of starch in the neighbourhood of a growing region. Grains of starch are of frequent occurrence in different parts of the bast, and particularly in the bundle-sheaths of certain regions. The explanation of their appearance there is simple; they are generally indications of such an interference with the supply and the demand as we have described. A checking of the demand by a cessation of the vigour of growth or nutrition is attended by an overaccumulation of the sugar, which is speedily changed into a storage form instead of being removed by the slow process of diffusion.

The transport of proteids follows the same course; the amido-acids are the travelling forms, and are conducted by the same forces to the growing points, or to reservoirs where accumulation of proteids takes place. Their deposition in storage forms along the pathway can also be detected, though these are not so widespread as those of carbohydrates. They can be observed generally in the sievetubes of the bast, which contain a curious modification of protoplasm in which proteid as such is present. It was formerly held that the sieve-tubes conduct proteid as such along the vascular bundles. Though there is not a very great improbability that such bodies may pass from cell to cell of the sieve-tube, on account of the protoplasmic or quasi-protoplasmic threads which extend throughout the openings of the sieve-plates, yet this method of transport must be necessarily very slow and subject to much hindrance. It seems more probable that the proteids in these vessels are constructed there from the amido-acids which reach them, and are to be regarded as temporary stores, like the starch grains already alluded to as being formed in different parts of the translocatory tract.

We have spoken of the bast as forming the pathway of the translocation of nutritive material or of the different food-stuffs which have been manufactured. The process by which they travel, we have seen, is mainly one of diffusion through the cell-walls, the latter being saturated with the 
cell-sap. It must not be forgotten, however, that the cellmembranes are all perforated by very delicate strands of protoplasm which extend from one protoplast to another. There is here a further means of transport which no doubt facilitates the passage.

We may find proofs that the pathway lies along the bast by experiments carried out on plants in which translocation is actively proceeding. If we cut a branch from a vigorously growing tree and remove from near its free end a ring of tissue extending inwards through the bark and cortex to the cambium, and then place all the lower part in water or moist earth, very marked effects follow. After some time, perhaps a few weeks, adventitious roots will be put out from near its end. Those which arise below the missing ring will be few and of small size ; those from above this region will be numerous and strong, and will continue to elongate. Any buds that may be on the part below the ring will not develop, while those above it will grow normally or even more freely than on an uninjured branch. The tissue immediately above the ring will become to some extent hypertrophied and show a decided swelling.

The continuance of the growth shows that the water supply has not been cut off, but the different behaviour of the parts above and below the excised tissue tells us that the supply of nutritive material to the latter region has been interfered with, and the buds and adventitious roots it bears gradually perish of inanition. The passage of any food or nutritive material across the ring has become impossible.

If a similar incision is made into another branch but is not carried so far inwards-if, that is, the ring of tissue removed consists only of the structures external to the bast-these appearances do not accompany or follow the wound. Evidently in this case the translocation path has not been interfered with. We may safely conclude therefore that the transport of elaborated products, chiefly food, is the principal function of the bast. 
To a certain extent the cortex of the plant shares the translocatory function. The contents of the cells include a certain amount of carbohydrate matter, but their reaction is distinctly acid, and this region is probably concerned much more definitely with the transport of vegetable acids, so far as it takes part in translocation at all. At the same time it is impossible to localise the transport of food exclusively in the bast.

Other parenchymatous tissues are sometimes the region of transport. In many germinating seeds there is a transference of large quantities of nutritive substance across the endosperm to the embryo, and in young seedlings similar transport takes place through both cortex and pith.

The vessels of the wood, which we have seen are the paths of the transpiration current, are probably not concerned normally in the translocation of manufactured products, though exceptionally they may contain certain amounts of proteids, amido-acids, \&c., in solution. Their function in this respect is, however, unimportant, and the presence of such bodies in them is mainly accidental.

It is doubtful how far the laticiferous systems which are present in many plants may be regarded as channels for translocation. No doubt latex contains many nutritive products, both nitrogenous and non-nitrogenous, but there is reason to think they are to be referred to the storage ,rather than to the transporting system. 


\section{CHAPTEP XVI}

THE STORAGE OF RESERVE MATERIALS

WE have seen that the large amount of food which is continually being manufactured by a normal green plant is very greatly in excess of its immediate requirements, and that there is a very extensive system of storage in such an organism, by the aid of which it is enabled to survive periods, often of some duration, in which the manufacturing processes are entirely suspended. We have considered further the mechanisms of transport, by which the various nutritive substances are transferred from the seats of their manufacture to the places in which they are laid up for longer or shorter periods.

The questions of transport and of storage are very intimately connected. Food once formed is not always moved at once to some place where, after a period of storage, it will be ultimately consumed. It is often transferred more than once, and may occupy several places in succession as the demand for it varies. Indeed, we may regard the surplus manufactured food, that is the quantity which is in excess of the immediate requirements of the constructive cells, as a single store, part of which is travelling about the plant, and part of which is from time to time withdrawn from the travelling stream and laid down in particular cells, either to rejoin the travelling current after a longer or shorter time, or to be separated from the parent plant, and serve as a starting point for the growth and nutrition of its offspring.

A very little consideration will show us that the forms in which the various food-stuffs are packed away in the 
storage reservoirs must be materially different from those in which they travel. We have.already seen that one of the conditions of the continuous formation of any one of them is the removal of it from the seat of its construction as soon as its amount exceeds a certain limit. If this is not secured, the sap of the constructing cells becomes saturated with the body in question, and then no more is made. The removal is dependent upon the deposition of the substance from the sap in some way which lessens the concentration of its solution in the latter. We find accordingly that the bulkier reserve materials are very frequently deposited in solid forms, sometimes amorphous, sometimes granular, and sometimes crystalline. Other cases are known as well, in which they remain in solution in the sap of particular cells, but in these cases they are retained in such cells through the difficulty or impossibility of diffusing through the plasmatic membranes of the cytoplasm. They are generally formed inside these cells from some particular constituent of the travelling stream, much as are those which become insoluble, and once formed, they are unable to pass out of the vacuole.

In considering the forms which the various reserve food materials assume in the reservoirs they occupy, we must then remember that they are not a simple accumulation of food pabulum in the form in which it is of immediate use. Granted that the plant in the first instance forms certain materials on which its living substance draws at the place where it is originally constructed, then, so long as the immediate needs are in excess of the amount prepared, there is no alteration in such materials; they are at once utilised by the living substance in the processes of nutrition and growth. But as soon as the supply exceeds the immediate demand, the surplus is not simply retained unchanged in the cell, nor does it overfiow unchanged to contiguous cells where demand exceeds supply, or where provision is made for storage. The storage forms, whether retained in the cells of construction or transferred to others, are different 
from and more complex than the originally prepared ones, and further energy has to be expended on them, either where they are made, or in the place of storage itself.

As we shall see later, when they come to be utilised in after time, a converse process takes place, which is comparable to the digestion which they undergo when, as so frequently happens, they are eaten by an animal. The surplus food of the plant exists thus in two conditions, the one suitable for travelling, the other for storage. The former is characterised by solubility and diffusibility, the latter generally by insolubility in the cell-sap, and always by an absence of the power to pass through the plasmatic membranes. The former usually consists of such substances as can at once be assimilated by the living material; the latter does not, but requires the digestive changes to take place before it becomes so.

The places where these reserve materials are deposited are more numerous than we are apt to suppose. Parts of the plant or definite structures, which ultimately serve as reproductive organs, readily occur to us as reservoirs which are adapted for a somewhat prolonged storage. Seeds, tubers, fleshy roots and branches, bulbs, corms, and rhizomes are instances of these, and in the short-lived plants which we group together roughly as herbaceous in their habit these are necessarily the most important reservoirs. But it is different with trees and shrubs which live for many years, and which do not form fleshy receptacles. We have in these forms stout stems or trunks, with numerous branches; large woody roots which continue to grow year after year, keeping pace with the parts aboveground. Though the primary use of these members is not to store food products, yet they have work of this kind to do. We have seen that in the cells which are the original seats of carbohydrate construction there is almost always an excess of such matter formed, which is partly deposited in the chloroplasts in the form of small granules of starch. These afford us an instance of a very 
transitory store, for the starch deposited there during exposure to sunlight is removed almost as soon as darkness supervenes. A plant which has been vigorously forming starch in its chloroplasts during a summer's day, will show that at evening there is a considerable amount accumulated there; if the leaves are examined again early next morning, the starch will be found to have disappeared. This is not brought about by its having been used in the metabolism of the cells during the night, for if the path of removal is obliterated, as it may be by severing the petiole in the evening, the leaf is found as full as ever in the morning. If a plant whose chloroplasts are charged with starch grains is kept for a time in an atmosphere free from carbon dioxide, the starch is gradually removed, whether it is kept in light or darkness, so that the removal of the starch can, and probably does, take place continuously, though it cannot be easily detected so long as construction is proceeding simultaneously.

The deposition of food in such other reservoirs in trees and shrubs as are not connected with the reproduction of the plant is generally of a transitory character, though not so markedly so as in the case of the leaves. These temporary storage places are found very widely distributed, and the reason for their occurrence is in each case traceable with comparative ease. A tree that has a trunk and a root which are growing in thickness is in need of a constant rather than an intermittent supply of food placed near the actively growing regions. The growth in thickness of such a trunk or root is brought about by the activity of a layer of delicate living cells, which are constantly dividing to produce new wood and new bast, and which appear quite early as a ring of cambium on the exterior of the woody mass (fig. 109, b). The new cells need a constant supply of nutritive material, at the expense of which they develop into the peculiar elements of wood and bast respectively. The cambium too is in continuous need of food, or it is perforce obliged to cease dividing, and so the 
growth in thickness of the trunk or root is stopped. Celldivision is indeed the result of cell-growth. When a cell of the cambium has attained its full size it divides into two, each of which then grows to its appropriate adult dimensions; some divide again, like those from which they sprang; others become transformed into wood or bast cells. In either case an immediate supply of food is needed, and from the condition of things this must be near at hand. The stream from the leaves is intermittent, and hence it is important that a certain reserve

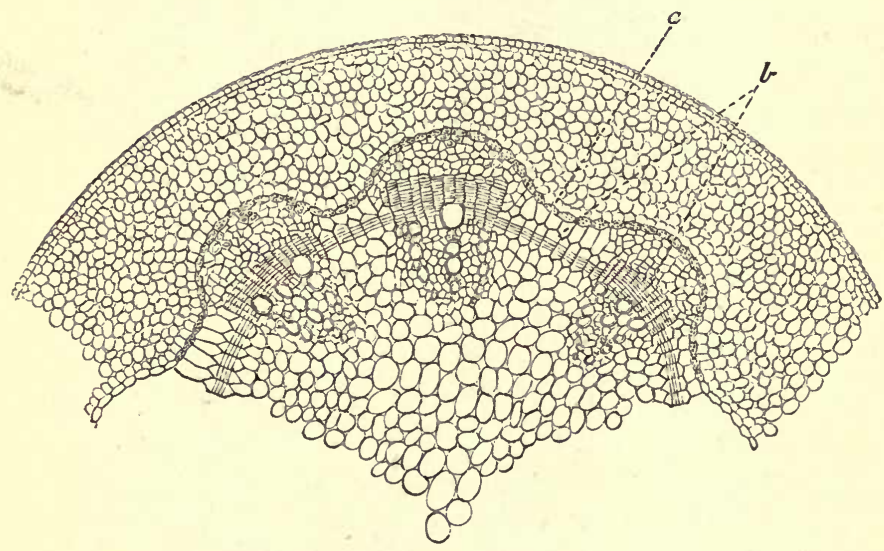

Fig. 109.-SEction of PART of Stem of Ricinus communis.

$a$, starch sheath ; at the extremities of the figure its cells are represented as empty; $b$, cambium layer.

shall be deposited not far from the growing cells, so that a slow continuous supply may be available. We find such reserves laid down near the cambium, either in the cells of definite sheaths surrounding the whole ring of new tissue (fig. 109, a), or in the spaces called medullary rays, which are found between the separate masses of wood and bast, these rays (fig. 110) being composed of cells which differ in shape from the typical forms of both wood and bast cells.

In stems of smaller girth which have not developed much wood, we find stores of food laid up in the region 
just underneath the surface, which constitutes what is called the cortex, and which gives place later on to the complex formation that is familiar to us under the name of bark.

The formation of the successive rings of cork deeper and deeper in the cortex, which ultimately constitutelthe bark, is attended by the same need of a continuous instead

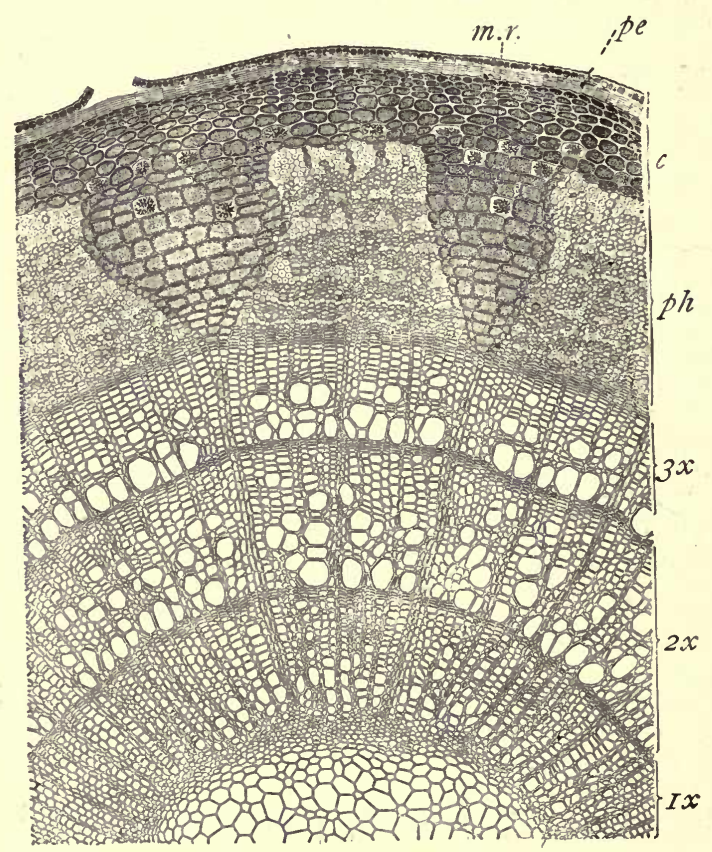

Fig. 110.-Section of Three-year-old Stem of Tilia, showing the MEDULlaRY RAYS RUNiNg THROUGH THE WOOD. $\times 50$. (After Kny.)

of an intermittent supply of food. We find, therefore, during the process of the construction of the bark, similar provision of food-containing tissue, which is situated near the cork layers. In some cases it takes the form of regular sheaths; in others the food is irregularly distributed through the cortex, which is the seat of the appearance of the formative layers of the cork. 
As the trunk grows older similar stores of food may be detected deeper in the wood. These generally occur in the medullary rays, either those which are the continuations of the primary ones, or others which are formed apparently for the purpose under discussion. These stores are especially for the nutrition of the more deeply placed wood-cells, when the ordinary constructive processes are in abeyance, as in the winter-time.

Transitory stores may also be detected near the growing points of the axis. These are due to intermission of growth and a consequent sudden cessation of the demand upon the translocation stream. The latter, instead of being diverted at once from the region to which it had been travelling, deposits in a suitably stable form the food which would have been consumed had not the check in the demand occurred. The supply is consequently ready to hand as soon as growth sets in again.

Deposits of reserve materials can be observed near the extremities of twigs as winter approaches. The output of the young leaves in the spring is greatly facilitated by the occurrence of such temporary storage. It is possible by appropriate pruning to influence to a considerable extent the locality and the extent of such deposition. This is of very common occurrence in horticulture, the nature of the pruning having in this way a very considerable influence upon the development of floral or foliage shoots.

Transitory deposits of food take place also in the floral organs. In many flowers which have long succulent styles, which must be perforated by the pollen tubes on their way to the ovules, there may be observed very frequently a deposition of food in the tissue of the style at the time when the germination of the pollen grain takes place upon the stigma. The food is then usually stored in the parenchymatous tissue which surrounds the vascular bundles of the organ.

Many of these reservoirs show by their structure that 
they are only intended to compensate for regular or accidental intermittence in the translocatory stream to the parts in question. The food is thrown down in the ordinary parenchymatous cells or in the sheaths of the conducting tissue, and no special arrangements are made to receive it. It is often of accidental occurrence-deposited suddenly and gradually or rapidly removed. Such deposition and reabsorption form, indeed, one of the features of the transporting mechanisms.

We may now pass to the consideration of the forms in which the different foods present themselves in these reservoirs of storage. It is not surprising that we find here a great deal of variety, even in any particular class of food. The more prolonged the stay in the reservoir, the more complex usually is the structure which the nutritive substance assumes.

We may deal in the first instance with the stores of carbohydrates. We have already noticed that in the great majority of cases these take the form of starch. In the chloroplasts in the leaf-cells the starch grains are laid down as minute bodies, showing hardly

(3)

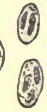

Fig. 111.-STARCH

Grains IN THE Bodies of ChloROPLASTS. $\times 250$. any trace of structure and crowded together in the substance of the plastid till they are almost in contact with each other (fig. 111). The deposition is due to the protoplasm or stroma of the plastid, and does not depend in any way upon the colouring matter, the presence of the latter influencing only the other function of the chloroplast, the synthesis of sugar, as we have already seen in a previous chapter. The process is thus one of true secretion, and the deposition of the starch originating at several centres in the plastid, several granules are coincidently formed. The number, however, is not constant.

In the more permanent reservoirs of starch it usually happens that the cells are so charged with the grains that they appear to contain nothing else. Fig. 112 shows a 
cell taken from the interior of a potato tuber. These grains of starch are much larger than those which occur in the chloroplasts of the leaf, and they have a complicated structure. Most of them are irregularly oval in shape, and their surfaces are marked by nearly concentric lines of striation, dividing them apparently into layers. The centre of these layers is not usually the geometrical centre of the grain, but lies near the small end, and the rings or layers are much narrower at that end than at the other (fig. 113).

In most cases the deposition of starch in these and similar cells is brought about by the agency of small protoplasmic corpuscles, which closely resemble the chloroplasts, except that they are colourless. They are known for this reason as leucoplasts; like the chloroplasts they

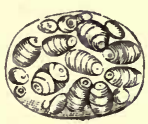

Fig. 112-Cell of Potato containing Starch Grains.

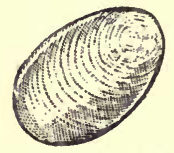

Fig. 113.-Starch Grain of Potato.

occur in considerable numbers in each cell, being situated usually near the nucleus. Their relationship to chloroplasts is shown by the fact that they turn green when they are exposed for a considerable time to light.

The leucoplasts behave very much like the chloroplasts. When a solution of sugar reaches the cell in which they lie, they absorb it as the chloroplasts do the excess of sugar manufactured in the cells of the leaf. They then secrete starch, which is at once deposited in their substance. If the point of deposition is the centre of the leucoplast, successive shells of starch are deposited concentrically upon the first-formed portion, and a symmetrical grain is produced which ultimately attains a relatively considerable size. It remains, however, surrounded by the leucoplast, which gradually becomes much stretched until there is 
merely a thin film of it surrounding the striated grain. It can frequently only be detected by delicate staining as the starch grain grows. If the point of deposition is near the side of the leucoplast, as is generally the case, the successive shells of starch are not of equal width, but are wider on the side of the grain which is in relation with the greater bulk of the plastid. The amount deposited on any part of the first-formed portion is proportional to the thickness of the plastid in contact with that part. An eccentric shape, often approximating to that of an oystershell, is consequently arrived at. Even the most eccentric grains can be shown by delicate staining to be covered entirely by the leucoplast, even the small free end which

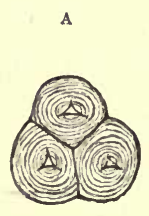

Fig. 114.-A, Compound, B, Semicompound Starch Grains From Potato.

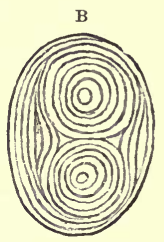

appears to protrude from the latter being clothed by a thin film of its substance.

Some grains often found in the potato are not so simple in their structure. These are represented in fig. 114, $\mathrm{A}$ and $\mathrm{B}$. The former arise by two or more grains originating in the interior of a leucoplast; as each grows by deposition of new layers they become closely pressed together, and constitute a compound grain. Fig. 114, B, shows what is often called a semi-compound grain. In such a formation the leucoplast commences deposition at two points, one towards each side. As the starch is deposited round each, the concentric grains come into contact, and the bulk of the leucoplast is reduced to a shell surrounding the mass. Its subsequent continued activity then forms new sheaths overlying the whole. The leucoplast, as in the first case, is gradually used up by its own activity, and it is finally reduced to a film of extreme tenuity, which surrounds the whole grain.

A very curious starch grain occurs in the latex of certain species of Euphorbia, having the appearance of a dumb-bell (fig. 115). This also is formed by a leucoplast; 
the latter is an elongated structure, and at first forms a rod of starch along its axis. As the deposition proceeds the leucoplast becomes very much stretched longitudinally, till its centre is reduced to a thin film round the rod of starch, while what is left of its substance is accumulated at the two ends. The further activity of these portions results in the development of the two heads of the dumbbell, the thin film connecting them ceasing to deposit any

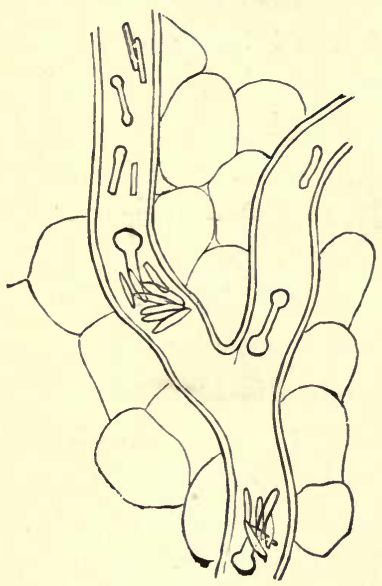

Fig. 115.-Laticiferous Cell FROM Euphorbia, CONTAINING DUMB - BELL - SHAPED STARCH GRAINS. starch along the centre of the rod.

It is not very easy to see the leucoplasts in the potato ; they can be detected,

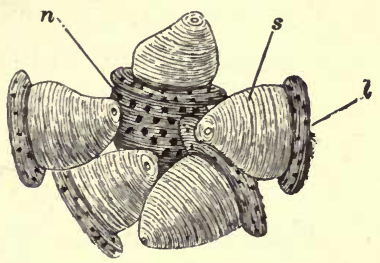

FIG. 116.-Group OF ROD-LIKE LEUCOPLASTS, $l$, EACH BEARING A STARCH GRAIN, $s$, COLLECTED ROUND THE NUCLEUS, $n$, OF A CELL OF THE PSEUDO-BULB OF AN ORCHW (Phajus grandifolius,$\times 500$ (After Schimper.)

however, more easily in other plants. Fig. 116 shows a group of them forming starch grains in a cell in one of the orchids. The greater bulk of each lies on the outside of the grain; they are disc-like in shape and not round as in the potato.

In the temporary reservoirs which we have already noticed, such as pollen grains and tubes, the sheaths of cells in various regions of the stem, the tissue of the style of the lily, \&c., the deposition of starch is not caused by leucoplasts but by the general protoplasm of the cell. In these cases immense numbers of very small grains, hardly larger 
than mere specks, make their appearance, while the highest powers of the microscope fail to enable an observer to detect the presence of any form of plastid before or during the deposition. Instead, the minute granules can be seen to arise in a homogeneous transparent hyaline protoplasm. The same phenomenon occurs in connection with the deposition of starch grains in the cells of young developing embryos, in the early stages of the formation of the seed. The protoplasm of the cells may be seen to have the form of a coarse network with many small meshes, which are empty spaces or contain only cell-sap. There is no leucoplast inside them, nor anything comparable to one. The starch grains originate in these meshes at some point in contact with the protoplasm and gradually increase in size till they fill them. In some cases simple, in others compound, grains of starch are thus developed.

In a large number of the Fungi which store up carbohydrate reserve materials, these take the form of glycogen. This is a substance which presents a somewhat close resemblance to starch, being readily converted into sugar in a manner almost, if not quite, identical with that which is characteristic of starch. It is coloured brown by iodine. It is usually deposited in amorphous form in the interior of the fungal hyphæ, or of particular cells of them. In a few cases there are definite granules, which to a certain extent resemble grains of starch, and which have been stated to originate in certain corpuscular bodies resembling leucoplasts. In most cases the deposition appears to be effected by the protoplasm.

Another carbohydrate which shows a certain resemblance to starch, though perhaps not a very close one, is inulin. The distribution of this material is much more limited than that of starch, but it is known to occur in several groups of plants, being conspicuous in many of the Compositce among the Dicotyledons, and in several species of the Liliacece, Amaryllidacece, and other allied orders among the Monocotyledons. Like starch and glycogen, it 
is capable of transformation into a sugar, though not the same sugar as in the other cases. It exists, in the plants mentioned, in solution in the cell-sap, but it can readily be made to crystallise out or to be precipitated in an amorphous condition by the application of alcohol (fig. 117).

We find many instances of the occurrence of various sugars as reserve materials. Cane-sugar is present in large quantities in the succulent parenchyma of the roots of the Beet and the Mangel-wurzel, and of the stems of the Sugarcane; grape-sugar is found in the leaves of the bulbs of the Onion and allied plants; small quantities of raffinose

A
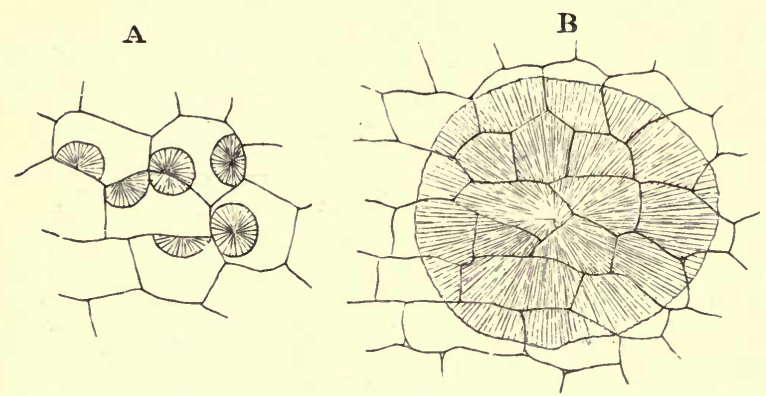

Fig. 117.-Sphero-crystals of InUlin from the Artichoke.

A, small crystals in the interior of cells treated with alcohol ; $\mathrm{B}$, large crystals extending through many cells.

are met with in the grains of barley and other cereals. These are all present in solution in the cell-sap, as has previously been mentioned.

In many cases carbohydrate reserve materials are found to take the form of considerable thickening of the cellwalls. That these are really deposited in seeds with a view to subsequent utilisation is evident from a study of the endosperm of many palms, the cells of which consist of little else; the walls are so thick that the cavities are almost obliterated, and the small space that is left between the thickened walls contains apparently nothing but 'a small amount of protoplasm with which some amorphous proteid matter is mixed. Even the unthickened cell-walls 
of most seeds must be looked upon as reserve food material, as they are used up in nourishing the embryo during the early stages of germination.

It is necessary, however, to mention that thickened cell-walls must not always be regarded as stores of food. In thickened sclerenchymatous tissue and in ordinary woodcells the deposit must be looked upon as a permanent strengthening of the skeleton of the plant.

These thickened cell-walls are not composed always of true cellulose. Our knowledge of their composition is not

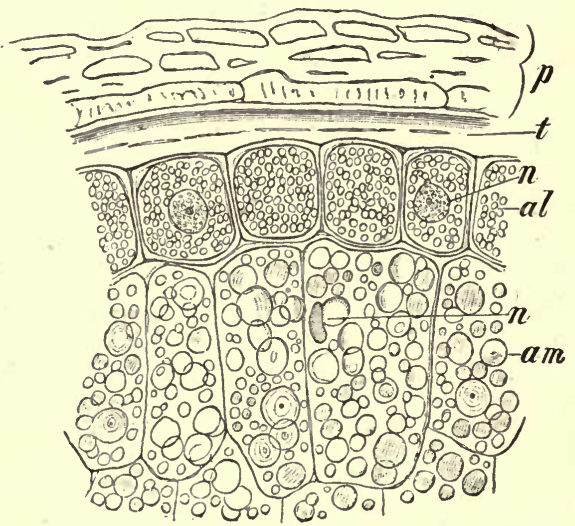

Fig. 118.-Section through external region of Grain of Barley.

$p$, pericarp of fruit; $t$, testa of seed; $a l$, layer of cells containing aleurone grains; am, cells of endosperm; $n$, nucleus. (After Strasburger.)

at all complete, but it extends so far as to show that both cellulose and pectic compounds may be present and in very different proportions in different cases. Layers of mucilage also are of frequent occurrence.

Nitrogenous material, like carbohydrate, is stored up in various places and in different forms. By far the commonest condition is that of some description of proteid. The most abundant deposits are found in seeds, in the cells of which they usually occur in the form of granules of varying sizes and often of complex composition. In certain cases, as in fleshy roots, the proteid may be dispersed in amorphous form in the substance of the protoplasm. 
When proteid is stored in the condition of granules these are known as aleurone grains. Like starch grains they may be deposited all through the substance of the seed, or they may occupy definite layers, as they do in the cereal grasses (fig. 118). They occur sometimes in the same cells as do starch grains, as in the pea or bean (fig. 119). In other cases they are found associated with a quantity of oil, as in the seed of the castor-oil plant.

An instance of the occurrence of aleurone grains of some size but yet of fairly simple composition is afforded by the Lupin, one of the Leguminose. This is of interest

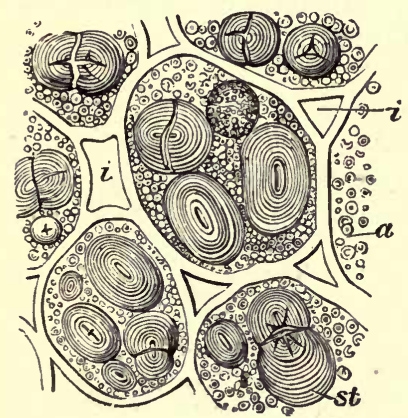

Fig. 119.-CeLLS OF EMBRyo of Pea. (After Sachs.) $a$, aleurone grains; st, starch grains.

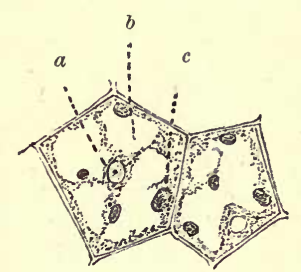

Fig. 120.-Cells of Seed of Lupinus, SHOWING COMMENCING FORMATION OF ALEURONE GRAINS. (After Rendle.)

$a$, nucleus; $b$, vacuole ; $c$, originating aleurone grain.

especially because the origin of the grain can be observed and its development traced. In this seed the aleurone grains begin to be formed at a very early period of the development, just as the growth of the embryo is sufficiently advanced to swell out the seed-coat. The cells of the embryo at that period show the protoplasm not sufficient in amount to fill each cell, so that a number of spaces or vacuoles occur, filled with sap. At certain places small projections from the protoplasm may be noticed which are of spherical or ovoid shape (fig. 120,c); these gradually increase in size, growing inwards into the protoplasm as well as outwards into the vacuole, till they can be seen to be in the form of grains embedded in the protoplasm, which 
in consequence of their development assumes the appearance of a coarse network. As this process continues, the original grains growing in size, and new ones being constantly formed, the original vacuoles become obliterated and the cell swollen out by its own deposits (fig. 121). While this mechanical process is going on chemical changes also take place in the material secreted. The protoplasm forms proteid originally at the expense of the amido-acids, sugars, \&c., brought down to the cell, but the variety originally constructed is not necessarily the same as that subsequently stored. At first the grains are not soluble in either 10 per cent., or saturated, solutions of common salt. Later on they can be dissolved by both of these fluids.

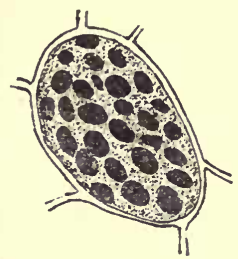

Fig. 121.-CELL OF RIPE SEED OF Lupinus, FILLED WITH ALEURONE GRAINS.

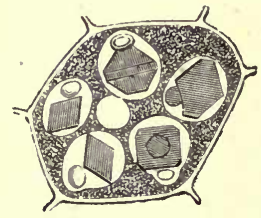

Fig. 122.-Cell of Ricivus Seed, CONTAINING FIVE ALEURONE GRAINS.

The deposition of aleurone grains in the cell is thus, like that of starch, a process of secretion carried out by the protoplasm : a process, that is, of manufacture of the grain by the latter, after it has been supplied with less highly organised material. It is so constructed by the intervention of the protoplasm itself, the grain growing at the apparent expense of the substance of the latter.

There is no doubt that the amorphous deposits of proteids in the cells of fleshy roots and stems are due to a similar process of secretion.

In many seeds, among which may be mentioned those of the Castor-oil plant and the Brazil nut, the aleurone grains possess a more complicated structure. Fig. 122 shows a section of one of the cells of a seed of the castor- 
oil plant in which some of them are lying. The figure represents the cell after treatment with alcohol, and subsequently with water. The alcohol removes the oil with which the cells are filled, and which obscures the appearance of the grains. The latter are of ovoid shape, and as they lie their structure is not apparent, Water dissolves part of the outer portion, leaving visible the ovoid body, which becomes transparent. Embedded in it are a large regular crystal of proteid matter, and a small rounded irregular mass of minute crystals of mineral matter. These two constituents are spoken of as the crystalloid and the globoid respectively. The part of the matrix which is not soluble in water will dissolve in a 10 per cent. solution of common salt, while the crystalloid is soluble only in a saturated solution. The globoid is a double phosphate of magnesium and calcium.

Examination of these grains and their reactions shows that several proteids can be detected in them. Those soluble in water are albumoses, while the others which dissolve only in salt solutions are globulins. In grains met with in other plants, proteids occur which are albuminates and dissolve only in dilute alkalis.

Crystals of proteid occur in other places than seeds. If we examine a young potato, we find, in certain cells lying a little below the epidermis, some regular transparent cubical crystals, which are composed apparently of the same material as' the crystalloids of the complex aleurone grains described. They are soluble in saturated solutions of common salt. Similar crystals are met with in the tissues of certain seaweeds. Many of them can be made to crystallise from the solvents which are used to extract them.

The seeds of the cereal grasses contain two other very curious reserve proteids, which give rise in the flour to a peculiar sticky material which is generally known as gluten. They do not appear to be present in the aleurone grains of the seeds, but to occur in the starch-containing 
cells. They have been called gliadin and glutenin; occurring separately in the seed they interact with one another in the presence of water and form the gluten of the flour. Like the zein of maize, these proteids belong to the peculiar class whose members are soluble in dilute alcohol.

In many cases the proteids of the reservoirs do not remain unchanged during the resting period which follows their deposition. This is especially the case with seeds, in which such changes are characteristic of the process known as ripening.

Proteids occur also in the temporary reservoirs to which allusion' has been made. Fleshy roots and stems contain them in amorphous form in their parenchyma; certain forms are met with in the sieve tubes, and are coagulable on boiling like the globulins of the seeds. The proteids which are constant constituents of latex are no doubt in great part reserve food-stuffs.

In many cases amido-acids such as asparagin may be detected in the sap of various cells. These may be reserve materials temporarily retained where they are found, or they may be only translocatory products. Their occurrence in some resting seeds suggests the former explanation of their presence. It is not easy to detect them in the cells, as they are dissolved in the sáp, but in many cases they can be caused to crystallise by placing a section of the tissue on a glass slip in glycerine.

A great many plants store quantities of complex substances known as glucosides. These are bodies which on decomposition give rise to some kind of sugar and some other compound or compounds, usually belonging to the aromatic series of carbon compounds. Among them may be mentioned amygdalin, which is found in the seed of the bitter almond. During germination it splits up into benzoyl aldehyde, hydrocyanic (prussic) acid, and grapesugar. Many such bodies are known, and they are somewhat widely distributed. Some occur in seeds, but they are more frequently represented in the reservoirs con- 
tained in fleshy roots and stems. Many plants belonging to the Cruciferce and several allied orders are particularly rich in reserve materials belonging to this group. Sinigrin, or myronate of potash, is the principal glucoside which they contain. It splits up into sulphocyanate of allyl, grape-sugar, and hydrogen-potassium-sulphate.

The nutritive value of these bodies is partly due to the sugar which they yield on decomposition. The evidence that the other products can minister to nutrition is not very complete, though it seems satisfactory in certain cases.

Fats or oils are frequently stored as reserve food-stuffs in different plants. The distribution of this material is very varied, though, as in so many other cases, the seed is the most general place of deposition. Many seeds, that for instance of the castor-oil plant, contain as much as 60 per cent. of their dry weight of oil, which is non-volatile. Others contain as little as 2 per cent., and between these limits very varying amounts may be found. When the oil is in great preponderance, it is usual for no other form of carbonaceous reserve to be present; in cases where but little oil occurs starch is usually found as well, as in so many of the Leguminosa. The Cruciferce as a group often contain oil in fairly large quantity. As a rule nitrogenous reserves in the shape of aleurone grains accompany the oil.

In other places than seeds large deposits of oil often occur, though their purpose is not so obvious. We have them in large amount in the pericarps of certain fruits, such as the olive; in the petals of many flowers, e.g. Funkia and Ornithogalum; in the leaves of some of the Agaves, the roots of Oncidium, \&c. They can hardly be regarded in some cases as truly reserve materials, being perhaps more strictly connected with the mechanisms of dispersion.

The mode of deposition of oil or fat is not at all well known. It is generally found saturating the protoplasm of 
the cell in which it lies, and not occupying a definite space as do aleurone and starch grains. Whether it is secreted from the substance of the protoplasm, or whether the materials of which it is made are taken to the latter in a state near the condition of the finished fat, is uncertain. It is formed by the combination of a fatty acid with glycerine. Both these bodies can be formed in the plant, but how they are finally presented to us in the shape of oil is still in need of elucidation. As the oil appears in the cell it seems to point to a process of breaking down of the protoplasm itself, and not to a direct combination of the antecedents mentioned. Thus if we stain cells which are forming fat with osmic acid, which colours fatty bodies brown or black, we see in the protoplasm small specks of fatty matter, which, while in the youngest cells mere dots, are in older ones larger, and can be recognised as droplets. In still older ones the blackness permeates the whole protoplasm, indicating that the latter is saturated with the oil, the droplets having run together in consequence of their number and dimensions.

The appearances are, however, not inconsistent with the view that the work of the protoplasm is only to effect the ultimate changes or interactions of the glycerine and the fatty acids which are transported separately to the cells or perhaps formed there from some antecedent.

The deposition of fat in some cases, particularly in leaves, has been stated to be effected by the agency of certain plastids corresponding to the leucoplasts already mentioned in connection with the formation of starch grains. These structures, which have been called elaïoplasts, are curious bodies of various shapes, sometimes round or oval, sometimes irregular in contour, which lie near the nucleus of the cell. Like the other plastids they consist of a spongy protoplasmic framework, in the meshes of which the oil is formed, much as it is in the protoplasm of the seeds already described.

All these bodies, when acted upon by a process analo- 
gous to the digestion known in the animal kingdom, are converted into materials which can directly nourish the living substance, or can be transported easily about the plant in a condition of which the latter can readily take advantage, needing indeed very little constructive change to fit them for actual assimilation. 


\section{CHAPTER XVII}

\section{DIGESTION}

WE have noticed in studying the deposition of reserve foodstuffs that the forms in which they exist in the reservoirs differ in many respects from those which they assume for purposes of transport or translocation. They are generally insoluble in water or cell-sap, and almost always indiffusible, whereas they travel in the form of soluble, diffusible bodies. The removal of them from the seats of storage takes place at times which are dependent on the resumption of activity of growth or development; and as such a removal involves the resumption of the travelling forms, they must undergo a process which, from analogy with similar processes in the animal body, may be described as digestion. Each must, after such treatment, be presented to the protoplasm of the growing cells in much the same form or condition as that in which it was first constructed from the simple bodies which the plant absorbed from its environment. This is necessary in all cases, because, as we have already noticed, the storage forms are not directly assimilable by the protoplasm, but have undergone a certain modification in the process of their deposition.

The process of digestion in plants is chiefly intracellular, and takes place in all cells in which reserve materials occur. It is only occasionally that we find it taking place on the exterior of the plant-that is, not in the interior of a cell. In a few cases we find it carried on in connection with the absorption of nitrogenous or proteid food, as has been already shown in a preceding 
chapter. Digestion, though most generally associated in plants with the utilisation of reserve materials, may thus occasionally be met with in connection with the absorption of food from without, when it is a process precisely similar to the digestive processes of the higher animals, though it is somewhat simpler in the details of its mechanism.

The intra-cellular digestion of plants agrees very closely with that of many of the humbler animals, and corresponds also to such processes in the higher forms as the utilisation of the glycogen of the liver and the fat of various regions.

We have seen that in a few rare cases proteid material is absorbed into the plant-body through various leaves or modified foliar organs. The insectivorous plants are materially assisted in their growth by capturing and digesting various insects, the products of the digestion being absorbed by the surface of the leaf or other organ concerned. We examined several of these mechanisms in some detail in Chapter XIV.

Absorption of food from without after preliminary digestion is much more frequently observed when we study the nutritive processes of the Fungi. Not only proteid, but also carbohydrate and fatty substances are thus digested outside the body of the plant, and the products of the digestion are subsequently absorbed.

We have then to inquire how these processes of digestion, whether internal or external, are brought about.

The protoplasm of the cell, among its many properties, no doubt has the power of setting up these decompositions, and probably in many of the very lowly plants, in which the whole organism consists of only a few protoplasts or perhaps a single one, the work is altogether effected by its instrumentality. The protoplast, in fact, carries out all the various processes of life by the interactions of its own living substance with the materials absorbed by it, aided in the constructive processes by the chlorophyll 
apparatus, if it possesses one. In such a protoplast we may observe at times the storage of such a reserve material as starch, and its digestion at the appropriate period.

Even in more complex plants it is certain that the living substance of every protoplast is in a constant state of change, initiating many decompositions in which its own substance takes part, as well as others into the course of which it does not itself enter. Among these decompositions we must include the various intra-cellular digestive processes.

Though all protoplasm has this power, it is not usual in plants, any more than in animals, to find it exclusively relying on it. The work of digestion, at any rate, is generally carried out by peculiar substances which it forms or secretes for the purpose. We have in plants a large number of these secretions, which are known as enzymes or soluble ferments.

The action of these enzymes is not at all completely understood. They appear not to enter into the composition of the substances which are formed by their activity, and they seem to be capable of carrying out an almost indefinite amount of such work without being used up in the process. They are inactive at very low temperatures, but effect the decompositions they set up freely at the ordinary temperature of the plant. As the temperature at which they are working is raised, their activity increases up to a certain point, which varies slightly for each enzyme, and is called its optimum point. This usually ranges between $30^{\circ}$ and $45^{\circ} \mathrm{C}$. If the temperature is raised above the optimum point, the enzyme becomes less and less active as it rises, and at about $60^{\circ}-70^{\circ} \mathrm{C}$. it is destroyed. The exact point, however, varies a good deal in the cases of different enzymes.

Enzymes work most advantageously in darkness or in a very subdued light; if they are exposed to bright sunshine they are gradually decomposed, the violet and ultra- 
violet rays being apparently most powerful in effecting their destruction. They are often injuriously affected by neutral salts, alkalis, or acids, though in this respect there exists considerable diversity throughout the group.

The enzymes are manufactured by the protoplasm of the various cells in which they occur, being produced from its own substance, in a manner somewhat similar to that of the formation of the cell-wall. Usually their presence is accompanied by a marked granularity of the protoplasm, due to the formation in it of an antecedent substanee, known as a zymogen, which is readily converted into the enzyme. This granularity does not, however, always occur, though we have reason to suppose that the secretion of the enzyme always takes place by successive stages. The zymogen has not, however, been definitely detected in all cases.

We find various degrees of completeness of differentiation of the cells which produce these enzymes. In the simplest cases, such as the mesophyll of the leaves of most plants, or the great majority of seeds, or the tubers of the potato, the enzyme is found in all the cells which contain the reserve materials, so that a rapid transformation of the latter is readily possible. In the Horse-radish and many allied plants the cells which secrete the enzyme do not themselves contain any reserve materials, but are situated among those which do, so that the enzyme has to pass from the seat of its formation to other cells in order to discharge its function. This is a very slow and gradual process, and is probably carried out through the agency of the delicate filaments of protoplasm which extend through the cell-walls, for enzymes are not eapable of dialysing through a membrane.

The occurrence of such cells, which are apparently set apart especially for the secretion of an enzyme, gives us, as it were, the starting-points of the special structures known as glands, whose function is similar but whose structure is more complex. In some of the plants belong- 
ing to the natural orders Capparidacece and Tropaolacece, the glandular cell divides several times to form a little mass or nodule of secreting cells, which must be regarded as a rudimentary gland, though it is not provided with any definite outlet or duct.

In the seed of the cereal grasses there is a special organ separating the embryo from the endosperm. This structure, which is a modification of part of the cotyledon, is known as the scutellum (fig. 123); its function is to effect the absorption of the nutritive material of the endosperm, and supply it to the growing embryo. This scutellum is covered on its outer face, which is in contact with the

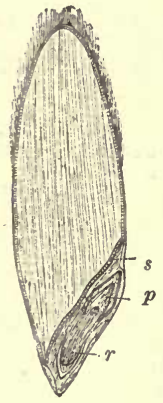

Fig. 123.-Section of Oat-grain. $p$, plumule; $r$, radicle; $s$, scutellum.

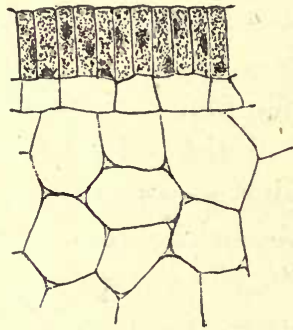

Fig. 124.--Section of PORTION OF ScUTELlum OF Barley, SHOWING THE SECRETING EPITHELIUM.

endosperm, by a layer of cylindrical cells, whose long axis is at right angles to the surface (fig. 124). These cells' are very granular in appearance, and form a very marked secreting structure, producing two enzymes, which are subsequently discharged into the endosperm to effect the digestion which must precede absorption. The aleurone layer of the same grain (fig. 125), which has already been described, is also a secreting layer, resembling the outer layer of the scutellum in several respects.

The tentacles of the leaves of Drosera, to which allusion has already been made, are very definitely secreting structures; in addition to preparing an enzyme they pro- 
duce a:weak acid, both of which are present in the glairy material that they pour out over the captured insect. These tentacles (fig. 126) and the secreting structures of

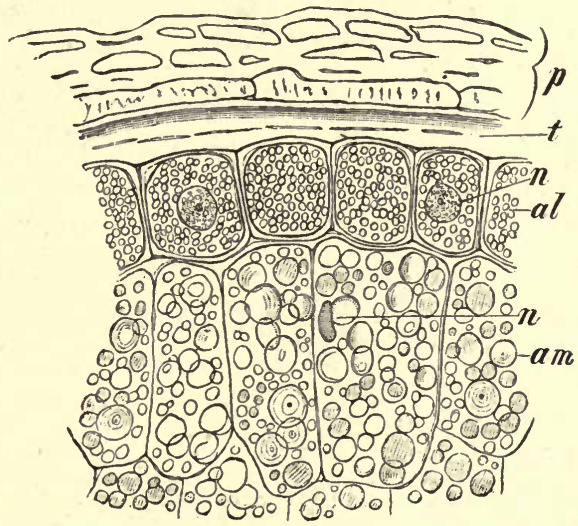

Fig. 125.-Section through external region of Grain of Barley.

$p$, pericarp of fruit; $t$, testa of seed ; $a l$, layer of cells containing aleurone grains; am, cells of endosperm; $n$, nucleus. (After Strasburger.)

the leaves of Dioncea and other plants, as well as the similar bodies which occur in the lining of the pitcher of Nepenthes, must be regarded as actual glands, comparable to those of the alimentary canal of the animal body, though less complex in structure. Glandular hairs, which consist of a few cells situated on a stalk, are found in great numbers on other plants, especially some species of Saxifraga.

There are many of these enzymes present in different plants, the function of some of which is still not understood. Many, however, have been investigated with some completeness. They are usually classified according to the materials on which they work. We may describe here four groups, the members

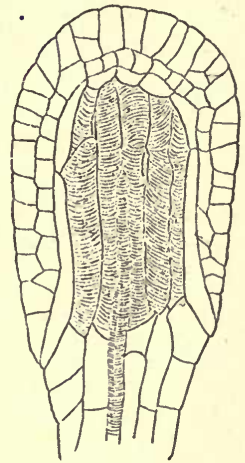

Fig. 126.-GLANDULAR Apex of a Tentacle of Drosera. of which take part in the digestion of reserve materials, as well as in the processes of external digestion. These 
decompose respectively carbohydrates, proteids, glucosides, and fats or oils. In nearly every case the action of these enzymes is one of hydration, the body acted upon being generally made to take up water, and to undergo a subsequent decomposition.

Of those which act upon carbohydrates we have two varieties of diastase, which convert starch into maltose, or malt-sugar; inulase, which forms another sugar, levulose or fructose, from inulin; invertase, which converts canesugar into glucose and fructose; glucase or maltase, which produces grape-sugar from maltose; and cytase, which hydrolyses cellulose. Another enzyme, which does not appear to be concerned with digestion so directly as the others, is known as pectase; it forms vegetable jelly from pectic substances occurring in the cell-wall.

The members of the second group act upon proteid substances, and are technically known as proteolytic enzymes. The principal members of this group are pepsin and the various trypsins, which convert albumins and globulins into peptones, the trypsins also decomposing certain peptones into amido-acids. Allied to these is rennet, which converts the caseinogen of milk into casein, the characteristic proteid of cheese. It occurs in a great many plants, but its function in vegetable metabolism is unknown.

The enzymes which act upon glucosides are many ; the best known are emulsin and myrosin ; others of less frequent occurrence are erythrozym, rhamnase, and gaultherase. Those which decompose fats have not been so fully investigated: they are known as lipases, but whether there are many different varieties or not has not at present been ascertained.

Diastase appears to exist in two varieties, distinguished from each other by their mode of action on the starch grain. One, called diastase of translocation, dissolves the grain slowly from without inwards, without altering its general appearance; the other, diastase of secretion, dis- 
integrates it by a process of corrosion before dissolving it (fig. 127). The first of the varieties has a very wide distribution in plants, being present almost everywhere. The second is the body formed by the glandular covering or epithelium of the scutellum of the grasses.

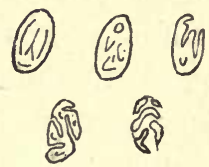

The great function of diastase in Fig. 127.-Starch Grains the plant is to transform starch (and probably glycogen where it occurs) IN PROCESS OF DIGESTION. ThE SUCCESSIVE FIGURES SHOW THE PROGRESS OF DISSOLUTION. into maltose or malt-sugar. Wherever starch is formed, whether in the living leaf or in the reservoir set apart for storage, it must be regarded as a reserve material, and its removal from the seats of deposition is preceded by its conversion into this sugar. The details of the transformation are not fully known at present, but a good deal of information has been obtained through the labours of many observers. Starch has a rather large molecule, but its exact formula is not thoroughly known. For a long time it was taken to be approximately $n\left(\mathrm{C}_{6} \mathrm{H}_{10} \mathrm{O}_{5}\right)$, and the value of $n$ was thought to be 5 . More recently the suggestion has been made that the molecule is much larger, and may be more truly represented by $5\left[\left(\mathrm{C}_{12} \mathrm{H}_{20} \mathrm{O}_{10}\right)_{20}\right]$, the view being based upon the formation of several complex substances during its decomposition. The starch molecule is possibly composed of four dextrin-like groups, each $\left(\mathrm{C}_{12} \mathrm{H}_{20} \mathrm{O}_{10}\right)_{20}$ arranged about a fifth. It has been suggested that the first action of the diastase is the liberation of these from one another; and that four of them by successive incorporations of water are converted, through a series of complex substances called malto-dextrins, into maltose, while the fifth withstands the action of the enzyme for a considerable time. After the action of the diastase has been proceeding for some time the resulting product is found to be four parts maltose and one part dextrin.

How far this series of decompositions represents what takes place in the plant is uncertain, but it is clear that the 
starch, which is insoluble, is converted into sugar, which can be removed to the parts of the plant where it is required for building up the protoplasm.

Inulase occurs in the tubers and tuberous roots of some of the Compositæ, in the bulbs of certain Monocotyledons, and in some of the Fungi. It converts inulin ultimately into levulose or fructose, but the action is not a very simple one, at least one intermediate body being formed during the process.

Invertase has a much wider distribution. It is easily extracted from the Yeast-plant, in which it is present in relatively considerable quantity. Other fungi which contain it are Fusarium and Aspergillus, besides certain bacteria. In flowering plants it has been found in seeds, buds, leaves, stems, roots, and pollen grains. Its action is the hydrolysis of cane-sugar, which it splits up into glucose and fructose, according to the equation

$$
\underset{\text { Cane-sugar }}{\mathrm{C}_{12} \mathrm{H}_{22} \mathrm{O}_{11}}+\underset{\text { Water }}{\mathrm{H}_{2} \mathrm{O}}=\underset{\text { Glucose }}{\mathrm{C}_{6} \mathrm{H}_{12} \mathrm{O}_{6}}+\underset{\text { Fructose }}{\mathrm{C}_{6} \mathrm{H}_{12} \mathrm{O}_{6}}
$$

It is a little difficult to understand why this decomposition of cane-sugar is necessary, as it can diffuse through membranes, and in many cases it has been found capable of assimilation by the protoplasm. Probably, however, each of the sugars concerned in the transformation has a special part to play in the metabolism of the plant, and neither can readily replace either of the others.

Glucase occurs in the grains of various cereals, being especially prominent in the Maize. It is also fairly abundant in the Yeast-plant. It has no action on canesugar, but splits up maltose into glucose, one molecule of the former taking up water and yielding at once two molecules of the latter.

Other sugars of similar constitution to maltose and cane-sugar are made to undergo similar transformations by enzymes of less widespread distribution. The chief of these are trehalase, melibiase, melizitase, and lactase.

There appear to be several varieties of cytase, which 
can be prepared from various seeds. The enzyme was first discovered in the germinating grain of the barley, in which it is located chiefly in the aleurone layer and to a less extent in the epithelium of the scutellum, where it exists side by side with diastase. It dissolves the walls of the cells of the endosperm, detaching them from each other and giving a curious mealy character to the grain. Its presence was first suspected in the Date-palm, where large reserves of cellulose are found in the hard cell-walls of the endosperm. The embryo dissolves these walls and absorbs their products, the work being effected by an epithelium which covers the part of the cotyledon which remains in the seed during the early processes of germination. This epithelium is composed of elongated cells arranged in a manner resembling that characteristic of those which form the secreting layer of the scutellum. It has recently been shown that cytase is formed in the embryo, probably in this layer, and passes thence into the endosperm. The amount of it that can be detected is very small, however, and the process of the decomposition of the cellulose is very slow and gradual. Cytase exists in considerable quantity in some of the higher fungi and in certain bacteria.

Pectase has recently been found to be very widespread in plants. Its function is not very clear, but it may assist cytase in the swelling up of the cell-wall which is antecedent to solution. It is recognised by its power of forming vegetable jelly from the pectic substances of the cell-wall. This jelly appears to be a compound of pectic acid and calcium.

The enzymes which digest proteids are frequently on that account spoken of as proteolytic enzymes. There are two main classes of them known at present. The first, represented by the pepsin of the stomach of the higher animals, converts albumins, globulins, and certain insoluble proteids into peptones, several intermediate bodies, known as proteoses or albumoses, being formed during the process. The second group, which may be represented by the 
trypsin of the pancreas, carries the digestion further and splits up certain peptones into amido-acids, of which the chief that have been observed are leucin, tyrosin, and asparagin.

It is not quite certain that representatives of the first class are to be met with in plants. It is for the present probable, however, that the enzyme of some insectivorous plants is a pepsin. It acts only in the presence of a weak acid, as does the pepsin of the stomach, but the products which it forms have not been accurately investigated. It is apparently only secreted when the gland has been stimulated by the absorption of nitrogenous matter.

Several varieties of vegetable trypsin have been discovered and their properties investigated. The earliest known enzyme belonging to the group is the papaïn which has been extracted from the Papau (Carica Papaya). It appears to exist in greatest quantity in the pulp of the fruit, but is present also in the sap which can be expressed from the stem and leaves. It is apparently associated in the juice with a peculiar proteose or albumose, and it is most energetic in a neutral solution, though it can act also in a faintly alkaline one. It is easily destroyed by a very small trace of free acid.

Another trypsin, which has been named bromelin, has been extracted from the fleshy pulp of the Pine-apple (Ananassa sativa). Like papain it is associated with a proteose. It acts most energetically in neutral and faintly acid solutions, alkalis in very small traces being prejudicial to it. Its activity varies a good deal according to the acid which is present, and to some extent according to the proteid which it is digesting.

Other vegetable trypsins have been extracted from the germinating seeds of the Lupin, the seedlings of several plants, the fruit of the Kachree gourd (Cucumis utilissimus), the juice of the Fig-tree (Ficus carica), and the leaves of certain species of Agave. How far these are identical, or whether they present specific differences, appears at present 
uncertain. They are all active in faintly acid solutions, but the most favourable concentration appears to vary. The enzyme of the Kachree gourd is most effective when the medium is faintly alkaline, whereas that of the lupin seed is inoperative under these conditions. Too much stress must not, however, be laid upon this point, as the enzymes have not been prepared in any case in anything like a pure condition.

The action of all these proteolytic enzymes is probably one of hydrolysis, though it is difficult to prove it by analysis.

Rennet occurs in many seeds, in some cases in the germinating, and in others in the resting, condition. It has also a wide distribution in the vegetative and floral parts of various plants. Whether it is really proteolytic in the vegetable organism it is hard to say, as the details of its action are unknown. It is so in the animal body.

The enzymes which decompose glucosides, as we have already seen, are numerous and varied in their distribution, occurring in various fungi and lichens as well as in the higher plants. Their action may be illustrated by the behaviour of emulsin, which exists in quantity in the seeds of the bitter Almond and in the vegetative parts of the Cherry-laurel (Prunus Laurocerasus). It splits up the glucoside amygdalin according to the equation

$$
\underset{\text { Amygdalin }}{\mathrm{C}_{20} \mathrm{H}_{27}} \mathrm{NO}_{11}+2 \mathrm{H}_{2} \mathrm{O}=\underset{\substack{\text { Benzoic } \\ \text { aldebyde }}}{\mathrm{C}_{7} \mathrm{H}_{4} \mathrm{O}}+\underset{\substack{\text { Prussic } \\ \text { acid }}}{\mathrm{HCN}}+\underset{\text { Glucose }}{2\left(\mathrm{C}_{6} \mathrm{H}_{12} \mathrm{O}_{6}\right)}
$$

This is, as in other cases, a process of hydrolysis. Myrosin, another of the group, is peculiar in that it effects its characteristic decomposition without causing the incorporation of water during the process, thus:

$$
\mathrm{C}_{10} \mathrm{H}_{18} \mathrm{NKS}_{\text {Sinigrin }} \mathrm{O}_{10}=\underset{\substack{\text { Sulpho-cyanate } \\ \text { of allyl }}}{\mathrm{C}_{3} \mathrm{H}_{5} \mathrm{CNS}}+\underset{\mathrm{C}_{6} \mathrm{H}_{12} \mathrm{O}_{6}}{\mathrm{Glucose}}+\underset{\substack{\text { Potassium- } \\ \text { lydrogen } \\ \text { sulphate }}}{\mathrm{KHSO}_{4}}
$$

Others, such as rhamnase, existing in the seeds of Rhamnus infectorius, erythrozym in the Madder, gaultherase in the 
bark of Betula lenta, act on various glucosides, after the manner of emulsin.

The digestion of the glucosides, we may notice, is always accompanied by the appearance of sugar, which is one of the products of their decomposition. The fate of the other bodies into which they split is not well ascertained, though there is some evidence that cyanogen compounds, even such as hydrocyanic or prussic acid, are used for nutritive purposes by certain plants.

The digestion of fat or oil has not been very fully investigated, though certain facts are known concerning its fate in germinating seeds. The digestion is generally accompanied by the appearance of starch grains in cells near the seat of digestion, and it was formerly considered that the starch arose directly from the oil. It appears now that the oil is split up by an enzyme, lipase, the result being the formation of a free fatty acid and glycerine. The fatty acid undergoes further decomposition, being oxidised into simpler acid bodies, which are crystalline instead of being viscid like the fatty acid first liberated. These pass into the general body of the seedling. The glycerine in its turn seems to be converted into some variety of sugar, from which the plastids of the seedling construct the starch which has been referred to, its formation indicating, as in other cases, a temporary surplus of carbohydrate supplies.

Within the last two years it has been ascertained that the production of alcohol from sugar is brought about by another soluble enzyme which has been prepared from yeast. Though the existence of this body has been long suspected, it is only quite recently that it has been demonstrated. Like the decomposition which is brought about by myrosin, the splitting up of the sugar is apparently not a process of hydrolysis. It may be expressed by the following equation:

$$
\mathrm{C}_{6} \mathrm{H}_{12} \mathrm{O}_{6}=2 \mathrm{CO}_{2}+2 \mathrm{CH}_{3} \mathrm{CH}_{2} \mathrm{OH}
$$

In the reaction the sugar is decomposed, alcohol is formed and carbon dioxide given off. 
This enzyme, which has been called zymase, has been proved to exist not only in yeast but in certain fruits, being formed there when the fruits are kept in an atmosphere which contains no oxygen.

The physiological explanation of this observation will be discussed more fully in a subsequent chapter.

There are other enzymes with a more restricted distribution, about whose value to the plant little or nothing is known at present. The cells of a particular microscopic organism, known as Micrococcus Urea, decompose urea with the formation of ammonium carbonate, and an enzyme, urease, having the same power, can be extracted from them. Many enzymes can be prepared from bacteria, which set up various changes in proteids, some resulting in the formation of peptone, and others producing toxic substances. Many bacteria excrete a variety of diastase.

Another class of enzymes has recently been discovered which do not apparently take any part in digestion, but which may be briefly alluded to here. They set up a process of oxidation in the substances they attack, and have consequently been named oxidases. They are apparently very widely distributed, and perform very various functions, being often concerned in bringing about the presence of particular colouring matters. They occur very prominently in Fungi, but are by no means confined to them. They have not at present been studied from the point of view of their utility to the plants which secrete them.

The conversion of zymogens into enzymes is much facilitated by a gentle warmth, particularly when a trace of free acid is present. The red rays of light exercise a similar influence in some cases.

The fermentative activity of protoplasm was alluded to at the opening of this chapter. The living substance of many cells is capable of setting up various fermentative decompositions, apparently identical with those that have been described. Various cells can convert starch into sugar, can peptonise proteids, and carry out other digestive 
processes, without the intervention of an enzyme. Though this property can easily be proved in the case of cells of the higher plants, it is especially prominent in many of the more lowly organisms such as the Bacteria. The processes of putrefaction generally depend on this property in the organisms which bring it about. Till quite recently the alcoholic fermentation of sugar was attributed to such an action in the yeast-cell, and in the cells of certain ripe fruits under particular conditions, the chief of which was the deprivation of oxygen. Such an action leads to the formation of acetic acid from alcohol by the microbe Mycoderma or Bacterium aceti. Similar protoplasmic action is responsible for the production of various acids in the cells of the higher plants. The dependence of these fermentations on the vital activity of the protoplasm is evident from the fact that no enzyme can be extracted from the cells which can set up the particular changes in question.

It is not difficult to prepare the enzymes from the tissues in which they work, but it would be extremely rash to say that they are in anything like a pure condition when obtained. Nor is it easy to say much about their purification, as they are not known except in close connection with the substances on which they act, or with the products of the decompositions they initiate. There is therefore no known test of their purity.

They can be extracted by treating the tissue, which should be very finely divided or ground in a mortar, with glycerine, or with a solution of common salt, or with water containing a trace of an antiseptic. After a period of ten or twelve hours the extract should be strained and subsequently filtered, when the enzyme may be precipitated from the filtrate by adding strong alcohol. It is very evident that this process will not yield it pure, for the solvents employed will dissolve many constituents of the tissue besides the enzymes, particularly proteids and sugars. The former will be thrown down with the enzyme by the alcohol. 
Any description of the process of digestion should naturally be followed by an account of the subsequent one of true assimilation or the construction of protoplasm from the food which is supplied to it as the result of digestion. Unfortunately but little can be said upon this subject, as such problems remain almost entirely unsolved. If we study the changes which take place in the growing points of plants, where such assimilation must necessarily be most active, we can find very little evidence of what is taking place. We can trace, for instance, the progress of sugar along the stem for a considerable distance, but just where it is assimilated our methods fail us. Sugar can no longer be detected, but in what way it has been incorporated into the living substance is still a mystery. Similar acknowledgment must be made in respect of the proteids. Amido-acids can be detected along the translocatory paths almost up to the locality of growth, but beyond that nothing can at present be said. We are unable also to explain the manner in which the food originally constructed ministers to the nutrition of the protoplasts or cells in which it is formed. 


\section{CHAPTER XVIII}

\section{METABOLISM}

WE have seen that the object of all the processes of construction and digestion that we have examined so far has been to present to the protoplasm materials which it can incorporate into its own substance. If we consider the processes which take place in a vegetable cell or protoplast, we find that they can be divided into those which minister to this construction or building up of the living substance, and those which are connected with its breaking down. The latter accompany or immediately follow the former, and the two together may be considered as the manifestation of the life of the protoplasm. The whole round of changes in which the living substance is concerned is generally spoken of as its metabolism. So many of the reactions as culminate in the construction of protoplasm are described as anabolic, while the changes which it initiates, or which are concerned in its decomposition, are termed katabolic.

We have been occupied mainly so far in discussing the anabolism of the protoplasts. The substances we have traced to the cells in which growth and repair are vigorous consist in far the greatest part of some form of sugar and of organic nitrogenous substances, either proteids themselves or the products of their decomposition, or substances constructed from simple materials with a view to the formation of proteids, such as the amido-acids asparagin or leucin. In the anabolic processes the protoplasm is continually reconstructing itself at the expense of such nutritive substances, which indeed constitute its food in the strict sense of the term. What is true of such cells as are 
actively growing and multiplying, which are found as we have seen in the special growing points or layers, is equally true of all cells so long as they are living. In all cases, though growth and division may not be evident, we have to do with processes of repair of the inevitable wasting of the living substance during the operations of its life. The same kind of change is evident in all cells, though the immediate results of such changes differ according to the part any particular cell takes in promoting the well-being of the whole organism.

If we turn from these anabolic processes we find we have proceeding, side by side with them, a decomposition of the protoplasm, involving a separation from its complex molecule of various substances which are of less complexity than the living material itself. These often, in the first instance, include such carbohydrate and nitrogenous substances as it made use of in building itself up. These can again be used in reconstruction of the protoplasm, or can be further broken down into simpler substances still. So long as the protoplasm is living, it is continually in a state of change or chemical activity, undergoing constant reconstruction and decomposition.

Besides initiating those chemical changes in which it takes this prominent part, it is also the seat of a large number of others into which its own molecule does not immediately enter. Processes of both oxidation and reduction are continually going on in its substance in which are involved the various materials which are found there, either in solution in the water which saturates it, or in amorphous form ; substances which have been transported from other cells, or have been formed in the processes of the selfdecomposition of the protoplasm.

These katabolic processes vary a great deal in the extent to which they are carried out. They may sometimes go on so far as to produce such simple bodies as carbon dioxide and water, which are given off from the organism. This is a very marked feature of the metabolism that may be 
observed in every living cell. Other katabolic changes, proceeding side by side with this very complete decomposition, are not so far-reaching, and a great accumulation of their products remains in the plant. Prominent among them we find such organic substances as woody or corky tissue. These must not be confused with what we have described as reserve materials, as the latter, unlike those now under discussion, are intended for ultimate consumption.

These changes involve the manufacture of great masses of material, whose construction, though ultimately dependent upon anabolism, is essentially a mark of the katabolic processes. The constructive processes indeed are both anabolic and katabolic, the former culminating in the formation of living substance, the latter marking the fabrication of its products. The great extent to which the constructive katabolic processes exceed such decomposition of protoplasm as is marked by the formation of carbon dioxide and water, finds its expression in the enormous bulk which many trees and other plants attain. This increase of the size of the plant-body is very much facilitated by the fact that the katabolic processes in question are not attended by the excretion of anything from the body of the organism. As a rule plants have no excreta except the gaseous bodies whose elimination we have already described, and these result in the main from the profounder decomposition of the living substance. Whatever a plant absorbs from the soil, except water, it nearly always retains within its tissues, so that increase of weight almost inevitably accompanies continuance of vitality.

It must not be inferred, however, that plants do not produce, during their constructive katabolic processes, any substances which are useless to them or which may even be deleterious. There are numerous products which come under this category, but from the mode of construction of the body of the plant they are not cast off as they would be from the animal organism under similar conditions. 
Instead of being eliminated entirely they are only removed to such localities as ensure their being withdrawn from the spheres of vital activity. They are generally deposited in such regions as leaves which are about to be shed, or the bark of trees, which is a collection mainly of dead matter; or they may be stored away in special cells, or in cell-walls, or intercellular passages, or elsewhere. These bodies really correspond to excreta, and the processes of their formation and deposition are called processes of excretion.

Most of the katabolic constructive processes are directly applied to the production of substances which are of great use to the plant. Emanating as these do directly from the protoplasm, their formation is generally termed secretion.

Though they originate however directly in and from the living substance, the latter does not always present them in the form in which they are found in the adult plant-body, for various changes both of the nature of oxidation and reduction may take place in them after they have been secreted.

The processes included under the general term katabolism are thus seen to be very varied. During the course of such changes many substances are frequently formed which seem to have no direct bearing on the vital processes, and whose meaning is still obscure. These are often spoken of as the bye-products of metabolism. .

We may now pass to consider in some detail some of the more prominent processes of secretion.

For many reasons the formation of such enzymes as are used during digestion may be regarded as the most typical of these. A cell which is about to secrete is generally found to be filled with colourless hyaline protoplasm in which certain vacuoles may be seen. Immediately before secretion begins an increase of the amount of the protoplasm can be observed, which is effected at the expense of various nutritive products which are transported to it. During the whole of the process, when this is prolonged, such a supply 
of nutritive material takes place. If during the secretion this supply is stopped, the process is rapidly suspended. This can be detected easily in the case of the epithelium of the scutellum of the barley grain, which we have seen produces considerable quantities of diastase. The first stage of the process is thus evidently anabolic. As soon as the nutrition of the cell has reached a certain point the appearance of the protoplasm undergoes a change. Minute granules begin to be formed in its substance, which increase in number until the hyaline character is replaced by a marked uniform granularity, the cell substance becoming somewhat like ground-glass in appearance. The growth of the protoplasm and this subsequent formation of granules lead to the obliteration of the vacuoles, till the cell is completely filled. After a time as the secretion leaves the cell the latter shrinks again; the granules are passed out in solution in the sap which is exuded, and the protoplasm is seen to be less plentiful and to become hyaline and vacuolated as at first.

Following the anabolic changes we have thus the breaking down of the protoplasm, attended by the appearance of the granules to which it has given rise. There is reason to believe that the granules consist of the zymogen rather than the enzyme, and that the final transformation of the former into the latter takes place just as the exudation of the sap occurs.

In glands in which the process of secretion is repeated more than once, similar changes may be traced. The secretion of the enzyme in these cases can be shown to take place by successive stages. The preliminary hyaline condition is followed by the granular one, and in this state the cell can remain for some time before the enzyme is discharged. When this has happened the hyaline condition is resumed.

The formation of the cell-wall which separates the cells is due to a similar activity of the protoplasm. The division of cells or the development of new protoplasts will be more 
fully considered in a subsequent chapter; it will suffice to say here that in all ordinary growing points this division of a protoplast into two is followed immediately by the formation of a new supporting membrane between them.

The division of the cell is preceded by the division of its nucleus, which is attended by a series of complicated movements of particular constituents of its substance. The two daughter-nuclei are situated at some little distance from each other and are connected by a number of delicate filaments which are gathered to a point at each end and spread out in the centre, forming what is called the nuclear spindle. This generally stretches completely across the long diameter of the cell.

During these introductory changes the hyaline protoplasm becomes more granular, and the granules, technically spoken of as microsomata, are attracted to the spindle fibres. They pass along these fibrils from both regions of the cell and form a plate of extreme tenuity across it, midway between the two new nuclei. This plate soon undergoes a transformation, the granules disappearing and the membrane becoming translucent, and so forming the ordinary substance of the cell-membrane, generally, though perhaps not strictly accurately, known as cellulose. The cell-wall is thus seen to be formed from the protoplasm, or to be secreted by it, the granules or microsomata of which it is at first composed being the result of decompositions set up in the living substance.

When cell-walls are growing in thickness or in surface a similar decomposition of the protoplasm can be observed. The microsomata or granules are formed in the protoplasm and are gradually deposited, often in oblique rows, upon the original membrane. They are subsequently changed in appearance and become the first thickening layer of cellulose. The occurrence of the rows of granules frequently leads to the striated appearance which can be noticed on the walls of many fibres, particularly those of the bast of the fibro-vascular bundles. 
In all cases therefore the formation of cellulose can be traced to the self-decomposition of the protoplasm, though whether the granules are actually cellulose or an intermediate substance is still uncertain.

A very similar phenomenon is observable in the formation of starch grains. In this case, as we have seen, we may either have to deal with the general protoplasm of the cell or, as is usual in reservoirs and in ordinary leaf parenchyma, with a definite plastid, either a chloroplast or a leucoplast. These structures, however, may be regarded as specially differentiated protoplasmic bodies. We have already discussed their behaviour and the formation of the starch grain by them. Building themselves up at the expense of sugar and probably of various nitrogenous compounds, either brought to them or remaining in their substance, they break down again to a certain extent, splitting off a quantity of starch, which is deposited in the interior of the plastid, sometimes at one point, sometimes at several. As the process goes on, successive laminæ or shells of starch are continually deposited round the original grain or granule till the structure of the fully formed starch grain is reached. In this case the process is somewhat clearer than the corresponding one in that of cellulose, as there is little doubt that each shell is composed of starch at the moment of its deposition.

The formation of starch is in these cases a secretion by the plastid, just as that of cellulose is a secretion by the protoplasm of the cell. The formation of the small starch grains by the general protoplasm of cells in which no plastid is present is of a similar character, though it is not so long continued and the formation of successive laminæ does not take place.

The method by which aleurone grains arise in the meshes of the cytoplasm is of a precisely similar character.

The formation of fat is due to similar behaviour on the part of the protoplasm. It can be observed most easily in 
the case of certain fungi when they are living under such conditions as prevent their being properly nourished. The protoplasm in the hyphæ diminishes in quantity, the vacuolation becomes considerable, and their cavities are found to contain large drops of oil. In the cells of seeds such as those of the castor-oil plant, in which large quantities of oil are stored as reserve materials, the deposition of fat can be studied. Sections of the cells should be stained with osmic acid, which colours fatty substances brown or black according to the quantity of them which is present. When fat is beginning to be formed the substance of the protoplasm becomes faintly granular, but the granules are more or less transparent and cannot be seen without staining. On the application of osmic acid they display their fatty character by becoming brown. In cells which are a little older the granularity is more marked, as the separate granules have increased in size and many have run together, forming small droplets. The staining is darker in these cells, the larger granules becoming quite black. In still older cells the whole substance becomes intensely black and the protoplasm can be seen to be saturated with the oil. If the latter is removed by treatment with ether, the living substance will be found to have diminished in amount. There has been a formation of fat by the protoplasm, and the latter has evidently produced it by a decomposition of its own substance, for it has become reduced in bulk.

The elaïoplasts, to which reference has been made, behave similarly, their substance diminishing at the same time that the fat or oil makes its appearance.

The decomposition of the protoplasm in the formation of fat is not accompanied by much reconstruction, so that it is soon very greatly diminished in amount, while the fat, the product of the katabolic processes, increases.

The appearance of fat in the two cases described seems to demand two different explanations. In the cells of the seeds and in the elaïoplasts it is to be regarded as a 
storage of reserve materials. In the starved hyphæ of the Fungus it appears to be due to the decomposition of protoplasm under conditions of grave disturbance of nutrition, if not of approaching death. In both cases, however, it is derived from the breaking down of the living substance, though the decomposition of the latter is due to such different causes in the two cases.

One of the most important of the secretions of plants is the green colouring matter, chlorophyll, which we have already seen is present in the form of a solution in the meshes of the chloroplasts. The formation of chlorophyll is a more specialised process than any of those which we have just been considering, and is dependent upon a variety of conditions. It probably involves not only the self-decomposition of the protoplasm, but also other processes which take place within the substance of the chloroplast.

The special conditions necessary for the formation of chlorophyll are-1st, access of light; 2nd, a particular range of temperature; 3rd, the presence of a minute quantity of iron in the plant; 4th, access of oxygen. There are a few exceptions to the rule that chlorophyll can be formed only in light; the embryo in the seed of Euonymus europaus is green at the time the seed is ripe, though it is surrounded by a thick red protecting coat which is opaque. Seedlings of Pinus also are green when they are raised from seeds in light which is insufficiently strong to enable chlorophyll to be formed in seedlings of Dicotyledons grown side by side with them. A few other cases also are known. If an ordinary plant is cultivated from the seed in darkness, the resulting seedling will not be green, but will have a yellowish-white colour. When its tissues are examined with a microscope, the plastids will be found in the cells, but they will be tinged with a pale yellow pigment known as etiolin. When the latter is exposed to light it will rapidly become green, being in fact converted into chlorophyll. The etiolin is in the first instance secreted by the 
protoplasm of the plastid, and subsequent changes take place about which very little is known, but which result in its conversion into chlorophyll. If the temperature is kept very low, the etiolin remains unchanged, even though light is admitted. Hence the first leaves of plants which spring up in winter or early spring are frequently yellow and not green. This peculiarity may easily be observed in the case of snowdrops and hyacinths which appear very early in the year.

The function of the iron is not understood ; plants which are cultivated in such a medium that this element is not supplied to them have an appearance much like that associated with etiolation. Their colour is even paler, indeed they are almost colourless, though the plastids are present. A supply of iron at once causes them to assume the normal appearance. Plants so suffering from the absence of iron are said to be chlorotic.

The influence, of a supply of oxygen is probably not a direct one. The failure of plants to form chlorophyll in its absence is most likely due to a pathological or unhealthy condition of the protoplasm, all whose activities are disturbed under such circumstances.

Another pigment which is of fairly widespread distribution in plants is the red colouring matter known as anthocyan. This is not associated with any plastids, but occurs in solution in the cell-sap. It is found very commonly in young developing shoots, on the illuminated side of leaves which appear during cold weather, on the petioles and midribs of leaves which are put out on twigs of many plants in sunny places, and in many tropical plants which grow in deep shade. In seedlings which are developed in spring or in cold weather, the anthocyan may appear somewhat irregularly in the leaves, but it is mainly found along the veins and on the leaf-stalk.

The function of anthocyan is not well understood. Many facts point to the probability that it aids in the transformation of starch into sugar in the leaves in which 
it occurs, rendering translocation more rapid. It has been found that the red rays of the solar spectrum which it allows to pass are instrumental in the formation of leafdiastase from its antecedent zymogen. The pigment, while allowing these red rays to pass into the leaf, acts as a screen preventing the passage of the violet ones which have a very destructive effect upon this enzyme.

Other views as to the significance of this pigment have been advanced. It has been suggested that it effects a conversion of light rays into heating ones, so facilitating the metabolic processes of the plant. Another hypothesis regards it as a protective screen to the chloroplasts and to the protoplasm, preserving them from injury from too intense light. Neither of these views can, however, be regarded as entirely satisfactory.

In many cases it is beneficial by absorbing the dark heat rays and so facilitating transpiration as well as general metabolism.

Anthocyan appears to be a derivative of tannin, an aromatic substance which, is very widely distributed in the vegetable organism. This substance has not generally been included among the secretions of plants, but rather as a bye-product of metabolism. It is not impossible that it may in some cases be a definite secretion for some particular purpose.

The distinction between definite processes of secretion, and such reactions as lead to the formation of the so-called bye-products of metabolism, is not at all well defined. In many cases substances are included in the latter category because nothing is known as to their function, and the classification can therefore be regarded only as provisional. In many cases it cannot yet be determined whether particular substances are formed by the direct decomposition of protoplasm, or by subsequent changes in the primary products of such decomposition. Till quite recently the formation of resin and allied bodies in the resin passages of the Conifers and in many glandular hairs was con- 
sidered a true secretion, the aromatic substances being held to arise in the cells. Recent investigations tend to show that this is not the mode of their origin at all, but that these substances are formed by a peculiar process of degradation of the cell-wall. The glandular hairs of Primula sinensis (fig. 128), and the more complex ones of the Hop (fig. 129) have long been known to form their resins in this way. It seems probable that we must now regard the resin-secreting organs of the Conifers as comparable with these.

The bye-products of metabolism are too numerous to be discussed in detail in the present treatise. Though they seem to be quite subordinate to the main products we

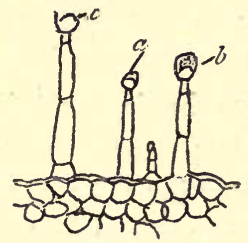

Fig. 128.-GLandular Hairs From Primula sinensis.

$a$, young hair ; $b$, hair showing secretion formed in the cell-wall of the terminal cell; $c$, hair after discharge of the secretion.

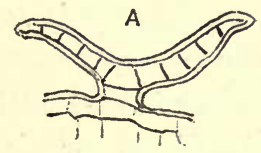

B

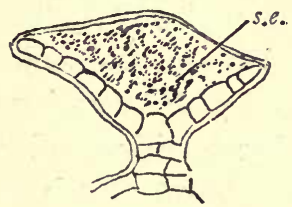

Fig. 129.-Glandular HaIrs From THE HOP.

A, young hair; B, mature hair; s.c, secretion under the cuticle.

have noticed, and to be formed indeed by decompositions which take place during the construction of the latter, we should not be warranted in ignoring their possible utility to the plant, nor the probability that many of them may be of nutritive value. We have seen that in the decomposition of amygdalin by its appropriate enzyme emulsin, besides the undoubtedly nutritive sugar there is a production of prussic acid and benzoic aldehyde. Some plants. have been shown to be capable of utilising the former of these, toxic as it is to animal life.

The bye-products include bodies of very varying degrees 
of complexity, some nitrogenous and others not. Among the former may be mentioned the great group of the alkaloids, which have not so far been found able to minister to the nutrition or growth of the plant, though their nitrogen is in organic combination. If a plant is supplied with them, but with no other form of combined nitrogen, it is rapidly starved. Many physiologists consider this group to belong rather to the definite excretions of the plant than even to its bye-products. They are always deposited in regions which are situated well away from the seats of active life, such as the bark of trees, the pericarps of fruits, \&c.' It is apparently very difficult to draw a distinct line of separation between excretions and byeproducts, just as it is to distinguish clearly between the latter and secretions.

The amidated fatty acids, as we have seen, generally occur in direct relation to nutrition. We have examined the part played by leucin and asparagin in proteid construction and metabolism. Several other related substances are met with in various plants, but how far they are available for nutrition and how far they are merely bye-products is uncertain. Such substances are xanthin and glycin, which can be extracted from various cells. The latex of plants frequently contains many of these substances. Caoutchouc is also a frequent constituent of latex.

Among the non-nitrogenous bye-products may be mentioned the great variety of yegetable acids. Conspicuous among these are tartaric, malic, citric, and acetic acids. They are usually regarded as arising in the course of the katabolic processes, but it is at least possible that some of them may be formed in the elaboration of food from the raw materials absorbed, having thus their origin in anabolism.

The bye-products include also a variety of aromatic substances. Mention has already been made of tannin, and its position discussed. In addition we may include phloroglucin and a variety of aromatic acids, such as 
benzoic, salicylic, \&c., but the nature of the processes which give rise to them is not well ascertained.

Certain decomposition products of cellulose may also be mentioned here. The lignin and suberin which are characteristic of woody and corky cell-walls arise in this way. During their formation, which takes place in the substance of the cell-wall, they can be removed by appropriate solvents, leaving the cellulose skeleton which they have been gradually replacing. These differ from most of the substances described in that they can be produced in the walls of cells that have lost their protoplasm, so that their formation is not directly dependent on metabolism.

We have again the odorous substances, and the colouring matters other than those already mentioned. Many colouring matters are products of the decomposition of chlorophyll, especially certain of those to which the autumnal tints of leaves are due. One of this group, xanthophyll, is a bright yellow pigment which is always associated with the chlorophyll, though in varying amount.

We have finally in connection with the metabolic processes to touch upon the excretions of plants. The term must be used in a wide sense to include all such substances as are undoubtedly withdrawn from the seats of active life, whether thrown off from the plant-body or not. The excreta which are completely eliminated are few; under normal conditions only the carbon dioxide and water which are products of respiration can be specified. Under abnormal conditions volatile compounds of ammonia or ammonia itself may be added to these. But there are certain other substances which are thrown off by a few plants, and for them some of these subserve definite purposes. Perhaps the most frequently occurring instance of these is the sugary solution known as the nectar, which is so common in flowers, and which is excreted usually to serve as an attraction to insect visitors. Mineral matters 'such as calcium carbonate are in some cases excreted on to the surface of the leaf, sometimes by special glands as in 
certain Saxifrages. In these it aids in the formation of a subsidiary water-absorbing apparatus as will be mentioned in a subsequent chapter.

In most cases the materials which we are discussing are not thrown off from the plant, but are removed to parts which are not concerned in the vital processes to any very great extent. Etherial oils are found deposited in special cavities in leaves, stems, and other parts (fig. 130). Mineral matters are often deposited in the substance of cell-walls. The oxalate of calcium occurs frequently in
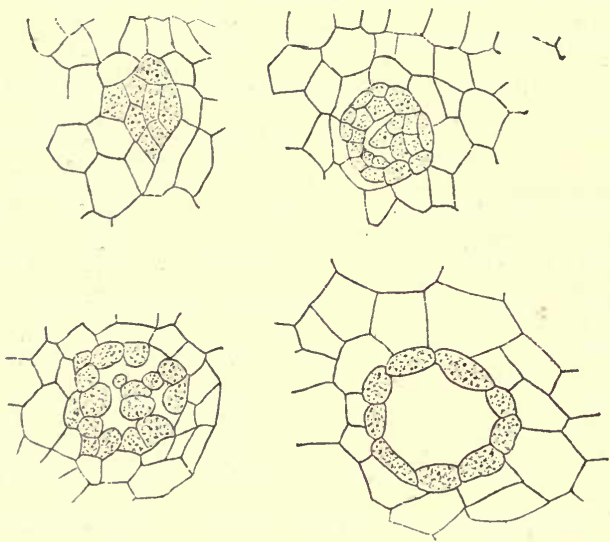

Fig. 130.-Development of Lysigenous Gland in STEM OF Hypericum. THE FOUR FIGURES REPRESENT SUCCESSIVE STAGES. $\times 250$.

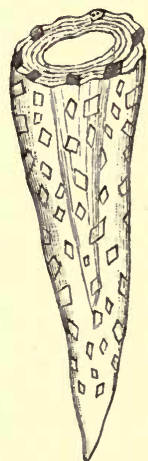

FIg. 131.-CRYSTALS OF CALCIUM OXALATE IN WaLL OF CELL OF THE BAST OF Ephedra.

this situation (fig. 131). In other cases it is deposited in special cells, where it forms clusters of crystals of characteristic shape (fig. 132, A, B). In these cases the cluster of crystals is usually invested by a delicate skin derived from the protoplasm, thus shutting it completely off from any participation in the metabolism of the cell in which it lies.

Carbonate of calcium may also be deposited in the substance of the cell-wall, or of protrusions from it, as in the cystoliths of Ficus, Urtica, and other plants (fig. 133). 
Silica again is accumulated in the epidermis of many grasses, and of the horsetails (Equisetum).

Though many of these substances, both excretions and bye-products, are of no value for nutrition, some of them may play a very important part in the defence of plants against their natural enemies, their nauseous smell or flavour preventing their being eaten by animals, \&c. Some odours and the nectar found in flowers are doubtless of great service in attracting insects, which assist in the
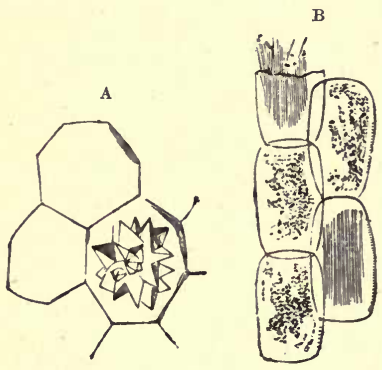

Fig. 132.-Crystals of Oxalate of Calcium. a, From Beet (Spharaphides); B, FROM ARUM (Raphides).

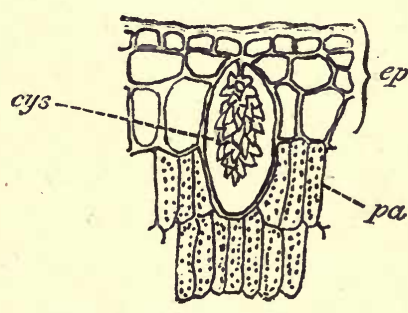

Fig. 133.-Section of Portion of LeAf of Ficus, showING Cystolith (cys) IN LARGE Cell OF THE THREeLAYERED EPIDERMIS $(e p)$.

process of cross-pollination, to be discussed in a subsequent chapter.

Though we cannot trace the formation of all these various substances, both bye-products and excretions, directly to the self-decomposition of the protoplasm, but must regard them as formed partly by the processes of oxidation and reduction, which we have seen are often associated with its activity, and partly by subsequent further decompositions of bodies originally thus formed, we can trace them ultimately to protoplasmic activity, and may consequently regard their formation as belonging to the katabolic processes going on in the organism. 


\section{CHAPTER XIX}

\section{THE ENERGY OF THE PLANT}

THe various operations which we have seen are continually going on in the body of the plant involve the execution of a considerable amount of work. This is very evident when we observe only the enormous development of a large tree, and compare it with the relatively small seed from which it has sprung. Such a process of construction has involved the preparation of a vast quantity of highly complex material from very simple chemical substances. The processes incident to life also, though they may not lead directly to the formation of such substances, cannot be conducted without involving a considerable amount of work, whether the plant is a minute body consisting of a single protoplast, or an organism of a much higher degree of complexity.

We must therefore turn our attention to the question of the supply and utilisation of the energy at the expense of which the various processes of life are carried out. At the outset it will be well to consider what demands for energy we find presented by the plant, or what are the ways in which energy is expended or lost.

Some of these have been incidentally alluded to in the preceding chapters, though we have not specially regarded them from this point of view. We may refer especially to the very great evaporation of water from the living cells into the intercellular spaces, which we have seen is in some cases supplemented by an evaporation from the general external surface, when this is not covered by any very distinct cuticle. It is evident that the great quantity of water which is given off by the leaves of a sunflower, to 
which allusion has been made in an earlier chapter, cannot be evaporated without the expenditure of a considerable amount of energy, which presumably takes the form of heat. It has been computed recently that 98 per cent. of the energy of the rays of light which are absorbed by the chlorophyll is expended in causing this transpiration.

The great accumulation of material which is so marked a feature of the life of a plant is the result of work which has been carried out in the plant on the simple substances which are absorbed. We may distinguish here between such products as are destined for immediate or ultimate consumption, and those which become incorporated into the actual substance of the plant. The accumulation of the latter is permanent, and the energy which is used in their construction is not subsequently made use of in the working of the organism. That it is stored however is evident from the fact that it can be re-converted into heat if the substance is burned. As we shall see later the products which are ultimately consumed in the nutritive processes may be regarded as stores of energy as well as of nutritive material. In both cases however their construction involves the expenditure of a considerable amount of energy before they assume their recognisable condition.

Closely allied to these constructive processes we have the phenomena of repair and of growth. As we have not yet studied the latter process in detail, we may be content with pointing out that there are involved in it many changes of various substances, which call for the execution of considerable amounts of work, which in turn demand the expenditure of energy. Many organs carry out their growth under conditions of pressure; roots, for instance, often penetrating through stiff soils. Not only is energy necessary to produce the growth itself, but the pressure. upon the growing organs must be counterbalanced by the internal forces they exhibit.

Many of the humbler plants possess a considerable 
power of active movement or locomotion. Zoospores of many of the Algæ and Fungi, and the antherozoids of most of the Cryptogams effect this locomotion by means of cilia which wave to and fro vigorously in the water in which they find themselves. The proportionate amount of energy which they expend in this way is very great compared with the total amount which they possess. Other movements which are not dependent upon ciliary action are not uncommon. The amœboid movements of the Myxomycetes or slime fungi, the rotation and circulation of the sap in many cells, the other internal movements of protoplasm, the hitherto unexplained movements of diatoms and the oscillations of certain filamentous Algæ, illustrate these. All alike are dependent upon a certain expenditure of energy.

The so-called movements of the growing parts of plants are frequently quoted in this connection. As we shall see hereafter however these are usually changes of position induced by variations in the processes of growth, and may rather be referred to expenditure of energy in connection with the latter than to actual movement. The movements of adult organs are also effected by causes which correspond in great measure to those which modify growth, being generally brought about by such variations in the turgescence of particular cells or groups of cells as those upon which we shall see growth largely depends. In this sense they are to be associated with modifications of the hydrostatic tensions in the parts concerned. A certain amount of expenditure of energy in the cells concerned is however most probable, though it is uncertain how far such changes as modify the resistance of the protoplasm to the passage of water through it involve the application of energy.

We have instances of what we may call the passive escape of energy in the shape of heat, and to a less extent in the manifestation of the so-called phenomena of phosphorescence. Heat is lost to the plant in many ways, one 
of which, the evaporation of the water of transpiration, has already been mentioned. Another almost equally important source of loss is radiation from the general surface. This is greatest from flattened members of the plant, such as leaves.

The temperature of the plant is very largely influenced by that of the air, and no doubt interchanges of heat take place in both directions. But it must not be concluded that the temperature of the plant and that of the air always vary together. On the contrary, radiation under some conditions may go on until the plant is several degrees colder than the surrounding air. This is probably the explanation of the ready formation of dew and hoar frost on the surfaces of leaves at certain seasons of the year. It is quite of frequent occurrence again that a plant or part of a plant may have a much higher temperature than the air, and hence a copious radiation may take place. During the processes of germination the temperature of the seed may be as much as $20^{\circ} \mathrm{C}$. above that of the air. The opening of flower buds is also attended by the attainment of a high temperature and a consequent escape of heat.

If we turn again to plants with a watery environment, the loss of heat may be observed under appropriate condi tions. It is well known that the processes of alcoholic fermentation provoked by the yeast-plant are attended by the liberation of heat, which is given off by the active cells, and causes a considerable rise of temperature in the fermenting liquid.

We may infer also from a consideration of the various processes we have studied, and from the fact that they are carried out most advantageously within a certain relatively small range of temperature, that the maintenance of such a temperature is a great desideratum to the plant. There is not a very complete mechanism in the plant to secure this. object, for the organism generally becomes of about the same temperature as the medium in which it grows, though the process of adjustment is often very slow, the tissues 
being generally very poor conductors of heat. Still it seems not improbable that a certain amount of energy is devoted to the attainment of the range which is most suitable for the vital processes. Though the dominating factor in the determination of the plant's temperature is to be looked for in the environment, the occurrence of the warmth of germination and of the expansion of the flowerbuds is an indication that it is not the only one.

A fuller consideration of the relations of the plant to heat must be deferred to a subsequent chapter.

The evolution of light by plants is a comparatively rare phenomenon, being probably confined to certain Fungi, though it has been attributed also to a few species of Algæ. It must call, however, for a certain expenditure of energy in such cases as have been authenticated.

If we turn now to consider the sources of this energy, it is evident that they must be in the first instance of external origin. The radiant energy of the sun indeed is the only possible source which can supply it to normal green plants. The question of the absorption of this energy has already been incidentally alluded to when we discussed the chlorophyll apparatus, but it may now be examined more closely.

The rays which emanate from the sun are generally alluded to as falling into three categories, those of the visible spectrum, those of the infra-red, and those of the ultra-violet. The second of these are frequently spoken of as heat rays, and the last as chemical.

The greatest absorption of energy appears to take place in consequence of the peculiarities of chlorophyll. As we have seen, this substance, whether in the plant or when in solution in various media, absorbs a large number of rays in the red and in the blue and violet regions of the spectrum, together with a few others in the yellow and the green.

The solar spectrum after the light has passed through a solution of chlorophyll is seen to be robbed of rays in these regions, and hence to present the appearance of a 
band of the different colours crossed by several dark bands (fig. 134). The greater part of the energy so obtained in the cells which contain the chloroplasts is at once expended, partly in constructing carbohydrate food materials and partly in evaporating the water of transpiration. The latter process is much the more expensive; recent observations have made it probable that 98 per cent. of the radiant energy actually absorbed during bright sunshine is at once devoted to this purpose.

When we speak of radiant energy we must remember that the rays of the visible spectrum do not supply all the

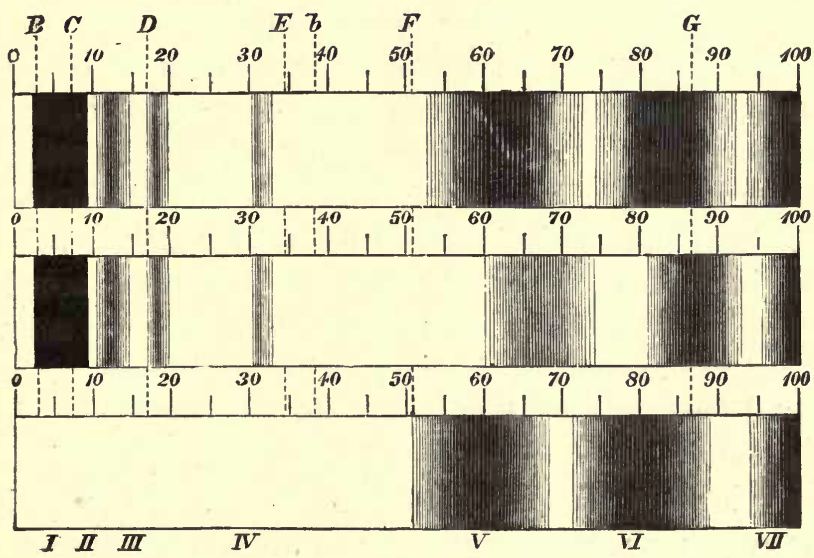

Fig. 134.-Absorption Spectra of Chlorophyll and XaNthophyll. (After Kraus.)

energy which the plant obtains. It has been suggested by several botanists with considerable plausibility that the ultra-violet or chemical rays can be absorbed and utilised by the protoplasm without the intervention of any pigment such as chlorophyll. There is some evidence pointing to this power in the cells of the higher plants. Certain bacteria also construct organic material from simple compounds of nitrogen and carbon dioxide, though it is not probable that they utilise radiant energy directly.

Finally we have evidence of the power of plants to avail themselves of the heat rays. The relations existing 
between the organism and its environment have already been mentioned. Not only can the air rob the plant of heat by radiation, but when its own temperature is high it can communicate heat to it in turn. Leaves have been proved to absorb heat with great avidity, particularly those which are succulent or fleshy, a difference of more than $20^{\circ} \mathrm{C}$. having been noted between their temperature and that of the air. The direct absorption of the rays of heat from the sun has also been noted, apart from the temperature of the air through which the rays were passing.

The supply of radiant energy is very much in excess of the amount which is needed for the internal work. Indeed its absorption by the leaves would be a source of considerable danger to the plant were it not for the cooling effect of transpiration, which we have seen dissipates 98 per cent. of it during bright sunshine. No doubt this dissipation is one of the chief benefits secured by transpiration.

It is evident, however, that in the general economy of the plant something further must be at work in connection with the supply of energy. The absorption of these external forms must take place at the exterior of the plant, while many of the processes of expenditure are carried out in parts which are more or less deep-seated. We are obliged to turn our attention, therefore, in this connection as in that of the construction and utilisation of food, to processes of accumulation, distribution, and economy.

We may ask ourselves what is the immediate fate of the energy absorbed. It enters the plant in what is known as the kinetic form. A very considerable part of the kinetic energy of the sun's rays, we have already seen, is devoted at once to the evaporation of the water of transpiration, but some of it is employed by the chloroplasts to construct some form of carbohydrate. The energy so applied can be again set free by the decomposition of this formed material. If the latter were burned its combustion would be attended by the evolution of a certain definite amount of heat. This heat would represent the energy that had 
been applied to the construction of the material so burned. Any accumulation of material in the body of the plant represents therefore not only a gain of weight or substance, but a storage of energy. This has disappeared from observation during the constructive processes, but can be liberated again during their decomposition and applied to other purposes. Energy which has thus been accumulated and stored is known as potential energy, to distinguish it from the actual or kinetic energy originally absorbed. The formation of material in the plant therefore involves a storage of energy in the potential form, and wherever such material is found there is an accumulation also of energy which can be liberated with a view to utilisation at any point to which the material has been transferred. The translocation of material therefore involves also a distribution of the energy which, originally absorbed as the kinetic energy of light or heat, has been applied to constructive processes, and has consequently been made potential.

From these considerations we may see that plants which absorb elaborated food from their environment have a source of energy therein. This food is a store of potential energy which is absorbed as such, and rendered kinetic subsequently.

It is this potential energy on which the plant depends for the various processes which go on in such cells as are not the recipients of external kinetic energy. Even in the cells which absorb the latter a certain amount of potential energy also is present, which represents what has been stored by them in the constructive processes they carry out, or has reached them in the shape of complex materials formed originally in other cells.

It is mainly on this store of energy that not only the whole organism but every cell depends for the execution of its vital processes. Each cell is practically the seat of the liberation of this potential energy, or its conversion into the kinetic form, during the decompositions which take place within it. 
The protoplasm itself contains a store of such potential energy. We have seen that it can only be constructed at the expense of food supplied to it. The formation of the protoplasm which follows the supply of food to the cell involves work, and the energy so used is partly changed from the kinetic to the potential condition. When the protoplasm undergoes what we have called its selfdecomposition, which is continually going on, a certain amount of this potential energy is liberated and can be observed and measured in various ways. When destructive metabolism is active we have already noticed that there is usually a rise of temperature, as in the processes of the germination of seeds. A certain amount of the liberated potential energy in this case manifests itself in the form of heat. A vegetable cell which obtains no direct radiant energy from without can consequently obtain the energy it needs from within itself, by setting up decomposition either of its own substance or of certain materials which have been accumulated within it.

The supply of elaborated material to a cell and that of available potential energy within it are not however exactly equivalent. A certain part of the transported material is devoted to the maintenance of the fabric of the cell. The protoplasm in a growing cell is permanently increased ; frequently its cell-wall is permanently thickened. In these cases the whole of such material is not subjected to subsequent decomposition, but much remains unchanged during the plant's life. The cell is consequently never found to be capable of giving up to the plant of which it is a member the whole of the potential energy which reaches it. If we consider the round of the metabolic changes which take place in such a cell, we find that energy is absorbed to construct its substance, and that as the latter undergoes self-decomposition energy is again liberated. But a certain part of what is supplied to it is permanently retained in potential form, and hence every cell is dependent for the maintenance of its energy upon a constant 
supply of complex material, whose subsequent decompositions will replace the amount of energy which has been utilised in the permanent increase of its substance.

The translocation of constructed materials which we have already considered must be regarded therefore not only as furnishing the materials for nutrition and growth, but as carrying or distributing throughout the plant-body the kinetic energy absorbed as light or heat by the cells to which these forces are originally supplied. The chlorophyll apparatus is an important piece of mechanism for the accumulation of energy which is subsequently distributed and utilised wherever need arises for it. This is true also of all cells which have the power of absorbing kinetic energy in any form.

The absorption and fixation of energy involved in the photosynthetic processes carried out by the chlorophyll apparatus can be easily observed, and the immediate fate of such energy can be readily determined. The accumulation of the energy of heat is not so easy to trace, but there is no doubt that it proceeds along similar lines. Part of it can travel as kinetic energy, as heat is slowly conducted along the tissues of the plant, but ultimately some at any rate becomes potential.

We see therefore that wherever any substance that has been manufactured by the plant is stored in a cell, that cell is thereby put in possession of a certain amount of potential energy corresponding to the quantity of such stored material. Even the living substance itself may be looked upon as a further store of energy, as it can liberate it by its own decomposition.

Each cell is thus supplied through the general activities of the whole plant not only with the food it needs for its nutrition, but also with the energy required for carrying out its vital processes. The ultimate utilisation of the stored energy is consequently a process which must be studied by a close scrutiny of the internal work of the cell itself. 
The transformation of potential into kinetic energy is associated with decomposition just as the converse process is bound up with construction. Destructive metabolism in the cell is then the means by which its energy is made available. We have seen that the processes of this katabolism go on in the interior of each cell. Each liberates at least as much energy as it requires for the maintenance of its life and the discharge of its particular functions.

The processes associated with the utilisation of the stored energy are, then, chemical decompositions in which various constituents of the cell are involved. We may divide them into two series, in the first of which the protoplasm itself takes part, and which comprise the processes in which its own breaking down takes place. In the second series it effects the splitting-up of other bodies without a necessary disruption of its own molecules.

We have already discussed the first of these two series, which involves the phenomena of respiration. We have seen that during the life of the cell oxygen is continuously absorbed by the protoplasm; the immediate result is its self-decomposition, attended by the formation of substances of very different degrees of complexity, the ultimate simplest ones being carbon dioxide and water. If the self-decomposition of protoplasm involved such a splitting-up as would lead to the formation of nothing but these, together with an equally simple form of combined nitrogen, nearly all the potential energy of the cell would be liberated. But we have seen that this does not take place. Carbon dioxide and water are accompanied by many other products of a fairly complex character. There is not, therefore, an evolution of nearly the possible amount of energy, and what is set free is mainly employed in the reconstruction of protoplasm from these products and the new food supplied. As carbon dioxide and water are continually escaping from the cell in appreciable quantities, it is evident that apart from any increase of its fabric the store of its potential energy is gradually diminished, and that it can only continue 
its life if new stores are supplied in the shape of further decomposable material which is capable of taking part in the renewed building-up of the living substance.

Respiration is thus to be looked upon as a process very largely connected with the utilisation of the store of energy which each cell possesses, and to be perhaps primarily concerned in the transformation of that energy from the potential to the kinetic form. The oxygen appears to be necessary mainly for the purpose of exciting those decompositions of the protoplasm which are so dependent upon its instability. It is not, however, certain that this is the only part it plays. It is possible that some of the products of the protoplasmic disruption are oxidisable substances, and that to a certain extent a direct oxidation of them takes place. There is undoubtedly some evidence pointing in that direction.

We have, besides the respiratory processes, another series of chemical decompositions going on in plants, in which the self-decomposition of protoplasm is not necessarily involved. We have seen already that many processes of oxidation and reduction are probably always taking place among the substances which are in solution in the water with which the cytoplasm is saturated. Besides these, other changes take place in which no oxidation is involved, and this whether oxygen is present or not. If the access of oxygen to a protoplast is interfered with, its normal respiration soon ceases, but very frequently other changes supervene, involving decompositions of a different character, which yield, at any rate for a time, the energy required for life.

Turning from the question of respiration to study other changes which subserve a similar purpose with regard to the local supply of energy, we may first examine such processes as are oxidative. In them all we cannot fail to mark the activity of the protoplasm in carrying them out. The living substance does not however act as a general oxidising agent, but different protoplasts possess specific powers. Certain micro-organisms can cause the oxidation 
of ammonia and the consequent formation of a nitrite; others can convert the nitrite into a nitrate, but neither can do the work of the other. Others have not such limited powers; a certain bacterium can cause the oxidation of alcohol to acetic acid, and after the exhaustion of what alcohol may be present, can further oxidise the acetic acid to carbon dioxide and water. The exact way in which the protoplasm acts as a carrier of the oxygen without apparently undergoing decomposition is very obscure. It may perhaps combine with the oxygen and pass it on to these oxidisable substances, acting as a carrier only.

It has recently been found that besides exerting a direct oxidative power, protoplasm can secrete an enzyme, or perhaps a variety of enzymes, each with a special peculiarity, through whose instrumentality the oxidation is effected. These enzymes have been termed oxidases, and they are probably wide-spread in the vegetable kingdom. A discussion of their peculiarities would be beyond the scope of this volume, but we may call attention to their general features.

The first one discovered is known as laccase; it has a very wide distribution, occurring in the roots, stems, and leaves of various plants, and in a very large number of fungi. It appears to oxidise various constituents of plants, but particularly the colouring matters. Another, known as tyrosinase, occurs in other fungi, and oxidises chiefly tyrosin. Others oxidise various colouring matters, together with tannin.

Many very complex disturbances set in when a normally. respiring plant is cut off from a supply of oxygen. Death does not immediately supervene as might almost be expected. Instead, the partial asphyxiation or suffocation stimulates the protoplasm to set up a new, and perhaps supplementary, series of decompositions, resulting in the liberation of energy as do those of the respiratory process. We have already noticed that under such circumstances the exhaling stream of carbon dioxide can still be observed. This led 
originally to the view that the protoplasm excited these decompositions of some complex substance in the cell to obtain oxygen from it, which should replace the oxygen whose access had been stopped. The ultimate changes were accordingly held to go on with but slight interruption, but the source of the oxygen taking part in them was different. On this account the process was termed intramolecular respiration. The term is rather an unfortunate one, for as we have seen the study of the ordinary respiratory processes has shown that the molecule of the living substance is the seat of the changes they involve, and hence that all respiration is intra-molecular. Moreover if the object of the decomposition is to provide oxygen to replace that which has been cut off, these transformations precede the actual respiration, which must then be set up as soon as the oxygen is liberated as suggested. Many botanists now prefer to speak of decompositions taking place in the absence of a supply of free oxygen as anaerobic respiration. They thus include as respiratory changes all the decompositions primarily intended to liberate energy, and divide them into those which are aerobic or dependent on oxygen, and those which are anaerobic. The latter need not involve the co-operation of oxygen in the disruption of the molecule. The object sought is energy and not oxygen.

It is uncertain how far the self-decomposition of protoplasm is concerned in these anaerobic respiratory processes. Probably not to any great extent; it is more likely that it secures the decomposition of other substances without being itself materially used up. Such a course would be much the more economical, not involving the consumption of much energy in reconstructive processes. This cannot however be regarded as finally established.

The substance which seems most readily available for this purpose is sugar. Under the conditions mentioned it becomes decomposed or broken up entirely, the resulting 'products being carbon dioxide and alcohol. The process of alcohol-formation which was for so long a time associated 
exclusively with the word fermentation, was first observed in connection with the life of the yeast-plant. It has however since been ascertained to be much more widespread, and to be indeed the most common of the anaerobic respiratory processes. In cases where the metabolic activities are very great, as in germinating peas, we find this process supplements the ordinary respiration, for alcohol can be detected in their cells in small quantities. The same thing has been noticed in the leaves of the vine. We must suppose here that the amount of oxygen absorbed is insufficient for their requirements, and that partial asphyxiation results.

Till quite recently it was held that alcoholic fermentation was conducted exclusively by the activity of the protoplasm of the cells in which it was observed. It has been ascertained however that it may also be caused by the action of an enzyme, which is secreted under conditions of incipient asphyxiation by many cells, and which is formed in the yeast-plant even in the absence of such stimulus.

Though the term fermentation was originally applied and confined to the formation of alcohol, it is now usual to extend it far more widely. Many other processes of similar nature have been discovered, nearly all of which at first were found to be carried out through the agency of microbes or higher fungi. Hence the meaning of the term has been extended to include them, and the organisms themselves have been called ferments. As however these processes have come to be recognised as normal in many of the higher plants, and to be carried out in them by the protoplasm of particular cells, this peculiarity is seen not to be special to the microbes and the fungi. The idea was soon transferred to the protoplasm in general, and this property of setting up anaerobic decomposition has become known as its fermentative power. The very similar processes set up through the enzymes which we have discussed in connection with digestion, show us another manifestation of the same fermentative power. All these processes can therefore be 
classed under the one term fermentation. We have seen that all the katabolic changes in which the self-decomposition of the protoplasm is not directly involved may be carried out either by the intervention of the living substance itself or an enzyme secreted by it. The oxidation of various matters is in some cases confined to the substance of the protoplasm itself, and is in others carried out in its vacuoles by an oxidase; alcoholic fermentation is in some cells a matter initiated and carried on by their protoplasm, and in others is due to the enzyme secreted by them. The digestive changes can similarly be conducted by enzymes or by the living substance without their intervention.

We must not however include all digestive fermentative changes among anaerobic respiratory phenomena, if such inclusion involves the acceptance of the view that this is their primary purpose. Though they do effect the conversion of potential into kinetic energy, this is wholly subsidiary to their function in connection with the nutrition of the plant. We have seen that in the processes of germination the energy they liberate is so far in excess of the requirements of the cells that a large amount escapes in the form of heat. For them to work indeed there must be an initial supply of energy, which is probably supplied to them in a similar form, for at $0^{\circ} \mathrm{C}$. they are incapable of effecting any decompositions.

We must not suppose that anaerobic respiration is capable permanently of taking the place of the normal aerobic process. Though the stoppage of oxygen can be to a certain extent compensated for, the vital mechanism gradually becomes exhausted, and life ceases if the cessation of the supply is prolonged. In the higher plants anaerobic is at the best only capable of supplementing aerobic respiration, and that for but a limited period. The commencing asphyxiation serves as a stimulus to the protoplasm, which responds by setting up the anaerobic changes, but like all stimulations the ultimate effect is exhaustion and a failure to continue the response. 
There are other plants however which are incapable of carrying on their life in the presence of oxygen. They are of a very humble type, and occur only among the Bacteria and Fungi. An instance may be found in the organisms which induce the formation of butyric acid from sugar or lactic acid. If a few of these are sown in a suitable liquid, and this is then enclosed in a hermetically sealed flask from which free oxygen has been removed, they multiply with extreme rapidity, until indeed either their food supply is exhausted, or the waste products of their metabolism accumulate to an inhibitory extent. If a little free oxygen is admitted their activity ceases and death ensues, or they pass into a resting condition, which lasts as long as oxygen is present. 


\section{CHAPTER XX}

\section{GROWTH}

Is studying the growth of plants we must bear in mind the relation which it bears to the processes of metabolism which we have already discussed. We have seen that the constructive processes, partly anabolic and partly katabolic, are much greater than those which lead to the disappearance of material from the plant-body. The result of this is that there is a conspicuous increase in the substance of the plant, as well as an accumulation of potential energy which can be made use of by the plant through various decompositions which its protoplasm can set up. The great permanent accumulation of material is what we associate with the processes of growth. Here however we must distinguish between the increase of the living substance, which is essentially an anabolic process, and that of the manufacture of the framework, the construction of eellulose, wood, cork, and other products, which is the result of katabolism.

The growth of the living substance is always the result of constructive metabolism, and is attended by an increase of bulk and weight. The growth of an organ sometimes appears to be independent of such increase of weight: indeed, a diminution of the weight of the whole structure is sometimes noticeable. Thus in the case of a potato tuber allowed to germinate under such conditions as prevent the absorption of food materials from without, we. meet with a marked change of form, but, owing to the loss of moisture by transpiration, and of carbon dioxide as a consequence of its respiration or the katabolic processes 
going on in its tissues, the resulting plant weighs much less than the original potato.

This difference is however rather apparent than real. We shall see that the actual growth, as well as the manufacture of new cells, is confined to certain regions. In these

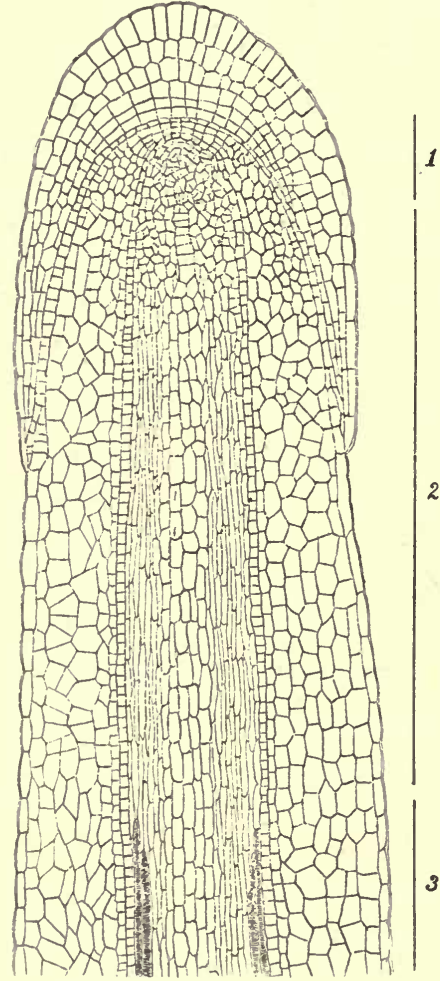

Fig. 135. - Longitudinal SECtion of Young ROOT, SHOWING STRUCTURE OF Growing Point. $\times 20$.

1 , zone of cell division ; 2 , region of greatest growth; 3 , region of complete differentiation. regions there is a considerable increase in bulk and weight, but as the materials which are used for the pur-

1 poses of this local growth are derived from substances stored up in the body of the tuber, the latter, the greater part of which is not at any time the seat of the growth, diminishes in weight and size to such an extent as more than to counterbalance the gain in the growing regions. Hence the whole plant weighs less than the tuber, though considerable growth may have taken place.

Mere increase of weight in an organ does not, on the other hand, necessarily imply any growth. The deposition of reserve materials in many seeds does not take place to any great extent till their mature dimensions are reached, and growth is therefore completed. It involves, however, a considerable increase of weight.

Growth is in the strict sense, then, always associated with the formation of new living substance, and is ry 
generally accompanied or immediately followed by additions to the framework of the growing cells or organs. It is in nearly all cases attended by a permanent change of form. This is perhaps not so evident in the case of axial organs as it is in that of leaves and their modifications, though even in them it can be detected to a certain extent. It is much more conspicuous in the case of leaves, for the latter, as they expand from the bud, have usually a different shape from that of the adult ones, and the assumption of the mature form is a gradual process, taking place as the age of the leaf increases.

This change of form can be seen not only in the case of an organ such as a leaf, but also in that of the indi-

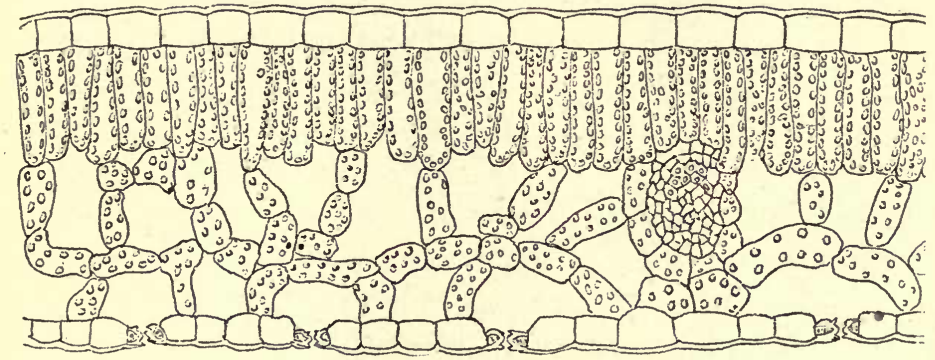

Fig. 136.-Section of Blade of Leaf, showing the Irregular Cells of the Spongy Mesophyll abutting on the lower epidermis.

vidual cells of which a plant consists. In the apical meristem of the root of a flowering plant the cells when first formed are almost cubical (fig. 135) ; after a little while we find many of them becoming elongated, and ultimately prosenchymatous. Many other cases can be noted, particularly the irregularly shaped cells of the spongy parenchyma of leaves (fig. 136), the stellate cells of the pith of certain rushes (fig. 137), the laticiferous cells of the Spurges, \&c.

Growth may, in the light of the considerations just advanced, be defined as permanent increase of bulk, attended by permanent change of form. We must not assume that every increase of bulk is necessarily growth; 
for, as we shall see, in growing cells and members there is a constant stretching of the cell or tissue by hydrostatic pressure or turgidity, which can be distinguished from growth by the fact that it can be removed, the result being a certain diminution of the size of the part under consideration.

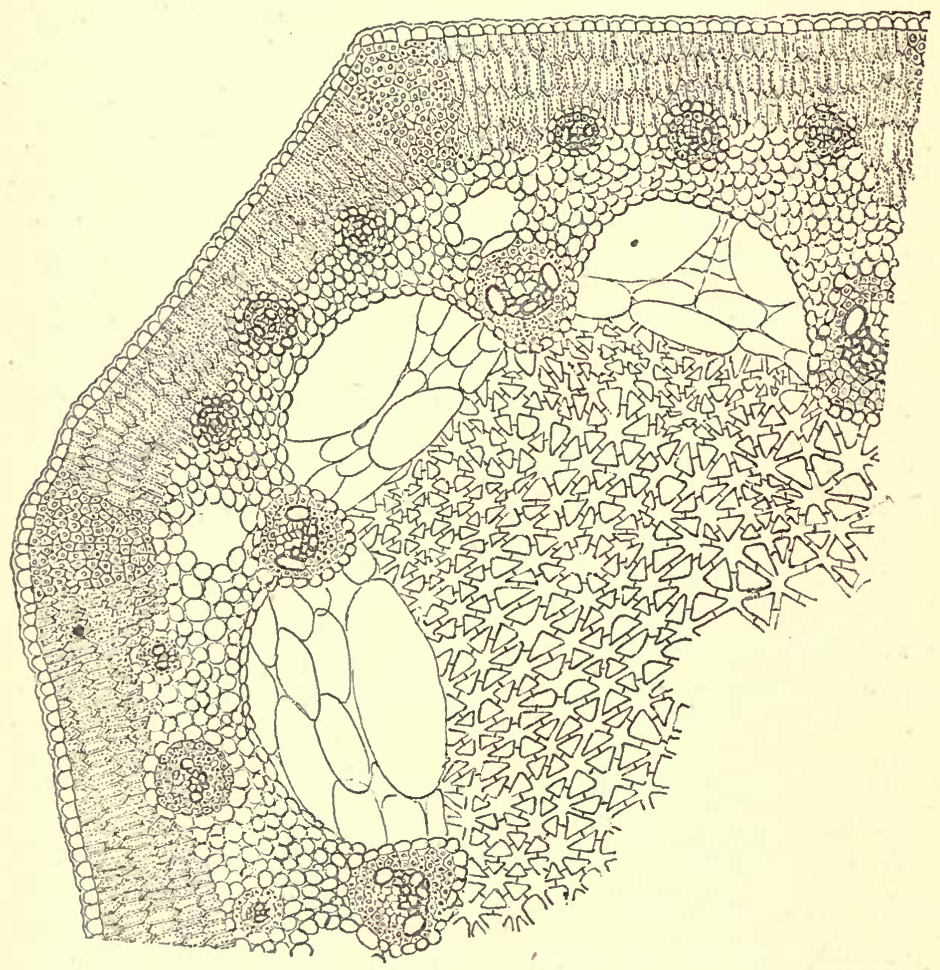

Fig. 137.-Pontion of Section of Stem of Rush, showing Stellate

Tissue of the Pith, with large Intercellular Spaces.

Growth in the lowliest plants may be co-extensive with the plant-body. In all plants of any considerable size however it is localised in particular regions, and in them it is associated with the formation of new protoplasts. We have already mentioned that in the sporophytes of all the higher plants there exist certain regions in which 
the cells are merismatic - that is, which have the power of cell-multiplication by means of division. In such regions, when a cell has reached a certain size, which varies with the individual, it divides into two, each of which increases to the original dimensions and then divides again. These regions have been called growing points (fig. 135); they may be apical or intercalary. In such stems and roots as grow in thickness there are other growing regions, which consist of cylindrical sheaths known as cambium layers or phellogens. By the multiplication of the protoplasts in these merismatic areas the substance of the plant is increased. In other words, as these growing regions consist of cells, the growth of the entire organ or plant will depend on the behaviour of the cells or protoplasts of which its merismatic tissues are composed.

The growth of such a cell will be found to depend mainly upon five conditions: (1) There must be a supply of nutritive or plastic materials, at the expense of which the increase of its protoplasm can take place, and which supply the needed potential energy. (2) There must be a supply of water to such an extent as to set up a certain hydrostatic pressure in the cell. This condition we have already considered in an earlier chapter, in which we discussed the relation of protoplasm to water. (3) The supply of water must be associated with the formation of osmotic substances in the cell, or it cannot be made to enter it. In the absence of the turgescence, which will be the result of the last two conditions, no growth is possible for reasons that will presently appear. (4) The cell must have a certain temperature, for, the activity of a protoplast is only possible within particular limits, which differ in the cases of different plants. (5) There must be a supply of oxygen to the growing cell, for, as we have seen, the protoplast is dependent upon this gas for the performance of its vital functions, and particularly for the liberation of the energy which is demanded in the constructive processes. This is evident also from the consideration that the growth 
of the cells is attended by the growth in surface of the cell-wall, and as the latter is a secretion from the protoplasm, a product, that is, of its katabolic activity, such a decomposition cannot readily take place unless oxygen is admitted to it.

Growth so far as it implies only the formation of living substance is thus a constructive process. It is however intimately associated with destructive metabolism or katabolism, the latter being involved in the construction of the increased bulk of the framework of the cell or cells, and being essential to supply the energy needed for the constructive processes.

When the conditions mentioned are present, the course of the growth of a cell appears to be the following: the young cell, immediately it is cut off from its fellow, absorbs water in consequence of the presence in it of osmotically

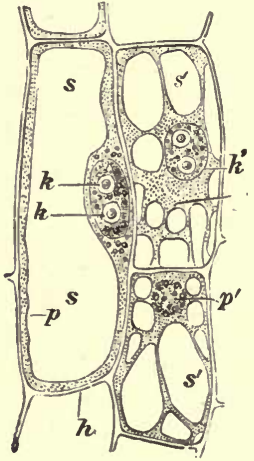

Fig. 138.-Adult Vegetable

Cells. $\times 500$. (After Sachs.)

$h$, cell-wall; $p$, protoplasm; $k k$, nucleus, with nucleoli; $s s^{\prime}$, vacuoles. active substances. With the water it takes in the various nutritive substances which the former contains in solution. There is set up at once a certain hydrostatic pressure due to the turgidity which ensues upon such absorption, and the extensible cell-wall stretches, at first in all directions. The growth of the protoplasm at the expense of the nutritive matter for a time keeps pace with the increased size of the cell, but by and by it becomes vacuolated as more and more water is attracted into the interior. Eventually the protoplasm usually forms only a lining layer to the cell-wall, and a large vacuole filled with cell-sap occupies the centre (fig. 138). The growtl of the protoplasm, though considerable, is therefore not commensurate with the increase in the size of the cell. The stretching of the cell-wall by the hydrostatic pressure is 
fixed by a secretion of new particles and their deposition upon the original wall, which as it becomes slightly thicker is capable of still greater extensibility, much in the same way as a thick band of india-rubber is capable of undergoing greater stretching than a thin one. The increase in surface of the cell-wall is thus due firstly to the stretching caused by turgidity, and secondly to the formation and deposition of new substance upon the old. The latter only is the growth of the cell-walls; the former can be removed by irrigating the cell with a solution of a substance, such as common salt, which will rob it of the water it contains. The constructive changes leading to the formation of new protoplasm are attended in this process by the katabolic formation of cell-wall and other substances, such as the osmotic bodies which are necessary to draw the water into the cell. The supply of oxygen is needed to allow the protoplasm to undergo these katabolic decompositions, enabling it thus to prepare the several products spoken of, and to gain from such decompositions the energy which must be expended upon the construction and reconstruction of the living substance, and used in the secondary chemical changes which supervene.

The process of the growth of a cell is limited in its extent, though the limits vary very widely in different cases. In some, cells grow only to a few times their original dimensions, in others they may attain a very considerable size. In any case, however, we can notice that the rate of growth varies regularly throughout the process; it begins slowly, increases to a maximum, and then becomes gradually slower till it stops. The time during which these regular changes in the rate can be observed is generally spoken of as the grand period of growth.

Changes in the shapes of cells arising during growth. depend upon two factors. The capacity of the cell to yield to hydrostatic pressure may be affected differently in different directions by the conditions of the cells which surround it. In the merismatic tissue of a growing point 
there is generally least resistance on the side of the free apex of the organ, and hence an increased protrusion of the latter results. Whatever may be the distribution of such pressure the growth of the cell will be greatest in the line of least resistance. If any internal cause should give rise to differences in the uniformity of hydrostatic pressure in all directions, the growth will be most extensive in the line of the greatest. In the second place the extensibility of the cell-wall may be locally modified by the protoplasm, so that a uniform internal hydrostatic pressure may affect one part more than another, and the growth consequently will become irregular, giving rise in many cases to cells of curious form.

If we consider the behaviour of a growing organ in the light of these facts, we shall see that, like the cell, it must show a grand period of growth. If we take the case of a root, in which the changes can be traced most easily on account of the simplicity of its structure, we find that just behind the apex the cells are all in active division. Growth is small and consists mainly in an increase of the quantity of protoplasm, for the cells divide again as soon as they have reached a certain size. As new cells are continually formed in the merismatic mass, those which are farthest from the apex gradually cease to divide and a different process of growth takes place in them, which is associated more particularly with the formation of the vacuoles and consequently with the establishment of considerable hydrostatic pressure, thus causing the bulk of the cells to be greatly enlarged, as we have described. Hence it is here that the actual extension in length of the root goes on, and the cells reach the maximum point of the grand period. They then gradually lose the power of growth, the oldest ones or those farthest from the apex parting with it first, and they pass gradually over into the condition of the permanent tissue (fig. 135). In this way each zone of the root which may be distinguished goes through a grand period of growth. At first when the cells are merismatic, growth is 
at a minimum, it gradually becomes accelerated, reaches a maximum, and slowly ceases, exactly as did that of the cell which we first considered. By careful examination of a growing root it can be found that the growth is greatest just behind the merismatic region. If a young root be taken and marked out into zones by a series of short lines at equal distances apart (fig. 139, A), and then allowed to continue its growth, it will be found that the lines remain close together at the apex and for a very short distance from it. Then they become separated by broader spaces (fig 139, B). Further back still the original intervals between the lines will again be found to

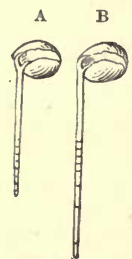

Fig. 139.-Germinating BEAN, SHOWING GROWTH of the RAdicle. be almost unaltered. The second region corresponds to the part where the cells are undergoing the enlargement described. The total growth of the root is, of course, the sum of the increments of all the zones so marked out.

The same order of events may be ascertained to take place in the stem, but in this region it is complicated by the occurrence of nodes and internodes. Growth is almost confined to the latter, each of which passes through a similar grand period. The growth of the stem is the algebraical sum of the growth of the internodes, many of which may be growing simultaneously and which will be at any particular moment therefore at different parts of their grand period. The region of growth in the stem is, as a rule, much longer than that in the root.

The growth of the leaf shows a little variation. The apical growth, as a rule, is not very long continued, and the subsequent enlargement of the leaf is due to an intercalary growing region near the base. This area has the merismatic cells at about its centre, and regions of greatest growth are on both sides of it. This can be traced more easily in the elongated leaves of Monocotyledons than in those of Dicotyledons. 
The grand period itself is not quite uniform, as the rates of growth in the active region may and do vary witl. changes in external conditions, and with differences in activity in the protoplasm from time to time. This can be observed very favourably in the case of a growing stem, which shows considerable differences in its rate of growth during twenty-four hours. The growth is greatest during the night and least during the day, and the variations in the rate are fairly regular, the total growth during successive periods of twenty-four hours being on the whole uniform. This regular variation of the rate constitutes what is known as the daily period of growth in length.

An instrument by which the progress of growth of such a structure as a stem can be ascertained and registered is

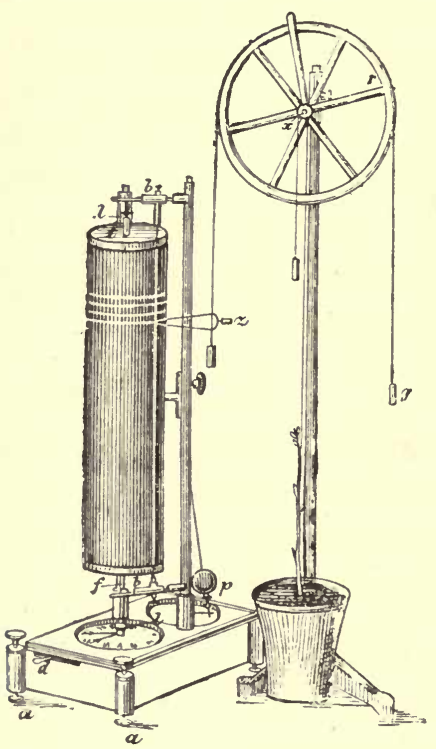

Fig. 140.-Pfeffer's automatically Registering Auxanometer. (After Detmer.) counterbalancing weight $g$.
The drum is caused to rotate slowly upon its axis by clockwork, so that the indicator traces a line along its surface.

A very convenient form which registers the gradual increase in length automatically, has been constructed by Pfeffer, and is represented in fig. 140. A thread attached to the plant passes over the small wheel $x$, which is cemented on the large wheel $r$, and accurately centred about the same axis. A thin lever $z$ is attached to another thread which is passed over the large wheel, and is made to write upon the smoked surface of a paper fastened round the cylindrical drum $t$. The string is kept tight by the

known as an auxanometer. 
So long as no growth takes place this line is horizontal, but as the indicator is displaced downwards by the descent of the small weight attached to the first cord, which is attendant upon any elongation of the axis of the plant, the line actually traced during growth is a spiral. The rate of the drum's revolution being known, the amount of the elongation of the axis per hour can easily be calculated. The actual augmentation of the plant's axis is magnified in the record, in a ratio dependent upon the ratio between the radii of the large and small wheels $r$ and $x$.

For the sake of simplicity of description it has been assumed, in what has already been said, that the turgidity of the cells in the growing member is uniform. This, however, is far from being the case. There is generally a fairly rhythmic variation in this turgidity in the different parts of the elongating member. The simplest case which we may consider is one which shows a difference in structure on two sides; such a member is described as dorsiventral. The two sides will often show a difference of degree of turgidity and consequently of rate of growth. If we consider a leaf of the common Fern, we find that in its young condition it is closely rolled up, the upper or ventral surface being quite concealed. As it gets older it gradually unfolds and expands into the adult form. This is due to the fact that in the young condition the turgidity and consequent growth are greater on the dorsal side of the leaf, so that it becomes rolled up as described. As it gets older the maximum turgidity and growth change to the upper side and so it becomes unfolded or expanded. These two conditions are generally described under the names of hyponasty and epinasty respectively.

These conditions are not confined to the leaves of ferns, but may be detected in those of other plants, though to a less conspicuous degree. It is in consequence of them that the leaves of the bud always fold over the apex of the stem from which they spring. The opening and closing of certain flowers, such as the Crocus, depend upon similar variations. 
Cylindrical organs may exhibit similar phenomena. One side of a stem may be more turgid than another, and the maximum turgidity with its consequent growth may alternate between two opposite sides. The greater turgidity of the cells is often accompanied by an increased extensibility of the cell-walls of the turgid region. The growing apex of such a stem will alternately incline first to one side and then to the other, exhibiting a kind of nodding movement in the two directions. This is known as nutation, and is of very frequent occurrence, particularly in such stems as are slightly flattened instead of being truly cylindrical.

The region of maximum turgidity instead of occurring alternately on two opposite sides may pass gradually round the growing zone. The apex in this case will describe a circle, or rather a spiral, as it is elongating all the time, pointing to all points of the compass in succession. This continuous change of position has been described by Darwin as circummutation, and has been said by him to be universal in all growing organs. The passage of the maximum turgidity round the stem may vary in rapidity at different places, causing the circle to be replaced by an ellipse. Indeed the simple nutation spoken of above may be regarded as only an extreme instance of the latter.

Circumnutation is exhibited during growth also by the hyphæ of many fungi, some of which have a cœnocytic structure. In these cases the movement appears to be due to a rhythmic variation in the extensibility of the membrane, induced probably by the protoplasm. It cannot be caused by differences of turgidity on the two sides of the hypha as this contains only one cavity.

By these movements of the growing apices - movements incident to growth, and proceeding altogether from internal causes-many advantages are secured by the plant. In the case of a climbing stem, the circumnutation enables it to reach a support, round which it twines, so that with but little expenditure of substance it can secure access to 
more light and air, than it could obtain in its absence. Roots by the same method are enabled more easily to make their way through the crevices of the soil. The axis of the embryo shows in one or other of its parts strong hyponastic curvature, forming an arch which enables it to leave the seedcoats and make its way through the soil without damage to the young delicate plumule, its progress being helped by simultaneous circumnutation. On reaching the surface epinastic growth causes it to assume an erect position, the arch opening out till the axis is straightened. Coincidently with this change, circumnutation of the apical region replaces that of the portion of the axis which was at first arched.

During the period of growth the young organ is extremely sensitive to changes in its environment, responding to such stimulating influences by further modifications of its behaviour. These will be considered in detail in a subsequent chapter,

Besides the hydrostatic tension set up in the cells of the growing regions, the processes of growth are accompanied by the development of other tensions in the interior of the growing member. These appear to depend upon differences between the turgidities of their several tissue systems as these develop, and upon different rates of growth of different internal parts. If a petiole of Rhubarb is taken, and a thin strip is peeled from one side, it will immediately curl outwards. If it is then placed in apposition with the part from which it was cut, it will be found to be appreciably shorter than the rest of the petiole. If the petiole is carefully measured, and then deprived of its cortical covering by the separation of successive strips, the central part will be found to be slightly longer than the original petiole. In such a petiole the central part is clearly compressed by the external portions, and when these are removed it undergoes an extension which is the expression of the amount of such compression. Similarly the external parts are stretched longitudinally by the 
central region, and when they are freed from it, the recoil is accompanied by a diminution of their length. There is thus a longitudinal tension in the petiole, due to the greater turgescence of the central part, which stretches the outer portions, and is itself compressed by their greater rigidity resisting the hydrostatic extension. This tension is not due to greater growth, but to more pronounced turgidity, for if such a petiole is soaked for a time in salt solution till the water is in great part removed from its interior, and it has become flaccid, removal of the cortex is not accompanied by the same changes of dimension. A similar experiment may be performed on the hollow flowerstalk of a Dandelion. If it is slit into two halves by a vertical cut, the two parts curl outwards from each other, showing a similar tension in the internal regions.

Transverse tensions in young growing axes can also be demonstrated. The cortex is found to be strained outwards by the central tissues, so that if a ring of it is cut out of such an axis and split longitudinally, it shortens. If the split ring is again put back in its original position, it will not completely surround the stem. The central tissues are in a state of compression, and the cortex in one of extension, laterally as well as longitudinally, as in the other case already quoted.

Transverse tensions of a similar kind are set up in the course of the thickening of stems and roots by the activity of the cambium layer, by the division of whose cells new bast is formed behind, and new wood in front of it. The bast and cortex are thus compressed outwards, and the wood and pith inwards, on account of the formation of the new material. The phellogens which form rings of cork at various depths in the cortex, give rise to similar strains. Sheaths of new cells are intercalated in the substance of the delicate tissue, which thus becomes greatly thickened. These tensions are due to growth, and not, like the others, to turgidity of the tissues. They cannot consequently be removed by treatment with salt solutions. 
These tensions are capable of demonstration all through the life of such stems and roots as increase in thickness. They give us a partial explanation of the structure of the annual rings of wood which are exhibited by such stems and roots, and of the ruptures that are generally noticeable in the exterior of such parts.

In the absence of various external stimulating influences, which will be discussed later, young growing members show a tendency to elongate uniformly, so that the direction of their growth is a straight line. Though the apex of any of them may continually show the movement of circumnutation, the mature part generally takes up a fixed position, growing vertically or horizontally as the case may be. This position is, however, usually due to the combined action of a number of external forces acting upon the growing member. The inherent tendency just spoken of can be satisfactorily seen when, by artificially eliminating the action of such forces, the plant is not exposed to their stimulating influences. Such a tendency has been called Rectipetality. It becomes apparent also in the case of a member which has become curved, owing to the action of one or other of the stimulating influences referred to. If it is removed from the influence of the stimulus, it becomes straight again. 


\section{CHAPTER XXI}

\section{TEMPERATURE AND ITS CONDITIONS}

THE various processes which are characteristic of vegetable life only take place so long as the plant is exposed to a particular range of temperature, which lies between the freezing point of water and about $50^{\circ} \mathrm{C}$., a few exceptions on both sides of that range however being met with. It is consequently essential to the well-being of the organism that its temperature shall be maintained within those limits. While life is possible within this range it is not equally well manifested at all the points which lie between the limits; each vital function indeed shows considerable variation in this respect. There is a certain point, lying generally near the freezing point, below which it cannot be observed. There is another point near the upper limit, beyond which it is not carried out, and somewhere between them there is a point at which it is manifested most advantageously. These three points are known respectively as the minimum, maximum, and optimum temperatures for that function. These temperatures vary for each function which accompanies the life of any particular plant. They are not, moreover, in the case of a particular function, necessarily identical in different plants.

The process of photosynthesis, for instance, commences in the grasses at about $2^{\circ} \mathrm{C}$., while in the Potamogetons it cannot be detected below $10^{\circ} \mathrm{C}$. The absorption of water by the roots of the Turnip and other cruciferous plants may begin when the soil has a temperature but slightly above the freezing point of water; in the case of the Tobaccoplant it must be at $12^{\circ} \mathrm{C}$ at least. The lowest temperature 
for the germination of the seed varies between $5^{\circ} \mathrm{C}$. for the Wheat and $13^{\circ} \mathrm{C}$. for the Vegetable Marrow. The upper limit for this function in the cases of these two plants has been ascertained to be $37^{\circ} \mathrm{C}$. for the former and $42^{\circ} \mathrm{C}$. for the latter. The optimum point for the growth of the roots of a seedling of Maize is $27^{\circ} \mathrm{C}$., while the corresponding temperature for that of the Barley and Wheat is about $23^{\circ} \mathrm{C}$. Respiration seems to show similar limits, but very few observations have been made upon it from this point of view. The optimum appears to be a little over $30^{\circ} \mathrm{C}$., and the maximum 25 degrees higher.

The temperature of a terrestrial plant is subject to great and frequent fluctuations, and there is considerable difficulty in securing for it for any length of time the optimum temperature for any of its vital functions, and indeed sometimes of maintaining it within the limits which are essential. As a rule such a plant only secures a general approximation to the optimum point. The difficulty is due to the fact that there is a continual and yet variable interchange of heat between itself and its environment. During the daytime it is constantly receiving supplies of radiant energy from the sun, and as the air surrounding it becomes warmer, a certain amount is absorbed by conduction. It is further continually expending heat on the maintenance of transpiration, losing it also from time to time by radiation and conduction. In its own metabolic processes it is sometimes rendering heat latent, and always liberating it by the processes of respiration, fermentation, \&c. Naturally, its temperature relationships are continually varying. On the whole, such a plant tends to approximate its temperature to that of its environment, but an equalisation is seldom reached, as both are varying simultaneously, and owing to the slowness of the conduction of heat along vegetable tissues, the processes of adjustment only take place with difficulty. The trunk of a tree is during the day often cooler than the air and warmer than the latter during the evening and night. The mean annual tempera- 
ture of such a tree trunk is, however, about equal to that of the air. Less bulky parts than the trunk, the leaves for instance, are very often much cooler than the air. This is made evident by the frequency with which dew or even hoar-frost may be detected on their surfaces. A thermometer placed upon grass often gives a much lower reading than one suspended in the air a little above the ground. This is, no doubt, due to the loss of heat by radiation from the leaves. Roots are often cooler than the air, losing heat by conduction to the soil, and by the evaporation which takes place into their intercellular spaces.

Aquatic plants are less subject to these disturbances than terrestrial ones. The range of temperature of the water surrounding them is smaller, and as they are practically in contact with water within and without, the internal changes of temperature incident to their metabolism are much more readily equalised.

In discussing the changes of temperature in the body of a plant we have to deal at the outset with the supplies of heat which it receives. We have already examined them from the point of view of the absorption of energy from without, but we may pursue still further here the question of the warming or cooling of the plant itself during such absorption.

The chief source from which heat is derived is the radiant energy of the sun. When bright sunlight falls upon a leaf about a quarter of its radiant energy is absorbed. A much larger relative amount is taken up when the light is less bright; in a strong diffuse light, such as that from a clear northern sky, the absorption amounts to about 96 per cent. of the incident energy. We cannot at present discriminate with any accuracy between the influence of the heat rays and that of those of the other parts of the spectrum. No doubt the relative proportions vary considerably during the year.

This radiant energy falling upon the leaf comes into 
relationship with it quite independently of the temperature of the air through which the rays pass, though the latter gradually rises also, particularly during bright sunshine. As we have seen already, three-quarters of the radiant energy during such conditions is not absorbed by the plant. The temperature of the air round the leaves under a diffuse illumination rises but slightly, as only about four per cent. of the radiant energy falling upon them remains unabsorbed by them.

In certain cases, particularly where the temperature of the air remains low for considerable periods, as in high latitudes and on mountains, many of the light rays appear to be transformed into heat. These are the rays which are most vigorously absorbed by chlorophyll and by anthocyan, and which cause the fluoreseence of those pigments. The importance of anthocyan in this respect especially may be noticed. It is of very frequent occurrence among plants which grow in deep shade, and which receive accordingly but little radiant energy. It is usually found on the underside only of foliage leaves, and in special leaves produced in summer upon the stems of deciduous shrubs which occur upon the outskirts of forests or in shady spots in their interior. It is found also at certain times on the upper sides of foliage leaves, particularly when the ordinary sources of heat are deficient, as in the cold weather of early spring. Many grasses which when in the lowlands are only green in colour develop a great amount of the red or purple anthocyan when they grow near the snow-line.

In both shady and alpine habitats the function of the anthocyan appears to be the same, to secure to the plant a certain amount of heat by the transformation of the light rays.

The absorption of heat from the environment by the processes of conduction is particularly noticeable in the case of aquatic plants. Indeed any alteration of the temperature of either the plant or the surrounding water 
is readily transmitted from the one to the other. Similar transmission of heat from the soil to the roots can take place and no doubt has a considerable effect in promoting the well-being of the latter, which as we have seen continually lose heat by the evaporation of water from their cells into their intercellular spaces. Here, however, as in the last case, the conduction of heat varies in direction according to the relative temperatures of soil and root.

The absorption of heat from the air in contact with the general surface must play a part very frequently in the heat interchanges. As in the other cases mentioned, however, the direction of this interchange is not constant.

While we can thus recognise these ultimate sources of heat supply, we find, no less evident, certain ways in which heat is given off by the plant in greater or less amount.

Of these losses the first and most important is the expenditure which is necessary in order to evaporate the water of transpiration. There can be no doubt that the amount of transpiration is very largely determined by the amount of the sun's rays which the plant receives. Not only are its stomata open widely in bright light, so that the vapour can readily diffuse into the air, but the actual evaporation from the cells into the intercellular passages is enormously accelerated during the absorption of the radiant energy. The amount of the latter which is taken up by a leaf has been computed to be nearly fifty times the amount which can be utilised in the process of photosynthesis; if the heat were allowed to accumulate in the leaf unchecked, it has been calculated that its temperature would rise during bright sunshine at the rate of more than $12^{\circ} \mathrm{C}$. per minute, with of course very rapidly fatal results. What is not used for photosynthesis is employed in the evaporation of the water of transpiration, the leaf being thus kept cool. It is noteworthy that whether the leaf is brightly or only moderately illuminated the same relative proportions of the 
total energy absorbed are devoted to the purposes of transpiration and photosynthesis.

When we review the phenomena of transpiration we find two very important considerations presenting themselves to us. On the one hand the suggestion comes that the enormous stream of water passing through a terrestrial plant is necessary in order that a sufficient amount of inorganic salts may be supplied to the leaves, and that the process of transpiration is maintained so that such a supply may be at the disposal of the protoplasts. The dilute solutions which are absorbed naturally involve the transport of a large amount of water with the salts. Transpiration seems thus to be subordinate to food supply.

On the other hand, the temperature relations which we have just examined appear to place transpiration upon quite a different plane. Instead of being a subordinate process, it appears to be imperative in order to prevent a fatal rise of temperature in the metabolic protoplasts; to be concerned primarily, that is, in the regulation of the conditions necessary for the maintenance of metabolism and life, rather than in the supply of material for metabolic purposes.

Which of these is the chief function of transpiration probably depends upon circumstances. The process serves the two purposes, sometimes one, sometimes the other, being the more prominent.

Another cause of loss of heat is found in radiation, which takes place to a very important extent from the surfaces of flattened organs such as leaves. This radiation is to a certain extent independent of the temperature of the surrounding air, and leads in some cases to a leaf being several degrees cooler than the latter. A thermometer placed on the grass will frequently show a temperature some nine or ten degrees lower than another one suspended a few inches above the surface of the ground. Evidence of the activity of radiation at night is afforded by the constant appearance of dew or hoar-frost on the leaves. 
The effects of excessive radiation often threaten to be disastrous and have led to the development of many protective adaptations by various plants. The masses of woolly hairs which are often found upon leaves, forming, indeed, in some cases a thick mantle, must generally be looked upon as such a defensive mechanism. The delicate leaves of buds are often protected by thick scale leaves, which in some cases are hairy, in others furnished with resinous excretions, to serve the same purpose. No doubt the thick cuticle of many leaves and twigs discharges a similar function.

Some plants secure a protection from excessive radiation from the upper surfaces of the leaves during the night, by folding them in various ways, so as always to expose as little surface as possible, and that surface the one which is least susceptible of injury by cooling. This so-called sleep or nyctitropic movement plays a most important part in the retention of heat, leaves that are prevented from carrying it out perishing very rapidly. The features of this behaviour will be examined more fully in a subsequent chapter.

Conduction of heat from the plant to its environment is of constant occurrence, but it is exhibited most clearly by plants that have an aquatic habit. The general interchanges that take place between a plant and the water in which it lives range usually through only a few degrees of temperature, and are so constantly going on that the temperature of both tends to become readjusted after every slight disturbance. In some cases however a very large amount of heat is dissipated by these means, as we may see in the fermentation of a saccharine solution by yeast. The metabolic processes of the latter, incident upon its nutrition and respiration, are so vigorous that a very large amount of energy is liberated by and during the decomposition of the sugar, and this takes very prominently the form of heat and passes from the plant to the sugary liquid in which it lives. 
Terrestrial plants show less direct evidence of the loss of heat by conduction. Their roots however no doubt give up a certain amount to the soil at different times, just as at others they absorb heat from the latter.

When we compare approximately the amount of heat absorbed by a green plant with that which is given off by it, we find that in all cases there is a certain excess of the former. Most plants thus show a certain gain of heat from their environment. This does not however usually manifest itself by a rise of temperature in the tissues. There is no uniformity in the absorption either; at times when there is the greatest balance in favour of absorption throughout the whole plant, parts of it may be giving off considerable quantities and may be cooler than the average temperature of the whole plant.

The gain of heat which is secured in this way is to be largely regarded, as we have already seen, as supplying energy to the plant. This is devoted at first to constructive processes, and thus much of it is rendered potential, being afterwards reconverted into the kinetic form and made to reappear, when it once more largely takes the shape of heat, and is subsequently devoted to purposes of growth, metabolism, repair of cell-substance, \&c., as we have already seen. But we may now lay a certain stress on the fact that at any rate a part of this liberated heat is devoted to a raising of the temperature of the cells which are the seat of its liberation.

We have thus an elementary though very incomplete mechanism for the regulation of the temperature of the plant. An excess of heat is absorbed: part is at once applied to purposes of growth, metabolism, \&c. ; part is retained, and the store is as it were economised, being liberated later with some reference to the temperature of the parts concerned in the vital processes.

This regulation of heat however is very rudimentary and imperfect. We do not find that an increased loss of heat stimulates metabolism in such a way as to set up 
destructive processes, which should liberate heat to com. pensate for the loss. On the contrary such increased decompositions are promoted by a rise instead of a fall of temperature. On the other hand again the processes of growth, repair, and constructive metabolism are also increased as the plant becomes warmer. We can rather speak of what takes place as a tendency to economise and distribute heat, than as a process of regulation. Even the distribution of heat, whether on its first absorption or after subsequent fixation and liberation, is so unequal that different parts of a plant may differ considerably as to their temperature.

As we have seen, life is possible within certain limits of temperature only. The maintenance of a healthy life depends upon the adequate discharge of various functions, each of which needs again a certain range. The limits within which life is possible do not necessarily coincide with those which are appropriate to every function. Outside the latter however a plant becomes unhealthy and eventually perishes, falling a victim to the attacks of internal or external adverse influences.

We do not find that all plants, or indeed all parts of plants, show the same amount of resistance to the extremes of heat and cold. The injury which any part of a plant experiences under such conditions, depends very much upon the amount of water which it contains. If more than a trace of the latter is present, the formation of ice which takes place below $\mathrm{O}^{\circ} \mathrm{C}$. may lead to rupture of the cells, the ice being usually deposited outside them. A considerable disturbance of the osmotic equilibrium of the sap may occur, setting up secondary injuries. The protoplasm becomes disorganised also at the low temperature.

After the freezing of a tissue has taken place, a subsequent rise of temperature leads to a process of thawing. This in many cases is more fatal to it than the freezing, but the effect depends largely on the rapidity of the thawing. If it is so gradual that the water can be re-absorbed into the 
cells, they may continue to live, but otherwise the organ is killed. The cells become flaccid and the protoplasm at once ceases to have the power of maintaining them in the turgid condition.

The effect of the absence of moisture in enabling vegetable organisms to resist cold has recently been examined in the case of seeds. Several kinds of these have been found to be capable of germinating after immersion for several hours in liquid hydrogen, the temperature of which is the lowest at present known.

A similar effect is found at the other end of the scale. If seeds are heated very gradually some will withstand a temperature of $98^{\circ} \mathrm{C}$. The gradual loss of water is a necessary condition for this immunity, for when the heating is conducted so quickly that the water is not driven off at a low or moderate temperature, the treatment is fatal in all cases. Under conditions of gradual heating, their temperature being maintained at $60^{\circ} \mathrm{C}$. for twenty-four hours, seeds have been found capable of germinating after a subsequent exposure to $98^{\circ} \mathrm{C}$. lasting for ten hours.

Spores of bacteria and of fungi have a great power of resisting high temperatures, and this is probably also associated with a considerable degree of dryness. They can withstand boiling in water for some time, but it is probable that the reason why they are not destroyed is that their walls successfully resist the passage of water into their interior.

We are unable at present to explain in detail the causes of the death of protoplasm under the conditions of extremes of temperature. We can only say that under these conditions living substance ceases to carry out the normal reactions which are characteristic of it so long as it is what we call 'living,' and that the power to resume them after the disappearance of the adverse conditions is not regained by it. The nature of life and the intimate causes and features of death are still beyond our knowledge. 


\section{CHAPTER XXII}

INFLUENCE OF THE ENVIRONMENT ON PLANTS

AcCoRding to the nature of their surroundings and the consequent differences in their mode of life, we find in many plants certain peculiarities of form and structure in which they differ from most of those which we have hitherto considered. Of these the vascular plants which live in water may be first discussed, as the direct influence of the environment is most conspicuous in their case.

These aquatic plants, most of which are Spermaphytes, but which include a few of the Pteridophytes, may be divided into two chief groups; those which are altogether submerged, and those which bear floating leaves as well as or instead of submerged ones.

In the former case the plant-body may be attached by roots to the bottom of the stream or pool in which it lives, or it may be altogether floating. The stems are generally long and slender, and easily swayed to and fro in the water. Some have however very short stems which give rise to numerous elongated ribbon-like leaves. These flexible stems depend for their support upon the nature of the medium in which they live, and though they possess a certain rigidity, this is not associated with any great development of woody tissue. Generally the latter is reduced to a minimum; the fibro-vascular bundles are usually few and contain few lignified elements. Their substance is largely parenchymatous and the cells have thin walls. The intercellular space system is often very complex, large lacunæ filled with air occupying considerable space in the distribution of the tissues (fig. 141). Their rigidity is secured by 
the turgescence of the parenchymatous cells, and buoyancy is much assisted by the air in the lacunæ.

The primary root is generally feebly developed, and, as a rule, does not persist through the life of the plant. The floating forms frequently have no roots, but in many cases adventitious roots are given off in large numbers from the various nodes of the stem. The root-hairs which are so

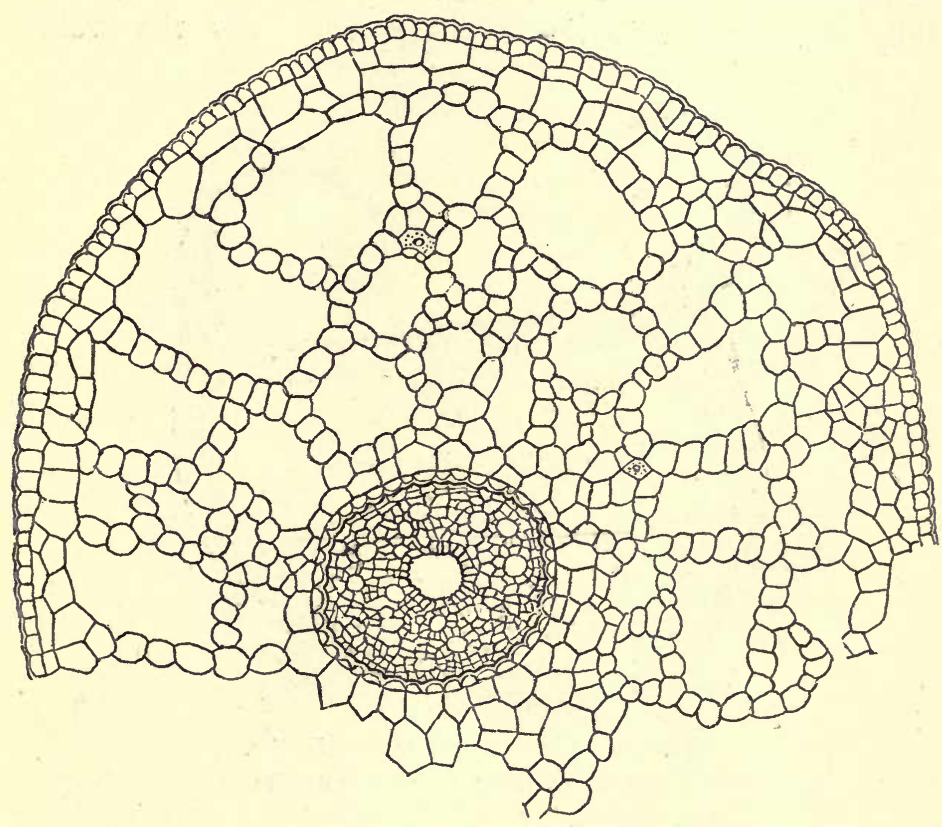

Fig. 141.-Section of Stem of Potamogeton, showing Air Passaǵes IN THE CoRTex.

characteristic of terrestrial roots are usually either very scanty or altogether absent.

The epidermis of both stem and root is not cuticularised, and therefore the cells remain capable of absorbing the water in which the plant is living. In the stem this tissue very frequently contains chloroplasts.

The character of the leaves differs according to the habitat. Those which grow in rapid streams are generally 
either long and thin, or are very much, and finely, divided, so that they offer, in either case, no resistance to the force of the current. In more sluggish water they may be long and ribbon-like, but are frequently broader, and sometimes attain a considerable size. The cell-walls of the former are often thickened, but in the latter the tissue is always very weak, the parenchyma of the mesophyll sometimes being greatly reduced. In Ouvirandra as the leaf becomes fully developed this tissue disappears, only the veins

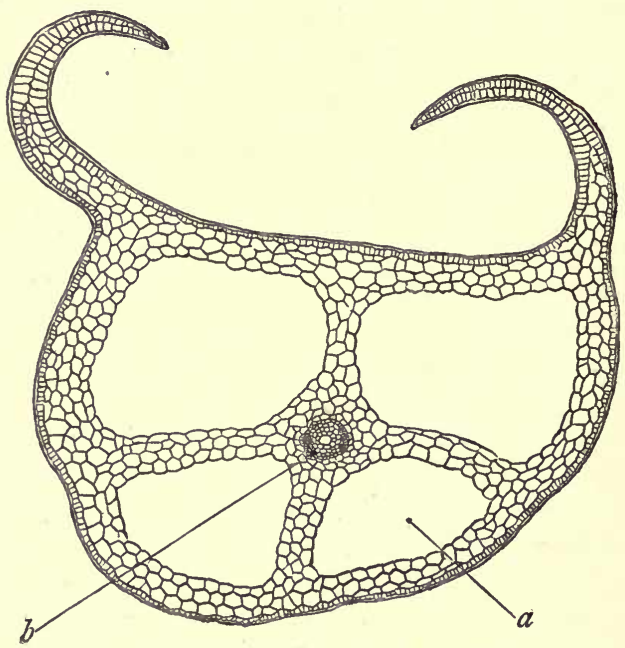

Fig. 142.-Section of Leaf of Isoëtes. $a$, lacunar cavities; $b$, vascular bundle.

remaining, so that it presents the appearance of a coarse grating or piece of lattice-work. The epidermis of a submerged leaf is never cuticularised, and it contains no stomata. In many cases large lacunæ are formed in the substance of the tissue, particularly when the lamina is somewhat stout, as in Isoëtes (fig. 142).

In plants with floating leaves the roots and stems are similar in character to those of the first class. The leaves however, which lie upon the top of the water, are usually tough and thick, their undersides being sometimes deeply 
rugose. They have not the much-divided outline characteristic of submerged leaves, but are usually simple and sometimes of considerable size. Those of the Victoria regia are often three feet in diameter, and are turned up at the edges, forming a rim, which helps to preserve the upper surface from being wetted. The upper epidermis of such floating leaves is often either strongly cuticularised, or

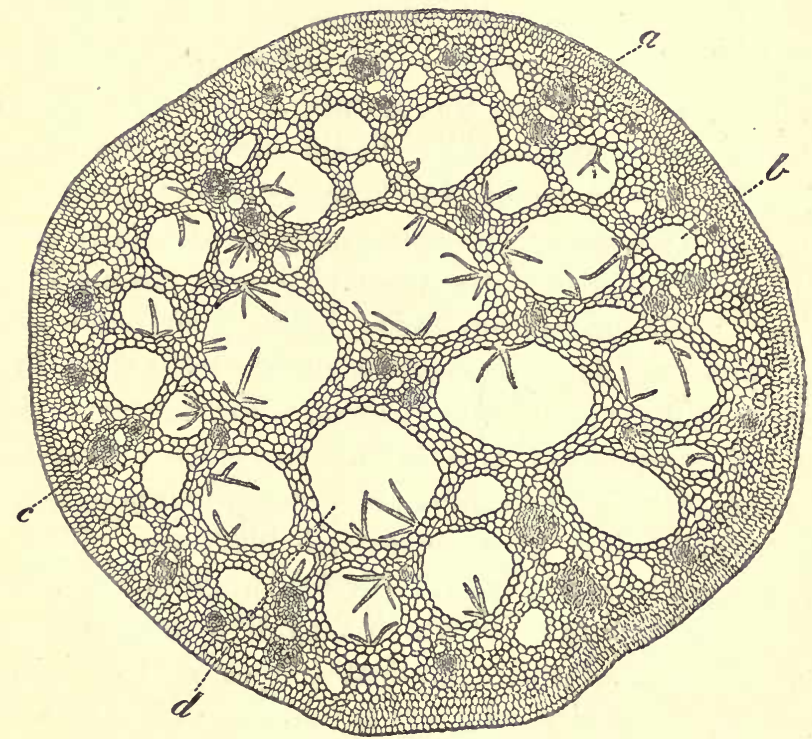

Fig. 143.-Section of Petiole of Water-Lily (Nymphaa alba). $a, c$, vascular bundles; $b, d$, air-chanuels.

impregnated with a waxy secretion serving the same purpose. The leaves are consequently shiny in appearance, and water will not adhere to them. These floating leaves bear their stomata upon the upper surface only.

The petioles are long and flexible, and possess a peculiar power of adapting themselves to varying depths of water. Should the stream in which they live become shallow, the leaves still remain floating, owing to the power of the petiole to become curved; should the water rise, the 
petioles respond by resuming their growth, so as always to keep pace with the increased depth. Their structure resembles that of the stem in that they are composed of turgid parenchyma and have little or no development of woody tissue. They also contain conspicuous lacunæ or air channels (fig. 143).

Vegetative reproduction is very common, branches becoming detached from the plant, which speedily put out adventitious roots of their own and form new plants.

Their watery environment explains the peculiarity of their structure. From the nature of their surroundings and their power of absorbing liquid through their epidermis we can easily explain the absence of the woody tissue, which we have seen to be, when present, especially devoted to the conducting of water from the roots throughout the plant. Their absorbing tissue being their whole superficial investment, such conduction is not called for for nutritive purposes. Their transpiration moreover is reduced to a minimum, and there is therefore no need of a provision for the rapid current of water which is so essential to the well-being of a terrestrial plant, in which this function is so prominent. Their food materials reach them dissolved in the water in which they live, and hence they have no need of the complicated root system with its absorbent root-hairs, which is so characteristic of a plant growing in ordinary soil. Gaseous absorption takes place through the general surface to a large extent, but this direct supply is insufficient for respiration. The ordinary arrangements for aeration, consisting of a network of intercellular spaces freely in communication with numerous stomata, are not exhibited by plants surrounded by water. We have seen that many of them have no stomata, the leaves being quite submerged; others have relatively few on the upper surfaces of the floating leaves. The gaseous interchange between the interior and the exterior is consequently greatly impeded. The large intercellular lacunæ form a mechanism by which this difficulty is 
surmounted, affording large reservoirs of air in the interior of all parts that are submerged, so that the slow rate of renewal of air does not impede the gaseous interchanges which the protoplasts require. These intercellular reservoirs are not confined to the vertical stems, petioles and leaves, but occur also in the more woody stems or rhizomes which many of these plants possess (fig. 144).

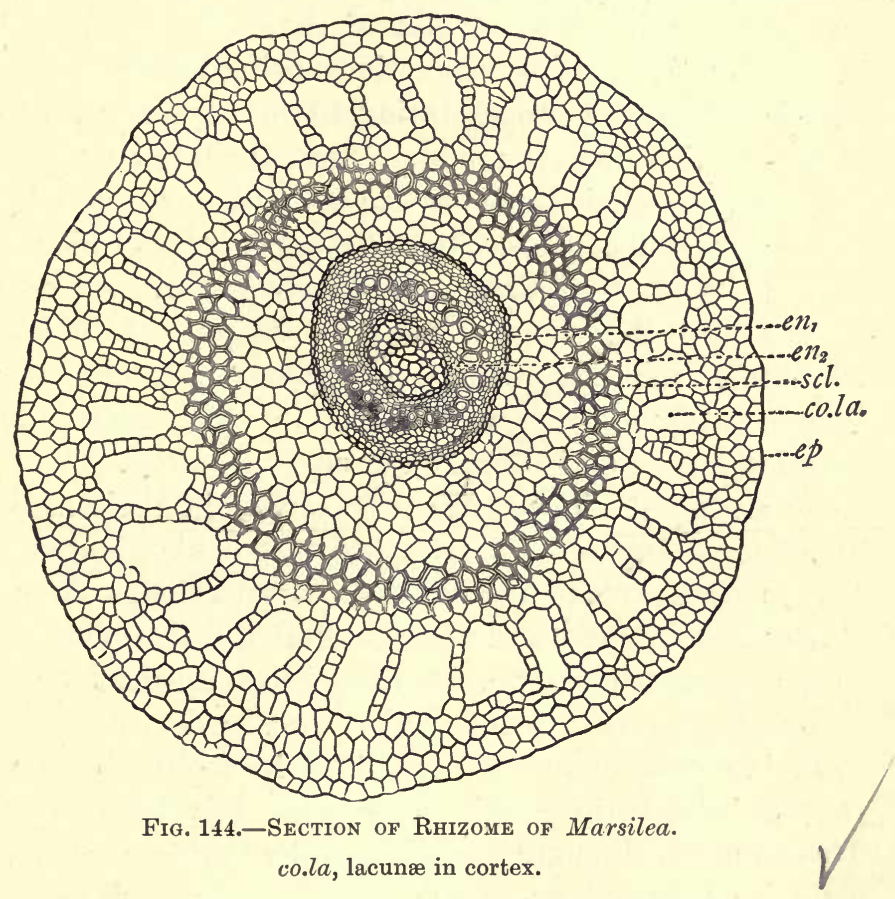

The absence of the transpiration current appears to be correlated with a comparatively small development of the plant-body. The large quantities of inorganic salts which the dilute solutions absorbed by the roots carry into the plant, in cases where the total absorption is very great owing to a large transpiration, lead to a large increase of constructive activity. In the absence of such an enormous absorption the plant-body does not receive the materials 
necessary for the acquirement of a considerable bulk. Aquatic vascular plants are consequently never very large.

The difference between the two groups of aquatic plants spoken of may be well seen in such forms as Cabomba, which bears both submerged and floating leaves. These show respectively the characteristics described in each case.

Some curious adaptations of the organism to its environments are exhibited by certain of these plants which live in marshy surroundings, sometimes being nearly or wholly submerged, and at others, owing to the drying up of the water, growing apon the mud. When the latter fate befalls them, such of their leaves as are adapted to an aquatic life become dried up, and perish. The upper leaves which have always been exposed to the air do not suffer. As growth continues, all the foliage which is produced is of the terrestrial type. On the other hand, when the plant-body is submerged the new leaves are all of the aquatic type. These plants are often spoken of as amphibious.

Some aquatic plants are saprophytic in their mode of life, flourishing best in water which is contaminated with sewage or with the products of putrefaction. They are chiefly certain species of Algæ or Fungi, but among them may be included a few Mosses and Phanerogams.

Another class of plants which show a definite response in their structure to the conditions in which they live is that to which the term Xerophytes has been applied. These inhabit different situations, all of which are characterised by presenting to the plant a very small supply of terrestrial water.' Many grow in sandy deserts, exposed to great heat, and frequently undergoing long periods of drought. Others grow upon a rocky substratum, and their roots are confined to the crannies and crevices which are present in the rock. Others are found in more temperate countries, occupying light sandy soils which cannot retain any considerable quantity of water. Such xerophytic plants as are woody in habit frequently show considerable ten- 
dency to diminish their leaf-surface, probably to reduce evaporation and conserve their stock of water. They often have many of their branches transformed inte thorns or spines, and very frequently their leaves show similar reduction. Others which contain little wood are succulent, and their surfaces are covered by a very thick and tough epidermis, which is strongly cuticularised. Many of those which grow upon rocks have leaves which show special structures for absorbing water from rain or dew. Several species of Saxifrage possess a number of glandular structures upon the teeth of their thick narrow leaves. Each

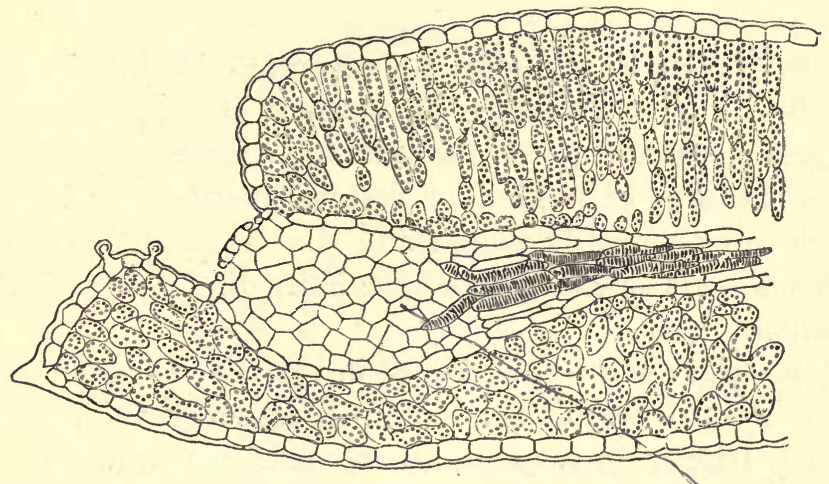

Fig. 145.-Leaf of Saxifraga incrustata, showing absorbing organ. $\quad \times 20$.

consists of a small mass of cells with delicate walls, which lie immediately under the epidermis of a small depression of the surface, and which communicate with the exterior by a few fine pores which perforate the latter. The epidermis of this depression is made up of cells with thin non-cuticularised walls. Each so-called gland is in contact with the end of a fibro-vascular bundle, whose sheath is carried forward over the general mass of delicate cells (fig. 145). The depression of the surface is filled with a mass of carbonate of lime, which is originally excreted by the leaf, and which is held in its place by a few papillæ which project from the epidermis. Such an arrangement 
serves a double purpose; any dew or rain which reaches the surface of the leaf is absorbed by the carbonate of lime and can make its way slowly into the gland, whence it passes into the fibro-vascular system; while, when the leaf is dry the incrusting mineral matter serves as a plug to the depression, and reduces transpiration.

Many plants which inhabit sandy deserts possess similar mechanisms; some excrete carbonate of lime, others crystalline accumulations of common salt. The

- latter can not only absorb dew and rain but can also condense and take up moisture from the air. They are found occurring in such sandy wastes as are by the seashore or near salt lakes.

Many trees which grow in temperate climates, in poor sandy soil on the margin of streams, show a somewhat similar mechanism, but the excretion from their leaves takes the form of a kind of resinous varnish or balsam which can be readily wetted and which can absorb water. In some cases so-called glandular hairs discharge a similar function.

The water which is absorbed in this way is rarely pure, but contains traces of sulphuric acid and ammonia, which, though trifling in amount, are no doubt of value in the nutritive processes. The adaptation to their environment which these plants exhibit is thus chiefly in the direction of economising a limited water supply.

The influence of the environment on the form of the plant can be seen equally well in the case of such plants as grow in Alpine regions, where the cold is usually intense, and the atmosphere for long periods so humid that transpiration is only occasionally possible, and where consequently the absorption of food materials is much impeded. Similar conditions mark the bleak moorlands of temperate climates. These show very great differences between the extremes of temperature which mark summer and winter respectively. The water supply also shows very great variations at different times of the year. The plants are generally of 
comparatively small size, and bear thick, often rolled-up, leaves which are evergreen. The thick exterior and the general hardness of the leaf are a response to, and a defence against, the cold. In the heaths, which may be regarded as typical moorland plants, transpiration is reduced to a minimum, large air-chambers in the leaf with only a few stomata, and those situated in a deep groove, providing for the aeration of the protoplasts. During the cold the closing of these almost hidden stomata guards the plant from the evaporation, which, if unchecked, would lead to a loss of heat that would be fatal to it. The metabolism being

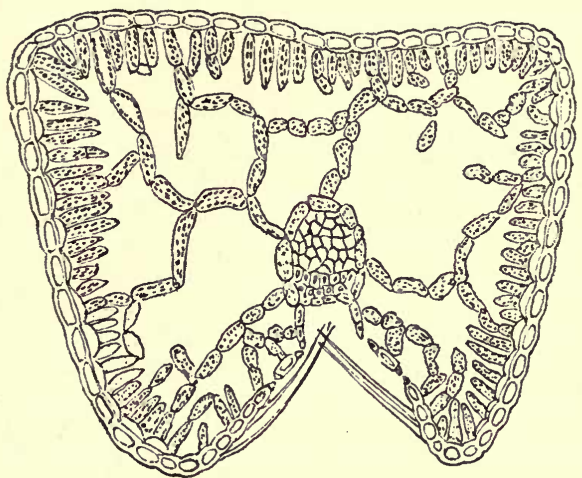

Fig. 146.-Transverse Section of Rolled Leaf of Heath.

reduced by the low temperature, the contents of the air reservoirs suffice for such interchanges of gases as are imperative, and for the coincident exhalation of watery vapour by the protoplasts, but as these contents are very slowly renewed the total evaporation is but slight. When, on the other hand, for a part of the year the temperature is high, the spacious reservoirs provide for a very rapid transpiration as soon as the stomata are open, a very large spongy mesophyll abutting on them (fig. 146). The evergreen leaves also are an expression of the struggle against the difficulty of the absorption of food materials, which in such atmospheric conditions is possible for only 
a limited period of the year. By preserving its leaves green the plant can take advantage not only of the light of summer, but also of those bright sunny days which occur occasionally during the cold season, and thus improve every opportunity afforded it.

Some lowland plants show a similar response to their environment, the form and structure of different individuals of the same species varying to a certain extent, according to their advantages or the reverse, in such conditions as sunlight or shade, drought or moisture, exposure to or protection from cold winds, \&c.

Epiphytic plants show some conspicuous modifications of their structure in consequence of their peculiar habit of life. They usually live upon the surfaces of trees, to which they cling by various means, but from which they derive no nourishment except such as is afforded by accumulations of débris, \&c. upon the trunks. They are not parasitic, but merely live upon the tree as other plants grow upon rocks or cliffs. Mosses and Liverworts are very largely epiphytic, as are certain species of Phanerogams; the latter are very specialised forms, and show most adaptation of form and structure. Perhaps the most remarkable feature about them is their aerial adventitious roots, which are given off in some cases from every node of the stem, so that each internode has its own supply. These are often long cord-like structures, which are of some thickness, often contain chloroplasts, and are either covered by a special epidermal development, or give rise to dense masses of root-hairs. In the first case, which is common among epiphytic orchids, the epidermis is many cells thick, and is known as the velamen. The cells are small tracheïds, with curious reticulated or spiral thickenings, and are often perforated. These peculiar tracheïds contain only air, and the velamen has consequently a curious glistening greenish appearance. The mass of tracheïds forms a kind of spongy covering to the root, and is capable of condensing and absorbing aqueous vapour from the moist atmosphere which 
usually surrounds it. At other times when the air is dry and there is a danger of evaporation from the root, this velamen acts as a protective membrane against loss of water in this way. The second case is illustrated by many aroids, and the dense plexus of root-hairs borne upon the aerial roots serves the same purpose as the velamen of the orchids. Besides these roots, thus adapted to absorb watery vapour from the air, epiphytes frequently have others which are closely applied to the surface of the bark on which they are growing. These are often strap-shaped, and cling very closely to the tree, absorbing from the bark the soluble products of its decomposition and any mineral débris that may be accidentally carried thither. The small amount of such food-stuffs available will explain the relatively large development of the root system, which is in much greater proportion than in ordinary terrestrial plants.

Parasites are another class of plants that have undergone much modification of structure in consequence of their mode of life. The parasitic habit is seen most completely in the group of Fungi, but it is by no means confined to them. We find many cases of partial or complete parasitism among flowering plants. In all cases we notice that the parasitic habit is associated with a degeneration of structure, which especially affects the vegetative organs.

The fungus which is parasitic in habit derives all its nourishment from the plant or animal whose tissues it has invaded. Other plants of the same group are not parasitic, but live upon decomposing organic matter, being known as saprophytes. Their mode of nutrition is, however, essentially the same. They have all lost the chlorophyll apparatus characteristic of the green plant, and cannot therefore work up the food materials that the latter absorbs from the air. Instead, therefore, of absorbing their carbon in the form of carbon dioxide, these plants take it in in the form of an organic compound of some complexity, which is usually some kind of sugar. Saprophytes can absorb nitrogen in the same combinations as a green 
plant, but they appear to utilise compounds of ammonia in preference to nitrates. No doubt their protoplasm is ultimately fed with the same substances as is that of the higher plants, but they lack a great deal of the constructive power of the latter.

The degradation of the structure of such plants is associated with the absence of the constructive processes which depend on the presence of chlorophyll. Their body is usually composed chiefly of delicate hyphæ, which

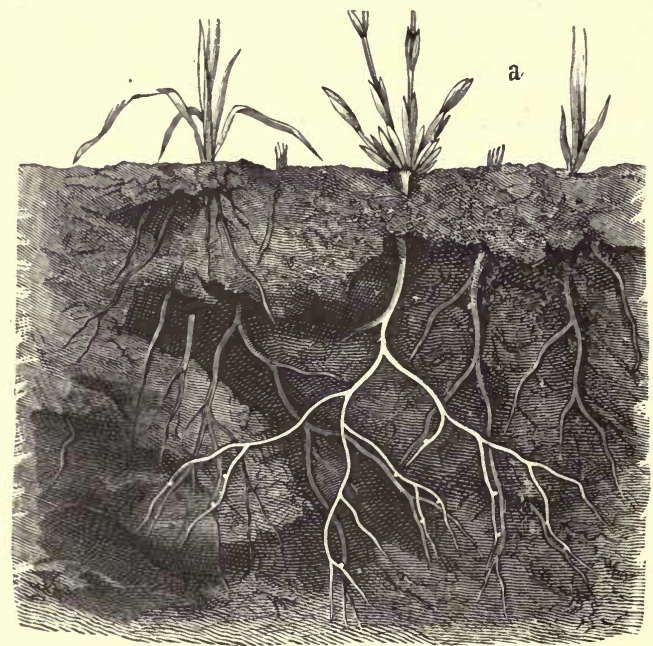

Fig. 147.-Thesium alpinum, SHOwING THE sUCKers oN THE RoOTS. (After Kerner.)

ramify in the nutrient either living or dead substratum, and which absorb elaborated products of some complexity freely by their whole surface. They have, therefore, no need of differentiated absorbing or conducting tissues, which are consequently not developed. A further consequence of the ease with which they obtain their food is the readiness with which vegetative and asexual reproduction is brought about; hence sexuality is in many cases non-existent among them.

Phanerogams which are completely parasitic show a 
similar degradation of structure. They possess no chloroplasts, their leaves are absent or reduced to the condition of scales, while their stems are often thick and succulent. Their roots are replaced by the so-called haustoria, which penetrate into the tissues of their hosts, complete fusion of the tissue of the host and the parasite frequently taking place. We have representatives of such parasites in the British flora in the Cuscutas and the Orobanchacea.

Many of the plants belonging to the Santalacece and the Scrophulariacea show a partial parasitism of this

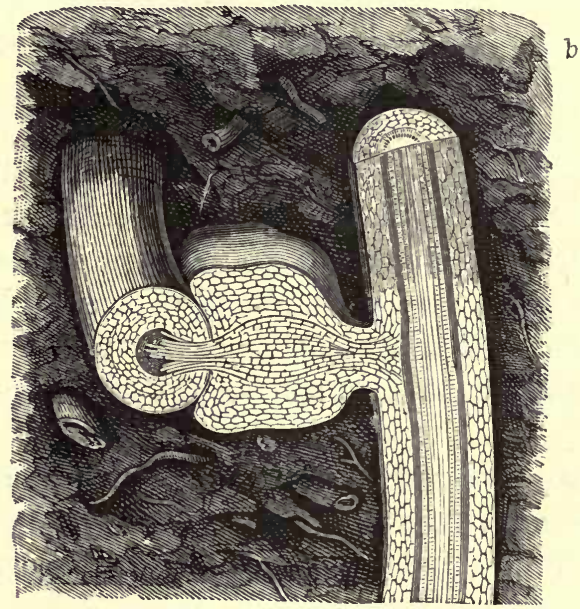

FIG. 148.-Thesium alpinum. PIECE of A ROOT WITH SUCKER IN SECTION. $\times \mathbf{3 5}$. (After Kerner.)

kind. They have short stems which bear green functional leaves, but are peculiar in that their roots become attached by curious sucker-like bodies to the roots of other plants growing near them (figs. 147, 148), and from these suckers absorbing cells are developed which penetrate into the substance of their hosts and draw nourishment from them. They are generally described as root parasites. The Mistletoe behaves similarly, striking its haustoria into the tissue of the branches of the apple, oak, poplar, \&c. The parasitism is partly compensated by the fact that its leaves 
remain green when the host has lost its foliage, and by their activity they to some extent assist the tree on which the mistletoe is growing. The relationship seems to be almost one of symbiosis rather than of parasitism. Probably the relationship of the root-parasites and their hosts is also one of mutual assistance rather than true parasitism.

The habit of capturing insects, which we have seen to be characteristic of several plants of very different forms, may also be looked upon as connected with their environment. Many of them, e.g. Drosera, grow upon a substratum which is largely composed of plants of Sphagnum, and which yields to them a very limited supply of nitrogenous compounds; others are found growing on the surface of rocky mountains, into the chinks of the stones of which their roots penetrate; others again flourish in the sandy soil of deserts; in all of which situations compounds of nitrogen exist only in very small amount. The organic substances yielded by the decomposing bodies of the captured insects must therefore form a valuable supplement to the ordinary sources of nitrogen.

Besides these responses to influences of the environment, which are very far-reaching, and modify very largely the shape and structure of the plants exposed to them, ordinary terrestrial plants also show great power of reacting to the different external conditions which they meet. These will be considered in subsequent chapters. 


\section{CHAPTER XXIII}

THE PROPERTIES OF VEGETABLE PROTOPLASM

The influence of the environment upon the structure of plants we have seen to be far-reaching. Different conditions of the surroundings are followed by differences of structure which are greater in proportion as the time during which those conditions act is more and more prolonged. The living substance of the plant is clearly the part influenced by the environment, for we have seen that the skeleton and other non-living parts of the plant owe their construction to its activity. We may therefore with advantage pause at this point to examine a little more closely the properties which are exhibited by vegetable protoplasm.

We have seen throughout all the foregoing chapters that all the processes which conduce to the well-being of the plant are, to a large extent, if not entirely, under the control of the living substance. Though the absorption of its food materials from the air and the soil is due to physical processes, these are nevertheless largely regulated by the behaviour of the protoplasm under all sorts of varying conditions. The manufacture of food from these crude materials, and its subsequent distribution, the accumulation and dissipation of energy, the processes of nutrition and growth, are all subject to the same regulation.

But there are also other properties of protoplasm which have not so far been more than incidentally referred to. The plant exhibits particularly the power of appreciating changes in its surroundings, and is capable of adapting itself in various ways to such changed conditions. 
In many cases the adaptation in question takes the form of a spontaneous movement, in which the living substance is concerned in a manner resembling the muscular contraction so characteristic of animal protoplasm. In others the response to such changes presents itself to us as a modification of the normal behaviour of the living substance with regard to the vital processes we have examined, and in particular to the entry of water into the vacuoles of the cells or its transmission outwards.

When we examine the phenomena of movement we find that though evidence of contractility is procurable, this phenomenon is of somewhat rare occurrence in plants.

Certain plants at particular times

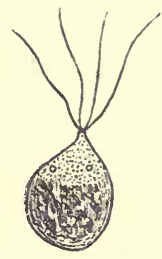

Fig.149.-Zoosípore of

Ulothrix. $\times 500$. emit from their body small masses of naked protoplasm which are furnished with a varying number of long filaments (fig. 149). These filaments, which are protoplasmic also, are ordinarily in a state of active vibration, causing currents in the water in which they live, which float them quickly from place to place. Among these free-swimming protoplasts may be mentioned the zoospores of the Algæ and Fungi, and the antherozoids of these and higher plants. The movement is a spontaneous one, the organisms being endowed with the property of locomotion, which they exercise in the discharge of their ordinary life-work. Though put forth in the absence of any external stimulation, the protoplasts are capable of receiving such impulses and modifying the vibratile action accordingly.

The mechanism of the movement is probably the contraction of each side of the filament or cilium alternately, or of the part of the cell just at the point of attachment. The impulse leading to the movement must be sought in some decomposition originating in the protoplasm itself, and not excited by any stimulation from without. The phenomenon is often spoken of as ciliary motion. 
Of a somewhat similar character is the curious creeping movement of the Myxomycetous Fungi. In a few cases the zoospores of these organisms are furnished with cilia or flagella, resembling those of the zoospores already mentioned, but more generally each consists of a minute mass of naked protoplasm, which makes its way over the surface of its substratum by putting out blunt processes of its own substance, known as pseudopodia (fig. 150). After a while a number of these zoospores become fused together to form a large jelly-like mass, known as a plasmodium. This colony of protoplasts then makes its
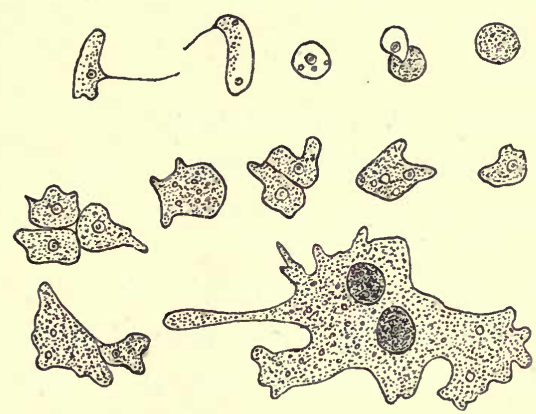

Fig. 150.-Stages in construction of the Plasmodium of a Myxomycete.

way slowly over its substratum by similar pseudopodial movements. Each pseudopodium is a protrusion of the ectoplasm, and the more fluid endoplasm is in some way drawn into the different protrusions, so that the rest of the cell or of the plasmodium follows the extension of the pseudopodium and is dragged after it. Which part of the operation corresponds to the act of contraction is disputed, but it seems probable that it is the second, and that the first protrusion is of the nature rather of relaxation. The movement, like that of ciliary action, is a property of the organism, and is used by it in the ordinary course of its life, even in the absence of stimulation.

Among the lowliest of the Algæ or seaweeds some other 
organisms are conspicuous by their power of locomotion. These are the Diatoms which are so prominent in ponds and sluggish streams. They are unicellular plants of very minute size, each of which consists of a protoplast encased in two silicified shells or valves which fit together very tightly, one overlapping the other by its edges. The cellwall which forms each valve is strongly impregnated with silica, the latter being deposited in patterns which are often of great regularity and beauty. The plants are not provided with cilia, nor so far as we know are the silicious valves perforated in any way. Each diatom is however capable of effecting a peculiar gliding and very rapid movement through the water, the mechanism of which is at present unexplained.

Certain filamentous Algæ, known as the Oscillatoria, also carry out a peculiar movement. They consist of long chains of protoplasts, each separated from its neighbour by a cell-wall, and the whole thread surrounded or coated by a peculiar semi-gelatinous sheath. Each chain is anchored to a substratum of stone or rock at one end, and the free portion is in constant waving or twisting motion to and fro, a movement which is quite independent of currents in the water, being exhibited in the total absence of such disturbance. The movement appears to resemble that of the Diatoms, but its mechanism is at present unexplained. Like the others so far discussed it is one of the features of the life of the organisms, and is carried out by their protoplasm without excitation by an external stimulus.

In certain organisms of still humbler type. another manifestation of the power of contractility can be observed. These are unicellular beings consisting of small unclothed masses of protoplasm. In their substance at some point there may be seen a clear space or vacuole which exhibits a more or less regular pulsation, assuming slowly the appearance of a nearly spherical cavity and then suddenly disappearing, recalling the active contraction of animal 
protoplasm. These pulsating or contractile vacuoles can be seen very well in Chlamydomonas.

The power of movement which is thus exhibited by many of the lowlier plants may be distinguished however from certain of the movements of portions of higher plants which have already been alluded to, and which will be discussed more fully subsequently. These movements include the circumnutation of growing organs, the closing of the leaves of Dionaa, the bending of the tentacles of Drosera, and many others. These are brought about in multicellular organs, and by a mechanism different from the one now under discussion, the movement being secondary and following indirectly on a change in the behaviour of the protoplasm of certain of the cells, which, instead of contracting, modifies its resistance to the escape of the water which they contain. In one or two cases, as in the curving of certain tendrils and in the drooping of certain leaves in response to stimulation, the hydrostatic disturbance seems to be attended by, and perhaps partly dependent upon, a contraction of the protoplasm of certain cells. These phenomena will be discussed in a subsequent chapter and need only be alluded to here as possibly showing the inherent power of contractility residing in the protoplasm.

Though the power of locomotion, which we have seen in many cases to exist, is an evidence of certain powers of movement or contractility possessed by living substance, it must not be inferred that only organisms which are free to move are possessed of these or similar properties. Locomotion is impossible to the great majority of plants on account of their relationship to their environment. There is however a certain amount of evidence to show that the instability which, in the cases discussed, finds its expression in movement, is a property of living substance in general. We find many cases in which movement of the living substance can be observed in the interior of ordinary cells. It can only be seen when the protoplasm is more or less 
filled with granules, as in their absence it is so transparent that it is impossible to say whether it is in motion or not. In the leaf of Elodea we find a very good instance of this movement. Each cell contains a considerable quantity of water, so that the protoplasm for the main part is found as a layer lining the cell-wall. This layer consists of two parts, an outer one in which are situated the chloroplasts, and an inner one in which are large numbers of fine granules. It is this inner layer which exhibits the move-

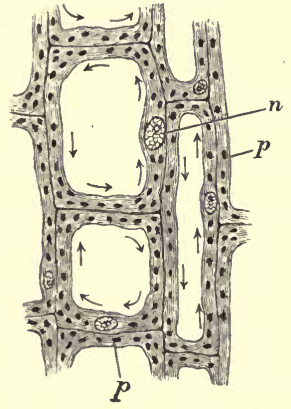

Fig. 151.-Cells From the Leaf of Elodea. $\times 300$.

$n$, nucleus; $p$, protoplasm, in which are embedded numerous chloroplasts. The arrows show the direction of the movement of the protoplasm.

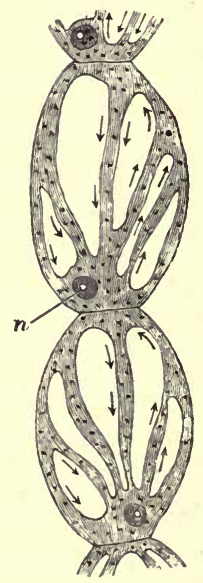

Fig. 152.-Two Cello from a Staninal HaIr of Tradescantia. $\times 300$.

The arrows show the direction of the movement of the protoplasm.

ment, which can be seen as a streaming motion of the granules, the whole layer flowing slowly round the cell (fig. 151).

In other cases, particularly in long pollen-tubes, where the distribution of the protoplasm is so far different that bands or bridles of it cross the vacuole in various directions, the movement has a more complicated course, streams of granules passing along these bridles as well as along the peripheral portions of the protoplasm. These two cases of 
streaming movements of protoplasm are spoken of as rotation and circulation respectively. There is no difference apparently between them, except what is involved in the different distribution of the protoplasm in the cells. Other instances are met with in the staminal hairs of Tradescantia (fig. 152), the leaves of Vallisneria, the internodal cells of Chara and Nitella, and the unicellular Desmids.

It is evident from the structure of most vegetable organisms that the possession of a power of active contractility, such as is possessed by most animals, would be of comparatively little use to them. Though flexible to a certain extent, they are possessed of a fair amount of rigidity, which under ordinary eonditions they do not relax. We have seen that one of the most important relations of their life is that which is maintained between the protoplasm and water. Each cell or protoplast is so organised as to contain its own appropriate store, upon the possession and renewal of which its efficiency as a member of the colony, if not its actual life, depends. The regulation of this supply of water is of the first importance to the plant, and it is not surprising therefore to find that such a regulatory power is one of the properties of vegetable protoplasm.

All healthy vegetable cells are during life in a condition which is known as turgor. The cell is overfull of water, so that a certain internal hydrostatic pressure is exerted on the whole surface of the limiting membrane, which is stretched accordingly. As the membrane possesses elasticity, the wall in turn exerts a pressure upon the fluid inside it, and during healthy life a certain equilibrium exists between these two pressures. Such a cell is called turgid, and the degree of its distension is the measure of its turgidity. This turgor can vary within fairly wide limits, consistently with the health of the cell. The turgor depends chiefly upon two factors, both of which are capable of control. The water is caused to enter the 
cell, as we have seen, by the formation of various osmotically active substances in its interior, which have an attraction for water, the quantity which enters depending upon the amount of such substance present. We have seen already that this regulation of osmotic material is controlled by the protoplasm. But besides this another important factor exists in the greater or less difficulty with which water is enabled to pass through the plasmatic membranes. The power of altering its permeability by water is a property of protoplasm which is of the highest importance in the mechanics of the cell. It takes the place practically which is held by the power of contractility in the living substance of animals. No doubt it can be called into play during life under constant conditions, but it becomes much more marked when the plant is subjected to particular kinds of stimulation. A ready instance of its employment under the former conditions is afforded by the variations of turgidity and subsequent growth which we have already spoken of as inducing circumnutation (p. 316). Instances of its following upon stimulation will be discussed more appropriately in a later chapter.

The facts thus briefly narrated impress upon us the belief that all protoplasm is the seat of active molecular movement, the intensity or vigour of which, as well as the forms of its manifestations, varies very greatly in different cases. Indeed, the life of the protoplasm is intimately bound up with such a motile condition. The manifestations are in all cases appropriate to the manner of life and the surroundings of the organism under observation; they may take the form of locomotion, of contractility, or of variation of permeability, leading to the regulation of turgescence.

If we look back to the behaviour of the contractile vacuole of Chlamydomonas, we are struck by the fact that its pulsations occur with a certain definite intermittence so long as they are not interfered with by external conditions. The vacuole dilates slowly, reaches a certain size, 
and suddenly disappears; then is gradually formed again, and the series of events is repeated. This regular intermittence constitutes what is often spoken of as rhythm.

The rhythm which is so easily seen in the case of pulsating vacuoles is characteristic also of those less obvious changes in protoplasmic motility which lead to the variations of turgidity in different organs, particularly in those which are growing. We have already seen that during the growth in length of a symmetrical organ, such as a stem or root, the apex points successively to all points of the compass, the successive changes of position being spoken of as circumnutation. This is the result of a rhythmic variation of the turgidity of the cells of the cortex. If we consider a longitudinal band of such cells, we find that at a certain moment the cells are at their point of maximum turgidity, and the growing apex is made to bend over in a direction diametrically opposite to this band. The turgidity of this band then gradually declines to a minimum, and again increases slowly to a maximum. If we conceive of the circumference of the organ as divided into a number of such bands, we can gain an idea of the changes in turgidity which cause the circumnutation. Each band is in a particular phase of its rhythm at any given moment, and the successive bands follow one another through the phases of their rhythm in orderly sequence, so that when one is at its maximum, another diametrically opposite to it is at its minimum. The phases of maximum and minimum turgidity thus pass rhythmically round the organ, and the apex is consequently compelled to describe a spiral line as it grows. If the stem or root is not circular in section, but is flattened in any direction, the steady sequence of the rhythmic changes will lead to the projection of this spiral assuming the form of an ellipse instead of a circle, and if the flattening is extreme the. movement will be a backward and forward one.

Modifications of the distribution of maximum and minimum turgescence in a radially symmetrical organ may 
lead to a similar nutation. It is not infrequent for the rhythmic change in the turgescence to affect only two sides, instead of passing regularly round it. The organ, though radially symmetrical in structure, will thus behave as a bilaterally symmetrical one, its organisation indeed being bilaterally symmetrical. Its changes will thus resemble those of a flattened organ which can only be made to oscillate backwards and forwards.

A similar rhythm can be noticed in the variations of the extensibility of the limiting membrane which characterise the circumnutation of a cœnocytic hypha. We must suppose these variations to be due to the protoplasm covering the wall, though we cannot explain the mechanism. The protoplasm has the power to soften the cell-membrane.

Rhythmic changes of this kind affect other processes than those of circumnutation. We have had occasion to notice that the behaviour of a growing organ during its grand period shows certain diurnal variations which we have called the daily periodicity of growth. Though no doubt we have to do here to a certain extent with changes in the behaviour of the protoplasm induced by the alternations of light and darkness, with coincident variations in temperature, this daily periodicity of the rhythm does not appear to be altogether dependent upon exposure to such alternations, for they persist for a longer or shorter time during continuous darkness. Their cessation after exposure to a period of darkness need not necessarily point to their dependence on the intermittent access of light and warmth, for, as we shall see later, prolonged deprivation of light leads to a peculiar condition of rigidity of the protoplasm which eventually causes its death. The cessation of the rhythm indeed appears to be a pathological phenomenon. The rhythm of the daily periodicity appears however to bear a certain relationship to the alternation of day and night, for plants which have been cultivated from seed in continuous darkness do not exhibit it. 
This rhythmic change in the protoplasm is not exhibited by organs during growth only ; in many cases it persists throughout their life. Very conspicuous instances of it are afforded by certain movements often exhibited by the leaves of particular plants. Perhaps the most familiar of these is the so-called Telegraph plant, Desmodium or Hedysarum gyrans. Its leaves are ternate, the terminal leaflet being very large in comparison with the two lateral ones (fig. 153). If the plant is watched while exposed to suitable temperature and illumination, the lateral leaflets are found to move up and down on the rachis, sometimes passing through an angle of $180^{\circ}$, and twisting slightly as they move. They thus describe a kind of ellipse, the duration of the movement being about two minutes. Many other instances of a similar kind are known, the Leguminosa furnishing many examples. All of them do not exhibit the movements with the same ease, as they are interfered with by other changes in position which result from external stimulation. They can often be made evident by

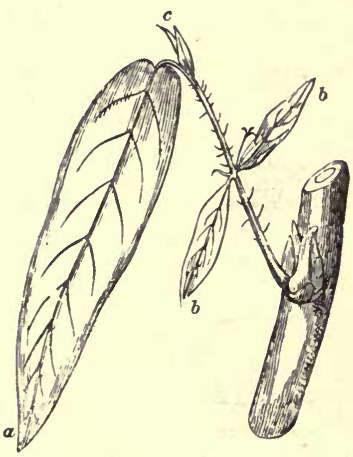
keeping the plant under constant external conditions. Darkness however if too prolonged causes their cessation, though in some cases they are made evident by deprivation of light for a short time. The mechanism of the movement in most of these cases is the rhythmically varying turgescence of particular organs known as pulvini, which are situated at the bases of the stalks of the leaves or leaflets. As in the cases already noticed the alternations in this turgescence are the expression of rhythmic changes in the protoplasm of the cells of each pulvinus. As these pulvini play a considerable part in the changes of position which are exhibited by many leaves under various conditions, their structure may well attract 
our attention here. Fig. 154 represents a longitudinal section through one of them, which occurs at the base of a leaflet of Mimosa. The stalk of the leaflet shows a swelling at the point of union with the rachis, the protuberance being greatest on the under side. Here there is a cushion of cells which are capable of containing a

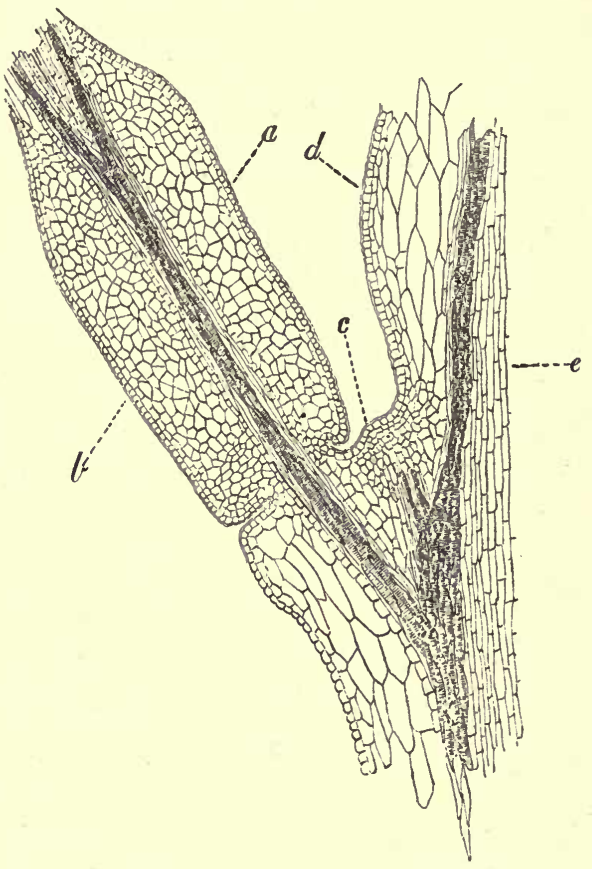

Fig. 154.-Pulvinus of Mimosa.

$a, b$, the succulent parenchyma of its upper and lower sides; $c$, bud;

$\bar{d}$, parenchyma of rachis; $e$, pith.

relatively considerable quantity of water. When turgid they swell out and force the leaf into an erect, or almost erect, position. When they part with their water and become flaccid the stalk of the leaf loses its support and the weight of the blade causes it to fall downwards. This is rendered more easy by the fact that the vascular strand 
or bundle which passes from the stele of the stem through the petiole is somewhat reduced, giving greater flexibility to the stalk at that point. The cells upon the upper side of the pulvinus in some cases play only a passive part in the phenomenon, the rhythmic variations only affecting those already described; in other cases the upper side or both sides may show the changes of turgidity.

The same tendency to rhythmic change is shown in what is called the periodicity of the various vital functions. If, for instance, the root-pressure of a plant is examined by the aid of the apparatus already described, in which the water taken up is made to support a column of mercury in a manometer, when the mercury has reached what we may call its mean or average height, it does not remain steady at that point, but begins to oscillate. It rises in the morning till about midday, then sinks somewhat, rises again during the evening, and falls during the night. There is thus a daily variation of the absorptive activity of the roots which is scarcely affected by changes in the environment. It is an instance of an automatic rhythm.

There is a similar daily variation in the bulk of a plant, the diameter of its various organs diminishing from night till some time in the afternoon, and increasing thenceforward till dawn. These variations largely depend upon the distribution of the water which the plant contains, which is regulated by the living substance in the way already described. This rhythm is under ordinary circumstances. very much affected by variations in transpiration, which we have seen is a process that is very soon modified by variations in illumination and temperature.

It is difficult to explain the occurrence of these various manifestations of rhythmic change in the protoplasm. Many of them suggest that they are the result of the influence of the alternations of light and darkness, and perhaps of the changes of the seasons, to which plants are exposed. But others are exhibited so regularly under constant conditions of the environment that they cannot be thus explained. 
They are now hereditary and to a certain extent independent of the changing conditions which the plants encounter. It may well be however that they have become impressed upon the organisation of vegetable protoplasm by the constant recurrence of these changes of the environment during the long ages of the past. This does not appear unlikely in the face of the fact that, as we shall see later, it is possible under appropriate conditions to impress a new rhythm upon particular organs. The manifestation of rhythmic change has however become one of the vital properties of protoplasm.

We saw in an earlier chapter that the peculiarities of form and structure which different plants possess are to be associated with the character of their environment. From such facts as were there discussed it is evident that a plant is capable of receiving impressions from without and responding to them in various ways. If we examine any plant which does not show such marked adaptation to its surroundings as those which were then more particularly under consideration, we can still find evidence of the possession of a similar power of appreciating differences in the external conditions in which it finds itself, and of modifying certain of its vital processes in response. When certain zoospores of some of the lower Algæ which swim freely in water are suddenly exposed to a brilliant light, they take up at once a definite position with regard to the incident rays. When a leaf of Mimosa pudica, the so-called sensitive plant, is roughly handled, it falls from its normal position and takes up a new one, while its leaflets become folded together. When a filament of Mesocarpus is exposed to an electric shock sent through the water in which it is floating, it is found not infrequently that it splits up into its constituent cells. This power of receiving impressions from without, to which we have had frequently to refer in discussing the phenomena of growth and rhythm, is another property of vegetable protoplasm and can be observed to belong, in a greater or less degree, to every vegetable 
organism. It is usually spoken of under the general term irritability.

This property, which is the most important of all, as it is particularly the one which keeps the organism in a proper relationship to its environment, is not always to be observed or demonstrated with equal ease. Indeed the protoplasm must be in a healthy condition to manifest it satisfactorily. It is easily injured if changes in the environment are too sudden or too severe. Consequently the adaptation of groups of plants to special environments has been a slow and difficult process, any single individual undergoing little change, but alterations of considerable extent having been effected by the continuous influencing of many generations.

The maintenance of the health of the individual is no doubt the great object of this sensitiveness ; and conversely it is only the healthy plant that manifests it in the greatest fulness. Health may be spoken of as the condition in which the reaction between an organism and its surroundings is a perfect one. In the case of the ordinary terrestrial plant these surroundings present especially three features which are subject to considerable variation. These are light, temperature, and moisture. A plant must exhibit a proper relationship to each of these conditions at any rate to be healthy. The condition in which the relationship to each of these factors is satisfactory is generally spoken of as one of tone, and the influence which each exerts when it affects the plant uniformly is spoken of as a tonic influence. When a dicotyledonous plant which has been growing under ordinary atmospheric conditions, exposed to diffused daylight, is removed into darkness and kept there for some time, it becomes incapable of being impressed by its surroundings. Nor is its irritability alone affected by the absence of light, for many of its parts, particularly its leaves, cease to grow under such conditions. The condition which is induced by light, and upon which the manifestations of irritability depend, is known as Phototonus. 
A corresponding condition marking an appropriate relationship between the plant and temperature may be called Thermotonus. This condition also is necessary for the manifestation of sensitiveness. If it is materially interfered with, the vital functions and the processes of growth and nutrition suffer seriously.

There must also be a satisfactory adjustment of the relations between a plant and moisture, though this is less restricted than the two already mentioned.

As the maintenance of health involves the continual adjustment of the plant to the changes in its environment, we must examine a little more closely the nature of the influence which the latter, and particularly the two factors of light and temperature, exert upon the organism. This influence is spoken of as a tonic or paratonic influence and leads to the establishment of a satisfactory condition of tone.

In order to study the tonic influence of light upon a plant we may first consider the features which characterise the growth of a plant in darkness. We find that such a plant is much modified both in form and structure. If we experiment with an ordinary dicotyledonous plant which has numerous leaves of moderate or small size upon an elongated stem, we find that these features become much exaggerated. The stem is very much elongated and remains slender ; it is more succulent than a normal stem, and bears extremely small leaves which grow out from it at a more acute angle than those which arise upon a normally illuminated stem. Certain Monocotyledons which have normally small stems and large broad leaves are differently affected. The great change in this case is in the leaves, which become much elongated and relatively narrower than normal ones. Certain phylloclades, such as those of some of the Cacti, become elongated and slender, instead of remaining broad.

The structure of the various parts also is modified; the woody and sclerenchymatous elements are much reduced, and the parenchyma of the cortex is increased in bulk. It 
becomes more succulent, and the reaction of its sap is much more acid. The chloroplasts do not become green, the pigment which they contain, known as etiolin, being a pale yellow. In the leaves the differentiation of the mesophyll into palisade and spongy parenchyma does not take place. The parenchymatous cells of the ground tissue of the elongated organs, whether they are stems or leaves, become altered in shape, their longitudinal diameter being considerably increased. Plants thus affected by darkness are said to be etiolated.

That these differences are to be attributed to the absence of the light can be seen by comparing two similar plants, the first cultivated in darkness and the second under ordinary conditions of illumination, the other conditions being kept the same for both.

The explanation of these changes is somewhat difficult. The absence of light is clearly the cause of the different colour, for, as we have seen in a preceding chapter, under such conditions the pigment chlorophyll is not formed, but is replaced by the yellowish-white etiolin. When an etiolated plant is exposed to light, the etiolin is soon replaced by chlorophyll, and the plant becomes green. Etiolin appears indeed to be the antecedent of chlorophyll. The question of the non-development of the woody elements and the generally increased succulence is more difficult to explain, and many hypotheses have been advanced to account for it. There is a disturbance of the normal course of the metabolism evidently, as shown by the greater production or accumulation of organic acids, to the osmotic properties of which the increased succulence is partly due. It is known that in plants possessing considerable succulence the free organic acids which are produced during the night undergo oxidation when light finds access to them. The reason for the disturbance in question is however not explained. Diminished transpiration may perhaps account for a good deal, for as we have seen in the absence of light the stomata remain shut and there is but little 
output of watery vapour. The increased turgidity of the tissues resulting from this factor may very probably upset the normal course of metabolism.

It is significant in this connection that the parts which show the excessive growth are in all cases those in which water accumulates as transpiration becomes checked.

If however the effects are admitted to be due to the disturbance of transpiration, this is no satisfactory explanation of the phenomenon, for, as we have seen, the actual evaporation of the water of transpiration into the intercellular spaces is under the regulating influence of the protoplasm, and the effect must therefore be traced back to some interference with the latter, caused by the absence of illumination. With the lowering of the tone which follows the absence of the light we have a failure of the protoplasm to exhibit its normal degree of permeability, and we find it retain in the cell more than the usual quantity of water.

We cannot easily explain the effects which we have seen are produced upon the structural elements of the plant. We do not know why the usual development of the woody and sclerenchymatous cells of the stem should be interfered with, nor can we explain the effect of light upon the degree of differentiation of the mesophyll of leaves. We find that palisade tissue is developed more readily under the influence of bright light; a phenomenon which may be easily ascertained by comparing the structure of two leaves from the same tree, one taken from a brilliantly lighted and the other from a deeply shaded part. Indeed, the differentiation of the mesophyll into palisade and spongy parenchyma may be traced to the difference of illumination which the two faces of a leaf receive, for when both are well lighted, palisade parenchyma appears upon both sides, while etiolated leaves, as we have seen, do not develop this tissue at all.

It is possible that this difference of structure on the two sides may be connected with the possibility of damage 
to the chloroplasts if they are too brilliantly illuminated. The arrangement of the palisade cells shields them to a extent.

If we pass to consider the effects of too intense an illumination we find that it is attended with considerable danger to the well-being of the protoplasm. When the leaves of certain plants, among which may be mentioned Oxalis acetosella, are kept exposed to very strong sunlight, and prevented from shading themselves as they normally do by changes in their position, they rapidly die, the duration of their life being reduced from two or three months to as many days. Bright sunlight has in other eases been found to check the growth in length of seedlings, the effect of different degrees of illumination having been compared by direct measurement. We find various arrangements in different plants which appear to be directed towards protecting them from the effects of too brilliant an insolation. Many which normally have their leaves so arranged as to expose their upper surfaces to the incident rays are found under bright sunlight to place them so that their edges and not their surfaces receive the light. This phenomenon has been called Paraheliotropism. It is exhibited normally by the leaves of Oxalis which have just been alluded to.

Another phenomenon, having for its purpose the protection of the chlorophyll, can be seen in many ordinary dorsiventral leaves. When brightly illuminated they are of a lighter green colour than when shaded, and this has been found to be due to a different arrangement of the chloroplasts in the two cases. In a leaf exposed to diffused light these are collected on the upper and lower walls of the cells just under the epidermis, and they present their broader surfaces to the incident rays. When the light is cut off altogether for a considerable time, and other conditions are unfavourable, they collect on the lateral and lower walls. When the leaf is brilliantly illuminated they place themselves upon the lateral walls only, and rotate on their long axis so as to present their edges instead of their 
surfaces to the light. In the first case the chloroplasts lie parallel to the surface of the leaf, and receive as much light as they can; in the last they lie at right angles to the surface so as to receive as little as possible. These two conditions are known as epistrophe and apostrophe respectively. When the conditions of the incidence of the light are altered, the chloroplasts change their positions accordingly.

The Alga Mesocarpus exhibits the phenomenon in a very striking manner. It consists of somewhat oblong or slightly elongated cells arranged in a filament. Each cell contains a single band-like chloroplast which lies nearly parallel to the long axis of the cell. In ordinary daylight it places itself so that the surface of the band is exposed to the illuminating rays, but if the light becomes intense, it revolves quickly upon its long axis, so that its edge is presented to them.

A different effect of a strong light is manifested by many dorsiventral structures, of which the thallus of Marchantia affords a good example. Whichever side of this organ is brilliantly illuminated, the dorsal or upper surface shows accelerated growth, so that the thallus exhibits epinasty. Some of the radially symmetrical structures which have been mentioned as bilaterally organised (page 354) behave similarly. Such are runners of Polygonum aviculare, and other plants of similar habit. This phenomenon has been called photo-epinasty, as the increased growth of the dorsal side is due to the access of light.

These facts may perhaps give us some idea of the influence of light upon protoplasm, and the condition of tone, one of whose chief features is the proper regulation of the permeability of the protoplasm by water. In darkness metabolism and growth are greatly affected, the latter being unduly accelerated. In the presence of too strong a light, a deleterious influence is exerted. An intermediate condition exists in which the vital processes of growth and nutrition and the sensitiveness to external influences are 
seen at their best. This is the condition of tone or phototonus, and its maintenance may be spoken of as due to the tonic influence of light. It is frequently said that light retards growth, and the tonic influence is associated with this retardation. This is however a somewhat incomplete presentation of the case. Retardation of the growth is not the only effect produced by the access of a proper degree of illumination. It is rather to be regarded as regulatory than retarding, and as it affects many other functions than growth, it seems more appropriate to consider the influence of the light as directed to the maintenance of this tone, which is really one of the conditions of health. How the actual effect upon the protoplasm is produced we cannot say ; it may be that the motility which is characteristic of healthy protoplasm and its control of its own permeability are adjusted to a particular relationship with the environment, of which phototonus is one condition.

The rays of the spectrum which exert this influence on the living substance appear to be those of high refrangibility, the blue and the violet. To these rays the protoplasm seems to be excessively sensitive. We do not explain their action when we say that they bring about a variation in the turgidity of the cells, or that they set up a change in the manner of their nutrition; the facts which we have called attention to can only be referred to the power of the protoplasm to respond to their influence.

The question of the influence of temperature upon the tone of the plant need not here be considered so fully, as in a preceding chapter we have discussed the phenomena of the general relations of temperature to the plant at some length. We may however again point out that plants are affected by variations in temperature in ways very similar to those depending on changes in light. It is not however always easy to ascertain the effects due to changes in temperature alone, as other conditions, such as light and moisture, usually vary at the same time as the temperature changes. 
As we have seen, the environment of the plant is partly the soil and partly the atmosphere, and the temperature of both may or may not vary simultaneously. We have seen that for each metabolic process there is a temperature at which it progresses to the greatest advantage. At lower and at higher points the protoplasm is less active, and in each case there is a point below which activity ceases, and one above which also it does not go on. The same thing we have seen is true of the processes of growth. We may say that for each plant there is a particular temperature at which it carries out the aggregate of its functions most advantageously, and it is when exposed to this temperature it is in a condition of the most complete thermotonus. This point is not the same for every plant, indeed considerable differences exist in this respect. We may say moreover that it is perhaps not so much a point as a range of temperature, for small divergences from the actual optimum point have but little effect upon the tone. Within this range the constant round of activity, chemical and physical, which is the expression of life, goes on most advantageously, below it it is injuriously affected, and at a minimum point it is suspended. At another point, higher in the scale, spoken of as the maximum temperature, the death of the protoplasm usually ensues.

We cannot explain the influence of temperature upon the protoplasm any more satisfactorily than we can that of light. All we know is that the two co-operate together to lieep the plant in the condition to which we have given the name of health.

The tone of the plant depends very greatly upon a proper adjustment of the relations between the protoplasts and water. For the maintenance of health it is essential that the normal turgidity of the cells shall not be disturbed. A definite amount of hydrostatic pressure inside such cells is necessary as we have seen for the due or efficient discharge of the processes of life. We may regard the maintenance of this relationship as one of the chief features of tone, 
for it involves a particular condition of the protoplasm with regard to its permeability. This condition may be regarded as a kind of effort, the living substance exerting some active living influence comparable to the condition of almost passive contraction, which is the normal condition of various muscular structures in the animal body. If this effort is increased, the existing hydrostatic pressure causes an excessive escape of water, and the cells become flaccid; if it is relaxed, the normal interchange of water between cells is diminished to their detriment, the permeability of the protoplasm becoming lessened.

A further aspect of tone may be seen to depend upon a constant and regular supply of oxygen. The function of this gas in vegetable life has already been discussed at some length in a preceding chapter. We have seen that if its access is interfered with the whole organism is for a time, if not permanently, upset, all the vital functions being thrown into disorder. The power of appreciating and responding to stimulation is also lost.

Another property which vegetable protoplasm possesses, and which is of the highest importance in adapting the organism to its environment, is what has been termed acclimatisation. This is manifested by the fact that after long continued applications of a particular stimulus the organism ceases to respond to it. This is shown by the fact that a plant, accustomed to live in light of but feeble intensity, if made to grow in a brighter region, though injuriously affected at first, will ultimately thrive in it as well as it did before. Similar phenomena in connection with temperature have been observed. 


\title{
CHAPTER XXIV
}

\author{
STIMULATION AND ITS RESULTS
}

WE may gather from what has just been said that there may exist for every plant, at any rate theoretically, a condition of adjustment when it is in absolute harmony with its environment, and when, consequently, its life is being regulated to the utmost advantage. We can see however that such a condition can be only momentary in any case, for the environment is in a constant state of change and the protoplasm of the organism is also exhibiting continual motility. For the maintenance of health or even of life it is essential that variations in one shall be adequately responded to by variations in the other, and the impossibility of securing indefinitely such a continual adjustment of relations is the cause of the cessation of life.

The responses which the organism makes to such alterations in its surroundings may now be considered in greater detail, and we may thereby form some acquaintance with the causes which have led to such great diversities in form, structure, and habit of life as we have already seen to characterise large groups of plants.

Any change in the environment which provokes some difference of behaviour on the part of a plant is spoken of as a stimulus, and the change of behaviour is to be looked upon as the result of stimulation. When we come to define more narrowly what we understand by the terms stimulus and stimulation we find it is not easy to restrict them however to such changes in the surroundings as we are able to observe and perhaps measure by even the most delicate instruments at our disposal. 
Many changes take place in protoplasm which escape our observation, originating perhaps in the condition of the protoplasm itself, or being due to disturbances in the interior of the plant. The normal course of metabolism may undergo a marked change in consequence of variation in the amount of some particular constituent of the food or of an alteration of the distribution or direction of the translocatory stream. Injury to the body of the plant may involve redistribution of energy or of material within its interior, which may have far-reaching effects upon the course of the vital processes. Variations in the supply of food, which may range between absolute starvation and over-engorgement, may produce very great changes not only in the outer life of the plant, but in the substances it produces in its metabolism and the energy which it liberates. The lack of oxygen may provoke an almost entirely new metabolism in connection with the production of such energy. These internal changes have been already discussed, and the effect of various factors at work in the organism have been examined, so that it is not necessary in the present connection to do more than emphasise the fact that we have in such matters evidence of stimulation and the response it provokes, evidence which points to the sensitiveness or irritability of protoplasm, as much as do the results of those changes in the environment which are purely external. The internal stimuli just noticed are largely chemical in character, and though chemical changes in the protoplasm are continuously occurring, many of them are directly instigated by such stimuli. Whether the automatic changes in organs and cells which we have already studied are due to stimulation is perhaps a little doubtful, but at any rate the nature of any stimulus provoking them has so far eluded investigation, and to all appearance they are not initiated in that way, but are independent of all stimulation.

Stimulation which is directly due to the physical conditions of the environment may be looked upon as the 
effect of any modification of the conditions which have induced tone. We have seen, for instance, that a particular degree or range of illumination sets up in a plant the condition of phototonus, which is one constituent of the healthy tone of the organism. Any modification of that illumination is followed by certain effects, the extremes of which we have already discussed. This alteration of the optimum illumination becomes at once a stimulating action, and we can speak of a stimulating influence of light, which is really any change in what we have called its tonic action. It can be in the direction of increase or decrease of the latter, but as it induces changes it must be regarded as stimulating.

What is true of light is also true of the other factors which combine to produce the healthy tone of the plant. Changes of temperature bring the organism nearer to or further from that optimum point at which it is in the most complete state of thermotonus and are responded to in various ways accordingly. Any alteration in the fluid contents of a cell brings about a change in what we may call the tonic tension of that cell, in which condition the permeability of the protoplasm exists at its best, and again an appropriate response is made.

In considering broadly the result of stimulation we must notice at the outset that it provokes a purposeful response. The living substance appears to have a definite aim; it may be to remove the stimulating cause if the latter affects it prejudicially; it may be to readjust its manifold forces to the new conditions to which the environment is suddenly or gradually subjecting it.

The means which the plant avails itself of are seldom abrupt and violent, like the manifestation of muscular contractility, but more frequently take the form of the modification of some rhythm which is characteristic of its behaviour. A few cases of sudden and sharp change are met with, as when the leaf of Mimosa droops on being touched, or when that of Dionaa rapidly closes over its 
captured prey. Less conspicuously purposeful are those changes in metabolism which are brought about in consequence of interference with the supply of food or oxygen, but even here evidence of purpose can be found if sought for.

To understand the purposeful changes in the behaviour of plants when they encounter modification of their surrounding conditions, we may consider briefly the nature of their environment. In the case of an ordinary terrestrial plant we find it to be as follows. The root system is embedded in the soil, among the particles of which the young root branches ramify as they grow, and to them the root-hairs become firmly attached; the soil undergoes usually only comparatively small changes of temperature, but is subject to a great deal of variation with respect to the amount of water it contains and the distribution of that water; it is composed of various materials, partly organic, partly inorganic, many of which are eagerly sought for by the plant, but others of them are of no use to it; of the former, some though valuable are not in a suitable condition for absorption. The stem rises vertically into the air and bears its branches and leaves; the air surrounding them contains a varying amount of aqueous vapour, together with a fairly constant quantity of carbon dioxide. The sub-aerial portion is subjected to the alternation of day and night, involving almost constant changes of degree of illumination, together with varying direction of the incident rays. During these times it meets with considerable variations of temperature and moisture as well as light. The whole plant is constantly acted on by the force of gravity. The subterranean portions are less affected by light, but they nevertheless receive a certain amount through the crevices between the particles of the soil, which varies from time to time both in amount and in direction. The environment, though to a certain extent constant, is nevertheless continually varying in these 
respects, so that no two plants are situated exactly similarly though they may be growing side by side.

The surroundings of an aquatic plant, though in some respects very different from those of a terrestrial one, exhibit the same general features and are subject to almost as frequent disturbances, though a watery environment is more uniform than a sub-aerial one.

We have considered already the effects which are produced by extremes. of light and darkness upon the behaviour and the structure of plants. We have however still to examine the rhythmic excitations to which plants are subjected by the variations of illumination which accompany the alternation of day and night. These are not accompanied in every case by conspicuous responses which can be easily observed, but certain plants exhibit a somewhat curious behaviour under these conditions. This is especially connected with the positions of their leaves, which assume different positions during the day and the night. This sensitiveness to the alternation of light and darkness is not however confined to ordinary foliage leaves, but is in many cases shared by cotyledons also. The degree of sensitiveness varies greatly in different plants.

This form of irritability is manifested in a very marked degree by many plants of the Leguminosa, the Oxalidacer, and a few other Natural Orders. Mimosa pudica may be mentioned as especially favourable for examination in this particular. When this plant is removed from light to darkness its leaflets droop, and the opposite pairs become closely approximated to one another, so that their upper surfaces are in contact. On being restored to light they separate again and attain their former expanded condition, but little time intervening before the change of position is assumed in either case. Another very good instance is afforded by Desmodium gyrans, the so-called Telegraph plant, the rhythmic movements of whose lateral leaflets have already been spoken of. During the day its leaves are extended almost at right angles to the stem (fig. 155, A); 
as night draws on, the terminal leaflets droop till they assume a position almost or quite parallel to the stem (fig. 155, в). The leaves of many others take up still more curious positions, in some cases becoming twisted on their petioles, or folded together in various ways. In some, as in Nicotiana glauca (fig. 156), they rise instead of falling and become somewhat closely approximated to each other.

These changes of position are generally spoken of as nyctitropic or sleep movements, though the latter term is

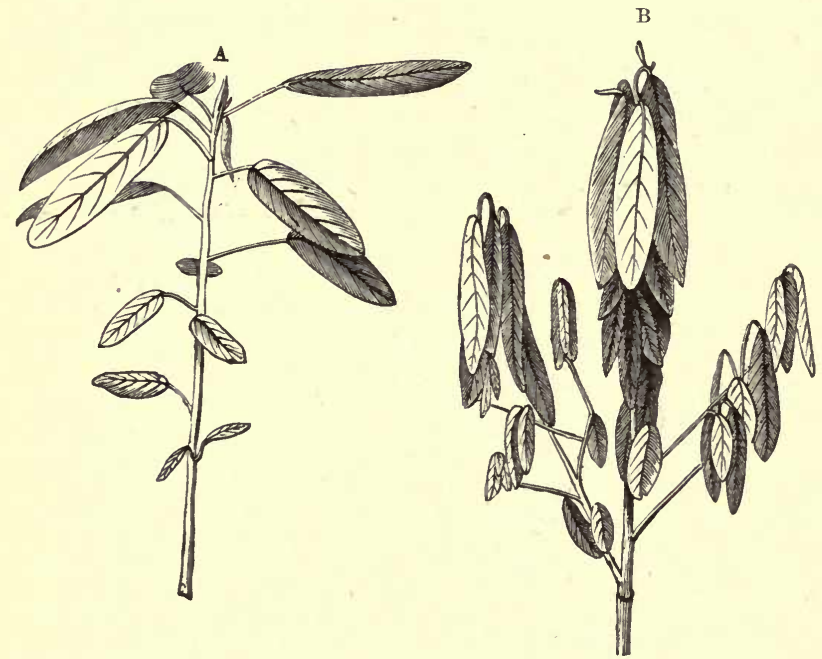

FIG. 155.-Desmodium gyrans. (After Darwin.)

A, stem with leaves as seen during the day; $\mathrm{B}$, a similar stem with leaves in the nocturnal position, pointing downwards.

misleading if it be interpreted to mean a sleep similar to that of animals. The latter phenomenon is attended by a temporary suspension of sensitiveness, which is not necessarily the case with the movements which we are discussing.

It is not difficult to prove that these curious changes of position are effected in response to the stimulation of the alternation of light and darkness, or to a rhythmic difference in the amount of light which they receive. The 
accompanying rhythmic variation of temperature no doubt in some cases also plays a part in the stimulation.

If a plant which changes the position of its leaves as described, is placed for a time under constant conditions such as darkness, the periodic movement is soon very much interfered with, even before the effect of darkness is evident in the loss of tone. If the rhythmic stimulus is not regularly applied the movement ultimately stops. The cessation is not however abrupt, but with most plants
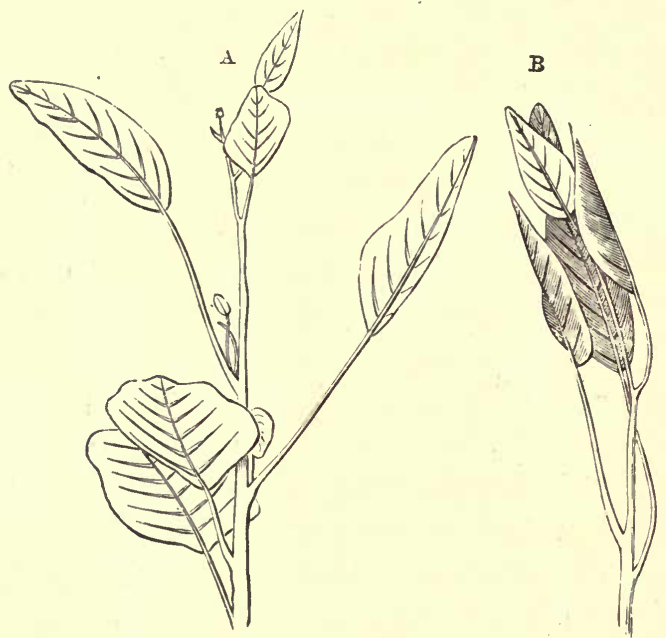

Frg. 156.-Nicotiana glauca. (After Darwin.)

$A$, shoots with leaves expanded during the day; $B$, the same in the nocturnal position.

the movements will continue for at least a day. The rhythm of the nyctitropic movement is excited by the stimulus, and is dependent for its permanency upon the continuation of the stimulating changes. Plants which are found in other countries to show this sensibility will, when cultivated in England, perform the movements at the normal hours, and not at times corresponding to the occurrence of day and night in the countries from which they come. Nor is it the mere alternation of day and 
night which they appreciate; it is rather the difference between the illumination they receive during the two periods which constitutes the stimulus, for some of them will not assume the nocturnal position unless they have been brilliantly illuminated during the day. The degree of sensitiveness in this case is not so great as in those where the diurnal and nocturnal positions are always regularly assumed.

The peculiar movements which the leaves perform in response to this stimulus are brought about by different mechanisms in different cases. In young leaves they are attendant upon growth, and are brought about by variations of turgescence upon the two sides of the leaf or its petiole, which are frequently followed by growth. We have seen that during growth the internal turgescence varies rhythmically, and leads to the curious movements of nutation or circumnutation. The actual nyctitropic movement is in these cases a modification of the extent of the circumnutation, the original rhythm being affected by the stimulus. The leaves which exhibit it can be seen by careful observation to be circumnutating during the day. When they assume their nocturnal position it is generally effected by their describing a much longer ellipse than that of their ordinary movement. In some cases only a single ellipse is described during the twentyfour hours; in others two ellipses, the nyctitropic one being much the greater in amplitude. In yet other cases, several ellipses may be described in the same time.

Adult leaves which show this movement do so by virtue of a special pulvinus, a kind of motile organ which is developed at that part of the leaf-stalk which joins the stem. This structure has special developments of parenchyma on its upper and lower sides (fig. 157), which become alternately turgid, and cause the leaf to droop and to rise accordingly. These leaves generally exhibit the movement for a much longer period than those in which it is brought about by variations of turgescence accom- 
panying or preceding growth. This naturally follows from the fact that the growth of leaves is not as a rule very prolonged.

That these movements are essentially dependent on the power of the protoplasm to receive impressions from without, or in other words upon its possession of tone, can

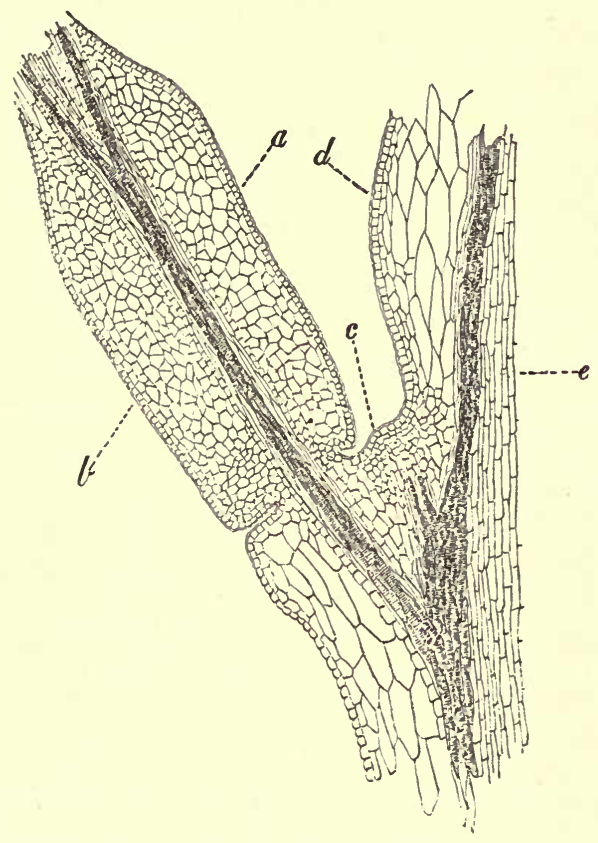

Fig. 157.-Pulvinus of Mimosa.

$a, b$, the succulent parenchyma of its upper and lower sides; $c$, bud;

$d$, parenchyma of Rachis; $e$, pith.

be seen from a study of the conditions under which they are performed. When the soil is too dry, or when from any other cause the protoplasm in the cells is not supplied with water in sufficient quantity, they cease. When the temperature is too low they are interfered with. Violent disturbance of the protoplasm by shaking the plant will 
in some cases prevent their occurrence for one or two nights.

The purpose of the movement is probably to protect the delicate leaves from excessive radiation, which affects them very prejudicially. Their upper surfaces are especially liable to be injured in this way, and it is noteworthy that in all cases these surfaces are most sheltered when they take up their nocturnal positions. Often the upper surfaces of leaflets are then closely approximated together; in Bauhinia the leaf folds itself upon its mid-rib as an axis, so as to hide completely the ventral face.

Movements which bear a striking superficial resemblance to the nyctitropic movements of leaves are those of the opening and closing of certain flowers, which take place with astonishing regularity and precision at certain hours of the morning and evening. Though they seem to be influenced by the alternation of light and darkness, it is more probable that they are really stimulated by the changes of temperature which accompany such alternation. These variations, to be effective, must lie however within the range already indicated as being necessary for the manifestation of irritability at all. The movement is due to rhythmically varying turgescence of the cells upon the two faces of the growing zone of the floral leaves, which is in these cases a narrow transverse band situated near their bases. This change of the turgescence is followed in many cases by actual growth, and as the latter is not of prolonged duration the flower can only open and close a few times while it is attaining its maturity.

Besides the general reactions of protoplasm to variations in those features of the environment which bring about modifications of its general tone, and which thus affect more or less the whole plant, we find instances of special sensitiveness in various parts to influences which are not appreciated by the whole of the living substance. Of these the most prominent are lateral light, gravity, 
contact with foreign bodies, moisture, and certain chemical stimuli. One or two other cases of special sensitiveness affecting only particular organisms may also be discussed.

LATERAL LIGHT. - The effect of the lateral incidence of light may be studied very easily in the case of young seedlings. When one of these is so placed that one side of its stem is more brightly illuminated than the opposite, a curvature soon appears in the part which is actively growing. This is of such a nature, and takes place to such an extent, as to cause the axis of the plant to take up a position in which it is parallel to the direction of the incident rays. It manifests itself in some cases very rapidly, in others more slowly. This response to the stimulus of a lateral illumination is not confined to the stems of seedlings, but may be seen to a greater or less degree in many adult plants. It is a matter of common observation that geraniums grown in a window all bend their stems and petioles towards the illuminated side.

In other cases the same stimulus may produce an opposite effect. When certain young roots are exposed to it, they curve so as to place themselves in the same position with regard to the incident rays, but with their growing apices in the opposite direction. Stems are said accordingly to grow towards, and roots away from, the light-source. This behaviour is not, however, confined to roots, it is exhibited by the tendrils of Bignoria capreolata, the peduncles of Cyclamen persicum, and by many other organs.

Leaves in many cases show a similar sensitiveness, but the position they assume is different again. They place themselves so as to present their upper surfaces at right angles to the incident rays.

These phenomena, thus associated with the incidence of a lateral light, are spoken of as heliotropism, apheliotropism, and diaheliotropism respectively. The advantages thus secured are in some cases very obvious; thus the heliotropism of a stem places its leaves in the most favourable position for the action of the chlorophyll in the 
process of photosynthesis of carbohydrate material; the same object is secured by the diaheliotropism of such leaves as exhibit it; the apheliotropism of a root assists it in penetrating into the erevices of the soil. The tendrils of Bignonia are aided by it in coming into contact with a support about which they can twine. I'he apheliotropism of the peduncles of Cyclamen, which are bent downwards in a hooked fashion, enables them to grow towards the soil, into which they press the capsule, thus burying the seeds.

The response to the stimulus varies sometimes with the age of the organ. The hypocotyl of the Ivy is heliotropic when young, but becomes apheliotropic when old.

The degree of sensitiveness varies very greatly in different organs. Some of the seedlings of Phalaris examined by Darwin responded to a degree of illumination so feeble that it was hardly sufficient to cast the shadow of a pencil upon a piece of white paper held close behind it. The rapidity of the response also varies, some organs bending almost immediately, while others do so much more slowly. To this point we shall return later. The movement of apheliotropism is usually much slower than that of heliotropism.

The bending is not caused by a direct interference of the light with the part actually growing. It would seem at first as if the retarding effect of light upon growth might explain the bending of the organ towards the light-source, the non-illuminated side continuing to grow and the illuminated one being prevented from doing so. This explanation is directly contradicted by the phenomenon of apheliotropism. It is moreover proved to be an insufficient explanation by the fact that the part which is sensitive to the stimulus is not the part which actually bends. Darwin showed this by preventing the access of the light to a small region about one-tenth of an inch in length close to the tip of the seedling, when he found that the heliotropic curvature did not take place, although the 
normally bending part was illuminated. Further, when the region normally curving under the influence of the stimulation is mechanically hindered from bending, the curvature takes place at a part a little lower down, which normally remains straight.

When the lateral light is fairly intense the resulting movement takes place uninterruptedly; when it is only weak the position is assumed by a series of zigzag movements, indicating that the new movement is an exaggeration of the ordinary circumnutation of the part. When the final position is reached the organ is found to circumnutate about the new direction of the axis.

A somewhat similar. response to the influence of a lateral light is exhibited by many unicellular organisms. When these are exposed to oblique illumination they take up a definite position with regard to the incident rays, placing their long axis parallel to them if the light is weak and at right angles to them if it is intense. This behaviour is known as phototaxis; it is exhibited by the zoospores of many of the Algæ and by certain Desmids.

Before leaving the subject of the effect of a lateral light in inducing these movements we may point out that the phenomena of heliotropism and apheliotropism must be distinguished from those of photo-epinasty and photohyponasty, which were alluded to in the last chapter (p. 364). The difference is easily seen, for in the latter cases the result of the access of the light is the same, whatever be the portion of the organ stimulated. The thallus of Marchantia becomes convex on the dorsal and concave on the ventral side, whether the light impinges on the one or the other. In the case of a heliotropic curvature the side which is stimulated always becomes concave ; in that of an apheliotropic one the stimulated side becomes convex.

Gravitation.-The force of gravitation exerts an influence upon plants which somewhat resembles that of lateral illumination. Most stems grow vertically upwards into the air; primary roots grow vertically downwards into the 
soil. A few organs, among which may be mentioned certain rhizomes and the runners of many plants, grow at right angles to the direction of gravity. When one of these is placed at an angle from the position which it usually assumes, a curvature of the growing organ results, which lasts till the normal attitude is regained. Thus when a young seedling is detached from the earth and laid upon its side, the stem gradually curves through an angle of $90^{\circ}$ and becomes erect, while the young root curves in the opposite direction till it points vertically downwards. Similarly when a runner is placed vertically, its apex is slowly deflected till it again grows parallel with the soil. These movements are termed apogeotropic, geotropic, and diageotropic respectively.

To prove these movements to be responses to the stimulus of gravitation it is necessary to eliminate the action of the latter force, and to observe the direction of growth under the new conditions. This can be done by causing the plant to grow supported upon an apparatus known as a Klinostat, one form of which, devised by Mr. Horace Darwin, is shown in fig. 158. The plant, growing in a flower-pot, is fixed in a wooden box $B$, which is secured by a thumb-screw th to the plate $p l$; the box is cubical in form and can be fixed either as shown in the figure, or with the axis of the pot at right angles to the spindle $k$ of the klinostat. The plate $p l$ is attached to this spindle, which ends in a point turning in the upper end of the left-hand support $s$. The spindle is also supported at $g$ on the friction wheel $\mathrm{fr}$. The spindle (with the plant attached) is made to rotate by means of a band of silk $d r$, passing round the wheel $w$, and also round a pulley on one of the axles of an American watch-action clock $c$, which is attached by means of the screw $R$ to the support $s$. By passing the driving-gear over the large pulley $W$, the spindle is made to rotate once in thirty minutes. By arranging wheels of different sizes at this point, the period of rotation can be made longer or shorter. 
In using the apparatus care must be taken that the centre of gravity of the plant and flower-pot shall coincide with the spindle, or the clock will have varying amounts of work to do in different parts of the rotation.

For ordinary purposes it is well to arrange the apparatus so that a complete revolution of the spindle may be made once in twenty minutes. When the plant is placed in a horizontal position on the revolving plate, every face of its axis comes successively under the influence of gravity, so that all parts of it are affected equally and similarly. It is then found that no curvature of the horizontal axis of the plant occurs in any direction.

Another experiment, due to Knight, pointing to the same conclusion, is that of growing a plant upon a rapidly revolving wheel mounted on a vertical axis. When the speed of the revolution is sufficiently great, though the plant is exposed all the time to the action of gravitation, the centrifugal force of the apparatus is so much greater than the force of gravity that the plant does not respond to the latter. Instead, it responds to the stimulus of the rapid rotation or centrifugal force, and the root grows outwards from the centre of the wheel while the stem grows inwards towards it. The force acts much like that of gravitation, and the plant responds to it in a similar way, the root growing in the direction of the force and the stem in one opposite to it. If the rotation is conducted at less speed, so that the centrifugal force is about equal to that of gravitation, the position assumed by the axis of the plant is that of a resultant between the two forces, in which it makes an angle of about $45^{\circ}$ with the vertical.

As in the case of heliotropic curvature, the part which receives, or is sensitive to, the stimulus is not the part which curves. In the case of a root it has been demonstrated by Darwin, and more recently by Pfeffer, that the sensitive part is the tip, while the curvature takes place at a point further back, where active growth is taking place. 


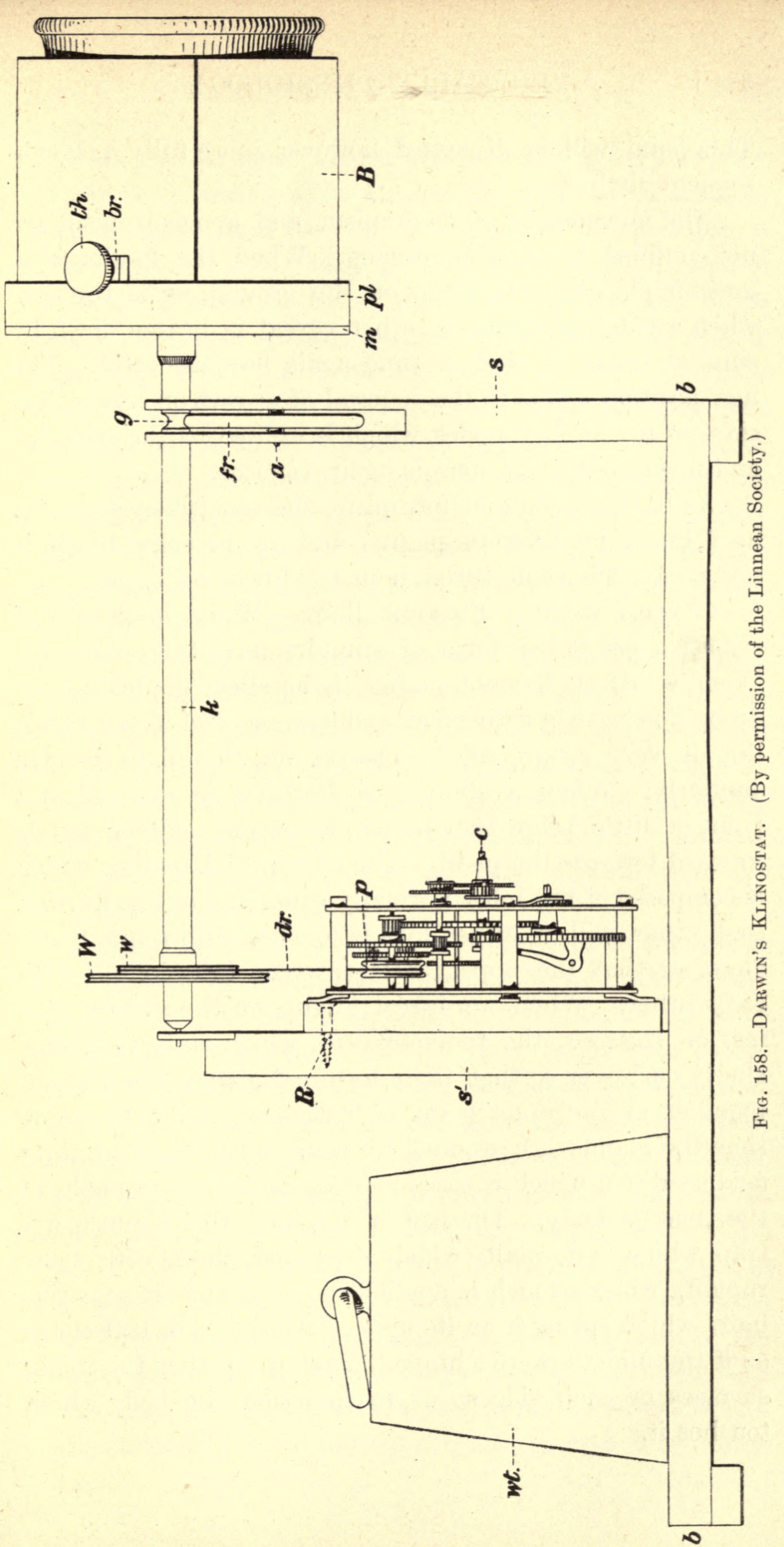


This point will be discussed however more fully in a subsequent chapter.

The movements of geotropism and apogeotropism are not confined to growing organs. When the haulm of a grass is placed horizontally on the ground, as is the case when a patch of wheat or other cereal is beaten down by wind or storm, it after a time again becomes erect. The new position is due to the renewal of growth on the undersides of the swollen nodes, which is excited by the stimulus and proceeds till the stem is again vertical.

As in the case of heliotropism, the sensitiveness varies very much in.different plants, and the response made is not always the same throughout the life of an organ.

Contact with a Foreign Body.-Many instances of sensitiveness to this form of stimulus have been observed. When a leaf of Mimosa pudica is handled, the leaflets all droop downwards with great suddenness, and if the handling is very rough, all the leaves on the plant behave similarly. When a stamen of Berberis is touched at a point a little below the anther, the whole stamen bends forward towards the pistil. The stigma of Mimulus, which is composed of two lobes normally extending outwards from each other, will, if either lobe is touched with a fine point, close, so that the upper surfaces come into contact with each other. When an insect alights on the surface of a leaf of Drosera, the tentacles with which it is furnished slowly curl over so that their terminal glands are brought together at the exact point of irritation, and at the same time the glands are excited to pour out a viscid slightly acid secretion which is capable of digesting the proteids of the insect's body. The leaf of Dioncea, the Venus's flytrap, which is normally widely expanded, closes with some rapidity when a touch is applied to one of the six sensitive hairs which spring from its upper surface. The leaf closes as if the mid-rib were a hinge, bringing together the upper surfaces on each side so as to imprison the body which touches it. 
This form of sensitiveness is exhibited in a very striking way by the growing apex of a young root. If a seedling bean is taken, and its tip is stimulated by pressing it lightly against some hard particle, or if a small piece of cardboard is attached by a drop of gum to one side of its apex, a curvature speedily results which causes the root to bend away from the irritating body. If the movement takes the sensitive part away from the latter the curvature is slight, but if, as in the case of the attached cardboard, the foreign body accompanies it in its displacement, the curvature will continue until the root is coiled completely round. The stimulus in the case of this movement must be prolonged, differing thus from the cases already noted, in which a mere touch is sufficient to bring it about.

The cause of this curvature must be the sensitiveness of the protoplasm to the stimulus of contact. The part which curves is some little distance from the apex, at which the capacity for receiving the stimulus is located, and the mechanism of the curvature is a modification of the distribution of turgescence of the cells in the zone of growth. It is only while that part is actively growing that the curvature can be caused.

Another kind of curvature can be detected in the course of the growth of young roots, which differs fundamentally from the one just described, and the two must be carefully distinguished from each other. If a young root comes into contact with an obstacle such as a small stone, so that the latter presses not upon the tip as in the case described, but upon the region of the growing cells some little distance further back, the resulting curvature causes the root to bend towards the obstacle instead of away from it. This appears to be due to the contact injuriously affecting the cells which are pressed upon, so that their growth is retarded or stopped. The cells on the other side of the root not being affected, a curvature results from their continued growth. These two capacities for curvature are of great assistance to a root during its growth downwards 
into the soil. On coming into contact with a particle of earth which is directly opposed to its progress, the tip becomes first stimulated and the subsequent curvature causes it to be deflected past the obstacle if it is not too large. A little further elongation, followed by an apogeotropic movement, brings the growing zone into contact with the particle and the converse curvature follows, so that the root grows round the obstacle, and then resumes its normal direction downwards, under the stimulus of gravity.

Perhaps the best instance of sensitiveness to slight contact is afforded by the behaviour of twining organs, tendrils, petioles, and climbing stems, the twining of these organs round their supports being altogether due to it. Very great differences of irritability are met with, tendrils generally possessing it in a very high degree, but climbing stems often exhibiting it very feebly ; indeed some observers deny that they possess this form of sensitiveness. In the most sensitive cases a very slight touch is sufficient to bring about a perceptible curvature in a very short space of time. Darwin found that the contact of a small loop of thread, weighing not more than $\frac{1}{32}$ grain, with one of the tendrils of Passiflora gracilis, caused it to bend, while a mere touch with a hard substance induced it to assume the form of a helix in about two minutes. This is perhaps the most sensitive tendril known; with others a stronger stimulus is needed, and the time taken for the response is longer, the irritability varying considerably. Slight rubbing is more effective than mere contact.

The behaviour of tendrils in twining is somewhat peculiar. When young they are generally circumnutating, and if in their movement they come into contact with any foreign body, they begin to curve round it. If the contact is not prolonged the tendril will curve for some time, but will ultimately straighten itself and move as before, till it touches something else. If, on the other hand, the body first touched is one round which the tendril can twine, it coils itself round it; the stimulus thus persists and the 
resulting eurvature inereases it, bringing more and more of the sensitive side into contact with the support, till the latter is encireled many times by the sensitive twiner. The coiling is seldom confined to the part of the tendril in contact with the support, but the free part between the latter and the axis of the plant also twists itself into a kind of helix. If the two are not very close together this helix usually shows two parts, the coils of which are in opposite directions. This is however only because the filamentous body is attached at both ends.

A tendril, though thus sensitive to contact, does not coil, according to Darwin, if its sensitive surface is struck by drops of rain, nor, in the case of the Passiflora already alluded to, if contact takes place between two tendrils.

The sensitive region varies in different tendrils, but it cannot be so strictly localised as in the case of a growing root. They are usually irritable on one side only, which is slightly concave, though in some cases the sensitiveness extends all round them. The lower part of a tendril is, as a rule, only sensitive to prolonged contact. Their susceptibility further varies with their age, being greatest when they are about three parts grown. The part which first responds to the stimulus is usually the part touched, but, as we have seen, the coiling also takes place nearer their bases, so that we have an evident transmission of the stimulus backwards, as in other cases noted. The method of response is usually increased turgidity upon the convex side, followed by greater growth. In many instances careful measurements have shown that both the concave and convex parts grow during the coiling, but in a few cases the concave side either does not grow or becomes actually shorter than before.

This sensitiveness to contact which is so markedly shown by tendrils is possessed also, though to a much smaller extent, by most climbing stems. These organs show the movement of circumnutation very conspicuously, the portion which takes part in the formation of the spiral 
being frequently of considerable length. This is of course a great advantage in enabling the stem to find a support. The continuation of the circumnutating movement after contact with such support, has given rise to the view that circumnutation alone will enable climbing to take place. Consideration of the behaviour of various twining stems with supports of various thickness has shown however that this is supplemented by changes resulting from the contact effected by circumnutation, and therefore from the possession of the sensitiveness under consideration.

Twining stems show individual peculiarities in the direction of their twisting, and in the nature and particularly the thickness of the support they need. The stem of the Hop twists in the direction taken by the hands of a watch; that of the Convolvulus in one diametrically opposite. The direction of the twining is not however always constant; Darwin noticed that it was not so always even in a single individual. In Scyphanthus elegans it was reversed in successive internodes of the same stem. Many of our ordinary climbers can twine up a support having only the thickness of a piece of string; other plants, particularly the climbers of tropical forests, need supports of some inches in diameter.

The twining of stems is often accompanied by a torsion of the stem, or a twisting round its own axis. This is not however of universal occurrence.

The stimulus of contact is sometimes followed by an outgrowth or hypertrophy of the part affected. This is seen in the tendrils of Ampelopsis Veitchi, which on prolonged stimulation develop little adhesive discs, that are closely adpressed to roughnesses in the surface of the support and, becoming mechanically attached to them, enable the plant to maintain a very strong hold upon the wall or other support to which it is elinging. The roots of Thesium show a similar property. When they come into contact with other roots growing near them they develop a swelling at the point of contact, from which certain cells 
grow out and penetrate the host, forming haustoria (fig. 159). The parasite Cuscuta, often found growing on clover, is affected in the same way, first twining round the clover stem and then putting out haustoria, which penetrate its tissues (fig. 160).

Another form of irritability is exhibited by many growing shoots, which is perhaps somewhat akin to sensitiveness to contact. If a shoot is gently struck laterally several times near its base, its apex curves over towards the side struck. If the blows are given

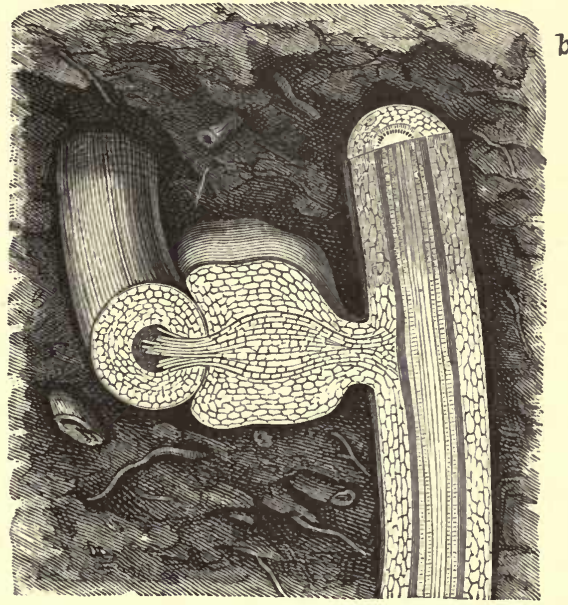

FIG. 159-Thesium alpinum. PIECE of A ROOT WITH SUCKER IN SECTION. $\times 35$. (After Kerner.)

near the apex, the resulting curvature is in the opposite direction.

The mechanism whereby the response to the stimulus of contact is brought about in growing organs we have seen to be an increased turgidity on the convex side, followed by growth. In those cases where the organ is mature it is evident that growth can have nothing to do with the movement. In these instances we have rather to do with a modification of turgescence, involving a redistribution of the water contained in the organ. The falling of the leaflets and leaves of Mimosa is due to a sudden change in the protoplasm of the cells on the lower sides of its pulvini, in consequence of which water escapes from them into the intercellular spaces between them. It is attended by a change of colour, the pulvinus becoming of a deeper green in consequence of the replacement of the air there by water. If a leaf is cut off just above the pulvinus and 
the plant allowed to recover from the effects of the injury, subsequent stimulation of an adjacent leaf causes water to

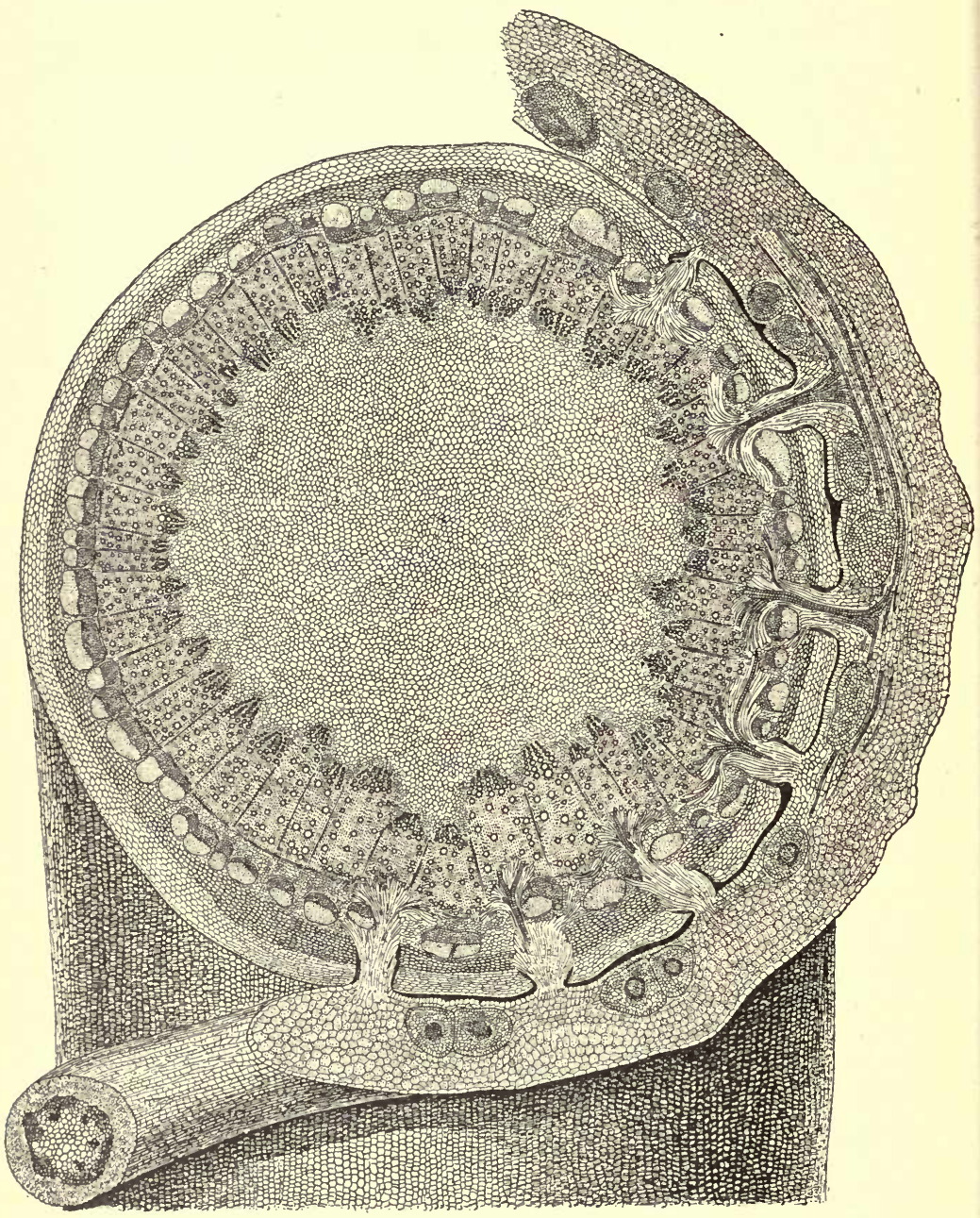

Fig. 160.-Section of Stem of Dicotyledonous Plant attacked by Haustoria of Cuscuta.

exude from the cut surface of the pulvinus. The cases of the irritable stamens and stigmas are probably to be 
explained similarly. The closing of the leaf of Dioncea (fig. 161) is due also to a redistribution of the water in the cells, brought about by a rapid change in the protoplasm, perhaps akin to contraction. In Drosera the inflexion of the tentacles has been found to be preceded by a peculiar churning movement of the protoplasm in the cells upon the side which becomes concave. This movement, which Darwin, who discovered it, called aggregation, is attended by a loss of turgidity.

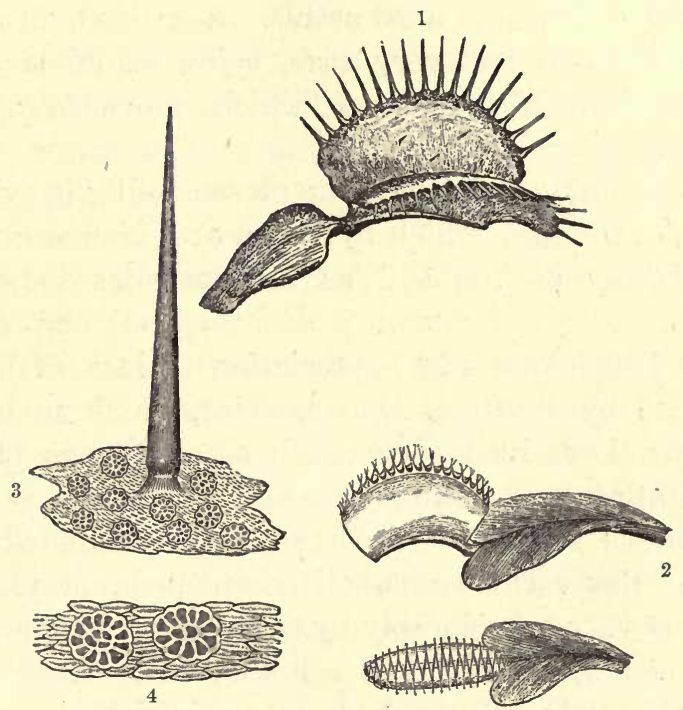

Fig. 161.-Leaf of Dioncea muscipula.

1 , open ; 2 , closed ; 3 , one of the sensitive spines $(\times 50) ; 4$, glands on the surface of the leaf $(\times 100)$.

Morsture. - Sensibility to variations in the moisture of the environment is not so widely distributed as are the forms of irritability hitherto discussed. It is exhibited among green plants chiefly by young roots and by the rhizoids of the Hepatica ; it also occurs in the hyphæ of certain Fungi. These organs tend to curve in the direction of a moist surface if they are growing near one. When young seedlings are cultivated in a vessel which 
contains moist sawdust or sand and is perforated so as to allow the rootlets to protrude, these at first grow vertically downwards, according to their geotropism. Soon after they protrude they curve to a greater or less extent towards the moist surface, as if seeking the moisture. This behaviour can be seen more easily if the vessel is inclined at an angle to the vertical. The phenomenon is known as hydrotropism. The root-tip, as in other cases, is the sensitive part, while the curvature takes place further back, where growth is most active. Negative hydrotropism or aphydrotropism is very rare, being exhibited only by some of the Myxomycetes, which move away from moisture.

The advantage of this form of sensibility is evident in the case of the root, which by virtue of it is drawn towards the moisture of the soil as it penetrates between its particles.

A curious instance of appreciation of lack of moisture is afforded by Porlieria hygrometrica, which under such conditions closes its leaflets much as nyctitropic plants do when light gives place to darkness.

Chemical stimuli. - We have already alluded to the fact that the various metabolic phenomena of plants are influenced very considerably by changes in the composition of the sap which the cells contain; that certain constituents stimulate the protoplasts to initiate or to alter particular reactions in those cells. Besides these responses to chemical stimuli there is evidence that vegetable protoplasm can modify its normal behaviour in other ways when exposed to similar influences. This form of sensitiveness is less widely distributed than those which we have just discussed, but instances of it are fairly abundant, especially among the more lowly forms of plants.

A certain number of unicellular organisms are strongly affected by the presence of free oxygen. The most interesting case of this sensibility is that of Bacterium termo ; when a number of these plants are placed in a drop of 
water upon a slip of glass and examined under the microscope, they are found to collect at the edge of the coverglass. If a small green Alga is placed in the drop of water with them, and the slide exposed to light of sufficient intensity to enable the decomposition of carbon dioxide to take place, the coincident evolution of oxygen attracts the bacteria, which at once swarm round the Alga. So sensitive are they to this attraction, that if the spectrum of sunlight is thrown upon the Alga, the bacteria accumulate at those parts which are illuminated by the red and blue rays, which we have seen to be capable of effecting the evolution of the oxygen. This response to the attraction of oxygen is not confined to these bacteria ; it is exhibited by many zoospores and also by the plasmodia of some of the Myxomycetes.

When the necks of the archegonia of the Bryophyta and Pteridophyta open with a view to the fertilisation of the oospheres which they contain, they discharge a certain mucilaginous fluid, which attracts to the organ the freeswimming antherozoïds. Careful experiments have been made in many cases to ascertain what is the nature of the attraction, and it has been found that the mucilage contains various substances which the antherozoïds seek. In the cases of the Ferns and some Selaginellas, it has been determined that the attractive body is malic acid. When a capillary tube containing a weak solution of this substance is inserted into water containing some of the antherozoïds, they make their way very quickly to the orifice of the tube. They are very sensitive to the presence of the acid, being guided apparently in their movements by very slight differences of concentration. When the acid exceeds a certain strength they avoid it as earnestly as they seek it when it is in greater dilution.

In the case of the Mosses the attractive substance is cane-sugar. Alkalies in any degree of concentration repel the antherozoïds of both groups.

A similar sensitiveness to chemical stimulation marks 
the plasmodia of the Myxomycetes. They move slowly towards a watery extract of tan, but retreat from a solution of sugar, glycerine, or certain neutral salts. The zoospores of Saprolegnia are attracted by a solution of extract of meat.

The sensitive tentacles of Drosera can respond not only to contact, as already described, but also to various substances placed upon the leaf. They are easily induced to bend by drops of liquid containing proteid matter, such as solution of albumin, or milk. Certain inorganic salts, especially carbonate of ammonia, produce the same effect.

A curious instance of this kind of irritability has been put on record by Miyoshi. He cultivated certain fungi in gelatin containing a small proportion of sugar. Under the stratum in which the hyphæ were ramifying, he placed another containing a larger proportion of sugar, and between the two arranged a membrane. The hyphæ very soon grew towards the stronger sugar solution, and to reach it penetrated the membrane.

Other instances of similar behaviour might be quoted. To this form of sensitiveness the name of chemotaxis has been given.

A few other forms of irritability have been observed in various plants. Certain plants growing in currents of water take up a definite position with regard to the direction of the current, some growing with it, others against it. Certain plants appreciate small differences of temperature and modify their growth accordingly. Almost all show a peculiar relationship to their substratum, stems growing out from it and roots into it in a direction at right angles to the surface. This can be seen by cultivating them so that they do not emerge in the normal direction but from the side of a cube of earth. They do not long maintain this direction, as they speedily feel the influence of light and gravity. If however appropriate means are adopted to eliminate these, the growth is always at right angles to the surface of the soil in which they live. 
If we now return to the study of the rhythmic changes which we have seen to be essentially characteristic of vegetable protoplasm, we see that while rhythm is no doubt inherent in plants it lends itself especially to such changes as are caused by stimulation. It is indeed this feature which is especially brought out by the various responses made to changes in the environment. While it occurs with some regularity when conditions are kept constant, it is easily affected by external causes. The effect of continuous darkness, or of too great cold, or other abnormal conditions, is that the rhythmic movements are made irregular and ultimately stop. In many cases differences in the degree of illumination during the day affect the readiness with which the nyctitropic movements of the leaves are brought about. After a day of brilliant sunshine they set in more quickly than after one of dull light.

These movements may show indeed a secondary induced rhythm superposed upon a normal one. The movements of heliotropism, geotropism, \&c. may be looked upon as instances of this. We have seen that they are based upon the ordinary movement of circumnutation, and are in fact exaggerations of it. As the latter is generally a manifestation of a rhythm of turgidity in the cells affected we have in them a case in point. In other cases the tendency to rhythmic change can be demonstrated by the production of an altogether artificial rhythm induced by submitting the plant to intermittent stimulation. F. Darwin and Pertz have described a very interesting experiment of this nature. A plant was fixed to a spindle placed horizontally, in a modification of the klinostat, and was by an arrangement of clockwork made to undergo a semi-revolution at intervals of thirty minutes. The force of gravity thus exerted its effect upon alternate sides for this interval of time, so that each side of the stem became slightly convex apogeotropically in turn. After a period of exposure upon the instrument the clockwork was stopped. Instead of the side which was then undermost increasing its convexity 
till the stem was vertical, the two sides continued to become alternately convex, as if the reversal of the instrument was still taking place. There was, in fact, an artificially induced rhythm manifested.

While the movements of heliotropism show the superposition of an induced rhythm upon a natural one, a conflict between the two can be observed in many organs. The heliotropic curvature is not brought about by a direct movement of the bending organ, but by its describing a series of ellipses. The organ at the time of the incidence of the light stimulus is performing its ordinary circumnutation, the apex describing a circle. The effect of the stimulus is to turn that circle into an ellipse; when the rhythmic impulse coincides with the stimulus of the light, the movement is accelerated and the resulting curve takes the direction of the long axis of the ellipse; when the two act in the opposite direction to each other, the short curve of the same figure is described. The same result is obtained under the stimulus of geotropism when the stem or root has by any means been inclined from the vertical. The ordinary rhythm of circumnutation is resumed when the new position has been assumed and the stimulus consequently no longer acts.

The slow response to the action of a stimulating force may frequently be explained in the same way. Often however the long delay is due to peculiarities in the protoplasm which will be discussed in the next chapter.

The various positions which are assumed by the various sub-aerial organs of plants are evidently those in which they can react most advantageously with their environment. It must be borne in mind however that in every case during natural life the plant is receiving coincidently several kinds of stimulation, the effect of some being not infrequently antagonistic to that of others. It is not easy to discriminate between these, nor to say how the influence of each helps to determine the resultant response. This is the more difficult as not only the stimuli themselves but their relative potencies differ continually. 


\section{CHAPTER XXV}

\section{THE NERVOUS MECHANISM OF PLANTS}

IT is difficult to refrain from coming to the conclusion, from a consideration of the facts which have been discussed in the last two chapters, that the nervous system of the animal kingdom is represented in the vegetable one. That plants are sensitive to variations in the conditions surrounding them, and that the responses they make to such variations are purposeful and conduce to the well-being of the organism, is abundantly evident. The response to any external stimulus, moreover, has been seen to be dependent upon the plant being in a condition of tone, that is of health and vigour. If its well-being has been interfered with by such disturbances as deprivation of light, or lack of oxygen, or exposure to too high or too low a temperature, no response is given, for its irritability is in abeyance or destroyed. The lack of response is not due to a failure in the motor mechanism by which the change is brought about, but by an absence of power to realise the altered conditions which would constitute a stimulus to an organism in a condition of full health. The age of the organism again has been seen to have an important influence upon its power of receiving impressions and its behaviour in responding to them.

We have already called attention to the fact that the responses made to stimuli of different character afford clear evidence of purpose. No reply is at all haphazard, but is devoted especially to some definite object which is closely related to the stimulus.

Another consideration which bears upon this question 
is that an extremely small stimulus is able to bring about a very considerable effect, and that there is no direct or simple ratio between the intensity of the stimulus and the extent of the response, whether this takes the form of movement or chemical change. The tendrils of Passiflora, already alluded to, can be caused to move by the contact with them of a small piece of thread, weighing not more than $\frac{1}{32}$ of a grain, and the resulting movement will be of considerable extent and prolonged for some time. The sensitive hair of the leaf of Dioncea needs only a touch to cause a rapid movement of the whole leaf-blade; the pricking of the staminal filament of Berberis causes a considerable movement of a relatively bulky body. The seedlings of Phalaris bend with some speed towards a light which is not sufficient to cause a visible shadow at the distance at which they are placed from it.

It can hardly be imagined that such slight disturbances can act mechanically upon the parts that move. This point is illustrated by the observation made by Wiesner, that if a part which responds only to the stimulus of lateral light is exposed for some time to such an illumination, and then, before the heliotropic curvature has begun, is removed into darkness, it will slowly bend towards the side which has been stimulated. The same observation has been made by other observers in the case of the stimulus of gravitation. There is no explanation possible other than that the stimulus brings about changes in the protoplasm of the cells of the moving part, which slowly modify their relation to the water of their contents, so that a great alteration of their turgidity results. Moreover, the separation of the part stimulated and the cells which are the seat of the resulting action, implies that there must be in the plant a means of rapidly conducting such external impressions from one part to another.

If then we admit that there is even a rudimentary nervous system in plants, we may proceed with an inquiry into the degree of its differentiation, and the completeness 
of the parallelism which it may be expected to show with the corresponding system in the animal kingdom.

The latter, in the most completely organised beings, can be shown to possess three distinct parts : one by means of which external stimulation is received and appreciated; another whereby movements, \&c. are caused; and a third which is a regulating and controlling part, and which can coordinate the responses to stimulation, or can initiate movements, \&c. in its absence. There are also definite paths or channels by which the three are brought into connection with each other, generally by impulses passing along such paths in definite directions. In the higher animals these are well differentiated from each other; we have the sense-organs, each devoted to and fitted for the appreciation of particular stimuli. We have various motor mechanisms, usually consisting of muscles or glands which are thrown into activity in consequence of the reception of impulses by senseorgans. It may appear to be straining matters somewhat to class these as part of the nervous system, but it does not appear wrong to do so in the sense that they are the means by which alone the working of the more particularly nervous elements of that system can be detected. The nervous and motor systems are indeed so closely connected that for the purposes of this discussion no inconvenience will result from classing them together. In the animal we have nerve-cells occurring singly or in groups, forming very large aggregations such as the brain, or smaller ones, the nerve ganglia. All such aggregations, or even single cells, are concerned in the task of co-ordinating stimuli and responses, or regulating the general life of the organism. Lastly, we have well-differentiated nerves which serve as the means of communicating between the three other factors already mentioned. Each nerve-fibre ends in a sense-organ or a motor mechanism, such as a muscle or a gland-cell, and in a nerve-cell belonging to the co-ordinating apparatus.

We can easily recognise in plants certain structures 
which may not inaptly be termed sense-organs, as we can localise in them the power of perception of stimulating influences. Darwin found that the seedlings of Phalaris were not sensitive to the faint light employed in his experiments, except at a small region extending about $\frac{1}{10}$ inch from the apex. If this part were covered by an opaque screen in the shape of a little blackened cap of not sufficient weight to cause any flexion of the stem, the seedlings no longer bent towards the light. We have already noticed that the root-tip at a very short distance from the apex is the only part which is appreciative of contact with a foreign body in such a way. as to cause the growing part to set up a curvature that will carry the tip away from the obstacle. The same region has been shown to possess the power of appreciating the stimulus of gravitation. This has been shown by Pfeffer in a very ingenious manner. He caused the roots of various seedlings, especially using Vicia faba, to grow into small and light glass tubes, closed at one end, and bent at a right angle about $\frac{1}{16}$ inch from that end. The cultivation was carried on on a klinostat for about twelve hours, when the root had penetrated to the end of the tube, and had consequently become sharply bent at a right angle about $\frac{1}{16}$ inch from the apex. Roots so prepared were then allowed to continue their growth after being placed in various positions. When the terminal portion was vertical, and the long part of the root consequently horizontal, the root continued to grow without any curvature; when these conditions were reversed a geotropic curvature resulted, which continued as long as the tip of the root was mechanically prevented from becoming vertical. Other observers have proved the same thing in different ways. Cisielski amputated the tips of certain rootlets, and laid them horizontally on a support. They did not then show any sensitiveness to gravitation, until they had recovered from the wound and a new root-tip was developed upon each. As soon as the new tip was formed, the rootlets showed a power of reacting to the stimulus of gravitation, and the curvature 
resulted in the usual place. If we turn to the reaction of the leaf of Dionæa to contact, we find that the whole leaf may be somewhat roughly handled without closing, so long as no contact is made with the hairs, three in number (fig. 162), which arise upon a particular portion of the blade. So soon, however, as one of these is touched, the leaf closes.

It is impossible to avoid the conclusion that we have to do in these instances, which are only representative ones,

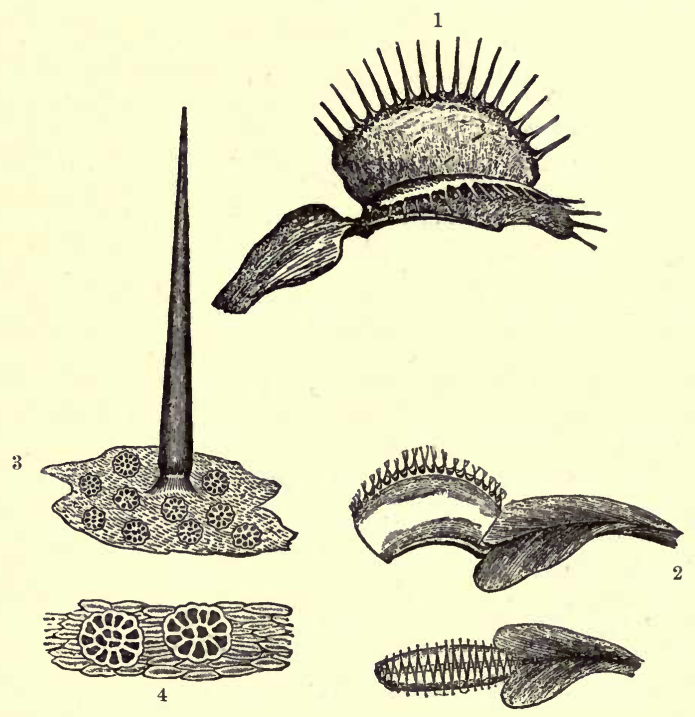

Fig. 162.-Leaf of Dionca muscipula.

1 , open; 2 , closed; 3 , one of the sensitive spines $(\times 50) ; 4$, glands on the surface of the leaf $(\times 100)$.

with a localisation of sensitiveness, or the differentiation of sense-organs. If we compare them with physiologically corresponding regions in the animal we find a certain agreement, though it must not be pressed too far. The power of sight is very complete in the higher animals, partly in consequence of the highly differentiated character of the eye, but in the lower animals it becomes less and less perfect, till in some it goes probably little further than the power of appreciating light. This power we have seen 
to be possessed by certain parts of the young seedlings of various plants in a very high degree, and by other organs to a less extent. The sense of touch may be compared with the power of responding to the stimulus of contact shown by tendrils and by the tips of roots; the muscular sense, or power of appreciating weight, is perhaps comparable to the property of responding to the attraction of gravitation, while the chemotactic behaviour of the organisms described in the last chapter suggests a rudimentary power of taste or smell, or both.

The differentiation of these mechanisms in plants is anatomically very slight. Indeed, no dissection will exhibit any special feature of the structure which can be associated visibly with the perception of the stimulus. It remains a property of the protoplasm of the cells in question, but is only one among many properties that the latter possesses. The direction of differentiation in vegetable protoplasm is not anatomical. But such a differentiation is very considerable physiologically. The degree of sensitiveness which many of these organs possess is extreme, as we have shown already by several examples.

Another somewhat remarkable fact, in view of the peculiar character of the differentiation of these organs, is that the same sense-organ is sensitive to many stimuli, though in different degrees. We have noticed in the case of the root that its tip appreciates contact, gravitation, and differences in hygrometric condition. There is nothing anatomical corresponding to this. If a sensitive organ is acted upon at the same time by two stimuli which both affect it, and which usually produce opposite movements, the resulting position is always that which would be caused by the stronger of the two. The organ is, in fact, able to receive both stimulations simultaneously, and to respond to each as if the other were not received.

If we turn to the second feature of the nervous system, we find that the motor mechanism of the plant seems at first to be entirely different from that of the animal. 
Closer consideration however lessens the difference considerably. The motor mechanism of an animal is very largely either muscular or glandular. The contractile power is but little developed in vegetable protoplasm, and when present it seems to be rather passive than active, to produce frequently recoil, rather than true contraction. Still, the latter is not entirely absent. We have seen that it can be detected in the pulsation of vacuoles, in ciliary motion, and in the crawling movements of the Myxomycetes. Its manifestation under an external stimulus seems to be evident when a filament of Mesocarpus splits up into its constituent cells as soon as an electric shock is sent through the water in which the plant is floating.

Though the power of contraction is comparatively seldom found, it has its representative in the power which vegetable protoplasm possesses of resisting or assisting the transit of water. The effect is really similar in both cases ; in the one the disturbance to the protoplasm leads to a contraction of its substance, in the other to its modifying its resistance to the passage of water through it. Each protoplasm responds in its own appropriate fashion, which is based upon the need of the organism of which it is part. The main requirement of most animals is freedom of locomotion or rapid assumption by the body of new positions. The most important duty of the plant is the regulation of the water supply upon which its constituent profoplasts are so dependent.

The effects of stimulation may be seen in glandular organs in plants as well as animals. Both Drosera and Dioncea are excited by contact to pour out on to the surface of their leaves acid digestive secretions, which are the result of changes in the activity of the gland-cells.

The conduction of the stimuli received is due in animals to the existence of differentiated nerves. The way in which it is carried out by plants has been much debated, but since the discovery of the continuity of the protoplasm through the cell-walls there is little doubt that we 
have here a similar mechanism. There is scarcely any differentiation, but the power of the protoplasm to conduct disturbances from one part of the cell to another is a matter of common observation. The connecting strands between adjacent cells (fig. 163) will suffice to suggest how impulses from the tip of the root may reach the growing cells.

The co-ordination of these factors we have seen is one of the most marked features of a highly differentiated nervous system. In this respect we cannot note anything in the plant which in its elaboration or in its peculiar

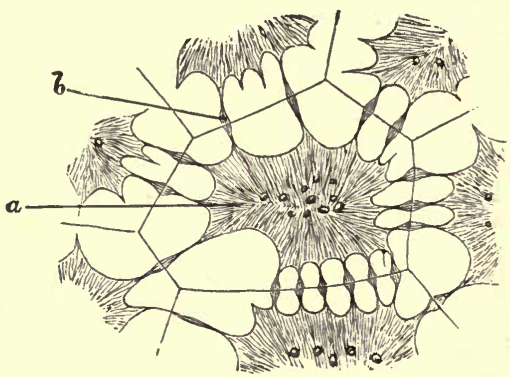

Fig. 163.-Continuity of the Protoplasm of contiguous Cells of the Endosperm of a Palm Seed (Bentinckia). Highly magnified. (After Gardiner.)

$a$, contracted protoplasm of a cell ; $b$, a group of delicate protoplasmic filaments passing through a pit in the cell-wall.

efficiency can be compared with the co-ordinating mechanism of animals. Certain responses to stimulation can be effected, but no definite regulation of any function shows any great completeness. We have seen this particularly in the case of the influence of temperature. Though a certain range of temperature is imperative for the plant's well-being, it has no power, or but little, to co-ordinate its own production or expenditure of heat with the variations of temperature to which it is exposed.

Neither anatomically nor physiologically do we find much differentiation in the direction of such co-ordination. The plant shows an almost complete absence of the 
differentiation which reaches its highest point in the nervecell. There is apparently no co-ordinating mechanism which receives the impulses from the sense-organs, and initiates in consequence the resulting movement. One case only has so far been put on record which even suggests a complexity of this kind. Attention has been called by Darwin to a peculiarity in the behaviour of the tentacles of Drosera, in which something of this nature is seen. When one of these organs is stimulated, its actual bending is preceded by a curious motility of the protoplasm of the cells of its stalk which has been called aggregation. If a tentacle on the surface of the leaf is excited, the tentacles of the margin are gradually inflected towards the excited spot. If the cells of one of these marginal tentacles are watched during the experiment, their contents are found to undergo this aggregation, but those nearest its apex manifest it first. If the aggregation were the direct effect of the stimulus, those which it reached first, i.e. those nearest the base of the tentacle, would respond first. The stimulus, apparently, has to travel up the gland, and a disturbance has to originate at its apex in response, this disturbance travelling down the tentacle in the direction of its base. Darwin has pointed out that this corresponds in a measure to the reflex action of the animal organism.

But though this co-ordinating power is very feebly developed we cannot deny that there is a power or property of protoplasm which represents it, even if in only rudimentary form. We have already alluded to the purposeful character of the responses to stimulation. There must be some means by which an appreciation of the character of the stimulus is communicated to the protoplasm, which suggests a certain possibility of perception, which must be the antecedent of co-ordination. We do not know whether the fact that the response is localised depends upon the possession of particular properties by the responding organ, so that while the impulses set up in the sense-organ travel 
in all directions through the plant, only certain cells can be excited to change in response to them, or whether the paths of the conduction of the impulses only take them to the responding organ. But the fact remains that the response bears a definite relationship to the stimulus, particularly to its locality, and to a less degree perhaps to its intensity. If a root-tip is brought into contact with an obstacle, the bending is invariably in such a direction as to enable the root to pass it. When one is allowed to impinge upon a small stone at right angles to its direction of growth, the curvature continues till the root has turned through a right angle, and can for a short distance, at any rate, grow parallel to the opposing surface, till, passing it, it can again respond to the influence of gravitation and grow vertically downwards. The stimulus causing the movement of hydrotropism serves to bring the root-hairs into contact with the moist surface, thus enabling them to discharge their appropriate function.

The behaviour of the tentacles of Drosera rotundifolia is very interesting in this connection. The leaf is of some size, and can therefore receive stimuli over a fairly large area. When the tentacles bend over in response to the alighting of an insect, they do not do so irregularly, but always place their glandular apices directly upon the spot which is the centre of the disturbance. This is very definitely purposeful, the invader being captured and digested wherever it alights, as all the tentacles are brought to bear upon it.

The purposeful character of heliotropic and diaheliotropic curvatures is also very evident, the leaves being always placed thereby in the position most favourable to the discharge of their functions.

The very rudimentary differentiation of any mechanism for co-ordination suggests a very immobile condition of the co-ordinating protoplasm. There are several considerations which support this view. In many cases the movement of heliotropism does not commence till a considerable time 
after the access of the lateral light, the actual time varying in different cases. Similarly the apogeotropic curvature of a stem placed horizontally may not be observable till the stimulus has lasted for more than an hour. We have what is generally called a long latent period before the manifestation of the irritability. The time is taken up in bringing about the response to the stimulus and not in appreciating it. The power of appreciation is generally rapid, as we should imagine when we remember the great degree of sensitiveness as measured by the smallness of the stimulus which is necessary to produce an effect. The sluggish nature of the co-ordinating mechanism can be seen from the fact that the removal of a stimulus before any response to it has become evident does not prevent that response from subsequently appearing. If young roots are laid upon their sides for about an hour and a half, and their tips are then carefully amputated so that they no longer perceive the stimulus of gravitation, they will nevertheless curve after a while towards the side which was downwards during the first exposure. The same curvature will be seen if they are placed in a vertical position after the amputation. The long delay in the response may no doubt be attributed partly to the disturbance set up by the amputation, but the fact that the response to the stimulus does eventually take place shows that the delay is due to slowness of changes in the responding protoplasm and not in the part which is sensitive.

An even more striking instance of action after the removal of the stimulus which has originated it-a socalled after-effect-may be seen by allowing a stimulus to operate for some time and then reversing its direction. This can be done by fastening a root horizontally in a damp atmosphere and, as soon as the curvature commences, inverting it so that the side showing the slight convexity is downwards. The curvature will continue in the original direction for some time and will only slowly cease and be replaced by one in the opposite direction. 
We can distinguish between the general condition of irritability or the state of tone, and these special forms of sensitiveness which we have examined. So long as the conditions remain favourable the general sensitiveness of the plant is maintained, but the power of responding to particular impressions may disappear from various causes without any disturbance of its sensibility to others. The power of appreciating differences in the environment varies with the age of the plant, disappearing in some cases from an organ while it still retains its power of circumnutating. The effect of a prolonged stimulation is sometimes failure to induce a movement. In the case of Dioncea this is very marked. If a leaf is for a time mechanically prevented from closing, repeated touching of one of the sensitive hairs brings about an exhaustion of its power to receive a stimulus, so that if the leaf is released a disturbance of that particular hair evokes no response. At first it may seem doubtful whether or no the interference with the free response of the leaf may have so injured the motor mechanism as to make it incapable of acting. The exhaustion however is shown to be that of the hair and not of the blade by the fact that touching another of the hairs at once causes closure.

The nervous sensitiveness is shown by this and many other similar experiments to be capable of fatigue. A similar suspension of power may be demonstrated by exposing the sensitive parts to anæsthetics, such as the vapour of chloroform or ether. The effect of these drugs at once suggests an action similar to that which they have on the nervous mechanism of an animal. When the effect of the fatigue or the anæsthetic has passed off, the organ again becomes capable of responding.

While we are able from these considerations to recognise in the plant a nervous system in some way comparable to that of an animal, we must clearly recognise the limitations under which it exists. It can only be regarded as rudimentary and as showing a very slight degree of 
differentiation. This we have seen is particularly noticeable with regard to its co-ordinating power. Another feature must be mentioned however before leaving the subject. We do not find in the plant any indication of anything corresponding to the higher functions of the nervous system of the higher animals. There is no evidence of anything which we may compare to consciousness or volition. Though many of the responses to stimulation are eminently purposeful we cannot regard them as in any way modified or held in check by any controlling power. A stimulus will produce its due effect although the manifestation of that effect at the particular moment may be followed by injurious consequences. The connection between the sense-organ and the motor mechanism is apparently a direct one, and there is no power to modify it possessed by the organism.

Nor, so far as we know, have we in plants any power of initiative. True, there are many movements and changes which are set up by causes that have their origin in some alteration of the protoplasm which we cannot explain, but there is no evidence of purpose in their origination. Even the locomotion of the Myxomycetes and the Diatoms shows no definite purpose except when it is clearly set up in response to some external stimulus.

Though there is no particular differentiation of an anatomical character in any of the sense-organs of a plant, there is nevertheless a differentiation of a physiological nature in the direction of sensitiveness which will equal if not surpass the powers of the sense-organs of an animal. The tendril of Passiflora appreciates and responds to a pressure which cannot be detected by even the human tongue; the seedlings of Phalaris readily obey the stimulus of an amount of light which is hardly perceptible by the human eye. Many plants readily detect and respond to the ultra-violet rays of the spectrum, which are utterly invisible to man.

The extent of the response to any stimulus is of course 
much less than that exhibited by an animal; but this, as we have seen, depends upon the differences in the motor mechanisms. In the vegetable protoplasm we have a much slower response, as well as one of a different kind, the effects taking as a rule longer before they are fully manifested and lasting for a longer time after the stimulus has been withdrawn. We have however, as in the animal mechanism, a much better response to a cumulative or prolonged stimulation than to one which is rapid and transitory. 


\section{CHAPTER XXVI}

\section{REPRODUCTION}

The phenomena we have hitherto been considering all concern the life of the individual plant. As this, however, at the best is comparatively limited in duration, we find plants possessed of the power of giving rise to new individuals. The process of originating each new individual from its parent or parents is known as reproduction.

We have seen that the life of the plant is essentially bound up with the individuality of the protoplasts which compose it. Many plants consist of but a single one of these organisms : others are composed of many, some of a very large number. We have seen reason to look upon each of these aggregations of protoplasts as a large colony whose members have become differentiated in various ways to carry out to the greatest advantage the vital processes of all. In the simplest forms, such as filaments of protoplasts like Spirogyra or Ulothrix, each protoplast is apparently independent in its behaviour, though mechanically attracted to its neighbours. In more complex and bulky forms this independence has been given up in favour of complete co-operation for the general welfare.

As every plant then is composed of either one protoplast or many, we may in the latter case distinguish between the colony and its constituents. The term individual is usually associated with the former, and we speak of reproduction as leading to the appearance of such individuals without making any reference to the protoplasts of which it consists. In dealing with reproduction however in the broad sense we must consider also the 
development of the protoplasts of the colony as well as of the appearance of new colonies or so-called individuals. Indeed in the case of unicellular plants such production of new protoplasts is the only form of reproduction possible.

It is important however to bear in mind the different individualities of the protoplast and of the colony of which it is part. In a filament of Ulothrix or other thread-like alga, each protoplast being like every other in all essential points, we may regard the formation of new protoplasts in the chain as a process of reproduction of the units; as the chain however grows by means of such multiplication of its constituent protoplasts, and has a distinct individuality as a filament, we may also regard the process of multiplication of the units as one of growth in the length of the chain. What is reproduction of the units of construction, the protoplasts, is growth of the individual, the colony. The same thing is seen in all plants which consist of more than a single cell.

We may study the method of multiplication of the protoplasts either in the cases in which they have an independent existence or in those in which each is part of a colony. In any case the process involves the division of the protoplast into two or many parts, each of which strictly resembles in all respects its progenitor. The cases in which two new protoplasts result from the fission are the most numerous, and they are classed together generally under the term cell-division. Of this there are various degrees of simplicity; the most primitive is illustrated by the behaviour of some of the lower fungi, such as the Saccharomycetes or yeasts. Each cell, which is rounded in form, puts out a lateral protuberance of small size, which grows until it is of nearly the same dimensions as the one from which it sprang, and is gradually cut off by the constriction of the cell-walls at the point of outgrowth. The new cell or protoplast becomes thus separated from its parent, which it resembles in all respects. This 
is known as gemmation or budding. It may go on so rapidly that the new cell in turn may put out a bud of its own before it is cut off from its parent, and in that way chains of cells may be produced (fig. 164).

A more general method of the division of the cell or protoplast is of a highly complicated character, and is preceded by a division of its nucleus. This structure we have seen consists essentially of a delicate network of fibrils of chromatin embedded in a hyaline substance, the whole being surrounded by a more or less well-defined outline derived from the cell-protoplasm, and known as the nuclear membrane. Associated with it in some cases are two small centrospheres. The process of division, which is known as Karyokinesis, or Mitosis, begins by the network

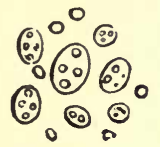

$a$

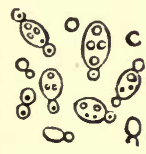

$b$

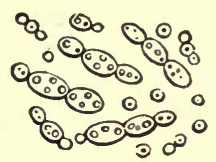

$c$

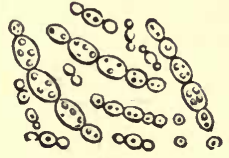

$d$

Fig. 164.-Saccharomyces Cerevisie, or Yeast-Plant, as developed DURING THE PROCESS OF Fermentation. $\times 300$.

$a, b, c, d$, successive stages of cell-multiplication.

of fibrils becoming coarser and gradually separating to form a long coiled fibre. The nucleoli disappear and the nuclear membrane ceases to be distinguishable. At the same time, in those cases in which centrospheres have been seen, they shift their position and come to lie on opposite sides of the nucleus at some little distance from it. The long coiled fibre of chromatin breaks up into a number of pieces, often V-shaped, which point towards the centre of the nucleus. The number of these varies in different cases, but is constant in the successive divisions of an individual. These pieces of the fibre are known as chromosomes. The chromatin in them is broken up into thin dises which are separated from each other by smaller discs of unstainable substance. 
Threads of a delicate character may next be seen to extend from one centrosphere to the other, forming a body known as the nuclear spinale. The positions of the centrospheres are called the poles of the nucleus. When no centrospheres can be detected the threads of the spindle nevertheless converge to two similarly situated poles. Some of the spindle fibres stretch uninterruptedly from pole to pole, while others become in some way attached to, or entangled with, the chromosomes. The latter travel along these threads, with which their points are in contact, till they form a disc across the spindle (fig. 165, b). This stage is constant in all cases of karyokinesis, though some
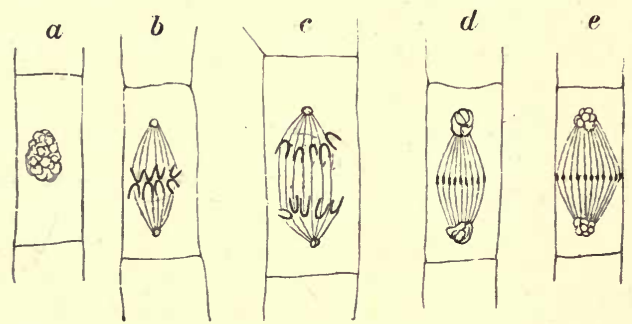

Fig. 165.-Stages in Karyokinetic Division of the Nucleus.

$a$, resting nucleus ; $b$, stage of equatorial plate ; $c$, separation of the chromosomes; $d$, commencement of formation of cell-wall; $e$, extension of nuclear spindle across the cell.

variations of the antecedent steps have been observed, the details of the formation of the disc not being always identical. This body is sometimes called the equatorial plate. After this stage is reached, or sometimes before it is quite completed, each chromosome splits longitudinally into two, and the bodies forming the equatorial plate separate into two sets in such a way that half of each original chromosome makes its way towards one pole, and the other half towards the other. The two sets of chromosomes so formed travel back along the spindle fibres, each going to one of the two poles of the nucleus, their positions as they go being such that their convex sides point towards the pole which they are approaching (fig. $165, c$ ). They thus collect 
into two places which are determined by the positions of the poles of the nucleus, or of the centrosomes if the latter are present, and they present there the appearance of two somewhat star-shaped aggregations. This is known as the diaster stage. The chromosomes at each pole next become united by their ends, and constitute two new nuclei, each gradually becoming well defined by the appearance of a nuclear membrane; the original appearance is completed by the development of nucleoli in each new nucleus. The mechanism of the movement of the chromosomes towards the poles is not fully understood at present, but it is held by some observers to be due to a contraction of the spindle fibres to which the chromosomes are attached.

This process of karyokinesis is followed in various ways by the production of a cell-wall between the two nuclei, which completes the division of the protoplast. In the cases in which the latter does not contain a relatively large vacuole and is of comparatively small diameter, the spindle fibres become increased in number, and form a barrel-shaped body whose short diameter stretches completely across the cell (fig. $165, d, e$ ) till the spindle is in contact with the lateral cell-walls. Granules which have been floating in the cell-protoplasm are to be seen streaming along the spindle fibres till they form a plate stretching across the cell from wall to wall. From this plate the septum of cellulose and its associated substances is formed.

If the protoplast has a large central vacuole, the spindle often does not reach completely across the cell. It is then at first in contact with one side only, and the new wall begins to be formed there in the same way as in the case described. It then detaches itself from the part of the new-formed wall which is in contact with the old membrane and moves gradually across to the opposite side of the cell, the new wall being completed as it goes. The spindle then disappears.

In some of the Thallophytes the new wall is formed without the intervention of a spindle. After the two new 
nuclei have taken up their positions, the new wall arises midway between them as a ring-like outgrowth from the original cell membrane, and gradually grows inwards till it is complete.

In the divisions of the protoplasts which constitute a cœnocyte the nuclear divisions are not followed by the construction of any cell-walls, so that the limits of each protoplast are not well defined ; in some cases indeed they are indistinguishable.

The reproduction of the protoplast is sometimes attended by the production of not two but several, which appear simultaneously. Such a case is illustrated by the formation of the endosperm in the embryo sac of the Phanerogams. It is, however, only a modification of the process already described. The division of the original nucleus is followed by the disappearance of the spindle; the daughter nuclei divide in turn, and the process is continued until a large number of free nuclei lie embedded in the protoplasm of the cell. These then become connected with each other by the simultaneous development of connecting fibrils or small spindles like the first, and cell plates, which later become cell membranes, arise across them as in the case described. The protoplasts so formed exhibit no differentiation among themselves, but are all alike in appearance, structure, and fate.

This modification of the process of reproduction of the protoplast is known as free cell formation, and in many cases it is attended by a specialisation of function, which will be alluded to a little later.

In many cases of the reproduction of such plants as consist of enormous numbers of protoplasts variously arranged and differentiated, we have to recognise essentially no other process than the multiplication of the protoplasts by such means as we have just described. Generally in these cases some part of the parent plant becomes detached and grows at once into the new individual. We have seen that this is the regular method of the multiplication of the 
yeast-plant, where each division of the protoplasts brings into being a new individual. The process can be noticed through all the families of the vegetable kingdom, though as we advance upwards in the scale, the separated body becomes more and more complex. We have the gemmæ of certain Algæ and Bryophyta, which are multicellular ; we have in certain Mosses branches which become detached by the dying off of the shoot behind them. Many Ferns develop buds upon the pinnæ of some of their leaves, which when separated from the latter grow into complete ferns. Among the Phanerogams we notice a great variety of this method of reproduction, many structures being developed normally to secure it, while others can be made to lead to it by artificial means. We have the propagation of plants normally by the formation and separation of tubers, buds, and corms; by the young plants which are developed from the nodes of runners and stolons. The artificial method of bringing it about is illustrated by cuttings, which are pieces of the stem, bearing buds; these when detached and planted in suitable soil, put out adventitious roots from the base of the cutting and develop into plants like the original one. Other instances are afforded by the buds which many leaves, notably those of Bryophyllum and certain Begonias, put out when wounded. These also develop adventitious roots, and young plants arise which become independent.

This method, in which we never meet with the preparation of cells which are specialised in the direction of reproductive powers, is usually spoken of as vegetative reproduction or propagation.

Some curious cases of it are known. In the embryo sac of Colebogyne there is no fertilisation of a sexual cell in the manner which will shortly be described, but still one or more embryos arise. This is caused by a vegetative budding of certain cells of the nucellus of the ovule which grow into the interior of the embryo sac, and develop into embryos. 
A feature of vegetative propagation which may here be emphasised, is that the new individual is developed continuously after its origination. There

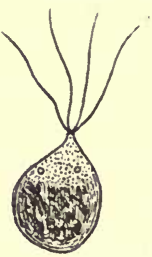

FIG. 166.-Zoospore oF

Ulothrix. $\times 500$. is no resting period, such as we find in most cases to mark the behaviour of the more specialised reproductive cells to be discussed below.

Apart from cases of vegetative propagation of the individual, we meet with two other methods of reproduction, both of which involve the preparation of special cells set apart for this purpose. The first of these is characterised by the fact that each cell so

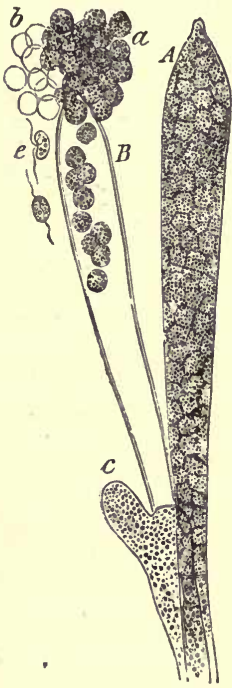

Fig, 167.-Two GoxiDANGIA OF Achlya.

$A$, closed; $\mathrm{B}$, ruptured, and allowing the zoogonidia $a$ to escape; $b$, mothercells of the latter, after escape of the zoogonidia from them. produced is able to grow, either at once, or after a short period of rest, into a new plant, which may or may not be exactly like the one from which the reproductive cell was formed. In plants exhibiting the simple organisation which we find among the seaweeds and the fungi, the parent and the offspring are in most cases precisely similar. The difference in this respect between them and plants higher in the seale will be discussed a little later. A good example of this mode of reproduction, which was probably the primitive form, is afforded by the common filamentous Alga Ulothrix. Any protoplast of the filament can divide into a number of separate pieces, each of ovoid shape with a pointed end and furnished there with four cilia (fig. 166). These new protoplasts swim about for a time in the water, then come to rest, and after a time grow out into new filaments. Not 
only the Algæ but the Fungi afford examples of the development of such cells, conspicuous among them being Saprolegnia and its allies (fig. 167). These free-swimming protoplasts are known as zoaspores or zoogonidia. Each on coming to rest clothes itself 'with a cell-wall, and can develop into a plant exactly like the one from which it arose. These zoogonidia are developed by the protoplasm of a single cell dividing up into a variable but often large number of separate protoplasts, the process being known as

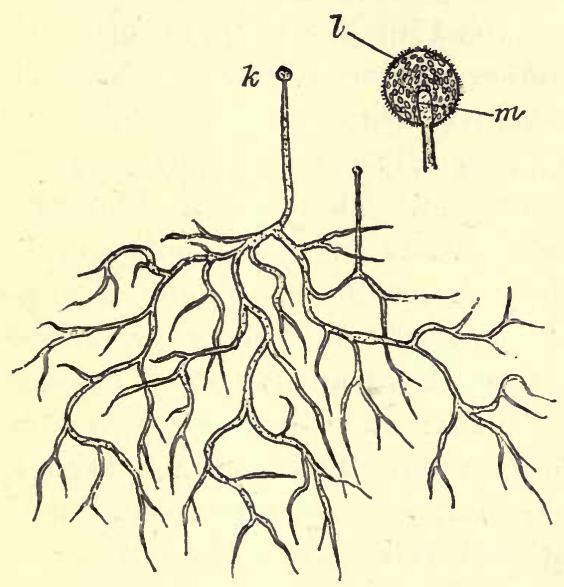

Fig. 168.-Cenocyte of Mucor, Bearing a GonidangiuM, $k$. This Is MORE HIGHLY MAGNIFIED IN THE FIGURE TO THE RIGHT. $m$, columella; $l$, gonidia.

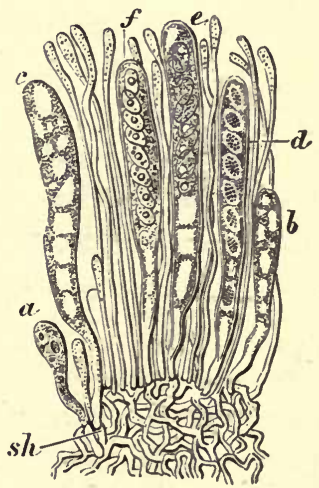

Fig. 169.-Asci. $a$, MIXED WITH BARREN HAIRS OR PARAPHYSES $e, f$; FROM HYMENIAL LAYER OF Peziza. $\times 250$.

free cell formation. Each protoplast possesses a nucleus derived from the original nucleus of the cell in which the formation takes place, in the manner already alluded to.

In most cases where these reproductive cells are met with they have not so simple a structure as those so far described, but each is furnished with a cell-wall. They are commonly called spores or gonidia, and arise in different ways upon the plant, often, or indeed generally, being developed in or on special organs, known as sporangia or gonidangia. 
The yeast-plant gives us perhaps the simplest form of this organ. Any cell can play this part; its protoplasm divides into a number of pieces, frequently four, each of which becomes rounded off and clothed with a new cellwall. After a time the four new cells are liberated by the breaking down of the original cell-wall. They are developed in more highly differentiated plants in special cells or chambers named asci (figs. 168 and 169), in very variable numbers, and are known as ascogonidia or ascospores. In other cases they are produced by abstriction from a cellular outgrowth of the thallus (fig. 170), and in these again the

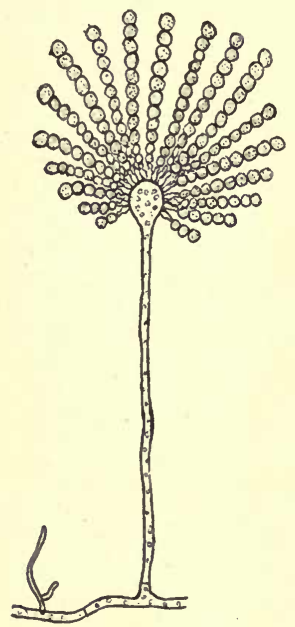

Fig. 170. - Strlogonidia of Eurotium, PRODUCED BY ABSTRICTION FROM STERIGMATA. number produced from a single cell may vary within wide limits. These are generally called stylogonidia or stylospores. There is an almost infinite variety of these bodies to be met with in different plants, but the variety affects only the conditions of their situation and does not indicate any difference in their own structure. They are unicellular bodies, or simple protoplasts, each clothed with a delicate cell-wall.

These asexual cells are usually spoken of as gonidia when they arise upon a gametophyte, and as spores when the sporophyte gives them origin.

The fact that they do not usually germinate till after a period of rest, though this is often not very prolonged, suggests that they originated in consequence of the plant needing certain cells which should possess the power of passing through times of exposure to unfavourable conditions without destruction. Such unfavourable conditions would be likely to kill the more delicate vegetative reproductive bodies. This view is supported by the fact that many of the lower plants, particularly Yeast, do not pro- 
duce gonidia when conditions are suitable for the life of the ordinary individual, but can be made to do so by cultivating them under adverse conditions of moisture, food supply, \&e.

A somewhat similar structure to the zoogonidia described is put out by the cœnocytic Alga Vaucheria. It appears as a mass of protoplasm, which is separated from the contents of a filament, and is set free by an opening at the apex of the latter. It is composed of several protoplasts which are arranged together as in the rest of the cœnocyte, but their individual outlines cannot be seen. The fact that it is cœenocytic is shown by the presence of a number of nuclei in the protoplasmic mass. A pair of cilia are given off opposite to each nucleus, so that it swims very readily in the water after its liberation. It is sometimes called a Zooccenocyte. After a period of motility it comes to rest, the cilia are withdrawn, and it becomes clothed by a cell-wall. The resting period lasts for a variable time, after which it develops into a new Vaucheria filament.

Besides these asexual reproductive bodies other cells are produced by the great majority of plants, which are incapable of giving rise to new individuals, unless two of them unite or fuse with one another. On account of this peculiarity they are known as sexual cells or gametes. In the lowliest forms, such as many filamentous Algæ, they are produced by the same filament as the asexual cells or gonidia. In the case of Ulothrix we find the first indication of these sexual cells. Besides the large zoogonidia with

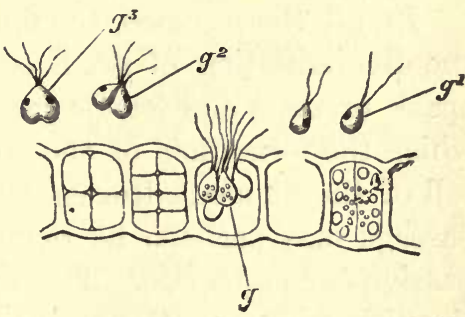

Fig. 171.-Part of a Fillament of Ulothrix, FRON WHICH THE GAMETES $g$ ARE ESCAPING.

$g^{1}$, free gamete $; g^{2}, g^{3}$, gametes conjugating.

their four cilia, other smaller free-swimming bodies are developed in certain cells of the filament. They are 
produced in larger numbers and have only two cilia each (fig. 171). After they are set free into the water they swim about for some time, and then they usually fuse together in pairs, nucleus joining nucleus and protoplasm uniting with protoplasm. The new body so formed is known as a zygospore. After a period of rest it can give rise to a new filament. As there is no difference between the cells which unite to form this structure, they are frequently called planogametes.

In the Zygnemea and the Mesocarpece the gametes are solitary and non-motile and do not escape from the cells in which they are formed. Two filaments take part in the fusion of the gametes; these are found lying close together in the water; from a cell of each filament a protrusion grows out towards the other and the two come into contact and join, the separating walls breaking down. The contents of one cell pass over into the other through the channel so formed, or the contents of both the cells meet in the middle of the passage ; fusion of the two takes place, and the new body, called as before the zygospore, clothes itself with a cell-wall. It is liberated after a while by the breaking down of the wall of the structure which encloses it, and can then give rise to a new individual. A similar process is characteristic of certain Fungi.

In all these cases, though the cells are sexual cells, the differentiation of sex is so slight that it is difficult to speak of male and female gametes. In the Zygnemeæ, in which the formation of the zygospore takes place in the cell of the filament, the gamete which passes through the passage may perhaps be regarded as male and the more passive one as female. This differentiation cannot be distinguished in the Mesocarpeæ, where both gametes meet in the connecting passage.

In Ulothrix the differentiation of sex is even more rudimentary, as it is not always necessary for the fusion to take place. If any cell escapes fusion it may develop into a new filament independently of this process. This 
fact suggests that the sexual cells have been derived from asexual ones, and are a later development, therefore, in the history of the race.

The more complete differentiation of the gametes into male and female can be observed among several of the families of the Algæ. In some species of Ectocarpus and Cutleria the gametes are much like those of Ulothrix, but some are smaller than the others. The larger ones come to rest soonest, and lose their cilia; one of the smaller more motile ones then fuses with each of the larger. We can in this case speak of the larger as female and the smaller as male. The differentiation is still very rudi-

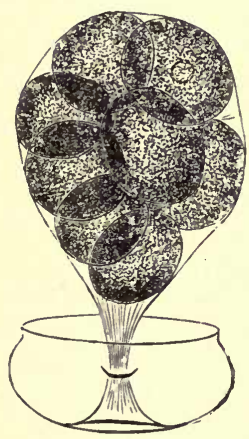

Fig. 172.-Oogonium of Fucus, conTAINING EIGHT OOSPHERES. (After Thuret.)

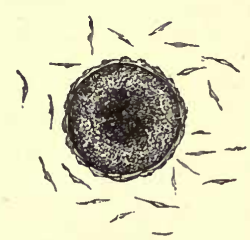

Fig. 173.-AN OosPhere of Fucus SURROUNDED BY ANTHEROZOIDS. (After Thuret.)

mentary, as in the event of no fusion taking place the female cell can still develop into a new plant.

The most complete differentiation of the gametes can be traced in the higher members of the Algæ. The females become larger and cease to develop cilia, the males remain small and motile. The former are then called oospheres and the latter antherozoids or spermatozoids. A good example of this stage of differentiation is afforded by Fucus (figs. 172 and 173).

The structures or organs in which the sexual cells of these plants are formed are known as gametangia. When the gametes are distinctly male and female the gametangia 
in which they are developed are termed antheridia and oogonia respectively.

In the group of Fungi similar differentiation of gametes occurs, but motile antherozoids are very rare, confined indeed to the genus Monoblepharis. In other cases they are generally undifferentiated masses of protoplasm which do not escape from their antheridia, but are conducted directly from it into the female organ, where the process of fusion takes place. In Pythium the oogonium is a swelling at the end of a hypha, which is cut off from the rest by a transverse wall. Its contents divide up into an oosphere and a certain amount of protoplasm, which surrounds the latter. The antheridium is another hyphal branch, which becomes closely pressed to the oogonium. A tube is put out by the antheridium, which perforates the wall of the oogonium, and the male cell, which is formed in the same way as the female one,

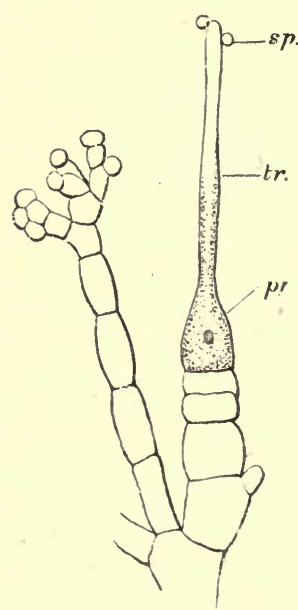

Fig. 174.-Procarpium of A RED SEAWEED. tr, trichogyne.

passes over into the female organ and fuses with the oosphere.

In some other Fungi a similar arrangement of the organs is brought about, but the male cell does not pass over into the oogonium.

A curious variation is seen in the red seaweeds, the Rhodophycea. The female organ, known as a procarpium, does not produce any differentiated oosphere, but the contents of the male cell pass by means of an elongated structure called a trichogyne (fig. 174) into its interior and apparently fuse with the whole of its protoplasm. The male cell in these plants is not naked as in other cases, but has a cell-wall. A somewhat similar condition is met with among the Ascomycetes, though whether fusion of the contents of the cells takes place is disputed. 
The gametangia of the plants above the Thallophytes are known as antheridia and archegonia respectively. An archegonium is a more complex structure than an oogonium, being composed of many cells and showing differentiation into a venter and a neck (fig. 175). It contains only a single oosphere.

The sexual cells differ from the great majority of asexual ones in never possessing cell-walls. The only cases in which they are clothed with them are those of the Rhodophyceæ and the Ascomycetes already alluded to. In both these groups the male gametes are the only ones that

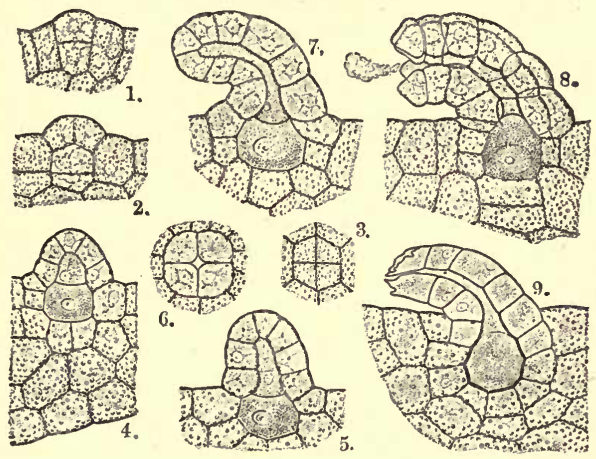

Fig. 175.-Development of the Archegonium of the Fern.

have them; the females, as we have seen, not being differentiated.

The fusion of the gametes is known as conjugation when they are alike, and as fertilisation when they are distinctly male and female. The resulting body is termed a zygote; it is a zygospore when it is produced by conjugation, and an oospore when it is the result of fertilisation.

In the more lowly organised forms it generally happens that both sexual and asexual reproductive cells may be produced upon the same individual. An exception is found in the Fucacea, the members of which do not develop any asexual cells. While it is possible however for many plants to produce both gonidia and gametes, it is 
more usual for them to bear the former only. So for a long series of individuals reproduction is brought about asexually by gonidia. Then for some reason an individual produces gametes, and the series is interrupted by the occurrence of sexual reproduction. This is generally followed by a further series like the first. We have here an instance of a kind of alternation of generations, which is however irregular and intermittent. As all the members of the series, whether producing gonidia or gametes, are essentially similar or homologous, this is often spoken of as homologous alternation of generations.

The forms which we have discussed appear all to be capable of producing gametes if conditions require them. They are accordingly termed gametophytes, and are distinguished as actual or potential as they do or do not give rise to sexual cells.

In plants which are higher in the scale the production of both sexual and asexual reproductive cells ceases to be possible upon the same individual, and we find consequently that the plant exhibits two phases in its life cycle, one of which is characterised by the production of sexual and the other of asexual cells. How this sharply marked separation arose is still a matter of controversy which we need not here enter into. The two forms however are not homologous, one being capable normally of producing only gametes, the other of giving rise only to spores. A further development also makes itself evident in that the zygote arising on the gametophyte is only capable of originating a form which bears spores, while the spore can only develop a form on which sexual cells arise. The asexual form is from this point upwards known as the sporophyte. The occurrence of them regularly in turn as described is known as antithetic alternation of generations. It is of constant and legular occurrence in all the groups of plants above the Thallophytes.

The existence of a sporophyte, or form which is never capable of bearing gametes, is still a matter of discussion 
as far as the Thallophytes are concerned. There are indications of its origination in that group, but they are extremely rudimentary, and occur in families which are widely separated from each other. The gametophyte was doubtless the primitive form of the plant, and in some way or other the sporophyte took its origin from it. Certain phenomena which may represent stages in the process can still be observed. In 2 dogonium the fertilised cell does not grow out into a new filament, but produces in its interior four zoospores which escape from it, and after a period of rest germinate and produce new plants. The fertilised cell here may perhaps represent the sporophyte, reduced however to a single sporangium. An even simpler stage of development may perhaps be recognised in Spirogyra, where the nucleus of the fertilised cell divides into four, though no definite cells are formed. On germination of the zygote however only one filament grows out. A more complex structure is formed in Coleochate; the zygote becomes invested with a covering derived from the adjacent cells, and after sinking to the bottom of the water, it germinates, producing inside its coating a small mass of cells, each one of which liberates a zoospore. Other complex structures are found as the result of the growth and development set up by fertilisation in the Rhodophyceæ. These are known as cystocarps, and they have been held to represent the sporophytes of those plants. It is important to notice however, both in their case and in that of Coleochæte, that only part of the structure in most cases is derived from the contents of the fertilised cells, the rest coming from other cells of the tissue of the gametophyte. As we have seen the sporophyte in the higher plants is entirely derived from the zygote.

The antithetic alternation of generations is seen most clearly in the groups of the Mosses and Ferns. In the former the Moss plant is the gametophyte, the so-called capsule or theca with its stalk is the sporophyte. In the Ferns the sporophyte is the predominant form and takes 
on the chief vegetative functions, while the gametophyte is the small prothallium (fig. 176).

Even without going beyond the Ferns we can notice as we pass through the several divisions of the vegetable kingdom that the predominant form of the plant has changed. In the Thallophyta it is always the gametophyte; the sporophyte is not universal there and is never more than a small structure, which nearly always remains attached to the gametophyte. In the Bryophyta the two phases are more nearly alike in degree of development; the gametophyte is always the vegetative body, while the

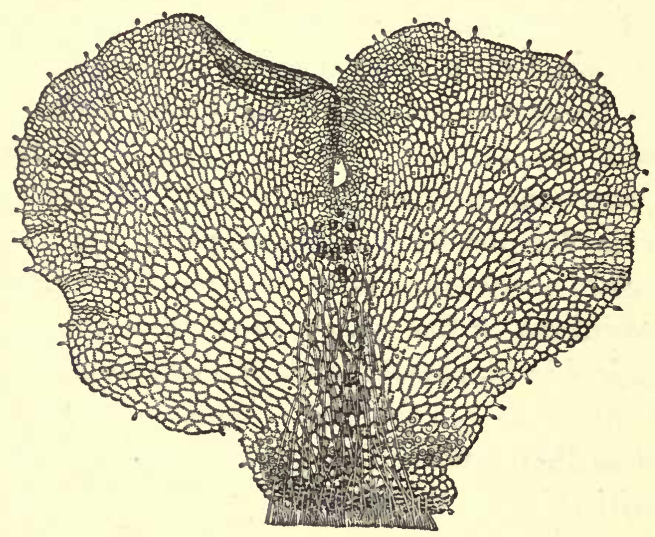

Fig. 176.-Prothallium (Gametophyte) of Fern.

sporophyte often shows the greater histological differentiation. It is always parasitic upon the gametophyte and never attains a higher degree of morphological value than a thallus. In the Pteridophyta the predominance of the sporophyte is very marked, and as higher and higher groups of plants are reached it becomes still more pronounced, the gametophyte ultimately being reduced to microscopic dimensions.

We encounter for the first time in the group of the Pteridophyta, the Ferns and their allies, a phenomenon which becomes of constant occurrence in all groups above 
them, and which leads to the production of the structure known as the seed, the latter being a special body produced by all members of the group of Phanerogams or flowering plants, and marking them off clearly from all below them. The phenomenon in question is known as heterospory. Plants which exhibit it bear two kinds of spore, which differ from each other mainly in their relative dimensions. Some are produced in large numbers in a sporangium and have usually the structure which has already been described. Others are much larger than these and are developed either singly or in small numbers, usually four in a sporangium. They are spoken of as microspores and megaspores respectively. In the Pteridophytes the megaspores when formed differ from the microspores chiefly in size; in the Phanerogams they are never liberated from the sporangium and have consequently thin and delicate walls.

The phenomenon of heterospory involves the production of two gametophytes to one sporophyte, as each of the spores produces its appropriate prothallium. The gametophyte arising from the microspore gives rise only to male gametes, that from the megaspore only to female ones. Such plants show in their life cycle, therefore, three forms, one sporophyte and two gametophytes, the latter occurring synchronously.

The gradual appearance or development of the seed can be examined by studying a series of forms. The earliest indication of it which we can find is ex-
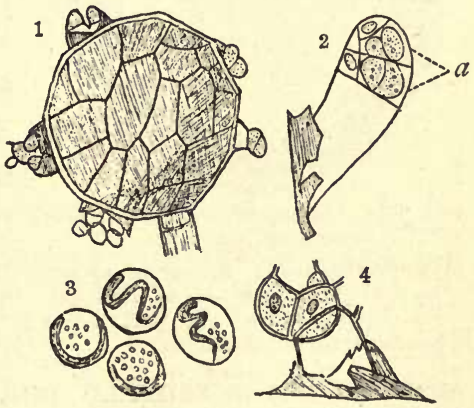

Fig. 177.-GERMination of a Mass of Microspores of Salvinia. (After Sachs.)

1, The mass protruding tubular prothalli from different spores; 2 , a prothallus more highly magnified, showing an antheridium, $a ; 3$, antherozoids in mother-cells; 4, ruptured antheridium.

hibited by the Hydropteridea, of which Salvinia is a characteristic type. Salvinia is a heterosporous form, each 
microspore of which gives rise to a very rudimentary prothallium bearing only one antheridium with four antherozoids (fig. 177). The megaspore, like the microspore, is liberated from the sporangium and on germination it produces a prothallium, part of which remains in the spore and part protrudes from it (fig. 178). The inclusion of part of the gametophyte within the spore is the first step towards the formation of the seed. The young sporophyte

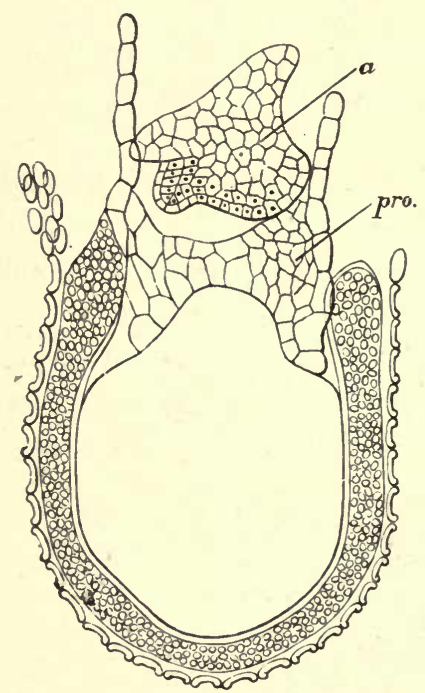

Fig. 178.-Germination of MegaSPORE OF Salvinia.

pro, prothallium; $a$, young sporophyte. The thick wall of the spore has been ruptured and part of the prothallium is protruding. arises upon this prothallium upon the exposed portion, originating as in other cases from the zygote produced in the archegonium in consequence of fertilisation.

A more advanced stage is seen in Selaginella, which also is a member of the Pteridophyta, though not a fern. The heterospory is just as pronounced as in Salvinia. When the megaspore is set free from the sporangium and its germination can be observed, it is found that more of the gametophyte remains inside the spore (fig. 179). The process of germination begins while the spore is still in the sporangium, and by the time the spore opens the prothallium has reached a fair degree of development.

A still further advance is shown by Isoëtes, in which the prothallium is developed inside the spore, which only opens a little at the apex when the archegonia are mature, in order that fertilisation may be possible.

When we pass to the Phanerogams two further advances may be seen. The spore never escapes from the sporangium, and the prothallium does not emerge even in 
part from the spore, which does not open. In these plants the megaspore is represented by the cell known formerly as the embryo-sac, the sporangium being the ovule. Among the Phanerogams we have two types of prothallium which are characteristic of the Gymnosperms and the Angiosperms respectively. Fig. 180 shows the structure in the former ; the spore or embryo-sac is filled with the prothallium, formerly called the endosperm, at the apex of which are several archegonia each containing a female gamete or oosphere. After fertilisation the resulting zygote

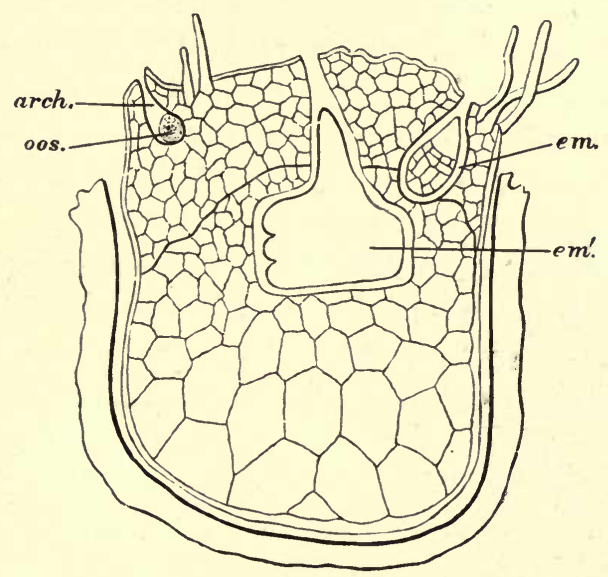

Fig. 179.-Germination of Megaspore of Selaginella.

arch, archegonia; oos, oospheres ; $\mathrm{em}^{\prime}$, embryo. The spore has been ruptured and the upper portion removed.

gives rise to a young sporophyte or embryo, which becomes embedded in the endosperm. The structure thus formed, consisting of the sporangium or ovule, with the solitary spore it contains, the latter having in its interior the embryo surrounded by the prothallus, constitutes the structure known as the seed. It becomes detached from the parent sporophyte and disseminated in various ways.

In the Angiosperms the formation of the seed is in the main similar to the process described, but it has certain peculiar features. The embryo-sac or megaspore has the 
same structure as in the Gymnosperms and remains enclosed in the sporangium or ovule. The development of the prothallium is different. The megaspore has a single nucleus as in other cases. When germination begins this divides into two, one of which travels to each end of the ovoid spore. Each of these gives rise by two successive divisions to a group of four nuclei, and a single nucleus from each group returns to the centre of the cell, where the two fuse together. These are often termed the polar nuclei.

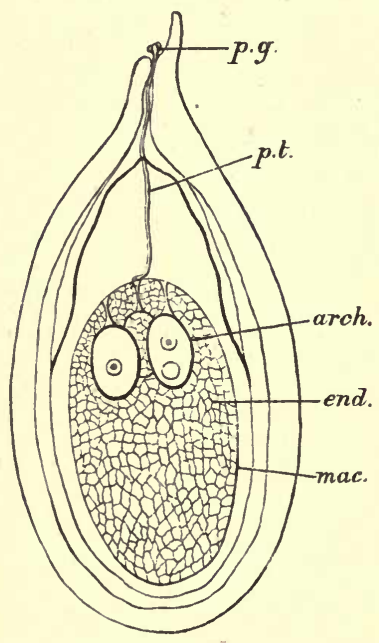

Fig. 180.-Ovule of Pinus, Showing THE PROTHALLIUM, end, IN THE MEGASPORE, mac.

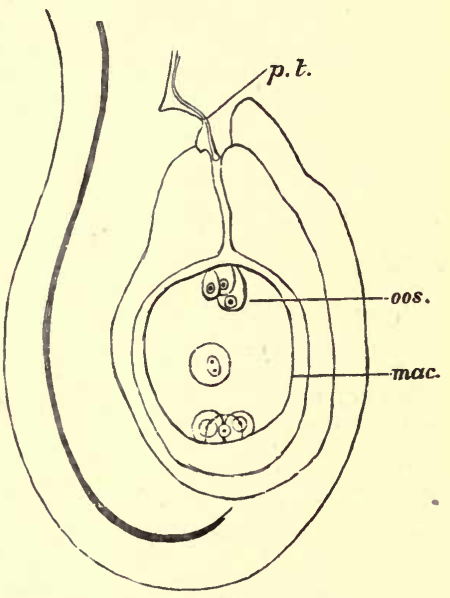

Fig. 181.-Ovule of AN ANGioSPERM SHOWING THE MEGASPORE, mac, WITH ITS PROTHALLIUM; oos, OOSPHERE.

arch, archegonia.

At this stage the prothallium ceases to undergo any change (fig. 181) ; it consists of a group of three nuclei at the apex, known as the egg apparatus; another group at the base, termed the antipodal cells; and the nucleus in the centre which is the result of the fusion of the polar nuclei, and is called the definitive mucleus of the embryo-sac. Each nucleus is surrounded by protoplasm, the egg apparatus in particular showing three well-defined naked or primordial cells. The antipodal cells become clothed with cell-walls. There 
is a certain amount of protoplasm existing in the spore, lying around the wall and forming bridles across it, connecting the peripheral substance with that in the centre in which the definitive nucleus is resting.

There are no apparent archegonia ; the oosphere is one of the three cells of the egg apparatus, the other two being known as the synergida. The oosphere is a product of the last division of the original upper nucleus, the other half being the polar nucleus which takes part in the fusion described.

After the fertilisation of the oosphere an embryo is developed from it, which remains enclosed in the spore. At the same time a large development of tissue takes place in consequence of repeated divisions of the definitive nucleus, so that the spore contains a massive so-called endosperm in addition to the embryo, the latter being embedded usually in the former. This so-called endosperm has as we have seen a different morphological value from the endosperm of the gymnospermous plant.

One of the most remarkable features about the structure and behaviour of the seed is the fact that soon after the embryo is formed it enters upon a period of rest, which in some cases is very prolonged. During this period the seed becomes detached from the parent plant. The resumption of its growth and development is known as the germination of the seed. This resting period does not occur during the development of the sporophyte in the Cryptogams.

The embryo frequently attains a considerable size before its resting period commences. In this case it absorbs the contents of the cells of a considerable part, or sometimes the whole, of the endosperm, so that it fills more or less completely the cavity of the spore.

The seed may thus be a very complex structure; it may consist of the following parts:

(1) The testa or skin, derived from the integuments of the ovule. 
(2) The perisperm, or remains of the body of the megasporangium.

(3) The embryo-sac or megaspore.

(4) The endosperm derived from the definitive nucleus.

(5) The embryo developed from the zygote.

The antipodal cells generally disappear during the development. (2) and (4) may be absent, having been absorbed by the megaspore or by the embryo respectively during their development. If either or both are present the seed is said to be albuminous, the term albumen embracing both perisperm and endosperm.

In the seeds of the Gymnosperms the endosperm represents the prothallium or gametophyte.

The formation of the seed we have seen to depend upon the fusion of the sexual cells or gametes. This

A

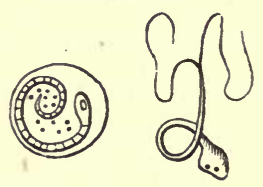

B

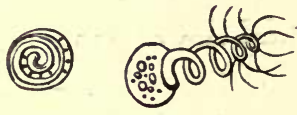

Fig. 182.-Antherozoids of Moss (A) ANd Fern (B).

process is a very widespread one and is the starting point of the development of the young sporophyte in all plants above the Thallophytes. The mode of bringing the gametes together varies with the habit of life of the plants. Where the male gamete is a motile antherozoild it makes its way to the oosphere by means of its cilia, which enable it to swim freely in water. In those forms with a terrestrial habit, such as the Bryophyta and Pteridophyta, in which the antherozoid is ciliated (fig. 182), fertilisation can only be brought about when the gametophytes are moistened, as is the case from time to time. The antherozoilds sometimes arise in antheridia upon the same gametophyte as the archegonia with their oospheres, sometimes upon different ones. In the heterosporous forms of course the latter is always 
the case. After their liberation they are attracted to the archegonia by some constituent of the mucilaginous matter which is excreted from their necks when they open (fig. 184). In the Mosses this has been ascertained to be cane-

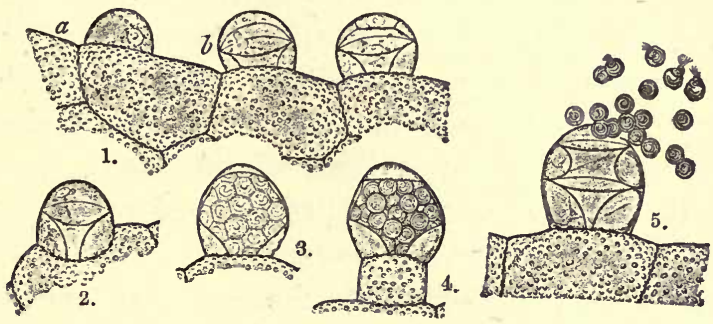

Fig. 183.-Development of the Antheridium in the Fern. (After Kny.)

sugar, in the Ferns it is malic acid or one of its salts. In the Rhodophyceæ and such Ascomycetes as exhibit sexual reproduction, the passive male gamete, often called a spermatium, instead of an antherozoild, is floated to the female organ or its trichogyne by currents in the water.

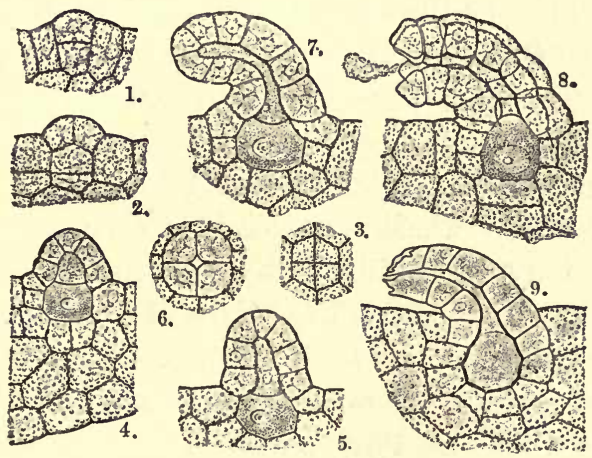

Fig. 184.-Development of the Archegonium of the Fern. (After Kny.)

In the Phanerogams, where the female gametophyte is always attached to the parent sporophyte, such a means of fertilisation is of course impossible. For fertilisation to take place it is necessary that the two gametophytes shall be produced in close propinquity to each other. This is 
effected by the bringing together of the two spores concerned in developing them. The microspore or pollen grain is carried by various means to the neighbourhood of the megasporangium; in the Gymnosperms it falls upon the megasporangium itself; in the Angiosperms upon the stigma of the pistil in which the megasporangia are hidden. When it germinates the prothallium or gametophyte takes the form of a long tube, which makes its way through the intervening tissues till it reaches the megaspore itself, close to the archegonium in the first case, and to the oosphere in the Angiosperms, where there is no archegonium. In the Gymnosperms the tube, the so-called pollen tube, contains a single antheridium, which produces two gametes, which are generally undifferentiated portions of protoplasm, but which in Ginkgo and in some species of Cycas have been found to be ciliated antherozoïds. In the Angiosperms there is no antheridium, but two gametes are produced, which show no differentiation. From the great preponderance of the nuclear matter they contain they are often spoken of as the generative nuclei.

Fusion of the latter, or of the antherozoid, with the oosphere, becomes possible by a deliquescence of the separating walls, and in all cases a single male gamete fuses with an oosphere. Where several oospheres are found upon the same prothallium, as in the Gymnosperms, more than one may be fertilised by gametes from the same pollen-tube. This occurs in certain of the Cupressinec; it is rendered possible by a multiplication of the male gametes, which takes place by ordinary processes of division exhibited by them as they pass down the tube. Several embryos may thus arise in the seed. Usually however only one of these undergoes a normal development.

In certain cases in the Angiosperms the second of the generative nuclei has been observed to fuse with the two polar nuclei or the definitive nucleus of the embryo-sac. The extent to which this takes place has not yet been determined and its interpretation is not at present easy. 


\section{CHAPTER XXVII}

REPRODUCTION (CONTINUED)

We have seen that the phenomena of fertilisation are preceded in the Phanerogams by an arrangement through which the two gametophytes which give rise respectively to the male and female sexual cells, are developed in such close proximity that they ultimately come into contact. That which is produced as the result of the germination of the microspore or pollen grain, is a tube of varying length, which bores its way through the tissue of certain parts of the sporophyte, being guided in some manner not yet fully understood, until it reaches some part, usually the apex, of the megaspore or embryo-sac, in which synchronously the prothallium which bears the oosphere has been developed. In the process of sexual reproduction in these plants we have two phenomena presented, which have often been treated of as if they were inseparably connected. The first of these, which is known as pollination, involves merely the transport of the pollen grain to an appropriate position on some part of the megasporophyll or of the megasporangium itself. The second, which may or may not follow the former one, is the actual fusion of the gametes which are produced upon the gametophytes to which the spores give rise, and which therefore must be considerably later in the time of its occurrence. This is what we have already described as fertilisation.

It is necessary to insist on the distinction between these two processes, as the phrase "the fertilisation of the flower' is frequently somewhat loosely and erroneously made use of when pollination is meant.

We have seen that cross-fertilisation is as a rule more 
advantageous to a plant than the fusion of gametes which are both produced by the same individual. In the same way certain advantages are secured by the process of crosspollination or the application of the pollen of one flower to the stigma of a different one of the same species. In the case of flowering plants or any others which are heterosporous, self-fertilisation is of course in the strictest sense impossible, as the male and female cells which fuse together are necessarily borne upon gametophytes which originate from different spores and cannot thus be derived immediately from the same individual. Self-pollination, or the transference of pollen from the stamens to the stigma of the same flower, is however possible, and in many cases occurs in the ordinary course of events. Cross-pollination, or the bringing together of spores from different flowers of the, same species, has been found to yield more and better seeds than self-pollination.

Very many mechanisms have been developed in different plants to secure this end. Pollen may be carried from flower to flower by wind or water, or by the agency of insects or other animals. From this point of view flowers have been classed as anemophilous, or wind-pollinated, hydrophilous, or water-pollinated, entomophilous, or insectpollinated, and zoophilous, or pollinated by other animals.

Of these methods of cross-pollination, the anemophilous and the entomophilous are most widespread. The former is the more primitive ; indeed, the latter has been gradually supplanting it. We find cases now of nearly allied genera which illustrate the transition from the one to the other. Among the Ranunculaceæ the flowers of the genus Thalictrum are pollinated by the wind, while those of the more specialised genera Aconitum and Delphinium depend upon insects. The Plantains also afford instances of the replacement of the one method by the other.

Anemophilous flowers exhibit certain structural features which are associated with their mode of transference of the pollen. It is produced in such flowers in great abun- 
dance, is extremely light and dry, and in some cases is furnished with bladders to aid its transport. The receptive organ is in some cases a bulky cone, the leaves of which are separated from each other, and from the common axis, by spaces into which the pollen may drop. In others it is a much-divided or plumose stigma, often furnished with hairs, so that pollen falling on it may be readily retained. The method however is a wasteful one and involves the production of a superabundance of pollen. On the other hand anemophilous flowers are always inconspicuous and of a comparatively humble type.

Flowers which are pollinated by insects are usually much larger and more showy, not infrequently possessing irregular corollas, and are often very highly coloured and provided with characteristic odours. Their perianths, and sometimes their sporophylls, are highly modified to adapt them to the habits of their insect visitors. As a further attraction to the latter they usually produce honey in some part of the flower, in such a situation as will lead to the removal of pollen by the insect in its search for the attractive liquid. The markings on the coloured perianth leaves are often arranged in such a way as to direct the insect towards the spot where the honey is concealed. The pollen itself also is often the object of the insect's visit. Many special mechanisms to secure the removal of the pollen from the microsporophyll and its deposition on the stigma of another flower are to be met with; indeed almost every Natural Order shows some modification of the structure of the flower in this direction. The consideration of them in detail, however, is beyond our present purpose.

Something akin to cross-pollination occurs in one of the Hydropterideæ, a family of Ferns with an aquatic habit. The plant in question, which is known as Azolla, is a small floating organism, consisting of a horizontal rhizome, sometimes copiously branched, on which are borne numerous very small leaves, which are partially submerged. It bears two kinds of spore, each produced in sporangia, which occur 
in definite groups or sori. There are numerous microspores in each microsporangium, which, when mature, are agglutinated together in masses. The contents of a sporangium usually exhibit two to eight of such masses, each of them being known as a massula. These are set free separately. A delicate skin surrounds each massula, and in some species this is furnished with a number of hairs bearing barbed processes or glochidia at their free ends. The mesgasporangium, which is solitary in its sorus, bears only a single megaspore. It is liberated from the sporangium, and is then found to be furnished on its. lower surface with large spongy bodies which are developed from its outer coat, and which serve as floats, enabling it to drift about in the water. The apex of the spore bears a number of delicate filaments extending between the floats. Both spores germinate after liberation, each producing its appropriate gametophyte. The glochidia of a massula of microspores generally catch in the filaments of a megaspore, which may have arisen on a different plant, and the massula thus becomes anchored to the megaspore. The gametophytes are thus brought together, so that the gametes can come into close propinquity to each other.

The mechanical adaptations which have been described are however not the only means we find to secure crosspollination. There are peculiarities connected with what we may call the receptivity of the pistil for any particular pollen. Of these the most generally occurring is dichogamy, or the maturing of the microsporophylls and the megasporophylls of a flower at different times. Two varieties of the condition are met with ; in the first, known as protandry, the stamens with their pollen are mature while the stigma is not sufficiently developed to be pollinated. Examples may be found in the Gentianacece, Onagracer, Campanulacece, Composita, \&c. In Parnassia the receptive surface of the stigma is not even formed until the anthers have discharged their pollen. The second condition is known as protogyny, and is the converse of the first, the stigma withering before 
the pollen is mature. This condition occurs in both anemophilous and entomophilous flowers ; certain of the Plantains (Plantago) and some grasses (Anthoxanthum, \&c.) show it in the former group, as does Scrophularia among the latter.

Something corresponding to dichogamy is found among the Ferns, where the antheridia and archegonia on a prothallium do not mature simultaneously. Cross-fertilisation must consequently be the only form possible. The same peculiarity may be observed among the Mosses.

Another means often observed to secure cross-pollination is diclinism, or the production of the stamens and carpels in different flowers. Diclinous plants may be monocious, where the staminate and pistillate flowers are on the same plant ; dicecious where they are on different plants ; or polygamous where a plant bears flowers with stamens and carpels as well as others which contain only one or the other kind of sporophyll.

The terms 'monœeious' and 'diøcious' are sometimes applied to the Cryptogams, when their sexual organs are upon the same or upon different plants. They then refer, of course, to the gametophytic and not to the sporophytic phase of the life cycle as in the cases just quoted.

Some flowers exhibit a peculiarity of form, which is an adaptation favouring cross-pollination. The plants possess flowers of two kinds, which are specially related to each other. The most familiar instance in our own flora is the common Primrose, which has five stamens and a club-shaped stigma. In some flowers the stigma is placed just in the throat of the corolla, and the stamens some little way down its tube. In the rest of the flowers the positions are reversed. We have here an adaptation to the visiting insect, for when it touches the stamens of a short-styled form, it covers with pollen the part of its body which will come into contact with the stigma of the next long-styled flower it alights upon. Another portion of its body will be dusted with the pollen from the latter, which will be suitably placed to be deposited upon the stigma of the 
next short-styled form it may visit. The best seeds are produced when each stigma is supplied with pollen from stamens occupying a corresponding position to itself. This method of cross-pollination is not thoroughly effective, as the insect after a short time will be carrying pollen from stamens of both lengths, having visited several flowers of both kinds. The size of the pollen grains in each case is, however, correlated with the features of the corresponding stigmatic surface, which helps to secure the most advantageous result.

This arrangement is termed heterostylism or dimorphism, of which however it is only one form. Lythrum Salicaria is trimorphic, bearing two sets of stamens of different lengths, and a style which differs from both. There are three modes of arrangement of these organs, and as in the Primrose, the most serviceable pollination is that which takes place when pollen from a stamen of a particular length is applied to a stigma of the same length.

Other arrangements are physiological rather than structural. Of these the strangest is what is called prepotency. When a stigma of a flower exhibiting this property is pollinated by pollen from its own stamens, and at the same time by pollen taken from another flower, the latter is always the originator of the gamete by which fertilisation is effected. Some flowers show self-sterility, that is fertilisation never takes place if they are only pollinated by pollen from their own stamens; in some few cases their own pollen acts as a poison to them.

Though cross-pollination is generally most advantageous it is not universal. Self-pollination occurs in many plants ; in some indeed, special means have been developed to secure it, either exclusively, or in cases in which crosspollination fails to be effected. Only one of these need here be alluded to ; this is cleistogamy, or the production of special flowers which do not open, in addition to the normal ones. The most conspicuous instances of this are afforded by several species of the genus Viola. In one of 
these flowers the pollen grains often put out their pollen tubes while they are in the sporangia, and the tubes grow towards the stigma, penetrating it and reaching the ovules as in the case of the normal flower, fertilisation resulting in the same way.

The process of pollination is followed in the ordinary course of events by the germination of the microspore or pollen grain. The facts that it grows upon the substratum of the stigmatic surface and that the resulting gametophyte or pollen tube is often of considerable length mark a great difference between it and the gametophytes of the vascular cryptogams. It becomes indeed a parasite feeding upon a host plant during the greater part of its development.

The course of events in the germination of the pollen grain appears to be the following. It at the outset absorbs water from the moist surface of the stigma and swells, its protoplasm becoming generally more granular. It almost simultaneously absorbs such food material as the surface of the stigma can supply, usually some kind of sugar. Most pollen grains contain a certain amount of reserve food material, frequently starch or sugar, or both. The process of absorption is followed by the secretion of enzymes, which can act upon these reserve materials, the most prominent of which are diastase and invertase. The former seems to be the most wide-spread, but the latter is far from uncommon. In some cases both enzymes are developed. The outer coat of the grain then bursts, and the inner one begins to protrude, probably in consequence of the hydrostatic pressure set up by the water that has been absorbed. Usually only one such tube protrudes, though occasionally several are developed. Intra-cellular digestion of the reserve materials follows, and the tube grows at their expense. The increased nutrition is followed by a further increase of the enzymes, which is sometimes preceded by a temporary diminution. This however does not last long, and soon a considerable increase can be observed. In some pollen tubes such as those of 
the Lily, in whose pollen starch granules are abundant, the process of the digestion of the starch can be observed taking place as the granules move along the tube during its elongation. Soon an excretion of the enzyme into the tissues of the style takes place, and the reserve materials which are stored in the style are gradually digested as the tube advances, thus ministering to its nutrition, absorption of the products of the digestion being effected by the tube. The latter in most cases makes its way to the micropyle of the ovule, and by this channel reaches the embryo-sac or megaspore. At this period the latter contains its gametophyte, or prothallium, at the apex of which the oosphere or female gamete occurs. The tip of the pollen tube comes in contact with the wall of the embryo-sac close to the oosphere. It then contains two gametes, which are undifferentiated masses of protoplasm, each with a very large nucleus. The separating walls become deliquescent and are absorbed, and one of the male gametes fuses with the oosphere, forming as before a zygote. The fate of the other male gamete is uncertain; it has generally been held to disintegrate and disappear, but in Lilium Martagon it has recently been seen to fuse with one or both of the polar nuclei which go to make up the definitive nucleus of the embryo-sac. How far this phenomenon is of general occurrence is not known.

In a few cases the pollen tube makes its way to the base of the embryo-sac and burrows through its contents, reaching the oosphere from below. This has been observed particularly in Casuarina and in certain of the forest trees.

A few variations of this process have been observed among the Gymnosperms. The pollen grain in these plants falls upon the micropyle of the ovule, there being no ovary and consequently no stigma. The growth of the tube is slow, sometimes extending over several months. Indeed in some cases the sporangium is detached from the parent plant before it has reached the embryo-sac, from which it is separated by a bulky portion of the nucellus or body of 
the sporangium. In Ginkgo and in a species of Cycas the male gametes are definite antherozoïds, furnished with cilia. In most of the Gymnosperms however this degree of differentiation has not been observed. The character of the female gametophyte has been already described.

Though cross-fertilisation is seen to be most advantageous throughout the vegetable kingdom, it is only possible within certain limits. For a new individual to be produced, the sexual cells taking part in the process must have a certain degree of relationship ; thus the antherozoïd of a moss cannot fertilise the oosphere of a fern. The most favourable degree of relationship is that the two gametes shall be produced by different plants of the same species. Such a union results in greater numbers of offspring and in the possession of greater vigour by them. Plants not so closely related may however produce offspring ; thus we may have the union of gametes of plants standing to each other in the relation of varieties of the same species, or very frequently of distinct species belonging to the same genus, or even of species of different genera. Such fertilisation is known as hybridisation.

Hybrids, the offspring of such fertilisation, generally exhibit peculiarities of form and structure intermediate between those of their parents; they are generally fertile with either of the parent species, but not usually so with another hybrid, or to a much smaller extent. When crossed with one of the parent forms the offspring tend to revert to that form.

The immediate result both of pollination and of fertilisation is generally to stimulate the parts concerned to increased growth. In some Orchids the ovules are not formed in the ovary until the stigma is pollinated, and seem to arise in consequence of that process. The stimulus of fertilisation is still more marked. In the Mosses its result is to cause not only the development of the sporophyte from the oosphere, but a considerable additional growth of the archegonium, forming the calyptra. The same 
thing may be noted in those Rhodophyceæ which produce a bulky cystocarp. The stimulus is however most easily observed in the Angiosperms, where it produces effects in several regions of both gametophyte and sporophyte. The oospore is excited to growth, and after a series of celldivisions becomes the embryo, while the definitive nucleus of the embryo-sac similarly inaugurates a series of divisions, ultimately giving rise to the endosperm, and other parts of the ovule undergo modification, so that the seed can shortly be recognised. Parts of the flower also exhibit renewed growth and further development, the carpels especially, though not exclusively, showing an almost coincident enlargement, which often attains considerable dimensions, so that a bulky structure known as the fruit is produced. The new tissue is usually ordinary parenchyma, and in most cases it becomes conspicuously succulent and frequently strongly acid. The attainment of its maximum development is followed by a process technically known as ripening. This may take one of two directions; the tissue may become dry and woody, the cells losing nearly all their water, and their walls becoming converted into lignin. On the other hand the succulence may persist and even increase; in such cases the acidity frequently becomes very much diminished and a considerable quantity of sugar is formed. Other changes in the cells lead to the appearance of various flavouring matters, and often of substances that are aromatic. Fruits thus acquire special characteristics of flavour and fragrance which they do not possess while they are young. The chemical changes which give rise to these peculiarities are very diverse, and cannot be said to be fully understood at present.

We have noticed that the asexual reproductive cell, whether spore or gonidium, is generally found to remain in a state of quiescence for some time after its formation. The same thing is seen, though not so constantly, in the case of the zygote. In the Thallophytes this resting period 
is sometimes a long one: in the higher Cryptogams it is not so noticeable, and in the Phanerogams or Spermaphytes, where the zygote is always developed inside the sporangium, it usually proceeds to active growth almost at once. In the latter plants, however, a resting period takes place later, after the seed is fully formed. The development of the young sporophyte, in fact, takes place in two stages, the one ending with what may be called the ripening of the seed, and the other beginning with the process of germination. Seeds when detached from the parent plant preserve their vitality for a variable length of time, sometimes even for years, and are capable of germinating freely when exposed to favourable conditions.

The germination of the dicotyledonous seed occurs in one of two methods. In the first of these, the cotyledons, which are thick and fleshy, remain underground. When kept warm and moist the seed absorbs water and swells, the radicle makes its way out of the micropyle, the testa bursts, and the plumule makes its way upwards, the epicotyl, or part between the cotyledons and the first foliage leaf or leaves, circumnutating and emerging in the form of an arch, owing to the greater growth of one side. After reaching the air the growth changes, the greatest increase passing to the opposite side, so that the epicotyl straightens itself. During this time it subsists upon the nourishment stored in the cotyledons in the shape of reserve materials. We have already discussed the means whereby these digestive and nutritive changes are brought about, the agencies which effect them, and the various transformations which are met with. As the cotyledons remain underground this process is called hypogean germination. In the other method-that of the so-called epigean germination - the cotyledons sooner or later rise above the ground and become green, the hypocotyl behaving as does the epicotyl in the first case. These are generally, though not always, albuminous seeds, and the nutritive matter is stored outside the embryo. In both cases the root makes 
its way into the soil by virtue of its geotropism and apheliotropism, aided by the movement of circumnutation, and by the adhesion of the root-hairs to particles of the soil.

In some Monocotyledons the upper part of the single cotyledon remains in the seed and absorbs the nutriment from the endosperm, while its base elongates and thrusts the young plant downwards.

Sometimes the usual alternation of sexual and asexual reproduction in the higher plants is interfered with by the substitution of the vegetative method for one of them. In the phenomenon of apospory, noticeable in some Ferns, we have small prothallia developed on the back of the leaves in the place of spores. This is a case of the production of a bud instead of an asexual cell. Apospory is also known to occur among the Mosses.

In the Ferns, again, the sporophyte sometimes arises as a bud or vegetative outgrowth upon the prothallium, a phenomenon known as apogamy.

There is another kind of apogamy known, which is generally termed parthenogenesis. It occurs among the Fungi, where, as in Saprolegnia, oospheres are formed in oogonia, which do not become fertilised, and yet have the power of growing out into new plants. In some species of Mucor which normally exhibit the fusion of particular hyphæ and the admixture of their contents, or gametes, a variation of the process is observed which comes under this category. Instead of two gametangia meeting, and their contents fusing, to form the zygospore, these organs are developed singly and do not coalesce. In this case the fertile cell, which should be a zygote, is produced parthenogenetically in each, and is known as an azygospore. Another variety of parthenogenesis, which resembles the apogamy of the Ferns, occurs in Colebogyne, where an embryo is produced in the embryo-sac but without pollination or fertilisation. No sexual cell is produced, but there occurs a vegetative budding of one or more of the cells of the nucellus of the ovule, the buds growing into the 
cavity of the megaspore and there developing into embryos. This is not quite the same thing as the apogamy of the Fern, as the new sporophyte arises as a bud upon part of the sporangium - that is, upon the parent sporophyte and not upon the gametophyte. It is really a curious variation of vegetative propagation. 



\section{N DEX}

Abnormal methods of food supply, 197

Absorption, of water 22, 69, of food materials 140, of salts 144, of gases 151, of organic food 197; conditions of continuous, 144 ; facilitated by $\mathrm{CO}_{2} 145$, by acid sap 145; strength of salts absorbed, 146; varied amounts of salts absorbed, 146, 147

Acclimatisation, 367

Acer, 87

Aconitum, 438

Ethalium, 9

After-effect of stimulation, 398, 407 Agave, 253

Aggregation, 391, 405

Air-chambers, in Isoëtes, 106 ; in Marsilea, 107 ; in submerged plants, 108; in grasses, 109 ; in leaf of heath, 112

Albumen, 434

Albumin, 175

Albuminates, $\mathbf{1 7 6}$

Albumoses, see Proteoses

Alburnum, 72

Alchemilla, 76, 83

Alcohol, 268, 301

Aleurone grains, 249, 251

Alkaloids, 284

Alternation of generations, 426

Aluminium, 143, 188

Amidated fatty acids, 284

Amido-acids, 180

Ampelopsis, 388

Amygdalin, 252, 267

Anabolism, 137, 272

Anaërobic plants, 129, 304

Anæsthetics, action of, 408

Analysis, destructive, 143

Ananassa, 266

Anemophilous flowers, 438

Antheridia, 424

Antherozoïds, 423 ; motion of, 434 ; attraction of, 435
Anthoceros, 210

Anthocyan, 281, 323

Anthoxanthum, 441

Antipodal cells, 432, 434

Apheliotropism, 378

Aphydrotropism, 392

Apogamy, 448

Apogeotropism, 381

Apospory, 448

Apostrophe, 364

Aquatic plants, structure of, 330

Arabinose, 42

Archegonia, 425

Ascending sap, 66

Ascidia, 201, 202, 203

Ascogonidia, 420

Ascomycetes, 424

Ascospores, 420

Asexual reproductive cells, 420

Ash of plants, composition of, 143, 184 ; effect of its constituents on vegetation, 195

Ash of proteids, 174

Asparagin, 180 ; as a reserve material, 252 ; as a product of digestion, 266

Aspergillus, 264

Asphyxiation, 300

Assimilation, 137, 271 ; (of carbon dioxide, see Photosynthesis)

Auxanometer, 314

Azolla, 210, 439

Azygospore, 448

BACH on photosynthesis, 168

Bacteria, 2, 3

Bacterium termo, relation to oxygen, 170, 392

Bambusa, 29

Bark, 20, 240

Bartsia, 204

Bast, 22, 52 ; function of, 227,230 , 232

Bauhinia, 377 
Bayer on decomposition of carbon dioxide, 165

Begonia, 417

Bentinckia, continuity of proto. plasm in, 16

Benzol, 182

Berberis, 384, 398

Bertholletia, 190

Betula lenta, 268

Biennial plants, 225

Bignonia, 378

Bleeding (of stems), 83, 86

Bloom (of fruits), 50

Bokorvy on action of chlorophyll apparatus, 164

Botrychium, 198

Bromeliacece, 210

Bromelin, 266

Bromine, 143, 188, 194

Brown and Morris on photosynthesis, 167

Bryophyllum, 417

Buckwheat, 186

Budding, 413

Buds, position of leaves in, 315

Bye-products, 275, 283

\section{Cabomba, 336}

Cactacere, 124

Cactus, 128

Calcium, in ash, 143, 188 ; mode of absorption of, 191; effect of, on herbage, 192

Calcium pectate, 46

Calcium, salts of, in cell-wall, 50, 286

Calyptra, 445

Cambium, 238, 309

Cane-sugar, as reserve material, 247 ; digestion of, 264 ; as attraction for antherozoïds, 393

Capillarity, 81

Capparidacea, 260

Carbohydrates, 40, 143 ; formation of, 163 ; theories of construction of, 165 ; course of process of construction of, 226 ; translocation of, 227; resting and travelling forms of, 229 ; storage of, 242

Carbon dioxide, absorption of, 114 , 152 ; exhalation of, 120 ; relation to oxygen absorbed, 125 ; incapable of nourishing protoplasm, 136; temperature at which decomposition by chloroplast begins, 164

Carbon monoxide as a stage in photosynthesis, 165
Carica Papaya, 266

Castor oil plant, see Ricinus

Casuarina, 160, 444

Cell, 2, 3; various applications of the term, 16

Cell-division, 412

Cell-wall, of protoplast, 2 ; properties of, 39 ; of Fungi, 40; theories of its composition, 43,44 ; thickening of, 44 ; stratification of, 44 ; formation of, 276,415

Cellulose, 39 ; properties of, 40 ; varieties of, 40 ; as reserve material, 247, 248

Centrifugal force, influence on direction of growth, 382

Centrospheres, 413

Cephalotus, 203, 204

Ceramium, 16

Chara, 351

Chelidonium, 7

Chemotaxis, 394

Chitin, 40

Chlamydomonas, 349,352

Chlorine, 188, 194

Chlorophyll, 135; condition in chloroplasts, 155; solvents of, 155 ; properties of, 156 ; spectrum of, 157; analyses of, 158; conditions of formation of, 162 ; action of, dependent on light, 163 ; relation to iron, 187 ; secretion of, 280

Chlorophyll apparatus, 155 ; action of, 163 ; absorption of energy by, 292

Chloroplasts, 138, 153, 155 ; distribution of, 159 ; structure of, 161 ; functions of the two components of, 170 ; effect of varying pressures of carbon dioxide on, 171 ; inhibition of, 171

Chlorosis, 163

Chlorotic plants, 281

Chromatin, 413

Chromosomes, 413

Chroococcus, 9

Cilia, 1, 2, 346

Ciliary motion, 346

Circulating food-stuffs, 226

Circumnutation, 316 ; dependent on rhythm, 353

Cladium, 28, 29

Cleistogamy, 442

Cobalt, 188

Cobalt chloride as test for watery vapour, 90

Colebogyne, 417, 448

Cœnocytes, structure of, 10, 11 ; 
skeleton of, 39 ; reproduction of, 416,421

Cold, injurious effects of, 328

Coleochate, 427

Collenchyma, 21, 52

Commensalism, 210

Conducting system, 21

Conjugation, 425

Constructive processes, anabolic, 272 ; katabolic, 274

Contact, stimulus of, 384

Convolvulus, 388

Copper, 143, 188

Cork, 20, 49

Cortex, 240

Crassulacece, 124, 128

Crato on photosynthesis, 168

Crystalloids, 251

Crystals of proteid, 251

Cucumis, 266

Cucurbita, 87

Cupuliferce, 212

Curvature (of stimulated roots), 385

Cuscuta, 219, 343, 389

Cuticle, 48 ; influence on transpiration, 90

Cuticularisation, 20, 48

Cutin, 49, 50

Cutleria, 423

Cycas (antherozoïds of), 436,445

Cyclamen (apheliotropism of peduncles of), 378

Cystocarps, 427

Cystoliths, 51, 286

Cytase, 262, 264

Cytoplasm, 5

DARWriv, on localisation of sensitiveness, 379,382 ; on tendrils, 386 ; on twining stems, 388 ; on Drosera, 391

Darwin, F., and Pertz on induced rhythm, 395

Day and night, influence of alternation of, 372

Delphinium, 438

Dermatosomes, 44

Descending sap, 231

Desmids, 380

Desmodium gyrans, 355, 372

Diageotropism, 381

Diaheliotropism, 378

Diastase, 262 ; function of, 263 ; in pollen, 443

Diaster stage in mitosis, 415

Diatoms, 348

Dichogamy, 440

Diclinism, 441
Digestion, 137 ; by Nepenthes, 203, 260 ; by Lathrea, 204; by Drosophyllum, 205; by Pinguicula, 206; by Dioncea, 208, 260 ; by Drosera, 207, 260; by Bacteria, 209 ; of reserve materials, 256 ; by Fungi, 257

Dimorphism, 442

Diœcious plants, 441

Dioncea, 149, 206, 208, 261, 349, $370,384,391,398,400,403,408$

Dodder, see Cuscuta

Dorsiventral structures, 315

Drosera, 149, 207, 261, 349, 384, 391, 394, 403, 405, 406

Drosophyllum, 205

Duramen, 72

Ectocarpus, 423

Ectoplasm, 5

Egg apparatus, 432

Elaïoplasts, 254, 279

Elodea, 6, 350

Embryo, nutrition of, 133

Embryo-sac, 431

Emulsin, 262, 267

Endodermis, 69

Endosperm, 431

Energy, expenditure of, in photosynthesis, 170, 288; expenditure of, in transpiration, 288, 294; in constructive processes, 288, 294 ; in growth, 289; in movement, 290 ; in production of heat 290 , of light 292; sources of, 292, 322 ; potential and kinetic forms of, 295 ; liberation of, 296 ; distribution of, 297

Engelvian, on evolution of oxygen in different parts of the spectrum, 170 ; on purple bacteria, 172

Entomophilous flowers, 438

Environment, nature of, 371

Enzymes, 138, 258; preparation of, 270 ; secretion of, 275 ; in pollen, 443

Ephedra, 50

Epidermis, characters of, 48

Epinasty, 315

Epiphytes, 210, 340

Epistrophe, 364

Equatorial plate, 414

Equisetacea, 50

Equisetum, sclerenchyma of, 27, 29; air-spaces in, 108; chloroplasts in, $\mathbf{1 6 0}$

Erianthus, 29

ErLenmeyer on photosynthesis, 167 
Erythrophyll, 158

Erythrozym, 262, 267

Etiolation, 361

Etiolin, 162; in photosynthesis, 171,187 ; antecedent of chlorophyll, 280, 361

Enonymus, 162, 280

Euphorbia, 245

EWART on inhibition of chlorophyll apparatus, 171

Exhaustion, 408

Exodermis, 21

Fat, 143 ; origin of, 183 ; as reserve material, 253 ; digestion of, 268 ; secretion of, 279

Fatigue, 408

Fermentation, 302, 326

Fermentative power of protoplasm, 302

Ferments (soluble), see Enzymes ; (microbic), 302

Fern, skeleton of, 25,26

Fertilisation, 425, 437 ; stimulating effect of, 445

Fimbristylis, 27, 29

Flaccidity, 64 ; removal by injecting water 91 , by checking transpiration 96

Flagella, 2

Flowers, opening and closing of, 315,377

Fluorine, 188

Food, its nature, 132 ; formation of, 155 ; conditions of continuous formation of, 236

Food materials, relation to food, 135 ; mode of absorption, 140, 142

Formaldehyde, as stage in photosynthesis, 165,168 ; in construction of proteid, 179

Formic acid, 167

Free-cell formation, 416

Frost; action of, 328

Fruit, formation of, 446

Fucacer, 425

Fungi, composition of cell-walls of, 40 ; constructive powers of, 197 ; digestive powers of, 209 ; haustoria of, 220

Funkia, 253

Fusarium, 264

Gametangia, 423

Gametes, 421

Gametophyte, 426
GarReau on absorption of oxygen by plants, 119

Gaseous interchanges, 103

Gases, mode of absorption of, 103 ; movements of, in plants, 113,114 ; currents of, affected by external conditions, 116; absolption of, 151

Gaultherase, 262, 267

GaUtier on chlorophyll, 158

Gelatin, 173

Gemmæ, 417

Gemmation, 413

Geotropism, 381, 395

Germination, of pollen, 443 ; of seeds, 447

Ginkgo, antherozoïds of, 436,445

Glands, 259

Gliadin, 177, 252

Globoids, 251

Globulin, 175

Glochidia, 440

Glucase, 262, 264

Glucosides as reserve material, 252

Gluten, 251

Glutenin, 177, 252

Glycogen, 246

Gonidangia, 419

Gonidia, 419

Grafting, 220

Grape-sugar as reserve material, 247

Grasses, construction of stem of, 109

Growing-points, 76, 309

Growth, 224, 305; localisation of, $306,309,313$; conditions of, 309 ; course of, 310 ; grand period of, 311,312 ; of leaf, 313 ; daily period of, 314,354

Gums, 51

Haustoria, 215, 219, 220, 343

Health, 359, 366

Heat, liberation of, 290 ; absorption of, 323; conduction of, 326 ; regulation of, 327 ; resistance to, 329

Heaths, rolled leaves of, 339

Hedysarum, 355 ; also see Desmo-

- dium

Helianthus, 91

Heliotropism, 378, 395, 396

Hepatica, 391

Heterospory, 429

Heterostylism, 442

Honey, 439

Hop, 388 
HopPE-SEYLER on chlorophyll, 158, 190

Humus, 67

Hybridisation, 445

Hydrocyanic acid, 181

Hydrogen peroxide in photosynthesis, 168

Hydrophilous flowers, 438

Hydrotropism, 392

Hypnum, 198

Hyponasty, 315

Individual, 411

Insectivorous plants, 149 ; Utricularia, 201; Sarracenia, 202 ; Nepenthes, 203 ; Cephalotus, 203; Bartsia, 204; Lathrae, 204; Drosophyllum, 205; Pinguicula, 206 ; Dioncea, 206, 208 ; Drosera, 207

Intercellular passages, 31, 32 ; formation of, 32, 104 function of, 34, 104; watery vapour in, 74; in Isoëtes, 106, 332 ; in Marsilea, 107, 335; relation to stomata, 111; ratio to cellular tissue in leaves, 113 ; composition of air in, 113,114 ; positive gaseous pressure in, 117 ; in aquatic plants, 330

Intercellular substance, 45

Inulase, 262, 264

Inulin, 246

Invertase, $262, \cdot 264,443$

Iodine, 143, 188, 194

Iron, in ash of plants, 143, 188; combinations absorbed, 150, 192 ; relation to chlorophyll, 163 ; function of, 191

Irritability, 359, 377, 394

Isoëtes, 106, 107, 332, 430

JuMELLE on decomposition of carbon dioxide, 164

Juncus, 28, 29

Kachree gourd, 266

Karyokinesis, 413

Katabolism, 137, 272, 298

Kephir, 211

Klinostat, 381

KNIGHT on action of centrifugal force on the direction of growth, 382

Laccase, 194, 300
Lactase, 264

Latent period of response to stimulation, 407

Lathrcea, 204, 217

Laticiferous systems, 234

Leaves, sclerenchyma of, 27 ; rolled, 112 ; irritability of, 372

Lecithin, 190

Iseguminosa, cotyledons of, 40 ; absorption of nitrogen by, 148, 178,211 ; irritability of leaves of, 372

Lenticels, 34, 76

Leucin, 180

Leucoplasts, 162, 243

Lichens, 210

Light, influence of, on transpiration, 95 ; absorption of, by chlorophyll, 156,292 ; necessary for formation of chlorophyll, 162 ; action in photosynthesis, 163, 170; tonic influence of, 360 ; effect of, on differentiation of leaves, 362 ; effect on growth, 365 ; lateral, 378

Lignin, 47, 50, 285

Lilium Martagon, fertilisation of, 444

Lipase, 262, 268

Lithium, 188

Locomotion, 290, 347, 348

Lycopodium, 40, 198

Lythrum, 442

Macallum on composition of nucleus, 191

Magnesium, in ash, 143, 188; mode of absorption of, 191

Malic acid, attraction for antherozoïds, 393

Maltase, 262

Malto-dextrin, 263

Manganese, 143, 194

Marchantia, photo-epinasty of, 364, 380

Marsilea, 51, 107

Massula of Azolla, 440

Medullary rays, influence of, on transpiration current, 81 ; as storehouses of reserve material, 239

Megaspores, 429

Melibiase, 264

Melizitase, 264

Mesocarpea, 422

Mesocarpus, 358, 364, 403

Metabolism, 6, 137, 272

Metapectic acid, 42

Metapectine, 42 
Methane, 182

Micellæ of cell-wall, 43

Micrococcus Urea, 269

Microspores, 429

Middle lamella, 45

Mimosa, 62, 356, 358, 370, 372, 384, 389

Mimulus, 384

Mistletoe, 213, 343

Mitosis, 413

Mrrosur on chemotaxis, 394

Monoblepharis, 424

Monocious flowers, 441

Monotropa, 213

Moorland plants, 338

Movements, of protoplasm, 6,351 ; for capture, 206, 208 ; of multicellular organs, 349

Mucilage, 51, 248

Mucor, 448

Mycoderma aceti, 270

Mycorhiza, 213

Myriophyllum, 117

Myronate of potash, see Sinigrin

Myrosin, 262, 267

Myxomycetes, 49, 347, 392, 393, 394

NAEgeli, theory of composition of cell-wall, 43

Nectar, 285

Nectary, 62, 439

Negative pressure, in wood-vessels, 89,91 ; influence of, on movements of gases, 116

Neottia, 198

Nepenthes, 149, 203, 261

Nervous mechanisms, 397

Nickel, 188

Nicotiana, 373

Nitella, 6, 351

Nitrates, 143, 193

Nitrification, 148, 171, 172

Nitrogen, absorption of, 113, 147 ; in proteids, 143; fixation of, by leguminous plants $148,178,211$, by Algæ 179

Nostoc, 12

Nuclear spindle, 414

Nuclein, 8, 188, 190

Nucleolus, 8

Nucleoplasm, 8

Nucleus, 5; position in cell, 7; structure of, 8 ; definitive, 432 ; generative, 436

Nutation, 316

Nyctitropic movements, 326, 373, 375,377

Nymphcea, 108, 333
Oil, see Fat

Oncidium, 253

Oogonia, 424

Oospheres, 423

Oospore, 425

Opuntia, 124, 128

Orchidacea, 210

Ornithogalum, 253

Orobanchacece, 343

Orobanche, 217

Orthocarbonic acid in photosynthesis, 168

Osmosis, 55; in adult cells, 60 ; from veins of leaf, 74 ; influence of, on transpiration- current, 100 , 102

Ouvirandra, 332

Ovule, 431

Oxalic acid, 129, 179

Oxalidacea, 372

Oxalis acetosella, 363

Oxidases, 269, 300

Oxidation, 299

Oxygen, mode of absorption by plants, 15, 31, 115 ; influence of, on action of roots, 85 ; proof of absorption of, 119 ; relation of absorption of, to exhalation of carbon dioxide, 125 ; variation in amount of, as affecting respiration, 130

Paonia, 40

Pangium, 181

Papain, 266

Paraheliotropism, 363

Parapectine, 42

Parasites, 135, 341

Parasitism, 198, 210, 214

Paratonic influence of light, 360

Parnassia, 440

Parthenogenesis, 448

Passiflora, 386, 398

Pectase, 262, 265

Pectic acid, 42 ; relation to middle lamella, 46

Pectine, 41

Pectose, 41

Pediastrum, 12

Pelvetia, 18

Pennisetum, 27, 29

Pepsin, 262, 265, 266

Peptone, 176

Perception, 405

Periodicity of root-pressure, 87,357

Perisperm, 434

Pfeffer, on water-culture, 185 ; on localisation of sensitiveness, 382 , 400 
Phajus, 245

Phalaris, 379, 398, 400

Phosphorus, in proteids, 143, 188 ; mode of absorption of, 150 ; associated with nucleus, 189

Photo-epinasty, 364

Photosynthesis, 164; theories of, 165 ; not carried out by fungi, 172

Phototaxis, 380

Phototonus, 359

Pinguicula, 206

Pitcher-plants, see Insectivcrous plants

Planogametes, 422

Plantago, 441

Plasmatic membranes, $6,57,59$

Plasmodium of Myxomycetes, 4, 9, 347

Plasmolysis, 59

Plastids, 5

Polar nuclei, 432, 444

Pollen grain, germination of, 443

Pollen tube, 436

Pollination, 437 ; methods of, 438

Polygamous plants, 441

Polygonum, photo-epinasty of, 364

Polymerisation of aldehydes, 166 , 167

Polytrichum, 26

Porlieria, 392

Positive pressure of gases in intercellular reservoirs, 117

Potamogeton, 31

Potassium, in ash, 143, 188 ; condition of, in soil, 150; mode of absorption of, 190; function of, 191, 192

Potometer, 97

Predominant form of plants in different groups, 428

Prepotency, 442

Primula, 283

Procarpium, 424

Protandry, 440

Proteids, 173; composition of, 174 ; crystals of, 174 ; reactions of, 174 ; coagulated, 177; construction of, 178,182 ; translocation of, 229 , 232 ; storage of, 230

Proteolytic enzymes, 262, 265

Proteoses, 176

Protococcus, 9

Protogyny, 440

Protoplasm, composition of, 6 ; movements of, 6,351 ; properties of, 12 ; continuity of, through cell-walls, 15, 404; regulation of osmosis by, 60,101 ; relation of, to respiration, 127; nutritive sub- stances for, 134; fermentative activity of, 257, 269, 302; permeability of, by water, 352,403

Protoplasts, structure of, 2 ; arrangement of, in multicellular plants, 3

Prunus, 87, 267

Prussic acid, 252

Pseudopodia of Myxomycetes, 4, 347

Pulvinus, 62, 355, 376, 389

Pythium, 424

Radiation, 291, 325

Raffinose, 247

Rafflesia, 219

Raphides, 287

Rectipetality, 319

Reflex action, 405

Rennet, 262, 267

Reproduction, 411 ; relation to growth, 412; vegetative, 417; asexual, 418 ; sexual, 421

Reserve materials, $\mathbf{2 3 6}$

Reservoirs, of air, 106 ; of food, 138, 237

Resin, 282

Respiration, gaseous interchanges of, 121 ; masked by photosynthesis, 122; loss of weight involved in, 122 ; intensity of, 123 ; nature of the process of, 126 ; relation of, to metabolism, 127; influence of external conditions on, 129 ; of seeds, 130 ; a source of energy, 298 ; intramolecular or anaërobic, 301

Respiratory quotient, 124

Rhamnase, 262, 267

Rhamnus, 267

Rhodophycea, 424, 427

Rhythm, of root-pressure, 83 ; nature of, 353 ; affected by stimulation, 395 ; induced, 295

Ricinus, 190, 239

Ripening, 446

Robinia, 62

Root-hairs, 68 ; action of, 69

Root-parasites, 214, 343

Root-pressure, 71, 81, 82 ; rhythm of, 83,87 ; method of measuring, 84,87 ; amount of, 85 ; periodicity of, 87

Rush, construction of stem of, 110

Saccharomyces, see Yeast

SACHS, on theory of transport of water in wood, 79 ; on a method of estimating rate of transpiration current, 81 
Salvinia, 107 ; germination of megaspore of, 429

Santalacea, 214, 343

Saprolegnia, 394, 419, 448

Saprophytes, 135, 198, 341

Sarracenia, 149, 202

Sadssure, De, on Opuntia, 128

Saxifraga, 261, 337

Saxifragacea, 124

Scirpus, 27, 29

Sclerenchyma, 21, 25, 29

Scrophularia, 441

Scrophulariacece, 214, 343

Scutellum, epithelium of, 260, 263

Scyphanthus, 388

Secretion, 275

Seed, 429 ; formation of, 431 ; structure of, 433 , germination of, 447

Selaginella, prothallium of, 430

Selective power, 188, 195

Self-sterility, 442

Sense organs, 400

Sensitiveness, variations of, 379 ; localisation of, $379,382,401$; delicacy of, 386,409 ; maintenance of, 408

Sex, differentiation of, 423

Sexual cells, 421

Sieve-tubes, 182, 232

Silica, 50, 287

Silicates, 150, 193

Silicic acid, 150

Silicon, 143, 150, 188, 193

Sinigrin, 253, 267

Skeleton, of plastid, 2, 37 ; of plant, 25,37

Sleep movements, see Nyctitropic movements

Slime fungi, see Myxomycetes

Sodium, 143, 150, 188, 192

Soil, composition of, 67

Sorus, 440

Spermatium, 435

Spermatozoids, 423

Sphagnum, 19

Spirogyra, 159, 166, 411, 427

Splachnum, 198

Sporangia, 419

Spores, 426

Sporophyte, 426

StahL on method of detecting escape of watery vapour, 90

Starch, appearance of, during photosynthesis, 166 ; probable eause of appearance of, in leaf, 169 ; formation of, as evidence of activity of chlorophyll apparatus, 170 ; deposition of, in chloroplasts, 228,242 ; removal of, from leaves, 238 ; storage of, 242 ; deposition of, by leucoplasts 243 , by protoplasm 245 ; digestion of, 263 ; secretion of, 279

Stele of root, 69

Stimulation, 368 ; nature of, 370 ; purposeful nature of responses to, $370,405,406$; localisation of, 379 , 387 ; after-effects of, 398 ; perception of, 405 ; latent period of, 407 Stimulus, nature of, 368 ; instances of rhythmic, 372,374 ; of lateral light, 378 ; of gravitation, 380 ; of contact, 384,389 ; of moisture, 391 ; of oxygen, 392 ; chemical, 392 ; relationship of response to, 406 ; of fertilisation and pollination, 445

Stomata, 33, 75, 92; number of, in different plants, 94 ; inode of action of, 94 ; regulating influence of, on transpiration, 95 ; relation to gaseous interchanges, 112, 117

Storage of food, 225, 238

Strasborger, on composition of cellwall, 43 ; on evaporation of water from cell-walls, 100

Stratification of cell-wall, 44

Style, reserve materials in, 241

Stylogonidia, 420

Stylospores, 420

Suberin, 285

Sugar, formation of, 166,169 ; fermentation of, 301

Sulphur, in proteids, 143, 188 ; mode of absorption of, 150

Sundew, see Drosera

Sunlight, influence of, on transpiration, 95

Surplus food, construction of, 222

Symbiosis, 198, 210

Synergidæ, 433

Tegumentary system, 19

Telegraph plant, see Desmodium

Temperature, influence of, on rootpressure, 85 ; on transpiration, 96 ; on respiration, 129 ; range of, for different functions, 320 ; fluctuations of, 321

Tendrils, 386

Tensions, in hollow stems, 30 ; in growing organs, 317

Testa of seed, 433

Thalictrum, 438

Thallophytes, 427

Theca, 427

Thermotonus, 360,366 
Thesium, 215, 388

Turriazerf on relative values of the rays of the spectrum in photosynthesis, 171

Tone, $359,364,367$

Torsion of stems, 388

Tradescantia, 7, 351

Translocation, of food, 223 ; direction of, 230 ; path of, 233,234

Transpiration, $75,81,88$; methods of demonstrating, 88; amount of, 91 ; effect of excessive, 91; influence of external conditions on, 95 ; suction of, 100 ; functions of, 324,325 ; relation to etiolation, 368

Transpiration current, 78, 80

Trees, conditions of life of, 24

Trehalase, 264

Treub on construction of proteid in Pangium, 181

Trichogyne, 424

Tropaolacea, 260

Tropcolum, 83, 167

Trypsin, 262, 265, 266

Turgescence, 61 ; importance of, 64 ; methods of restoring, in flaccid tissue, 91; affected by shaking branches, 97

Turgor, 351; also see Turgescence

Twigs, reserve materials in, 241

Twining organs, 386

Typha, 27, 29

Tyrosin, 180

Tyrosinase, 300

Vlothrix, 1, 411, 418, 421, 422

UNGER on relative volumes of air and cellular tissue in leaves, 113

Unicellular plants, 2

Urea, 149

Urease, 269

Utricularia, 199

Vacuole, 4; formation of, 13,56 ; function of, 14 ; occasional absence of, 54 ; pulsating, 348, 352

Vallisneria, 6, 351

Vascular bundles, 22, 27

Vaucheria, 421

Vegetable acids, 284
Vegetative propagation, 417

Veins of leaf, 22, 72

Velamen, 340

Venus's fly-trap, see Dionca

Vicia, 400

Victoria regia, 333

Vines on photosynthesis, 168

Viola, 442

Vitis, 87

Volvox, 9, 10

Water, importance of, to protoplasm 13,14, to plant in general 14 ; absorption of, by aquatic plants, 22; function of, in cell, 54 ; circulation of, in plant, 54 ; exhalation of vapour of, 60,125 ; hygroscopic, 68; absorption by root-hairs, 69 ; transport in root 70 , in stem 72 ; excretion of, 76 ; mode of transport in wood, 79 ; exudation of, under pressure, 83 ; exudation affected by salts, 86

Water-culture, 140, 185

Water-glands, 337

Wax in cell-walls, 50

Wiesner, on composition of cellwall, 44; on heliotropism, 398

Withering, 91

Wood, 22

Xanthophyll, 157, 285

Xerophytes, 336

Yeast, 2, 302, 412, 420

Zein, 177

Zinc, 143, 188

Zirconium, 188

Zoocœnocyte, 421

Zoogonidia, 419

Zoophilous flowers, 438

Zoospores, 1, 419

Zygnema, 159, 422

Zygospore, 422, 425

Zygote, 425

Zymase, 269

Zymogen, 259

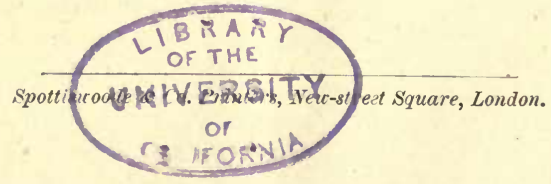




$\therefore$ THIS BOOK IS DUE ON THE LAST DATE STAMPED BELOW

AN INITIAL FINE OF 25 CENTS WILL BE ASSESSED FOR FAILURE TO RETURN THIS BOOK ON THE DATE DUE. THE PENALTY WILL INCREASE TO 50 CENTS ON THE FOURTH DAY AND TO \$1.00 ON THE SEVENTH DAY OVERDUE.

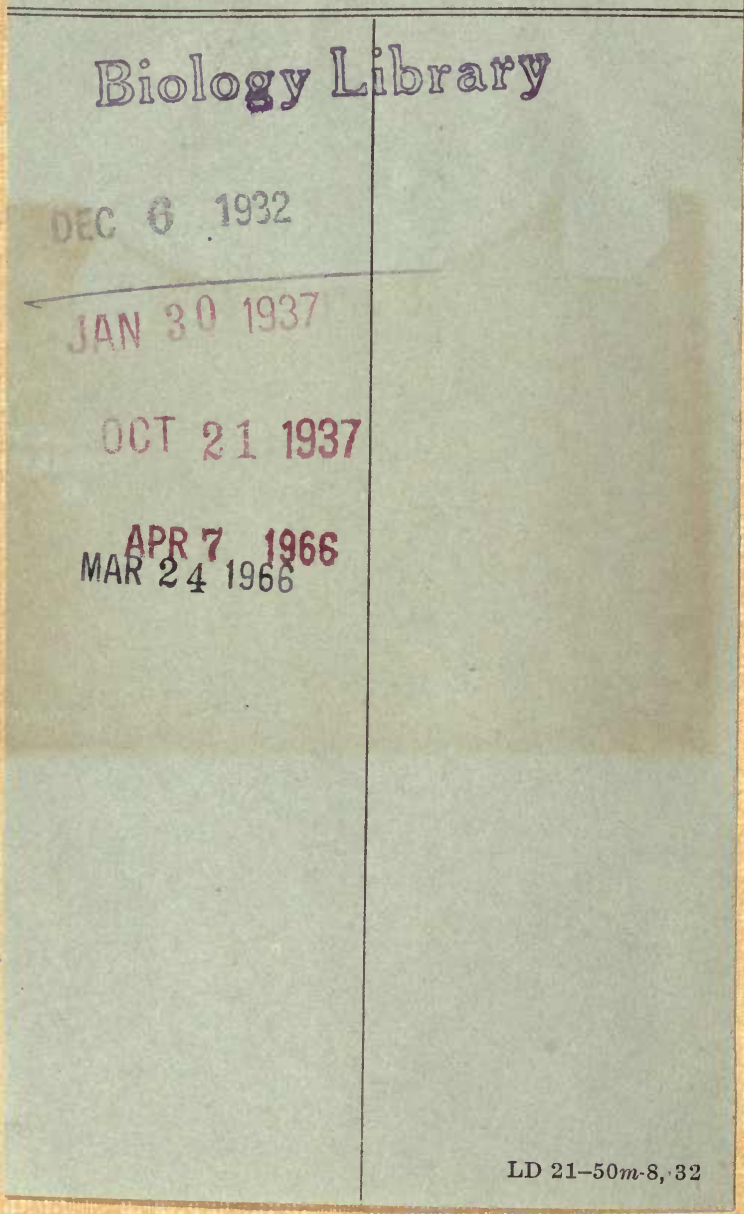




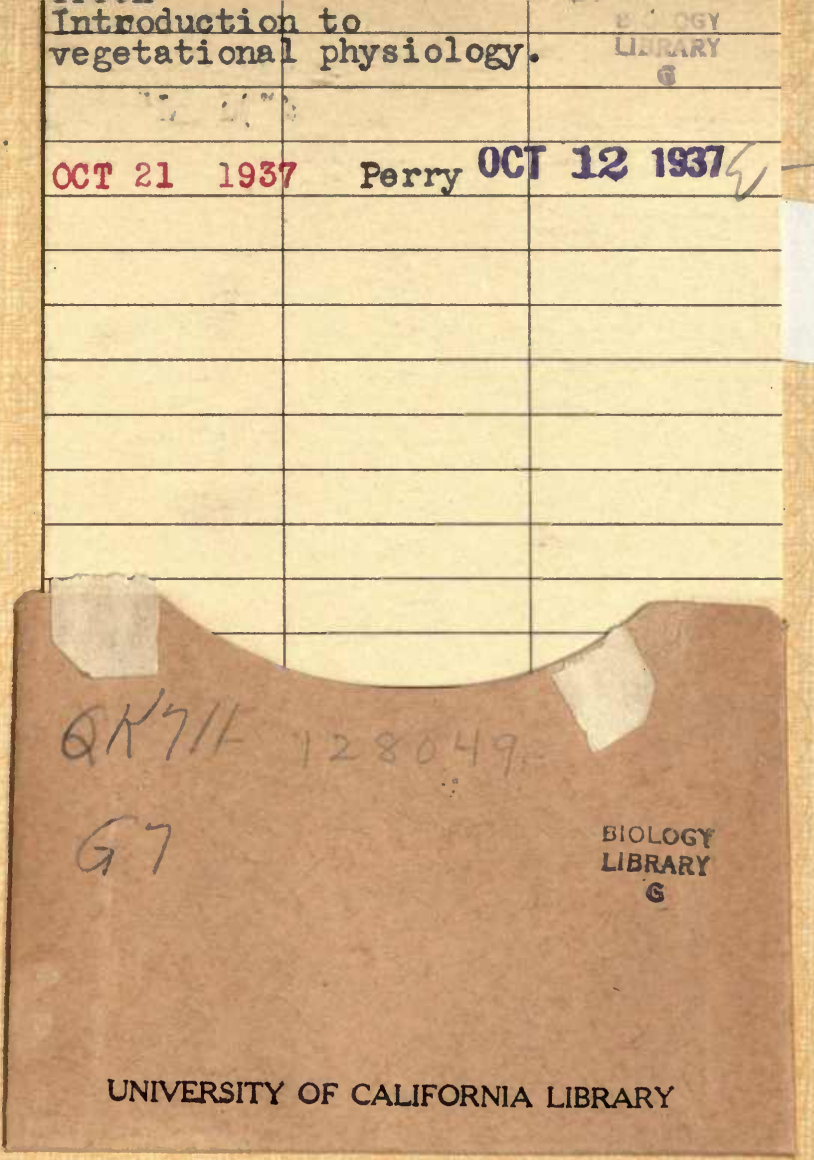




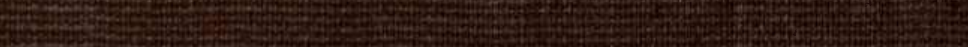

tow

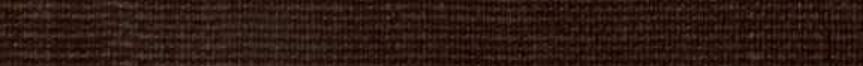

tintes

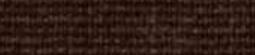

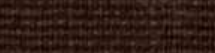

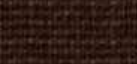

2.

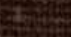

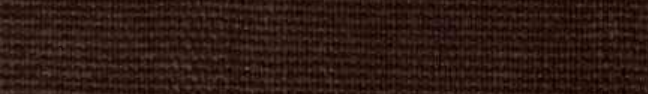

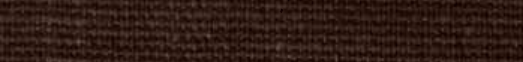

30.

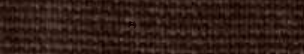

+

610

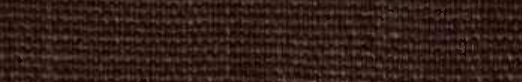

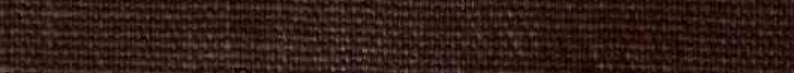

